\title{
The Impact of Stress Shadowing with variations in Cluster Spacing on the Production for Wells Completed in the Marcellus Shale
}

Joshua R. Dietz

jrdietz@mix.wvu.edu

Follow this and additional works at: https://researchrepository.wvu.edu/etd

Part of the Other Engineering Commons

\section{Recommended Citation}

Dietz, Joshua R., "The Impact of Stress Shadowing with variations in Cluster Spacing on the Production for Wells Completed in the Marcellus Shale" (2020). Graduate Theses, Dissertations, and Problem Reports. 7865.

https://researchrepository.wvu.edu/etd/7865

This Thesis is protected by copyright and/or related rights. It has been brought to you by the The Research Repository @ WVU with permission from the rights-holder(s). You are free to use this Thesis in any way that is permitted by the copyright and related rights legislation that applies to your use. For other uses you must obtain permission from the rights-holder(s) directly, unless additional rights are indicated by a Creative Commons license in the record and/ or on the work itself. This Thesis has been accepted for inclusion in WVU Graduate Theses, Dissertations, and Problem Reports collection by an authorized administrator of The Research Repository @ WVU. For more information, please contact researchrepository@mail.wvu.edu. 


\title{
The Impact of Stress Shadowing with variations in Cluster Spacing on the Production for Wells Completed in the Marcellus Shale
}

\author{
Joshua Dietz
}

Thesis Submitted to the

Benjamin M. Statler College of Engineering and Mineral Resources at

West Virginia University

In partial fulfillment of the requirements for the degree of

\author{
Master of Science in \\ Petroleum and Natural Gas Engineering \\ Kashy Aminian, Ph.D., Chair \\ Samuel Ameri, M.S. \\ Ebrahim Fathi, Ph.D. \\ Department of Petroleum and Natural Gas Engineering
}

Morgantown, West Virginia

December 3, 2020

Keywords: Stress Shadowing; Hydraulic Fracturing, Marcellus Shale Copyright 2020 Joshua Dietz 


\begin{abstract}
The Impact of Stress Shadowing on The Production from the Horizontal

Wells Completed in Marcellus Shale
\end{abstract}

\title{
Joshua Dietz
}

The design of the well stimulation treatment has significantly changed over the last decade because of the advancement of hydraulic fracturing. The cluster and stage spacing design component of hydraulic fracturing has been a key factor in enhancing the production from the horizontal wells. Recently, the treatment designs are moving towards lower cluster spacing. The reduction of the spacing alters the in-situ stresses in the formation and begin to impact the propagation of next set of future fractures and the properties of previous fractures. These stress alterations from hydraulic fracturing is referred to as stress shadowing.

The objective of study was to investigate the impact of the cluster spacing and the completion design on the production from a horizontal shale gas well. The available completion, drilling, and reservoir data from a horizontal shale gas well located at Marcellus Shale Energy and Environment Laboratory (MSEEL) in Morgantown, WV was used to develop the base model for this study using GOHFER 3D fracture modeling software coupled with CMG reservoir simulation software. Subsequently, different the cluster spacings and the completion designs were modeled with GOHFER 3D to evaluate the impact that stress shadowing has on fractures properties. The predicted fractures properties were then imported into CMG reservoir simulation to predict the production performance. The results of the study provided a better understanding of the stress shadowing in shale gas reservoirs relative to the cluster spacing of the completion design. 


\section{Table of Contents}

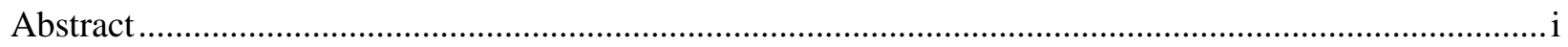

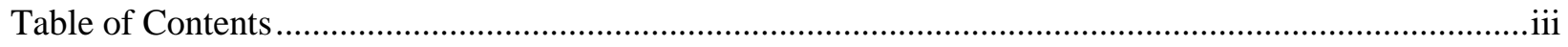

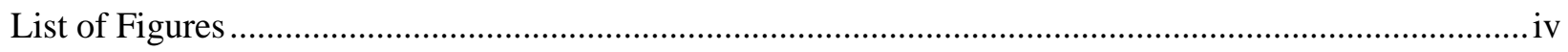

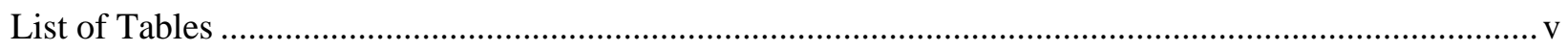

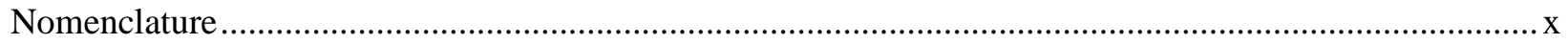

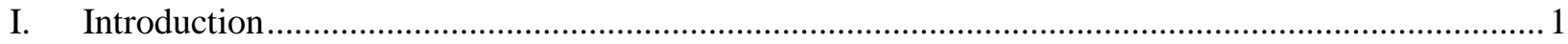

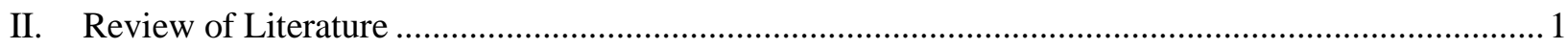

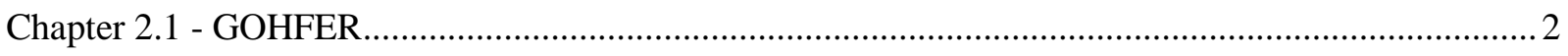

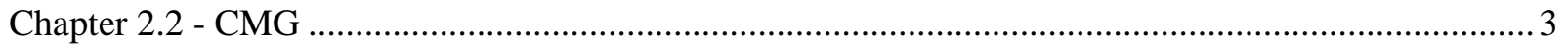

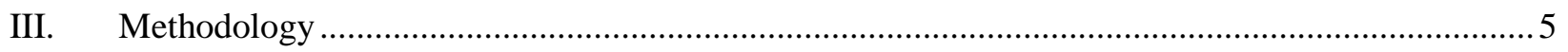

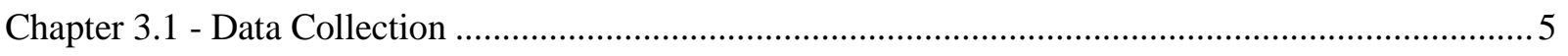

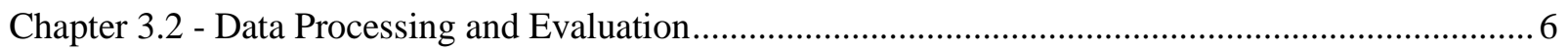

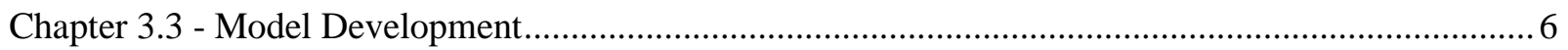

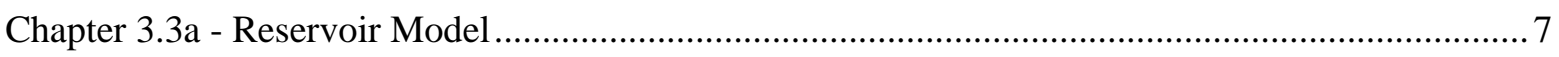

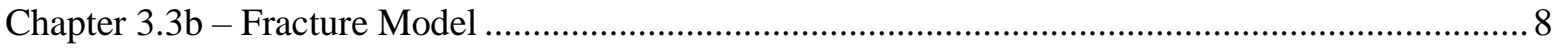

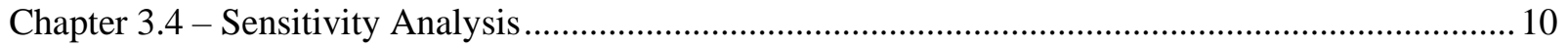

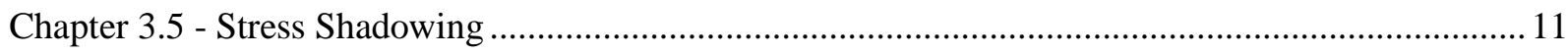

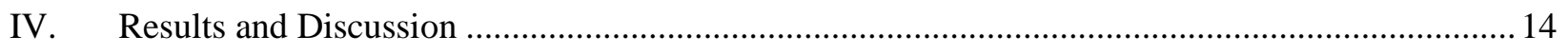

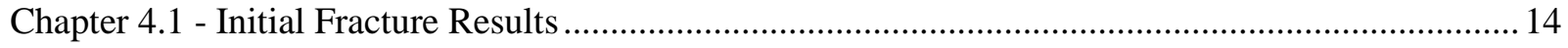

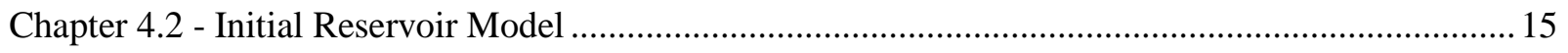

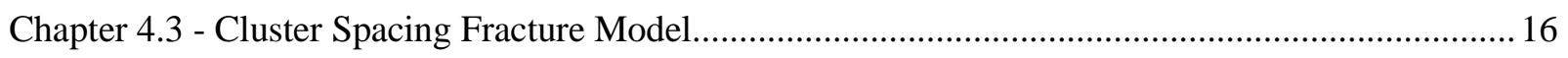

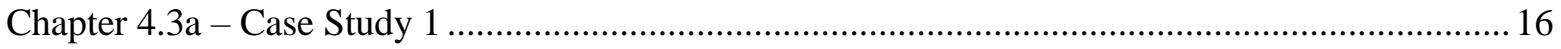

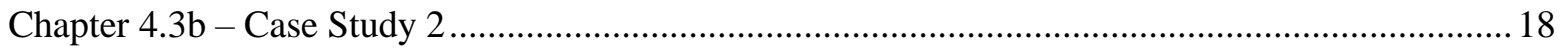

Chapter 4.4 - Cluster Spacing Reservoir Model ............................................................................. 19

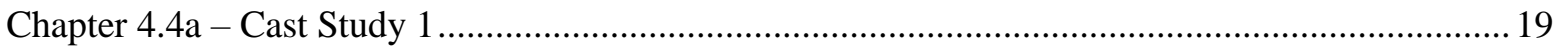

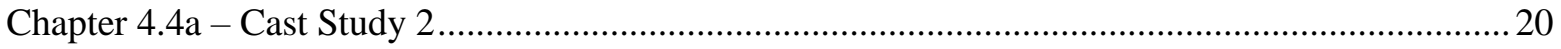

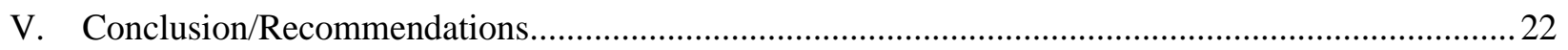

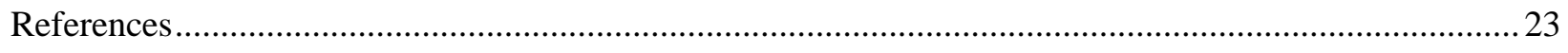

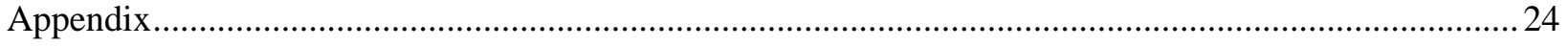




\section{List of Figures}

Figure 1: Relationship between Closure Stress increase and distance away from fracture (stress shadow) as a function of fracture height (Fisher, et al., 2004) .............................................................................. 2

Figure 2: Illustration of "stress shadowing" for multiple fractures (Barree, 2015). .................................2

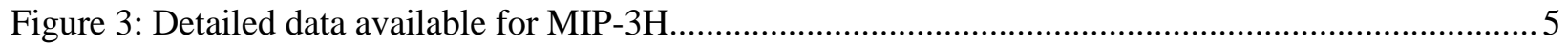

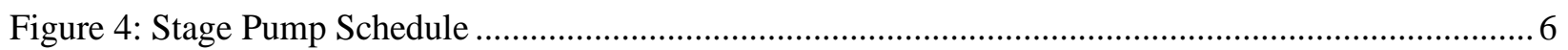

Figure 5: Well Trajectory (MSEEL - Research - MIP Wells, 2018) …................................................. 7

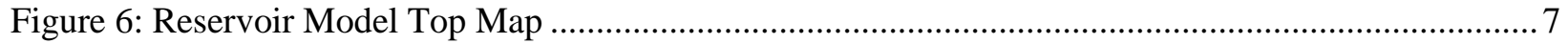

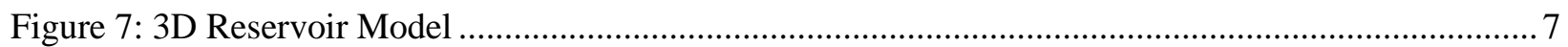

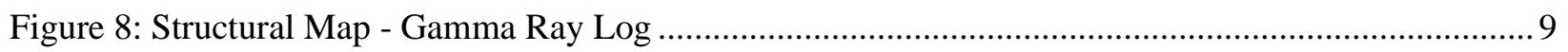

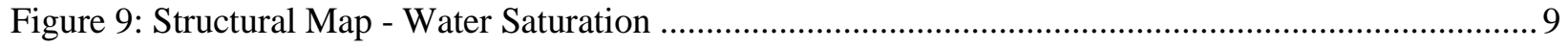

Figure 10: Actual Reservoir Pressure vs CMG Reservoir Pressure ........................................................ 10

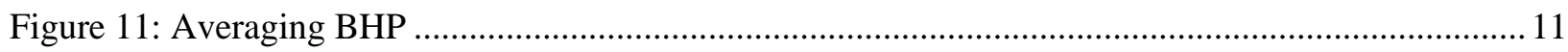

Figure 12: Case Study 1 - 45ft Cluster Spacing Design......................................................................... 12

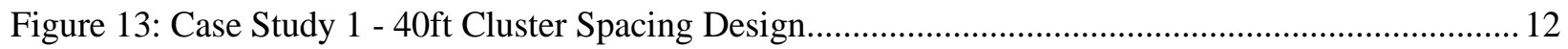

Figure 14: Case Study 1 - 35ft Cluster Spacing Design...................................................................... 12

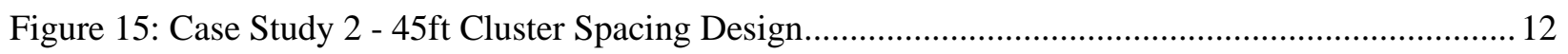

Figure 16: Case Study 2 - 40ft Cluster Spacing Design....................................................................... 13

Figure 17: Case Study 2 - 35ft Cluster Spacing Design....................................................................... 13

Figure 18: Comparison of Average Fracture Width for Base Model...................................................... 14

Figure 19: Comparison of Average Fracture Length for Base Model ................................................... 14

Figure 20: Comparison of Average Fracture Height for Base Model ..................................................... 15

Figure 21: Comparison of Average Proppant Concentration for Base Model .......................................... 15

Figure 22: Production Matching with Pressure Constraint ................................................................... 15

Figure 23: Pressure Matching with Flow Constraint …......................................................................... 16

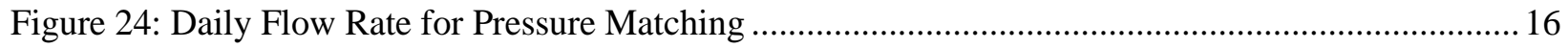

Figure 25: Comparison of Average Fracture Length for Different Cluster Spacings (Stage 1-14) ........... 16

Figure 26: Comparison of Average Fracture Length for Different Cluster Spacings (Stage 15-28) .......... 16

Figure 27: Comparison of Average Fracture Height for Different Cluster Spacings (Stage 1-14)............ 17

Figure 28: Comparison of Average Fracture Height for Different Cluster Spacings (Stage 15-28)........... 17

Figure 29: Comparison of Average Fracture Width for Different Cluster Spacings (Stage 1-14) ............. 17 
Figure 30: Comparison of Average Fracture Width for Different Cluster Spacings (Stage 15-28) ........... 17

Figure 31: Comparison of Average Fracture Length for Different Cluster Spacings (Stage 1-14) ............ 18

Figure 32: Comparison of Average Fracture Length for Different Cluster Spacings (Stage 15-28) .......... 18

Figure 33: Comparison of Average Fracture Height for Different Cluster Spacings (Stage 1-14)............ 18

Figure 34: Comparison of Average Fracture Height for Different Cluster Spacings (Stage 15-28)........... 18

Figure 35: Comparison of Average Fracture Width for Different Cluster Spacings (Stage 1-14) ............. 19

Figure 36: Comparison of Average Fracture Width for Different Cluster Spacings (Stage 15-28) ........... 19

Figure 37: Case Study 1 - Production Results for Different Cluster Spacings ........................................20

Figure 38: Case Study 2 - Production Results for Different Cluster Spacings .....................................21

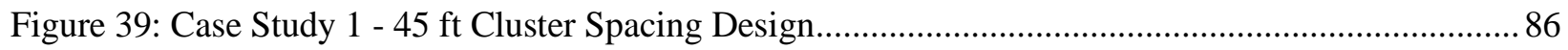

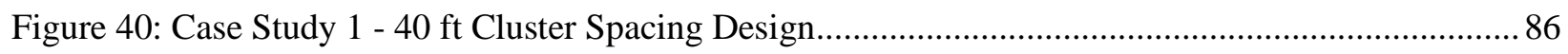

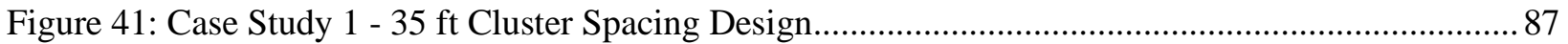

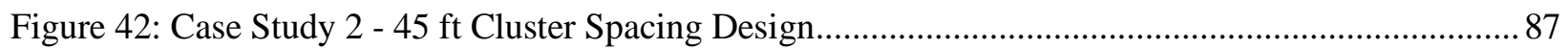

Figure 43: Case Study 2 - 40 ft Cluster Spacing Design.................................................................... 87

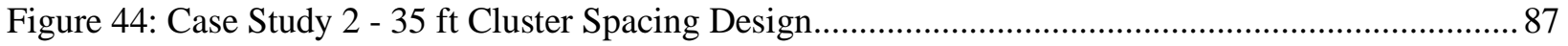

\section{List of Tables}

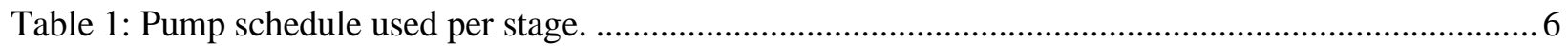

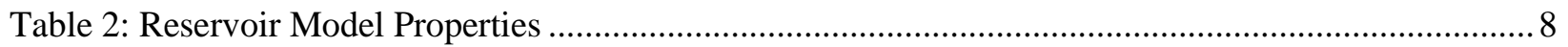

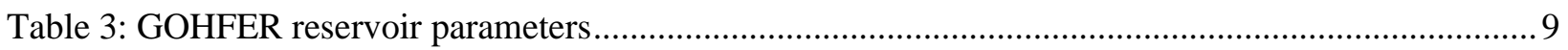

Table 4: Example of perforation placement for a single stage ................................................................ 9

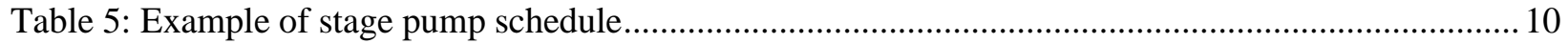

Table 6: Final Reservoir Model Parameter Values ..................................................................................... 11

Table 7: Average Pressures over Production Periods ............................................................................. 12

Table 8: Average Fracture Geometric Properties Comparison Base Models .......................................... 15

Table 9: Case Study 1 - Average Fracture Geometric Properties for Stages ........................................... 18

Table 10: Case Study 2 - Average Fracture Geometric Properties for Stages ......................................... 19

Table 11: Case Study 1 - Detailed Final Production for Different Cluster Spacing Models ......................20

Table 12: Case Study 2 - Detailed Final Production for Different Cluster Spacing Models .....................21

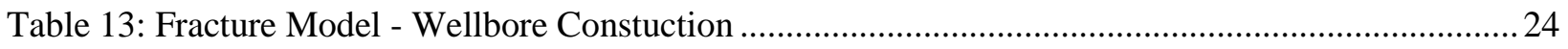

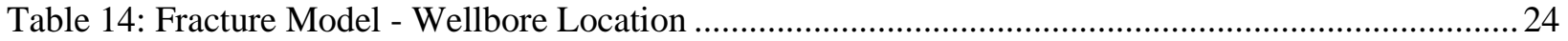




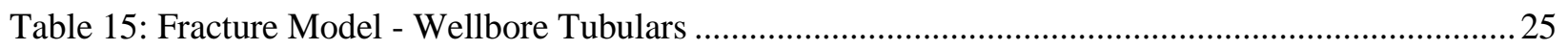

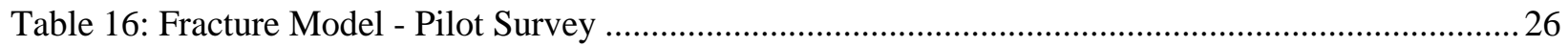

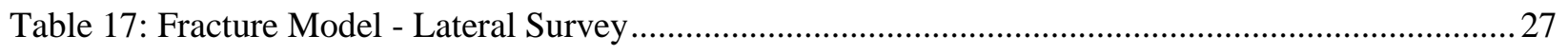

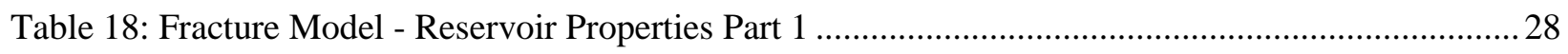

Table 19: Fracture Model - Reservoir Properties Part 2 …..................................................................28

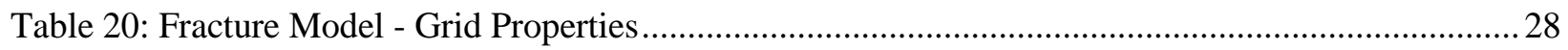

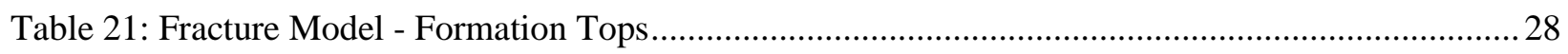

Table 22: Fracture Model - Overview Stage 1 .....................................................................................29

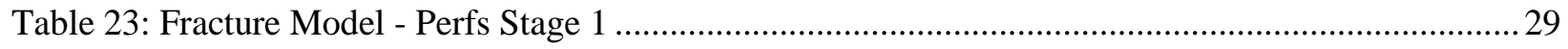

Table 24: Fracture Model - Pump Schedule Stage 1............................................................................2 29

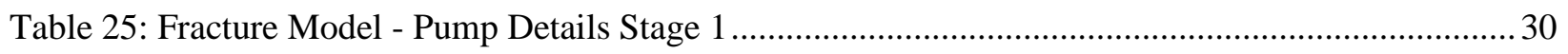

Table 26: Fracture Model - Overview Stage 2 ….................................................................................. 30

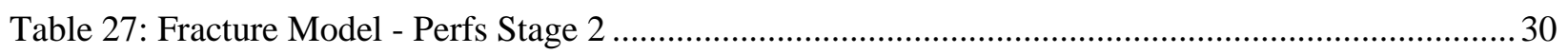

Table 28: Fracture Model - Pump Schedule Stage 2 ............................................................................ 31

Table 29: Fracture Model - Pump Details Stage 2 …............................................................................. 31

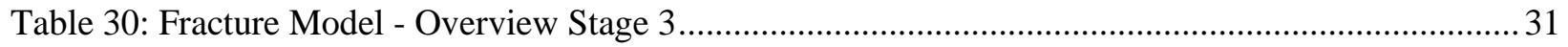

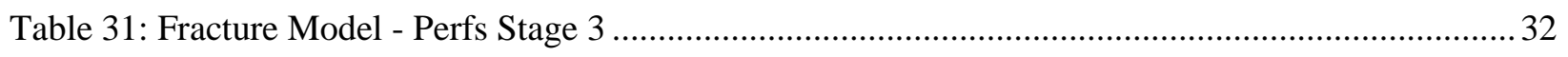

Table 32: Fracture Model - Pump Schedule Stage 3 ........................................................................... 32

Table 33: Fracture Model - Pump Details Stage 3 ….............................................................................. 32

Table 34: Fracture Model - Overview Stage 4 .................................................................................... 33

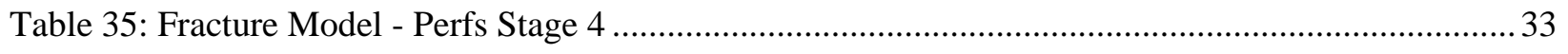

Table 36: Fracture Model - Pump Schedule Stage 4................................................................................ 33

Table 37: Fracture Model - Pump Details Stage 4 …............................................................................ 34

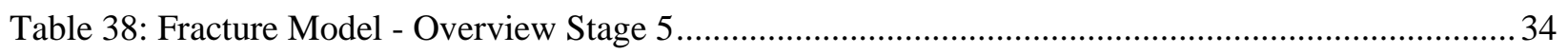

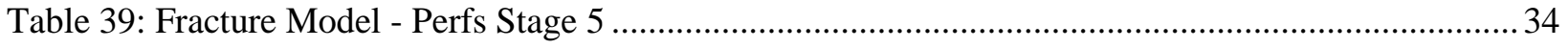

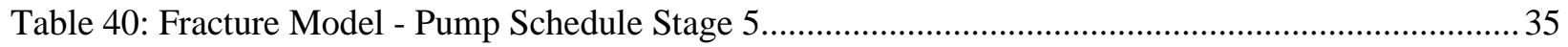

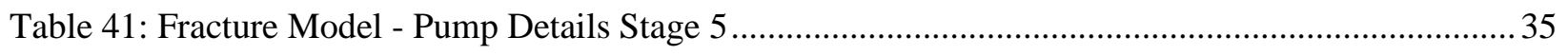

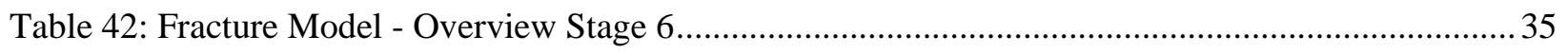

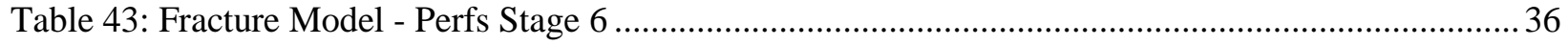

Table 44: Fracture Model - Pump Schedule Stage 6............................................................................. 36

Table 45: Fracture Model - Pump Details Stage 6 ............................................................................. 36 


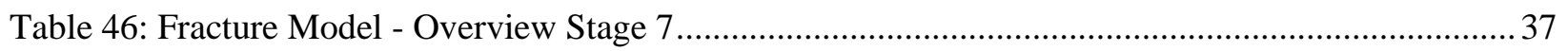

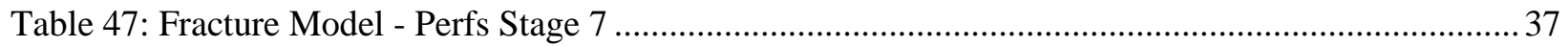

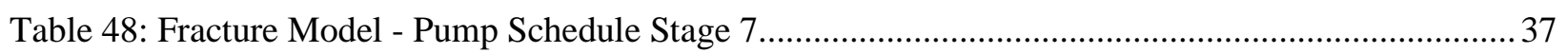

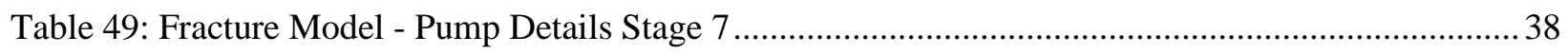

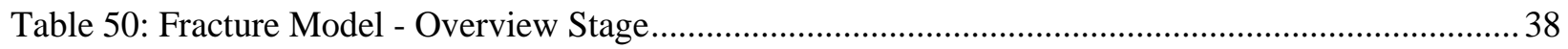

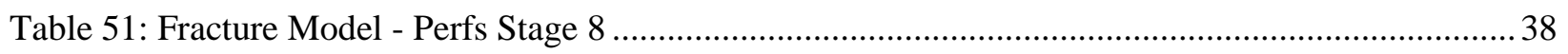

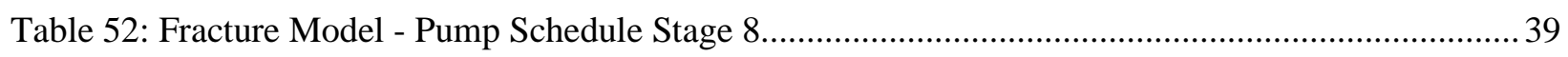

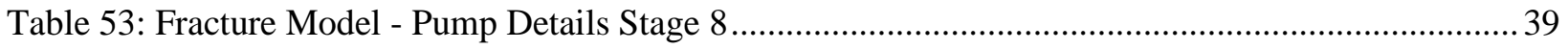

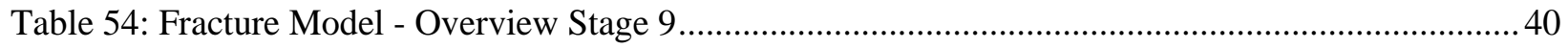

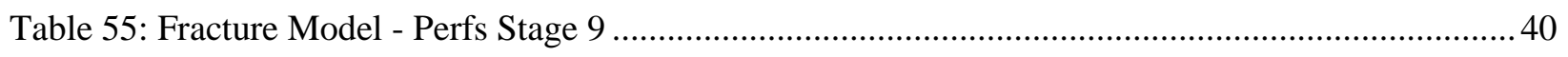

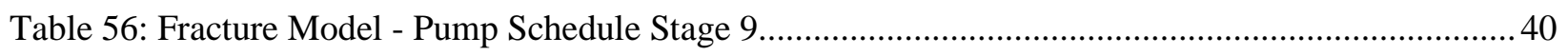

Table 57: Fracture Model - Pump Details Stage 9 …............................................................................. 41

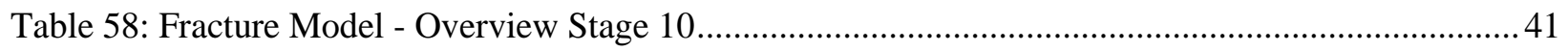

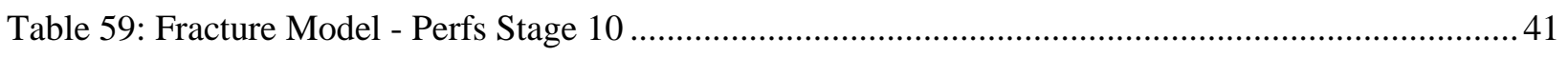

Table 60: Fracture Model - Pump Schedule Stage 10..........................................................................42

Table 61: Fracture Model - Pump Details Stage 10 ................................................................................. 42

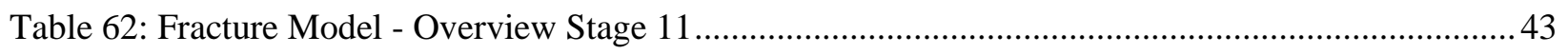

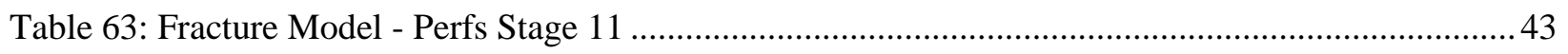

Table 64: Fracture Model - Pump Schedule Stage 11............................................................................ 43

Table 65: Fracture Model - Pump Details Stage 11 …......................................................................... 44

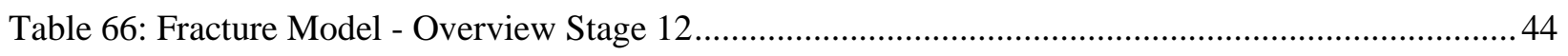

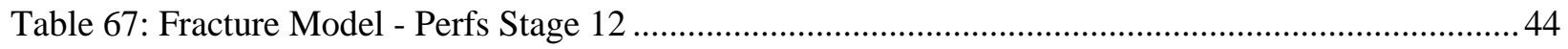

Table 68: Fracture Model - Pump Schedule Stage 12 ........................................................................ 45

Table 69: Fracture Model - Pump Details Stage 12 …......................................................................45

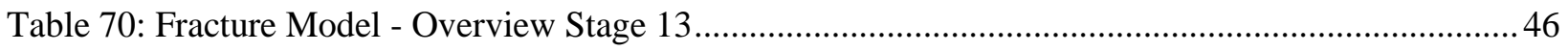

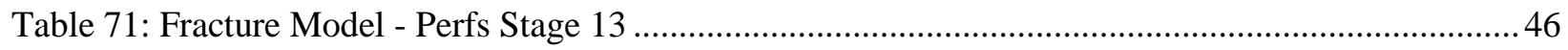

Table 72: Fracture Model - Pump Schedule Stage 13 ......................................................................... 46

Table 73: Fracture Model - Pump Details Stage 13 …...................................................................... 47

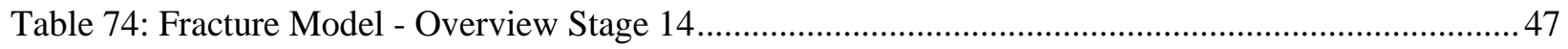

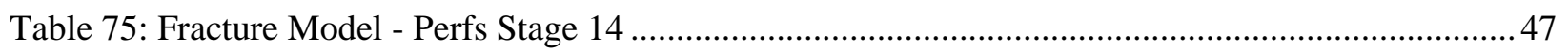

Table 76: Fracture Model - Pump Schedule Stage 14........................................................................ 48 


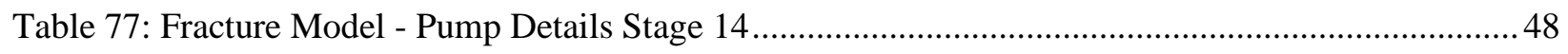

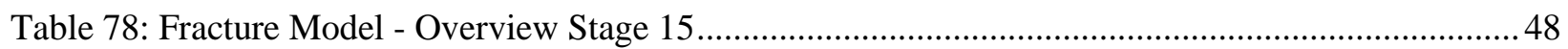

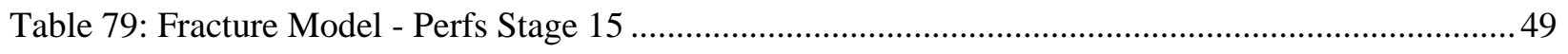

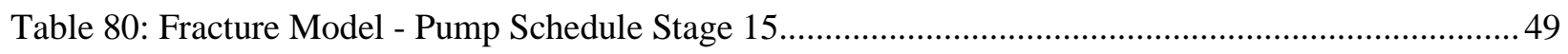

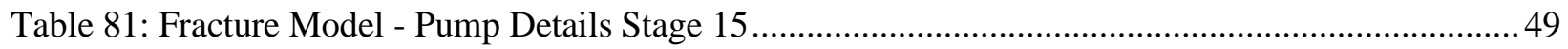

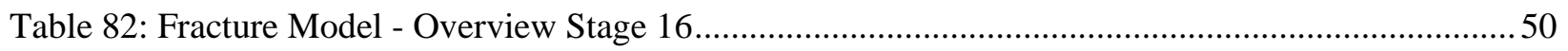

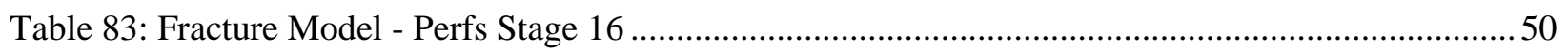

Table 84: Fracture Model - Pump Schedule Stage 16.........................................................................50

Table 85: Fracture Model - Pump Details Stage 16 ...............................................................................51

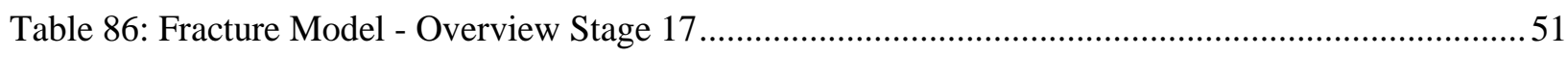

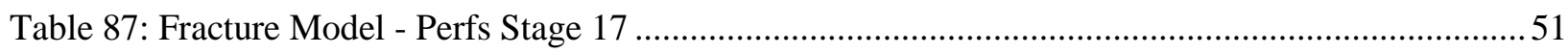

Table 88: Fracture Model - Pump Schedule Stage 17...........................................................................52

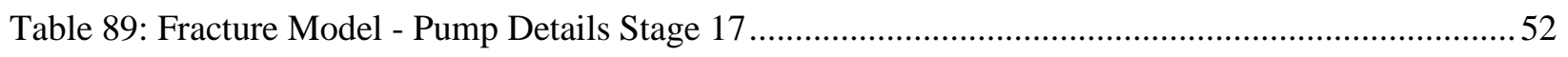

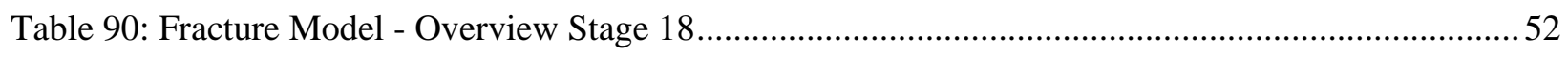

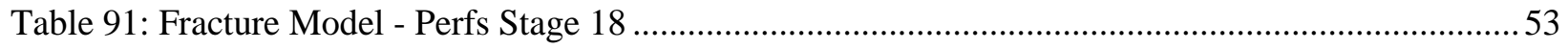

Table 92: Fracture Model - Pump Schedule Stage 18 .............................................................................53

Table 93: Fracture Model - Pump Details Stage 18 ….......................................................................53

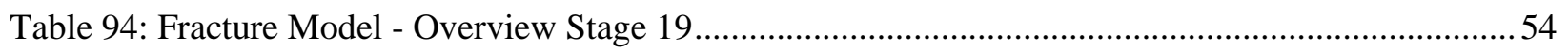

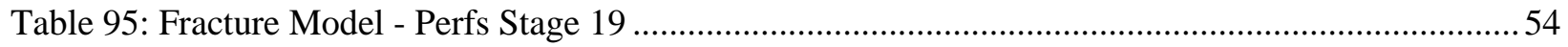

Table 96: Fracture Model - Pump Schedule Stage 19..........................................................................54

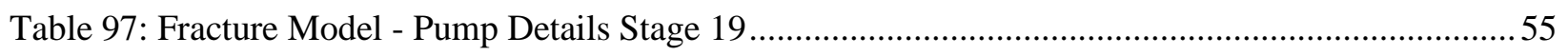

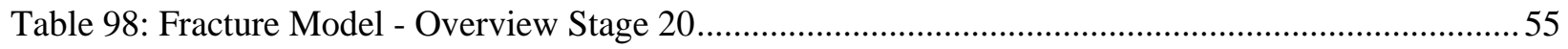

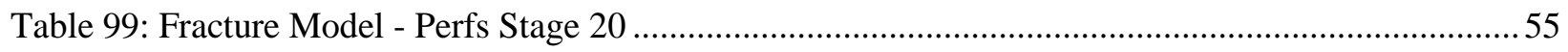

Table 100: Fracture Model - Pump Schedule Stage 20......................................................................56

Table 101: Fracture Model - Pump Details Stage 20 ............................................................................56

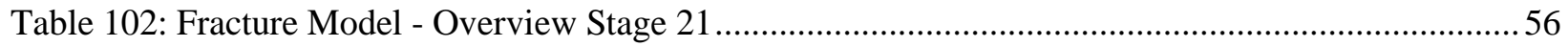

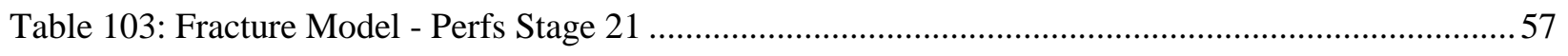

Table 104: Fracture Model - Pump Schedule Stage 21 .........................................................................57

Table 105: Fracture Model - Pump Details Stage 21 …...........................................................................57

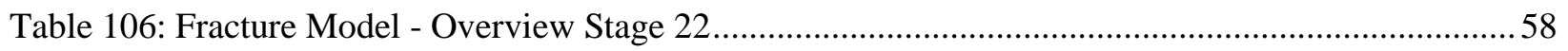

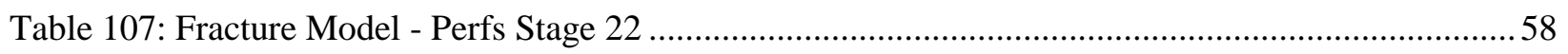


Table 108: Fracture Model - Pump Schedule Stage 22 ........................................................................58

Table 109: Fracture Model - Pump Details Stage 22 ............................................................................59

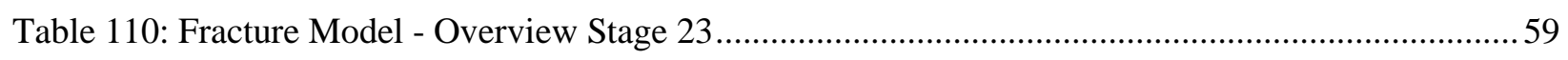

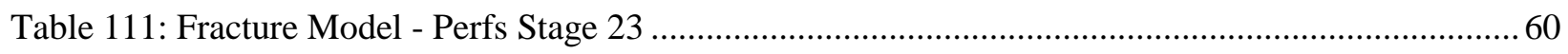

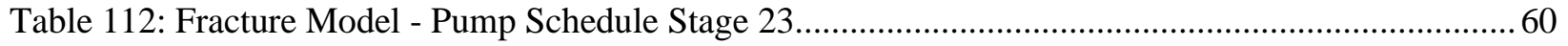

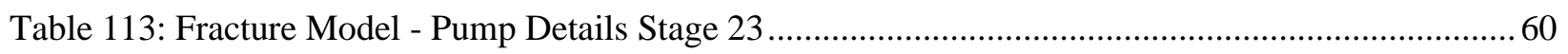

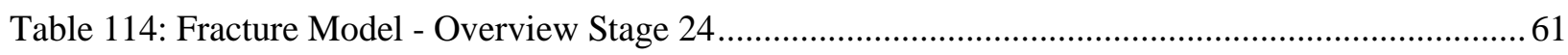

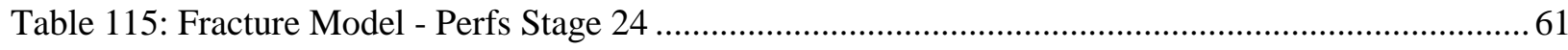

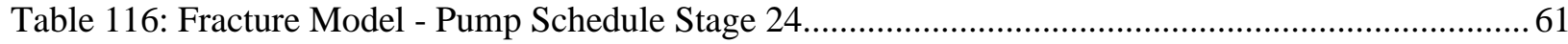

Table 117: Fracture Model - Pump Details Stage 24 …......................................................................62

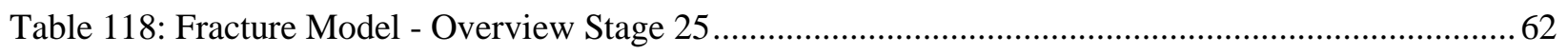

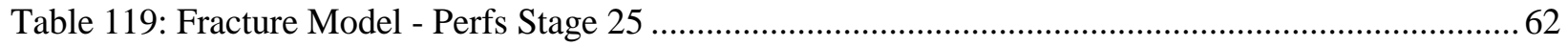

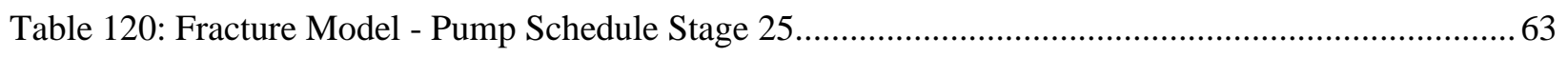

Table 121: Fracture Model - Pump Details Stage 25 …....................................................................... 63

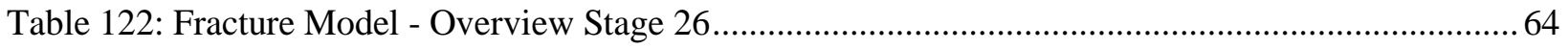

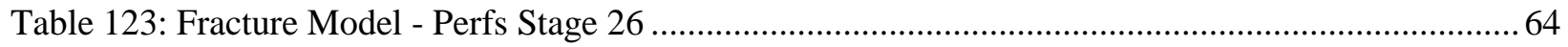

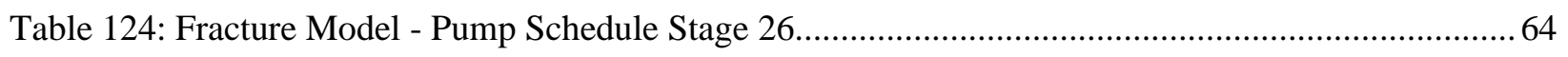

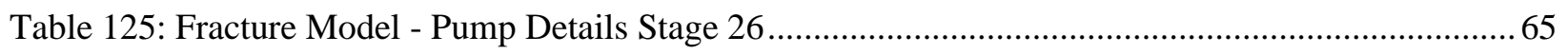

Table 126: Fracture Model - Overview Stage 27 ................................................................................. 65

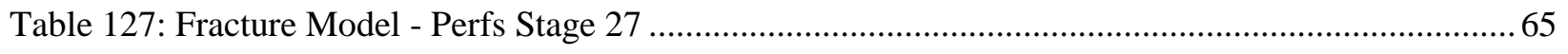

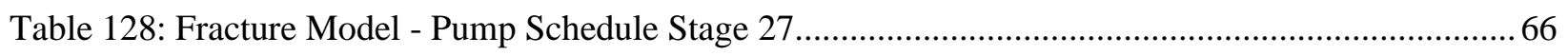

Table 129: Fracture Model - Pump Details Stage 27 ............................................................................. 66

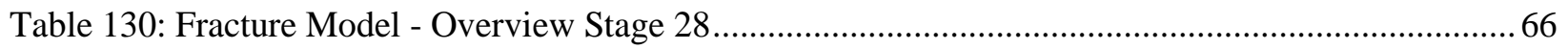

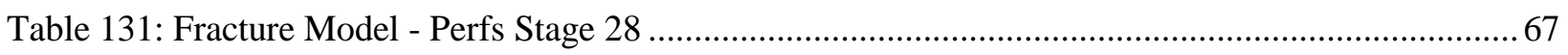

Table 132: Fracture Model - Pump Schedule Stage 28 ….................................................................... 67

Table 133: Fracture Model - Pump Details Stage 28 …......................................................................... 67

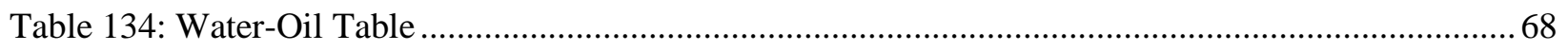

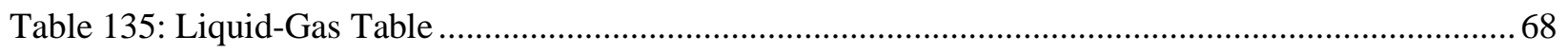

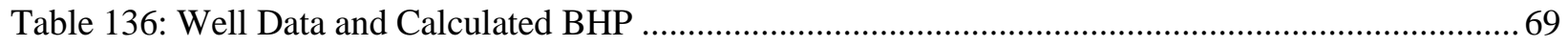

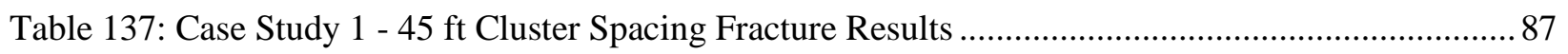

Table 138: Case Study 1 - 40 ft Cluster Spacing Fracture Results ........................................................ 89 
Table 139: Case Study 1 - 35 ft Cluster Spacing Fracture Results ...................................................... 91

Table 140: Case Study 2 - 45 ft Cluster Spacing Fracture Results ........................................................92

Table 141: Case Study 2 - 40 ft Cluster Spacing Fracture Results ........................................................94

Table 142: Case Study 2 - 35 ft Cluster Spacing Fracture Results ......................................................96

Table 143: Case Study 1 - 45 ft Cluster Spacing Average Fracture Results.............................................99

Table 144: Case Study 1 - 40 ft Cluster Spacing Average Fracture Results............................................99

Table 145: Case Study 1 - 35 ft Cluster Spacing Average Fracture Results........................................... 100

Table 146: Case Study 2 - 45 ft Cluster Spacing Average Fracture Results.......................................... 101

Table 147: Case Study 2 - 40 ft Cluster Spacing Average Fracture Results.......................................... 101

Table 148: Case Study 2 - 35 ft Cluster Spacing Average Fracture Results.......................................... 102

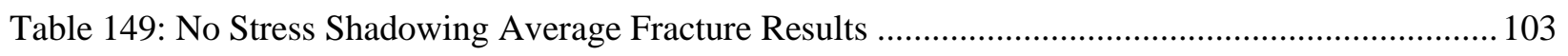

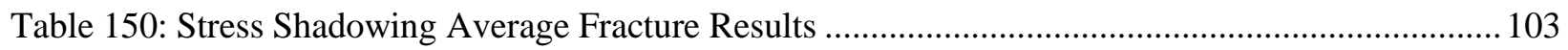

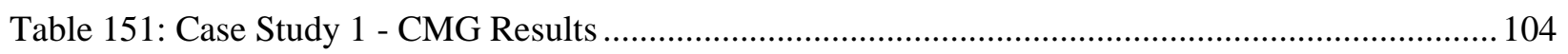

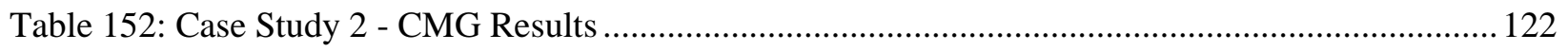

\section{Nomenclature}

$$
\begin{array}{ll}
\gamma_{g} & =\text { Specific Gravity of Gas, frac. } \\
Z & =\text { True Vertical Depth, feet } \\
\bar{Z} & =\text { Average } \text {-factor } \\
\bar{T} & =\text { Average Temperature, }{ }^{\circ} R \\
P_{c} & =\text { closure pressure, } p \text { si } \\
v & =\text { Poisson's Ratio } \\
D_{t v} & =\text { True Vertical Depth, feet } \\
\gamma_{o b} & =\text { Overburden Stress Gradient, psi/ft } \\
\gamma_{p} & =\text { Pore Fluid Gradient, psi/ft } \\
\alpha_{v} & =\text { Vertical Biot's Poroelastic Constant } \\
\alpha_{h} & =\text { Horizontal Biot's Poroelastic Constant } \\
P_{o f f} & =\text { Pore Pressure Offset, psi } \\
\varepsilon_{x} & =\text { Regional Horizontal Strain, microstrains } \\
E & =\text { Young's } \text { Modulus, million psi } \\
\sigma_{t} & =\text { Regional Horizontal Tectonic Stress } \\
D & =\text { depth }
\end{array}
$$




$$
\begin{array}{ll}
f_{i j} & =\text { fugacity of component } i \text { in phase } j \\
F & =\text { function } \\
g & =\text { phase-equilibrium function } \\
n_{b} & =\text { number of gridblocks } \\
n_{c} & =\text { number of components } \\
N_{i} & =\text { moles of component } i \text { per unit block volume } \\
p & =\text { pressure } \\
P_{c o g} & =\text { oil-gas capillary pressure } \\
P_{c w o} & =\text { water-oil capillary pressure } \\
q & =\text { injection/production rate } \\
t & =\text { time } \\
T_{j} & =\text { transmissibility of phase } j \\
V & =\text { gridblock volume } \\
y_{i j} & =\text { mole fraction of component } i \text { in phase } j \\
\gamma & =\text { specific gravity or gravity term in flow equation } \\
\Delta t & =\text { timestep } \\
\rho_{m} & =\text { molar density of phase } \\
\phi & =\text { porosity } \\
\psi & =\text { function }
\end{array}
$$




\section{Introduction}

The recent boom in gas production from the Marcellus shale has led the oil and gas industry into a technology advancement growth. The rapid growth in hydraulic fracturing technology has enabled the ability to drill longer laterals and more perforations. As time goes on, more and more companies are moving to smaller cluster spacing designs to be able to gain access to more of the shale reservoir. With knowing, hydraulic fracturing changes the in-situ stresses of the shale formation when being stimulated, there is a point that these stress changes will start effecting future and/or previous stimulated fractures. This effect that the changes in stresses has on the propagation of future or previous fractures is defined as "stress shadowing."

Through studying literature, there are not many studies conducted on the impact of stress shadowing in shale reservoirs. The potential for stress shadowing to impact the production when reducing cluster spacings is an important issue to investigate. By understanding this problem, potential guidance for cluster spacings could be established which would help maximize production for a well. However, this is a very complex problem that is impacted by many factors. The focus of this study is to look at just the impact that stress shadowing has on the production based on the cluster spacing.

\section{Review of Literature}

The propagation of a fracture that is created during a hydraulic fracture treatment is dependent on the formation stress field. A fracture will always propagate perpendicular to the minimum horizontal stress. During the propagation of a fracture, the local stress field around the fracture will change. This change in stress is also referred to as stress shadowing. More specifically stress shadowing is, when a hydraulic fracture is opened the compressive stress normal to the fracture faces is increased above the initial in-situ stress by an amount equal to the net fracturing pressure. Figure 1 depicts the stress shadow that created by a stimulated hydraulic fracture (Fisher, et al., 2004). 


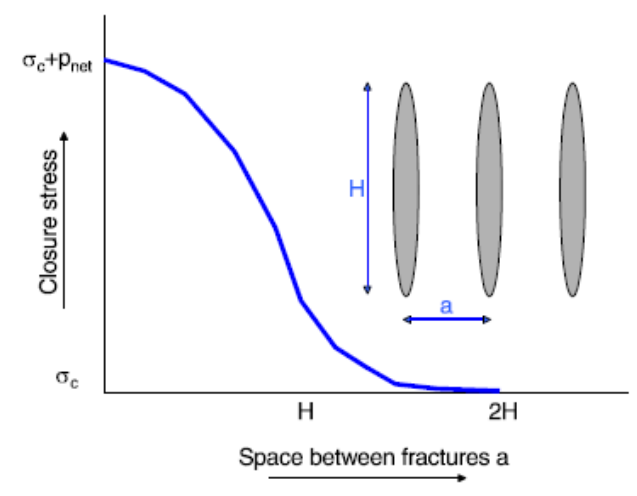

Figure 1: Relationship between Closure Stress increase and distance away from fracture (stress shadow) as a function of fracture height (Fisher, et al., 2004)

Stress shadowing can lead to restrictions on the propagation of future fractions, deviations in future fractures or damage to previously created fractures. Also, for stages with multiple clusters ( 2 or more), the interior fractures see the effects of stress shadowing (Fisher, et al., 2004). This is because of the lower net pressure inside the fracture, which causes a smaller aperture, which generates a lower transmissibility, causing less fluid to enter, and retarding the rate of growth of the interior fractures (Barree, 2015). Figure 2 illustrates stress shadowing when there are multiple fractures in a close proximity.

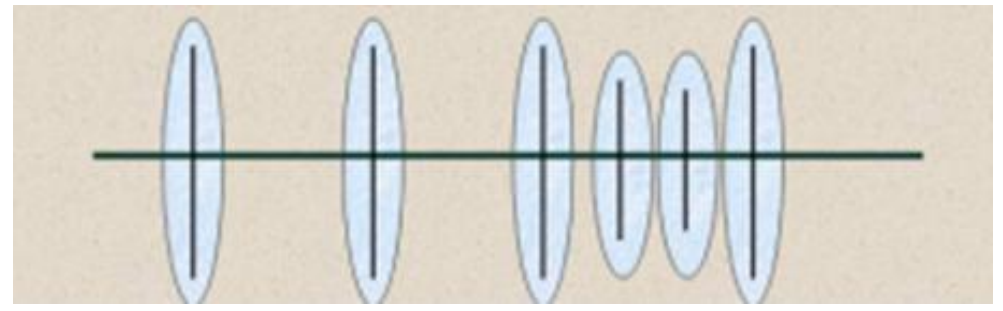

Figure 2: Illustration of "stress shadowing” for multiple fractures (Barree, 2015).

\section{Chapter 2.1 - GOHFER}

The fracture software used for this study was GOHFER (Grid Oriented Hydraulic Fracture Extension Replicator). It is a planar 3-D geometry fracture simulator with a fully coupled fluid/solid transport simulator. The main building blocks of the fracture model comes from well logs to be able to model fracture growth and post-frac production. Depending on the type of fracture model created, either synthetic or actual field well logs are used. GOHFER can directly import log data (LAS files) and has a log analysis tool to help create a lithologic description. The log analysis creates an in-situ stress profile that is internally calculated from pore pressure, poroelasticity, elastic moduli, and geologically consistent boundary conditions (Barree, 2018). 
The way that GOHFER simulates their fracture models is by using a grid system similar to what reservoir models use. The grid system for GOHFER is used for elastic rock displacement calculations and planar finite difference for the fluid flow solutions. Fracture properties such as, fluid composition, proppant concentration, shear, leakoff, width, pressure, viscosity, etc. are defined in each grid block generated for the model created. In-situ stress is one of the primary factors for determining fracture characteristics. When modeling in-situ stress, GOHFER internally calculates in-situ stress from pore pressure, poroelasticity, elastic moduli and geologically consistent boundary conditions. The calculation is completed using Equation 2.1.1 (Barree, 2018).

$$
P_{c}=\frac{v}{1-v}\left[D_{t v}-\alpha_{v}\left(D_{t v} \gamma_{p}+P_{o f f}\right)\right]+\alpha_{h}\left(D_{t v} \gamma_{p}+P_{o f f}\right)+\varepsilon_{x} E+\sigma_{t}
$$

Fracture extension and proppant transport is best explained by the following from GOHFER's software manual:

Fracture extension is based on a smoothly closing tip model and eliminates the fictitious singularity at the tip as well as the stress intensity factor. The fracture extension and deformation model in GOHFER is based on a formulation that expects the formation to fail in shear and be essentially decoupled. Most models assume linear-elastic deformation of a fully coupled rock mass. In shales, the assumption of shear is critical. Along with this GOHFER accounts for pressure dependent leakoff, transverse storage in horizontal and off-angle fracture components, and pressure dependent modulus of the bulk rock mass. All these things happen as the formation is stressed to the shear failure point. The result is higher treating pressures, smaller fracture heights, and more difficulty in placing proppant. The proppant transport model in GOHFER also includes modeling of nonuniform solid and liquid velocities, solid holdup, and variable transmissibility linked to fracture offsets and irregularities. (Barree, 2018, p. 2).

\section{Chapter 2.2- CMG}

The reservoir modeling software used for this study was CMG GEM, which is a multidimensional equationof-state computational simulator. GEM utilizes either the Peng-Robinson or the Soave- Redlich-Kwong equation of state to predict the phase equilibrium compositions and densities of the oil and gas phases, and supports various schemes for computing related properties such as oil and gas viscosities. CMG Builder is used to create the physical reservoir model with creating and importing grids and grid properties, locating wells, importing well production data, importing or creating fluid models, rock-fluid properties, and initial conditions (Builder User Guide: Simulation Model Building Application, 2018).

The principle equations used for CMG GEM, from CMG GEM manual, are defined below (GEM User Guide: Conventional \& Unconventional Simulator, 2018): 
- Flow Equations:

$$
\begin{aligned}
& \begin{array}{l}
\psi_{i} \equiv \Delta T_{o}^{m} y_{i o}^{m}\left(\Delta p^{n+1}-\gamma_{o}^{m} \Delta D\right)+\Delta T_{g}^{m} y_{i g}^{m}\left(\Delta p^{n+1}+\Delta P_{c o g}^{m}-\gamma_{g}^{m} \Delta D\right) \\
\quad+q_{i}^{m}-\frac{V}{\Delta t}\left[N_{i}^{n+1}-N_{i}^{n}\right]=0
\end{array} \\
& \begin{array}{l}
\psi_{n_{c}+1} \equiv \Delta T_{w}^{m}\left(\Delta p^{n+1}+\Delta P_{c w o}^{m}-\gamma_{w}^{m} \Delta D\right)+q_{n_{c}+1}^{m}-\frac{V}{\Delta t}\left[N_{n_{c}+1}^{n+1}-N_{n_{c}+1}^{n}\right]=0 \\
N_{i}=\phi\left(\rho_{o} S_{o} y_{i o}+\rho_{g} S_{g} y_{i g}\right)
\end{array} \\
& N_{n_{c}+1}=\phi \rho_{w} S_{w}
\end{aligned}
$$

- Phase-Equilibrium Equations

$$
\begin{aligned}
& g_{i} \equiv \ln f_{i g}-\ln f_{i o}=0 \\
& N_{i o}=N_{i}-N_{i g}
\end{aligned}
$$

- Saturation Equation

$$
\begin{aligned}
& S_{w}=N_{n_{c}+1} /\left(\phi \rho_{w}\right) \\
& S_{o}=\left(1-S_{w}\right) \frac{N_{o} / \rho_{o}}{N_{o} / \rho_{o}+N_{g} / \rho_{g}} \\
& S_{g}=\left(1-S_{w}\right) \frac{N_{g} / \rho_{g}}{N_{o} / \rho_{o}+N_{g} / \rho_{g}}=1-S_{w}-S_{o}
\end{aligned}
$$

- Mole or Volume Consistency Equations

$$
\psi_{p} \equiv V \frac{\sum_{i=1}^{n_{C}+1} N_{i}^{n+1}}{\left(\rho_{o} S_{o}+\rho_{g} S_{g}+\rho_{w} S_{w}\right)^{n+1}}-V \phi^{n+1}=0
$$

The primary setup in CMG required selecting which simulator to build from and the type of porosity model. The porosity model could be single porosity, dual porosity, dual permeability, subdomain or minc depending on the specified type of model desired. Dual permeability was used for this study. The grid system is the next major component of building the reservoir model. This is because the size of the size and location of the grids is directly related to the transmissibility calculations. The grids being to large will miss critical data and the grids too small with require large computational power. Along with the type of grid selected between cartesian, orthogonal corner point, non-orthogonal corner point, or radial (Builder User Guide: Simulation Model Building Application, 2018). 


\section{Methodology}

\section{Chapter 3.1 - Data Collection}

The data used in this study was gathered from Marcellus Shale Energy and Environment Laboratory (MSEEL). The study started with analyzing and modeling well MIP-6H and MIP-4H but focused on well MIP-3H due to the vast amount of data available in drilling, completions, reservoir engineering and fiber optics. All the data available may be seen in Figure 3.
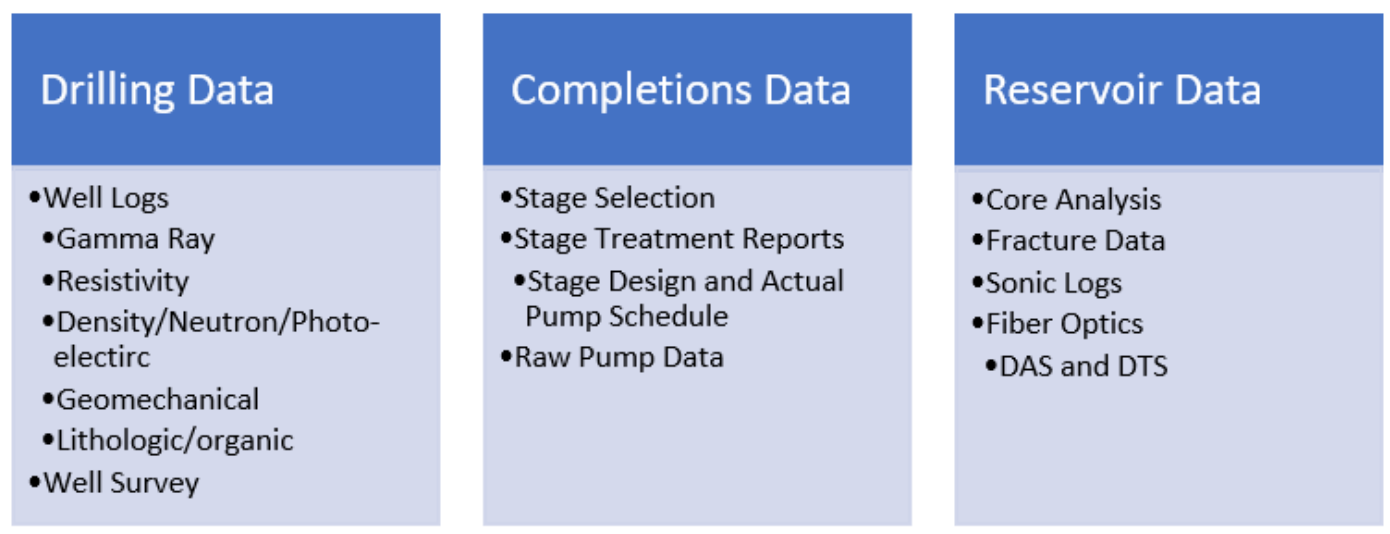

Figure 3: Detailed data available for MIP-3H

For this study, 1,434 days of daily production records were available for MIP-3H. The well was completed with twenty-eight stages and a lateral length of $6,058 \mathrm{ft}$. The stimulation treatment was conducted with an average injection pressure of 8,500 psi. There were four different pump schedules used to stimulate the well. The pump schedule for each stage may be seen in Table 1 and Figure 4.

- $\quad$ Pump schedule A used a slurry rate of $90 \mathrm{bpm}, 8,430$ barrels of slick water, 155,200 pounds of 100-mesh, 284,800 pounds of 40/70-mesh.

- $\quad$ Pump schedule B used a slurry rate of $90 \mathrm{bpm}, 8,184$ barrels of slick water, 330,000 pounds of 100-mesh, 110,000 pounds of 40/70-mesh.

- $\quad$ Pump schedule $C$ used a slurry rate of 72 bpm, 7,090 barrels of slick water, 127,600 pounds of 100-mesh, 232,400 pounds of 40/70-mesh.

- $\quad$ Pump schedule D used a slurry rate of $90 \mathrm{bpm}, 7,424$ barrels of slick water, 155,200 pounds of 100-mesh, 284,800 pounds of 40/70-mesh. 
Table 1: Pump schedule used per stage.

\begin{tabular}{|c|c|c|c|}
\hline $\begin{array}{c}\text { Stage } \\
\text { Number }\end{array}$ & $\begin{array}{c}\text { Pump } \\
\text { Schedule }\end{array}$ & $\begin{array}{c}\text { Stage } \\
\text { Number }\end{array}$ & $\begin{array}{c}\text { Pump } \\
\text { Schedule }\end{array}$ \\
\hline 1 & A & 15 & C \\
\hline 2 & A & 16 & C \\
\hline 3 & A & 17 & C \\
\hline 4 & A & 18 & C \\
\hline 5 & A & 19 & C \\
\hline 6 & A & 20 & D \\
\hline 7 & B & 21 & D \\
\hline 8 & B & 22 & A \\
\hline 9 & B & 23 & A \\
\hline 10 & B & 24 & B \\
\hline 11 & B & 25 & B \\
\hline 12 & B & 26 & B \\
\hline 13 & A & 27 & B \\
\hline 14 & A & 28 & C \\
\hline
\end{tabular}

Pump Schedule A

Pump Schedule B

Pump Schedule C

Pump Schedule D

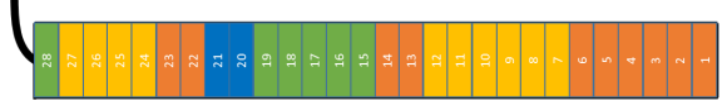

Figure 4: Stage Pump Schedule

\section{Chapter 3.2 - Data Processing and Evaluation}

The data available required processing to be able to use in the for further studying. The main processing occurred with the pump data for the fracture treatment. In order to have a consistent reporting for the stage pump schedule, a python code was generated to process the pump data for each stage and generate a pump schedule based on the proppant and stage times.

\section{Chapter 3.3 - Model Development}

This study required a reservoir model, created through CMG, that would be able to use different fracture model data, generated using GOHFER, to study the impact of stress shadowing with variations in cluster spacing on the well's production.. The first section goes through the full development of the base reservoir model, while the second section goes through the development of the base fracture model. 


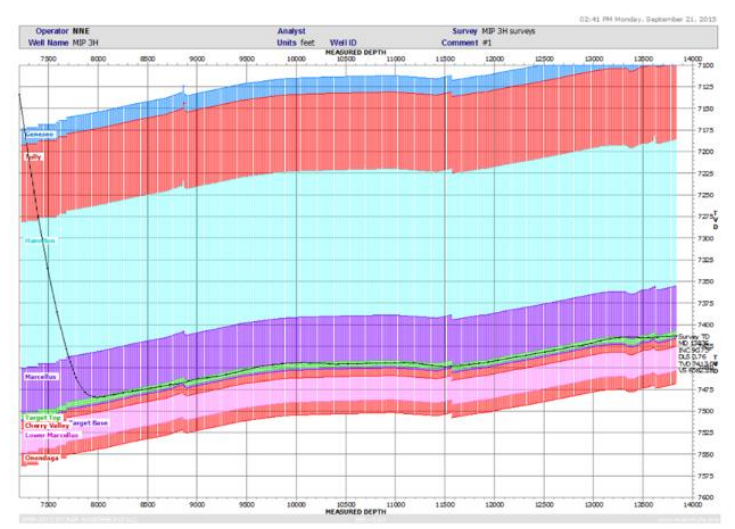

Figure 5: Well Trajectory (MSEEL - Research - MIP Wells, 2018)

Before the reservoir model was designed, a formation top mesh map was created using well trajectory and available geological data. This was first done by using a grid system of 90 x 60 with a grid size of $100 \mathrm{ft}$ x 100ft. and then implementing the formation top depths across the field as seen in Figure 6.

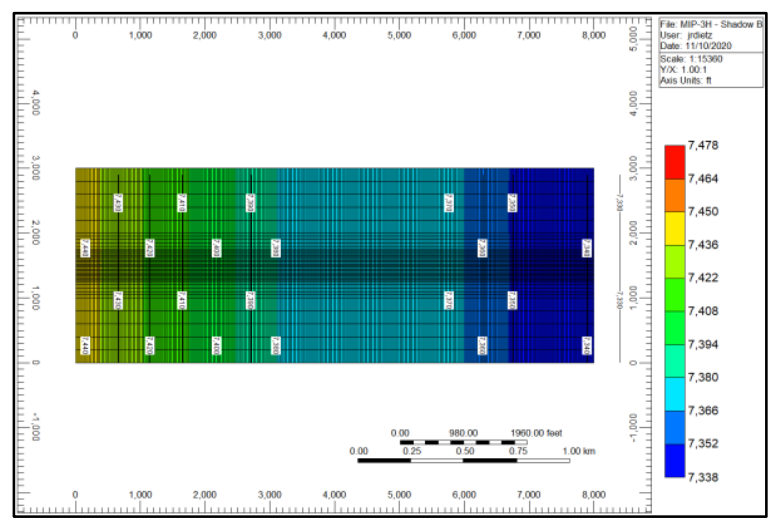

Figure 6: Reservoir Model Top Map

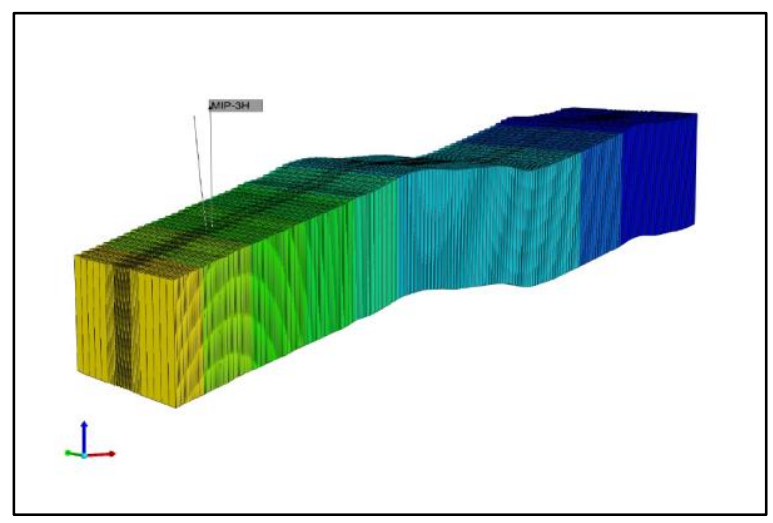

Figure 7: 3D Reservoir Model

The top map was then implemented into the reservoir model with a more refined grid of $400 \mathrm{x} 45 \mathrm{x} 1 \mathrm{using}$ corner point style grids. The refined grid was used to help with reducing the computational processing the reservoir mode would require. The reservoir parameters used for the model are provided in Table 2. 
Table 2: Reservoir Model Properties

\begin{tabular}{|l|c|c|}
\hline \multicolumn{1}{|c|}{ Reservoir Parameters } & Values & Units \\
\hline Model Dimensions (LxWxH) & $8000 \times 3000 \times 90$ & $\mathrm{ft}$. \\
\hline Initial Reservoir Pressure & 4850 & $\mathrm{psi}$ \\
\hline Initial Temperature & 146 & ${ }^{\circ} \mathrm{F}$ \\
\hline Fissure Porosity & 0.0001 & Fraction \\
\hline Matrix Porosity & 0.07 & Fraction \\
\hline Water Saturation & 0.15 & Fraction \\
\hline Fissure Permeability (i,j,k) & $0.001,0.001,0.0001$ & $\mathrm{md}$ \\
\hline Matrix Permeability (i,j,k) & $0.0004,0.0004,0.00004$ & $\mathrm{md}$ \\
\hline Langmuir Pressure & 400 & $\mathrm{psi}$ \\
\hline Langmuir Volume & 0.12 & gmol/lb \\
\hline
\end{tabular}

The well trajectory and well perforations were then imported into CMG for MIP-3H. The well trajectory can be seen and checked in 3D view as seen in Figure 7. The fracture data would be imported into CMG using a .cmg file created by the GOHFER fracture model. To make sure that all the files imported correctly, the well trajectory, well perforations, and hydraulic fractures needed to be manually verified.

The last step in building the reservoir model was to set well constraints. There are many different types of constraints that can be set for the well, such as minimum bottom-hole pressure, maximum flow rate, water cut and more. For this study, the important constraint for this model was setting the well constraint for flow rate. These constraints were used because, the best way to make sure that the model would accurately predict was to set the flow constraint to the real daily production and match the pressure from the model to the actual field pressure. MIP-3H shut-in dates were also added to this model to help build a strong predictive reservoir model.

\section{Chapter 3.3b - Fracture Model}

With all the available data properly gathered and processed, the fracture model for well MIP-3H could be created. The base fracture model created was designed specifically using all the raw data from MIP-3H to enhance the reservoir model's accuracy in predicting production and pressure.

The fracture model development started with constructing a well with a vertical section using pilot surveys and a lateral section using lateral surveys. The different surveys and their respective well logs were then implemented into the software. For this study, eight well logs were used with the pilot section, which included gamma ray, resistivity, density/neutron/photo-electric, geomechanical and lithological/organic well $\operatorname{logs}$ and a gamma ray log was used for the lateral section. Table 3 provides the values used to develop the fracture model, which were gathered from the well log analysis. 
Table 3: GOHFER reservoir parameters

\begin{tabular}{|l|r|c|}
\hline \multicolumn{1}{|c|}{ Reservoir Parameters } & Values & Units \\
\hline Initial Reservoir Pressure & 4850 & $\mathrm{psi}$ \\
\hline Initial Temperature & 146 & ${ }^{\circ} \mathrm{F}$ \\
\hline Matrix Porosity & 0.07 & Fraction \\
\hline Water Saturation & 0.15 & Fraction \\
\hline Gas Saturation & 0.85 & Fraction \\
\hline PDL Coefficient & 0.0002 & $1 / \mathrm{psi}$ \\
\hline Transverse Storage Coefficient & 0.0005 & $1 / \mathrm{psi}$ \\
\hline
\end{tabular}

The formation tops were then implemented into the software and used, along with horizontal well logs, to help created a three-dimensional map for the formations. Gamma ray logs and water saturation logs were used to help create the structural map for the formations as seen in Figure 8 and Figure 9.

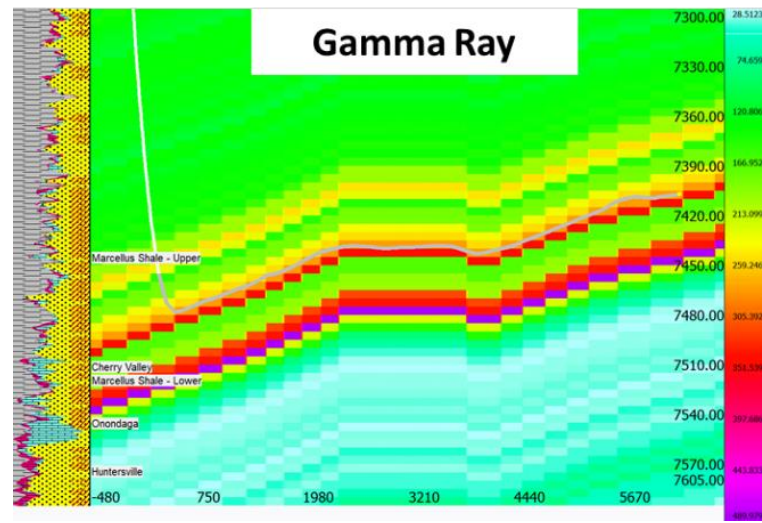

Figure 8: Structural Map - Gamma Ray Log

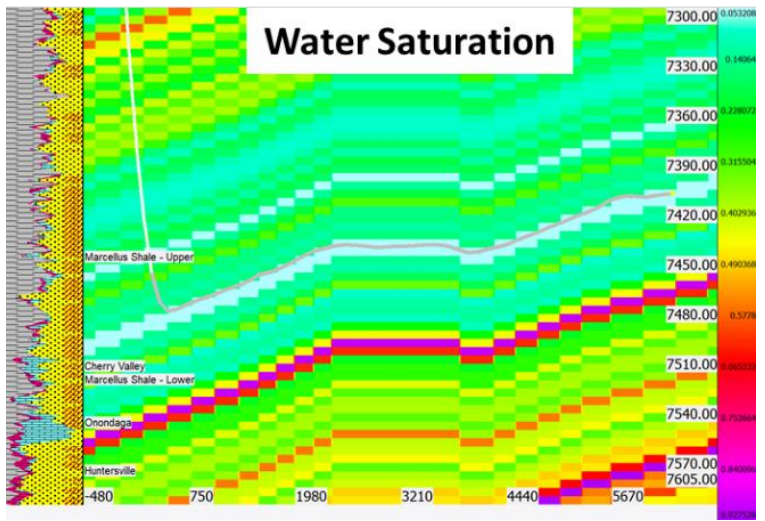

Figure 9: Structural Map - Water Saturation

This led into creating a treatment design for the well using the actual perforation and pumping schedule, with each stage designed separately. Table 4 is an example of a perforation design for a stage and Table 5 is an example of a pump schedule design for a stage.

Table 4: Example of perforation placement for a single stage.

\begin{tabular}{|ccccc|}
\hline \multicolumn{5}{c|}{ Perfs } \\
\hline Cluster & Start.FT & Stop.FT & Perf_Diam.IN & Perf_Shots. \\
\hline 5 & 13625.00 & 13627.00 & 0.42 & 10 \\
4 & 13672.00 & 13674.00 & 0.42 & 10 \\
3 & 13720.00 & 13722.00 & 0.42 & 10 \\
2 & 13767.00 & 13769.00 & 0.42 & 10 \\
1 & 13809.00 & 13811.00 & 0.42 & 10 \\
\hline
\end{tabular}


Table 5: Example of stage pump schedule

\begin{tabular}{|c|c|c|c|c|c|c|c|c|c|c|c|}
\hline Stage & $\begin{array}{l}\text { Tot Time } \\
\text { mm:ss }\end{array}$ & $\begin{array}{l}\text { Stage Time } \\
\mathrm{mm}: \mathrm{ss}\end{array}$ & Fluid & $\begin{array}{l}\text { Clean Stg Vol } \\
\text { (gal) }\end{array}$ & $\begin{array}{l}\text { Cum Cln Vol } \\
\text { (gal) }\end{array}$ & Proppant & $\begin{array}{l}\text { Slurry Conc } \\
\text { (ppa) }\end{array}$ & $\begin{array}{l}\text { Cum Prop } \\
\text { (lbs) }\end{array}$ & $\begin{array}{l}\text { Slurry Rate } \\
\text { (bbl/min) }\end{array}$ & $\begin{array}{l}\text { Cum Slurry } \\
\text { (bbl) }\end{array}$ & $\begin{array}{l}\text { Clean Rate } \\
\text { (bbl/min) }\end{array}$ \\
\hline 1 & 0.00 & 1.41 & SlickWater & 824.60 & 824.60 & $<$ None $>$ & 0.00 & 0.00 & 11.82 & 19.90 & 11.66 \\
\hline 2 & 1.41 & 4.29 & $\mathrm{HCl} 7.5 \%$ & 2999.99 & 3824.59 & $<$ None $>$ & 0.00 & 0.00 & 15.93 & 91.33 & 15.93 \\
\hline 3 & 6.10 & 17.04 & SlickWater & 41986.72 & 45811.30 & $<$ None $>$ & 0.00 & 0.00 & 58.63 & 1092.02 & 58.58 \\
\hline 4 & 23.14 & 4.14 & SlickWater & 13163.08 & 58974.38 & 100 Mesh Sand & 0.24 & 3212.00 & 74.82 & 1408.76 & 74.03 \\
\hline 5 & 27.28 & 5.13 & SlickWater & 14018.61 & 72992.99 & 100 Mesh Sand & 0.49 & 10140.00 & 65.40 & 1749.92 & 63.98 \\
\hline 6 & 32.41 & 6.23 & SlickWater & 18566.17 & 91559.16 & 100 Mesh Sand & 0.74 & 23914.00 & 71.67 & 2207.40 & 69.25 \\
\hline 7 & 39.04 & 6.23 & SlickWater & 23066.84 & 114625.99 & 100 Mesh Sand & 1.00 & 46879.00 & 89.91 & 2781.34 & 86.04 \\
\hline 8 & 45.27 & 9.56 & SlickWater & 35311.51 & 149937.51 & 100 Mesh Sand & 1.50 & 99752.00 & 90.39 & 3679.26 & 84.64 \\
\hline 9 & 55.23 & 8.11 & SlickWater & 28478.54 & 178416.05 & 100 Mesh Sand & 1.99 & 156394.00 & 90.36 & 4418.69 & 82.86 \\
\hline 10 & 63.34 & 6.29 & SlickWater & 23970.17 & 202386.22 & 40/70 White & 0.53 & 169066.00 & 90.13 & 5003.01 & 88.03 \\
\hline 11 & 70.03 & 5.04 & SlickWater & 18531.22 & 220917.44 & 40/70 White & 0.75 & 182897.00 & 90.05 & 5459.26 & 87.08 \\
\hline 12 & 75.07 & 5.09 & SlickWater & 18556.08 & 239473.52 & 40/70 White & 1.00 & 201382.00 & 89.67 & 5921.05 & 85.79 \\
\hline 13 & 80.16 & 10.10 & SlickWater & 36301.45 & 275774.97 & 40/70 White & 1.33 & 249521.00 & 90.14 & 6837.51 & 85.02 \\
\hline 14 & 90.26 & 4.32 & SlickWater & 16455.77 & 292230.74 & $<$ None $>$ & 0.00 & 249521.00 & 86.42 & 7229.26 & 86.43 \\
\hline 15 & 94.58 & 7.00 & Gel (WF110) & 23851.14 & 316081.88 & 40/70 White & 0.49 & 261201.00 & 82.93 & 7809.79 & 81.13 \\
\hline 16 & 101.58 & 5.14 & Gel (WF110) & 17025.18 & 333107.06 & 40/70 White & 0.74 & 273837.00 & 80.08 & 8228.87 & 77.46 \\
\hline 17 & 107.12 & 11.51 & Gel (WF110) & 38269.91 & 371376.97 & 40/70 White & 1.00 & 312035.00 & 80.39 & 9181.51 & 76.89 \\
\hline 18 & 119.03 & 7.44 & Gel (WF110) & 24431.64 & 395808.61 & 40/70 White & 1.49 & 348473.00 & 80.34 & 9802.81 & 75.22 \\
\hline 19 & 126.47 & 12.41 & Gel (WF110) & 39455.71 & 435264.32 & 40/70 White & 1.89 & 423076.00 & 80.44 & 10823.05 & 74.07 \\
\hline 20 & 139.28 & 4.02 & SlickWater & 13021.04 & 448285.36 & $<$ None $>$ & 0.00 & 423076.00 & 76.56 & 11131.83 & 76.87 \\
\hline
\end{tabular}

The model was then processed, and the results provided the fracture half-length based on proppant concentration and fracture conductivity. These results were imported into a reservoir model created using CMG.

\section{Chapter 3.4-Sensitivity Analysis}

The actual field pressure was calculated using daily wellhead pressure and production data available in SCADA. Utilizing this data in Equation 3.4.1, the reservoir pressure could be calculated and compared to the reservoir pressure generated from $\mathrm{CMG}$.

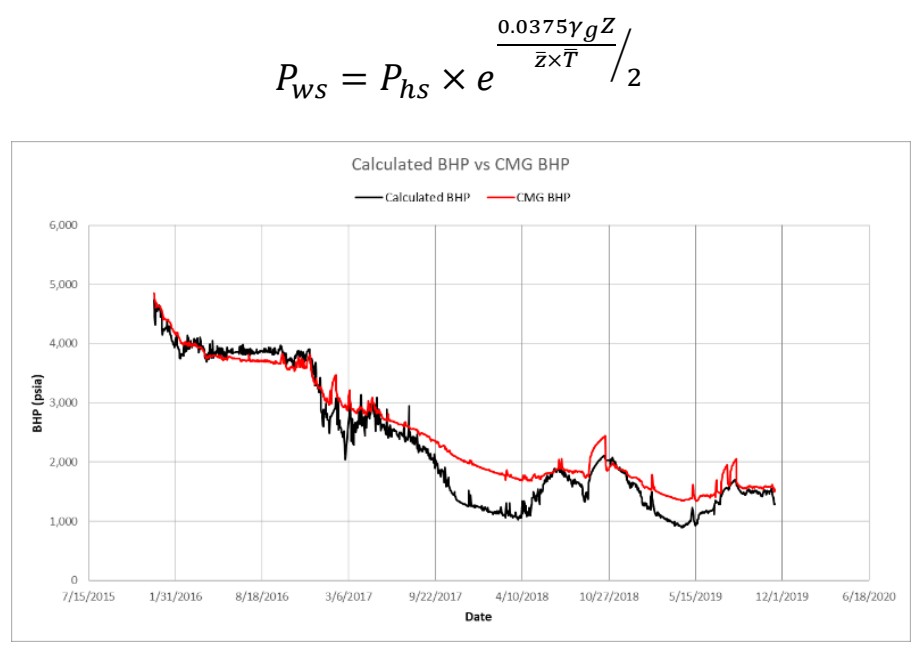

Figure 10: Actual Reservoir Pressure vs CMG Reservoir Pressure

Figure 10 shows the comparison of the calculated reservoir pressure versus the CMG modeled reservoir pressure. A sensitivity analysis was conducted for this reservoir model. Some of the key parameters tested were fissure/matrix permeability, fracture conductivity, and reservoir porosity. Table 6 represents the final 
parameters used for the reservoir model. After running the sensitivity analysis, it was determined that the model created would be sufficient for this study.

Table 6: Final Reservoir Model Parameter Values

\begin{tabular}{|l|c|c|}
\hline \multicolumn{1}{|c|}{ Reservoir Parameters } & Values & Units \\
\hline Model Dimensions (LxWxH) & $8000 \times 3000 \times 90$ & $\mathrm{ft}$. \\
\hline Initial Reservoir Pressure & 4850 & $\mathrm{psi}$ \\
\hline Initial Temperature & 146 & ${ }^{\circ} \mathrm{F}$ \\
\hline Fissure Porosity & 0.0001 & Fraction \\
\hline Matrix Porosity & 0.07 & Fraction \\
\hline Water Saturation & 0.15 & Fraction \\
\hline Fissure Permeability $(\mathrm{i}, \mathrm{j}, \mathrm{k})$ & $0.0025,0.0025,0.00025$ & $\mathrm{md}$ \\
\hline Matrix Permeability $(\mathrm{i}, \mathrm{j}, \mathrm{k})$ & $0.000435,0.000435,0.0000435$ & $\mathrm{md}$ \\
\hline Langmuir Pressure & 400 & $\mathrm{psi}$ \\
\hline Langmuir Volume & 0.12 & gmol/lb \\
\hline
\end{tabular}

Chapter 3.5 - Stress Shadowing

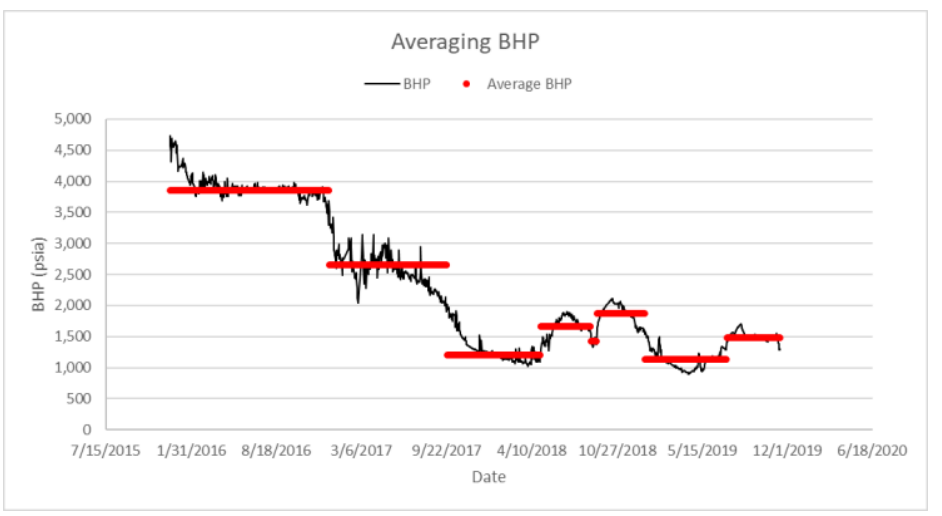

Figure 11: Averaging BHP

Once the initial reservoir model was created and met the desired match, the next step was designing the fracture model for stress shadowing and change the reservoir model from a flow constraint to a pressure constraint. Changing to a pressure constraint allows for the model to show the potential of the well when there is not a restriction on the daily flow. To help simplify the model, average pressures were used for different time periods, as seen in Figure 11. 


\begin{tabular}{|c|c|r|}
\hline Start Date & End Date & Average Pressure (psia) \\
\hline $12 / 12 / 2015$ & $12 / 19 / 2016$ & 3,850 \\
\hline $12 / 20 / 2016$ & $09 / 22 / 2017$ & 2,660 \\
\hline $09 / 23 / 2017$ & $04 / 30 / 2018$ & 1,196 \\
\hline $05 / 01 / 2018$ & $08 / 26 / 2018$ & 1,665 \\
\hline $08 / 27 / 2018$ & $09 / 09 / 2018$ & 1,421 \\
\hline $09 / 10 / 2018$ & $12 / 31 / 2018$ & 1,873 \\
\hline $01 / 01 / 2019$ & $07 / 11 / 2019$ & 1,129 \\
\hline $07 / 12 / 2019$ & $11 / 14 / 2019$ & 1,486 \\
\hline
\end{tabular}

Two case studies were decided upon for studying the effect of stress shadowing on production when changing the cluster spacing per stage. The first case had three designs with cluster spacing of $45 \mathrm{ft}$. for each stage, $40 \mathrm{ft}$. for each stage and $35 \mathrm{ft}$. for each stage. To implement these options, the first thing to consider was keeping the number of clusters per stage the same and keep the same pump schedules. Then inside each stage change the location of each perforation based on the stage length as seen in Figure 12, Figure 13, and Figure 14. The second case had the same design, expect additional clusters would be added to the stage when room permitted as seen in Figure 15, Figure 16, and Figure 17.

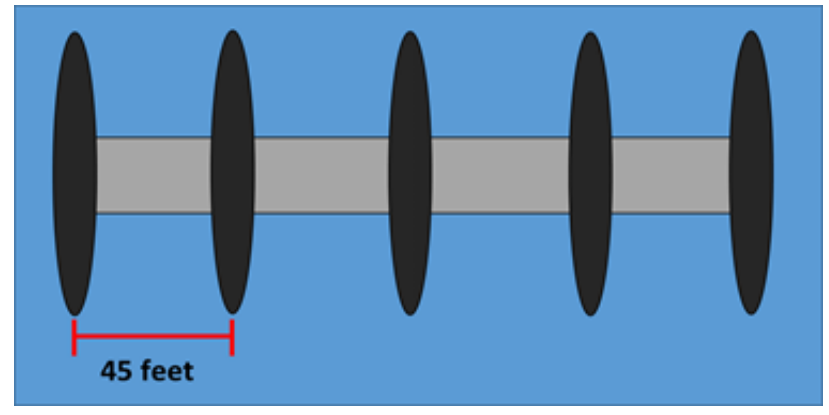

Figure 12: Case Study 1 - 45ft Cluster Spacing Design

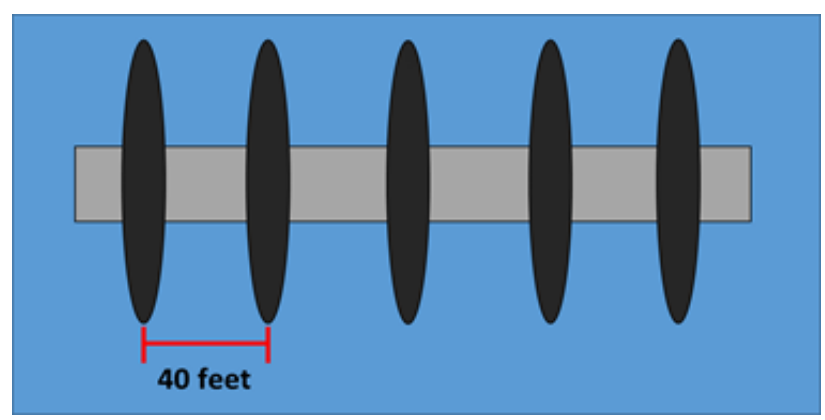

Figure 13: Case Study 1 - 40ft Cluster Spacing Design

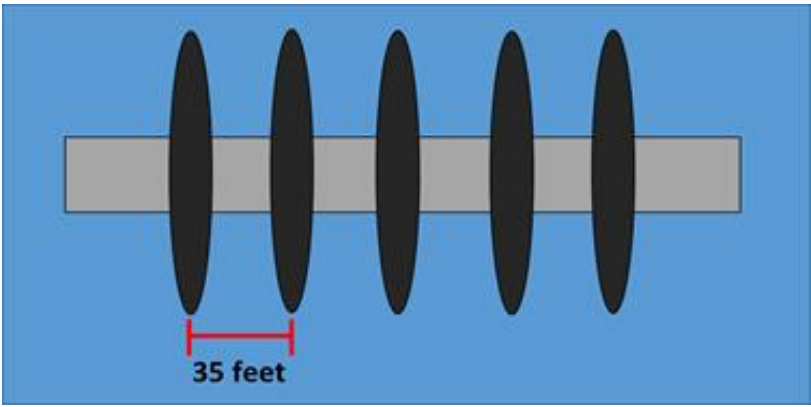

Figure 14: Case Study 1 - 35ft Cluster Spacing Design

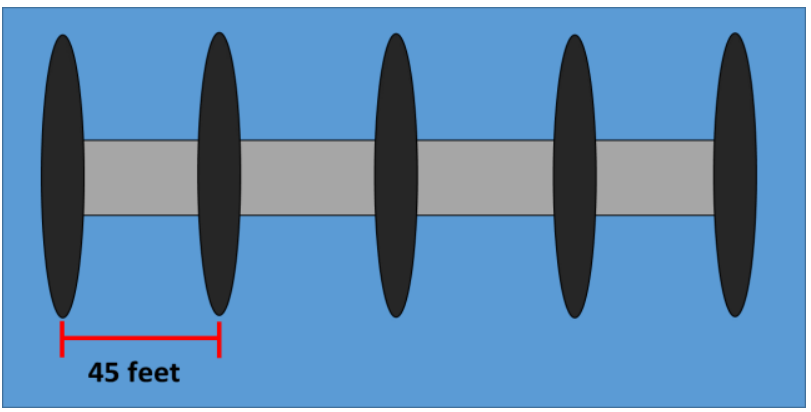

Figure 15: Case Study 2 - 45ft Cluster Spacing Design 


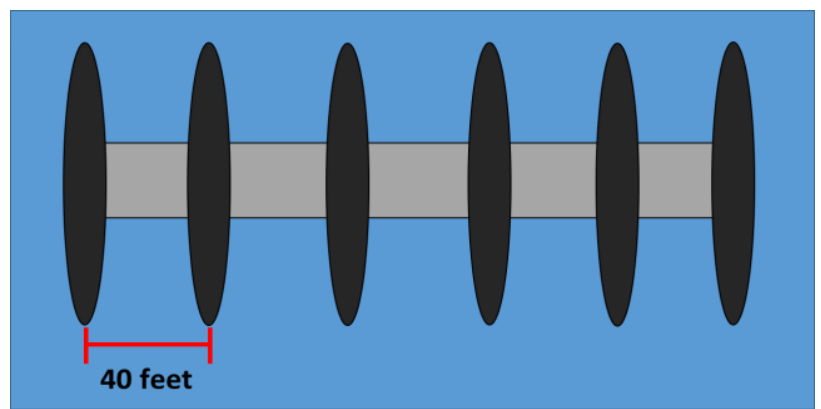

Figure 16: Case Study 2 - 40ft Cluster Spacing Design

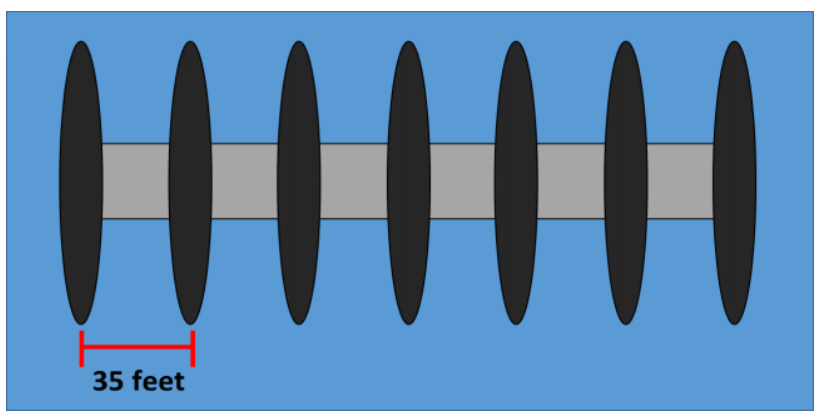

Figure 17: Case Study 2 - 35ft Cluster Spacing Design 


\section{Results and Discussion}

\section{Chapter 4.1 - Initial Fracture Results}

The first part of the study was generating hydraulic fractures using log data in GOHFER and studying the difference between a model implementing stress shadowing and a model without implementing stress shadowing. After the models were created and processed, the average geometrical properties of the stages were gathered and visually compared through Figure 18, Figure 19, Figure 20 and Figure 21.

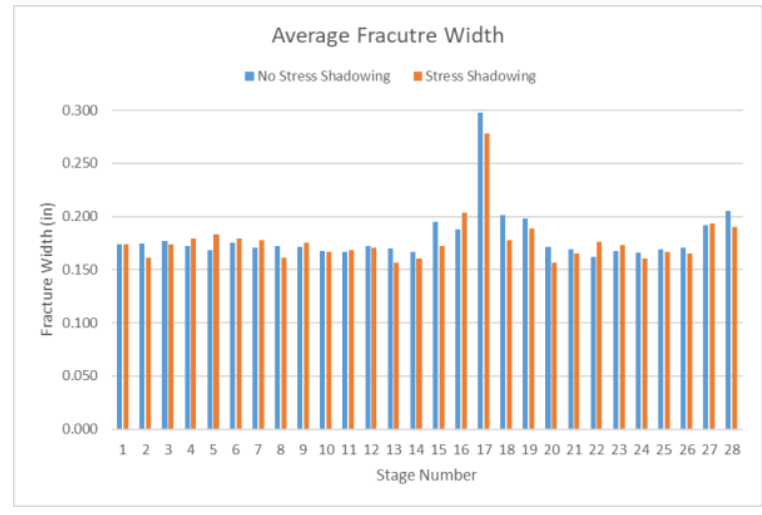

Figure 18: Comparison of Average Fracture Width for Base Model

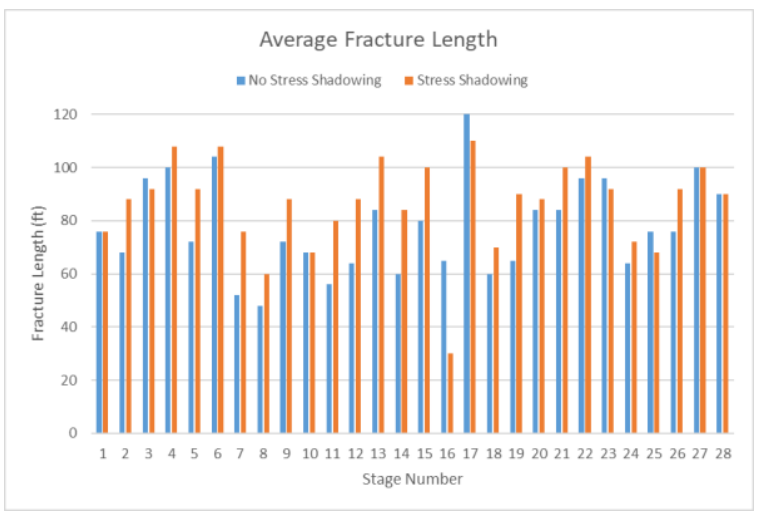

Figure 19: Comparison of Average Fracture Length for Base Model

Figure 18 shows the average fracture width of each stage's cluster and the main observation was that there is little change between the model with stress shadowing and the model without. Figure 19 shows the average fracture length of each stage's cluster and there is a clear indication that the stress shadowing model will generate longer fracture lengths compared to the model without stress shadowing. This is because of the geomechanical properties, when the in-situ stress is greater in the region the fractures will want to propagate longer, but with less of a fracture height. This can be seen through Figure 20 with the stress shadowing model having a smaller average fracture height. Another property that was considered was the average proppant concentration of each stage's cluster. This property also provided insight into how the stress shadowing affects the proppant placement. 


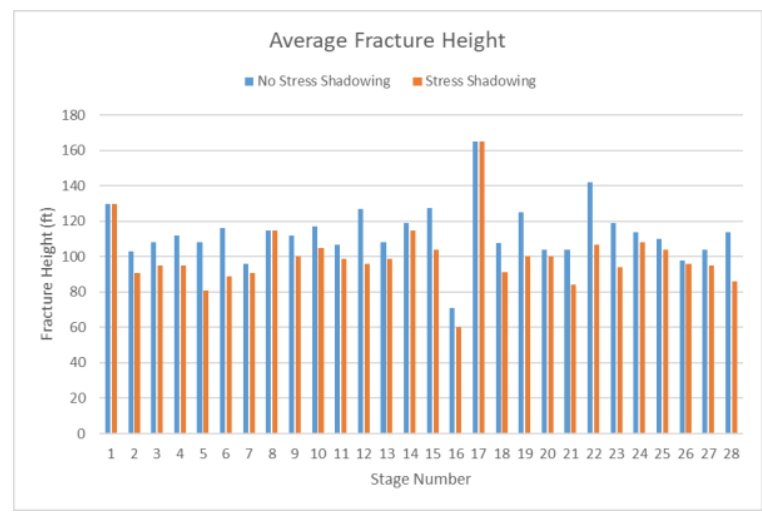

Figure 20: Comparison of Average Fracture Height for

$$
\text { Base Model }
$$

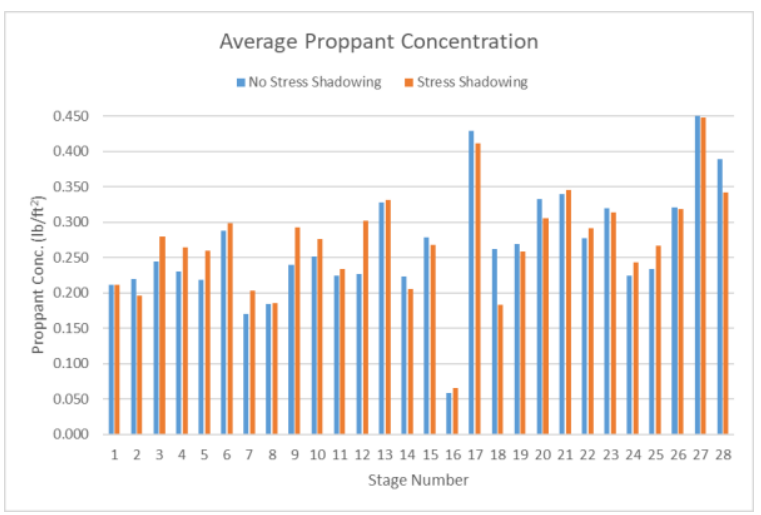

Figure 21: Comparison of Average Proppant

Concentration for Base Model

Looking deeper into the results between stress shadowing and no stress shadowing models. The average fracture properties were taken for all stages as seen in Table 8. The main conclusion from this is that the fracture length (proppant cuttoff length) is generally longer when stress shadowing is implemented, yet the fracture height is reduced.

Table 8: Average Fracture Geometric Properties Comparison Base Models

\begin{tabular}{|c|c|c|c|c|c|c|}
\hline \multicolumn{7}{|c|}{ Average of Fracture Geometric Properties } \\
\hline Model & $\begin{array}{c}\text { Proppant } \\
\text { Cutoff } \\
\text { Length } \\
\text { (ft) }\end{array}$ & $\begin{array}{c}\text { Est Flowing } \\
\text { Frac Length } \\
\text { (ft) }\end{array}$ & $\begin{array}{c}\text { Est Inf } \\
\text { Conductivity } \\
\text { Length } \\
\text { (ft) }\end{array}$ & $\begin{array}{c}\text { Fracture } \\
\text { Height } \\
\text { (ft) }\end{array}$ & $\begin{array}{c}\text { Average } \\
\text { Proppant } \\
\text { Conc } \\
\left(\mathbf{l b} / \mathbf{f t}^{2}\right)\end{array}$ & $\begin{array}{c}\text { Average } \\
\text { Fracture } \\
\text { Width } \\
\text { (in) }\end{array}$ \\
\hline No Stress Shadowing & 77.71 & 9.61 & 9.55 & 113.67 & 0.266 & 0.180 \\
\hline Stress Shadowing & 86.36 & 9.94 & 9.87 & 99.83 & 0.271 & 0.177 \\
\hline
\end{tabular}

\section{Chapter 4.2 - Initial Reservoir Model}

The next part of this study was to create a reservoir model that closely matched the field production/pressure. The first model created was made with a pressure constraint and tried to match field production. The results from this can be seen in Figure 22. The model created with pressure constraint did not show reliable results, so the model was adjusted to flow constraints.

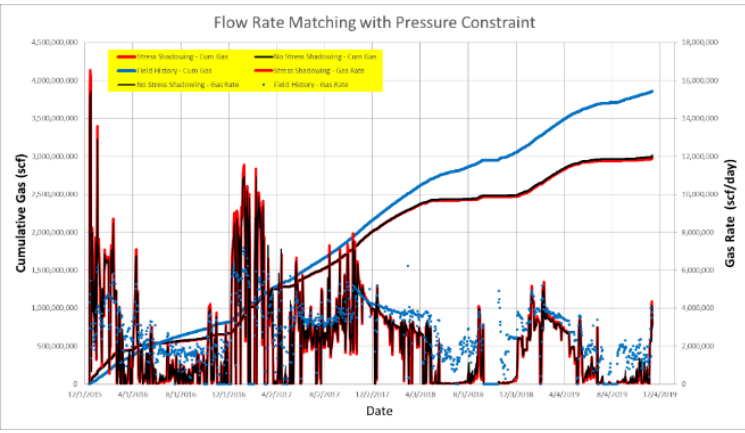

Figure 22: Production Matching with Pressure Constraint 
This led into the making of a model with a flow constraint instead of a pressure constraint. The results are seen in Figure 23 with pressure matching the field and Figure 24 showing the daily production for each model and the field. While both models follow similar trends, the percent error between the field and the no stress shadowing model is $17.2 \%$ and $15.9 \%$ for the stress shadowing model.

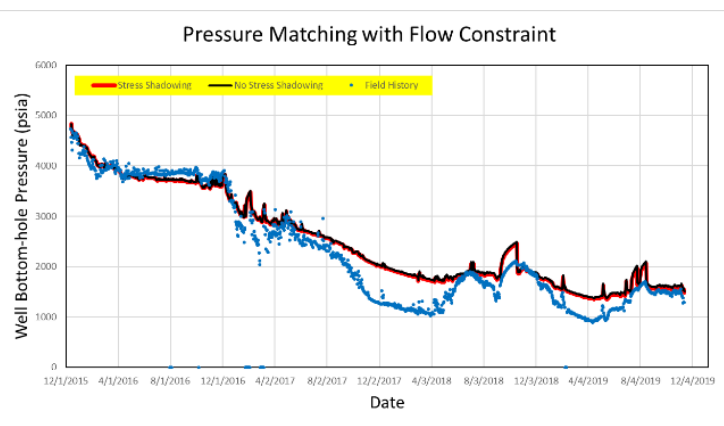

Figure 23: Pressure Matching with Flow Constraint

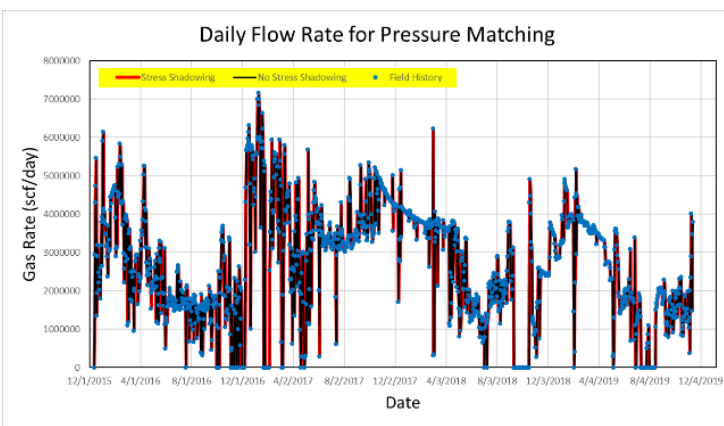

Figure 24: Daily Flow Rate for Pressure Matching

\section{Chapter 4.3 - Cluster Spacing Fracture Model}

With the base reservoir model completed, the next step was generating hydraulic fracturing models that simulated different cluster spacings while keeping other properties constant. As in the first fracture model created for the base model, the geometric properties of the stage's cluster were analyzed after the models were completed.

Chapter 4.3a - Case Study 1

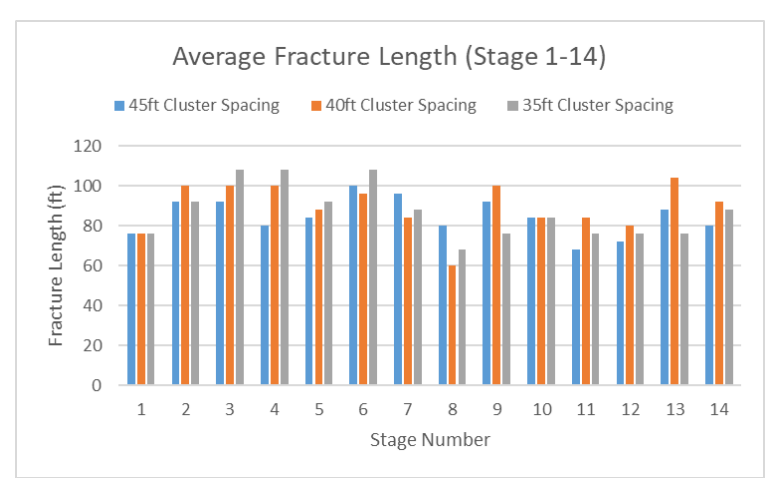

Figure 25: Comparison of Average Fracture Length for Different Cluster Spacings (Stage 1-14)

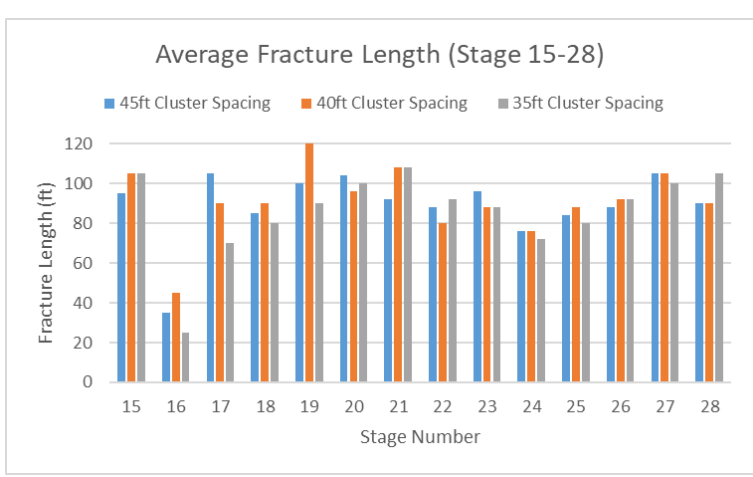

Figure 26: Comparison of Average Fracture Length for Different Cluster Spacings (Stage 15-28)

Figure 25 shows the average fracture length of each stage's cluster for stages 1-14 and Figure 26 shows the average fracture length of each stage's cluster for stages 15-28. The results show that there are minimal changes between the variation of cluster spacings and that there is no clear indication for the effect that changing the cluster spacings have on the average fracture length. 


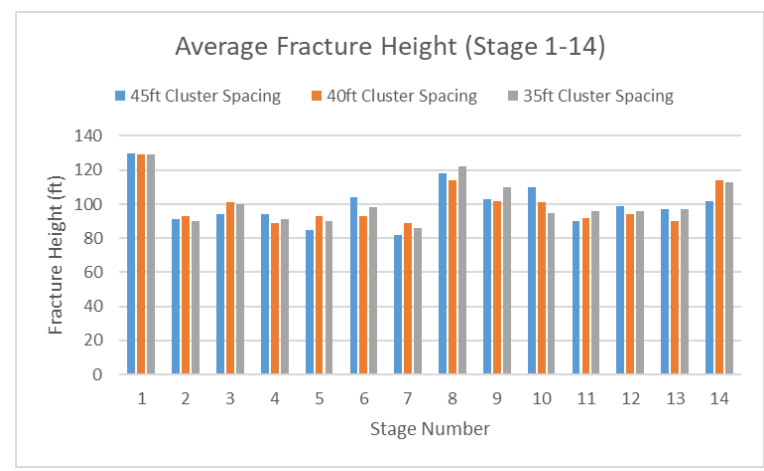

Figure 27: Comparison of Average Fracture Height for Different Cluster Spacings (Stage 1-14)

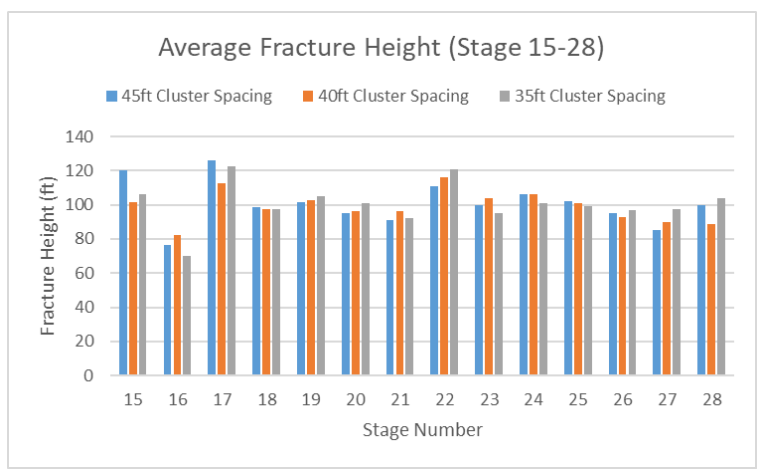

Figure 28: Comparison of Average Fracture Height for Different Cluster Spacings (Stage 15-28)

Figure 27 shows the average fracture height of each stage's cluster for stages 1-14 and Figure 28 shows the average fracture height of each stage's cluster for stages 15-28. The results show that there are minimal changes between the variation of cluster spacings and that there is no clear indication for the effect that changing the cluster spacings have on the average fracture height.

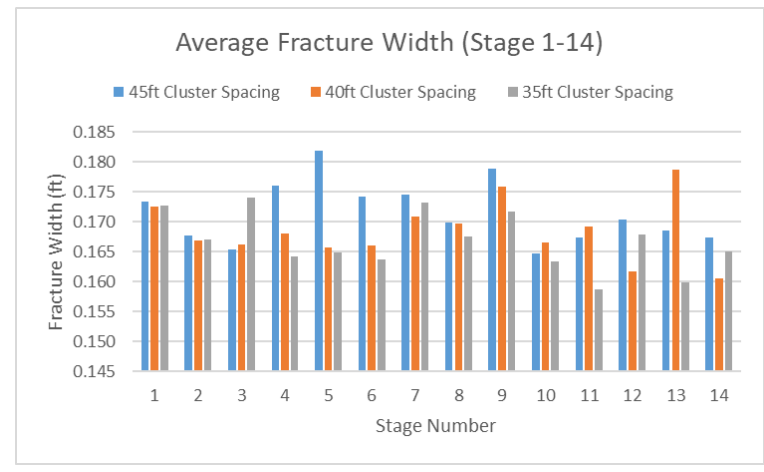

Figure 29: Comparison of Average Fracture Width for Different Cluster Spacings (Stage 1-14)

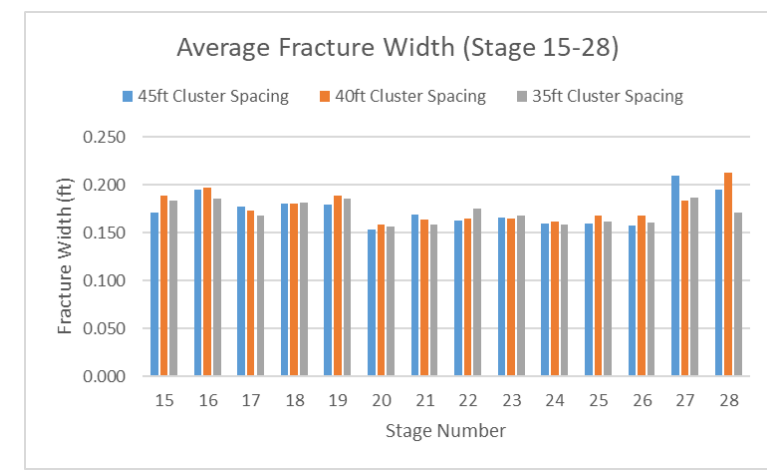

Figure 30: Comparison of Average Fracture Width for

Different Cluster Spacings (Stage 15-28)

Figure 29 shows the average fracture width of each stage's cluster for stages 1-14 and Figure 30 shows the average fracture width of each stage's cluster for stages 15-28. The results show that there are minimal changes between the variation of cluster spacings and that there is no clear indication for the effect that changing the cluster spacings have on the average fracture width. 
Table 9: Case Study 1 - Average Fracture Geometric Properties for Stages

\begin{tabular}{|c|c|c|c|c|c|c|c|}
\hline \multicolumn{2}{|c|}{ Average of Fracture Geometric Properties } \\
\hline $\begin{array}{c}\text { Case } \\
\text { Study }\end{array}$ & $\begin{array}{c}\text { Cluster } \\
\text { Spacing }\end{array}$ & $\begin{array}{c}\text { Proppant } \\
\text { Cutoff } \\
\text { Length } \\
\text { (ft) }\end{array}$ & $\begin{array}{c}\text { Est Flowing } \\
\text { Frac Length } \\
\text { (ft) }\end{array}$ & $\begin{array}{c}\text { Est Inf } \\
\text { Conductivity } \\
\text { Length } \\
\text { (ft) }\end{array}$ & $\begin{array}{c}\text { Fracture } \\
\text { Height } \\
\text { (ft) }\end{array}$ & $\begin{array}{c}\text { Average } \\
\text { Proppant } \\
\text { Conc } \\
\left(\mathbf{l b} / \mathbf{f t}^{\mathbf{2}}\right)\end{array}$ & $\begin{array}{c}\text { Average } \\
\text { Fracture } \\
\text { Width } \\
\text { (in) }\end{array}$ \\
\hline \multirow{3}{*}{ Case 1 } & $\mathbf{4 5} \mathbf{f t}$ & 87 & 9.85 & 9.79 & 100 & 0.259 & 0.173 \\
\cline { 2 - 9 } & $\mathbf{4 0} \mathbf{f t}$ & 90 & 9.91 & 9.85 & 99 & 0.261 & 0.172 \\
\cline { 2 - 9 } & $\mathbf{3 5} \mathbf{f t}$ & 87 & 9.83 & 9.77 & 101 & 0.255 & 0.169 \\
\hline
\end{tabular}

The average fracture properties were taken for all stages as seen in Table 9.Table 8 The main conclusion from this is that the fracture geometric properties do not significantly change through this case study.

Chapter 4.3b - Case Study 2

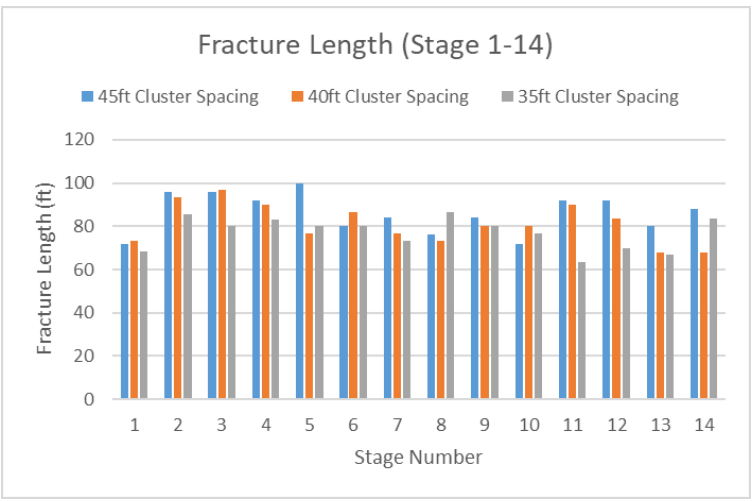

Figure 31: Comparison of Average Fracture Length for Different Cluster Spacings (Stage 1-14)

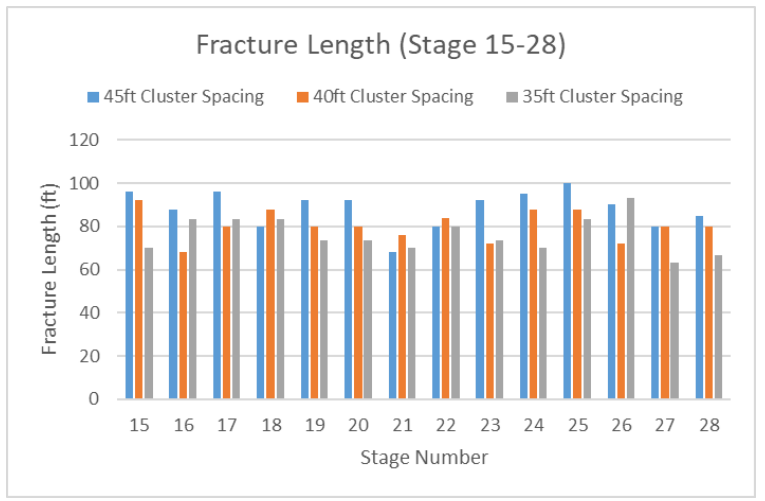

Figure 32: Comparison of Average Fracture Length for Different Cluster Spacings (Stage 15-28)

Figure 31 shows the average fracture length of each stage's cluster for stages 1-14 and Figure 32 shows the average fracture length of each stage's cluster for stages 15-28. The results show that as the cluster spacing decreases in length, the average fracture length also decreases.

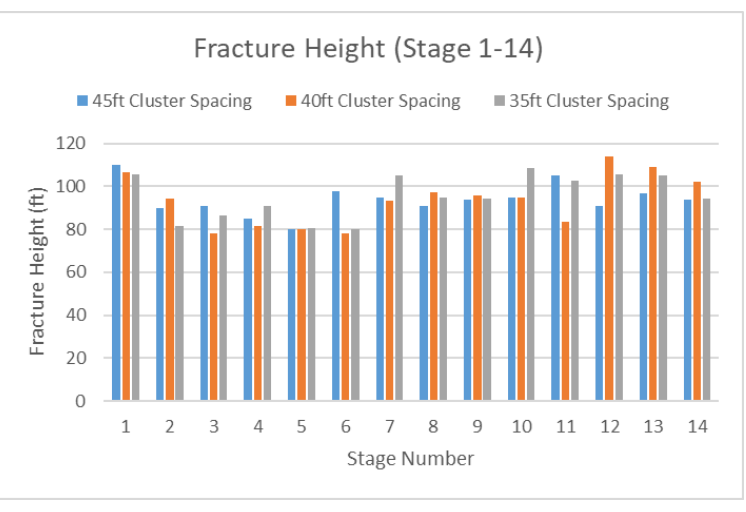

Figure 33: Comparison of Average Fracture Height for Different Cluster Spacings (Stage 1-14)

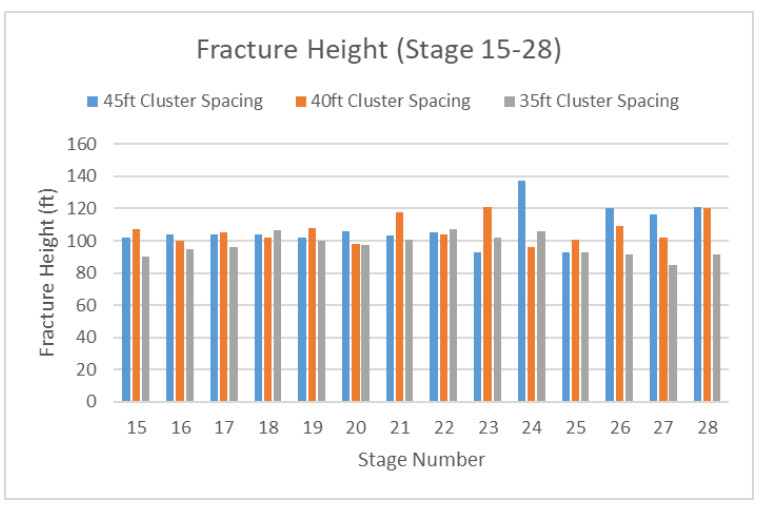

Figure 34: Comparison of Average Fracture Height for Different Cluster Spacings (Stage 15-28) 
Figure 33 shows the average fracture height of each stage's cluster for stages 1-14 and Figure 34 shows the average fracture height of each stage's cluster for stages 15-28. The results show that as the cluster spacing decreases in length, the average fracture height does not significantly change between the different cluster spacings.

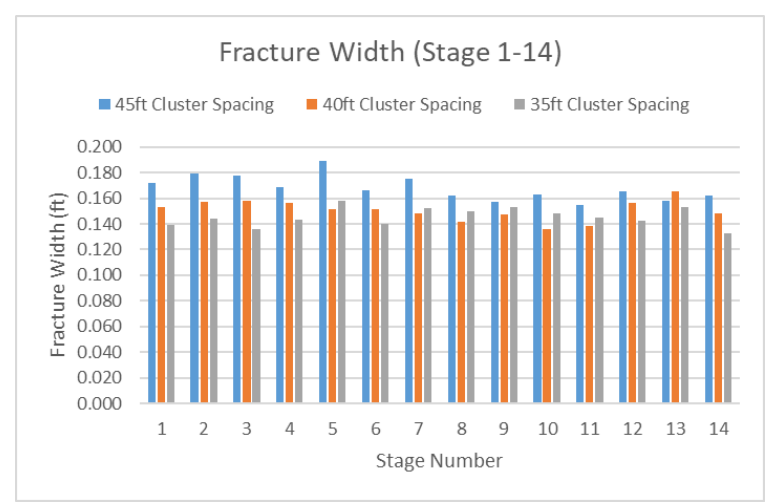

Figure 35: Comparison of Average Fracture Width for Different Cluster Spacings (Stage 1-14)

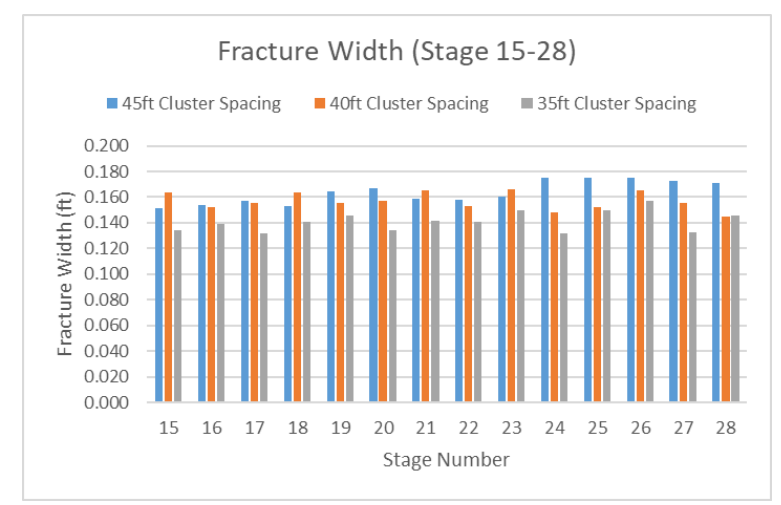

Figure 36: Comparison of Average Fracture Width for Different Cluster Spacings (Stage 15-28)

Figure 35 shows the average fracture width of each stage's cluster for stages 1-14 and Figure 36 shows the average fracture width of each stage's cluster for stages 15-28. The results show that as the cluster spacing decreases in length, the average fracture width also decreases.

Table 10: Case Study 2 - Average Fracture Geometric Properties for Stages

\begin{tabular}{|c|c|c|c|c|c|c|c|}
\hline \multicolumn{8}{|c|}{ Average of Fracture Geometric Properties } \\
\hline $\begin{array}{c}\text { Case } \\
\text { Study }\end{array}$ & $\begin{array}{c}\text { Cluster } \\
\text { Spacing }\end{array}$ & $\begin{array}{c}\text { Proppant } \\
\text { Cutoff } \\
\text { Length } \\
\text { (ft) }\end{array}$ & $\begin{array}{c}\text { Est Flowing } \\
\text { Frac Length } \\
\text { (ft) }\end{array}$ & $\begin{array}{c}\text { Est Inf } \\
\text { Conductivity } \\
\text { Length } \\
\text { (ft) }\end{array}$ & $\begin{array}{c}\text { Fracture } \\
\text { Height } \\
\text { (ft) }\end{array}$ & $\begin{array}{c}\text { Average } \\
\text { Proppant } \\
\text { Conc } \\
\left(\mathbf{l b} / \mathbf{f t}^{\mathbf{2}}\right)\end{array}$ & $\begin{array}{c}\text { Average } \\
\text { Fracture } \\
\text { Width } \\
\text { (in) }\end{array}$ \\
\hline \multirow{3}{*}{ Case 2 } & $\mathbf{4 5} \mathbf{f t}$ & 87 & 9.92 & 9.85 & 101 & 0.271 & 0.166 \\
\cline { 2 - 9 } & $\mathbf{4 0} \mathbf{f t}$ & 81 & 9.67 & 9.60 & 100 & 0.253 & 0.154 \\
\cline { 2 - 9 } & $\mathbf{3 5} \mathbf{f t}$ & 77 & 9.56 & 9.50 & 96 & 0.238 & 0.143 \\
\hline
\end{tabular}

The average fracture properties were taken for all stages as seen in Table 10. The main conclusion from this is that the fracture geometric properties do not significantly change through this case study.

\section{Chapter 4.4 - Cluster Spacing Reservoir Model}

The fracture results were then imported into the CMG reservoir model and ran for the current life of the well.

\section{Chapter 4.4a - Cast Study 1}

The results are seen in Figure 37 and detailed in Table 11. The first observation was that with smaller cluster spacings, there is a larger amount of early produced gas rate. However, this rate falls off very quickly when the pressure settles in the reservoir. This leads to the understanding that if there are no changes in operation 
conditions with controlling flowing pressure, the production of a well will be slightly less for smaller cluster spacings. The actual change in production when decreasing the cluster spacings is seen in Table 11 with a negative $0.55 \%$ change in production from $45 \mathrm{ft}$. cluster spacing to $40 \mathrm{ft}$. cluster spacing and a negative $0.67 \%$ change in production from $45 \mathrm{ft}$. cluster spacing to $35 \mathrm{ft}$. cluster spacing.

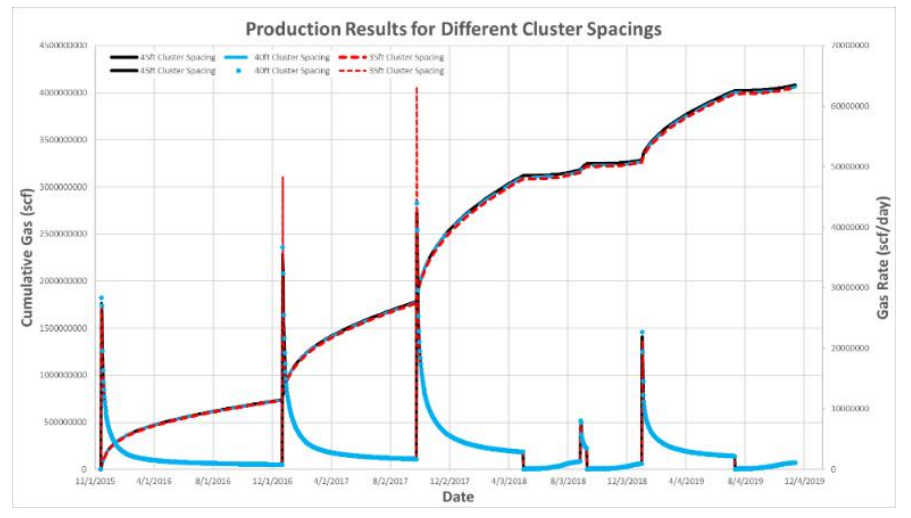

Figure 37: Case Study 1 - Production Results for Different Cluster Spacings

Table 11: Case Study 1 - Detailed Final Production for Different Cluster Spacing Models

\begin{tabular}{|c|c|c|}
\hline Model & Final Production (scf) & Production Change \\
\hline 45ft Cluster Spacing & $4,083,804,672$ & \\
\hline 40ft Cluster Spacing & $4,061,278,464$ & $-0.55 \%$ \\
\hline 35ft Cluster Spacing & $4,056,321,536$ & $-0.67 \%$ \\
\hline
\end{tabular}

\section{Chapter $4.4 a$ - Cast Study 2}

The results are seen in Figure 38 and detailed in Table 12. The first observation was that with smaller cluster spacings, there is a larger amount of early produced gas rate. However, this rate falls off very quickly when the pressure settles in the reservoir. This case study, the smaller cluster spacing with greater number of clusters has more production, except for the $40 \mathrm{ft}$. cluster spacing design. The actual change in production when decreasing the cluster spacings is seen in Table 12 with a $-0.51 \%$ change in production from $45 \mathrm{ft}$. cluster spacing to $40 \mathrm{ft}$. cluster spacing and a $5.77 \%$ change in production from $45 \mathrm{ft}$. cluster spacing to $35 \mathrm{ft}$. cluster spacing. 


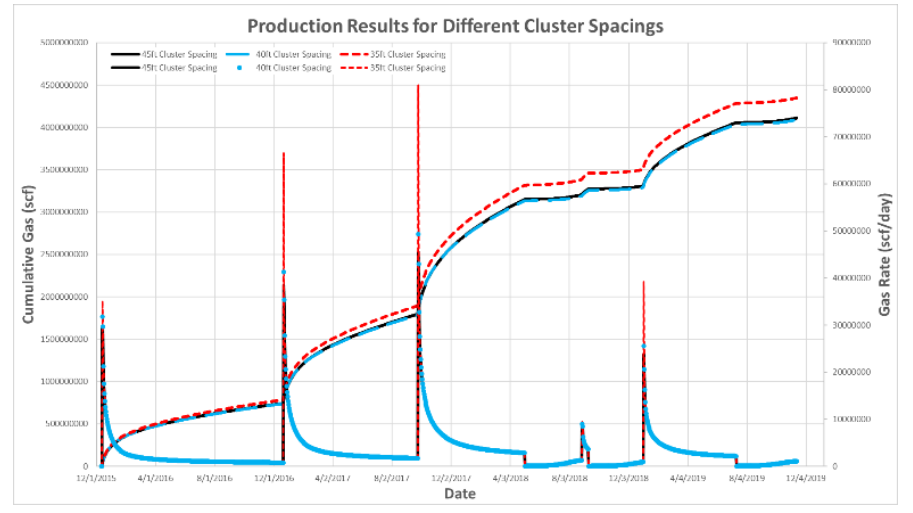

Figure 38: Case Study 2 - Production Results for Different Cluster Spacings

Table 12: Case Study 2 - Detailed Final Production for Different Cluster Spacing Models

\begin{tabular}{|c|r|r|}
\hline Model & Final Production (scf) & $\begin{array}{c}\text { Production } \\
\text { Change }\end{array}$ \\
\hline $45 \mathrm{ft}$ Cluster Spacing & $4,111,501,824$ & \\
\hline $40 \mathrm{ft}$ Cluster Spacing & $4,090,338,560$ & $-0.51 \%$ \\
\hline $35 \mathrm{ft}$ Cluster Spacing & $4,348,771,328$ & $5.77 \%$ \\
\hline
\end{tabular}




\section{Conclusion/Recommendations}

- When building a reservoir model with hydraulic fracture data, Stress Shadowing must be included for more accurate results

- Stress shadowing has a slight impact on production when only reducing the length of cluster spacing $(\sim<1 \%$ change)

- When incorporating more clusters when reducing the cluster spacing, the overall fracture properties are reduced while production still increases because of the increase in reservoir stimulation

- This is a very complex problem that requires future studies. Some ways to improve the study would be to incorporate:

○ Engineering Design Cluster Spacing

- Fracture Flowback

- Applying Same Study to other Marcellus Wells 


\section{References}

Barree, R. D. (2015, May). Stress Shadowing and Fracture Interference in GOHFER. Lakewood, Colorado. Retrieved from https://barree.net

Barree, R. D. (2018). GOHFER 3D User Manual. Lakewood, Colorado. Retrieved from https://barree.net/

Computing Modelling Group LTD. (2018). Builder User Guide: Simulation Model Building Application. Calgary, Alberta, Canada.

Computing Modelling Group LTD. (2018). GEM User Guide: Conventional \& Unconventional Simulator. Calgary, Alberta, Canada.

Fisher, M. K., Heinze, J. R., Harris, C. D., Davidson, B. M., Wright, C. A., \& Dunn, K. P. (2004, September 26-29). Optimizing Horizontal Completion Techniques in the Barnett Shale Using Microseismic Fracture Mapping. SPE Annual Technical Conference and Exhibition, Houston, Texas, 26-29 September: SPE-90051-MS.

MSEEL - Research - MIP Wells. (2018, January). Retrieved from MSEEL: http://www.mseel.org/ 


\section{Appendix}

Fracture Model

Table 13: Fracture Model - Wellbore Constuction

\begin{tabular}{|c|c|}
\hline \multicolumn{2}{|c|}{ Well Construction } \\
\hline \multicolumn{2}{|c|}{ Overview } \\
\hline \multicolumn{2}{|c|}{ Site Information } \\
\hline $\begin{array}{c}\text { Customer Name } \\
\text { Site Name }\end{array}$ & $\begin{array}{l}\text { WVU } \\
\text { MIP }\end{array}$ \\
\hline \multicolumn{2}{|c|}{ Site Location } \\
\hline $\begin{array}{c}\text { Coordinate System } \\
\text { Easting.Ft } \\
\text { Northing.Ft } \\
\text { Elevation.Ft } \\
\text { UTM Zone }\end{array}$ & $\begin{array}{c}\text { State Plane } \\
1834344.90 \\
401656.60 \\
1058.00 \\
\text { WV N-4701 }\end{array}$ \\
\hline $\begin{array}{c}\text { Lattitude } \\
\text { Longitude } \\
\text { Coordinate Reference System } \\
\text { Zone } \\
\end{array}$ & $\begin{array}{c}\text { N 39०36' 6.420070" } \\
\text { W 79 } 58^{\prime} 34.04266^{\prime \prime} \\
\text { NAD } 83 \\
\text { N-4701 }\end{array}$ \\
\hline $\begin{array}{c}\text { Lat.dec } \\
\text { Lon.dec } \\
\text { Well Azimuth. } \\
\text { Azimuth of Max Stress }\end{array}$ & $\begin{array}{c}39.6017834 \\
-79.9761230 \\
0 \\
0 \\
\end{array}$ \\
\hline \multicolumn{2}{|c|}{ Project Settings } \\
\hline $\begin{array}{c}\text { Depth Units } \\
\text { Well Units } \\
\text { Sea Water Depth.ft } \\
\text { Sea Water Gradiet.psi/ft } \\
\text { OBG.psi/ft } \\
\text { Stress Anistropy.frac }\end{array}$ & $\begin{array}{c}\text { Feet } \\
\text { Oil Field } \\
0.00 \\
0.44 \\
1.00 \\
\text { <CFOP> }\end{array}$ \\
\hline
\end{tabular}

Table 14: Fracture Model - Wellbore Location

\begin{tabular}{|c|c|c|c|}
\hline \multicolumn{4}{|c|}{ Well 1 - MIP 3H } \\
\hline \multicolumn{2}{|c|}{ MIP 3H } & \multicolumn{2}{|c|}{ MIP 3H - Pilot } \\
\hline \multicolumn{2}{|c|}{ Wellbore String } & \multicolumn{2}{|c|}{ Wellbore String } \\
\hline $\begin{array}{c}\text { Pump down Annulus } \\
\text { CLAT.Ft }\end{array}$ & $\begin{array}{c}\text { FALSE } \\
0.00\end{array}$ & & \\
\hline \multicolumn{2}{|c|}{ Well Information } & \multicolumn{2}{|c|}{ Well Information } \\
\hline $\begin{array}{l}\text { Well Identity } \\
\text { Field }\end{array}$ & $\begin{array}{c}\text { MIP 3H } \\
\text { Marcellus }\end{array}$ & $\begin{array}{l}\text { Well Identity } \\
\text { Field }\end{array}$ & $\begin{array}{c}\text { MIP 3H } \\
\text { Marcellus }\end{array}$ \\
\hline \multicolumn{2}{|c|}{ Site Location } & \multicolumn{2}{|c|}{ Site Location } \\
\hline $\begin{array}{c}\text { Coordinate System } \\
\text { Easting.Ft } \\
\text { Northing.Ft } \\
\text { Elevation.Ft } \\
\text { UTM Zone } \\
\end{array}$ & $\begin{array}{c}\text { State Plane } \\
1834344.90 \\
401656.60 \\
1058.00 \\
\text { WV N-4701 }\end{array}$ & $\begin{array}{c}\text { Coordinate System } \\
\text { Easting.Ft } \\
\text { Northing.Ft } \\
\text { Elevation.Ft } \\
\text { UTM Zone } \\
\end{array}$ & $\begin{array}{c}\text { State Plane } \\
1834344.90 \\
401656.60 \\
1058.00 \\
\text { WV N-4701 }\end{array}$ \\
\hline $\begin{array}{c}\text { Lattitude } \\
\text { Longitude } \\
\text { Coordinate Reference System } \\
\text { Zone } \\
\end{array}$ & $\begin{array}{c}\text { N 39 } 36^{\circ} 6.420070^{\prime \prime} \\
\text { W 79०58' } 34.04266^{\prime \prime} \\
\text { NAD83 } \\
\text { N-4701 }\end{array}$ & $\begin{array}{c}\text { Lattitude } \\
\text { Longitude } \\
\text { Coordinate Reference System } \\
\text { Zone } \\
\end{array}$ & 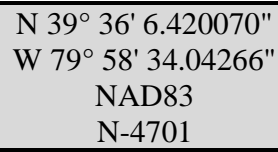 \\
\hline $\begin{array}{c}\text { Elevation Offset.Ft } \\
\text { North Offset.Ft } \\
\text { East Offset.Ft } \\
\text { Lat.dec } \\
\text { Lon.dec } \\
\text { Well Azimuth. }\end{array}$ & $\begin{array}{c}0 \\
0 \\
0 \\
39.6017834 \\
-79.976123 \\
321.361 \\
\end{array}$ & $\begin{array}{c}\text { Elevation Offset.Ft } \\
\text { North Offset.Ft } \\
\text { East Offset.Ft } \\
\text { Lat.dec } \\
\text { Lon.dec } \\
\text { Well Azimuth. }\end{array}$ & $\begin{array}{c}0 \\
0 \\
0 \\
39.6017834 \\
-79.976123 \\
0\end{array}$ \\
\hline
\end{tabular}


Table 15: Fracture Model - Wellbore Tubulars

\begin{tabular}{|c|c|c|c|c|c|c|}
\hline \multicolumn{7}{|c|}{ Wellbore Tubulars } \\
\hline Casing & $\begin{array}{c}\text { Hole } \\
\text { Size (in) }\end{array}$ & $\begin{array}{c}\text { Casing } \\
\text { Size (in) }\end{array}$ & $\begin{array}{c}\text { Casing } \\
\text { Grade }\end{array}$ & Lb / Ft & $\begin{array}{c}\text { I.D. } \\
\text { (in) }\end{array}$ & $\begin{array}{c}\text { Setting Depth } \\
\text { (ft) }\end{array}$ \\
\hline Conductor & 24 & 20 & STD & 78.67 & 19.25 & 42 \\
\hline Surface & 17.5 & 13.375 & J-55 & 54.5 & 12.615 & 508 \\
\hline Intermediate & 12.25 & 9.625 & J-55 & 40 & 8.835 & 1803 \\
\hline Production & 8.75 & 5.5 & P-110 & 20 & 4.778 & 13869 \\
\hline
\end{tabular}


Table 16: Fracture Model - Pilot Survey

\begin{tabular}{|c|c|c|c|c|c|}
\hline \multicolumn{3}{|c|}{ Pilot Survey (Part 1) } & \multicolumn{3}{|c|}{ Pilot Survey (Part 2) } \\
\hline MD & Incl & Azim Grid & MD & Incl & Azim Grid \\
\hline (ft) & $\left({ }^{\circ}\right)$ & $\left({ }^{\circ}\right)$ & $(\mathrm{ft})$ & $\left({ }^{\circ}\right)$ & $\left({ }^{\circ}\right)$ \\
\hline 0.00 & 0.00 & 0.00 & 4012.00 & 1.62 & 301.18 \\
\hline 112.00 & 0.42 & 156.90 & 4112.00 & 1.35 & 1.72 \\
\hline 212.00 & 0.63 & 185.61 & 4212.00 & 1.83 & 95.88 \\
\hline 312.00 & 0.69 & 182.45 & 4312.00 & 1.81 & 297.96 \\
\hline 412.00 & 0.88 & 231.58 & 4412.00 & 1.87 & 293.98 \\
\hline 512.00 & 0.28 & 276.25 & 4512.00 & 1.95 & 92.56 \\
\hline 612.00 & 0.29 & 271.92 & 4612.00 & 1.96 & 86.72 \\
\hline 712.00 & 0.44 & 270.09 & 4712.00 & 1.69 & 81.17 \\
\hline 812.00 & 0.45 & 262.83 & 4812.00 & 1.84 & .39 \\
\hline 912.00 & 0.41 & 227.92 & 4912.00 & 1.90 & 91.08 \\
\hline 1012.00 & 0.63 & 235.53 & 5012.00 & 2.30 & 301.03 \\
\hline 1112.00 & 0.82 & 250.18 & 5112.00 & 2.25 & 306.39 \\
\hline 1212.00 & 0.94 & 258.21 & 521 & 1.88 & 4.40 \\
\hline 1312.00 & 1.08 & 39 & 53 & 1.49 & .76 \\
\hline 1412.00 & 1.12 & 246.63 & 5412.00 & 1.57 & 317.94 \\
\hline 1512.00 & 1.14 & 243.23 & 5512.00 & 1.42 & 317.22 \\
\hline 1612.00 & 1.12 & 216.81 & 5612.00 & 1.12 & 333.06 \\
\hline 1712.00 & 0.67 & 208.16 & 5712.00 & 0.52 & 323.22 \\
\hline 1812.00 & 0.72 & 248.96 & 5812.00 & 0.72 & .11 \\
\hline 1912.00 & 0.70 & 1.83 & 59 & 0.51 & 0.49 \\
\hline 2012.00 & 0.45 & 231.01 & 6012.00 & 0.32 & 13.28 \\
\hline 2112.00 & 0.39 & 257.87 & 6112.00 & 0.27 & 14.02 \\
\hline 2212.00 & 0.38 & 279.40 & 6212.00 & 0.52 & 357.71 \\
\hline 2312.00 & 0.83 & 288.12 & 6312.00 & 0.79 & 329.34 \\
\hline 2412.00 & 1.12 & 285.37 & 6412.00 & 1.05 & 305.37 \\
\hline 2512.00 & 0.95 & 283.79 & 6512.00 & 0.66 & 309.59 \\
\hline 2612.00 & 0.94 & 287.25 & 6612.00 & 0.76 & 307.88 \\
\hline 2712.00 & 1.09 & 300.82 & 6712.00 & 0.73 & 288.99 \\
\hline 2812.00 & 1.20 & 282.59 & 6812.00 & 1.18 & 295.18 \\
\hline 2912.00 & 1.33 & 286.36 & 6890.00 & 1.30 & 287.01 \\
\hline 3012.00 & 1.39 & 289.94 & 6911.00 & 1.14 & 289.50 \\
\hline 3112.00 & 1.47 & 266.38 & 7005.00 & 2.37 & 267.49 \\
\hline 3212.00 & 1.31 & 297.88 & 7099.00 & 3.87 & 274.48 \\
\hline 3312.00 & 1.57 & 286.93 & 7194.00 & 4.92 & 277.43 \\
\hline 3412.00 & 1.22 & 288.22 & 7239.00 & 5.45 & 281.76 \\
\hline 3512.00 & 1.43 & 294.24 & 7288.00 & 4.48 & 285.77 \\
\hline 3612.00 & 1.63 & 293.51 & 7383.00 & 2.81 & 282.40 \\
\hline 3712.00 & 1.30 & 286.90 & 7398.00 & 2.55 & 286.08 \\
\hline 3812.00 & 1.26 & 285.87 & 7445.00 & 1.20 & 281.15 \\
\hline 3912.00 & 1.45 & 299.91 & 8003.00 & 0.00 & 275.00 \\
\hline
\end{tabular}


Table 17: Fracture Model - Lateral Survey

\begin{tabular}{|c|c|c|c|c|c|c|c|c|c|c|c|}
\hline \multicolumn{3}{|c|}{ Treatment Survey (Part 1) } & \multicolumn{3}{|c|}{ Treatment Survey (Part 2) } & \multicolumn{3}{|c|}{ Treatment Survey (Part 3) } & \multicolumn{3}{|c|}{ Treatment Survey (Part 4) } \\
\hline $\begin{array}{l}\mathrm{MD} \\
(\mathrm{ft}) \\
\end{array}$ & $\begin{array}{l}\text { Incl } \\
\left({ }^{\circ}\right) \\
\end{array}$ & $\begin{array}{c}\text { Azim Grid } \\
\left({ }^{\circ}\right)\end{array}$ & $\begin{array}{l}\mathrm{MD} \\
\text { (ft) } \\
\end{array}$ & $\begin{array}{l}\text { Incl } \\
\left({ }^{\circ}\right) \\
\end{array}$ & $\begin{array}{c}\text { Azim Grid } \\
\left({ }^{\circ}\right)\end{array}$ & $\begin{array}{l}\mathrm{MD} \\
\text { (ft) } \\
\end{array}$ & $\begin{array}{l}\text { Incl } \\
\left({ }^{\circ}\right) \\
\end{array}$ & $\begin{array}{c}\text { Azim Grid } \\
\left({ }^{\circ}\right)\end{array}$ & $\begin{array}{l}\mathrm{MD} \\
\text { (ft) } \\
\end{array}$ & $\begin{array}{l}\text { Incl } \\
\left({ }^{\circ}\right) \\
\end{array}$ & $\begin{array}{c}\text { Azim Grid } \\
\left({ }^{\circ}\right)\end{array}$ \\
\hline 0.00 & 0.00 & 0.00 & 4012.00 & 1.62 & 301.18 & 7871.00 & 84.42 & 325.25 & 11656.00 & 90.57 & 322.73 \\
\hline 112.00 & 0.42 & 156.90 & 4112.00 & 1.35 & 301.72 & 7966.00 & 89.16 & 327.68 & 11751.00 & 90.84 & 322.39 \\
\hline 212.00 & 0.63 & 185.61 & 4212.00 & 1.83 & 295.88 & 8061.00 & 91.71 & 325.82 & 11845.00 & 90.48 & 323.37 \\
\hline 312.00 & 0.69 & 182.45 & 4312.00 & 1.81 & 297.96 & 8156.00 & 91.01 & 324.72 & 11940.00 & 91.45 & 324.81 \\
\hline 412.00 & 0.88 & 231.58 & 4412.00 & 1.87 & 293.98 & 8251.00 & 91.54 & 322.60 & 12034.00 & 91.10 & 324.90 \\
\hline 512.00 & 0.28 & 276.25 & 4512.00 & 1.95 & 292.56 & 8346.00 & 90.66 & 323.35 & 12129.00 & 91.28 & 324.96 \\
\hline 612.00 & 0.29 & 271.92 & 4612.00 & 1.96 & 286.72 & 8441.00 & 91.28 & 324.41 & 12224.00 & 90.92 & 324.68 \\
\hline 712.00 & 0.44 & 270.09 & 4712.00 & 1.69 & 281.17 & 8535.00 & 91.10 & 324.27 & 12319.00 & 91.28 & 324.72 \\
\hline 812.00 & 0.45 & 262.83 & 4812.00 & 1.84 & 279.39 & 8630.00 & 91.28 & 323.32 & 12413.00 & 91.28 & 324.55 \\
\hline 912.00 & 0.41 & 227.92 & 4912.00 & 1.90 & 291.08 & 8725.00 & 90.92 & 323.60 & 12508.00 & 91.01 & 324.35 \\
\hline 1012.00 & 0.63 & 235.53 & 5012.00 & 2.30 & 301.03 & 8819.00 & 91.01 & 324.20 & 12603.00 & 91.36 & 324.98 \\
\hline 1112.00 & 0.82 & 250.18 & 5112.00 & 2.25 & 306.39 & 8913.00 & 92.15 & 324.34 & 12697.00 & 91.28 & 324.43 \\
\hline 1212.00 & 0.94 & 258.21 & 5212.00 & 1.88 & 304.40 & 9008.00 & 91.28 & 324.06 & 12792.00 & 91.19 & 325.29 \\
\hline 1312.00 & 1.08 & 248.39 & 5312.00 & 1.49 & 305.76 & 9101.00 & 90.48 & 322.17 & 12886.00 & 90.92 & 325.39 \\
\hline 1412.00 & 1.12 & 246.63 & 5412.00 & 1.57 & 317.94 & 9196.00 & 90.92 & 324.34 & 12981.00 & 91.80 & 323.86 \\
\hline 1512.00 & 1.14 & 243.23 & 5512.00 & 1.42 & 317.22 & 9291.00 & 91.71 & 323.86 & 13075.00 & 91.98 & 324.14 \\
\hline 1612.00 & 1.12 & 216.81 & 5612.00 & 1.12 & 333.06 & 9386.00 & 91.45 & 323.89 & 13170.00 & 90.75 & 324.86 \\
\hline 1712.00 & 0.67 & 208.16 & 5712.00 & 0.52 & 323.22 & 9480.00 & 91.63 & 324.29 & 13264.00 & 90.75 & 324.73 \\
\hline 1812.00 & 0.72 & 248.96 & 5812.00 & 0.72 & 2.11 & 9575.00 & 91.80 & 324.90 & 13359.00 & 89.78 & 324.91 \\
\hline 1912.00 & 0.70 & 221.83 & 5912.00 & 0.51 & 60.49 & 9670.00 & 90.84 & 322.95 & 13454.00 & 89.69 & 325.04 \\
\hline 2012.00 & 0.45 & 231.01 & 6012.00 & 0.32 & 13.28 & 9765.00 & 90.57 & 322.42 & 13549.00 & 89.96 & 325.61 \\
\hline 2112.00 & 0.39 & 257.87 & 6112.00 & 0.27 & 14.02 & 9860.00 & 90.92 & 322.92 & 13643.00 & 90.92 & 323.98 \\
\hline 2212.00 & 0.38 & 279.40 & 6212.00 & 0.52 & 357.71 & 9954.00 & 90.13 & 322.97 & 13738.00 & 90.04 & 323.07 \\
\hline 2312.00 & 0.83 & 288.12 & 6259.00 & 1.32 & 179.82 & 10049.00 & 90.22 & 324.03 & 13836.00 & 90.75 & 323.29 \\
\hline 2412.00 & 1.12 & 285.37 & 6355.00 & 9.41 & 200.86 & 10143.00 & 89.52 & 325.14 & 13874.00 & 90.75 & 323.29 \\
\hline 2512.00 & 0.95 & 283.79 & 6450.00 & 10.38 & 213.06 & 10238.00 & 90.13 & 324.89 & & & \\
\hline 2612.00 & 0.94 & 287.25 & 6544.00 & 17.24 & 195.08 & 10332.00 & 89.08 & 324.25 & & & \\
\hline 2712.00 & 1.09 & 300.82 & 6639.00 & 21.28 & 186.82 & 10426.00 & 90.40 & 323.91 & & & \\
\hline 2812.00 & 1.20 & 282.59 & 6733.00 & 19.43 & 208.22 & 10521.00 & 90.04 & 323.18 & & & \\
\hline 2912.00 & 1.33 & 286.36 & 6828.00 & 23.30 & 227.97 & 10616.00 & 90.22 & 322.93 & & & \\
\hline 3012.00 & 1.39 & 289.94 & 6922.00 & 27.44 & 243.12 & 10710.00 & 90.13 & 323.54 & & & \\
\hline 3112.00 & 1.47 & 266.38 & 7017.00 & 29.02 & 262.98 & 10805.00 & 90.04 & 323.20 & & & \\
\hline 3212.00 & 1.31 & 297.88 & 7111.00 & 31.22 & 272.68 & 10899.00 & 90.40 & 322.42 & & & \\
\hline 3312.00 & 1.57 & 286.93 & 7206.00 & 36.32 & 281.48 & 10993.00 & 89.78 & 322.35 & & & \\
\hline 3412.00 & 1.22 & 288.22 & 7302.00 & 40.89 & 291.21 & 11088.00 & 90.31 & 321.45 & & & \\
\hline 3512.00 & 1.43 & 294.24 & 7396.00 & 48.36 & 296.42 & 11183.00 & 89.43 & 321.53 & & & \\
\hline 3612.00 & 1.63 & 293.51 & 7491.00 & 54.69 & 305.18 & 11277.00 & 88.99 & 321.68 & & & \\
\hline 3712.00 & 1.30 & 286.90 & 7586.00 & 60.67 & 315.50 & 11372.00 & 88.99 & 322.59 & & & \\
\hline 3812.00 & 1.26 & 285.87 & 7680.00 & 65.42 & 320.01 & 11466.00 & 89.43 & 323.04 & & & \\
\hline 3912.00 & 1.45 & 299.91 & 7776.00 & 74.39 & 322.89 & 11561.00 & 90.84 & 323.22 & & & \\
\hline
\end{tabular}


Table 18: Fracture Model - Reservoir Properties Part 1

\begin{tabular}{|c|c|c|c|c|c|}
\hline \multicolumn{6}{|c|}{ Treatment Configuration } \\
\hline \multicolumn{6}{|c|}{ Marcellus - No Shading } \\
\hline \multicolumn{3}{|c|}{ Simulation Mode: } & \multicolumn{3}{|c|}{ Transverse + Longitudinal } \\
\hline \multicolumn{6}{|c|}{ Est Reservoir Properties } \\
\hline Zone: & Marcellus & & Fraction $\mathrm{CO} 2:$ & 0.00 & (fraction) \\
\hline Reservoir Porosisty: & 0.07 & (fraction) & Fraction N2: & 0.00 & (fraction) \\
\hline TVD at Pore Pressure: & 7412.23 & $(\mathrm{ft})$ & Fraction $\mathrm{H} 2 \mathrm{~S}$ : & 0.00 & (fraction) \\
\hline Fluid Gradient (Well): & 0.44 & (psi/ft) & OBG: & 1.00 & $(p s i / f t)$ \\
\hline Reservoir Pore Pressure: & 4850.00 & (psi) & Oil Compressibility: & $1.53 \mathrm{E}-06$ & (1/psi) \\
\hline Water Saturation: & 0.15 & (fraction) & Water Compressibility: & $2.77 \mathrm{E}-06$ & (1/psi) \\
\hline Gas Saturation: & 0.85 & (fraction) & Gas Compressibility: & $1.16 \mathrm{E}-04$ & (1/psi) \\
\hline Oil Saturation: & 0.00 & (fraction) & Rock Compressibility: & $1.25 \mathrm{E}-05$ & (1/psi) \\
\hline Oil Specific Gravity: & 35.00 & (API) & Total Compressibility: & $1.12 \mathrm{E}-04$ & (1/psi) \\
\hline Gas Specific Gravity: & 0.70 & & Gas Viscosity: & 0.0284 & (cp) \\
\hline Reservoir Temperature: & 146.00 & $\left({ }^{\circ} \mathrm{F}\right)$ & Oil Viscosity: & 0.4814 & (cp) \\
\hline Reservoir Conduction Factor: & 1.00 & & Fluid Viscosity: & 0.0284 & (cp) \\
\hline
\end{tabular}

Table 19: Fracture Model - Reservoir Properties Part 2

\begin{tabular}{|ccc|}
\hline \multicolumn{3}{|c|}{ Stage - Reservoir Properties } \\
\hline PDL Coefficient & 0.0002 & $(1 / \mathrm{psi})$ \\
Transverse Storage Coefficient & 0.0005 & $(1 / \mathrm{psi})$ \\
Rel Perm Factor & 5.00 & \\
Coefficient of Discharge & 0.70 & \\
Tortuosity Factor & 0.00 & $(\mathrm{psi} / \mathrm{Vbpm})$ \\
Tortuosity Erosion Factor & 1.00 & \\
Transverse Exponent & 1.20 & \\
Width Exponent & 3.20 & \\
BHP Datum & Grid Top & $(\mathrm{ft})$ \\
Modulus Stiffness Factor & $7.00 \mathrm{E}-06$ & $(1 / \mathrm{psi})$ \\
\hline
\end{tabular}

Table 20: Fracture Model - Grid Properties

\begin{tabular}{|c|c|}
\hline \multicolumn{2}{|c|}{ Grid Properties } \\
\cline { 1 - 1 } Property & Value \\
\cline { 1 - 1 } Perf Type & Asymmetrical \\
\cline { 1 - 1 } Node Size.ft & 5.00 \\
\cline { 1 - 1 } Aspect Ratio & 2.00 \\
\cline { 1 - 1 } \# Columns & 50 \\
\cline { 1 - 1 } Total Columns & 738 \\
\cline { 1 - 1 } Length.ft & 2000 \\
\cline { 1 - 1 } Transverse Columns & 100 \\
\cline { 1 - 1 } Transverse Aspect & 4.00 \\
\cline { 1 - 1 } Grid Top.ft & 7300.00 \\
\cline { 1 - 1 } Frid Bottom.ft & 7600.00 \\
\cline { 1 - 1 } Angle Around Hole.deg & 45.00 \\
\cline { 1 - 1 } Show Regional Dip & FALSE \\
\cline { 1 - 1 } Regional Dip & 0.00 \\
\hline
\end{tabular}

Table 21: Fracture Model - Formation Tops

\begin{tabular}{|c|c|}
\hline \multicolumn{2}{|c|}{ Formation Tops } \\
\hline Description & TVD.FT \\
\hline Genesea & 7176.00 \\
\cline { 1 - 1 } Tully & 7196.00 \\
\cline { 1 - 1 } Hamilton & 7284.00 \\
\cline { 1 - 1 } Marcellus Shale - Upper & 7454.00 \\
\cline { 1 - 1 } Cherry Valley & 7520.00 \\
\cline { 1 - 1 } Marcellus Shale - Lower & 7528.00 \\
\cline { 1 - 1 } Onondaga & 7554.00 \\
\cline { 1 - 1 } Huntersville & 7583.00 \\
\hline
\end{tabular}


Table 22: Fracture Model - Overview Stage 1

\begin{tabular}{|c|c|c|}
\hline \multicolumn{3}{|c|}{ Stage 01} \\
\hline \multicolumn{3}{|c|}{ Treatment Summary } \\
\hline Formation & Marcellus & \\
\hline Formation Type & Shale & \\
\hline \multicolumn{3}{|c|}{ Stage Data } \\
\hline Report Date & $11 / 6 / 2015$ & \\
\hline Stage Length & 233.00 & $\mathrm{ft}$ \\
\hline Frac Gradient & 1.22 & $\mathrm{psi} / \mathrm{ft}$ \\
\hline ISIP & 5867.00 & psi \\
\hline 5 Min ISIP & 5314.00 & psi \\
\hline 10 Min ISIP & 5126.00 & psi \\
\hline 15 Min ISIP & 4986.00 & psi \\
\hline Breakdown Rate & 15.00 & bpm \\
\hline Breakdown Pressure & 6995.00 & psi \\
\hline Breakdown Volume & 10.00 & bbls \\
\hline Cut Short & $\mathrm{x}$ & $\mathrm{x}$ \\
\hline Screen Out & & $\mathrm{x}$ \\
\hline \multicolumn{3}{|c|}{ Fluid and Proppant Data } \\
\hline Total Clean Fluid & 10671.00 & bbls \\
\hline Prop.1-Type & 100 Mesh & \\
\hline Prop.2-Type & 40/70 White & \\
\hline Total Proppant Amount & 420600.00 & Ibs \\
\hline Proppant Concentration & 1805.15 & $\mathrm{lb} / \mathrm{ft}$ \\
\hline Proppant Concentration & 0.94 & $\mathrm{lb} / \mathrm{gal}$ \\
\hline \multicolumn{3}{|c|}{ Chemical Data } \\
\hline Acid \% Strength & 15.00 & \\
\hline Total Acid & 6000.00 & gal \\
\hline Total Slickwater & 7195.00 & bbls \\
\hline Total Linear & 3405.00 & bbls \\
\hline Total XL & 0.00 & bbls \\
\hline FR Amount & 388.00 & gal \\
\hline Gel Amount & 1440.00 & gal or lbs \\
\hline Breaker Amount & 85.00 & gal or lbs \\
\hline Scale Inhibitor & 45.00 & gal \\
\hline Biocide & 132.00 & gal \\
\hline
\end{tabular}

Table 23: Fracture Model - Perfs Stage 1

\begin{tabular}{|ccccc|}
\hline \multicolumn{5}{|c|}{ Perfs } \\
\hline Cluster & Start.FT & Stop.FT & Perf_Diam.IN & Perf_Shots. \\
\hline 5 & 13625.00 & 13627.00 & 0.42 & 10 \\
4 & 13672.00 & 13674.00 & 0.42 & 10 \\
3 & 13720.00 & 13722.00 & 0.42 & 10 \\
2 & 13767.00 & 13769.00 & 0.42 & 10 \\
1 & 13809.00 & 13811.00 & 0.42 & 10 \\
\hline
\end{tabular}

Table 24: Fracture Model - Pump Schedule Stage 1

\begin{tabular}{|c|c|c|c|c|c|c|c|c|c|c|c|}
\hline Stage & $\begin{array}{c}\text { Tot Time } \\
\text { mm:ss }\end{array}$ & $\begin{array}{l}\text { Stage Time } \\
\text { mm:ss }\end{array}$ & Fluid & $\begin{array}{c}\text { Clean Stg Vol } \\
\text { (gal) }\end{array}$ & $\begin{array}{c}\text { Cum Cln Vol } \\
\text { (gal) }\end{array}$ & Proppant & $\begin{array}{c}\text { Slurry Conc } \\
\text { (ppa) }\end{array}$ & $\begin{array}{l}\text { Cum Prop } \\
\text { (Ibs) }\end{array}$ & $\begin{array}{c}\text { Slurry Rate } \\
\text { (bbl/min) }\end{array}$ & $\begin{array}{c}\text { Cum Slurry } \\
\text { (bbl) }\end{array}$ & $\begin{array}{r}\text { Clean Rate } \\
\text { (bbl/min) }\end{array}$ \\
\hline 1 & 0.00 & 1.41 & SlickWater & 824.60 & 824.60 & $<$ None $>$ & 0.00 & 0.00 & 11.82 & 19.90 & 11.66 \\
\hline 2 & 1.41 & 4.29 & $\mathrm{HCl} 7.5 \%$ & 2999.99 & 3824.59 & $<$ None $>$ & 0.00 & 0.00 & 15.93 & 91.33 & 15.93 \\
\hline 3 & 6.10 & 17.04 & SlickWater & 41986.72 & 45811.30 & $<$ None $>$ & 0.00 & 0.00 & 58.63 & 1092.02 & 58.58 \\
\hline 4 & 23.14 & 4.14 & SlickWater & 13163.08 & 58974.38 & 100 Mesh Sand & 0.24 & 3212.00 & 74.82 & 1408.76 & 74.03 \\
\hline 5 & 27.28 & 5.13 & SlickWater & 14018.61 & 72992.99 & 100 Mesh Sand & 0.49 & 10140.00 & 65.40 & 1749.92 & 63.98 \\
\hline 6 & 32.41 & 6.23 & SlickWater & 18566.17 & 91559.16 & 100 Mesh Sand & 0.74 & 23914.00 & 71.67 & 2207.40 & 69.25 \\
\hline 7 & 39.04 & 6.23 & SlickWater & 23066.84 & 114625.99 & 100 Mesh Sand & 1.00 & 46879.00 & 89.91 & 2781.34 & 86.04 \\
\hline 8 & 45.27 & 9.56 & SlickWater & 35311.51 & 149937.51 & 100 Mesh Sand & 1.50 & 99752.00 & 90.39 & 3679.26 & 84.64 \\
\hline 9 & 55.23 & 8.11 & SlickWater & 28478.54 & 178416.05 & 100 Mesh Sand & 1.99 & 156394.00 & 90.36 & 4418.69 & 82.86 \\
\hline 10 & 63.34 & 6.29 & SlickWater & 23970.17 & 202386.22 & 40/70 White & 0.53 & 169066.00 & 90.13 & 5003.01 & 88.03 \\
\hline 11 & 70.03 & 5.04 & SlickWater & 18531.22 & 220917.44 & 40/70 White & 0.75 & 182897.00 & 90.05 & 5459.26 & 87.08 \\
\hline 12 & 75.07 & 5.09 & SlickWater & 18556.08 & 239473.52 & 40/70 White & 1.00 & 201382.00 & 89.67 & 5921.05 & 85.79 \\
\hline 13 & 80.16 & 10.10 & SlickWater & 36301.45 & 275774.97 & 40/70 White & 1.33 & 249521.00 & 90.14 & 6837.51 & 85.02 \\
\hline 14 & 90.26 & 4.32 & SlickWater & 16455.77 & 292230.74 & $<$ None $>$ & 0.00 & 249521.00 & 86.42 & 7229.26 & 86.43 \\
\hline 15 & 94.58 & 7.00 & Gel (WF110) & 23851.14 & 316081.88 & 40/70 White & 0.49 & 261201.00 & 82.93 & 7809.79 & 81.13 \\
\hline 16 & 101.58 & 5.14 & Gel (WF110) & 17025.18 & 333107.06 & 40/70 White & 0.74 & 273837.00 & 80.08 & 8228.87 & 77.46 \\
\hline 17 & 107.12 & 11.51 & Gel (WF110) & 38269.91 & 371376.97 & $40 / 70$ White & 1.00 & 312035.00 & 80.39 & 9181.51 & 76.89 \\
\hline 18 & 119.03 & 7.44 & Gel (WF110) & 24431.64 & 395808.61 & 40/70 White & 1.49 & 348473.00 & 80.34 & 9802.81 & 75.22 \\
\hline 19 & 126.47 & 12.41 & Gel (WF110) & 39455.71 & 435264.32 & 40/70 White & 1.89 & 423076.00 & 80.44 & 10823.05 & 74.07 \\
\hline 20 & 139.28 & 4.02 & SlickWater & 13021.04 & 448285.36 & $<$ None $>$ & 0.00 & 423076.00 & 76.56 & 11131.83 & 76.87 \\
\hline
\end{tabular}


Table 25: Fracture Model - Pump Details Stage 1

\begin{tabular}{|c|c|c|c|c|c|c|c|}
\hline Step\# & Name & Avg S Rate & Max S Rate & Avg Tr Pres & Max Tr Pres & Min Tr Pressure & Calc Avg TR P \\
\hline & & (bbl/min) & (bbl/min) & (psi) & (psi) & (psi) & (psi) \\
\hline 1 & Rate & 14.3 & 16.0 & 6501 & 6994 & 3944 & 6010 \\
\hline 2 & $7.5 \%$ HC & 15.9 & 16.0 & 6789 & 6965 & 6675 & 6785 \\
\hline 3 & PAD & 63.4 & 80.8 & 9019 & 9258 & 6674 & 8957 \\
\hline 4 & 0.25 PPA & 75.2 & 78.9 & 9187 & 9240 & 9132 & 9183 \\
\hline 5 & 0.5 PPA & 65.6 & 70.0 & 9189 & 9258 & 9106 & 9188 \\
\hline 6 & 0.75 PPA & 72.4 & 89.7 & 8889 & 9232 & 8370 & 8898 \\
\hline 7 & 1.00 PPA & 90.0 & 90.5 & 8899 & 9028 & 8835 & 8898 \\
\hline 8 & 1.5 PPA & 90.4 & 91.0 & 8856 & 8906 & 8806 & 8857 \\
\hline 9 & 2.00 PPA & 90.4 & 90.5 & 9014 & 9093 & 8902 & 9015 \\
\hline 10 & 0.50 PPA & 90.1 & 90.5 & 9026 & 9149 & 8922 & 9025 \\
\hline 11 & 0.75 PPA & 90.1 & 90.2 & 8916 & 8937 & 8898 & 8916 \\
\hline 12 & 1.0 PPA & 89.7 & 90.4 & 8905 & 8919 & 8886 & 8905 \\
\hline 13 & 1.5 PPA & 90.1 & 90.6 & 8917 & 9223 & 8829 & 8919 \\
\hline 14 & Flush & 86.5 & 89.0 & 9173 & 9242 & 9048 & 9171 \\
\hline 15 & 0.50 PPA & 83.1 & 85.8 & 9131 & 9250 & 9016 & 9132 \\
\hline 16 & 0.75 PPA & 80.1 & 80.5 & 8923 & 9054 & 8858 & 8921 \\
\hline 17 & 1.0 PPA & 80.4 & 80.6 & 8797 & 8864 & 8743 & 8797 \\
\hline 18 & 1.5 PPA & 80.3 & 80.5 & 8684 & 8754 & 8633 & 8683 \\
\hline 19 & 2.0 PPA & 80.4 & 80.6 & 8665 & 8813 & 8566 & 8666 \\
\hline 20 & Flush & 79.3 & 80.5 & 8865 & 9088 & 5663 & 8723 \\
\hline
\end{tabular}

Table 26: Fracture Model - Overview Stage 2

\begin{tabular}{|c|c|c|}
\hline \multicolumn{3}{|c|}{ Stage 02} \\
\hline \multicolumn{3}{|c|}{ Treatment Summary } \\
\hline Formation & Marcellus & \\
\hline Formation Type & Shale & \\
\hline \multicolumn{3}{|c|}{ Stage Data } \\
\hline Report Date & $11 / 7 / 2015$ & \\
\hline Stage Length & 223.00 & $\mathrm{ft}$ \\
\hline Frac Gradient & 1.24 & $\mathrm{psi} / \mathrm{ft}$ \\
\hline ISIP & 6048.00 & psi \\
\hline 5 Min ISIP & 5311.00 & psi \\
\hline 10 Min ISIP & 5135.00 & psi \\
\hline 15 Min ISIP & 5015.00 & psi \\
\hline Breakdown Rate & 15.00 & bpm \\
\hline Breakdown Pressure & 6078.00 & psi \\
\hline Breakdown Volume & 288.00 & bbls \\
\hline Cut Short & $x$ & $\mathrm{X}$ \\
\hline Screen Out & & $\mathrm{x}$ \\
\hline \multicolumn{3}{|c|}{ Fluid and Proppant Data } \\
\hline Total Clean Fluid & 7912.00 & bbls \\
\hline Prop.1-Type & 100 Mesh & \\
\hline Prop.2-Type & 40/70 White & \\
\hline Total Proppant Amount & 346380.00 & Ibs \\
\hline Proppant Concentration & 1553.27 & $\mathrm{lb} / \mathrm{ft}$ \\
\hline Proppant Concentration & 1.04 & $\mathrm{lb} / \mathrm{gal}$ \\
\hline \multicolumn{3}{|c|}{ Chemical Data } \\
\hline Acid \% Strength & 15.00 & \\
\hline Total Acid & 3000.00 & gal \\
\hline Total Slickwater & 7841.00 & bbls \\
\hline Total Linear & 0.00 & bbls \\
\hline Total XL & 0.00 & bbls \\
\hline FR Amount & 249.00 & gal \\
\hline Gel Amount & 0.00 & gal or lbs \\
\hline Breaker Amount & 0.00 & gal or lbs \\
\hline Scale Inhibitor & 27.00 & gal \\
\hline Biocide & 76.00 & gal \\
\hline
\end{tabular}

Table 27: Fracture Model - Perfs Stage 2

\begin{tabular}{|ccccc|}
\hline \multicolumn{5}{|c|}{ Perfs } \\
\hline Cluster & Start.FT & Stop.FT & Perf_Diam.IN & Perf_Shots. \\
\hline 5 & 13398.00 & 13400.00 & 0.42 & 10 \\
4 & 13443.00 & 13445.00 & 0.42 & 10 \\
3 & 13490.00 & 13492.00 & 0.42 & 10 \\
2 & 13532.00 & 13534.00 & 0.42 & 10 \\
1 & 13576.00 & 13578.00 & 0.42 & 10 \\
\hline
\end{tabular}


Table 28: Fracture Model - Pump Schedule Stage 2

\begin{tabular}{|c|c|c|c|c|c|c|c|c|c|c|c|}
\hline Stage & $\begin{array}{c}\text { Tot Time } \\
\text { mm:ss }\end{array}$ & $\begin{array}{c}\text { Stage Time } \\
\text { mm:ss }\end{array}$ & Fluid & $\begin{array}{c}\text { Clean Stg Vol } \\
\text { (gal) }\end{array}$ & $\begin{array}{c}\text { Cum Cln Vol } \\
\text { (gal) }\end{array}$ & Proppant & $\begin{array}{c}\text { Slurry Conc } \\
\text { (ppa) }\end{array}$ & $\begin{array}{c}\text { Cum Prop } \\
\text { (Ibs) }\end{array}$ & $\begin{array}{c}\text { Slurry Rate } \\
\text { (bbl/min) }\end{array}$ & $\begin{array}{c}\text { Cum Slurry } \\
\text { (bbl) }\end{array}$ & $\begin{array}{l}\text { Clean Rate } \\
\text { (bbl/min) }\end{array}$ \\
\hline 1 & 0.00 & 1.32 & SlickWater & 594.16 & 594.16 & $<$ None $>$ & 0.00 & 0.00 & 9.40 & 14.42 & 9.23 \\
\hline 2 & 1.32 & 4.49 & $\mathrm{HCl} 15 \%$ & 3008.39 & 3602.55 & $<$ None $>$ & 0.00 & 0.00 & 14.87 & 86.03 & 14.87 \\
\hline 3 & 6.21 & 24.55 & SlickWater & 41981.66 & 45584.21 & <None $>$ & 0.00 & 0.00 & 40.15 & 1086.55 & 40.12 \\
\hline 4 & 31.16 & 3.45 & SlickWater & 13160.62 & 58744.83 & 100 Mesh Sand & 0.24 & 3211.00 & 84.51 & 1403.46 & 83.56 \\
\hline 5 & 35.01 & 3.51 & SlickWater & 13989.72 & 72734.55 & 100 Mesh Sand & 0.49 & 10108.00 & 88.48 & 1744.12 & 86.52 \\
\hline 6 & 38.52 & 5.03 & SlickWater & 18541.20 & 91275.75 & 100 Mesh Sand & 0.74 & 23881.00 & 90.37 & 2200.50 & 87.42 \\
\hline 7 & 43.55 & 6.22 & SlickWater & 23117.66 & 114393.41 & 100 Mesh Sand & 0.99 & 46866.00 & 90.36 & 2775.77 & 86.45 \\
\hline 8 & 50.17 & 9.58 & SlickWater & 35296.55 & 149689.96 & 100 Mesh Sand & 1.50 & 99660.00 & 90.05 & 3673.28 & 84.32 \\
\hline 9 & 60.15 & 7.28 & SlickWater & 25762.78 & 175452.74 & 100 Mesh Sand & 2.00 & 151061.00 & 89.58 & 4342.14 & 82.15 \\
\hline 10 & 67.43 & 6.30 & SlickWater & 24009.72 & 199462.46 & 40/70 White & 0.53 & 163691.00 & 90.03 & 4927.35 & 87.95 \\
\hline 11 & 74.13 & 5.05 & SlickWater & 18563.71 & 218026.17 & 40/70 White & 0.74 & 177493.00 & 89.87 & 5384.18 & 86.95 \\
\hline 12 & 79.18 & 5.07 & SlickWater & 18510.50 & 236536.67 & 40/70 White & 0.99 & 195896.00 & 90.04 & 5844.90 & 86.14 \\
\hline 13 & 84.25 & 9.08 & SlickWater & 32393.75 & 268930.41 & 40/70 White & 1.50 & 244343.00 & 90.19 & 6668.64 & 84.45 \\
\hline 14 & 93.33 & 14.48 & SlickWater & 50467.16 & 319397.57 & 40/70 White & 1.93 & 341566.00 & 88.30 & 7975.41 & 81.19 \\
\hline 15 & 108.21 & 5.09 & SlickWater & 12945.94 & 332343.51 & $<$ None $>$ & 0.00 & 341566.00 & 59.61 & 8282.41 & 59.85 \\
\hline
\end{tabular}

Table 29: Fracture Model - Pump Details Stage 2

\begin{tabular}{|c|c|c|c|c|c|c|c|}
\hline Step\# & Name & Avg S Rate & Max S Rate & Avg Tr Pres & Max Tr Pres & Min Tr Pressure & Calc Avg TR P \\
\hline & & (bbl/min) & (bbl/min) & (psi) & (psi) & (psi) & (psi) \\
\hline 1 & Rate & 12.2 & 16.0 & 5552 & 6313 & 3702 & 5089 \\
\hline 2 & Acid 15\% HC & 15.0 & 16.0 & 6194 & 6321 & 6022 & 6187 \\
\hline 3 & PAD & 70.4 & 81.2 & 8779 & 8946 & 5878 & 7395 \\
\hline 4 & 0.25 PPA & 84.6 & 85.7 & 8440 & 8510 & 8245 & 8434 \\
\hline 5 & 0.5 PPA & 88.5 & 90.5 & 8598 & 8702 & 8452 & 8599 \\
\hline 6 & 0.75 PPA & 90.4 & 90.5 & 8731 & 8746 & 8696 & 8732 \\
\hline 7 & 1.00 PPA & 90.4 & 90.5 & 8748 & 8765 & 8730 & 8748 \\
\hline 8 & 1.50 PPA & 90.1 & 90.5 & 8734 & 8825 & 8501 & 8731 \\
\hline 9 & 2.00 PPA & 89.6 & 91.1 & 8811 & 8920 & 8730 & 8812 \\
\hline 10 & 0.50 PPA & 90.0 & 90.1 & 8897 & 9032 & 8783 & 8896 \\
\hline 11 & 0.75 PPA & 89.9 & 90.6 & 8749 & 8783 & 8726 & 8749 \\
\hline 12 & 1.00 PPA & 90.0 & 90.2 & 8758 & 8779 & 8740 & 8758 \\
\hline 13 & 1.50 PPA & 90.2 & 91.1 & 8823 & 8892 & 8752 & 8824 \\
\hline 14 & 2.00 PPA & 88.5 & 91.1 & 8932 & 9248 & 8687 & 8936 \\
\hline 15 & Flush & 64.5 & 74.8 & 9046 & 9258 & 6066 & 8902 \\
\hline
\end{tabular}

Table 30: Fracture Model - Overview Stage 3

\begin{tabular}{|c|c|c|}
\hline \multicolumn{3}{|c|}{ Stage 03} \\
\hline \multicolumn{3}{|c|}{ Treatment Summary } \\
\hline Formation & Marcellus & \\
\hline Formation Type & Shale & \\
\hline \multicolumn{3}{|c|}{$\begin{array}{c}\text { Stage Data } \\
\end{array}$} \\
\hline Report Date & $11 / 7 / 2015$ & \\
\hline Stage Length & 238.00 & $\mathrm{ft}$ \\
\hline Frac Gradient & 1.20 & $\mathrm{psi} / \mathrm{ft}$ \\
\hline ISIP & 5703.00 & psi \\
\hline 5 Min ISIP & 5242.00 & psi \\
\hline 10 Min ISIP & 5144.00 & psi \\
\hline 15 Min ISIP & 5070.00 & psi \\
\hline Breakdown Rate & 15.00 & bpm \\
\hline Breakdown Pressure & 6214.00 & psi \\
\hline Breakdown Volume & 315.00 & bbls \\
\hline Cut Short & $\mathrm{x}$ & $\mathrm{x}$ \\
\hline Screen Out & & $\mathrm{x}$ \\
\hline \multicolumn{3}{|c|}{ Fluid and Proppant Data } \\
\hline Total Clean Fluid & 8537.00 & bbls \\
\hline Prop.1-Type & 100 Mesh & \\
\hline Prop.2-Type & 40/70 White & \\
\hline Total Proppant Amount & 399320.00 & Ibs \\
\hline Proppant Concentration & 1677.82 & $\mathrm{lb} / \mathrm{ft}$ \\
\hline Proppant Concentration & 1.11 & $\mathrm{lb} / \mathrm{gal}$ \\
\hline \multicolumn{3}{|c|}{ Chemical Data } \\
\hline Acid \% Strength & 7.50 & \\
\hline Total Acid & 3000.00 & gal \\
\hline Total Slickwater & 8465.00 & bbls \\
\hline Total Linear & 0.00 & bbls \\
\hline Total XL & 0.00 & bbls \\
\hline FR Amount & 300.00 & gal \\
\hline Gel Amount & 0.00 & gal or Ibs \\
\hline Breaker Amount & 0.00 & gal or lbs \\
\hline Scale Inhibitor & 35.00 & gal \\
\hline Biocide & 95.00 & gal \\
\hline
\end{tabular}


Table 31: Fracture Model - Perfs Stage 3

\begin{tabular}{|ccccc|}
\hline \multicolumn{5}{c|}{ Perfs } \\
\hline Cluster & Start.FT & Stop.FT & Perf_Diam.IN & Perf_Shots. \\
\hline 5 & 13162.00 & 13164.00 & 0.42 & 10 \\
4 & 13212.00 & 13214.00 & 0.42 & 10 \\
3 & 13259.00 & 13261.00 & 0.42 & 10 \\
2 & 13305.00 & 13307.00 & 0.42 & 10 \\
1 & 13353.00 & 13355.00 & 0.42 & 10 \\
\hline
\end{tabular}

Table 32: Fracture Model - Pump Schedule Stage 3

\begin{tabular}{|c|c|c|c|c|c|c|c|c|c|c|c|}
\hline Stage & $\begin{array}{c}\text { Tot Time } \\
\mathrm{mm}: \mathrm{ss}\end{array}$ & $\begin{array}{c}\text { Stage Time } \\
\text { mm:ss }\end{array}$ & Fluid & $\begin{array}{c}\text { Clean Stg Vol } \\
\text { (gal) }\end{array}$ & $\begin{array}{c}\text { Cum Cln Vol } \\
\text { (gal) }\end{array}$ & Proppant & $\begin{array}{c}\text { Slurry Conc } \\
\text { (ppa) }\end{array}$ & $\begin{array}{c}\text { Cum Prop } \\
\text { (Ibs) }\end{array}$ & $\begin{array}{c}\text { Slurry Rate } \\
\text { (bbl/min) }\end{array}$ & $\begin{array}{c}\text { Cum Slurry } \\
\text { (bbl) }\end{array}$ & $\begin{array}{l}\text { Clean Rate } \\
\text { (bbl/min) }\end{array}$ \\
\hline 1 & 0.00 & 1.33 & SlickWater & 827.75 & 827.75 & $<$ None $>$ & 0.00 & 0.00 & 12.88 & 19.97 & 12.72 \\
\hline 2 & 1.33 & 2.18 & $\mathrm{HCl} 7.5 \%$ & 1509.20 & 2336.95 & $<$ None $>$ & 0.00 & 0.00 & 15.62 & 55.90 & 15.62 \\
\hline 3 & 3.51 & 3.12 & SlickWater & 2100.91 & 4437.86 & $<$ None $>$ & 0.00 & 0.00 & 15.63 & 105.92 & 15.63 \\
\hline 4 & 7.03 & 2.18 & $\mathrm{HCl} 7.5 \%$ & 1509.97 & 5947.83 & $<$ None $>$ & 0.00 & 0.00 & 15.63 & 141.88 & 15.63 \\
\hline 5 & 9.21 & 23.31 & SlickWater & 41982.75 & 47930.57 & $<$ None $>$ & 0.00 & 0.00 & 42.56 & 1142.71 & 42.51 \\
\hline 6 & 32.52 & 3.31 & SlickWater & 13172.24 & 61102.81 & 100 Mesh Sand & 0.24 & 3206.00 & 90.18 & 1459.84 & 89.18 \\
\hline 7 & 36.23 & 3.47 & SlickWater & 14011.75 & 75114.56 & 100 Mesh Sand & 0.49 & 10105.00 & 90.16 & 1800.93 & 88.18 \\
\hline 8 & 40.10 & 5.03 & SlickWater & 18501.27 & 93615.83 & 100 Mesh Sand & 0.74 & 23843.00 & 90.17 & 2256.29 & 87.23 \\
\hline 9 & 45.13 & 6.23 & SlickWater & 23130.53 & 116746.36 & 100 Mesh Sand & 0.99 & 46855.00 & 90.18 & 2831.92 & 86.28 \\
\hline 10 & 51.36 & 9.57 & SlickWater & 35282.16 & 152028.52 & 100 Mesh Sand & 1.50 & 99648.00 & 90.17 & 3729.06 & 84.43 \\
\hline 11 & 61.33 & 8.31 & SlickWater & 29575.61 & 181604.12 & 100 Mesh Sand & 1.99 & 158597.00 & 90.17 & 4496.98 & 82.68 \\
\hline 12 & 70.04 & 6.31 & SlickWater & 23989.60 & 205593.73 & 40/70 White & 0.53 & 171264.00 & 89.74 & 5081.75 & 87.65 \\
\hline 13 & 76.35 & 5.04 & SlickWater & 18555.38 & 224149.10 & 40/70 White & 0.74 & 185049.00 & 90.16 & 5538.58 & 87.20 \\
\hline 14 & 81.39 & 5.07 & SlickWater & 18531.43 & 242680.54 & 40/70 White & 1.00 & 203503.00 & 90.15 & 5999.83 & 86.23 \\
\hline 15 & 86.46 & 11.28 & SlickWater & 40648.12 & 283328.66 & 40/70 White & 1.49 & 264253.00 & 90.16 & 7033.70 & 84.40 \\
\hline 16 & 98.14 & 21.11 & SlickWater & 72235.74 & 355564.41 & 40/70 White & 1.94 & 404588.00 & 88.36 & 8905.44 & 81.19 \\
\hline 17 & 119.25 & 4.00 & SlickWater & 11139.32 & 366703.73 & $<$ None $>$ & 0.00 & 404588.00 & 66.16 & 9170.08 & 66.31 \\
\hline
\end{tabular}

Table 33: Fracture Model - Pump Details Stage 3

\begin{tabular}{|c|c|c|c|c|c|c|c|}
\hline Step\# & Name & Avg S Rate & Max S Rate & Avg Tr Pres & Max Tr Pres & Min Tr Pressure & Calc Avg TR P \\
\hline & & (bbl/min) & (bbl/min) & (psi) & (psi) & (psi) & (psi) \\
\hline 1 & Rate & 14.5 & 15.8 & 6409 & 6787 & 4020 & 6088 \\
\hline 2 & Acid 7.5\% HC & 15.6 & 15.7 & 6620 & 6746 & 6531 & 6614 \\
\hline 3 & Spacer & 15.6 & 15.7 & 6415 & 6524 & 6288 & 6410 \\
\hline 4 & Acid 7.5\% HC & 15.6 & 15.7 & 6288 & 6312 & 6264 & 6288 \\
\hline 5 & PAD & 63.6 & 90.3 & 8154 & 9113 & 6125 & 7347 \\
\hline 6 & 0.25 PPA & 90.2 & 90.3 & 8666 & 8676 & 8657 & 8667 \\
\hline 7 & 0.5 PPA & 90.2 & 90.2 & 8672 & 8687 & 8651 & 8672 \\
\hline 8 & 0.75 PPA & 90.2 & 90.3 & 8663 & 8693 & 8642 & 8663 \\
\hline 9 & 1.00 PPA & 90.2 & 90.3 & 8670 & 8693 & 8651 & 8670 \\
\hline 10 & 1.5 PPA & 90.2 & 90.3 & 8735 & 8822 & 8661 & 8736 \\
\hline 11 & 2.0 PPA & 90.2 & 90.3 & 8960 & 9130 & 8822 & 8963 \\
\hline 12 & .50 PPA & 89.7 & 90.3 & 9138 & 9216 & 9072 & 9138 \\
\hline 13 & .75 PPA & 90.2 & 90.3 & 9035 & 9091 & 9007 & 9035 \\
\hline 14 & 1.0 PPA & 90.2 & 90.3 & 9011 & 9032 & 8992 & 9011 \\
\hline 15 & 1.5 PPA & 90.2 & 90.3 & 9018 & 9055 & 8953 & 9018 \\
\hline 16 & 2.0 PPA & 88.4 & 90.3 & 8890 & 9188 & 8717 & 8890 \\
\hline 17 & Flush & 67.1 & 82.8 & 8922 & 9229 & 6678 & 8996 \\
\hline
\end{tabular}


Table 34: Fracture Model - Overview Stage 4

\begin{tabular}{|c|c|c|}
\hline \multicolumn{3}{|c|}{ Stage 04} \\
\hline \multicolumn{3}{|c|}{ Treatment Summary } \\
\hline Formation & Marcellus & \\
\hline Formation Type & Shale & \\
\hline \multicolumn{3}{|c|}{ Stage Data } \\
\hline Report Date & $11 / 7 / 2015$ & \\
\hline Stage Length & 230.00 & $\mathrm{ft}$ \\
\hline Frac Gradient & 1.24 & $\mathrm{psi} / \mathrm{ft}$ \\
\hline ISIP & 5978.00 & psi \\
\hline 5 Min ISIP & 5372.00 & psi \\
\hline 10 Min ISIP & 5256.00 & psi \\
\hline 15 Min ISIP & 5176.00 & psi \\
\hline Breakdown Rate & 15.00 & bpm \\
\hline Breakdown Pressure & 5940.00 & psi \\
\hline Breakdown Volume & 280.00 & bbls \\
\hline Cut Short & & $\mathrm{x}$ \\
\hline Screen Out & & $\mathrm{x}$ \\
\hline \multicolumn{3}{|c|}{ Fluid and Proppant Data } \\
\hline Total Clean Fluid & 9135.00 & bbls \\
\hline Prop.1-Type & 100 Mesh & \\
\hline Prop.2-Type & 40/70 White & \\
\hline Total Proppant Amount & 440060.00 & Ibs \\
\hline Proppant Concentration & 1913.30 & $\mathrm{lb} / \mathrm{ft}$ \\
\hline Proppant Concentration & 1.15 & $\mathrm{lb} / \mathrm{gal}$ \\
\hline \multicolumn{3}{|c|}{ Chemical Data } \\
\hline Acid \% Strength & 7.50 & \\
\hline Total Acid & 3000.00 & gal \\
\hline Total Slickwater & 9063.00 & bbls \\
\hline Total Linear & 0.00 & bbls \\
\hline Total XL & 0.00 & bbls \\
\hline FR Amount & 299.00 & gal \\
\hline Gel Amount & 0.00 & gal or lbs \\
\hline Breaker Amount & 0.00 & gal or lbs \\
\hline Scale Inhibitor & 40.00 & gal \\
\hline Biocide & 95.00 & gal \\
\hline
\end{tabular}

Table 35: Fracture Model - Perfs Stage 4

\begin{tabular}{|ccccc|}
\hline \multicolumn{5}{c|}{ Perfs } \\
\hline Cluster & Start.FT & Stop.FT & Perf_Diam.IN & Perf_Shots. \\
\hline 5 & 12932.00 & 12934.00 & 0.42 & 10 \\
4 & 12977.00 & 12979.00 & 0.42 & 10 \\
3 & 13025.00 & 13027.00 & 0.42 & 10 \\
2 & 13068.00 & 13070.00 & 0.42 & 10 \\
1 & 13118.00 & 13120.00 & 0.42 & 10 \\
\hline
\end{tabular}

Table 36: Fracture Model - Pump Schedule Stage 4

\begin{tabular}{|c|c|c|c|c|c|c|c|c|c|c|c|}
\hline Stage & $\begin{array}{c}\text { Tot Time } \\
\mathrm{mm}: \mathrm{ss}\end{array}$ & $\begin{array}{c}\text { Stage Time } \\
\text { mm:ss }\end{array}$ & Fluid & $\begin{array}{c}\text { Clean Stg Vol } \\
\text { (gal) }\end{array}$ & $\begin{array}{c}\text { Cum Cln Vol } \\
\text { (gal) }\end{array}$ & Proppant & $\begin{array}{l}\text { Slurry Conc } \\
\text { (ppa) }\end{array}$ & $\begin{array}{l}\text { Cum Prop } \\
\text { (lbs) }\end{array}$ & $\begin{array}{l}\text { Slurry Rate } \\
\text { (bbl/min) }\end{array}$ & $\begin{array}{l}\text { Cum Slurry } \\
\text { (bbl) }\end{array}$ & $\begin{array}{l}\text { Clean Rate } \\
\text { (bbl/min) }\end{array}$ \\
\hline 1 & 0.00 & 2.56 & SlickWater & 824.88 & 824.88 & $<$ None $>$ & 0.00 & 0.00 & 6.78 & 19.89 & 6.70 \\
\hline 2 & 2.56 & 2.20 & $\mathrm{HCl} 7.5 \%$ & 1511.37 & 2336.25 & $<$ None $>$ & 0.00 & 0.00 & 15.43 & 55.89 & 15.42 \\
\hline 3 & 5.16 & 3.15 & SlickWater & 2105.46 & 4441.71 & $<$ None $>$ & 0.00 & 0.00 & 15.42 & 106.01 & 15.42 \\
\hline 4 & 8.31 & 2.20 & $\mathrm{HCl} 7.5 \%$ & 1512.84 & 5954.55 & $<$ None $>$ & 0.00 & 0.00 & 15.44 & 142.03 & 15.44 \\
\hline 5 & 10.51 & 22.52 & SlickWater & 41971.85 & 47926.40 & $<$ None $>$ & 0.00 & 0.00 & 43.76 & 1142.62 & 43.70 \\
\hline 6 & 33.43 & 3.31 & SlickWater & 13234.97 & 61161.36 & 100 Mesh Sand & 0.24 & 3205.00 & 90.60 & 1461.22 & 89.61 \\
\hline 7 & 37.14 & 3.45 & SlickWater & 13955.96 & 75117.33 & 100 Mesh Sand & 0.49 & 10070.00 & 90.59 & 1800.95 & 88.61 \\
\hline 8 & 40.59 & 5.02 & SlickWater & 18523.04 & 93640.36 & 100 Mesh Sand & 0.74 & 23814.00 & 90.58 & 2256.86 & 87.62 \\
\hline 9 & 46.01 & 6.21 & SlickWater & 23113.98 & 116754.35 & 100 Mesh Sand & 0.99 & 46802.00 & 90.58 & 2832.06 & 86.67 \\
\hline 10 & 52.22 & 9.54 & SlickWater & 35266.49 & 152020.84 & 100 Mesh Sand & 1.50 & 99585.00 & 90.58 & 3728.81 & 84.82 \\
\hline 11 & 62.16 & 7.52 & SlickWater & 27443.78 & 179464.61 & 100 Mesh Sand & 1.99 & 154177.00 & 90.58 & 4441.39 & 83.06 \\
\hline 12 & 70.08 & 6.28 & SlickWater & 24032.04 & 203496.65 & 40/70 White & 0.53 & 166842.00 & 90.59 & 5027.23 & 88.48 \\
\hline 13 & 76.36 & 5.02 & SlickWater & 18517.31 & 222013.96 & 40/70 White & 0.75 & 180657.00 & 90.57 & 5483.13 & 87.59 \\
\hline 14 & 81.38 & 5.06 & SlickWater & 18557.96 & 240571.92 & 40/70 White & 1.00 & 199143.00 & 90.58 & 5945.08 & 86.64 \\
\hline 15 & 86.44 & 9.06 & SlickWater & 32414.56 & 272986.48 & 40/70 White & 1.49 & 247526.00 & 90.59 & 6769.47 & 84.81 \\
\hline 16 & 95.50 & 27.41 & SlickWater & 95048.85 & 368035.34 & 40/70 White & 2.00 & 437390.00 & 89.19 & 9238.47 & 81.75 \\
\hline 17 & 123.31 & 1.02 & SlickWater & 3522.05 & 371557.39 & 40/70 White & 1.47 & 442570.00 & 86.42 & 9327.76 & 81.15 \\
\hline 18 & 123.31 & 3.29 & SlickWater & 12340.79 & 383898.18 & $<$ None $>$ & 0.00 & 442570.00 & 83.94 & 9620.16 & 84.35 \\
\hline
\end{tabular}


Table 37: Fracture Model - Pump Details Stage 4

\begin{tabular}{|c|c|c|c|c|c|c|c|}
\hline Step\# & Name & Avg S Rate & Max S Rate & Avg Tr Pres & Max Tr Pres & Min Tr Pressure & Calc Avg TR P \\
\hline & & (bbl/min) & (bbl/min) & (psi) & (psi) & (psi) & (psi) \\
\hline 1 & Rate & 7.4 & 10.6 & 5372 & 6072 & 3933 & 5229 \\
\hline 2 & Acid 7.5\% HC & 15.4 & 15.5 & 6094 & 6246 & 6011 & 6089 \\
\hline 3 & Spacer & 15.4 & 15.5 & 5966 & 6010 & 5948 & 5965 \\
\hline 4 & Acid 7.5\% HC & 15.4 & 15.5 & 5935 & 5953 & 5915 & 5934 \\
\hline 5 & PAD & 90.6 & 91.4 & 8631 & 8951 & 5133 & 7294 \\
\hline 6 & 0.25 PPA & 90.6 & 90.8 & 8640 & 8657 & 8616 & 8640 \\
\hline 7 & 0.5 PPA & 90.6 & 90.8 & 8606 & 8632 & 8584 & 8605 \\
\hline 8 & 0.75 PPA & 90.6 & 90.7 & 8576 & 8597 & 8553 & 8577 \\
\hline 9 & 1.00 PPA & 90.6 & 90.7 & 8583 & 8594 & 8563 & 8583 \\
\hline 10 & 1.5 PPA & 90.6 & 90.7 & 8580 & 8606 & 8528 & 8580 \\
\hline 11 & 2.0 PPA & 90.6 & 90.7 & 8621 & 8709 & 8553 & 8622 \\
\hline 12 & .50 PPA & 90.6 & 90.7 & 8724 & 8849 & 8633 & 8723 \\
\hline 13 & .75 PPA & 90.6 & 90.7 & 8585 & 8637 & 8553 & 8584 \\
\hline 14 & 1.00 PPA & 90.6 & 90.7 & 8563 & 8587 & 8542 & 8563 \\
\hline 15 & 1.5 PPA & 90.6 & 90.7 & 8589 & 8624 & 8537 & 8590 \\
\hline 16 & 2.00 PPA & 89.2 & 90.7 & 8898 & 9195 & 8620 & 8899 \\
\hline 17 & 2.5 PPA & 86.4 & 86.5 & 8872 & 8912 & 8859 & 8875 \\
\hline 18 & Flush & 85.7 & 86.5 & 8990 & 9138 & 5507 & 8875 \\
\hline
\end{tabular}

Table 38: Fracture Model - Overview Stage 5

\begin{tabular}{|c|c|c|}
\hline \multicolumn{3}{|c|}{ Stage 05} \\
\hline \multicolumn{3}{|c|}{ Treatment Summary } \\
\hline Formation & Marcellus & \\
\hline Formation Type & Shale & \\
\hline \multicolumn{3}{|c|}{ Stage Data } \\
\hline Report Date & $11 / 8 / 2015$ & \\
\hline Stage Length & 234.00 & $\mathrm{ft}$ \\
\hline Frac Gradient & $\# \mathrm{~N} / \mathrm{A}$ & $\mathrm{psi} / \mathrm{ft}$ \\
\hline ISIP & $\# \mathrm{~N} / \mathrm{A}$ & psi \\
\hline 5 Min ISIP & $\# \mathrm{~N} / \mathrm{A}$ & psi \\
\hline 10 Min ISIP & $\# \mathrm{~N} / \mathrm{A}$ & psi \\
\hline 15 Min ISIP & $\# \mathrm{~N} / \mathrm{A}$ & psi \\
\hline Breakdown Rate & 15.00 & bpm \\
\hline Breakdown Pressure & 6222.00 & psi \\
\hline Breakdown Volume & 286.00 & bbls \\
\hline Cut Short & $x$ & $x$ \\
\hline Screen Out & $x$ & $x$ \\
\hline \multicolumn{3}{|c|}{ Fluid and Proppant Data } \\
\hline Total Clean Fluid & 8037.00 & bbls \\
\hline Prop.1-Type & 100 Mesh & \\
\hline Prop.2-Type & 40/70 White & \\
\hline Total Proppant Amount & 337620.00 & Ibs \\
\hline Proppant Concentration & 1442.82 & $\mathrm{Ib} / \mathrm{ft}$ \\
\hline Proppant Concentration & 1.00 & $\mathrm{lb} / \mathrm{gal}$ \\
\hline \multicolumn{3}{|c|}{ Chemical Data } \\
\hline Acid \% Strength & 7.50 & \\
\hline Total Acid & 3000.00 & gal \\
\hline Total Slickwater & 7966.00 & bbls \\
\hline Total Linear & 0.00 & bbls \\
\hline Total XL & 0.00 & bbls \\
\hline FR Amount & 240.00 & gal \\
\hline Gel Amount & 0.00 & gal or lbs \\
\hline Breaker Amount & 0.00 & gal or lbs \\
\hline Scale Inhibitor & 35.00 & gal \\
\hline Biocide & 85.00 & gal \\
\hline
\end{tabular}

Table 39: Fracture Model - Perfs Stage 5

\begin{tabular}{|ccccc|}
\hline \multicolumn{5}{c|}{ Perfs } \\
\hline Cluster & Start.FT & Stop.FT & Perf_Diam.IN & Perf_Shots. \\
\hline 5 & 12697.00 & 12699.00 & 0.42 & 10 \\
4 & 12746.00 & 12748.00 & 0.42 & 10 \\
3 & 12793.00 & 12795.00 & 0.42 & 10 \\
2 & 12837.00 & 12839.00 & 0.42 & 10 \\
1 & 12884.00 & 12886.00 & 0.42 & 10 \\
\hline
\end{tabular}


Table 40: Fracture Model - Pump Schedule Stage 5

\begin{tabular}{|c|c|c|c|c|c|c|c|c|c|c|c|}
\hline Stage & $\begin{array}{c}\text { Tot Time } \\
\text { mm:ss }\end{array}$ & $\begin{array}{c}\text { Stage Time } \\
\text { mm:ss }\end{array}$ & Fluid & $\begin{array}{c}\text { Clean Stg Vol } \\
\text { (gal) }\end{array}$ & $\begin{array}{c}\text { Cum Cln Vol } \\
\text { (gal) }\end{array}$ & Proppant & $\begin{array}{c}\text { Slurry Conc } \\
\text { (ppa) }\end{array}$ & $\begin{array}{c}\text { Cum Prop } \\
\text { (Ibs) }\end{array}$ & $\begin{array}{c}\text { Slurry Rate } \\
\text { (bbl/min) }\end{array}$ & $\begin{array}{c}\text { Cum Slurry } \\
\text { (bbl) }\end{array}$ & $\begin{array}{l}\text { Clean Rate } \\
\text { (bbl/min) }\end{array}$ \\
\hline 1 & 0.00 & 1.38 & SlickWater & 833.49 & 833.49 & $<$ None $>$ & 0.00 & 0.00 & 12.31 & 20.10 & 12.15 \\
\hline 2 & 1.38 & 3.19 & $\mathrm{HCl} 7.5 \%$ & 2158.24 & 2991.73 & $<$ None $>$ & 0.00 & 0.00 & 15.49 & 71.49 & 15.49 \\
\hline 3 & 3.19 & 21.07 & Slickwater & 26605.97 & 29597.70 & $<$ None $>$ & 0.00 & 0.00 & 29.99 & 704.71 & 30.00 \\
\hline 4 & 21.07 & 10.11 & Slickwater & 33589.98 & 63187.68 & $<$ None $>$ & 0.00 & 0.00 & 78.68 & 1505.97 & 78.54 \\
\hline 5 & 10.11 & 3.32 & Slickwater & 13204.03 & 76391.71 & 100 Mesh Sand & 0.24 & 3206.00 & 89.97 & 1823.85 & 88.98 \\
\hline 6 & 3.32 & 3.47 & Slickwater & 13984.39 & 90376.11 & 100 Mesh Sand & 0.49 & 10088.00 & 89.98 & 2164.27 & 88.01 \\
\hline 7 & 3.47 & 5.04 & Slickwater & 18521.57 & 108897.68 & 100 Mesh Sand & 0.74 & 23830.00 & 89.97 & 2620.14 & 87.04 \\
\hline 8 & 5.04 & 6.24 & Slickwater & 23138.27 & 132035.94 & 100 Mesh Sand & 0.99 & 46851.00 & 89.97 & 3195.95 & 86.08 \\
\hline 9 & 6.24 & 9.58 & Slickwater & 35264.54 & 167300.48 & 100 Mesh Sand & 1.50 & 99616.00 & 89.97 & 4092.62 & 84.24 \\
\hline 10 & 9.58 & 6.24 & Slickwater & 22172.59 & 189473.07 & 100 Mesh Sand & 1.99 & 143774.00 & 89.95 & 4668.31 & 82.49 \\
\hline 11 & 6.24 & 6.30 & Slickwater & 23989.16 & 213462.23 & 40/70 White & 0.53 & 156401.00 & 89.96 & 5253.05 & 87.87 \\
\hline 12 & 6.30 & 5.04 & Slickwater & 18515.14 & 231977.37 & 40/70 White & 0.75 & 170228.00 & 89.97 & 5708.90 & 87.01 \\
\hline 13 & 5.04 & 5.08 & Slickwater & 18551.75 & 250529.12 & 40/70 White & 1.00 & 188712.00 & 89.95 & 6170.65 & 86.05 \\
\hline 14 & 5.08 & 9.09 & Slickwater & 32365.25 & 282894.37 & 40/70 White & 1.49 & 237097.00 & 89.96 & 6993.78 & 84.22 \\
\hline 15 & 9.09 & 14.20 & Slickwater & 48579.70 & 331474.07 & 40/70 White & 1.89 & 329111.00 & 87.63 & 8249.82 & 80.70 \\
\hline 16 & 14.20 & 2.05 & Slickwater & 5023.83 & 336497.90 & $<$ None $>$ & 0.00 & 329111.00 & 56.83 & 8368.23 & 57.42 \\
\hline
\end{tabular}

Table 41: Fracture Model - Pump Details Stage 5

\begin{tabular}{|c|c|c|c|c|c|c|c|}
\hline Step\# & Name & Avg S Rate & Max S Rate & Avg Tr Pres & Max Tr Pres & Min Tr Pressure & Calc Avg TR P \\
\hline & & (bbl/min) & (bbl/min) & (psi) & (psi) & (psi) & (psi) \\
\hline 1 & Rate & 14.5 & 15.5 & 5283 & 5711 & 3747 & 5044 \\
\hline 2 & $7.5 \%$ HC & 15.5 & 15.5 & 5936 & 5996 & 5722 & 5922 \\
\hline 3 & PAD & 52.5 & 90.1 & 7501 & 8847 & 30 & 6601 \\
\hline 4 & PAD & 85.7 & 90.1 & 8475 & 8753 & 4468 & 8194 \\
\hline 5 & 0.25 PPA & 90.0 & 90.1 & 8596 & 8630 & 8548 & 8594 \\
\hline 6 & 0.5 PPA & 90.0 & 90.1 & 8496 & 8547 & 8457 & 8495 \\
\hline 7 & 0.75 PPA & 90.0 & 90.1 & 8460 & 8480 & 8441 & 8460 \\
\hline 8 & 1.00 PPA & 90.0 & 90.1 & 8443 & 8459 & 8425 & 8443 \\
\hline 9 & 1.5 PPA & 90.0 & 90.1 & 8455 & 8490 & 8409 & 8456 \\
\hline 10 & 2.0 PPA & 90.0 & 90.1 & 8531 & 8651 & 8465 & 8533 \\
\hline 11 & .50 PPA & 90.0 & 90.1 & 8768 & 8902 & 8651 & 8769 \\
\hline 12 & .75 PPA & 90.0 & 90.0 & 8674 & 8709 & 8619 & 8673 \\
\hline 13 & 1.0 PPA & 90.0 & 90.1 & 8601 & 8621 & 8582 & 8601 \\
\hline 14 & 1.5 PPA & 90.0 & 90.1 & 8634 & 8701 & 8571 & 8635 \\
\hline 15 & 2.00 PPA & 87.9 & 90.1 & 8797 & 9039 & 8646 & 8797 \\
\hline 16 & Flush & 53.7 & 72.7 & 8616 & 9498 & 6711 & 8884 \\
\hline
\end{tabular}

Table 42: Fracture Model - Overview Stage 6

\begin{tabular}{|c|c|c|}
\hline \multicolumn{3}{|c|}{ Stage 06} \\
\hline \multicolumn{3}{|c|}{ Treatment Summary } \\
\hline Formation & Marcellus & \\
\hline Formation Type & Shale & \\
\hline \multicolumn{3}{|c|}{ Stage Data } \\
\hline Report Date & $11 / 8 / 2015$ & \\
\hline Stage Length & 245.00 & $\mathrm{ft}$ \\
\hline Frac Gradient & 1.27 & $\mathrm{psi} / \mathrm{ft}$ \\
\hline ISIP & 6205.00 & psi \\
\hline $5 \mathrm{Min} I S I P$ & 5469.00 & psi \\
\hline 10 Min ISIP & 5348.00 & psi \\
\hline 15 Min ISIP & 5266.00 & psi \\
\hline Breakdown Rate & 15.00 & bpm \\
\hline Breakdown Pressure & 6005.00 & psi \\
\hline Breakdown Volume & 185.00 & bbls \\
\hline Cut Short & & $\mathrm{x}$ \\
\hline Screen Out & & $x$ \\
\hline \multicolumn{3}{|c|}{ Fluid and Proppant Data } \\
\hline Total Clean Fluid & 9544.00 & bbls \\
\hline Prop.1-Type & 100 Mesh & \\
\hline Prop.2-Type & 40/70 White & \\
\hline Total Proppant Amount & 440180.00 & Ibs \\
\hline Proppant Concentration & 1796.65 & $\mathrm{lb} / \mathrm{ft}$ \\
\hline Proppant Concentration & 1.10 & $\mathrm{lb} / \mathrm{gal}$ \\
\hline \multicolumn{3}{|c|}{ Chemical Data } \\
\hline Acid \% Strength & 7.50 & \\
\hline Total Acid & 3000.00 & gal \\
\hline Total Slickwater & 9473.00 & bbls \\
\hline Total Linear & 0.00 & bbls \\
\hline Total XL & 0.00 & bbls \\
\hline FR Amount & 350.00 & gal \\
\hline Gel Amount & 0.00 & gal or lbs \\
\hline Breaker Amount & 0.00 & gal or lbs \\
\hline Scale Inhibitor & 35.00 & gal \\
\hline Biocide & 95.00 & gal \\
\hline
\end{tabular}


Table 43: Fracture Model - Perfs Stage 6

\begin{tabular}{|ccccc|}
\hline \multicolumn{5}{c|}{ Perfs } \\
\hline Cluster & Start.FT & Stop.FT & Perf_Diam.IN & Perf_Shots. \\
\hline 5 & 12452.00 & 12454.00 & 0.42 & 10 \\
4 & 12512.00 & 12514.00 & 0.42 & 10 \\
3 & 12558.00 & 12560.00 & 0.42 & 10 \\
2 & 12606.00 & 12608.00 & 0.42 & 10 \\
1 & 12650.00 & 12652.00 & 0.42 & 10 \\
\hline
\end{tabular}

Table 44: Fracture Model - Pump Schedule Stage 6

\begin{tabular}{|c|c|c|c|c|c|c|c|c|c|c|c|}
\hline Stage & $\begin{array}{c}\text { Tot Time } \\
\mathrm{mm}: \mathrm{ss}\end{array}$ & $\begin{array}{c}\text { Stage Time } \\
\text { mm:ss }\end{array}$ & Fluid & $\begin{array}{c}\text { Clean Stg Vol } \\
\text { (gal) }\end{array}$ & $\begin{array}{c}\text { Cum Cln Vol } \\
\text { (gal) }\end{array}$ & Proppant & $\begin{array}{c}\text { Slurry Conc } \\
\text { (ppa) }\end{array}$ & $\begin{array}{c}\text { Cum Prop } \\
\text { (Ibs) }\end{array}$ & $\begin{array}{c}\text { Slurry Rate } \\
\text { (bbl/min) }\end{array}$ & $\begin{array}{c}\text { Cum Slurry } \\
\text { (bbl) }\end{array}$ & $\begin{array}{l}\text { Clean Rate } \\
\text { (bbl/min) }\end{array}$ \\
\hline 1 & 0.00 & 1.34 & SlickWater & 833.56 & 833.56 & $<$ None $>$ & 0.00 & 0.00 & 12.83 & 20.10 & 12.67 \\
\hline 2 & 1.34 & 4.38 & $\mathrm{HCl} 7.5 \%$ & 2994.18 & 3827.74 & $<$ None $>$ & 0.00 & 0.00 & 15.39 & 91.39 & 15.39 \\
\hline 3 & 6.12 & 33.18 & SlickWater & 71354.42 & 75182.15 & $<$ None $>$ & 0.00 & 0.00 & 51.06 & 1791.55 & 51.02 \\
\hline 4 & 39.30 & 3.31 & SlickWater & 13155.59 & 88337.74 & 100 Mesh Sand & 0.24 & 3200.00 & 90.06 & 2108.25 & 89.07 \\
\hline 5 & 43.01 & 3.48 & SlickWater & 14003.16 & 102340.90 & 100 Mesh Sand & 0.49 & 10129.00 & 89.69 & 2449.06 & 87.74 \\
\hline 6 & 46.49 & 5.04 & SlickWater & 18571.05 & 120911.95 & 100 Mesh Sand & 0.74 & 23948.00 & 90.21 & 2906.15 & 87.27 \\
\hline 7 & 51.53 & 6.23 & SlickWater & 23097.38 & 144009.33 & 100 Mesh Sand & 1.00 & 46934.00 & 90.05 & 3480.95 & 86.15 \\
\hline 8 & 58.16 & 9.57 & SlickWater & 35244.37 & 179253.70 & 100 Mesh Sand & 1.50 & 99663.00 & 90.07 & 4377.15 & 84.34 \\
\hline 9 & 68.13 & 7.54 & SlickWater & 27398.13 & 206651.82 & 100 Mesh Sand & 1.99 & 154268.00 & 90.04 & 5088.49 & 82.57 \\
\hline 10 & 76.07 & 6.30 & SlickWater & 24013.28 & 230665.10 & 40/70 White & 0.53 & 166933.00 & 90.06 & 5673.91 & 87.96 \\
\hline 11 & 82.37 & 5.03 & SlickWater & 18470.47 & 249135.56 & 40/70 White & 0.75 & 180720.00 & 90.05 & 6128.64 & 87.08 \\
\hline 12 & 87.40 & 5.07 & SlickWater & 18511.62 & 267647.18 & 40/70 White & 1.00 & 199153.00 & 90.05 & 6589.42 & 86.14 \\
\hline 13 & 92.47 & 9.09 & SlickWater & 32401.23 & 300048.41 & 40/70 White & 1.49 & 247566.00 & 90.05 & 7413.42 & 84.31 \\
\hline 14 & 101.56 & 15.37 & SlickWater & 54139.58 & 354187.99 & 40/70 White & 2.00 & 355829.00 & 90.06 & 8819.79 & 82.54 \\
\hline 15 & 117.33 & 10.23 & SlickWater & 34329.40 & 388517.39 & 40/70 White & 2.42 & 438921.00 & 87.38 & 9727.11 & 78.72 \\
\hline 16 & 127.56 & 4.09 & SlickWater & 12265.83 & 400783.22 & $<$ None $>$ & 0.00 & 438921.00 & 70.04 & 10017.76 & 70.37 \\
\hline
\end{tabular}

Table 45: Fracture Model - Pump Details Stage 6

\begin{tabular}{|c|c|c|c|c|c|c|c|}
\hline Step\# & Name & Avg S Rate & Max S Rate & Avg Tr Pres & Max Tr Pres & Min Tr Pressure & Calc Avg TR P \\
\hline & & (bbl/min) & (bbl/min) & (psi) & (psi) & (psi) & (psi) \\
\hline 1 & Rate & 14.5 & 15.4 & 5497 & 5848 & 3954 & 5313 \\
\hline 2 & Acid 7.5\% HC & 15.4 & 15.5 & 5967 & 6002 & 5856 & 5968 \\
\hline 3 & PAD & 90.0 & 90.8 & 8431 & 9002 & 5096 & 7173 \\
\hline 4 & 0.25 PPA & 90.1 & 90.1 & 8401 & 8424 & 8381 & 8401 \\
\hline 5 & 0.5 PPA & 89.7 & 91.0 & 8373 & 8402 & 8349 & 8372 \\
\hline 6 & 0.75 PPA & 90.2 & 91.1 & 8372 & 8388 & 8342 & 8372 \\
\hline 7 & 1.00 PPA & 90.0 & 90.1 & 8384 & 8400 & 8367 & 8384 \\
\hline 8 & 1.5 PPA & 90.1 & 90.2 & 8405 & 8456 & 8342 & 8406 \\
\hline 9 & 2.0 PPA & 90.0 & 90.2 & 8537 & 8651 & 8434 & 8539 \\
\hline 10 & .50 PPA & 90.1 & 90.2 & 8650 & 8791 & 8539 & 8648 \\
\hline 11 & .75 PPA & 90.1 & 90.2 & 8520 & 8549 & 8501 & 8520 \\
\hline 12 & 1.0 PPA & 90.0 & 90.2 & 8516 & 8533 & 8498 & 8516 \\
\hline 13 & 1.5 PPA & 90.0 & 90.1 & 8557 & 8641 & 8490 & 8558 \\
\hline 14 & 2.00 PPA & 90.1 & 90.2 & 8703 & 8889 & 8594 & 8704 \\
\hline 15 & 2.5 PPA & 87.5 & 90.2 & 8725 & 9142 & 8355 & 8719 \\
\hline 16 & Flush & 74.6 & 83.7 & 8974 & 9272 & 6879 & 8919 \\
\hline
\end{tabular}


Table 46: Fracture Model - Overview Stage 7

\begin{tabular}{|c|c|c|}
\hline \multicolumn{3}{|c|}{ Stage 07} \\
\hline \multicolumn{3}{|c|}{ Treatment Summary } \\
\hline Formation & Marcellus & \\
\hline Formation Type & Shale & \\
\hline \multicolumn{3}{|c|}{ Stage Data } \\
\hline Report Date & $11 / 9 / 2015$ & \\
\hline Stage Length & 224.00 & $\mathrm{ft}$ \\
\hline Frac Gradient & 1.22 & $\mathrm{psi} / \mathrm{ft}$ \\
\hline ISIP & 5867.00 & psi \\
\hline 5 Min ISIP & 5538.00 & psi \\
\hline $10 \mathrm{Min}$ ISIP & 5418.00 & psi \\
\hline 15 Min ISIP & 5340.00 & psi \\
\hline Breakdown Rate & 15.00 & bpm \\
\hline Breakdown Pressure & 6053.00 & psi \\
\hline Breakdown Volume & 288.00 & bbls \\
\hline Cut Short & & $x$ \\
\hline Screen Out & & $x$ \\
\hline \multicolumn{3}{|c|}{ Fluid and Proppant Data } \\
\hline Total Clean Fluid & 9212.00 & bbls \\
\hline Prop.1-Type & 100 Mesh & \\
\hline Prop.2-Type & 40/70 White & \\
\hline Total Proppant Amount & 440880.00 & Ibs \\
\hline Proppant Concentration & 1968.21 & $\mathrm{lb} / \mathrm{ft}$ \\
\hline Proppant Concentration & 1.14 & $\mathrm{Ib} / \mathrm{gal}$ \\
\hline \multicolumn{3}{|c|}{ Chemical Data } \\
\hline Acid \% Strength & 7.50 & \\
\hline Total Acid & 3000.00 & gal \\
\hline Total Slickwater & 9140.00 & bbls \\
\hline Total Linear & 0.00 & bbls \\
\hline Total XL & 0.00 & bbls \\
\hline FR Amount & 261.00 & gal \\
\hline Gel Amount & 0.00 & gal or Ibs \\
\hline Breaker Amount & 0.00 & gal or lbs \\
\hline Scale Inhibitor & 40.00 & gal \\
\hline Biocide & 116.00 & gal \\
\hline
\end{tabular}

Table 47: Fracture Model - Perfs Stage 7

\begin{tabular}{|ccccc|}
\hline \multicolumn{5}{|c|}{ Perfs } \\
\hline Cluster & Start.FT & Stop.FT & Perf_Diam.IN & Perf_Shots. \\
\hline 5 & 12239.00 & 12241.00 & 0.42 & 10 \\
4 & 12285.00 & 12287.00 & 0.42 & 10 \\
3 & 12325.00 & 12327.00 & 0.42 & 10 \\
2 & 12367.00 & 12369.00 & 0.42 & 10 \\
1 & 12418.00 & 12420.00 & 0.42 & 10 \\
\hline
\end{tabular}

Table 48: Fracture Model - Pump Schedule Stage 7

\begin{tabular}{|c|c|c|c|c|c|c|c|c|c|c|c|}
\hline Stage & $\begin{array}{c}\text { Tot Time } \\
\mathrm{mm}: \mathrm{ss}\end{array}$ & $\begin{array}{c}\text { Stage Time } \\
\text { mm:ss }\end{array}$ & Fluid & $\begin{array}{c}\text { Clean Stg Vol } \\
\text { (gal) }\end{array}$ & $\begin{array}{c}\text { Cum Cln Vol } \\
\text { (gal) }\end{array}$ & Proppant & $\begin{array}{c}\text { Slurry Conc } \\
\text { (ppa) }\end{array}$ & $\begin{array}{c}\text { Cum Prop } \\
\text { (lbs) }\end{array}$ & $\begin{array}{c}\text { Slurry Rate } \\
\text { (bbl/min) }\end{array}$ & $\begin{array}{c}\text { Cum Slurry } \\
\text { (bbl) }\end{array}$ & $\begin{array}{l}\text { Clean Rate } \\
\text { (bbl/min) }\end{array}$ \\
\hline 1 & 0.00 & 1.03 & SlickWater & 401.10 & 401.10 & $<$ None $>$ & 0.00 & 0.00 & 9.33 & 9.80 & 9.10 \\
\hline 2 & 1.03 & 4.47 & $\mathrm{HCl} 7.5 \%$ & 2999.63 & 3400.73 & $<$ None $>$ & 0.00 & 0.00 & 14.93 & 81.22 & 14.93 \\
\hline 3 & 5.50 & 19.37 & SlickWater & 24934.54 & 28335.27 & $<$ None $>$ & 0.00 & 0.00 & 30.32 & 676.05 & 30.26 \\
\hline 4 & 25.27 & 6.02 & SlickWater & 22016.57 & 50351.85 & 100 Mesh Sand & 0.25 & 5434.00 & 87.86 & 1206.14 & 86.88 \\
\hline 5 & 31.29 & 8.55 & SlickWater & 33057.66 & 83409.51 & 100 Mesh Sand & 0.50 & 21845.00 & 90.27 & 2011.00 & 88.27 \\
\hline 6 & 40.24 & 10.00 & SlickWater & 36676.71 & 120086.22 & 100 Mesh Sand & 0.75 & 49247.00 & 90.29 & 2913.92 & 87.33 \\
\hline 7 & 50.24 & 12.44 & SlickWater & 46193.70 & 166279.92 & 100 Mesh Sand & 1.00 & 95415.00 & 90.30 & 4063.71 & 86.38 \\
\hline 8 & 63.08 & 11.23 & SlickWater & 40351.31 & 206631.23 & 100 Mesh Sand & 1.49 & 155737.00 & 90.13 & 5089.70 & 84.40 \\
\hline 9 & 74.31 & 2.25 & SlickWater & 8451.24 & 215082.48 & 100 Mesh Sand & 1.86 & 171454.00 & 90.31 & 5307.94 & 83.26 \\
\hline 10 & 76.56 & 10.27 & SlickWater & 36589.52 & 251671.99 & 100 Mesh Sand & 1.75 & 235554.00 & 90.00 & 6248.43 & 83.37 \\
\hline 11 & 87.23 & 8.20 & SlickWater & 28829.24 & 280501.23 & 100 Mesh Sand & 2.00 & 293162.00 & 89.84 & 6997.12 & 82.37 \\
\hline 12 & 95.43 & 4.35 & SlickWater & 15566.39 & 296067.62 & 100 Mesh Sand & 2.49 & 331924.00 & 90.01 & 7409.69 & 80.86 \\
\hline 13 & 100.18 & 2.00 & SlickWater & 7342.86 & 303410.48 & 40/70 White & 0.62 & 336447.00 & 89.77 & 7589.24 & 87.41 \\
\hline 14 & 102.18 & 3.01 & SlickWater & 10966.42 & 314376.89 & 40/70 White & 0.74 & 344590.00 & 89.49 & 7859.19 & 86.55 \\
\hline 15 & 105.19 & 4.10 & SlickWater & 15022.64 & 329399.53 & 40/70 White & 0.99 & 359518.00 & 89.74 & 8233.11 & 85.84 \\
\hline 16 & 109.29 & 4.15 & SlickWater & 15014.16 & 344413.69 & 40/70 White & 1.49 & 381853.00 & 89.82 & 8614.86 & 84.11 \\
\hline 17 & 113.44 & 5.39 & SlickWater & 19577.39 & 363991.08 & 40/70 White & 1.99 & 420823.00 & 89.99 & 9123.32 & 82.50 \\
\hline 18 & 119.23 & 1.28 & SlickWater & 4981.27 & 368972.35 & 40/70 White & 2.47 & 433107.00 & 89.99 & 9255.30 & 80.86 \\
\hline 19 & 120.51 & 0.54 & SlickWater & 2996.07 & 371968.42 & 40/70 White & 2.95 & 441952.00 & 89.97 & 9336.28 & 79.26 \\
\hline 20 & 121.45 & 4.10 & SlickWater & 15068.83 & 387037.25 & $<$ None $>$ & 0.28 & 446204.00 & 86.83 & 9698.08 & 86.11 \\
\hline
\end{tabular}


Table 49: Fracture Model - Pump Details Stage 7

\begin{tabular}{|c|c|c|c|c|c|c|c|}
\hline Step\# & Name & Avg S Rate & Max S Rate & Avg Tr Pres & Max Tr Pres & Min Tr Pressure & Calc Avg TR P \\
\hline & & (bbl/min) & (bbl/min) & (psi) & (psi) & (psi) & (psi) \\
\hline 1 & Rate & 12.5 & 15.3 & 5640 & 6489 & 3952 & 5203 \\
\hline 2 & Acid 7.5\% HC & 14.9 & 15.0 & 6212 & 6589 & 5937 & 6204 \\
\hline 3 & PAD & 53.8 & 85.5 & 7549 & 9210 & 5808 & 6523 \\
\hline 4 & 0.25 PPA & 88.0 & 91.7 & 8249 & 8601 & 8026 & 8241 \\
\hline 5 & 0.5 PPA & 90.3 & 90.5 & 8247 & 8275 & 8220 & 8247 \\
\hline 6 & 0.75 PPA & 90.3 & 90.7 & 8255 & 8283 & 8222 & 8256 \\
\hline 7 & 1.00 PPA & 90.3 & 90.6 & 8286 & 8321 & 8228 & 8285 \\
\hline 8 & 1.50 PPA & 90.1 & 90.8 & 8318 & 8368 & 8215 & 8316 \\
\hline 9 & 2.00 PPA & 90.3 & 90.7 & 8342 & 8358 & 8309 & 8339 \\
\hline 10 & 1.75 PPA & 90.0 & 90.6 & 8416 & 8509 & 8311 & 8416 \\
\hline 11 & 2.00 PPA & 89.8 & 90.7 & 8541 & 8598 & 8441 & 8539 \\
\hline 12 & 2.50 PPA & 90.0 & 90.5 & 8612 & 8691 & 8528 & 8610 \\
\hline 13 & 0.50 PPA & 89.8 & 90.5 & 8717 & 8807 & 8669 & 8722 \\
\hline 14 & 0.75 PPA & 89.5 & 89.8 & 8719 & 8860 & 8535 & 8713 \\
\hline 15 & 1.00 PPA & 89.7 & 90.0 & 8529 & 8544 & 8514 & 8530 \\
\hline 16 & 1.50 PPA & 89.8 & 90.2 & 8519 & 8550 & 8475 & 8518 \\
\hline 17 & 2.00 PPA & 90.0 & 90.3 & 8568 & 8630 & 8520 & 8568 \\
\hline 18 & 2.5 PPA & 90.0 & 90.4 & 8532 & 8593 & 8447 & 8524 \\
\hline 19 & 3.0 PPA & 90.0 & 90.1 & 8410 & 8446 & 8346 & 8401 \\
\hline 20 & Flush & 88.5 & 89.7 & 8435 & 8721 & 5531 & 8315 \\
\hline
\end{tabular}

Table 50: Fracture Model - Overview Stage

\begin{tabular}{|c|c|c|}
\hline \multicolumn{3}{|c|}{ Stage 08} \\
\hline \multicolumn{3}{|c|}{ Treatment Summary } \\
\hline Formation & Marcellus & \\
\hline Formation Type & Shale & \\
\hline \multicolumn{3}{|c|}{ Stage Data } \\
\hline Report Date & $11 / 9 / 2015$ & \\
\hline Stage Length & 222.00 & $\mathrm{ft}$ \\
\hline Frac Gradient & 1.24 & $\mathrm{psi} / \mathrm{ft}$ \\
\hline ISIP & 5992.00 & psi \\
\hline 5 Min ISIP & 5624.00 & psi \\
\hline 10 Min ISIP & 5505.00 & psi \\
\hline 15 Min ISIP & 5419.00 & psi \\
\hline Breakdown Rate & 15.00 & bpm \\
\hline Breakdown Pressure & 6458.00 & psi \\
\hline Breakdown Volume & 167.00 & bbls \\
\hline Cut Short & & $\mathrm{X}$ \\
\hline Screen Out & & $x$ \\
\hline \multicolumn{3}{|c|}{ Fluid and Proppant Data } \\
\hline Total Clean Fluid & 8773.00 & bbls \\
\hline Prop.1-Type & 100 Mesh & \\
\hline Prop.2-Type & 40/70 White & \\
\hline Total Proppant Amount & 440500.00 & Ibs \\
\hline Proppant Concentration & 1984.23 & $\mathrm{lb} / \mathrm{ft}$ \\
\hline Proppant Concentration & 1.20 & lb/gal \\
\hline \multicolumn{3}{|c|}{ Chemical Data } \\
\hline Acid \% Strength & 7.50 & \\
\hline Total Acid & 3000.00 & gal \\
\hline Total Slickwater & 8702.00 & bbls \\
\hline Total Linear & 0.00 & bbls \\
\hline Total XL & 0.00 & bbls \\
\hline FR Amount & 262.00 & gal \\
\hline Gel Amount & 0.00 & gal or Ibs \\
\hline Breaker Amount & 0.00 & gal or Ibs \\
\hline Scale Inhibitor & 37.00 & gal \\
\hline Biocide & 110.00 & gal \\
\hline
\end{tabular}

Table 51: Fracture Model - Perfs Stage 8

\begin{tabular}{|ccccc|}
\hline \multicolumn{5}{c|}{ Perfs } \\
\hline Cluster & Start.FT & Stop.FT & Perf_Diam.IN & Perf_Shots. \\
\hline 5 & 12015.00 & 12017.00 & 0.42 & 10 \\
4 & 12058.00 & 12060.00 & 0.42 & 10 \\
3 & 12098.00 & 12100.00 & 0.42 & 10 \\
2 & 12143.00 & 12145.00 & 0.42 & 10 \\
1 & 12194.00 & 12196.00 & 0.42 & 10 \\
\hline
\end{tabular}


Table 52: Fracture Model - Pump Schedule Stage 8

\begin{tabular}{|c|c|c|c|c|c|c|c|c|c|c|c|}
\hline Stage & $\begin{array}{c}\text { Tot Time } \\
\text { mm:ss }\end{array}$ & $\begin{array}{c}\text { Stage Time } \\
\text { mm:ss }\end{array}$ & Fluid & $\begin{array}{c}\text { Clean Stg Vol } \\
\text { (gal) }\end{array}$ & $\begin{array}{c}\text { Cum Cln Vol } \\
\text { (gal) }\end{array}$ & Proppant & $\begin{array}{c}\text { Slurry Conc } \\
\text { (ppa) }\end{array}$ & $\begin{array}{c}\text { Cum Prop } \\
\text { (Ibs) }\end{array}$ & $\begin{array}{c}\text { Slurry Rate } \\
\text { (bbl/min) }\end{array}$ & $\begin{array}{c}\text { Cum Slurry } \\
\text { (bbl) }\end{array}$ & $\begin{array}{l}\text { Clean Rate } \\
\text { (bbl/min) }\end{array}$ \\
\hline 1 & 0.00 & 1.42 & SlickWater & 829.78 & 829.78 & $<$ None $>$ & 0.00 & 0.00 & 11.77 & 20.01 & 11.62 \\
\hline 2 & 1.42 & 4.46 & $\mathrm{HCl} 7.5 \%$ & 3003.35 & 3833.13 & $<$ None $>$ & 0.00 & 0.00 & 15.00 & 91.52 & 15.00 \\
\hline 3 & 6.28 & 17.39 & SlickWater & 24919.86 & 28752.99 & <None $>$ & 0.00 & 0.00 & 33.70 & 686.24 & 33.62 \\
\hline 4 & 24.07 & 5.19 & SlickWater & 21996.30 & 50749.28 & 100 Mesh Sand & 0.25 & 5589.00 & 99.65 & 1216.06 & 98.51 \\
\hline 5 & 29.26 & 4.00 & SlickWater & 16405.90 & 67155.18 & 100 Mesh Sand & 0.50 & 13749.00 & 99.86 & 1615.51 & 97.65 \\
\hline 6 & 33.26 & 4.56 & SlickWater & 20018.03 & 87173.21 & 100 Mesh Sand & 0.74 & 28579.00 & 99.87 & 2108.18 & 96.61 \\
\hline 7 & 38.22 & 11.32 & SlickWater & 46266.93 & 133440.13 & 100 Mesh Sand & 1.00 & 74694.00 & 99.84 & 3259.63 & 95.51 \\
\hline 8 & 49.54 & 12.01 & SlickWater & 47180.21 & 180620.34 & 100 Mesh Sand & 1.50 & 145335.00 & 99.84 & 4459.33 & 93.48 \\
\hline 9 & 61.55 & 11.41 & SlickWater & 45025.09 & 225645.43 & 100 Mesh Sand & 1.75 & 224065.00 & 99.04 & 5616.42 & 91.76 \\
\hline 10 & 73.36 & 7.47 & SlickWater & 28779.87 & 254425.30 & 100 Mesh Sand & 2.00 & 281523.00 & 96.02 & 6363.75 & 88.04 \\
\hline 11 & 81.23 & 5.46 & SlickWater & 20928.76 & 275354.06 & 100 Mesh Sand & 2.49 & 333715.00 & 96.19 & 6918.44 & 86.41 \\
\hline 12 & 87.09 & 1.52 & SlickWater & 7326.76 & 282680.83 & 40/70 White & 0.62 & 338290.00 & 96.07 & 7097.76 & 93.45 \\
\hline 13 & 89.01 & 2.52 & SlickWater & 11026.26 & 293707.08 & 40/70 White & 0.79 & 347009.00 & 94.89 & 7369.77 & 91.58 \\
\hline 14 & 91.53 & 3.47 & SlickWater & 14976.01 & 308683.09 & 40/70 White & 1.00 & 361935.00 & 98.54 & 7742.60 & 94.25 \\
\hline 15 & 95.40 & 3.52 & SlickWater & 15020.18 & 323703.28 & 40/70 White & 1.49 & 384271.00 & 98.75 & 8124.44 & 92.49 \\
\hline 16 & 99.32 & 5.14 & SlickWater & 19508.80 & 343212.08 & 40/70 White & 1.99 & 423095.00 & 96.81 & 8631.05 & 88.76 \\
\hline 17 & 104.46 & 1.44 & SlickWater & 6329.82 & 349541.90 & 40/70 White & 2.47 & 438710.00 & 96.73 & 8798.71 & 86.95 \\
\hline 18 & 106.30 & 1.51 & SlickWater & 7244.16 & 356786.06 & 40/70 White & 1.34 & 448439.00 & 98.91 & 8981.70 & 93.23 \\
\hline 19 & 108.21 & 2.58 & SlickWater & 11653.60 & 368439.65 & $<$ None $>$ & 0.00 & 448439.00 & 92.98 & 9257.53 & 93.53 \\
\hline
\end{tabular}

Table 53: Fracture Model - Pump Details Stage 8

\begin{tabular}{|c|c|c|c|c|c|c|c|}
\hline Step\# & Name & Avg S Rate & Max S Rate & Avg Tr Pres & Max Tr Pres & Min Tr Pressure & Calc Avg TR P \\
\hline & & $(\mathbf{b b l} / \mathbf{m i n})$ & $\mathbf{( b b l / m i n )}$ & (psi) & (psi) & (psi) & (psi) \\
\hline 1 & Rate & 13.8 & 15.0 & 5470 & 5833 & 4303 & 5270 \\
\hline 2 & Acid 7.5\% HC & 15.0 & 15.1 & 6028 & 6106 & 5839 & 6032 \\
\hline 3 & PAD & 57.2 & 98.2 & 7996 & 9143 & 6028 & 6965 \\
\hline 4 & 0.25 PPA & 99.6 & 100.0 & 8991 & 9089 & 8900 & 8990 \\
\hline 5 & 0.5 PPA & 99.9 & 100.0 & 8877 & 8902 & 8855 & 8878 \\
\hline 6 & 0.75 PPA & 99.9 & 100.0 & 8913 & 8948 & 8893 & 8914 \\
\hline 7 & 1.00 PPA & 99.8 & 100.0 & 8951 & 8988 & 8915 & 8952 \\
\hline 8 & 1.5 PPA & 99.8 & 100.0 & 9058 & 9150 & 8944 & 9059 \\
\hline 9 & 1.75 PPA & 99.1 & 100.0 & 9153 & 9197 & 9124 & 9153 \\
\hline 10 & 2.0 PPA & 96.1 & 98.3 & 9066 & 9200 & 8361 & 9059 \\
\hline 11 & 2.5 PPA & 96.2 & 96.6 & 9127 & 9195 & 9059 & 9127 \\
\hline 12 & .50 PPA & 96.1 & 96.2 & 9139 & 9190 & 9096 & 9141 \\
\hline 13 & .75 PPA & 94.9 & 96.8 & 9040 & 9219 & 8889 & 9036 \\
\hline 14 & 1.0 PPA & 98.5 & 99.2 & 9129 & 9188 & 9004 & 9132 \\
\hline 15 & 1.50 PPA & 98.8 & 99.2 & 9171 & 9203 & 9135 & 9170 \\
\hline 16 & 2.00 PPA & 96.8 & 97.9 & 9123 & 9182 & 9047 & 9122 \\
\hline 17 & 2.5 PPA & 96.7 & 96.9 & 8937 & 9074 & 8777 & 8926 \\
\hline 18 & 3.0 PPA & 98.8 & 99.3 & 8904 & 9037 & 8770 & 8914 \\
\hline 19 & Flush & 95.6 & 99.3 & 9059 & 9167 & 6093 & 8900 \\
\hline
\end{tabular}


Table 54: Fracture Model - Overview Stage 9

\begin{tabular}{|c|c|c|}
\hline \multicolumn{3}{|c|}{ Stage 09} \\
\hline \multicolumn{3}{|c|}{ Treatment Summary } \\
\hline Formation & Marcellus & \\
\hline Formation Type & Shale & \\
\hline \multicolumn{3}{|c|}{ Stage Data } \\
\hline Report Date & $11 / 9 / 2015$ & \\
\hline Stage Length & 237.00 & $\mathrm{ft}$ \\
\hline Frac Gradient & 1.27 & $\mathrm{psi} / \mathrm{ft}$ \\
\hline ISIP & 6210.00 & psi \\
\hline 5 Min ISIP & 5600.00 & psi \\
\hline 10 Min ISIP & 5455.00 & psi \\
\hline 15 Min ISIP & 5363.00 & psi \\
\hline Breakdown Rate & 15.00 & bpm \\
\hline Breakdown Pressure & 6553.00 & psi \\
\hline Breakdown Volume & 254.00 & bbls \\
\hline Cut Short & & $x$ \\
\hline Screen Out & & $\mathrm{x}$ \\
\hline \multicolumn{3}{|c|}{ Fluid and Proppant Data } \\
\hline Total Clean Fluid & 8696.00 & bbls \\
\hline Prop.1-Type & 100 Mesh & \\
\hline Prop.2-Type & 40/70 White & \\
\hline Total Proppant Amount & 440960.00 & Ibs \\
\hline Proppant Concentration & 1860.59 & $\mathrm{lb} / \mathrm{ft}$ \\
\hline Proppant Concentration & 1.21 & $\mathrm{Ib} / \mathrm{gal}$ \\
\hline \multicolumn{3}{|c|}{ Chemical Data } \\
\hline Acid \% Strength & 7.50 & \\
\hline Total Acid & 3000.00 & gal \\
\hline Total Slickwater & 8622.00 & bbls \\
\hline Total Linear & 0.00 & bbls \\
\hline Total XL & 0.00 & bbls \\
\hline FR Amount & 305.00 & gal \\
\hline Gel Amount & 0.00 & gal or Ibs \\
\hline Breaker Amount & 0.00 & gal or Ibs \\
\hline Scale Inhibitor & 36.00 & gal \\
\hline Biocide & 91.00 & gal \\
\hline
\end{tabular}

Table 55: Fracture Model - Perfs Stage 9

\begin{tabular}{|ccccc|}
\hline \multicolumn{5}{c|}{ Perfs } \\
\hline Cluster & Start.FT & Stop.FT & Perf_Diam.IN & Perf_Shots. \\
\hline 5 & 11782.00 & 11784.00 & 0.42 & 10 \\
4 & 11828.00 & 11830.00 & 0.42 & 10 \\
3 & 11880.00 & 11882.00 & 0.42 & 10 \\
2 & 11925.00 & 11927.00 & 0.42 & 10 \\
1 & 11971.00 & 11973.00 & 0.42 & 10 \\
\hline
\end{tabular}

Table 56: Fracture Model - Pump Schedule Stage 9

\begin{tabular}{|c|c|c|c|c|c|c|c|c|c|c|c|}
\hline Stage & $\begin{array}{c}\text { Tot Time } \\
\mathrm{mm}: \mathrm{ss}\end{array}$ & $\begin{array}{c}\text { Stage Time } \\
\text { mm:ss }\end{array}$ & Fluid & $\begin{array}{c}\text { Clean Stg Vol } \\
\text { (gal) }\end{array}$ & $\begin{array}{c}\text { Cum Cln Vol } \\
\text { (gal) }\end{array}$ & Proppant & $\begin{array}{l}\text { Slurry Conc } \\
\text { (ppa) }\end{array}$ & $\begin{array}{l}\text { Cum Prop } \\
\text { (lbs) }\end{array}$ & $\begin{array}{l}\text { Slurry Rate } \\
\text { (bbl/min) }\end{array}$ & $\begin{array}{c}\text { Cum Slurry } \\
\text { (bbl) }\end{array}$ & $\begin{array}{l}\text { Clean Rate } \\
\text { (bbl/min) }\end{array}$ \\
\hline 1 & 0.00 & 1.29 & SlickWater & 830.97 & 830.97 & $<$ None $>$ & 0.00 & 0.00 & 13.51 & 20.04 & 13.34 \\
\hline 2 & 1.29 & 2.18 & $\mathrm{HCl} 7.5 \%$ & 1513.47 & 2344.44 & $<$ None $>$ & 0.00 & 0.00 & 15.67 & 56.08 & 15.67 \\
\hline 3 & 3.47 & 3.11 & SlickWater & 2095.94 & 4440.38 & $<$ None $>$ & 0.00 & 0.00 & 15.68 & 105.98 & 15.68 \\
\hline 4 & 6.58 & 2.18 & $\mathrm{HCl} 7.5 \%$ & 1513.40 & 5953.78 & $<$ None $>$ & 0.00 & 0.00 & 15.67 & 142.02 & 15.67 \\
\hline 5 & 9.16 & 14.31 & SlickWater & 24952.28 & 30906.06 & $<$ None $>$ & 0.00 & 0.00 & 41.02 & 737.51 & 40.93 \\
\hline 6 & 23.47 & 5.19 & SlickWater & 21965.39 & 52871.45 & 100 Mesh Sand & 0.25 & 5403.00 & 99.47 & 1266.37 & 98.37 \\
\hline 7 & 29.06 & 4.20 & SlickWater & 17722.34 & 70593.79 & 100 Mesh Sand & 0.50 & 14179.00 & 99.57 & 1697.86 & 97.38 \\
\hline 8 & 33.26 & 4.48 & SlickWater & 19431.31 & 90025.10 & 100 Mesh Sand & 0.74 & 28578.00 & 99.65 & 2176.18 & 96.39 \\
\hline 9 & 38.14 & 11.33 & SlickWater & 46240.85 & 136265.94 & 100 Mesh Sand & 1.00 & 74701.00 & 99.64 & 3327.05 & 95.32 \\
\hline 10 & 49.47 & 10.18 & SlickWater & 40362.56 & 176628.51 & 100 Mesh Sand & 1.50 & 135098.00 & 99.65 & 4353.41 & 93.30 \\
\hline 11 & 60.05 & 11.49 & SlickWater & 44936.65 & 221565.15 & 100 Mesh Sand & 1.75 & 213691.00 & 97.73 & 5508.31 & 90.54 \\
\hline 12 & 71.54 & 7.33 & SlickWater & 28935.07 & 250500.22 & 100 Mesh Sand & 2.00 & 271436.00 & 99.52 & 6259.68 & 91.25 \\
\hline 13 & 79.27 & 6.19 & SlickWater & 23714.20 & 274214.42 & 100 Mesh Sand & 2.49 & 330429.00 & 99.49 & 6888.10 & 89.39 \\
\hline 14 & 85.46 & 1.51 & SlickWater & 7380.66 & 281595.08 & 40/70 White & 0.62 & 335001.00 & 97.53 & 7068.53 & 94.99 \\
\hline 15 & 87.37 & 2.51 & SlickWater & 11012.75 & 292607.83 & 40/70 White & 0.79 & 343689.00 & 95.32 & 7340.20 & 92.00 \\
\hline 16 & 90.28 & 3.48 & SlickWater & 14979.09 & 307586.92 & 40/70 White & 1.00 & 358600.00 & 98.09 & 7712.95 & 93.85 \\
\hline 17 & 94.16 & 3.51 & SlickWater & 15023.33 & 322610.26 & 40/70 White & 1.49 & 380922.00 & 99.21 & 8094.90 & 92.91 \\
\hline 18 & 98.07 & 4.00 & SlickWater & 15266.22 & 337876.48 & 40/70 White & 1.99 & 411247.00 & 99.10 & 8491.29 & 90.87 \\
\hline 19 & 102.07 & 1.24 & SlickWater & 5170.48 & 343046.96 & 40/70 White & 2.46 & 423958.00 & 97.82 & 8628.24 & 87.93 \\
\hline 20 & 103.31 & 2.57 & SlickWater & 10894.38 & 353941.34 & 40/70 White & 2.39 & 450026.00 & 97.42 & 8915.64 & 87.93 \\
\hline 21 & 103.31 & 3.12 & SlickWater & 11443.46 & 365384.80 & $<$ None $>$ & 0.00 & 450026.00 & 84.64 & 9186.49 & 85.14 \\
\hline
\end{tabular}


Table 57: Fracture Model - Pump Details Stage 9

\begin{tabular}{|c|c|c|c|c|c|c|c|}
\hline Step\# & Name & Avg S Rate & Max S Rate & Avg Tr Pres & Max Tr Pres & Min Tr Pressure & Calc Avg TR P \\
\hline & & (bbl/min) & (bbl/min) & (psi) & (psi) & (psi) & (psi) \\
\hline 1 & Rate & 14.8 & 15.7 & 5373 & 5766 & 4017 & 5245 \\
\hline 2 & Acid 7.5\% HC & 15.7 & 15.7 & 5964 & 6081 & 5772 & 5972 \\
\hline 3 & spacer & 15.7 & 15.7 & 6026 & 6057 & 6008 & 6026 \\
\hline 4 & Acid 7.5\% HC & 15.7 & 15.7 & 6063 & 6085 & 6041 & 6064 \\
\hline 5 & PAD & 99.5 & 99.6 & 8995 & 9125 & 6017 & 7348 \\
\hline 6 & 0.25 PPA & 99.5 & 99.6 & 8963 & 9002 & 8919 & 8962 \\
\hline 7 & 0.5 PPA & 99.6 & 99.8 & 8883 & 8919 & 8858 & 8883 \\
\hline 8 & 0.75 PPA & 99.6 & 99.8 & 8875 & 8892 & 8857 & 8874 \\
\hline 9 & 1.00 PPA & 99.6 & 99.8 & 8901 & 8956 & 8860 & 8902 \\
\hline 10 & 1.5 PPA & 99.7 & 99.8 & 8988 & 9080 & 8907 & 8989 \\
\hline 11 & 1.75 PPA & 97.8 & 99.9 & 8965 & 9240 & 8620 & 8961 \\
\hline 12 & 2.0 PPA & 99.5 & 99.7 & 8940 & 8991 & 8889 & 8941 \\
\hline 13 & 2.5 PPA & 99.5 & 99.7 & 8980 & 9182 & 8885 & 8982 \\
\hline 14 & .50 PPA & 97.7 & 99.5 & 9164 & 9195 & 9124 & 9163 \\
\hline 15 & .75 PPA & 95.3 & 97.0 & 9087 & 9182 & 9018 & 9084 \\
\hline 16 & 1.0 PPA & 98.1 & 99.4 & 9075 & 9113 & 9030 & 9076 \\
\hline 17 & 1.50 PPA & 99.2 & 99.4 & 9125 & 9169 & 9095 & 9126 \\
\hline 18 & 2.00 PPA & 99.1 & 99.4 & 9157 & 9194 & 9131 & 9157 \\
\hline 19 & 2.5 PPA & 97.8 & 98.0 & 9166 & 9177 & 9147 & 9165 \\
\hline 20 & 3.0 PPA & 97.5 & 97.8 & 9151 & 9184 & 9122 & 9152 \\
\hline
\end{tabular}

Table 58: Fracture Model - Overview Stage 10

\begin{tabular}{|c|c|c|}
\hline \multicolumn{3}{|c|}{ Stage 10} \\
\hline \multicolumn{3}{|c|}{ Treatment Summary } \\
\hline Formation & Marcellus & \\
\hline Formation Type & Shale & \\
\hline \multicolumn{3}{|c|}{ Stage Data } \\
\hline Report Date & $11 / 9 / 2015$ & \\
\hline Stage Length & 227.00 & $\mathrm{ft}$ \\
\hline Frac Gradient & 1.27 & $\mathrm{psi} / \mathrm{ft}$ \\
\hline ISIP & 6241.00 & psi \\
\hline 5 Min ISIP & 5730.00 & psi \\
\hline 10 Min ISIP & 5515.00 & psi \\
\hline 15 Min ISIP & 5390.00 & psi \\
\hline Breakdown Rate & 15.00 & bpm \\
\hline Breakdown Pressure & 6785.00 & psi \\
\hline Breakdown Volume & 254.00 & bbls \\
\hline Cut Short & & $\mathrm{X}$ \\
\hline Screen Out & & $\mathrm{x}$ \\
\hline \multicolumn{3}{|c|}{ Fluid and Proppant Data } \\
\hline Total Clean Fluid & 8408.00 & bbls \\
\hline Prop.1-Type & 100 Mesh & \\
\hline Prop.2-Type & 40/70 White & \\
\hline Total Proppant Amount & 440020.00 & Ibs \\
\hline Proppant Concentration & 1938.41 & $\mathrm{lb} / \mathrm{ft}$ \\
\hline Proppant Concentration & 1.25 & $\mathrm{lb} / \mathrm{gal}$ \\
\hline \multicolumn{3}{|c|}{ Chemical Data } \\
\hline Acid \% Strength & 7.50 & \\
\hline Total Acid & 3000.00 & gal \\
\hline Total Slickwater & 8336.00 & bbls \\
\hline Total Linear & 0.00 & bbls \\
\hline Total XL & 0.00 & bbls \\
\hline FR Amount & 249.00 & gal \\
\hline Gel Amount & 0.00 & gal or lbs \\
\hline Breaker Amount & 0.00 & gal or lbs \\
\hline Scale Inhibitor & 40.00 & gal \\
\hline Biocide & 108.00 & gal \\
\hline
\end{tabular}

Table 59: Fracture Model - Perfs Stage 10

\begin{tabular}{|ccccc|}
\hline \multicolumn{5}{|c|}{ Perfs } \\
\hline Cluster & Start.FT & Stop.FT & Perf_Diam.IN & Perf_Shots. \\
\hline 5 & 11557.00 & 11559.00 & 0.42 & 10 \\
4 & 11601.00 & 11603.00 & 0.42 & 10 \\
3 & 11649.00 & 11651.00 & 0.42 & 10 \\
2 & 11695.00 & 11697.00 & 0.42 & 10 \\
1 & 11735.00 & 11737.00 & 0.42 & 10 \\
\hline
\end{tabular}


Table 60: Fracture Model - Pump Schedule Stage 10

\begin{tabular}{|c|c|c|c|c|c|c|c|c|c|c|c|}
\hline Stage & $\begin{array}{c}\text { Tot Time } \\
\text { mm:ss }\end{array}$ & $\begin{array}{c}\text { Stage Time } \\
\text { mm:ss }\end{array}$ & Fluid & $\begin{array}{c}\text { Clean Stg Vol } \\
\text { (gal) }\end{array}$ & $\begin{array}{c}\text { Cum Cln Vol } \\
\text { (gal) }\end{array}$ & Proppant & $\begin{array}{c}\text { Slurry Conc } \\
\text { (ppa) }\end{array}$ & $\begin{array}{c}\text { Cum Prop } \\
\text { (Ibs) }\end{array}$ & $\begin{array}{c}\text { Slurry Rate } \\
\text { (bbl/min) }\end{array}$ & $\begin{array}{c}\text { Cum Slurry } \\
\text { (bbl) }\end{array}$ & $\begin{array}{l}\text { Clean Rate } \\
\text { (bbl/min) }\end{array}$ \\
\hline 1 & 0.00 & 1.10 & SlickWater & 424.13 & 424.13 & $<$ None $>$ & 0.00 & 0.00 & 8.88 & 10.36 & 8.66 \\
\hline 2 & 1.10 & 2.23 & $\mathrm{HCl} 7.5 \%$ & 1508.01 & 1932.14 & $<$ None $>$ & 0.00 & 0.00 & 15.06 & 46.25 & 15.07 \\
\hline 3 & 3.33 & 3.19 & SlickWater & 2094.33 & 4026.47 & <None $>$ & 0.00 & 0.00 & 15.04 & 96.12 & 15.03 \\
\hline 4 & 6.52 & 2.23 & $\mathrm{HCl} 7.5 \%$ & 1505.63 & 5532.10 & $<$ None $>$ & 0.00 & 0.00 & 15.04 & 131.97 & 15.04 \\
\hline 5 & 9.15 & 15.56 & SlickWater & 24913.66 & 30445.76 & $<$ None $>$ & 0.00 & 0.00 & 37.30 & 726.31 & 37.23 \\
\hline 6 & 25.11 & 4.58 & SlickWater & 20357.81 & 50803.57 & 100 Mesh Sand & 0.25 & 5015.00 & 98.74 & 1216.73 & 97.59 \\
\hline 7 & 30.09 & 3.16 & SlickWater & 13464.70 & 64268.26 & 100 Mesh Sand & 0.49 & 11598.00 & 100.33 & 1544.46 & 98.14 \\
\hline 8 & 33.25 & 3.11 & SlickWater & 12976.67 & 77244.93 & 100 Mesh Sand & 0.74 & 21177.00 & 100.32 & 1863.80 & 97.06 \\
\hline 9 & 36.36 & 11.28 & SlickWater & 46229.10 & 123474.03 & 100 Mesh Sand & 1.00 & 67211.00 & 100.33 & 3014.27 & 95.99 \\
\hline 10 & 48.04 & 10.14 & SlickWater & 40376.26 & 163850.29 & 100 Mesh Sand & 1.50 & 127594.00 & 100.32 & 4040.93 & 93.94 \\
\hline 11 & 58.18 & 11.31 & SlickWater & 44955.40 & 208805.69 & 100 Mesh Sand & 1.75 & 206153.00 & 100.31 & 5196.21 & 92.94 \\
\hline 12 & 69.49 & 11.08 & SlickWater & 43008.49 & 251814.18 & 100 Mesh Sand & 2.00 & 292058.00 & 100.32 & 6313.08 & 91.98 \\
\hline 13 & 80.57 & 3.59 & SlickWater & 15078.34 & 266892.52 & 100 Mesh Sand & 2.49 & 329608.00 & 100.33 & 6712.72 & 90.13 \\
\hline 14 & 84.56 & 1.48 & SlickWater & 7384.30 & 274276.82 & 40/70 White & 0.62 & 334187.00 & 100.32 & 6893.29 & 97.68 \\
\hline 15 & 86.44 & 1.56 & SlickWater & 7882.07 & 282158.89 & 40/70 White & 0.73 & 339979.00 & 100.33 & 7087.26 & 97.07 \\
\hline 16 & 88.40 & 3.44 & SlickWater & 15050.22 & 297209.10 & 40/70 White & 0.99 & 354896.00 & 100.32 & 7461.80 & 95.98 \\
\hline 17 & 92.24 & 3.48 & SlickWater & 14994.36 & 312203.47 & 40/70 White & 1.49 & 377215.00 & 100.33 & 7843.07 & 93.95 \\
\hline 18 & 96.12 & 3.57 & SlickWater & 15258.19 & 327461.66 & 40/70 White & 1.99 & 407546.00 & 100.31 & 8239.31 & 91.97 \\
\hline 19 & 100.09 & 0.55 & SlickWater & 3474.87 & 330936.53 & 40/70 White & 2.43 & 415979.00 & 100.28 & 8331.24 & 90.26 \\
\hline 20 & 101.04 & 2.57 & SlickWater & 11209.52 & 342146.05 & 40/70 White & 2.42 & 443054.00 & 100.34 & 8627.24 & 90.47 \\
\hline 21 & 101.04 & 2.42 & SlickWater & 10930.57 & 353076.62 & $<$ None $>$ & 0.00 & 443054.00 & 95.78 & 8885.84 & 96.39 \\
\hline
\end{tabular}

Table 61: Fracture Model - Pump Details Stage 10

\begin{tabular}{|c|c|c|c|c|c|c|c|}
\hline Step\# & Name & Avg S Rate & Max S Rate & Avg Tr Pres & Max Tr Pres & Min Tr Pressure & Calc Avg TR P \\
\hline & & (bbl/min) & (bbl/min) & (psi) & (psi) & (psi) & (psi) \\
\hline 1 & Rate & 11.4 & 15.6 & 5268 & 5778 & 4447 & 5050 \\
\hline 2 & Acid 7.5\% HC & 15.1 & 15.6 & 6031 & 6171 & 5787 & 6042 \\
\hline 3 & Spacer & 15.0 & 15.1 & 6199 & 6212 & 6174 & 6200 \\
\hline 4 & Acid 7.5\% HC & 15.0 & 15.1 & 6241 & 6261 & 6214 & 6242 \\
\hline 5 & PAD & 87.5 & 89.2 & 8729 & 8905 & 6140 & 7247 \\
\hline 6 & 0.25 PPA & 98.7 & 100.4 & 8226 & 8749 & 8106 & 8222 \\
\hline 7 & 0.5 PPA & 100.3 & 100.4 & 8107 & 8126 & 8095 & 8107 \\
\hline 8 & 0.75 PPA & 100.3 & 100.4 & 8117 & 8128 & 8106 & 8117 \\
\hline 9 & 1.00 PPA & 100.3 & 100.5 & 8134 & 8175 & 8103 & 8134 \\
\hline 10 & 1.50 PPA & 100.3 & 100.4 & 8209 & 8269 & 8154 & 8210 \\
\hline 11 & $1.75 P P A$ & 100.3 & 100.5 & 8315 & 8347 & 8265 & 8315 \\
\hline 12 & 2.00 PPA & 100.3 & 100.5 & 8349 & 8382 & 8312 & 8349 \\
\hline 13 & 2.50 PPA & 100.3 & 100.4 & 8384 & 8408 & 8366 & 8385 \\
\hline 14 & 0.50 PPA & 100.3 & 100.4 & 8407 & 8448 & 8377 & 8409 \\
\hline 15 & 0.75 PPA & 100.3 & 100.4 & 8410 & 8482 & 8312 & 8406 \\
\hline 16 & 1.00 PPA & 100.3 & 100.4 & 8261 & 8315 & 8223 & 8259 \\
\hline 17 & 1.50 PPA & 100.3 & 100.5 & 8191 & 8232 & 8158 & 8191 \\
\hline 18 & 2.00 PPA & 100.3 & 100.4 & 8171 & 8198 & 8136 & 8171 \\
\hline 19 & 2.50 PPA & 100.3 & 100.4 & 8199 & 8208 & 8187 & 8199 \\
\hline 20 & 3.00 PPA & 100.3 & 100.5 & 8180 & 8234 & 8154 & 8181 \\
\hline 21 & Flush & 99.3 & 100.4 & 8370 & 8578 & 5706 & 8242 \\
\hline
\end{tabular}


Table 62: Fracture Model - Overview Stage 11

\begin{tabular}{|c|c|c|}
\hline \multicolumn{3}{|c|}{ Stage 11} \\
\hline \multicolumn{3}{|c|}{ Treatment Summary } \\
\hline Formation & Marcellus & \\
\hline Formation Type & Shale & \\
\hline \multicolumn{3}{|c|}{ Stage Data } \\
\hline Report Date & $11 / 11 / 2015$ & \\
\hline Stage Length & 232.00 & $\mathrm{ft}$ \\
\hline Frac Gradient & 1.24 & $\mathrm{psi} / \mathrm{ft}$ \\
\hline ISIP & 6022.00 & psi \\
\hline 5 Min ISIP & 5510.00 & psi \\
\hline 10 Min ISIP & 5277.00 & psi \\
\hline 15 Min ISIP & 5116.00 & psi \\
\hline Breakdown Rate & 15.00 & bpm \\
\hline Breakdown Pressure & 5930.00 & psi \\
\hline Breakdown Volume & 255.00 & bbls \\
\hline Cut Short & & $\mathrm{x}$ \\
\hline Screen Out & & $\mathrm{x}$ \\
\hline \multicolumn{3}{|c|}{ Fluid and Proppant Data } \\
\hline Total Clean Fluid & 8052.00 & bbls \\
\hline Prop.1-Type & 100 Mesh & \\
\hline Prop.2-Type & 40/70 White & \\
\hline Total Proppant Amount & 440520.00 & Ibs \\
\hline Proppant Concentration & 1898.79 & $\mathrm{lb} / \mathrm{ft}$ \\
\hline Proppant Concentration & 1.30 & $\mathrm{lb} / \mathrm{gal}$ \\
\hline \multicolumn{3}{|c|}{ Chemical Data } \\
\hline Acid \% Strength & 7.50 & \\
\hline Total Acid & 3000.00 & gal \\
\hline Total Slickwater & 7981.00 & bbls \\
\hline Total Linear & 0.00 & bbls \\
\hline Total XL & 0.00 & bbls \\
\hline FR Amount & 253.00 & gal \\
\hline Gel Amount & 0.00 & gal or lbs \\
\hline Breaker Amount & 0.00 & gal or lbs \\
\hline Scale Inhibitor & 38.00 & gal \\
\hline Biocide & 116.00 & gal \\
\hline
\end{tabular}

Table 63: Fracture Model - Perfs Stage 11

\begin{tabular}{|ccccc|}
\hline \multicolumn{5}{c|}{ Perfs } \\
\hline Cluster & Start.FT & Stop.FT & Perf_Diam.IN & Perf_Shots. \\
\hline 5 & 11324.00 & 11326.00 & 0.42 & 10 \\
4 & 11371.00 & 11373.00 & 0.42 & 10 \\
3 & 11416.00 & 11418.00 & 0.42 & 10 \\
2 & 11457.00 & 11459.00 & 0.42 & 10 \\
1 & 11509.00 & 11511.00 & 0.42 & 10 \\
\hline
\end{tabular}

Table 64: Fracture Model - Pump Schedule Stage 11

\begin{tabular}{|c|c|c|c|c|c|c|c|c|c|c|c|}
\hline Stage & $\begin{array}{c}\text { Tot Time } \\
\mathrm{mm}: \mathrm{ss}\end{array}$ & $\begin{array}{c}\text { Stage Time } \\
\text { mm:ss }\end{array}$ & Fluid & $\begin{array}{c}\text { Clean Stg Vol } \\
\text { (gal) }\end{array}$ & $\begin{array}{c}\text { Cum Cln Vol } \\
\text { (gal) }\end{array}$ & Proppant & $\begin{array}{l}\text { Slurry Conc } \\
\text { (ppa) }\end{array}$ & $\begin{array}{l}\text { Cum Prop } \\
\text { (Ibs) }\end{array}$ & $\begin{array}{l}\text { Slurry Rate } \\
\text { (bbl/min) }\end{array}$ & $\begin{array}{c}\text { Cum Slurry } \\
\text { (bbl) }\end{array}$ & $\begin{array}{l}\text { Clean Rate } \\
\text { (bbl/min) }\end{array}$ \\
\hline 1 & 0.00 & 1.22 & SlickWater & 544.39 & 544.39 & $<$ None $>$ & 0.00 & 0.00 & 9.68 & 13.22 & 9.48 \\
\hline 2 & 1.22 & 4.31 & $\mathrm{HCl} 7.5 \%$ & 3008.25 & 3552.64 & $<$ None $>$ & 0.00 & 0.00 & 15.86 & 84.84 & 15.86 \\
\hline 3 & 5.53 & 16.44 & SlickWater & 24966.10 & 28518.73 & $<$ None $>$ & 0.00 & 0.00 & 35.60 & 680.54 & 35.52 \\
\hline 4 & 22.37 & 3.16 & SlickWater & 13657.63 & 42176.37 & 100 Mesh Sand & 0.24 & 3315.00 & 100.66 & 1009.36 & 99.55 \\
\hline 5 & 25.53 & 3.26 & SlickWater & 14208.26 & 56384.63 & 100 Mesh Sand & 0.49 & 10288.00 & 100.73 & 1355.21 & 98.53 \\
\hline 6 & 29.19 & 1.44 & SlickWater & 7090.16 & 63474.79 & 100 Mesh Sand & 0.73 & 15443.00 & 100.62 & 1529.62 & 97.39 \\
\hline 7 & 31.03 & 8.36 & SlickWater & 34799.94 & 98274.73 & 100 Mesh Sand & 0.99 & 50023.00 & 100.70 & 2395.65 & 96.35 \\
\hline 8 & 39.39 & 10.11 & SlickWater & 40380.36 & 138655.08 & 100 Mesh Sand & 1.50 & 110408.00 & 100.82 & 3422.39 & 94.41 \\
\hline 9 & 49.50 & 11.33 & SlickWater & 45005.47 & 183660.55 & 100 Mesh Sand & 1.75 & 189059.00 & 100.14 & 4578.97 & 92.78 \\
\hline 10 & 61.23 & 13.04 & SlickWater & 50398.15 & 234058.70 & 100 Mesh Sand & 2.00 & 289710.00 & 100.16 & 5887.71 & 91.83 \\
\hline 11 & 74.27 & 4.16 & SlickWater & 16135.76 & 250194.47 & 100 Mesh Sand & 2.49 & 329884.00 & 100.24 & 6315.39 & 90.04 \\
\hline 12 & 78.43 & 1.48 & SlickWater & 7350.07 & 257544.54 & 40/70 White & 0.62 & 334452.00 & 99.85 & 6495.11 & 97.22 \\
\hline 13 & 80.31 & 2.43 & SlickWater & 11007.64 & 268552.18 & 40/70 White & 0.74 & 342575.00 & 99.73 & 6766.06 & 96.47 \\
\hline 14 & 83.14 & 3.45 & SlickWater & 15032.92 & 283585.10 & 40/70 White & 0.99 & 357487.00 & 99.77 & 7140.18 & 95.45 \\
\hline 15 & 86.59 & 3.48 & SlickWater & 14947.39 & 298532.48 & 40/70 White & 1.49 & 379720.00 & 100.02 & 7520.24 & 93.66 \\
\hline 16 & 90.47 & 3.53 & SlickWater & 14966.42 & 313498.91 & 40/70 White & 1.99 & 409434.00 & 100.07 & 7908.85 & 91.76 \\
\hline 17 & 94.40 & 1.00 & SlickWater & 3790.71 & 317289.62 & 40/70 White & 2.44 & 418673.00 & 100.34 & 8009.19 & 90.26 \\
\hline 18 & 95.40 & 2.34 & SlickWater & 9835.84 & 327125.46 & 40/70 White & 2.14 & 439757.00 & 100.05 & 8265.98 & 91.24 \\
\hline 19 & 98.14 & 2.45 & SlickWater & 11103.47 & 338228.93 & $<$ None $>$ & 0.00 & 439757.00 & 95.61 & 8528.91 & 96.13 \\
\hline
\end{tabular}


Table 65: Fracture Model - Pump Details Stage 11

\begin{tabular}{|c|c|c|c|c|c|c|c|}
\hline Step\# & Name & Avg S Rate & Max S Rate & Avg Tr Pres & Max Tr Pres & Min Tr Pressure & Calc Avg TR P \\
\hline & & $(\mathbf{b b l} / \mathbf{m i n})$ & $\mathbf{( b b l / m i n )}$ & (psi) & (psi) & (psi) & (psi) \\
\hline 1 & Rate & 11.7 & 15.8 & 5653 & 6322 & 3754 & 5272 \\
\hline 2 & Acid 7.5\% HC & 15.9 & 15.9 & 6151 & 6317 & 6041 & 6147 \\
\hline 3 & PAD & 94.7 & 100.3 & 8250 & 8689 & 5874 & 6652 \\
\hline 4 & 0.25 PPA & 100.6 & 100.9 & 8158 & 8178 & 8143 & 8159 \\
\hline 5 & 0.5 PPA & 100.7 & 100.9 & 8116 & 8168 & 8095 & 8114 \\
\hline 6 & 0.75 PPA & 100.6 & 100.9 & 8097 & 8110 & 8085 & 8097 \\
\hline 7 & 1.00 PPA & 100.7 & 100.6 & 8098 & 8111 & 8074 & 8098 \\
\hline 8 & 1.50 PPA & 100.8 & 100.4 & 8069 & 8101 & 8025 & 8069 \\
\hline 9 & 1.75 PPA & 100.1 & 100.6 & 8113 & 8183 & 8001 & 8113 \\
\hline 10 & 2.00 PPA & 100.2 & 100.7 & 8219 & 8248 & 8182 & 8219 \\
\hline 11 & 2.50 PPA & 100.2 & 100.8 & 8199 & 8229 & 8175 & 8199 \\
\hline 12 & 0.50 PPA & 99.9 & 100.8 & 8220 & 8271 & 8188 & 8222 \\
\hline 13 & 0.75 PPA & 99.7 & 99.9 & 8210 & 8309 & 8130 & 8206 \\
\hline 14 & 1.00 PPA & 99.8 & 100.2 & 8116 & 8150 & 8091 & 8115 \\
\hline 15 & 1.50 PPA & 100.0 & 100.3 & 8090 & 8118 & 8063 & 8090 \\
\hline 16 & 2.00 PPA & 100.1 & 100.5 & 8088 & 8124 & 8056 & 8088 \\
\hline 17 & 2.50 PPA & 100.3 & 100.7 & 8113 & 8125 & 8092 & 8111 \\
\hline 18 & 3.00 PPA & 100.1 & 100.7 & 8066 & 8111 & 8043 & 8067 \\
\hline 19 & Flush & 98.7 & 99.9 & 8169 & 8434 & 5525 & 8061 \\
\hline
\end{tabular}

Table 66: Fracture Model - Overview Stage 12

\begin{tabular}{|c|c|c|}
\hline \multicolumn{3}{|c|}{ Stage 12} \\
\hline \multicolumn{3}{|c|}{ Treatment Summary } \\
\hline Formation & Marcellus & \\
\hline Formation Type & Shale & \\
\hline \multicolumn{3}{|c|}{ Stage Data } \\
\hline Report Date & $11 / 11 / 2015$ & \\
\hline Stage Length & 231.00 & $\mathrm{ft}$ \\
\hline Frac Gradient & 1.23 & $\mathrm{psi} / \mathrm{ft}$ \\
\hline ISIP & 5898.00 & psi \\
\hline 5 Min ISIP & 5580.00 & $\mathrm{psi}$ \\
\hline 10 Min ISIP & 5457.00 & psi \\
\hline 15 Min ISIP & 5360.00 & psi \\
\hline Breakdown Rate & 15.00 & $\mathrm{bpm}$ \\
\hline Breakdown Pressure & 6085.00 & psi \\
\hline Breakdown Volume & 147.00 & bbls \\
\hline Cut Short & & $x$ \\
\hline Screen Out & & $\mathrm{x}$ \\
\hline \multicolumn{3}{|c|}{ Fluid and Proppant Data } \\
\hline Total Clean Fluid & 8984.00 & bbls \\
\hline Prop.1-Type & 100 Mesh & \\
\hline Prop.2-Type & 40/70 Resin & \\
\hline Total Proppant Amount & 440680.00 & Ibs \\
\hline Proppant Concentration & 1907.71 & $\mathrm{Ib} / \mathrm{ft}$ \\
\hline Proppant Concentration & 1.17 & $\mathrm{lb} / \mathrm{gal}$ \\
\hline \multicolumn{3}{|c|}{ Chemical Data } \\
\hline Acid \% Strength & 7.50 & \\
\hline Total Acid & 3000.00 & gal \\
\hline Total Slickwater & 8913.00 & bbls \\
\hline Total Linear & 0.00 & bbls \\
\hline Total XL & 0.00 & bbls \\
\hline FR Amount & 188.00 & gal \\
\hline Gel Amount & 0.00 & gal or Ibs \\
\hline Breaker Amount & 0.00 & gal or lbs \\
\hline Scale Inhibitor & 30.00 & gal \\
\hline Biocide & 75.00 & gal \\
\hline
\end{tabular}

Table 67: Fracture Model - Perfs Stage 12

\begin{tabular}{|ccccc|}
\hline \multicolumn{5}{|c|}{ Perfs } \\
\hline Cluster & Start.FT & Stop.FT & Perf_Diam.IN & Perf_Shots. \\
\hline 5 & 11093.00 & 11095.00 & 0.42 & 10 \\
4 & 11140.00 & 11142.00 & 0.42 & 10 \\
3 & 11187.00 & 11189.00 & 0.42 & 10 \\
2 & 11230.00 & 11232.00 & 0.42 & 10 \\
1 & 11277.00 & 11279.00 & 0.42 & 10 \\
\hline
\end{tabular}


Table 68: Fracture Model - Pump Schedule Stage 12

\begin{tabular}{|c|c|c|c|c|c|c|c|c|c|c|c|}
\hline Stage & $\begin{array}{c}\text { Tot Time } \\
\text { mm:ss }\end{array}$ & $\begin{array}{c}\text { Stage Time } \\
\text { mm:ss }\end{array}$ & Fluid & $\begin{array}{c}\text { Clean Stg Vol } \\
\text { (gal) }\end{array}$ & $\begin{array}{c}\text { Cum Cln Vol } \\
\text { (gal) }\end{array}$ & Proppant & $\begin{array}{c}\text { Slurry Conc } \\
\text { (ppa) }\end{array}$ & $\begin{array}{c}\text { Cum Prop } \\
\text { (Ibs) }\end{array}$ & $\begin{array}{c}\text { Slurry Rate } \\
\text { (bbl/min) }\end{array}$ & $\begin{array}{c}\text { Cum Slurry } \\
\text { (bbl) }\end{array}$ & $\begin{array}{l}\text { Clean Rate } \\
\text { (bbl/min) }\end{array}$ \\
\hline 1 & 0.00 & 1.54 & SlickWater & 826.70 & 826.70 & $<$ None $>$ & 0.00 & 0.00 & 10.48 & 19.92 & 10.36 \\
\hline 2 & 1.54 & 5.06 & $\mathrm{HCl} 7.5 \%$ & 3003.07 & 3829.77 & $<$ None $>$ & 0.00 & 0.00 & 14.02 & 91.42 & 14.02 \\
\hline 3 & 6.60 & 25.46 & SlickWater & 47208.62 & 51038.39 & <None $>$ & 0.00 & 0.00 & 43.68 & 1216.86 & 43.62 \\
\hline 4 & 32.46 & 2.35 & SlickWater & 10627.26 & 61665.65 & 100 Mesh Sand & 0.24 & 2547.00 & 99.02 & 1472.67 & 97.95 \\
\hline 5 & 35.21 & 3.28 & SlickWater & 14100.95 & 75766.60 & 100 Mesh Sand & 0.49 & 9488.00 & 99.02 & 1815.93 & 96.85 \\
\hline 6 & 38.49 & 5.10 & SlickWater & 20787.84 & 96554.44 & 100 Mesh Sand & 0.74 & 24942.00 & 99.04 & 2327.62 & 95.80 \\
\hline 7 & 43.59 & 11.37 & SlickWater & 46215.57 & 142770.01 & 100 Mesh Sand & 1.00 & 71023.00 & 99.01 & 3477.81 & 94.72 \\
\hline 8 & 55.36 & 10.22 & SlickWater & 40355.90 & 183125.91 & 100 Mesh Sand & 1.50 & 131415.00 & 98.99 & 4503.98 & 92.69 \\
\hline 9 & 65.58 & 11.40 & SlickWater & 44944.55 & 228070.46 & 100 Mesh Sand & 1.75 & 209988.00 & 99.01 & 5659.05 & 91.72 \\
\hline 10 & 77.38 & 7.34 & SlickWater & 28848.40 & 256918.86 & 100 Mesh Sand & 2.00 & 267585.00 & 99.01 & 6408.20 & 90.78 \\
\hline 11 & 85.12 & 8.06 & SlickWater & 30194.57 & 287113.43 & 100 Mesh Sand & 2.51 & 343251.00 & 98.84 & 7208.79 & 88.76 \\
\hline 12 & 93.18 & 1.49 & SlickWater & 7351.33 & 294464.76 & 40/70 White & 0.63 & 347867.00 & 99.00 & 7388.63 & 96.35 \\
\hline 13 & 95.07 & 2.45 & SlickWater & 11038.72 & 305503.48 & 40/70 White & 0.78 & 356529.00 & 99.00 & 7660.87 & 95.57 \\
\hline 14 & 97.52 & 3.47 & SlickWater & 15048.24 & 320551.72 & 40/70 White & 0.99 & 371418.00 & 99.00 & 8035.40 & 94.70 \\
\hline 15 & 101.39 & 3.51 & SlickWater & 14993.85 & 335545.57 & 40/70 White & 1.49 & 393703.00 & 99.02 & 8416.64 & 92.73 \\
\hline 16 & 105.30 & 4.49 & SlickWater & 18362.83 & 353908.39 & 40/70 White & 1.99 & 430231.00 & 99.00 & 8893.50 & 90.77 \\
\hline 17 & 110.19 & 1.21 & SlickWater & 5045.11 & 358953.50 & 40/70 White & 2.46 & 442663.00 & 99.01 & 9027.16 & 88.98 \\
\hline 18 & 111.40 & 2.03 & SlickWater & 7756.56 & 366710.06 & 40/70 White & 2.20 & 459743.00 & 99.00 & 9230.11 & 90.09 \\
\hline 19 & 113.43 & 2.36 & SlickWater & 10637.76 & 377347.82 & $<$ None $>$ & 0.00 & 459743.00 & 97.05 & 9482.44 & 97.42 \\
\hline
\end{tabular}

Table 69: Fracture Model - Pump Details Stage 12

\begin{tabular}{|c|c|c|c|c|c|c|c|}
\hline Step\# & Name & Avg S Rate & Max S Rate & Avg Tr Pres & Max Tr Pres & Min Tr Pressure & Calc Avg TR P \\
\hline & & $(\mathbf{b b l} / \mathbf{m i n})$ & $(\mathbf{b b l} / \mathbf{m i n})$ & (psi) & (psi) & (psi) & (psi) \\
\hline 1 & Rate & 12.6 & 14.1 & 5194 & 5644 & 3952 & 4959 \\
\hline 2 & Acid 7.5\% HC & 14.0 & 14.1 & 5902 & 6010 & 5648 & 5906 \\
\hline 3 & PAD & 69.3 & 99.2 & 7545 & 9105 & 5124 & 6780 \\
\hline 4 & 0.25 PPA & 99.0 & 99.2 & 8063 & 8077 & 8053 & 8062 \\
\hline 5 & 0.5 PPA & 99.0 & 99.1 & 8059 & 8083 & 8037 & 8059 \\
\hline 6 & 0.75 PPA & 99.0 & 99.2 & 8036 & 8054 & 8014 & 8035 \\
\hline 7 & 1.00 PPA & 99.0 & 99.1 & 8009 & 8052 & 7968 & 8009 \\
\hline 8 & 1.5 PPA & 99.0 & 99.1 & 8049 & 8082 & 8020 & 8062 \\
\hline 9 & 1.75 PPA & 99.0 & 99.2 & 8061 & 8102 & 8016 & 8061 \\
\hline 10 & 2.00 PPA & 99.0 & 99.1 & 8103 & 8131 & 8072 & 8103 \\
\hline 11 & 2.5 PPA & 98.9 & 100.7 & 8210 & 8297 & 8130 & 8212 \\
\hline 12 & .50 PPA & 99.0 & 99.1 & 8283 & 8328 & 8254 & 8285 \\
\hline 13 & .75 PPA & 99.0 & 99.1 & 8256 & 8370 & 8176 & 8252 \\
\hline 14 & 1.0 PPA & 99.0 & 99.1 & 8169 & 8200 & 8151 & 8168 \\
\hline 15 & 1.50 PPA & 99.0 & 99.1 & 8161 & 8182 & 8127 & 8161 \\
\hline 16 & 2.00 PPA & 99.0 & 99.2 & 8167 & 8190 & 8145 & 8167 \\
\hline 17 & 2.5 PPA & 99.0 & 99.1 & 8204 & 8220 & 8179 & 8207 \\
\hline 18 & 3.0 PPA & 99.0 & 99.1 & 8309 & 8394 & 8219 & 8315 \\
\hline 19 & Flush & 98.6 & 99.1 & 8531 & 8727 & 5756 & 8424 \\
\hline
\end{tabular}


Table 70: Fracture Model - Overview Stage 13

\begin{tabular}{|c|c|c|}
\hline \multicolumn{3}{|c|}{ Stage 13} \\
\hline \multicolumn{3}{|c|}{ Treatment Summary } \\
\hline Formation & Marcellus & \\
\hline Formation Type & Shale & \\
\hline \multicolumn{3}{|c|}{ Stage Data } \\
\hline Report Date & $11 / 11 / 2015$ & \\
\hline Stage Length & 230.00 & $\mathrm{ft}$ \\
\hline Frac Gradient & 1.24 & $\mathrm{psi} / \mathrm{ft}$ \\
\hline ISIP & 6015.00 & psi \\
\hline 5 Min ISIP & 5535.00 & psi \\
\hline 10 Min ISIP & 5424.00 & psi \\
\hline 15 Min ISIP & 5337.00 & psi \\
\hline Breakdown Rate & 15.00 & bpm \\
\hline Breakdown Pressure & 6312.00 & psi \\
\hline Breakdown Volume & 134.00 & bbls \\
\hline Cut Short & & $x$ \\
\hline Screen Out & & $x$ \\
\hline \multicolumn{3}{|c|}{ Fluid and Proppant Data } \\
\hline Total Clean Fluid & 7466.00 & bbls \\
\hline Prop.1-Type & 100 Mesh & \\
\hline Prop.2-Type & 40/70 White & \\
\hline Total Proppant Amount & 360380.00 & Ibs \\
\hline Proppant Concentration & 1566.87 & $\mathrm{Ib} / \mathrm{ft}$ \\
\hline Proppant Concentration & 1.15 & Ib/gal \\
\hline \multicolumn{3}{|c|}{ Chemical Data } \\
\hline Acid \% Strength & 7.50 & \\
\hline Total Acid & 3000.00 & gal \\
\hline Total Slickwater & 7391.00 & bbls \\
\hline Total Linear & 0.00 & bbls \\
\hline Total XL & 0.00 & bbls \\
\hline FR Amount & 140.00 & gal \\
\hline Gel Amount & 0.00 & gal or lbs \\
\hline Breaker Amount & 0.00 & gal or lbs \\
\hline Scale Inhibitor & 30.00 & gal \\
\hline Biocide & 75.00 & gal \\
\hline
\end{tabular}

Table 71: Fracture Model - Perfs Stage 13

\begin{tabular}{|ccccc|}
\hline \multicolumn{5}{c|}{ Perfs } \\
\hline Cluster & Start.FT & Stop.FT & Perf_Diam.IN & Perf_Shots. \\
\hline 5 & 10863.00 & 10865.00 & 0.42 & 6 \\
4 & 10909.00 & 10911.00 & 0.42 & 6 \\
3 & 10955.00 & 10957.00 & 0.42 & 6 \\
2 & 11000.00 & 11002.00 & 0.42 & 6 \\
1 & 11046.00 & 11048.00 & 0.42 & 6 \\
\hline
\end{tabular}

Table 72: Fracture Model - Pump Schedule Stage 13

\begin{tabular}{|c|c|c|c|c|c|c|c|c|c|c|c|}
\hline Stage & $\begin{array}{c}\text { Tot Time } \\
\text { mm:ss }\end{array}$ & $\begin{array}{c}\text { Stage Time } \\
\text { mm:ss }\end{array}$ & Fluid & $\begin{array}{c}\text { Clean Stg Vol } \\
\text { (gal) }\end{array}$ & $\begin{array}{c}\text { Cum Cln Vol } \\
\text { (gal) }\end{array}$ & Proppant & $\begin{array}{c}\text { Slurry Conc } \\
\text { (ppa) }\end{array}$ & $\begin{array}{l}\text { Cum Prop } \\
\text { (Ibs) }\end{array}$ & $\begin{array}{l}\text { Slurry Rate } \\
\text { (bbl/min) }\end{array}$ & $\begin{array}{c}\text { Cum Slurry } \\
\text { (bbl) }\end{array}$ & $\begin{array}{l}\text { Clean Rate } \\
\text { (bbl/min) }\end{array}$ \\
\hline 1 & 0.00 & 1.47 & SlickWater & 828.80 & 828.80 & $<$ None $>$ & 0.00 & 0.00 & 11.20 & 19.97 & 11.07 \\
\hline 2 & 1.47 & 5.05 & $\mathrm{HCl} 7.5 \%$ & 2993.27 & 3822.07 & $<$ None $>$ & 0.00 & 0.00 & 14.02 & 91.24 & 14.02 \\
\hline 3 & 6.52 & 22.23 & SlickWater & 41934.85 & 45756.92 & $<$ None $>$ & 0.00 & 0.00 & 44.66 & 1090.82 & 44.61 \\
\hline 4 & 29.15 & 3.27 & SlickWater & 12020.34 & 57777.25 & 100 Mesh Sand & 0.24 & 2914.00 & 83.87 & 1380.18 & 82.96 \\
\hline 5 & 32.42 & 3.18 & SlickWater & 12036.08 & 69813.33 & 100 Mesh Sand & 0.49 & 8852.00 & 88.85 & 1673.39 & 86.84 \\
\hline 6 & 36.00 & 3.55 & SlickWater & 15255.73 & 85069.06 & 100 Mesh Sand & 0.74 & 20090.00 & 95.87 & 2048.86 & 92.74 \\
\hline 7 & 39.55 & 4.52 & SlickWater & 18917.98 & 103987.05 & 100 Mesh Sand & 0.99 & 38876.00 & 96.73 & 2519.62 & 92.55 \\
\hline 8 & 44.47 & 7.35 & SlickWater & 28850.13 & 132837.18 & 100 Mesh Sand & 1.49 & 81990.00 & 96.73 & 3253.19 & 90.58 \\
\hline 9 & 52.22 & 6.20 & SlickWater & 23592.07 & 156429.25 & 100 Mesh Sand & 1.99 & 128990.00 & 96.72 & 3865.76 & 88.69 \\
\hline 10 & 58.42 & 4.45 & SlickWater & 18842.66 & 175271.91 & $40 / 70$ - Treated & 0.54 & 139121.00 & 96.73 & 4325.24 & 94.45 \\
\hline 11 & 63.27 & 3.50 & SlickWater & 15061.69 & 190333.60 & $40 / 70$ - Treated & 0.74 & 150288.00 & 96.72 & 4695.99 & 93.55 \\
\hline 12 & 67.17 & 3.54 & SlickWater & 15158.29 & 205491.89 & 40/70 - Treated & 0.99 & 165334.00 & 96.73 & 5073.24 & 92.54 \\
\hline 13 & 71.11 & 6.57 & SlickWater & 26431.65 & 231923.54 & 40/70 White & 1.49 & 204820.00 & 96.72 & 5745.47 & 90.55 \\
\hline 14 & 78.08 & 10.09 & SlickWater & 37798.28 & 269721.82 & 40/70 White & 1.99 & 280205.00 & 96.73 & 6727.24 & 88.67 \\
\hline 15 & 88.17 & 6.17 & SlickWater & 22627.68 & 292349.50 & 40/70 White & 2.49 & 336542.00 & 95.47 & 7327.09 & 85.74 \\
\hline 16 & 94.34 & 3.05 & SlickWater & 10895.43 & 303244.93 & 40/70 White & 2.58 & 364661.00 & 93.94 & 7616.74 & 84.13 \\
\hline 17 & 97.39 & 2.41 & SlickWater & 10371.13 & 313616.06 & $<$ None $>$ & 0.00 & 364661.00 & 91.69 & 7862.79 & 92.02 \\
\hline
\end{tabular}


Table 73: Fracture Model - Pump Details Stage 13

\begin{tabular}{|c|c|c|c|c|c|c|c|}
\hline Step\# & Name & Avg S Rate & Max S Rate & Avg Tr Pres & Max Tr Pres & Min Tr Pressure & Calc Avg TR P \\
\hline & & (bbl/min) & (bbl/min) & (psi) & (psi) & (psi) & (psi) \\
\hline 1 & Rate & 12.9 & 14.1 & 5237 & 5554 & 4313 & 5096 \\
\hline 2 & Acid 7.5\% HC & 14.0 & 14.1 & 5809 & 5915 & 5562 & 5813 \\
\hline 3 & PAD & 84.7 & 96.4 & 9135 & 9188 & 5794 & 7388 \\
\hline 4 & 0.25 PPA & 83.9 & 84.0 & 9031 & 9114 & 8965 & 9028 \\
\hline 5 & 0.5 PPA & 88.8 & 94.9 & 8832 & 9044 & 8546 & 8829 \\
\hline 6 & 0.75 PPA & 95.9 & 96.8 & 8885 & 8954 & 8813 & 8882 \\
\hline 7 & 1.00 PPA & 96.7 & 96.9 & 8791 & 8871 & 8741 & 8789 \\
\hline 8 & 1.50 PPA & 96.7 & 96.9 & 8714 & 8752 & 8688 & 8714 \\
\hline 9 & 2.0 PPA & 96.7 & 96.8 & 8713 & 8735 & 8673 & 8714 \\
\hline 10 & .50 PPA & 96.7 & 96.9 & 8628 & 8714 & 8517 & 8626 \\
\hline 11 & .75 PPA & 96.7 & 96.8 & 8532 & 8549 & 8514 & 8531 \\
\hline 12 & 1.0 PPA & 96.7 & 96.8 & 8527 & 8544 & 8508 & 8527 \\
\hline 13 & 1.50 PPA & 96.7 & 96.8 & 8521 & 8545 & 8489 & 8521 \\
\hline 14 & 2.00 PPA & 96.7 & 96.8 & 8569 & 8632 & 8506 & 8570 \\
\hline 15 & 2.5 PPA & 95.5 & 96.9 & 8762 & 9016 & 8609 & 8761 \\
\hline 16 & 3.0 PPA & 93.9 & 94.0 & 8563 & 8630 & 8515 & 8564 \\
\hline 17 & Flush & 93.1 & 94.1 & 8778 & 8963 & 6630 & 8699 \\
\hline
\end{tabular}

Table 74: Fracture Model - Overview Stage 14

\begin{tabular}{|c|c|c|}
\hline \multicolumn{3}{|c|}{ Stage 14} \\
\hline \multicolumn{3}{|c|}{ Treatment Summary } \\
\hline Formation & Marcellus & \\
\hline Formation Type & Shale & \\
\hline \multicolumn{3}{|c|}{ Stage Data } \\
\hline Report Date & $11 / 11 / 2015$ & \\
\hline Stage Length & 228.00 & $\mathrm{ft}$ \\
\hline Frac Gradient & 1.22 & $\mathrm{psi} / \mathrm{ft}$ \\
\hline ISIP & 5867.00 & psi \\
\hline 5 Min ISIP & 5488.00 & psi \\
\hline 10 Min ISIP & 5369.00 & psi \\
\hline 15 Min ISIP & 5273.00 & psi \\
\hline Breakdown Rate & 15.00 & bpm \\
\hline Breakdown Pressure & 6905.00 & psi \\
\hline Breakdown Volume & 233.00 & bbls \\
\hline Cut Short & $x$ & $\mathrm{x}$ \\
\hline Screen Out & & $x$ \\
\hline \multicolumn{3}{|c|}{ Fluid and Proppant Data } \\
\hline Total Clean Fluid & 8788.00 & bbls \\
\hline Prop.1-Type & 100 Mesh & \\
\hline Prop.2-Type & 40/70 White & \\
\hline Total Proppant Amount & 437060.00 & Ibs \\
\hline Proppant Concentration & 1916.93 & $\mathrm{lb} / \mathrm{ft}$ \\
\hline Proppant Concentration & 1.18 & $\mathrm{lb} / \mathrm{gal}$ \\
\hline \multicolumn{3}{|c|}{ Chemical Data } \\
\hline Acid \% Strength & 7.50 & \\
\hline Total Acid & 3000.00 & gal \\
\hline Total Slickwater & 8724.00 & bbls \\
\hline Total Linear & 0.00 & bbls \\
\hline Total XL & 0.00 & bbls \\
\hline FR Amount & 225.00 & gal \\
\hline Gel Amount & 0.00 & gal or lbs \\
\hline Breaker Amount & 0.00 & gal or lbs \\
\hline Scale Inhibitor & 35.00 & gal \\
\hline Biocide & 85.00 & gal \\
\hline
\end{tabular}

Table 75: Fracture Model - Perfs Stage 14

\begin{tabular}{|ccccc|}
\hline \multicolumn{5}{c|}{ Perfs } \\
\hline Cluster & Start.FT & Stop.FT & Perf_Diam.IN & Perf_Shots. \\
\hline 5 & 10639.00 & 10641.00 & 0.42 & 6 \\
4 & 10683.00 & 10685.00 & 0.42 & 6 \\
3 & 10731.00 & 10733.00 & 0.42 & 6 \\
2 & 10774.00 & 10776.00 & 0.42 & 6 \\
1 & 10822.00 & 10824.00 & 0.42 & 6 \\
\hline
\end{tabular}


Table 76: Fracture Model - Pump Schedule Stage 14

\begin{tabular}{|c|c|c|c|c|c|c|c|c|c|c|c|}
\hline Stage & $\begin{array}{l}\text { Tot Time } \\
\text { mm:ss }\end{array}$ & $\begin{array}{c}\text { Stage Time } \\
\text { mm:ss }\end{array}$ & Fluid & $\begin{array}{c}\text { Clean Stg Vol } \\
\text { (gal) }\end{array}$ & $\begin{array}{c}\text { Cum Cln Vol } \\
\text { (gal) }\end{array}$ & Proppant & $\begin{array}{l}\text { Slurry Conc } \\
\text { (ppa) }\end{array}$ & $\begin{array}{l}\text { Cum Prop } \\
\text { (lbs) }\end{array}$ & $\begin{array}{l}\text { Slurry Rate } \\
\text { (bbl/min) }\end{array}$ & $\begin{array}{l}\text { Cum Slurry } \\
\text { (bbl) }\end{array}$ & $\begin{array}{l}\text { Clean Rate } \\
\text { (bbl/min) }\end{array}$ \\
\hline 1 & 0.00 & 1.06 & SlickWater & 454.02 & 454.02 & $\langle$ None $>$ & 0.00 & 0.00 & 10.06 & 11.07 & 9.83 \\
\hline 2 & 1.06 & 4.11 & $\mathrm{HCl} 7.5 \%$ & 2676.38 & 3130.40 & $<$ None $>$ & 0.00 & 0.00 & 15.23 & 74.79 & 15.23 \\
\hline 3 & 5.17 & 21.30 & SlickWater & 41964.53 & 45094.93 & $<$ None $>$ & 0.00 & 0.00 & 46.54 & 1075.33 & 46.47 \\
\hline 4 & 26.47 & 3.14 & SlickWater & 13197.65 & 58292.58 & 100 Mesh Sand & 0.23 & 3095.00 & 98.23 & 1392.92 & 97.18 \\
\hline 5 & 30.01 & 3.25 & SlickWater & 13984.32 & 72276.90 & 100 Mesh Sand & 0.48 & 9829.00 & 99.60 & 1733.21 & 97.45 \\
\hline 6 & 33.26 & 4.34 & SlickWater & 18519.05 & 90795.95 & 100 Mesh Sand & 0.74 & 23622.00 & 99.83 & 2189.08 & 96.55 \\
\hline 7 & 37.60 & 5.46 & SlickWater & 23135.21 & 113931.16 & 100 Mesh Sand & 0.99 & 46610.00 & 99.84 & 2764.81 & 95.52 \\
\hline 8 & 43.46 & 8.59 & SlickWater & 35272.70 & 149203.86 & 100 Mesh Sand & 1.49 & 99330.00 & 99.83 & 3661.64 & 93.49 \\
\hline 9 & 52.45 & 6.13 & SlickWater & 23899.26 & 173103.12 & 100 Mesh Sand & 1.99 & 146979.00 & 99.82 & 4282.19 & 91.53 \\
\hline 10 & 58.58 & 5.51 & SlickWater & 23957.97 & 197061.09 & 40/70 - Treated & 0.53 & 159648.00 & 99.83 & 4866.21 & 97.51 \\
\hline 11 & 64.49 & 4.35 & SlickWater & 18590.73 & 215651.81 & 40/70 - Treated & 0.74 & 173426.00 & 99.84 & 5323.81 & 96.58 \\
\hline 12 & 69.24 & 4.37 & SlickWater & 18515.43 & 234167.25 & 40/70 - Treated & 0.99 & 191816.00 & 99.82 & 5784.64 & 95.49 \\
\hline 13 & 74.01 & 8.15 & SlickWater & 32384.38 & 266551.62 & 40/70 White & 1.49 & 240222.00 & 99.83 & 6608.26 & 93.46 \\
\hline 14 & 82.16 & 24.30 & SlickWater & 92406.60 & 358958.22 & 40/70 White & 1.96 & 421088.00 & 97.79 & 9004.16 & 89.80 \\
\hline 15 & 106.46 & 3.08 & SlickWater & 10137.75 & 369095.97 & $<$ None $>$ & 0.00 & 421088.00 & 76.61 & 9244.21 & 77.03 \\
\hline
\end{tabular}

Table 77: Fracture Model - Pump Details Stage 14

\begin{tabular}{|c|c|c|c|c|c|c|c|}
\hline Step\# & Name & Avg S Rate & Max S Rate & Avg Tr Pres & Max Tr Pres & Min Tr Pressure & Calc Avg TR P \\
\hline & & (bbl/min) & (bbl/min) & (psi) & (psi) & (psi) & (psi) \\
\hline 1 & Rate & 12.9 & 15.2 & 5339 & 5774 & 4340 & 5110 \\
\hline 2 & Acid 7.5\% HC & 15.2 & 15.3 & 6139 & 6272 & 5782 & 6147 \\
\hline 3 & PAD & 94.4 & 98.3 & 8779 & 9011 & 6046 & 7346 \\
\hline 4 & 0.25 PPA & 98.2 & 98.3 & 8887 & 8938 & 8861 & 8888 \\
\hline 5 & 0.5 PPA & 99.6 & 99.9 & 8831 & 8927 & 8809 & 8828 \\
\hline 6 & 0.75 PPA & 99.8 & 99.9 & 8775 & 8821 & 8748 & 8774 \\
\hline 7 & 1.00 PPA & 99.8 & 100.0 & 8717 & 8747 & 8699 & 8716 \\
\hline 8 & 1.50 PPA & 99.8 & 100.0 & 8695 & 8723 & 8652 & 8695 \\
\hline 9 & 2.00 PPA & 99.8 & 100.0 & 8704 & 8749 & 8646 & 8704 \\
\hline 10 & 0.50 PPA & 99.8 & 99.9 & 8660 & 8765 & 8539 & 8658 \\
\hline 11 & 0.75 PPA & 99.8 & 100.0 & 8518 & 8546 & 8497 & 8517 \\
\hline 12 & 1.00 PPA & 99.8 & 99.9 & 8504 & 8521 & 8492 & 8503 \\
\hline 13 & 1.50 PPA & 99.8 & 99.9 & 8513 & 8569 & 8457 & 8513 \\
\hline 14 & 2.00 PPA & 97.9 & 100.0 & 8721 & 9083 & 8396 & 8726 \\
\hline 15 & Flush & 78.3 & 82.5 & 8950 & 9094 & 6564 & 8830 \\
\hline
\end{tabular}

Table 78: Fracture Model - Overview Stage 15

\begin{tabular}{|c|c|c|}
\hline \multicolumn{3}{|c|}{ Stage 15} \\
\hline \multicolumn{3}{|c|}{ Treatment Summary } \\
\hline Formation & Marcellus & \\
\hline Formation Type & Shale & \\
\hline \multicolumn{3}{|c|}{ Stage Data } \\
\hline Report Date & $11 / 12 / 2015$ & \\
\hline Stage Length & 186.00 & $\mathrm{ft}$ \\
\hline Frac Gradient & 1.20 & $\mathrm{psi} / \mathrm{ft}$ \\
\hline ISIP & 5681.00 & psi \\
\hline 5 Min ISIP & 4909.00 & psi \\
\hline 10 Min ISIP & 4788.00 & psi \\
\hline 15 Min ISIP & 4712.00 & psi \\
\hline Breakdown Rate & 15.00 & bpm \\
\hline Breakdown Pressure & 7138.00 & psi \\
\hline Breakdown Volume & 228.00 & bbls \\
\hline Cut Short & & $\mathrm{X}$ \\
\hline Screen Out & & $x$ \\
\hline \multicolumn{3}{|c|}{ Fluid and Proppant Data } \\
\hline Total Clean Fluid & 8590.00 & bbls \\
\hline Prop.1-Type & 100 Mesh & \\
\hline Prop.2-Type & 40/70 White & \\
\hline Total Proppant Amount & 359960.00 & Ibs \\
\hline Proppant Concentration & 1935.27 & $\mathrm{lb} / \mathrm{ft}$ \\
\hline Proppant Concentration & 1.00 & $\mathrm{lb} / \mathrm{gal}$ \\
\hline \multicolumn{3}{|c|}{ Chemical Data } \\
\hline Acid \% Strength & 7.50 & \\
\hline Total Acid & 3000.00 & gal \\
\hline Total Slickwater & 8520.00 & bbls \\
\hline Total Linear & 0.00 & bbls \\
\hline Total XL & 0.00 & bbls \\
\hline FR Amount & 210.00 & gal \\
\hline Gel Amount & 0.00 & gal or Ibs \\
\hline Breaker Amount & 0.00 & gal or Ibs \\
\hline Scale Inhibitor & 40.00 & gal \\
\hline Biocide & 85.00 & gal \\
\hline
\end{tabular}


Table 79: Fracture Model - Perfs Stage 15

\begin{tabular}{|ccccc|}
\hline \multicolumn{5}{c|}{ Perfs } \\
\hline Cluster & Start.FT & Stop.FT & Perf_Diam.IN & Perf_Shots. \\
\hline 4 & 10456.00 & 10458.00 & 0.42 & 6 \\
3 & 10503.00 & 10505.00 & 0.42 & 6 \\
2 & 10547.00 & 10549.00 & 0.42 & 8 \\
1 & 10594.00 & 10596.00 & 0.42 & 6 \\
\hline
\end{tabular}

Table 80: Fracture Model - Pump Schedule Stage 15

\begin{tabular}{|c|c|c|c|c|c|c|c|c|c|c|c|}
\hline Stage & $\begin{array}{l}\text { Tot Time } \\
\mathrm{mm}: \mathrm{ss}\end{array}$ & $\begin{array}{l}\text { Stage Time } \\
\text { mm:ss }\end{array}$ & Fluid & $\begin{array}{c}\text { Clean Stg Vol } \\
\text { (gal) }\end{array}$ & $\begin{array}{c}\text { Cum Cln Vol } \\
\text { (gal) }\end{array}$ & Proppant & $\begin{array}{l}\text { Slurry Conc } \\
\text { (ppa) }\end{array}$ & $\begin{array}{l}\text { Cum Prop } \\
\text { (Ibs) }\end{array}$ & $\begin{array}{c}\text { Slurry Rate } \\
\text { (bbl/min) }\end{array}$ & $\begin{array}{c}\text { Cum Slurry } \\
\text { (bbl) }\end{array}$ & $\begin{array}{l}\text { Clean Rate } \\
\text { (bbl/min) }\end{array}$ \\
\hline 1 & 0.00 & 1.27 & SlickWater & 723.10 & 723.10 & $<$ <None $>$ & 0.00 & 0.00 & 12.02 & 17.43 & 11.87 \\
\hline 2 & 1.27 & 4.37 & $\mathrm{HCl} 7.5 \%$ & 2928.32 & 3651.42 & $<$ None $>$ & 0.00 & 0.00 & 15.10 & 87.15 & 15.10 \\
\hline 3 & 6.04 & 22.38 & SlickWater & 39985.72 & 43637.14 & $<$ None> & 0.00 & 0.00 & 42.11 & 1040.27 & 42.06 \\
\hline 4 & 28.42 & 3.37 & SlickWater & 12003.11 & 55640.25 & 100 Mesh Sand & 0.24 & 2921.00 & 79.90 & 1329.24 & 79.02 \\
\hline 5 & 32.19 & 3.40 & SlickWater & 12035.30 & 67675.55 & 100 Mesh Sand & 0.49 & 8831.00 & 79.90 & 1622.23 & 78.15 \\
\hline 6 & 35.59 & 4.41 & SlickWater & 15199.52 & 82875.07 & 100 Mesh Sand & 0.75 & 20171.00 & 79.90 & 1996.42 & 77.27 \\
\hline 7 & 40.40 & 5.54 & SlickWater & 18941.62 & 101816.69 & 100 Mesh Sand & 1.00 & 39043.00 & 79.90 & 2467.84 & 76.44 \\
\hline 8 & 46.34 & 9.11 & SlickWater & 28858.10 & 130674.79 & 100 Mesh Sand & 1.49 & 82176.00 & 79.90 & 3201.60 & 74.82 \\
\hline 9 & 55.45 & 5.54 & SlickWater & 18157.44 & 148832.23 & 100 Mesh Sand & 1.99 & 118344.00 & 79.91 & 3673.05 & 73.27 \\
\hline 10 & 61.39 & 5.46 & SlickWater & 18898.98 & 167731.21 & 40/70 - Treated & 0.53 & 128305.00 & 79.89 & 4133.75 & 78.03 \\
\hline 11 & 67.25 & 4.38 & SlickWater & 15038.31 & 182769.53 & 40/70 - Treated & 0.74 & 139482.00 & 79.90 & 4503.95 & 77.28 \\
\hline 12 & 72.03 & 4.42 & SlickWater & 15087.89 & 197857.42 & 40/70 - Treated & 0.99 & 154474.00 & 79.90 & 4879.47 & 76.43 \\
\hline 13 & 76.45 & 8.25 & SlickWater & 26441.48 & 224298.90 & 40/70 White & 1.49 & 193965.00 & 79.89 & 5551.91 & 74.80 \\
\hline 14 & 85.10 & 7.52 & SlickWater & 24395.44 & 248694.34 & 40/70 White & 1.80 & 237973.00 & 79.89 & 6180.36 & 73.84 \\
\hline 15 & 93.02 & 2.58 & SlickWater & 9955.20 & 258649.54 & $<$ None $>$ & 0.00 & 237973.00 & 79.90 & 6417.39 & 79.90 \\
\hline 16 & 96.00 & 0.07 & SlickWater & 391.44 & 259040.98 & $<$ None $>$ & 0.00 & 237973.00 & 79.87 & 6426.71 & 79.89 \\
\hline 17 & 96.07 & 4.00 & SlickWater & 13130.66 & 272171.63 & 40/70 White & 0.48 & 244308.00 & 79.89 & 6746.27 & 78.16 \\
\hline 18 & 100.07 & 4.17 & SlickWater & 13753.96 & 285925.60 & 40/70 White & 0.99 & 257860.00 & 79.90 & 7088.50 & 76.45 \\
\hline 19 & 104.24 & 4.41 & SlickWater & 14717.86 & 300643.45 & 40/70 White & 1.49 & 279731.00 & 79.90 & 7462.69 & 74.82 \\
\hline 20 & 109.05 & 4.42 & SlickWater & 14459.54 & 315103.00 & 40/70 White & 1.99 & 308498.00 & 79.90 & 7838.22 & 73.25 \\
\hline 21 & 113.47 & 4.25 & SlickWater & 13310.70 & 328413.70 & 40/70 White & 2.49 & 341587.00 & 79.89 & 8191.09 & 71.76 \\
\hline 22 & 118.12 & 2.06 & SlickWater & 6442.87 & 334856.57 & 40/70 White & 2.09 & 355043.00 & 79.90 & 8358.88 & 73.05 \\
\hline 23 & 120.18 & 3.01 & SlickWater & 9904.45 & 344761.01 & $<$ None $>$ & 0.00 & 355043.00 & 77.91 & 8593.92 & 78.17 \\
\hline
\end{tabular}

Table 81: Fracture Model - Pump Details Stage 15

\begin{tabular}{|c|c|c|c|c|c|c|c|}
\hline Step\# & Name & Avg S Rate & Max S Rate & Avg Tr Pres & Max Tr Pres & Min Tr Pressure & Calc Avg TR P \\
\hline & & $\mathbf{( b b l / m i n ) ~}$ & $\mathbf{( b b l / m i n )}$ & (psi) & (psi) & (psi) & (psi) \\
\hline 1 & Rate & 13.4 & 15.1 & 5661 & 6268 & 4109 & 5452 \\
\hline 2 & Acid 7.5\% HC & 15.1 & 15.2 & 6420 & 6458 & 6270 & 6423 \\
\hline 3 & PAD & 79.7 & 80.0 & 8388 & 8807 & 6147 & 7543 \\
\hline 4 & 0.25 PPA & 79.9 & 80.0 & 8444 & 8475 & 8422 & 8445 \\
\hline 5 & 0.5 PPA & 79.9 & 80.0 & 8267 & 8475 & 8154 & 8261 \\
\hline 6 & 0.75 PPA & 79.9 & 80.0 & 8115 & 8161 & 8081 & 8114 \\
\hline 7 & 1.00 PPA & 79.9 & 80.0 & 8036 & 8082 & 8007 & 8035 \\
\hline 8 & 1.50 PPA & 79.9 & 80.0 & 7958 & 8021 & 7858 & 7956 \\
\hline 9 & 2.00 PPA & 79.9 & 80.0 & 7876 & 7960 & 7780 & 7877 \\
\hline 10 & 0.50 PPA & 79.9 & 80.0 & 8042 & 8154 & 7912 & 8042 \\
\hline 11 & 0.75 PPA & 79.9 & 80.0 & 7985 & 8028 & 7956 & 7984 \\
\hline 12 & $1.00 P P A$ & 79.9 & 80.0 & 7954 & 7974 & 7896 & 7952 \\
\hline 13 & 1.50 PPA & 79.9 & 80.0 & 7915 & 7975 & 7871 & 7915 \\
\hline 14 & 2.00 PPA & 79.9 & 80.0 & 8102 & 8486 & 7955 & 8106 \\
\hline 15 & Flush & 79.9 & 80.0 & 8652 & 8761 & 8487 & 8653 \\
\hline 16 & 0.75 PPA & 79.9 & 79.9 & 8534 & 8549 & 8508 & 8499 \\
\hline 17 & 0.50 PPA & 79.9 & 80.0 & 8410 & 8500 & 8375 & 8408 \\
\hline 18 & 1.00 PPA & 79.9 & 80.0 & 8322 & 8390 & 8275 & 8320 \\
\hline 19 & 1.50 PPA & 79.9 & 80.0 & 8197 & 8301 & 8118 & 8195 \\
\hline 20 & 2.00 PPA & 79.9 & 80.0 & 8123 & 8162 & 8079 & 8123 \\
\hline 21 & 2.5 PPA & 79.9 & 80.0 & 8170 & 8229 & 8139 & 8171 \\
\hline 22 & 3.0 PPA & 79.9 & 80.0 & 8195 & 8234 & 8131 & 8193 \\
\hline 23 & Flush & 79.2 & 79.9 & 8427 & 8717 & 6373 & 8352 \\
\hline
\end{tabular}


Table 82: Fracture Model - Overview Stage 16

\begin{tabular}{|c|c|c|}
\hline \multicolumn{3}{|c|}{ Stage 16} \\
\hline \multicolumn{3}{|c|}{ Treatment Summary } \\
\hline Formation & Marcellus & \\
\hline Formation Type & Shale & \\
\hline \multicolumn{3}{|c|}{ Stage Data } \\
\hline Report Date & $11 / 12 / 2015$ & \\
\hline Stage Length & 178.00 & $\mathrm{ft}$ \\
\hline Frac Gradient & 1.26 & $\mathrm{psi} / \mathrm{ft}$ \\
\hline ISIP & 5961.00 & psi \\
\hline 5 Min ISIP & 5024.00 & psi \\
\hline $10 \mathrm{Min} I S I P$ & 4848.00 & psi \\
\hline 15 Min ISIP & 4746.00 & psi \\
\hline Breakdown Rate & 15.00 & bpm \\
\hline Breakdown Pressure & 7161.00 & psi \\
\hline Breakdown Volume & 223.00 & bbls \\
\hline Cut Short & $x$ & $x$ \\
\hline Screen Out & & $\mathrm{x}$ \\
\hline \multicolumn{3}{|c|}{ Fluid and Proppant Data } \\
\hline Total Clean Fluid & 6137.00 & bbls \\
\hline Prop.1-Type & 100 Mesh & \\
\hline Prop.2-Type & 40/70 White & \\
\hline Total Proppant Amount & 188460.00 & Ibs \\
\hline Proppant Concentration & 1058.76 & $\mathrm{lb} / \mathrm{ft}$ \\
\hline Proppant Concentration & 0.73 & $\mathrm{lb} / \mathrm{gal}$ \\
\hline \multicolumn{3}{|c|}{ Chemical Data } \\
\hline Acid \% Strength & 7.50 & \\
\hline Total Acid & 3000.00 & gal \\
\hline Total Slickwater & 6066.00 & bbls \\
\hline Total Linear & 0.00 & bbls \\
\hline Total XL & 0.00 & bbls \\
\hline FR Amount & 100.00 & gal \\
\hline Gel Amount & 0.00 & gal or lbs \\
\hline Breaker Amount & 0.00 & gal or lbs \\
\hline Scale Inhibitor & 25.00 & gal \\
\hline Biocide & 80.00 & gal \\
\hline
\end{tabular}

Table 83: Fracture Model - Perfs Stage 16

\begin{tabular}{|ccccc|}
\hline \multicolumn{5}{c|}{ Perfs } \\
\hline Cluster & Start.FT & Stop.FT & Perf_Diam.IN & Perf_Shots. \\
\hline 4 & 10278.00 & 10280.00 & 0.42 & 6 \\
3 & 10316.00 & 10318.00 & 0.42 & 8 \\
2 & 10365.00 & 10367.00 & 0.42 & 6 \\
1 & 10408.00 & 10410.00 & 0.42 & 6 \\
\hline
\end{tabular}

Table 84: Fracture Model - Pump Schedule Stage 16

\begin{tabular}{|c|c|c|c|c|c|c|c|c|c|c|c|}
\hline Stage & $\begin{array}{l}\text { Tot Time } \\
\text { mm:ss }\end{array}$ & $\begin{array}{l}\text { Stage Time } \\
\text { mm:ss }\end{array}$ & Fluid & $\begin{array}{c}\text { Clean Stg Vol } \\
\text { (gal) }\end{array}$ & $\begin{array}{c}\text { Cum Cln Vol } \\
\text { (gal) }\end{array}$ & Proppant & $\begin{array}{l}\text { Slurry Conc } \\
\text { (ppa) }\end{array}$ & $\begin{array}{l}\text { Cum Prop } \\
\text { (Ibs) }\end{array}$ & $\begin{array}{l}\text { Slurry Rate } \\
\text { (bbl/min) }\end{array}$ & $\begin{array}{c}\text { Cum Slurry } \\
\text { (bbl) }\end{array}$ & $\begin{array}{l}\text { Clean Rate } \\
\text { (bbl/min) }\end{array}$ \\
\hline 1 & 0.00 & 1.10 & SlickWater & 446.67 & 446.67 & $<$ <None $>$ & 0.00 & 0.00 & 9.34 & 10.90 & 9.12 \\
\hline 2 & 1.10 & 4.38 & $\mathrm{HCl} 7.5 \%$ & 2999.85 & 3446.52 & $<$ None $>$ & 0.00 & 0.00 & 15.41 & 82.31 & 15.42 \\
\hline 3 & 5.48 & 21.24 & SlickWater & 41976.16 & 45422.67 & $<$ None> & 0.00 & 0.00 & 46.75 & 1082.80 & 46.70 \\
\hline 4 & 27.12 & 3.40 & SlickWater & 11984.08 & 57406.75 & 100 Mesh Sand & 0.24 & 2921.00 & 78.68 & 1371.31 & 77.82 \\
\hline 5 & 30.52 & 3.43 & SlickWater & 12012.71 & 69419.46 & 100 Mesh Sand & 0.49 & 8836.00 & 78.68 & 1663.74 & 76.96 \\
\hline 6 & 34.35 & 4.45 & SlickWater & 15183.61 & 84603.06 & 100 Mesh Sand & 0.74 & 20121.00 & 78.68 & 2037.47 & 76.11 \\
\hline 7 & 39.20 & 5.59 & SlickWater & 18915.40 & 103518.46 & 100 Mesh Sand & 1.00 & 38980.00 & 78.68 & 2508.25 & 75.27 \\
\hline 8 & 45.19 & 9.20 & SlickWater & 28882.03 & 132400.49 & 100 Mesh Sand & 1.49 & 82155.00 & 78.68 & 3242.62 & 73.68 \\
\hline 9 & 54.39 & 9.40 & SlickWater & 29288.48 & 161688.97 & 100 Mesh Sand & 1.99 & 140545.00 & 78.67 & 4003.12 & 72.14 \\
\hline 10 & 64.19 & 5.50 & SlickWater & 18823.80 & 180512.77 & 40/70 - Treated & 0.53 & 150565.00 & 78.67 & 4462.04 & 76.83 \\
\hline 11 & 70.09 & 4.43 & SlickWater & 15072.52 & 195585.29 & 40/70 - Treated & 0.74 & 161787.00 & 78.67 & 4833.10 & 76.09 \\
\hline 12 & 74.52 & 4.46 & SlickWater & 15065.20 & 210650.49 & 40/70 White & 1.00 & 176805.00 & 78.67 & 5208.11 & 75.25 \\
\hline 13 & 79.38 & 9.27 & SlickWater & 29357.01 & 240007.49 & 40/70 White & 1.38 & 217304.00 & 78.60 & 5950.84 & 73.97 \\
\hline 14 & 89.05 & 6.27 & SlickWater & 17783.73 & 257791.22 & $<$ None $>$ & 0.00 & 217304.00 & 65.46 & 6373.03 & 65.65 \\
\hline
\end{tabular}


Table 85: Fracture Model - Pump Details Stage 16

\begin{tabular}{|c|c|c|c|c|c|c|c|}
\hline Step\# & Name & Avg S Rate & Max S Rate & Avg Tr Pres & Max Tr Pres & Min Tr Pressure & Calc Avg TR P \\
\hline & & $(\mathbf{b b l} / \mathbf{m i n})$ & $(\mathbf{b b l} / \mathbf{m i n})$ & $(\mathbf{p s i})$ & $(\mathbf{p s i})$ & (psi) & (psi) \\
\hline 1 & Rate & 11.9 & 15.6 & 5009 & 5628 & 3914 & 4759 \\
\hline 2 & Acid 7.5\% HC & 15.4 & 15.5 & 6196 & 6337 & 5642 & 6204 \\
\hline 3 & PAD & 78.8 & 80.5 & 9016 & 9189 & 6183 & 7671 \\
\hline 4 & 0.25 PPA & 78.7 & 78.7 & 8963 & 8979 & 8949 & 8962 \\
\hline 5 & 0.5 PPA & 78.7 & 78.8 & 8719 & 8950 & 8615 & 8711 \\
\hline 6 & 0.75 PPA & 78.7 & 78.7 & 8538 & 8615 & 8475 & 8535 \\
\hline 7 & 1.00 PPA & 78.7 & 78.8 & 8415 & 8499 & 8353 & 8413 \\
\hline 8 & 1.50 PPA & 78.7 & 78.8 & 8285 & 8365 & 8206 & 8283 \\
\hline 9 & 2.00 PPA & 78.7 & 78.7 & 8246 & 8451 & 8189 & 8247 \\
\hline 10 & 0.50 PPA & 78.7 & 78.8 & 8614 & 8701 & 8451 & 8614 \\
\hline 11 & $0.75 P P A$ & 78.7 & 78.7 & 8555 & 8600 & 8523 & 8552 \\
\hline 12 & 1.00 PPA & 78.7 & 78.7 & 8441 & 8523 & 8390 & 8439 \\
\hline 13 & 1.50 PPA & 78.6 & 78.8 & 8555 & 9241 & 8395 & 8562 \\
\hline 14 & Flush & 66.5 & 74.5 & 9003 & 9241 & 5786 & 8950 \\
\hline
\end{tabular}

Table 86: Fracture Model - Overview Stage 17

\begin{tabular}{|c|c|c|}
\hline \multicolumn{3}{|c|}{ Stage 17} \\
\hline \multicolumn{3}{|c|}{ Treatment Summary } \\
\hline Formation & Marcellus & \\
\hline Formation Type & Shale & \\
\hline \multicolumn{3}{|c|}{$\begin{array}{l}\text { Stage Data } \\
\end{array}$} \\
\hline Report Date & $11 / 12 / 2015$ & \\
\hline Stage Length & 181.00 & $\mathrm{ft}$ \\
\hline Frac Gradient & $\# \mathrm{~N} / \mathrm{A}$ & $\mathrm{psi} / \mathrm{ft}$ \\
\hline ISIP & $\# \mathrm{~N} / \mathrm{A}$ & psi \\
\hline 5 Min ISIP & $\# \mathrm{~N} / \mathrm{A}$ & psi \\
\hline 10 Min ISIP & $\# \mathrm{~N} / \mathrm{A}$ & psi \\
\hline 15 Min ISIP & $\# \mathrm{~N} / \mathrm{A}$ & psi \\
\hline Breakdown Rate & 15.00 & bpm \\
\hline Breakdown Pressure & 7960.00 & psi \\
\hline Breakdown Volume & 218.00 & bbls \\
\hline Cut Short & $\mathrm{x}$ & $x$ \\
\hline Screen Out & $\mathrm{x}$ & $\mathrm{x}$ \\
\hline \multicolumn{3}{|c|}{ Fluid and Proppant Data } \\
\hline Total Clean Fluid & 6976.00 & bbls \\
\hline Prop.1-Type & 100 Mesh & \\
\hline Prop.2-Type & 40/70 White & \\
\hline Total Proppant Amount & 337760.00 & Ibs \\
\hline Proppant Concentration & 1866.08 & $\mathrm{Ib} / \mathrm{ft}$ \\
\hline Proppant Concentration & 1.15 & Ib/gal \\
\hline \multicolumn{3}{|c|}{ Chemical Data } \\
\hline Acid \% Strength & 7.50 & \\
\hline Total Acid & 3000.00 & gal \\
\hline Total Slickwater & 6910.00 & bbls \\
\hline Total Linear & 0.00 & bbls \\
\hline Total XL & 0.00 & bbls \\
\hline FR Amount & 190.00 & gal \\
\hline Gel Amount & 0.00 & gal or lbs \\
\hline Breaker Amount & 0.00 & gal or lbs \\
\hline Scale Inhibitor & 25.00 & gal \\
\hline Biocide & 65.00 & gal \\
\hline
\end{tabular}

Table 87: Fracture Model - Perfs Stage 17

\begin{tabular}{|ccccc|}
\hline \multicolumn{5}{c|}{ Perfs } \\
\hline Cluster & Start.FT & Stop.FT & Perf_Diam.IN & Perf_Shots. \\
\hline 4 & 10090.00 & 10092.00 & 0.42 & 8 \\
3 & 10092.00 & 10094.00 & 0.42 & 8 \\
2 & 10134.00 & 10136.00 & 0.42 & 8 \\
1 & 10139.00 & 10141.00 & 0.42 & 8 \\
\hline
\end{tabular}


Table 88: Fracture Model - Pump Schedule Stage 17

\begin{tabular}{|c|c|c|c|c|c|c|c|c|c|c|c|}
\hline Stage & $\begin{array}{c}\text { Tot Time } \\
\text { mm:ss }\end{array}$ & $\begin{array}{c}\text { Stage Time } \\
\text { mm:ss }\end{array}$ & Fluid & $\begin{array}{c}\text { Clean Stg Vol } \\
\text { (gal) }\end{array}$ & $\begin{array}{c}\text { Cum Cln Vol } \\
\text { (gal) }\end{array}$ & Proppant & $\begin{array}{c}\text { Slurry Conc } \\
\text { (ppa) }\end{array}$ & $\begin{array}{c}\text { Cum Prop } \\
\text { (Ibs) }\end{array}$ & $\begin{array}{c}\text { Slurry Rate } \\
\text { (bbl/min) }\end{array}$ & $\begin{array}{c}\text { Cum Slurry } \\
\text { (bbl) }\end{array}$ & $\begin{array}{l}\text { Clean Rate } \\
\text { (bbl/min) }\end{array}$ \\
\hline 1 & 0.00 & 1.19 & SlickWater & 549.01 & 549.01 & $<$ None $>$ & 0.00 & 0.00 & 10.12 & 13.33 & 9.93 \\
\hline 2 & 1.19 & 4.23 & $\mathrm{HCl} 7.5 \%$ & 2786.84 & 3335.85 & $<$ None $>$ & 0.00 & 0.00 & 15.14 & 79.68 & 15.14 \\
\hline 3 & 5.42 & 25.19 & SlickWater & 42018.75 & 45354.61 & $<$ None $>$ & 0.00 & 0.00 & 39.55 & 1080.97 & 39.52 \\
\hline 4 & 31.01 & 4.24 & SlickWater & 12013.96 & 57368.57 & 100 Mesh Sand & 0.24 & 2925.00 & 65.73 & 1370.20 & 65.01 \\
\hline 5 & 35.25 & 5.37 & SlickWater & 15132.47 & 72501.04 & 100 Mesh Sand & 0.49 & 10408.00 & 65.60 & 1738.62 & 64.15 \\
\hline 6 & 41.02 & 5.02 & SlickWater & 15234.39 & 87735.43 & 100 Mesh Sand & 0.74 & 21721.00 & 74.54 & 2113.83 & 72.06 \\
\hline 7 & 46.04 & 5.53 & SlickWater & 18906.23 & 106641.66 & 100 Mesh Sand & 1.00 & 40550.00 & 79.98 & 2584.36 & 76.51 \\
\hline 8 & 51.57 & 9.10 & SlickWater & 28834.22 & 135475.88 & 100 Mesh Sand & 1.49 & 83633.00 & 79.98 & 3317.49 & 74.89 \\
\hline 9 & 61.07 & 6.10 & SlickWater & 18993.77 & 154469.64 & 100 Mesh Sand & 1.99 & 121492.00 & 79.98 & 3810.69 & 73.34 \\
\hline 10 & 67.17 & 5.44 & SlickWater & 18807.09 & 173276.73 & 40/70 White & 0.53 & 131463.00 & 79.97 & 4269.18 & 78.10 \\
\hline 11 & 73.01 & 4.38 & SlickWater & 15050.01 & 188326.74 & 40/70 White & 0.74 & 142647.00 & 79.96 & 4639.67 & 77.34 \\
\hline 12 & 77.39 & 4.43 & SlickWater & 15153.12 & 203479.86 & 40/70 White & 0.99 & 157697.00 & 79.96 & 5016.80 & 76.49 \\
\hline 13 & 82.22 & 8.25 & SlickWater & 26467.53 & 229947.39 & 40/70 White & 1.49 & 197204.00 & 79.97 & 5689.89 & 74.87 \\
\hline 14 & 90.47 & 12.16 & SlickWater & 37760.90 & 267708.29 & 40/70 White & 2.00 & 272644.00 & 79.97 & 6670.82 & 73.29 \\
\hline 15 & 103.03 & 8.35 & SlickWater & 24810.43 & 292518.72 & 40/70 White & 2.29 & 329436.00 & 75.92 & 7322.48 & 68.82 \\
\hline 16 & 111.38 & 0.35 & SlickWater & 559.16 & 293077.88 & $<$ None $>$ & 0.00 & 329436.00 & 21.63 & 7335.09 & 22.82 \\
\hline
\end{tabular}

Table 89: Fracture Model - Pump Details Stage 17

\begin{tabular}{|c|c|c|c|c|c|c|c|}
\hline Step\# & Name & Avg S Rate & Max S Rate & Avg Tr Pres & Max Tr Pres & Min Tr Pressure & Calc Avg TR P \\
\hline & & $(\mathbf{b b l} / \mathbf{m i n})$ & $\mathbf{( b b l / m i n )}$ & $(\mathbf{p s i})$ & $\mathbf{( p s i )}$ & (psi) & (psi) \\
\hline 1 & Rate & 12.8 & 15.1 & 6647 & 7514 & 3827 & 6004 \\
\hline 2 & Acid 7.5\% HC & 15.1 & 15.2 & 7454 & 7520 & 7363 & 7451 \\
\hline 3 & PAD & 51.0 & 65.8 & 8606 & 9157 & 6989 & 8198 \\
\hline 4 & 0.25 PPA & 65.7 & 65.8 & 8889 & 8958 & 8804 & 8887 \\
\hline 5 & 0.5 PPA & 65.6 & 67.8 & 9001 & 9184 & 8799 & 9001 \\
\hline 6 & 0.75 PPA & 79.4 & 80.0 & 8751 & 8930 & 8711 & 8781 \\
\hline 7 & 1.00 PPA & 80.0 & 80.0 & 8532 & 8739 & 8356 & 8528 \\
\hline 8 & 1.50 PPA & 80.0 & 80.1 & 8199 & 8359 & 8095 & 8198 \\
\hline 9 & 2.0 PPA & 80.0 & 80.1 & 8051 & 8127 & 8002 & 8049 \\
\hline 10 & 0.50 PPA & 80.0 & 80.0 & 8005 & 8062 & 7946 & 8005 \\
\hline 11 & 0.75 PPA & 80.0 & 80.1 & 7932 & 7999 & 7901 & 7931 \\
\hline 12 & 1.00 PPA & 80.0 & 80.0 & 7871 & 7903 & 7853 & 7870 \\
\hline 13 & 1.50 PPA & 80.0 & 80.1 & 7824 & 7857 & 7794 & 7824 \\
\hline 14 & 2.00 PPA & 80.0 & 80.1 & 7863 & 8179 & 7737 & 7866 \\
\hline 15 & 2.5 PPA & 76.8 & 80.1 & 8631 & 9198 & 8151 & 8660 \\
\hline 16 & Flush & 31.1 & 48.9 & 9086 & 9342 & 1 & 9154 \\
\hline
\end{tabular}

Table 90: Fracture Model - Overview Stage 18

\begin{tabular}{|c|c|c|}
\hline \multicolumn{3}{|c|}{ Stage 18} \\
\hline \multicolumn{3}{|c|}{ Treatment Summary } \\
\hline Formation & Marcellus & \\
\hline Formation Type & Shale & \\
\hline \multicolumn{3}{|c|}{ Stage Data } \\
\hline Report Date & $11 / 13 / 2015$ & \\
\hline Stage Length & 180.00 & $\mathrm{ft}$ \\
\hline Frac Gradient & 1.30 & $\mathrm{psi} / \mathrm{ft}$ \\
\hline ISIP & 6427.00 & $\mathrm{psi}$ \\
\hline 5 Min ISIP & 5695.00 & $\mathrm{psi}$ \\
\hline 10 Min ISIP & 5393.00 & psi \\
\hline 15 Min ISIP & 5200.00 & psi \\
\hline Breakdown Rate & 15.00 & bpm \\
\hline Breakdown Pressure & 6236.00 & psi \\
\hline Breakdown Volume & 222.00 & bbls \\
\hline Cut Short & $\mathrm{x}$ & $\mathrm{x}$ \\
\hline Screen Out & & $\mathrm{x}$ \\
\hline \multicolumn{3}{|c|}{ Fluid and Proppant Data } \\
\hline Total Clean Fluid & 6904.00 & bbls \\
\hline Prop.1-Type & 100 Mesh & \\
\hline Prop.2-Type & 40/70 White & \\
\hline Total Proppant Amount & 291860.00 & Ibs \\
\hline Proppant Concentration & 1621.44 & $\mathrm{Ib} / \mathrm{ft}$ \\
\hline Proppant Concentration & 1.01 & $\mathrm{Ib} / \mathrm{gal}$ \\
\hline \multicolumn{3}{|c|}{ Chemical Data } \\
\hline Acid \% Strength & 7.50 & \\
\hline Total Acid & 3000.00 & gal \\
\hline Total Slickwater & 6832.00 & bbls \\
\hline Total Linear & 0.00 & bbls \\
\hline Total XL & 0.00 & bbls \\
\hline FR Amount & 130.00 & gal \\
\hline Gel Amount & 0.00 & gal or lbs \\
\hline Breaker Amount & 0.00 & gal or lbs \\
\hline Scale Inhibitor & 30.00 & gal \\
\hline Biocide & 90.00 & gal \\
\hline
\end{tabular}


Table 91: Fracture Model - Perfs Stage 18

\begin{tabular}{|ccccc|}
\hline \multicolumn{5}{c|}{ Perfs } \\
\hline Cluster & Start.FT & Stop.FT & Perf_Diam.IN & Perf_Shots. \\
\hline 4 & 9912.00 & 9914.00 & 0.42 & 8 \\
3 & 9959.00 & 9961.00 & 0.42 & 8 \\
2 & 10002.00 & 10004.00 & 0.42 & 8 \\
1 & 10048.00 & 10050.00 & 0.42 & 8 \\
\hline
\end{tabular}

Table 92: Fracture Model - Pump Schedule Stage 18

\begin{tabular}{|c|c|c|c|c|c|c|c|c|c|c|c|}
\hline Stage & $\begin{array}{c}\text { Tot Time } \\
\mathrm{mm}: \mathrm{ss}\end{array}$ & $\begin{array}{c}\text { Stage Time } \\
\text { mm:ss }\end{array}$ & Fluid & $\begin{array}{c}\text { Clean Stg Vol } \\
\text { (gal) }\end{array}$ & $\begin{array}{c}\text { Cum Cln Vol } \\
\text { (gal) }\end{array}$ & Proppant & $\begin{array}{c}\text { Slurry Conc } \\
\text { (ppa) }\end{array}$ & $\begin{array}{l}\text { Cum Prop } \\
\text { (Ibs) }\end{array}$ & $\begin{array}{c}\text { Slurry Rate } \\
\text { (bbl/min) }\end{array}$ & $\begin{array}{c}\text { Cum Slurry } \\
\text { (bbl) }\end{array}$ & $\begin{array}{l}\text { Clean Rate } \\
\text { (bbl/min) }\end{array}$ \\
\hline 1 & 0.00 & 0.50 & SlickWater & 295.82 & 295.82 & $<$ None $>$ & 0.00 & 0.00 & 8.75 & 7.29 & 8.45 \\
\hline 2 & 0.50 & 2.23 & $\mathrm{HCl} 7.5 \%$ & 1508.78 & 1804.60 & $<$ None $>$ & 0.00 & 0.00 & 15.07 & 43.22 & 15.07 \\
\hline 3 & 3.13 & 3.18 & SlickWater & 2090.69 & 3895.29 & $<$ None $>$ & 0.00 & 0.00 & 15.08 & 93.00 & 15.08 \\
\hline 4 & 6.31 & 2.23 & $\mathrm{HCl} 7.5 \%$ & 1508.71 & 5404.00 & $<$ None $>$ & 0.00 & 0.00 & 15.07 & 128.92 & 15.07 \\
\hline 5 & 8.54 & 18.18 & SlickWater & 41967.16 & 47371.16 & $<$ None $>$ & 0.00 & 0.00 & 54.67 & 1129.34 & 54.60 \\
\hline 6 & 27.12 & 3.19 & SlickWater & 12004.23 & 59375.39 & 100 Mesh Sand & 0.24 & 2908.00 & 87.13 & 1418.33 & 86.18 \\
\hline 7 & 30.31 & 3.19 & SlickWater & 11993.11 & 71368.50 & 100 Mesh Sand & 0.49 & 8818.00 & 88.04 & 1710.34 & 86.10 \\
\hline 8 & 33.50 & 4.04 & SlickWater & 15155.76 & 86524.26 & 100 Mesh Sand & 0.74 & 20050.00 & 91.74 & 2083.42 & 88.73 \\
\hline 9 & 37.54 & 5.02 & SlickWater & 18930.03 & 105454.29 & 100 Mesh Sand & 0.99 & 38863.00 & 93.59 & 2554.50 & 89.55 \\
\hline 10 & 42.56 & 7.52 & SlickWater & 28956.07 & 134410.36 & 100 Mesh Sand & 1.49 & 82038.00 & 93.59 & 3290.76 & 87.64 \\
\hline 11 & 50.48 & 6.14 & SlickWater & 22470.44 & 156880.81 & 100 Mesh Sand & 1.99 & 126788.00 & 93.60 & 3874.18 & 85.83 \\
\hline 12 & 57.02 & 4.54 & SlickWater & 18807.46 & 175688.27 & 40/70 White & 0.54 & 136887.00 & 93.59 & 4332.79 & 91.39 \\
\hline 13 & 61.56 & 3.58 & SlickWater & 15081.78 & 190770.05 & 40/70 White & 0.74 & 148101.00 & 93.60 & 4704.06 & 90.53 \\
\hline 14 & 65.54 & 4.01 & SlickWater & 15104.95 & 205875.00 & 40/70 White & 0.99 & 163113.00 & 93.60 & 5080.01 & 89.54 \\
\hline 15 & 69.55 & 9.08 & SlickWater & 33611.13 & 239486.12 & 40/70 White & 1.50 & 213374.00 & 93.60 & 5934.86 & 87.62 \\
\hline 16 & 79.03 & 11.23 & SlickWater & 41117.50 & 280603.62 & 40/70 White & 1.86 & 290055.00 & 93.29 & 6996.85 & 86.00 \\
\hline 17 & 90.26 & 2.33 & SlickWater & 9388.12 & 289991.74 & $<$ None $>$ & 0.00 & 290055.00 & 87.06 & 7218.86 & 87.66 \\
\hline
\end{tabular}

Table 93: Fracture Model - Pump Details Stage 18

\begin{tabular}{|c|c|c|c|c|c|c|c|}
\hline Step\# & Name & Avg S Rate & Max S Rate & Avg Tr Pres & Max Tr Pres & Min Tr Pressure & Calc Avg TR P \\
\hline & & $(\mathbf{b b l} / \mathbf{m i n})$ & $(\mathbf{b b l} / \mathbf{m i n})$ & $(\mathbf{p s i})$ & (psi) & (psi) & (psi) \\
\hline 1 & Rate & 12.6 & 15.7 & 5682 & 6459 & 3866 & 5127 \\
\hline 2 & Acid 7.5\% HC & 15.1 & 15.1 & 6554 & 6615 & 6477 & 6554 \\
\hline 3 & Spacer & 15.1 & 15.1 & 6370 & 6508 & 6276 & 6365 \\
\hline 4 & Acid 7.5\% HC & 15.1 & 15.1 & 6276 & 6291 & 6265 & 6275 \\
\hline 5 & PAD & 87.1 & 87.2 & 8764 & 8999 & 6133 & 7793 \\
\hline 6 & 0.25 PPA & 87.1 & 87.3 & 8788 & 8838 & 8709 & 8785 \\
\hline 7 & 0.5 PPA & 88.0 & 90.5 & 8473 & 8694 & 8370 & 8469 \\
\hline 8 & 0.75 PPA & 91.7 & 93.7 & 8548 & 8642 & 8462 & 8548 \\
\hline 9 & 1.00 PPA & 93.6 & 93.7 & 8578 & 8620 & 8556 & 8578 \\
\hline 10 & 1.50 PPA & 93.6 & 93.8 & 8533 & 8563 & 8504 & 8533 \\
\hline 11 & 2.00 PPA & 93.6 & 93.7 & 8564 & 8616 & 8520 & 8564 \\
\hline 12 & 0.50 PPA & 93.6 & 93.7 & 8542 & 8608 & 8485 & 8540 \\
\hline 13 & $0.75 P P A$ & 93.6 & 93.7 & 8507 & 8521 & 8493 & 8507 \\
\hline 14 & 1.00 PPA & 93.6 & 93.7 & 8513 & 8524 & 8503 & 8514 \\
\hline 15 & 1.50 PPA & 93.6 & 93.8 & 8493 & 8533 & 8402 & 8492 \\
\hline 16 & 2.00 PPA & 93.3 & 93.7 & 8472 & 8873 & 8367 & 8476 \\
\hline 17 & Flush & 90.6 & 91.7 & 8909 & 9091 & 6080 & 8755 \\
\hline
\end{tabular}


Table 94: Fracture Model - Overview Stage 19

\begin{tabular}{|c|c|c|}
\hline \multicolumn{3}{|c|}{ Stage 19} \\
\hline \multicolumn{3}{|c|}{ Treatment Summary } \\
\hline Formation & Marcellus & \\
\hline Formation Type & Shale & \\
\hline \multicolumn{3}{|c|}{ Stage Data } \\
\hline Report Date & $11 / 13 / 2015$ & \\
\hline Stage Length & 180.00 & $\mathrm{ft}$ \\
\hline Frac Gradient & 1.24 & $\mathrm{psi} / \mathrm{ft}$ \\
\hline ISIP & 5992.00 & psi \\
\hline 5 Min ISIP & 5667.00 & psi \\
\hline 10 Min ISIP & 5555.00 & psi \\
\hline 15 Min ISIP & 5463.00 & psi \\
\hline Breakdown Rate & 15.00 & bpm \\
\hline Breakdown Pressure & 6001.00 & psi \\
\hline Breakdown Volume & 211.00 & bbls \\
\hline Cut Short & & $x$ \\
\hline Screen Out & & $x$ \\
\hline \multicolumn{3}{|c|}{ Fluid and Proppant Data } \\
\hline Total Clean Fluid & 7807.00 & bbls \\
\hline Prop.1-Type & 100 Mesh & \\
\hline Prop.2-Type & 40/70 White & \\
\hline Total Proppant Amount & 360720.00 & Ibs \\
\hline Proppant Concentration & 2004.00 & $\mathrm{Ib} / \mathrm{ft}$ \\
\hline Proppant Concentration & 1.10 & Ib/gal \\
\hline \multicolumn{3}{|c|}{ Chemical Data } \\
\hline Acid \% Strength & 7.50 & \\
\hline Total Acid & 3000.00 & gal \\
\hline Total Slickwater & 6000.00 & bbls \\
\hline Total Linear & 1408.00 & bbls \\
\hline Total XL & 0.00 & bbls \\
\hline FR Amount & 105.00 & gal \\
\hline Gel Amount & 1117.00 & gal or lbs \\
\hline Breaker Amount & 132.00 & gal or lbs \\
\hline Scale Inhibitor & 37.00 & gal \\
\hline Biocide & 100.00 & gal \\
\hline
\end{tabular}

Table 95: Fracture Model - Perfs Stage 19

\begin{tabular}{|ccccc|}
\hline \multicolumn{5}{c|}{ Perfs } \\
\hline Cluster & Start.FT & Stop.FT & Perf_Diam.IN & Perf_Shots. \\
\hline 4 & 9732.00 & 9734.00 & 0.42 & 6 \\
3 & 9779.00 & 9781.00 & 0.42 & 6 \\
2 & 9823.00 & 9825.00 & 0.42 & 6 \\
1 & 9869.00 & 9871.00 & 0.42 & 6 \\
\hline
\end{tabular}

Table 96: Fracture Model - Pump Schedule Stage 19

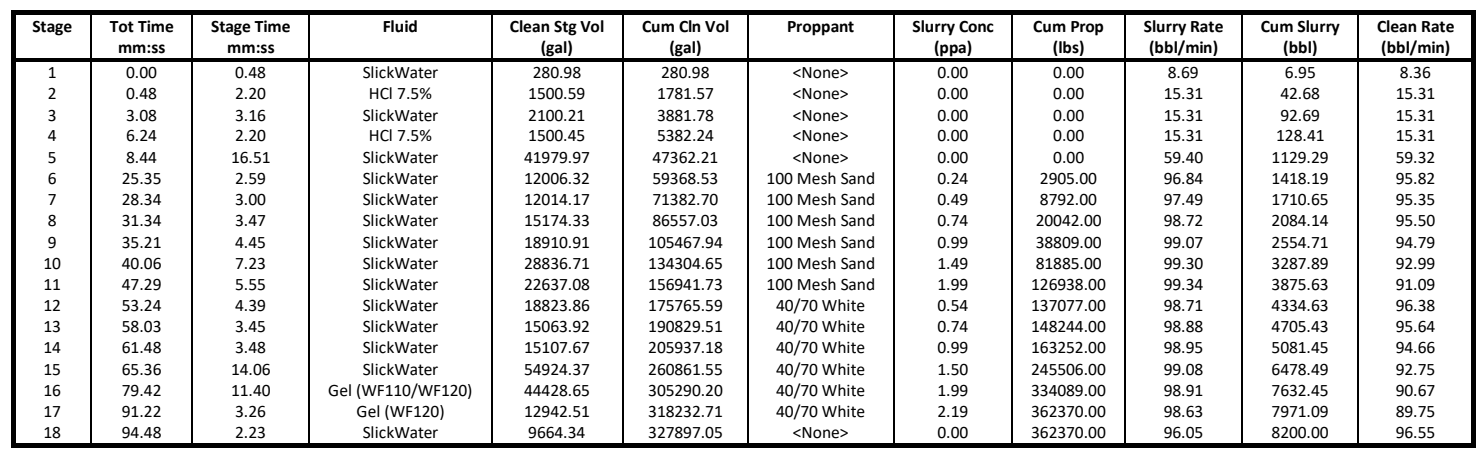


Table 97: Fracture Model - Pump Details Stage 19

\begin{tabular}{|c|c|c|c|c|c|c|c|}
\hline Step\# & Name & Avg S Rate & Max S Rate & Avg Tr Pres & Max Tr Pres & Min Tr Pressure & Calc Avg TR P \\
\hline & & (bbl/min) & (bbl/min) & (psi) & (psi) & (psi) & (psi) \\
\hline 1 & Rate & 12.3 & 15.5 & 5108 & 5619 & 3966 & 4774 \\
\hline 2 & Acid 7.5\% HC & 15.3 & 15.4 & 5993 & 6062 & 5651 & 6004 \\
\hline 3 & Spacer & 15.3 & 15.3 & 5979 & 6050 & 5942 & 5977 \\
\hline 4 & Acid 7.5\% HC & 15.3 & 15.4 & 5954 & 5977 & 5933 & 5955 \\
\hline 5 & PAD & 97.2 & 99.7 & 8910 & 8977 & 5864 & 7773 \\
\hline 6 & 0.25 PPA & 96.9 & 97.3 & 8953 & 8988 & 8916 & 8955 \\
\hline 7 & 0.5 PPA & 97.5 & 97.6 & 8768 & 8988 & 8708 & 8762 \\
\hline 8 & 0.75 PPA & 98.7 & 100.2 & 8717 & 8790 & 8628 & 8715 \\
\hline 9 & 1.00 PPA & 99.1 & 99.4 & 8642 & 8718 & 8605 & 8641 \\
\hline 10 & 1.50 PPA & 99.3 & 99.7 & 8589 & 8610 & 8558 & 8589 \\
\hline 11 & 2.00 PPA & 99.3 & 99.8 & 8610 & 8658 & 8573 & 8611 \\
\hline 12 & 0.50 PPA & 98.7 & 99.8 & 8607 & 8672 & 8545 & 8606 \\
\hline 13 & 0.75 PPA & 98.9 & 99.1 & 8541 & 8551 & 8530 & 8541 \\
\hline 14 & 1 & 99.0 & 99.3 & 8534 & 8546 & 8519 & 8533 \\
\hline 15 & 1.50 PPA & 99.1 & 99.7 & 8494 & 8598 & 8391 & 8493 \\
\hline 16 & 2.00 PPA & 98.9 & 99.4 & 8439 & 8630 & 8283 & 8440 \\
\hline 17 & 2.5 PPA & 98.6 & 98.9 & 8615 & 8644 & 8579 & 8614 \\
\hline 18 & Flush & 97.9 & 98.7 & 8550 & 8759 & 5492 & 8442 \\
\hline
\end{tabular}

Table 98: Fracture Model - Overview Stage 20

\begin{tabular}{|c|c|c|}
\hline \multicolumn{3}{|c|}{ Stage 20} \\
\hline \multicolumn{3}{|c|}{ Treatment Summary } \\
\hline Formation & Marcellus & \\
\hline Formation Type & Shale & \\
\hline \multicolumn{3}{|c|}{ Stage Data } \\
\hline Report Date & $11 / 14 / 2015$ & \\
\hline Stage Length & 240.00 & $\mathrm{ft}$ \\
\hline Frac Gradient & 1.22 & $\mathrm{psi} / \mathrm{ft}$ \\
\hline ISIP & 5867.00 & psi \\
\hline 5 Min ISIP & 5685.00 & psi \\
\hline 10 Min ISIP & 5630.00 & psi \\
\hline $15 \mathrm{Min}$ ISIP & 5578.00 & psi \\
\hline Breakdown Rate & 15.00 & bpm \\
\hline Breakdown Pressure & 6332.00 & psi \\
\hline Breakdown Volume & 205.00 & bbls \\
\hline Cut Short & & $\mathrm{x}$ \\
\hline Screen Out & & $x$ \\
\hline \multicolumn{3}{|c|}{ Fluid and Proppant Data } \\
\hline Total Clean Fluid & 7497.00 & bbls \\
\hline Prop.1-Type & 100 Mesh & \\
\hline Prop.2-Type & 40/70 White & \\
\hline Total Proppant Amount & 439320.00 & Ibs \\
\hline Proppant Concentration & 1830.50 & $\mathrm{lb} / \mathrm{ft}$ \\
\hline Proppant Concentration & 1.40 & $\mathrm{lb} / \mathrm{gal}$ \\
\hline \multicolumn{3}{|c|}{ Chemical Data } \\
\hline Acid \% Strength & 7.50 & \\
\hline Total Acid & 3000.00 & gal \\
\hline Total Slickwater & 2314.00 & bbls \\
\hline Total Linear & 5109.00 & bbls \\
\hline Total XL & 0.00 & bbls \\
\hline FR Amount & 250.00 & gal \\
\hline Gel Amount & 0.00 & gal or lbs \\
\hline Breaker Amount & 0.00 & gal or lbs \\
\hline Scale Inhibitor & 29.00 & gal \\
\hline Biocide & 85.00 & gal \\
\hline
\end{tabular}

Table 99: Fracture Model - Perfs Stage 20

\begin{tabular}{|ccccc|}
\hline \multicolumn{5}{c|}{ Perfs } \\
\hline Cluster & Start.FT & Stop.FT & Perf_Diam.IN & Perf_Shots. \\
\hline 5 & 9504.00 & 9506.00 & 0.42 & 8 \\
4 & 9552.00 & 9554.00 & 0.42 & 8 \\
3 & 9594.00 & 9596.00 & 0.42 & 8 \\
2 & 9639.00 & 9641.00 & 0.42 & 8 \\
1 & 9685.00 & 9687.00 & 0.42 & 8 \\
\hline
\end{tabular}


Table 100: Fracture Model - Pump Schedule Stage 20

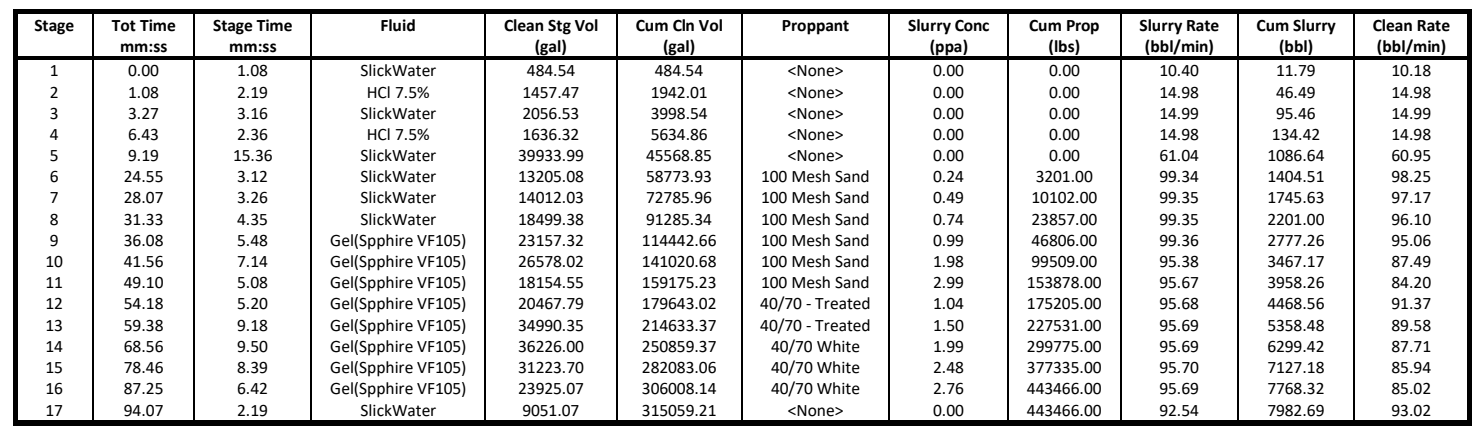

Table 101: Fracture Model - Pump Details Stage 20

\begin{tabular}{|c|c|c|c|c|c|c|c|}
\hline Step\# & Name & Avg S Rate & Max S Rate & Avg Tr Pres & Max Tr Pres & Min Tr Pressure & Calc Avg TR P \\
\hline & & (bbl/min) & (bbl/min) & (psi) & (psi) & (psi) & (psi) \\
\hline 1 & Rate & 13.4 & 15.0 & 5398 & 5692 & 4440 & 5177 \\
\hline 2 & Acid 7.5\% HC & 15.0 & 15.0 & 5911 & 6021 & 5702 & 5920 \\
\hline 3 & Spacer & 15.0 & 15.0 & 6021 & 6039 & 6011 & 6021 \\
\hline 4 & Acid 7.5\% HC & 15.0 & 15.0 & 6029 & 6054 & 6012 & 6030 \\
\hline 5 & PAD & 98.6 & 99.5 & 8212 & 8536 & 5967 & 7425 \\
\hline 6 & 0.25 PPA & 99.3 & 99.4 & 8594 & 8680 & 8529 & 8596 \\
\hline 7 & 0.5 PPA & 99.3 & 99.5 & 8593 & 8634 & 8564 & 8592 \\
\hline 8 & 0.75 PPA & 99.4 & 99.5 & 8561 & 8585 & 8535 & 8560 \\
\hline 9 & 1.00 PPA & 99.4 & 99.5 & 8489 & 8537 & 8452 & 8488 \\
\hline 10 & 2.00 PPA & 95.5 & 99.5 & 8255 & 8459 & 8003 & 8250 \\
\hline 11 & 3.0 PPA & 95.7 & 95.8 & 8287 & 8324 & 8245 & 8287 \\
\hline 12 & 1.0 PPA & 95.7 & 95.9 & 8215 & 8320 & 8135 & 8213 \\
\hline 13 & 1.50 PPA & 95.7 & 95.9 & 8127 & 8163 & 8095 & 8127 \\
\hline 14 & 2.0 PPA & 95.7 & 95.9 & 8112 & 8145 & 8065 & 8112 \\
\hline 15 & 2.5 PPA & 95.7 & 95.9 & 8140 & 8176 & 8087 & 8141 \\
\hline 16 & 3.0 PPA & 95.7 & 95.8 & 8219 & 8331 & 8159 & 8221 \\
\hline 17 & Flush & 94.8 & 95.8 & 8299 & 8471 & 5405 & 8176 \\
\hline
\end{tabular}

Table 102: Fracture Model - Overview Stage 21

\begin{tabular}{|c|c|c|}
\hline \multicolumn{3}{|c|}{ Stage 21} \\
\hline \multicolumn{3}{|c|}{ Treatment Summary } \\
\hline Formation & Marcellus & \\
\hline Formation Type & Shale & \\
\hline \multicolumn{3}{|c|}{ Stage Data } \\
\hline Report Date & $11 / 14 / 2015$ & \\
\hline Stage Length & 218.00 & $\mathrm{ft}$ \\
\hline Frac Gradient & 1.22 & $\mathrm{psi} / \mathrm{ft}$ \\
\hline ISIP & 5898.00 & psi \\
\hline 5 Min ISIP & 5654.00 & psi \\
\hline 10 Min ISIP & 5592.00 & psi \\
\hline 15 Min ISIP & 5513.00 & psi \\
\hline Breakdown Rate & 15.00 & bpm \\
\hline Breakdown Pressure & 6506.00 & psi \\
\hline Breakdown Volume & 197.00 & bbls \\
\hline Cut Short & & $\mathrm{x}$ \\
\hline Screen Out & & $\mathrm{x}$ \\
\hline \multicolumn{3}{|c|}{ Fluid and Proppant Data } \\
\hline Total Clean Fluid & 7279.00 & bbls \\
\hline Prop.1-Type & 100 Mesh & \\
\hline Prop.2-Type & 40/70 White & \\
\hline Total Proppant Amount & 441000.00 & lbs \\
\hline Proppant Concentration & 2022.94 & $\mathrm{lb} / \mathrm{ft}$ \\
\hline Proppant Concentration & 1.44 & $\mathrm{lb} / \mathrm{gal}$ \\
\hline \multicolumn{3}{|c|}{ Chemical Data } \\
\hline Acid \% Strength & 7.50 & \\
\hline Total Acid & 3000.00 & gal \\
\hline Total Slickwater & 3356.00 & bbls \\
\hline Total Linear & 3851.00 & bbls \\
\hline Total XL & 0.00 & bbls \\
\hline FR Amount & 306.00 & gal \\
\hline Gel Amount & 0.00 & gal or lbs \\
\hline Breaker Amount & 0.00 & gal or lbs \\
\hline Scale Inhibitor & 41.00 & gal \\
\hline Biocide & 90.00 & gal \\
\hline
\end{tabular}


Table 103: Fracture Model - Perfs Stage 21

\begin{tabular}{|ccccc|}
\hline \multicolumn{5}{c|}{ Perfs } \\
\hline Cluster & Start.FT & Stop.FT & Perf_Diam.IN & Perf_Shots. \\
\hline 5 & 9271.00 & 9273.00 & 0.42 & 8 \\
4 & 9318.00 & 9320.00 & 0.42 & 8 \\
3 & 9365.00 & 9367.00 & 0.42 & 8 \\
2 & 9410.00 & 9412.00 & 0.42 & 8 \\
1 & 9445.00 & 9447.00 & 0.42 & 8 \\
\hline
\end{tabular}

Table 104: Fracture Model - Pump Schedule Stage 21

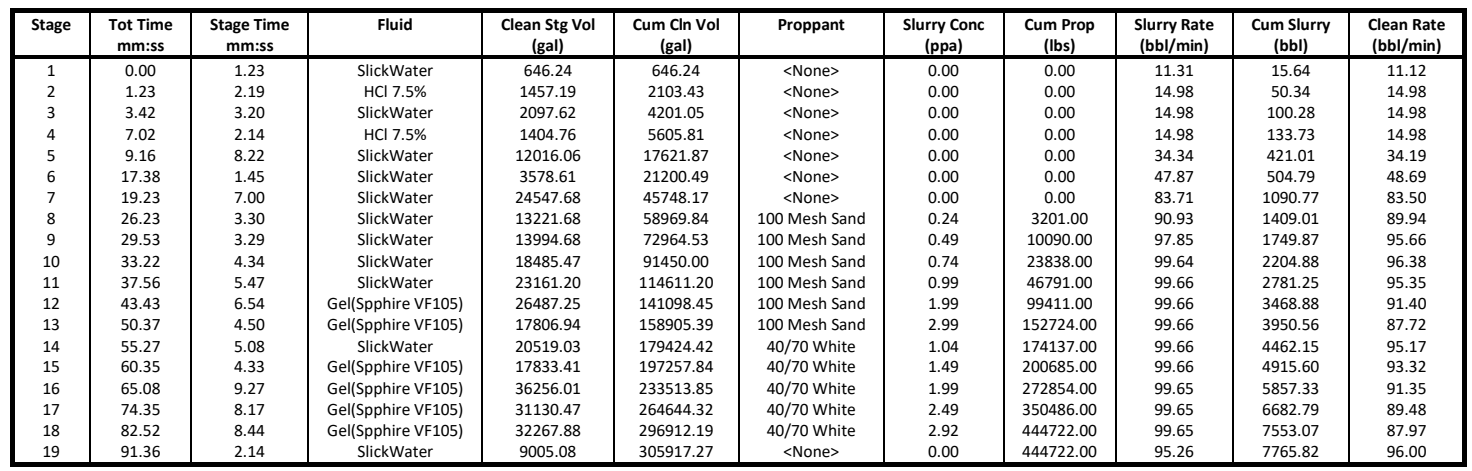

Table 105: Fracture Model - Pump Details Stage 21

\begin{tabular}{|c|c|c|c|c|c|c|c|}
\hline Step\# & Name & Avg S Rate & Max S Rate & Avg Tr Pres & Max Tr Pres & Min Tr Pressure & Calc Avg TR P \\
\hline & & $(\mathbf{b b l} / \mathbf{m i n})$ & $\mathbf{( b b l / m i n )}$ & (psi) & (psi) & (psi) & (psi) \\
\hline 1 & Rate & 13.7 & 15.0 & 5385 & 5640 & 4602 & 5242 \\
\hline 2 & Acid 7.5\% HC & 15.0 & 15.0 & 5752 & 5821 & 5644 & 5757 \\
\hline 3 & spacer & 15.0 & 15.0 & 5824 & 5840 & 5816 & 5824 \\
\hline 4 & Acid 7.5\% HC & 15.0 & 15.0 & 5843 & 5881 & 5823 & 5845 \\
\hline 5 & PAD & 50.3 & 85.9 & 7670 & 8513 & 5838 & 6918 \\
\hline 6 & Stepdown & 61.6 & 86.1 & 7242 & 8300 & 5266 & 6712 \\
\hline 7 & PAD & 87.6 & 91.1 & 8500 & 8638 & 5280 & 8378 \\
\hline 8 & 0.25 PPA & 91.0 & 91.0 & 8488 & 8519 & 8417 & 8485 \\
\hline 9 & 0.5 PPA & 97.8 & 99.7 & 8658 & 8740 & 8417 & 8659 \\
\hline 10 & 0.75 PPA & 99.6 & 99.8 & 8667 & 8703 & 8627 & 8666 \\
\hline 11 & 1.00 PPA & 99.7 & 99.8 & 8605 & 8629 & 8572 & 8604 \\
\hline 12 & 2.00 PPA & 99.7 & 99.8 & 8550 & 8595 & 8515 & 8550 \\
\hline 13 & 3.0 PPA & 99.6 & 99.8 & 8518 & 8550 & 8469 & 8518 \\
\hline 14 & 1.0 PPA & 99.7 & 99.9 & 8475 & 8546 & 8412 & 8473 \\
\hline 15 & 1.50 PPA & 99.7 & 99.8 & 8348 & 8412 & 8308 & 8347 \\
\hline 16 & 2.00 PPA & 99.7 & 99.8 & 8325 & 8353 & 8295 & 8325 \\
\hline 17 & 2.50 PPA & 99.7 & 99.8 & 8315 & 8331 & 8295 & 8315 \\
\hline 18 & 3.0 PPA & 99.6 & 99.8 & 8326 & 8400 & 8295 & 8327 \\
\hline 19 & Flush & 98.9 & 99.7 & 8424 & 8597 & 5444 & 8279 \\
\hline
\end{tabular}


Table 106: Fracture Model - Overview Stage 22

\begin{tabular}{|c|c|c|}
\hline \multicolumn{3}{|c|}{ Stage 22} \\
\hline \multicolumn{3}{|c|}{ Treatment Summary } \\
\hline Formation & Marcellus & \\
\hline Formation Type & Shale & \\
\hline \multicolumn{3}{|c|}{ Stage Data } \\
\hline Report Date & $11 / 14 / 2015$ & \\
\hline Stage Length & 220.00 & $\mathrm{ft}$ \\
\hline Frac Gradient & 1.25 & $\mathrm{psi} / \mathrm{ft}$ \\
\hline ISIP & 6022.00 & psi \\
\hline 5 Min ISIP & 5771.00 & psi \\
\hline 10 Min ISIP & 5604.00 & psi \\
\hline 15 Min ISIP & 5498.00 & psi \\
\hline Breakdown Rate & 15.00 & bpm \\
\hline Breakdown Pressure & 6837.00 & psi \\
\hline Breakdown Volume & 195.00 & bbls \\
\hline Cut Short & & $x$ \\
\hline Screen Out & & $x$ \\
\hline \multicolumn{3}{|c|}{ Fluid and Proppant Data } \\
\hline Total Clean Fluid & 10779.00 & bbls \\
\hline Prop.1-Type & 100 Mesh & \\
\hline Prop.2-Type & 40/70 White & \\
\hline Total Proppant Amount & 439940.00 & Ibs \\
\hline Proppant Concentration & 1999.73 & $\mathrm{Ib} / \mathrm{ft}$ \\
\hline Proppant Concentration & 0.97 & Ib/gal \\
\hline \multicolumn{3}{|c|}{ Chemical Data } \\
\hline Acid \% Strength & 7.50 & \\
\hline Total Acid & 3000.00 & gal \\
\hline Total Slickwater & 8284.00 & bbls \\
\hline Total Linear & 2423.00 & bbls \\
\hline Total XL & 0.00 & bbls \\
\hline FR Amount & 140.00 & gal \\
\hline Gel Amount & 1843.00 & gal or lbs \\
\hline Breaker Amount & 88.00 & gal or lbs \\
\hline Scale Inhibitor & 55.00 & gal \\
\hline Biocide & 128.00 & gal \\
\hline
\end{tabular}

Table 107: Fracture Model - Perfs Stage 22

\begin{tabular}{|ccccc|}
\hline \multicolumn{5}{|c|}{ Perfs } \\
\hline Cluster & Start.FT & Stop.FT & Perf_Diam.IN & Perf_Shots. \\
\hline 5 & 9051.00 & 9053.00 & 0.42 & 8 \\
4 & 9087.00 & 9089.00 & 0.42 & 8 \\
3 & 9136.00 & 9138.00 & 0.42 & 8 \\
2 & 9183.00 & 9185.00 & 0.42 & 8 \\
1 & 9227.00 & 9229.00 & 0.42 & 8 \\
\hline
\end{tabular}

Table 108: Fracture Model - Pump Schedule Stage 22

\begin{tabular}{|c|c|c|c|c|c|c|c|c|c|c|c|}
\hline Stage & $\begin{array}{c}\text { Tot Time } \\
\text { mm:ss }\end{array}$ & $\begin{array}{c}\text { Stage Time } \\
\text { mm:ss }\end{array}$ & Fluid & $\begin{array}{c}\text { Clean Stg Vol } \\
\text { (gal) }\end{array}$ & $\begin{array}{c}\text { Cum Cln Vol } \\
\text { (gal) }\end{array}$ & Proppant & $\begin{array}{c}\text { Slurry Conc } \\
\text { (ppa) }\end{array}$ & $\begin{array}{l}\text { Cum Prop } \\
\text { (Ibs) }\end{array}$ & $\begin{array}{c}\text { Slurry Rate } \\
\text { (bbl/min) }\end{array}$ & $\begin{array}{c}\text { Cum Slurry } \\
\text { (bbl) }\end{array}$ & $\begin{array}{l}\text { Clean Rate } \\
\text { (bbl/min) }\end{array}$ \\
\hline 1 & 0.00 & 0.52 & SlickWater & 325.50 & 325.50 & $<$ None $>$ & 0.00 & 0.00 & 9.23 & 8.00 & 8.94 \\
\hline 2 & 0.52 & 2.22 & $\mathrm{HCl} 7.5 \%$ & 1506.26 & 1831.76 & $<$ None $>$ & 0.00 & 0.00 & 15.15 & 43.86 & 15.15 \\
\hline 3 & 2.22 & 3.18 & Slickwater & 2100.49 & 3932.25 & $<$ None $>$ & 0.00 & 0.00 & 15.16 & 93.87 & 15.16 \\
\hline 4 & 3.18 & 2.21 & $\mathrm{HCl} 7.5 \%$ & 1495.27 & 5427.52 & $<$ None $>$ & 0.00 & 0.00 & 15.15 & 129.47 & 15.15 \\
\hline 5 & 2.21 & 16.15 & Slickwater & 41916.43 & 47343.94 & $<$ None $>$ & 0.00 & 0.00 & 61.50 & 1128.77 & 61.42 \\
\hline 6 & 16.15 & 3.19 & Slickwater & 13265.14 & 60609.08 & 100 Mesh Sand & 0.24 & 3214.00 & 96.32 & 1448.25 & 95.23 \\
\hline 7 & 3.19 & 3.26 & Slickwater & 14009.94 & 74619.03 & 100 Mesh Sand & 0.49 & 10080.00 & 99.32 & 1789.26 & 97.16 \\
\hline 8 & 3.26 & 4.37 & Slickwater & 18591.31 & 93210.33 & 100 Mesh Sand & 0.74 & 23904.00 & 99.12 & 2246.86 & 95.88 \\
\hline 9 & 4.37 & 5.48 & Slickwater & 23102.19 & 116312.52 & 100 Mesh Sand & 0.99 & 46865.00 & 99.12 & 2821.75 & 94.84 \\
\hline 10 & 5.48 & 9.03 & Slickwater & 35278.17 & 151590.69 & 100 Mesh Sand & 1.50 & 99618.00 & 99.12 & 3718.76 & 92.81 \\
\hline 11 & 9.03 & 7.03 & Slickwater & 26955.46 & 178546.15 & 100 Mesh Sand & 1.99 & 153340.00 & 99.28 & 4418.69 & 91.03 \\
\hline 12 & 7.03 & 5.53 & Slickwater & 24009.51 & 202555.65 & 40/70 White & 0.53 & 166017.00 & 99.47 & 5003.94 & 97.17 \\
\hline 13 & 5.53 & 4.35 & Slickwater & 18522.20 & 221077.86 & 40/70 White & 0.74 & 179746.00 & 99.47 & 5459.85 & 96.22 \\
\hline 14 & 4.35 & 4.38 & Slickwater & 18521.08 & 239598.94 & 40/70 White & 0.99 & 198124.00 & 99.49 & 5920.80 & 95.18 \\
\hline 15 & 4.38 & 16.47 & Slickwater & 59330.34 & 298929.28 & 40/70 White & 1.39 & 280515.00 & 89.44 & 7421.95 & 84.17 \\
\hline 16 & 16.47 & 6.11 & Slickwater & 14100.65 & 313029.92 & 40/70 White & 0.00 & 280515.00 & 54.15 & 7756.76 & 54.30 \\
\hline 17 & 6.11 & 0.02 & Slickwater & 0.07 & 313029.99 & $<$ None> & 0.00 & 280515.00 & 0.15 & 7756.77 & 0.05 \\
\hline 18 & 0.02 & 8.31 & Slickwater & 25165.25 & 338195.25 & $<$ None $>$ & 0.00 & 280515.00 & 70.53 & 8357.46 & 70.35 \\
\hline 19 & 8.31 & 6.16 & Slickwater & 23344.91 & 361540.16 & $<$ None $>$ & 0.49 & 291975.00 & 90.69 & 8925.78 & 88.70 \\
\hline 20 & 6.16 & 3.54 & Slickwater & 14371.76 & 375911.92 & 40/70 White & 0.74 & 302631.00 & 90.71 & 9279.55 & 87.74 \\
\hline 21 & 3.54 & 2.48 & WF115 & 10204.11 & 386116.03 & 40/70 White & 0.99 & 312729.00 & 90.69 & 9533.49 & 86.77 \\
\hline 22 & 2.48 & 3.07 & WF115 & 11118.94 & 397234.97 & 40/70 White & 1.48 & 329168.00 & 90.68 & 9816.11 & 84.94 \\
\hline 23 & 3.07 & 6.25 & WF115 & 22406.13 & 419641.10 & 40/70 Treated & 1.99 & 373787.00 & 90.69 & 10398.04 & 83.14 \\
\hline 24 & 6.25 & 2.10 & WF115 & 7398.65 & 427039.75 & 40/70 White & 2.51 & 392332.00 & 90.70 & 10594.55 & 81.30 \\
\hline 25 & 2.10 & 1.30 & WF115 & 5121.83 & 432161.58 & 40/70 White & 2.54 & 405361.00 & 90.69 & 10730.58 & 81.30 \\
\hline 26 & 1.30 & 3.32 & WF115 & 11973.29 & 444134.87 & 40/70 White & 2.74 & 438127.00 & 90.68 & 11050.98 & 80.68 \\
\hline 27 & 3.32 & 2.28 & Slickwater & 8735.16 & 452870.03 & $<$ None $>$ & 0.00 & 438127.00 & 83.74 & 11257.54 & 84.32 \\
\hline
\end{tabular}


Table 109: Fracture Model - Pump Details Stage 22

\begin{tabular}{|c|c|c|c|c|c|c|c|}
\hline Step\# & Name & Avg S Rate & Max S Rate & Avg Tr Pres & Max Tr Pres & Min Tr Pressure & Calc Avg TR P \\
\hline & & (bbl/min) & (bbl/min) & (psi) & (psi) & (psi) & (psi) \\
\hline 1 & Rate & 12.0 & 15.2 & 5238 & 5458 & 4727 & 5104 \\
\hline 2 & Acid 7.5\% HC & 15.2 & 15.2 & 5627 & 5702 & 5469 & 5634 \\
\hline 3 & Spacer & 15.2 & 15.2 & 5772 & 5825 & 5701 & 5775 \\
\hline 4 & Acid 7.5\% HC & 15.1 & 15.2 & 5867 & 5900 & 5825 & 5869 \\
\hline 5 & PAD & 88.2 & 92.5 & 8894 & 9129 & 5785 & 7970 \\
\hline 6 & 0.25 PPA & 96.3 & 99.9 & 8479 & 8772 & 8360 & 8470 \\
\hline 7 & 0.5 PPA & 99.3 & 99.9 & 8272 & 8356 & 8231 & 8270 \\
\hline 8 & 0.75 PPA & 99.1 & 99.2 & 8200 & 8241 & 8168 & 8199 \\
\hline 9 & 1.00 PPA & 99.1 & 99.3 & 8159 & 8173 & 8142 & 8159 \\
\hline 10 & 1.50 PPA & 99.1 & 99.3 & 8159 & 8176 & 8122 & 8159 \\
\hline 11 & 2.0 PPA & 99.3 & 99.7 & 8193 & 8240 & 8124 & 8193 \\
\hline 12 & 0.50 PPA & 99.5 & 99.6 & 8148 & 8215 & 8097 & 8147 \\
\hline 13 & 0.75 PPA & 99.5 & 99.6 & 8094 & 8106 & 8083 & 8094 \\
\hline 14 & 1.00 PPA & 99.5 & 99.6 & 8086 & 8095 & 8073 & 8086 \\
\hline 15 & 1.50 PPA & 95.7 & 99.7 & 7964 & 8258 & 5092 & 7776 \\
\hline 16 & Flush & 55.2 & 0.0 & 6713 & 5928 & 5928 & 6689 \\
\hline 17 & Rate & 5.4 & 0.0 & 5645 & 5928 & 4175 & 4175 \\
\hline 18 & Flush & 81.3 & 90.8 & 8200 & 8740 & 4175 & 7684 \\
\hline 19 & .5 PPA & 90.7 & 90.8 & 8278 & 8562 & 8106 & 8279 \\
\hline 20 & .75 PPA & 90.7 & 90.8 & 8016 & 8393 & 7881 & 8009 \\
\hline 21 & 1.0 PPA & 90.7 & 90.8 & 7912 & 7962 & 7857 & 7910 \\
\hline 22 & 1.5 PPA & 90.7 & 90.8 & 7847 & 7873 & 7817 & 7847 \\
\hline 23 & 2.0 PPA & 90.7 & 90.8 & 7823 & 7849 & 7792 & 7823 \\
\hline 24 & 2.5 PPA & 90.7 & 90.8 & 7810 & 7826 & 7798 & 7809 \\
\hline 25 & 2.5 PPA & 90.7 & 90.8 & 7815 & 7847 & 7796 & 7817 \\
\hline 26 & 3.0 PPA & 90.7 & 90.8 & 7844 & 7877 & 7814 & 7844 \\
\hline 27 & Flush & 89.3 & 90.8 & 8017 & 8289 & 5810 & 7854 \\
\hline
\end{tabular}

Table 110: Fracture Model - Overview Stage 23

\begin{tabular}{|c|c|c|}
\hline \multicolumn{3}{|c|}{ Stage 23} \\
\hline \multicolumn{3}{|c|}{ Treatment Summary } \\
\hline Formation & Marcellus & \\
\hline Formation Type & Shale & \\
\hline \multicolumn{3}{|c|}{ Stage Data } \\
\hline Report Date & $11 / 14 / 2015$ & \\
\hline Stage Length & 237.00 & $\mathrm{ft}$ \\
\hline Frac Gradient & 1.27 & $\mathrm{psi} / \mathrm{ft}$ \\
\hline ISIP & 6223.00 & psi \\
\hline 5 Min ISIP & 5885.00 & psi \\
\hline 10 Min ISIP & 5777.00 & psi \\
\hline 15 Min ISIP & 5698.00 & psi \\
\hline Breakdown Rate & 15.00 & $\mathrm{bpm}$ \\
\hline Breakdown Pressure & 7562.00 & psi \\
\hline Breakdown Volume & 188.00 & bbls \\
\hline Cut Short & & $\mathrm{x}$ \\
\hline Screen Out & & $x$ \\
\hline \multicolumn{3}{|c|}{ Fluid and Proppant Data } \\
\hline Total Clean Fluid & 8646.00 & bbls \\
\hline Prop.1-Type & 100 Mesh & \\
\hline Prop.2-Type & 40/70 White & \\
\hline Total Proppant Amount & 436160.00 & Ibs \\
\hline Proppant Concentration & 1840.34 & $\mathrm{lb} / \mathrm{ft}$ \\
\hline Proppant Concentration & 1.20 & $\mathrm{lb} / \mathrm{gal}$ \\
\hline \multicolumn{3}{|c|}{ Chemical Data } \\
\hline Acid \% Strength & 7.50 & \\
\hline Total Acid & 3000.00 & gal \\
\hline Total Slickwater & 6772.00 & bbls \\
\hline Total Linear & 1944.00 & bbls \\
\hline Total XL & 0.00 & bbls \\
\hline FR Amount & 160.00 & gal \\
\hline Gel Amount & 1451.00 & gal or lbs \\
\hline Breaker Amount & 82.00 & gal or lbs \\
\hline Scale Inhibitor & 36.00 & gal \\
\hline Biocide & 85.00 & gal \\
\hline
\end{tabular}


Table 111: Fracture Model - Perfs Stage 23

\begin{tabular}{|ccccc|}
\hline \multicolumn{5}{c|}{ Perfs } \\
\hline Cluster & Start.FT & Stop.FT & Perf_Diam.IN & Perf_Shots. \\
\hline 5 & 8814.00 & 8816.00 & 0.42 & 8 \\
4 & 8863.00 & 8865.00 & 0.42 & 8 \\
3 & 8910.00 & 8912.00 & 0.42 & 8 \\
2 & 8959.00 & 8961.00 & 0.42 & 8 \\
1 & 9005.00 & 9007.00 & 0.42 & 8 \\
\hline
\end{tabular}

Table 112: Fracture Model - Pump Schedule Stage 23

\begin{tabular}{|c|c|c|c|c|c|c|c|c|c|c|c|}
\hline Stage & $\begin{array}{c}\text { Tot Time } \\
\mathrm{mm}: \mathrm{ss}\end{array}$ & $\begin{array}{c}\text { Stage Time } \\
\text { mm:ss }\end{array}$ & Fluid & $\begin{array}{c}\text { Clean Stg Vol } \\
\text { (gal) }\end{array}$ & $\begin{array}{c}\text { Cum Cln Vol } \\
\text { (gal) }\end{array}$ & Proppant & $\begin{array}{c}\text { Slurry Conc } \\
\text { (ppa) }\end{array}$ & $\begin{array}{c}\text { Cum Prop } \\
\text { (Ibs) }\end{array}$ & $\begin{array}{c}\text { Slurry Rate } \\
\text { (bbl/min) }\end{array}$ & $\begin{array}{l}\text { Cum Slurry } \\
\text { (bbl) }\end{array}$ & $\begin{array}{c}\text { Clean Rate } \\
\text { (bbl/min) }\end{array}$ \\
\hline 1 & 0.00 & 0.50 & SlickWater & 432.32 & 432.32 & $<$ None $>$ & 0.00 & 0.00 & 12.59 & 10.49 & 12.35 \\
\hline 2 & 0.50 & 2.18 & $\mathrm{HCl} 7.5 \%$ & 1454.74 & 1887.06 & $<$ None $>$ & 0.00 & 0.00 & 15.06 & 45.13 & 15.06 \\
\hline 3 & 3.08 & 3.13 & SlickWater & 2034.06 & 3921.12 & $<$ None $>$ & 0.00 & 0.00 & 15.06 & 93.56 & 15.06 \\
\hline 4 & 6.21 & 2.20 & $\mathrm{HCl} 7.5 \%$ & 1476.09 & 5397.21 & $<$ None $>$ & 0.00 & 0.00 & 15.06 & 128.70 & 15.06 \\
\hline 5 & 8.41 & 15.10 & SlickWater & 41943.37 & 47340.58 & $<$ None $>$ & 0.00 & 0.00 & 65.94 & 1128.77 & 65.85 \\
\hline 6 & 23.51 & 3.11 & SlickWater & 13204.02 & 60544.60 & 100 Mesh Sand & 0.24 & 3142.00 & 99.83 & 1446.56 & 98.76 \\
\hline 7 & 27.02 & 3.25 & SlickWater & 14021.13 & 74565.74 & 100 Mesh Sand & 0.48 & 9914.00 & 99.86 & 1787.75 & 97.71 \\
\hline 8 & 30.27 & 4.34 & SlickWater & 18522.62 & 93088.35 & 100 Mesh Sand & 0.74 & 23656.00 & 99.83 & 2243.64 & 96.57 \\
\hline 9 & 35.01 & 5.45 & SlickWater & 23065.78 & 116154.13 & 100 Mesh Sand & 0.99 & 46578.00 & 99.82 & 2817.62 & 95.51 \\
\hline 10 & 40.46 & 8.59 & SlickWater & 35257.93 & 151412.06 & 100 Mesh Sand & 1.50 & 99308.00 & 99.80 & 3714.15 & 93.45 \\
\hline 11 & 49.45 & 7.19 & SlickWater & 28120.40 & 179532.45 & 100 Mesh Sand & 1.99 & 155340.00 & 99.79 & 4444.29 & 91.51 \\
\hline 12 & 57.04 & 5.52 & SlickWater & 24016.07 & 203548.52 & 40/70 White & 0.53 & 168027.00 & 99.78 & 5029.70 & 97.47 \\
\hline 13 & 62.56 & 4.34 & SlickWater & 18515.55 & 222064.07 & 40/70 White & 0.74 & 181744.00 & 99.80 & 5485.43 & 96.54 \\
\hline 14 & 67.30 & 4.37 & SlickWater & 18512.48 & 240576.56 & 40/70 White & 0.99 & 200122.00 & 99.80 & 5946.18 & 95.47 \\
\hline 15 & 72.07 & 8.15 & SlickWater & 32372.47 & 272949.02 & 40/70 White & 1.50 & 248524.00 & 99.80 & 6769.51 & 93.43 \\
\hline 16 & 80.22 & 6.39 & Gel (WF115) & 25553.72 & 298502.75 & 40/70 White & 1.99 & 299285.00 & 99.80 & 7433.20 & 91.49 \\
\hline 17 & 87.01 & 8.47 & Gel (WF115) & 33059.80 & 331562.55 & 40/70 - Treated & 2.49 & 381676.00 & 99.80 & 8309.76 & 89.62 \\
\hline 18 & 95.48 & 6.12 & Gel (WF115) & 23085.15 & 354647.70 & $40 / 70$ - Treated & 2.77 & 445643.00 & 99.80 & 8928.54 & 88.65 \\
\hline 19 & 102.00 & 2.05 & SlickWater & 8535.03 & 363182.72 & $<$ None $>$ & 0.00 & 445643.00 & 96.83 & 9130.26 & 97.54 \\
\hline
\end{tabular}

Table 113: Fracture Model - Pump Details Stage 23

\begin{tabular}{|c|c|c|c|c|c|c|c|}
\hline Step\# & Name & Avg S Rate & Max S Rate & Avg Tr Pres & Max Tr Pres & Min Tr Pressure & Calc Avg TR P \\
\hline & & (bbl/min) & (bbl/min) & (psi) & (psi) & (psi) & (psi) \\
\hline 1 & Rate & 13.5 & 15.1 & 5339 & 5597 & 4607 & 5280 \\
\hline 2 & Acid 7.5\% HC & 15.1 & 15.1 & 5870 & 6034 & 5605 & 5882 \\
\hline 3 & Spacer & 15.1 & 15.1 & 6084 & 6100 & 6035 & 6085 \\
\hline 4 & Acid 7.5\% HC & 15.1 & 15.1 & 6124 & 6176 & 6098 & 6126 \\
\hline 5 & PAD & 99.8 & 100.0 & 8403 & 8680 & 6098 & 7779 \\
\hline 6 & 0.25 PPA & 99.8 & 100.0 & 8107 & 8133 & 8080 & 8108 \\
\hline 7 & 0.5 PPA & 99.9 & 100.0 & 8131 & 8246 & 8089 & 8134 \\
\hline 8 & 0.75 PPA & 99.8 & 99.9 & 8332 & 8367 & 8253 & 8333 \\
\hline 9 & 1.00 PPA & 99.8 & 100.0 & 8342 & 8378 & 8284 & 8343 \\
\hline 10 & 1.50 PPA & 99.8 & 99.9 & 8367 & 8426 & 8323 & 8368 \\
\hline 11 & 2.0 PPA & 99.8 & 99.9 & 8527 & 8640 & 8392 & 8529 \\
\hline 12 & 0.50 PPA & 99.8 & 99.9 & 8564 & 8683 & 8491 & 8562 \\
\hline 13 & 0.75 PPA & 99.8 & 99.9 & 8484 & 8500 & 8470 & 8484 \\
\hline 14 & 1.00 PPA & 99.8 & 99.9 & 8487 & 8497 & 8474 & 8487 \\
\hline 15 & 1.50 PPA & 99.8 & 99.9 & 8422 & 8516 & 8313 & 8422 \\
\hline 16 & 2.00 PPA & 99.8 & 99.9 & 8428 & 8511 & 8287 & 8429 \\
\hline 17 & 2.5 PPA & 99.8 & 99.9 & 8473 & 8517 & 8427 & 8473 \\
\hline 18 & 3.0 PPA & 99.8 & 100.0 & 8466 & 8497 & 8441 & 8466 \\
\hline 19 & Flush & 99.0 & 99.9 & 8533 & 8735 & 6368 & 8401 \\
\hline
\end{tabular}


Table 114: Fracture Model - Overview Stage 24

\begin{tabular}{|c|c|c|}
\hline \multicolumn{3}{|c|}{ Stage 24} \\
\hline \multicolumn{3}{|c|}{ Treatment Summary } \\
\hline Formation & Marcellus & \\
\hline Formation Type & Shale & \\
\hline \multicolumn{3}{|c|}{ Stage Data } \\
\hline Report Date & $11 / 15 / 2015$ & \\
\hline Stage Length & 222.00 & $\mathrm{ft}$ \\
\hline Frac Gradient & 1.29 & $\mathrm{psi} / \mathrm{ft}$ \\
\hline ISIP & 6333.00 & psi \\
\hline 5 Min ISIP & 5848.00 & psi \\
\hline $10 \mathrm{Min} I S I P$ & 5687.00 & psi \\
\hline 15 Min ISIP & 5585.00 & psi \\
\hline Breakdown Rate & 15.00 & bpm \\
\hline Breakdown Pressure & 6373.00 & psi \\
\hline Breakdown Volume & 181.00 & bbls \\
\hline Cut Short & & $x$ \\
\hline Screen Out & & $\mathrm{x}$ \\
\hline \multicolumn{3}{|c|}{ Fluid and Proppant Data } \\
\hline Total Clean Fluid & 8264.00 & bbls \\
\hline Prop.1-Type & 100 Mesh & \\
\hline Prop.2-Type & 40/70 White & \\
\hline Total Proppant Amount & 440280.00 & Ibs \\
\hline Proppant Concentration & 1983.24 & $\mathrm{lb} / \mathrm{ft}$ \\
\hline Proppant Concentration & 1.27 & $\mathrm{lb} / \mathrm{gal}$ \\
\hline \multicolumn{3}{|c|}{ Chemical Data } \\
\hline Acid \% Strength & 7.50 & \\
\hline Total Acid & 3000.00 & gal \\
\hline Total Slickwater & 8197.00 & bbls \\
\hline Total Linear & 0.00 & bbls \\
\hline Total XL & 0.00 & bbls \\
\hline FR Amount & 205.00 & gal \\
\hline Gel Amount & 0.00 & gal or lbs \\
\hline Breaker Amount & 0.00 & gal or lbs \\
\hline Scale Inhibitor & 35.00 & gal \\
\hline Biocide & 90.00 & gal \\
\hline
\end{tabular}

Table 115: Fracture Model - Perfs Stage 24

\begin{tabular}{|ccccc|}
\hline \multicolumn{5}{c|}{ Perfs } \\
\hline Cluster & Start.FT & Stop.FT & Perf_Diam.IN & Perf_Shots. \\
\hline 5 & 8587.00 & 8589.00 & 0.42 & 8 \\
4 & 8634.00 & 8636.00 & 0.42 & 8 \\
3 & 8678.00 & 8680.00 & 0.42 & 8 \\
2 & 8724.00 & 8726.00 & 0.42 & 8 \\
1 & 8763.00 & 8765.00 & 0.42 & 8 \\
\hline
\end{tabular}

Table 116: Fracture Model - Pump Schedule Stage 24

\begin{tabular}{|c|c|c|c|c|c|c|c|c|c|c|c|}
\hline Stage & $\begin{array}{c}\text { Tot Time } \\
\mathrm{mm}: \mathrm{ss}\end{array}$ & $\begin{array}{l}\text { Stage Time } \\
\text { mm:ss }\end{array}$ & Fluid & $\begin{array}{c}\text { Clean Stg Vol } \\
\text { (gal) }\end{array}$ & $\begin{array}{c}\text { Cum Cln Vol } \\
\text { (gal) }\end{array}$ & Proppant & $\begin{array}{l}\text { Slurry Conc } \\
\text { (ppa) }\end{array}$ & $\begin{array}{l}\text { Cum Prop } \\
\text { (lbs) }\end{array}$ & $\begin{array}{l}\text { Slurry Rate } \\
\text { (bbl/min) }\end{array}$ & $\begin{array}{l}\text { Cum Slurry } \\
\text { (bbl) }\end{array}$ & $\begin{array}{l}\text { Clean Rate } \\
\text { (bbl } / \mathrm{min})\end{array}$ \\
\hline 1 & 0.00 & 0.55 & SlickWater & 353.85 & 353.85 & $<$ None> & 0.00 & 0.00 & 9.47 & 8.68 & 9.19 \\
\hline 2 & 0.55 & 2.18 & $\mathrm{HCl} 7.5 \%$ & 1446.97 & 1800.82 & $<$ None> & 0.00 & 0.00 & 14.98 & 43.14 & 14.98 \\
\hline 3 & 3.13 & 3.17 & SlickWater & 2065.91 & 3866.73 & $<$ None $>$ & 0.00 & 0.00 & 14.98 & 92.32 & 14.98 \\
\hline 4 & 6.30 & 2.11 & $\mathrm{HCl} 7.5 \%$ & 1374.03 & 5240.76 & $<$ None $>$ & 0.00 & 0.00 & 14.98 & 125.04 & 14.98 \\
\hline 5 & 8.41 & 11.01 & SlickWater & 24910.54 & 30151.30 & $<$ None $>$ & 0.00 & 0.00 & 53.95 & 719.37 & 53.84 \\
\hline 6 & 19.42 & 4.26 & SlickWater & 16650.42 & 46801.72 & 100 Mesh Sand & 0.24 & 4077.00 & 90.44 & 1120.32 & 89.42 \\
\hline 7 & 24.08 & 3.53 & SlickWater & 15235.91 & 62037.63 & 100 Mesh Sand & 0.49 & 11570.00 & 95.52 & 1491.24 & 93.41 \\
\hline 8 & 28.01 & 4.28 & SlickWater & 17645.38 & 79683.01 & 100 Mesh Sand & 0.74 & 24610.00 & 97.23 & 1925.54 & 94.06 \\
\hline 9 & 32.29 & 9.07 & SlickWater & 36541.67 & 116224.68 & 100 Mesh Sand - Treated & 1.00 & 60982.00 & 99.75 & 2834.91 & 95.43 \\
\hline 10 & 41.36 & 10.17 & SlickWater & 40342.17 & 156566.85 & 100 Mesh Sand & 1.50 & 121322.00 & 99.75 & 3860.68 & 93.41 \\
\hline 11 & 51.53 & 11.36 & SlickWater & 45014.33 & 201581.18 & 100 Mesh Sand & 1.75 & 199997.00 & 99.73 & 5017.52 & 92.39 \\
\hline 12 & 63.29 & 11.30 & SlickWater & 44159.19 & 245740.37 & 100 Mesh Sand & 1.99 & 288079.00 & 99.72 & 6164.28 & 91.43 \\
\hline 13 & 74.59 & 4.49 & SlickWater & 18110.83 & 263851.20 & 100 Mesh Sand & 2.50 & 333422.00 & 99.71 & 6644.53 & 89.52 \\
\hline 14 & 79.48 & 1.48 & SlickWater & 7337.47 & 271188.67 & 40/70 White & 0.63 & 338018.00 & 99.72 & 6824.02 & 97.06 \\
\hline 15 & 81.36 & 2.43 & SlickWater & 11005.19 & 282193.86 & 40/70 White & 0.74 & 346127.00 & 99.70 & 7094.87 & 96.45 \\
\hline 16 & 84.19 & 3.45 & SlickWater & 15028.59 & 297222.45 & 40/70 White & 0.99 & 361024.00 & 99.73 & 7468.85 & 95.42 \\
\hline 17 & 88.04 & 3.49 & SlickWater & 14968.24 & 312190.70 & 40/70 White & 1.49 & 383288.00 & 99.72 & 7849.44 & 93.38 \\
\hline 18 & 91.53 & 3.44 & SlickWater & 14338.53 & 326529.22 & 40/70 White & 1.99 & 411752.00 & 99.73 & 8221.75 & 91.44 \\
\hline 19 & 95.37 & 1.20 & SlickWater & 5022.36 & 331551.58 & 40/70 White & 2.44 & 424030.00 & 99.70 & 8354.69 & 89.69 \\
\hline 20 & 96.57 & 1.54 & SlickWater & 7261.45 & 338813.04 & 40/70 White & 2.14 & 439573.00 & 99.75 & 8544.21 & 91.00 \\
\hline 21 & 98.51 & 2.01 & SlickWater & 8261.61 & 347074.64 & $<$ None $>$ & 0.00 & 439573.00 & 97.05 & 8739.93 & 97.54 \\
\hline
\end{tabular}


Table 117: Fracture Model - Pump Details Stage 24

\begin{tabular}{|c|c|c|c|c|c|c|c|}
\hline Step\# & Name & Avg S Rate & Max S Rate & Avg Tr Pres & Max Tr Pres & Min Tr Pressure & Calc Avg TR P \\
\hline & & (bbl/min) & (bbl/min) & (psi) & (psi) & (psi) & (psi) \\
\hline 1 & Rate & 12.5 & 15.0 & 5599 & 5855 & 4930 & 5430 \\
\hline 2 & Acid 7.5\% HC & 15.0 & 15.0 & 6085 & 6188 & 5860 & 6094 \\
\hline 3 & Spacer & 15.0 & 15.0 & 6163 & 6197 & 6055 & 6161 \\
\hline 4 & Acid 7.5\% HC & 15.0 & 15.0 & 6023 & 6054 & 6007 & 6022 \\
\hline 5 & PAD & 93.0 & 95.1 & 9029 & 9110 & 5900 & 7618 \\
\hline 6 & 0.25 PPA & 90.4 & 95.1 & 8870 & 9016 & 8719 & 8866 \\
\hline 7 & 0.5 PPA & 95.5 & 96.9 & 8771 & 8856 & 8713 & 8769 \\
\hline 8 & 0.75 PPA & 97.2 & 99.8 & 8678 & 8776 & 8634 & 8678 \\
\hline 9 & 1.00 PPA & 99.7 & 99.8 & 8707 & 8769 & 8642 & 8706 \\
\hline 10 & 1.50 PPA & 99.7 & 99.9 & 8598 & 8679 & 8538 & 8597 \\
\hline 11 & 1.75 PPA & 99.7 & 99.9 & 8582 & 8609 & 8548 & 8582 \\
\hline 12 & 2.00 PPA & 99.7 & 99.9 & 8441 & 8617 & 8390 & 8440 \\
\hline 13 & 2.5 PPA & 99.7 & 99.8 & 8454 & 8489 & 8419 & 8455 \\
\hline 14 & 0.50 PPA & 99.7 & 99.8 & 8497 & 8558 & 8451 & 8500 \\
\hline 15 & 0.75 PPA & 99.7 & 99.8 & 8522 & 8617 & 8478 & 8520 \\
\hline 16 & 1.00 PPA & 99.7 & 99.9 & 8433 & 8482 & 8395 & 8432 \\
\hline 17 & 1.50 PPA & 99.7 & 99.8 & 8385 & 8414 & 8353 & 8385 \\
\hline 18 & 2.00 PPA & 99.7 & 99.9 & 8339 & 8392 & 8289 & 8337 \\
\hline 19 & 2.5 PPA & 99.7 & 99.8 & 8313 & 8319 & 8306 & 8313 \\
\hline 20 & 3.0 PPA & 99.7 & 99.9 & 8282 & 8315 & 8257 & 8281 \\
\hline 21 & Flush & 99.0 & 99.8 & 8371 & 8572 & 6203 & 8258 \\
\hline
\end{tabular}

Table 118: Fracture Model - Overview Stage 25

\begin{tabular}{|c|c|c|}
\hline \multicolumn{3}{|c|}{ Stage 25} \\
\hline \multicolumn{3}{|c|}{ Treatment Summary } \\
\hline Formation & Marcellus & \\
\hline Formation Type & Shale & \\
\hline \multicolumn{3}{|c|}{ Stage Data } \\
\hline Report Date & $11 / 15 / 2015$ & \\
\hline Stage Length & 231.00 & $\mathrm{ft}$ \\
\hline Frac Gradient & 1.30 & $\mathrm{psi} / \mathrm{ft}$ \\
\hline ISIP & 6459.00 & psi \\
\hline 5 Min ISIP & 6004.00 & psi \\
\hline 10 Min ISIP & 5846.00 & psi \\
\hline 15 Min ISIP & 5741.00 & psi \\
\hline Breakdown Rate & 15.00 & bpm \\
\hline Breakdown Pressure & 6640.00 & $\mathrm{psi}$ \\
\hline Breakdown Volume & 175.00 & bbls \\
\hline Cut Short & & $\mathrm{x}$ \\
\hline Screen Out & & $x$ \\
\hline \multicolumn{3}{|c|}{ Fluid and Proppant Data } \\
\hline Total Clean Fluid & 8066.00 & bbls \\
\hline Prop.1-Type & 100 Mesh & \\
\hline Prop.2-Type & 40/70 White & \\
\hline Total Proppant Amount & 442700.00 & lbs \\
\hline Proppant Concentration & 1916.45 & $\mathrm{lb} / \mathrm{ft}$ \\
\hline Proppant Concentration & 1.31 & lb/gal \\
\hline \multicolumn{3}{|c|}{ Chemical Data } \\
\hline Acid \% Strength & 7.50 & \\
\hline Total Acid & 3000.00 & gal \\
\hline Total Slickwater & 8546.00 & bbls \\
\hline Total Linear & 0.00 & bbls \\
\hline Total XL & 0.00 & bbls \\
\hline FR Amount & 165.00 & gal \\
\hline Gel Amount & 0.00 & gal or lbs \\
\hline Breaker Amount & 0.00 & gal or lbs \\
\hline Scale Inhibitor & 35.00 & gal \\
\hline Biocide & 90.00 & gal \\
\hline
\end{tabular}

Table 119: Fracture Model - Perfs Stage 25

\begin{tabular}{|ccccc|}
\hline \multicolumn{5}{c|}{ Perfs } \\
\hline Cluster & Start.FT & Stop.FT & Perf_Diam.IN & Perf_Shots. \\
\hline 5 & 8356.00 & 8358.00 & 0.42 & 8 \\
4 & 8400.00 & 8402.00 & 0.42 & 8 \\
3 & 8448.00 & 8450.00 & 0.42 & 8 \\
2 & 8495.00 & 8497.00 & 0.42 & 8 \\
1 & 8541.00 & 8543.00 & 0.42 & 8 \\
\hline
\end{tabular}


Table 120: Fracture Model - Pump Schedule Stage 25

\begin{tabular}{|c|c|c|c|c|c|c|c|c|c|c|c|}
\hline Stage & $\begin{array}{c}\text { Tot Time } \\
\mathrm{mm}: \mathrm{ss}\end{array}$ & $\begin{array}{l}\text { Stage Time } \\
\text { mm:ss }\end{array}$ & Fluid & $\begin{array}{c}\text { Clean Stg Vol } \\
\text { (gal) }\end{array}$ & $\begin{array}{c}\text { Cum Cln Vol } \\
\text { (gal) }\end{array}$ & Proppant & $\begin{array}{l}\text { Slurry Conc } \\
\text { (ppa) }\end{array}$ & $\begin{array}{c}\text { Cum Prop } \\
\text { (lbs) }\end{array}$ & $\begin{array}{l}\text { Slurry Rate } \\
\text { (bbl/min) }\end{array}$ & $\begin{array}{l}\text { Cum Slurry } \\
\text { (bbl) }\end{array}$ & $\begin{array}{l}\text { Clean Rate } \\
\text { (bbl/min) }\end{array}$ \\
\hline 1 & 0.00 & 0.43 & SlickWater & 313.18 & 313.18 & <None> & 0.00 & 0.00 & 10.74 & 7.70 & 10.40 \\
\hline 2 & 0.43 & 2.18 & $\mathrm{HCl} 7.5 \%$ & 1447.25 & 1760.43 & $<$ None $>$ & 0.00 & 0.00 & 14.98 & 42.15 & 14.98 \\
\hline 3 & 3.01 & 3.21 & SlickWater & 2107.21 & 3867.64 & $<$ None $>$ & 0.00 & 0.00 & 14.98 & 92.32 & 14.98 \\
\hline 4 & 6.22 & 2.37 & HCl $7.5 \%$ & 1646.12 & 5513.76 & $<$ None $>$ & 0.00 & 0.00 & 14.98 & 131.52 & 14.98 \\
\hline 5 & 8.59 & 11.37 & SlickWater & 24934.07 & 30447.83 & $<$ None> & 0.00 & 0.00 & 51.21 & 726.39 & 51.10 \\
\hline 6 & 20.36 & 3.23 & SlickWater & 12257.62 & 42705.46 & 100 Mesh Sand & 0.24 & 2977.00 & 87.22 & 1021.50 & 86.26 \\
\hline 7 & 23.59 & 3.55 & SlickWater & 15206.60 & 57912.06 & 100 Mesh Sand & 0.49 & 10429.00 & 94.55 & 1391.84 & 92.44 \\
\hline 8 & 27.54 & 3.20 & SlickWater & 13478.50 & 71390.55 & 100 Mesh Sand & 0.74 & 20368.00 & 99.51 & 1723.54 & 96.27 \\
\hline 9 & 31.14 & 7.45 & SlickWater & 31061.88 & 102452.44 & 100 Mesh Sand - Treated & 0.99 & 51258.00 & 99.74 & 2496.49 & 95.43 \\
\hline 10 & 38.59 & 10.17 & SlickWater & 40330.07 & 142782.51 & 100 Mesh Sand & 1.50 & 111601.00 & 99.73 & 3522.01 & 93.38 \\
\hline 11 & 49.16 & 11.36 & SlickWater & 45019.70 & 187802.21 & 100 Mesh Sand & 1.75 & 190269.00 & 99.74 & 4678.96 & 92.40 \\
\hline 12 & 60.52 & 12.03 & SlickWater & 46277.81 & 234080.02 & 100 Mesh Sand & 2.00 & 282612.00 & 99.73 & 5880.75 & 91.44 \\
\hline 13 & 72.55 & 5.20 & SlickWater & 20058.57 & 254138.59 & 100 Mesh Sand & 2.51 & 332872.00 & 99.74 & 6412.67 & 89.55 \\
\hline 14 & 78.15 & 1.48 & SlickWater & 7336.14 & 261474.73 & 40/70 White & 0.63 & 337477.00 & 99.70 & 6592.13 & 97.04 \\
\hline 15 & 80.03 & 2.43 & SlickWater & 11006.66 & 272481.39 & 40/70 White & 0.74 & 345581.00 & 99.72 & 6863.03 & 96.47 \\
\hline 16 & 82.46 & 3.45 & SlickWater & 15025.30 & 287506.69 & 40/70 White & 0.99 & 360480.00 & 99.72 & 7236.96 & 95.40 \\
\hline 17 & 86.31 & 3.49 & SlickWater & 14969.30 & 302475.99 & 40/70 White & 1.49 & 382745.00 & 99.73 & 7617.58 & 93.38 \\
\hline 18 & 90.20 & 4.05 & SlickWater & 15680.01 & 318156.00 & 40/70 White & 1.99 & 413891.00 & 99.72 & 8024.77 & 91.43 \\
\hline 19 & 94.25 & 1.20 & SlickWater & 5024.25 & 323180.25 & 40/70 White & 2.44 & 426166.00 & 99.74 & 8157.75 & 89.72 \\
\hline 20 & 95.45 & 2.01 & SlickWater & 7695.45 & 330875.70 & 40/70 White & 2.18 & 442922.00 & 99.74 & 8358.90 & 90.86 \\
\hline 21 & 97.46 & 1.57 & SlickWater & 7991.20 & 338866.90 & $<$ None $>$ & 0.00 & 442922.00 & 97.07 & 8548.18 & 97.57 \\
\hline
\end{tabular}

Table 121: Fracture Model - Pump Details Stage 25

\begin{tabular}{|c|c|c|c|c|c|c|c|}
\hline Step\# & Name & Avg S Rate & Max S Rate & Avg Tr Pres & Max Tr Pres & Min Tr Pressure & Calc Avg TR P \\
\hline & & $\mathbf{( b b l / m i n )}$ & $\mathbf{( b b l / m i n )}$ & (psi) & (psi) & (psi) & (psi) \\
\hline 1 & Rate & 12.6 & 15.0 & 5358 & 5562 & 4757 & 5270 \\
\hline 2 & Acid 7.5\% HC & 15.0 & 15.0 & 5898 & 6078 & 5573 & 5913 \\
\hline 3 & spacer & 15.0 & 15.0 & 6132 & 6163 & 6080 & 6133 \\
\hline 4 & Acid 7.5\% HC & 15.0 & 15.0 & 6124 & 6147 & 6108 & 6125 \\
\hline 5 & PAD & 85.8 & 87.2 & 8856 & 9023 & 6077 & 7896 \\
\hline 6 & 0.25 PPA & 87.2 & 89.1 & 8872 & 9057 & 8716 & 8863 \\
\hline 7 & 0.5 PPA & 94.6 & 98.0 & 8771 & 8816 & 8677 & 8768 \\
\hline 8 & 0.75 PPA & 99.5 & 99.9 & 8817 & 8871 & 8751 & 8817 \\
\hline 9 & 1.00 PPA & 99.7 & 99.9 & 8695 & 8751 & 8654 & 8695 \\
\hline 10 & 1.50 PPA & 99.7 & 99.9 & 8624 & 8671 & 8557 & 8623 \\
\hline 11 & 1.75 PPA & 99.7 & 99.9 & 8582 & 8597 & 8565 & 8582 \\
\hline 12 & 2.00 PPA & 99.7 & 99.9 & 8548 & 8651 & 8401 & 8547 \\
\hline 13 & 2.50 PPA & 99.7 & 99.9 & 8417 & 8437 & 8387 & 8417 \\
\hline 14 & 0.50 PPA & 99.7 & 99.9 & 8421 & 8465 & 8389 & 8423 \\
\hline 15 & 0.75 PPA & 99.7 & 99.8 & 8399 & 8497 & 8351 & 8397 \\
\hline 16 & 1.00 PPA & 99.7 & 99.8 & 8346 & 8374 & 8328 & 8346 \\
\hline 17 & 1.50 PPA & 99.7 & 99.9 & 8325 & 8348 & 8298 & 8324 \\
\hline 18 & 2.00 PPA & 99.7 & 99.8 & 8326 & 8348 & 8298 & 8326 \\
\hline 19 & 2.5 PPA & 99.8 & 99.9 & 8348 & 8356 & 8340 & 8348 \\
\hline 20 & 3.0 PPA & 99.8 & 99.8 & 8354 & 8376 & 8344 & 8357 \\
\hline 21 & Flush & 99.0 & 99.8 & 8531 & 8793 & 6612 & 8437 \\
\hline
\end{tabular}


Table 122: Fracture Model - Overview Stage 26

\begin{tabular}{|c|c|c|}
\hline \multicolumn{3}{|c|}{ Stage 26} \\
\hline \multicolumn{3}{|c|}{ Treatment Summary } \\
\hline Formation & Marcellus & \\
\hline Formation Type & Shale & \\
\hline \multicolumn{3}{|c|}{ Stage Data } \\
\hline Report Date & $11 / 15 / 2015$ & \\
\hline Stage Length & 225.00 & $\mathrm{ft}$ \\
\hline Frac Gradient & 1.27 & $\mathrm{psi} / \mathrm{ft}$ \\
\hline ISIP & 6205.00 & psi \\
\hline 5 Min ISIP & 5985.00 & psi \\
\hline 10 Min ISIP & 5860.00 & psi \\
\hline 15 Min ISIP & 5765.00 & psi \\
\hline Breakdown Rate & 15.00 & bpm \\
\hline Breakdown Pressure & 6945.00 & psi \\
\hline Breakdown Volume & 142.00 & bbls \\
\hline Cut Short & & $x$ \\
\hline Screen Out & & $x$ \\
\hline \multicolumn{3}{|c|}{ Fluid and Proppant Data } \\
\hline Total Clean Fluid & 7240.00 & bbls \\
\hline Prop.1-Type & 100 Mesh & \\
\hline Prop.2-Type & 40/70 White & \\
\hline Total Proppant Amount & 440500.00 & Ibs \\
\hline Proppant Concentration & 1957.78 & $\mathrm{Ib} / \mathrm{ft}$ \\
\hline Proppant Concentration & 1.45 & Ib/gal \\
\hline \multicolumn{3}{|c|}{ Chemical Data } \\
\hline Acid \% Strength & 7.50 & \\
\hline Total Acid & 3000.00 & gal \\
\hline Total Slickwater & 4472.00 & bbls \\
\hline Total Linear & 2696.00 & bbls \\
\hline Total XL & 0.00 & bbls \\
\hline FR Amount & 338.00 & gal \\
\hline Gel Amount & 0.00 & gal or lbs \\
\hline Breaker Amount & 0.00 & gal or lbs \\
\hline Scale Inhibitor & 40.00 & gal \\
\hline Biocide & 125.00 & gal \\
\hline
\end{tabular}

Table 123: Fracture Model - Perfs Stage 26

\begin{tabular}{|ccccc|}
\hline \multicolumn{5}{c|}{ Perfs } \\
\hline Cluster & Start.FT & Stop.FT & Perf_Diam.IN & Perf_Shots. \\
\hline 5 & 8128.00 & 8130.00 & 0.42 & 8 \\
4 & 8175.00 & 8177.00 & 0.42 & 8 \\
3 & 8220.00 & 8222.00 & 0.42 & 8 \\
2 & 8264.00 & 8266.00 & 0.42 & 8 \\
1 & 8310.00 & 8312.00 & 0.42 & 8 \\
\hline
\end{tabular}

Table 124: Fracture Model - Pump Schedule Stage 26

\begin{tabular}{|c|c|c|c|c|c|c|c|c|c|c|c|}
\hline Stage & $\begin{array}{c}\text { Tot Time } \\
\mathrm{mm}: \mathrm{ss}\end{array}$ & $\begin{array}{c}\text { Stage Time } \\
\mathrm{mm}: \mathrm{ss}\end{array}$ & Fluid & $\begin{array}{c}\text { Clean Stg Vol } \\
\text { (gal) }\end{array}$ & $\begin{array}{c}\text { Cum Cln Vol } \\
\text { (gal) }\end{array}$ & Proppant & $\begin{array}{c}\text { Slurry Conc } \\
\text { (ppa) }\end{array}$ & $\begin{array}{c}\text { Cum Prop } \\
\text { (Ibs) }\end{array}$ & $\begin{array}{c}\text { Slurry Rate } \\
\text { (bbl/min) }\end{array}$ & $\begin{array}{c}\text { Cum Slurry } \\
\text { (bbl) }\end{array}$ & $\begin{array}{l}\text { Clean Rate } \\
\text { (bbl/min) }\end{array}$ \\
\hline 2 & 1.10 & 2.20 & $\mathrm{HCl} 7.5 \%$ & 1517.95 & 2012.43 & $<$ None $>$ & 0.00 & 0.00 & 15.49 & 48.18 & 15.49 \\
\hline 3 & 3.30 & 3.08 & SlickWater & 2037.21 & 4049.64 & $<$ None $>$ & 0.00 & 0.00 & 15.48 & 96.68 & 15.48 \\
\hline 5 & 8.58 & 8.21 & SlickWater & 24960.19 & 30527.22 & $<$ None $>$ & 0.00 & 0.00 & 71.34 & 728.51 & 71.17 \\
\hline 6 & 17.19 & 5.34 & SlickWater & 23053.51 & 53580.73 & 100 Mesh Sand & 0.25 & 5676.00 & 99.71 & 1283.55 & 98.60 \\
\hline 7 & 22.53 & 7.11 & SlickWater & 29428.48 & 83009.21 & 100 Mesh Sand & 0.49 & 20227.00 & 99.73 & 1999.97 & 97.54 \\
\hline 11 & 45.21 & 10.57 & SlickWater & 42055.48 & 185996.93 & 100 Mesh Sand & 1.99 & 171885.00 & 99.74 & 4616.28 & 91.44 \\
\hline 12 & 56.18 & 10.03 & Gel(Spphire VF105) & 37790.90 & 223787.83 & 100 Mesh Sand & 2.51 & 266561.00 & 99.72 & 5618.42 & 89.53 \\
\hline 13 & 66.21 & 6.14 & Gel(Spphire VF105) & 22974.77 & 246762.60 & 100 Mesh Sand & 3.00 & 335489.00 & 99.72 & 6239.98 & 87.76 \\
\hline 14 & 72.35 & 3.17 & Gel(Spphire VF105) & 12587.75 & 259350.35 & 40/70 White & 2.04 & 361121.00 & 99.72 & 6567.39 & 91.28 \\
\hline 15 & 75.52 & 5.37 & Gel(Spphire VF105) & 21114.25 & 280464.60 & 40/70 White & 2.49 & 413763.00 & 99.71 & 7127.45 & 89.51 \\
\hline 16 & 81.29 & 4.15 & Gel(Spphire VF105) & 15823.21 & 296287.81 & 40/70 White & 2.75 & 457326.00 & 99.70 & 7551.17 & 88.65 \\
\hline
\end{tabular}


Table 125: Fracture Model - Pump Details Stage 26

\begin{tabular}{|c|c|c|c|c|c|c|c|}
\hline Step\# & Name & Avg S Rate & Max S Rate & Avg Tr Pres & Max Tr Pres & Min Tr Pressure & Calc Avg TR P \\
\hline & & (bbl/min) & (bbl/min) & (psi) & (psi) & (psi) & (psi) \\
\hline 1 & Rate & 13.5 & 15.7 & 5567 & 5824 & 4862 & 5395 \\
\hline 2 & Acid 7.5\% & 15.5 & 15.5 & 6048 & 6179 & 5825 & 6059 \\
\hline 3 & spacer & 15.5 & 15.5 & 6215 & 6262 & 6180 & 6216 \\
\hline 4 & Acid 7.5\% & 15.5 & 15.5 & 6305 & 6347 & 6263 & 6305 \\
\hline 5 & PAD & 82.7 & 99.8 & 8328 & 8848 & 6226 & 8190 \\
\hline 6 & 0.25 PPA & 99.7 & 99.8 & 8575 & 8650 & 8371 & 8568 \\
\hline 7 & 0.5 PPA & 99.7 & 99.9 & 8546 & 8576 & 8523 & 8546 \\
\hline 8 & 0.75 PPA & 99.7 & 99.9 & 8518 & 8533 & 8506 & 8517 \\
\hline 9 & 1.00 PPA & 99.7 & 99.9 & 8501 & 8516 & 8484 & 8501 \\
\hline 10 & 1.5 PPA & 99.7 & 99.8 & 8488 & 8502 & 8457 & 8488 \\
\hline 11 & 2.00 PPA & 99.7 & 100.0 & 8487 & 8535 & 8455 & 8488 \\
\hline 12 & 2.5 PPA & 99.7 & 99.8 & 8488 & 8528 & 8435 & 8487 \\
\hline 13 & 3.0 PPA & 99.7 & 99.9 & 8460 & 8480 & 8441 & 8460 \\
\hline 14 & 2.00 PPA & 99.7 & 99.8 & 8400 & 8461 & 8336 & 8397 \\
\hline 15 & 2.5 PPA & 99.7 & 99.8 & 8344 & 8370 & 8310 & 8344 \\
\hline 16 & 3.0 PPA & 99.7 & 99.8 & 8374 & 8422 & 8344 & 8375 \\
\hline 17 & Flush & 97.9 & 99.8 & 8399 & 8600 & 5654 & 8279 \\
\hline
\end{tabular}

Table 126: Fracture Model - Overview Stage 27

\begin{tabular}{|c|c|c|}
\hline \multicolumn{3}{|c|}{ Stage 27} \\
\hline \multicolumn{3}{|c|}{ Treatment Summary } \\
\hline Formation & Marcellus & \\
\hline Formation Type & Shale & \\
\hline \multicolumn{3}{|c|}{ Stage Data } \\
\hline Report Date & $11 / 15 / 2015$ & \\
\hline Stage Length & 184.00 & $\mathrm{ft}$ \\
\hline Frac Gradient & 1.29 & $\mathrm{psi} / \mathrm{ft}$ \\
\hline ISIP & 6335.00 & psi \\
\hline 5 Min ISIP & 6003.00 & psi \\
\hline 10 Min ISIP & 5911.00 & psi \\
\hline 15 Min ISIP & 5827.00 & psi \\
\hline Breakdown Rate & 15.00 & bpm \\
\hline Breakdown Pressure & 6895.00 & psi \\
\hline Breakdown Volume & 166.00 & bbls \\
\hline Cut Short & & $\mathrm{x}$ \\
\hline Screen Out & & $\mathrm{x}$ \\
\hline \multicolumn{3}{|c|}{ Fluid and Proppant Data } \\
\hline Total Clean Fluid & 6538.00 & bbls \\
\hline Prop.1-Type & 100 Mesh & \\
\hline Prop.2-Type & 40/70 White & \\
\hline Total Proppant Amount & 453260.00 & Ibs \\
\hline Proppant Concentration & 2463.37 & $\mathrm{Ib} / \mathrm{ft}$ \\
\hline Proppant Concentration & 1.65 & $\mathrm{Ib} / \mathrm{gal}$ \\
\hline \multicolumn{3}{|c|}{ Chemical Data } \\
\hline Acid \% Strength & 7.50 & \\
\hline Total Acid & 3000.00 & gal \\
\hline Total Slickwater & 3618.00 & bbls \\
\hline Total Linear & 2840.00 & bbls \\
\hline Total XL & 0.00 & bbls \\
\hline FR Amount & 202.00 & $\mathrm{gal}$ \\
\hline Gel Amount & 0.00 & gal or lbs \\
\hline Breaker Amount & 0.00 & gal or lbs \\
\hline Scale Inhibitor & 40.00 & gal \\
\hline Biocide & 105.00 & gal \\
\hline
\end{tabular}

Table 127: Fracture Model - Perfs Stage 27

\begin{tabular}{|ccccc|}
\hline \multicolumn{5}{c|}{ Perfs } \\
\hline Cluster & Start.FT & Stop.FT & Perf_Diam.IN & Perf_Shots. \\
\hline 4 & 7944.00 & 7946.00 & 0.42 & 10 \\
3 & 7993.00 & 7995.00 & 0.42 & 10 \\
2 & 8042.00 & 8044.00 & 0.42 & 10 \\
1 & 8085.00 & 8087.00 & 0.42 & 10 \\
\hline
\end{tabular}


Table 128: Fracture Model - Pump Schedule Stage 27

\begin{tabular}{|c|c|c|c|c|c|c|c|c|c|c|c|}
\hline Stage & $\begin{array}{c}\text { Tot Time } \\
\text { mm:ss }\end{array}$ & $\begin{array}{c}\text { Stage Time } \\
\text { mm:ss }\end{array}$ & Fluid & $\begin{array}{c}\text { Clean Stg Vol } \\
\text { (gal) }\end{array}$ & $\begin{array}{c}\text { Cum Cln Vol } \\
\text { (gal) }\end{array}$ & Proppant & $\begin{array}{c}\text { Slurry Conc } \\
\text { (ppa) }\end{array}$ & $\begin{array}{c}\text { Cum Prop } \\
\text { (lbs) }\end{array}$ & $\begin{array}{c}\text { Slurry Rate } \\
\text { (bbl/min) }\end{array}$ & $\begin{array}{c}\text { Cum Slurry } \\
\text { (bbl) }\end{array}$ & $\begin{array}{l}\text { Clean Rate } \\
\text { (bbl/min) }\end{array}$ \\
\hline 2 & 1.07 & 2.19 & $\mathrm{HCl} 7.5 \%$ & 1515.08 & 2005.57 & $<$ None $>$ & 0.00 & 0.00 & 15.57 & 47.99 & 15.57 \\
\hline 5 & 8.58 & 9.53 & SlickWater & 24918.03 & 30538.40 & $<$ None $>$ & 0.00 & 0.00 & 60.17 & 728.75 & 60.03 \\
\hline 6 & 18.51 & 5.50 & SlickWater & 24155.39 & 54693.79 & 100 Mesh Sand & 0.25 & 5950.00 & 99.70 & 1310.34 & 98.59 \\
\hline 7 & 24.41 & 4.07 & SlickWater & 16845.23 & 71539.02 & 100 Mesh Sand & 0.49 & 14275.00 & 99.62 & 1720.45 & 97.43 \\
\hline 10 & 37.17 & 10.58 & SlickWater & 41411.87 & 146551.80 & 100 Mesh Sand & 2.00 & 138656.00 & 98.11 & 3641.42 & 89.91 \\
\hline 11 & 48.15 & 12.03 & Gel(Spphire VF105) & 42012.40 & 188564.20 & 100 Mesh Sand & 2.50 & 243732.00 & 92.41 & 4755.02 & 83.01 \\
\hline 12 & 60.18 & 9.52 & Gel(Spphire VF105) & 34318.68 & 222882.88 & 100 Mesh Sand & 3.00 & 346785.00 & 94.15 & 5683.94 & 82.82 \\
\hline 13 & 70.10 & 3.27 & Gel(Spphire VF105) & 12632.19 & 235515.08 & 40/70 White & 2.03 & 372458.00 & 95.24 & 6012.51 & 87.18 \\
\hline 14 & 73.37 & 3.29 & Gel(Spphire VF105) & 12551.70 & 248066.78 & 40/70 White & 2.50 & 403831.00 & 95.58 & 6345.45 & 85.79 \\
\hline 15 & 77.06 & 2.42 & Gel(Spphire VF105) & 9533.93 & 257600.70 & 40/70 White & 2.98 & 432249.00 & 95.58 & 6603.51 & 84.07 \\
\hline 16 & 79.48 & 2.47 & Gel(Spphire VF105) & 9445.87 & 267046.57 & 40/70 White & 4.02 & 470187.00 & 95.58 & 6869.53 & 80.80 \\
\hline
\end{tabular}

Table 129: Fracture Model - Pump Details Stage 27

\begin{tabular}{|c|c|c|c|c|c|c|c|}
\hline Step\# & Name & Avg S Rate & Max S Rate & Avg Tr Pres & Max Tr Pres & Min Tr Pressure & Calc Avg TR P \\
\hline & & (bbl/min) & (bbl/min) & (psi) & (psi) & (psi) & (psi) \\
\hline 1 & Rate & 13.9 & 15.7 & 5361 & 5658 & 4465 & 5148 \\
\hline 2 & Acid 7.5\% & 15.6 & 15.7 & 5891 & 6015 & 5664 & 5901 \\
\hline 3 & Spacer & 15.5 & 15.6 & 6061 & 6105 & 6016 & 6063 \\
\hline 4 & Acid 7.5\% & 15.6 & 15.6 & 6129 & 6158 & 6105 & 6129 \\
\hline 5 & PAD & 76.4 & 99.9 & 8548 & 8932 & 6067 & 8082 \\
\hline 6 & 0.25 PPA & 99.7 & 100.0 & 8729 & 8822 & 8634 & 8727 \\
\hline 7 & 0.5 PPA & 99.6 & 99.8 & 8606 & 8641 & 8584 & 8606 \\
\hline 8 & 1.00 PPA & 99.6 & 99.7 & 8554 & 8607 & 8520 & 8553 \\
\hline 9 & 1.5 PPA & 99.6 & 99.7 & 8519 & 8548 & 8495 & 8519 \\
\hline 10 & 2.00 PPA & 99.3 & 99.8 & 8529 & 8598 & 8471 & 8530 \\
\hline 11 & 2.5 PPA & 92.5 & 97.8 & 8500 & 8958 & 8261 & 8497 \\
\hline 12 & 3.0 PPA & 94.2 & 94.3 & 8416 & 8460 & 8399 & 8415 \\
\hline 13 & $2.00 \mathrm{ppa}$ & 95.2 & 95.7 & 8426 & 8454 & 8374 & 8426 \\
\hline 14 & $2.5 \mathrm{ppa}$ & 95.6 & 95.7 & 8399 & 8438 & 8365 & 8400 \\
\hline 15 & $3.0 \mathrm{ppa}$ & 95.6 & 95.7 & 8401 & 8435 & 8383 & 8401 \\
\hline 16 & $4.0 \mathrm{PPA}$ & 95.6 & 95.7 & 8476 & 8675 & 8414 & 8482 \\
\hline 17 & Flush & 94.1 & 95.7 & 8599 & 8781 & 5999 & 8429 \\
\hline
\end{tabular}

Table 130: Fracture Model - Overview Stage 28

\begin{tabular}{|c|c|c|}
\hline \multicolumn{3}{|c|}{ Stage 28} \\
\hline \multicolumn{3}{|c|}{ Treatment Summary } \\
\hline Formation & Marcellus & \\
\hline Formation Type & Shale & \\
\hline \multicolumn{3}{|c|}{ Stage Data } \\
\hline Report Date & $11 / 15 / 2015$ & \\
\hline Stage Length & 191.00 & $\mathrm{ft}$ \\
\hline Frac Gradient & 1.29 & $\mathrm{psi} / \mathrm{ft}$ \\
\hline ISIP & 6335.00 & psi \\
\hline 5 Min ISIP & 5612.00 & psi \\
\hline 10 Min ISIP & 5370.00 & $\mathrm{psi}$ \\
\hline 15 Min ISIP & 5269.00 & psi \\
\hline Breakdown Rate & 15.00 & bpm \\
\hline Breakdown Pressure & 7185.00 & psi \\
\hline Breakdown Volume & 157.00 & bbls \\
\hline Cut Short & & $\mathrm{x}$ \\
\hline Screen Out & & $\mathrm{x}$ \\
\hline \multicolumn{3}{|c|}{ Fluid and Proppant Data } \\
\hline Total Clean Fluid & 7376.00 & bbls \\
\hline Prop.1-Type & 100 Mesh & \\
\hline Prop.2-Type & 40/70 White & \\
\hline Total Proppant Amount & 360560.00 & Ibs \\
\hline Proppant Concentration & 1887.75 & $\mathrm{lb} / \mathrm{ft}$ \\
\hline Proppant Concentration & 1.16 & $\mathrm{lb} / \mathrm{gal}$ \\
\hline \multicolumn{3}{|c|}{ Chemical Data } \\
\hline Acid \% Strength & 7.50 & \\
\hline Total Acid & 3000.00 & gal \\
\hline Total Slickwater & 5764.00 & bbls \\
\hline Total Linear & 1539.00 & bbls \\
\hline Total XL & 0.00 & bbls \\
\hline FR Amount & 105.00 & gal \\
\hline Gel Amount & 820.00 & gal or lbs \\
\hline Breaker Amount & 88.00 & gal or lbs \\
\hline Scale Inhibitor & 35.00 & gal \\
\hline Biocide & 100.00 & gal \\
\hline
\end{tabular}


Table 131: Fracture Model - Perfs Stage 28

\begin{tabular}{|ccccc|}
\hline \multicolumn{5}{c|}{ Perfs } \\
\hline Cluster & Start.FT & Stop.FT & Perf_Diam.IN & Perf_Shots. \\
\hline 4 & 7753.00 & 7755.00 & 0.42 & 10 \\
3 & 7803.00 & 7805.00 & 0.42 & 10 \\
2 & 7852.00 & 7854.00 & 0.42 & 10 \\
1 & 7901.00 & 7903.00 & 0.42 & 10 \\
\hline
\end{tabular}

Table 132: Fracture Model - Pump Schedule Stage 28

\begin{tabular}{|c|c|c|c|c|c|c|c|c|c|c|c|}
\hline Stage & $\begin{array}{c}\text { Tot Time } \\
\mathrm{mm}: \mathrm{ss}\end{array}$ & $\begin{array}{l}\text { Stage Time } \\
\text { mm:ss }\end{array}$ & Fluid & $\begin{array}{c}\text { Clean Stg Vol } \\
\text { (gal) }\end{array}$ & $\begin{array}{c}\text { Cum Cln Vol } \\
\text { (gal) }\end{array}$ & Proppant & $\begin{array}{l}\text { Slurry Conc } \\
\text { (ppa) }\end{array}$ & $\begin{array}{c}\text { Cum Prop } \\
\text { (Ibs) }\end{array}$ & $\begin{array}{l}\text { Slurry Rate } \\
\text { (bbl/min) }\end{array}$ & $\begin{array}{c}\text { Cum Slurry } \\
\text { (bbl) }\end{array}$ & $\begin{array}{l}\text { Clean Rate } \\
\text { (bbl/min) }\end{array}$ \\
\hline 1 & 0.00 & 1.26 & SlickWater & 540.82 & 540.82 & $\langle$ <None $>$ & 0.00 & 0.00 & 9.16 & 13.13 & 8.98 \\
\hline 2 & 1.26 & 2.22 & $\mathrm{HCl} 7.5 \%$ & 1518.86 & 2059.68 & $\langle$ None $>$ & 0.00 & 0.00 & 15.28 & 49.30 & 15.28 \\
\hline 3 & 3.48 & 3.16 & SlickWater & 2097.69 & 4157.37 & $<$ None $>$ & 0.00 & 0.00 & 15.29 & 99.24 & 15.29 \\
\hline 4 & $\begin{array}{l}7.40^{\circ}-04 \\
\end{array}$ & 2.43 & $\mathrm{HCl} 7.5 \%$ & 1565.76 & 5723.13 & $<$ None> & 0.00 & 0.00 & 13.72 & 136.52 & 13.72 \\
\hline 5 & 9.47 & 14.05 & SlickWater & 41938.76 & 47661.89 & $\langle$ None $>$ & 0.00 & 0.00 & 70.98 & 1136.15 & 70.90 \\
\hline 6 & 23.52 & 3.24 & SlickWater & 12037.13 & 59699.02 & 100 Mesh Sand & 0.24 & 2925.00 & 85.23 & 1425.94 & 84.29 \\
\hline 7 & 27.16 & 3.26 & SlickWater & 12020.40 & 71719.43 & 100 Mesh Sand & 0.49 & 8849.00 & 85.23 & 1718.57 & 83.36 \\
\hline 8 & 30.42 & 4.03 & SlickWater & 15214.08 & 86933.51 & 100 Mesh Sand & 0.74 & 20143.00 & 92.51 & 2093.21 & 89.44 \\
\hline 9 & 34.45 & 4.55 & SlickWater & 18896.01 & 105829.52 & 100 Mesh Sand & 0.99 & 38906.00 & 95.64 & 2563.42 & 91.51 \\
\hline 10 & 39.40 & 7.41 & SlickWater & 28900.61 & 134730.13 & 100 Mesh Sand - Treated & 1.49 & 81981.00 & 95.63 & 3298.21 & 89.56 \\
\hline 11 & 47.21 & 7.33 & SlickWater & 27796.51 & 162526.64 & 100 Mesh Sand & 1.99 & 137291.00 & 95.60 & 4019.98 & 87.66 \\
\hline 12 & 54.54 & 4.52 & SlickWater & 19083.67 & 181610.31 & 40/70 White & 0.53 & 147334.00 & 95.60 & 4485.23 & 93.36 \\
\hline 13 & 59.46 & 3.54 & SlickWater & 15151.29 & 196761.60 & 40/70 White & 0.74 & 158508.00 & 95.62 & 4858.15 & 92.50 \\
\hline 14 & 63.40 & 3.56 & SlickWater & 15112.24 & 211873.84 & 40/70 White & 0.99 & 173504.00 & 95.62 & 5234.26 & 91.48 \\
\hline 15 & 67.36 & 7.05 & SlickWater & 26630.39 & 238504.22 & 40/70 White & 1.49 & 213097.00 & 95.61 & 5911.51 & 89.51 \\
\hline 16 & 74.41 & 5.57 & Gel (WF115) & 21908.22 & 260412.44 & 40/70 White & 1.98 & 256583.00 & 95.62 & 6480.48 & 87.67 \\
\hline 17 & 80.38 & 4.32 & Gel (WF115) & 16349.64 & 276762.09 & 40/70 White & 2.49 & 297280.00 & 95.62 & 6913.97 & 85.87 \\
\hline 18 & 85.10 & 2.45 & Gel (WF115) & 9699.97 & 286462.05 & 40/70 White & 3.01 & 326525.00 & 95.62 & 7176.93 & 83.98 \\
\hline 19 & 87.55 & 2.58 & Gel (WF115) & 10462.06 & 296924.11 & 40/70 White & 3.05 & 358403.00 & 95.60 & 7460.55 & 83.97 \\
\hline 20 & 90.53 & 1.45 & Gel (WF115) & 6283.34 & 303207.45 & 40/70 White & 2.65 & 375049.00 & 95.64 & 7627.92 & $\begin{array}{l}85.49 \\
85.97\end{array}$ \\
\hline 21 & 92.38 & 1.50 & SlickWater & 7277.69 & 310485.14 & $<$ None $>$ & 0.00 & 375049.00 & 94.20 & 7800.62 & 94.52 \\
\hline
\end{tabular}

Table 133: Fracture Model - Pump Details Stage 28

\begin{tabular}{|c|c|c|c|c|c|c|c|}
\hline Step\# & Name & Avg S Rate & Max S Rate & Avg Tr Pres & Max Tr Pres & Min Tr Pressure & Calc Avg TR P \\
\hline & & (bbl/min) & (bbl/min) & (psi) & (psi) & (psi) & (psi) \\
\hline 1 & Rate & 13.5 & 15.7 & 5565 & 5857 & 4697 & 5283 \\
\hline 2 & Acid 7.5\% HC & 15.3 & 15.3 & 6059 & 6133 & 5866 & 6066 \\
\hline 3 & PAD & 15.3 & 15.3 & 6151 & 6182 & 6131 & 6152 \\
\hline 4 & acid 7.5\% & 14.3 & 15.3 & 6142 & 6233 & 5817 & 6116 \\
\hline 5 & PAD & 85.2 & 89.3 & 8582 & 9042 & 6179 & 8447 \\
\hline 6 & 0.25 PPA & 85.2 & 85.3 & 8305 & 8392 & 8201 & 8301 \\
\hline 7 & 0.5 PPA & 85.2 & 85.3 & 8056 & 8200 & 7982 & 8052 \\
\hline 8 & 0.75 PPA & 92.6 & 95.8 & 8278 & 8412 & 7980 & 8276 \\
\hline 9 & $1.00 \mathrm{PPA}$ & 95.6 & 95.7 & 8361 & 8372 & 8346 & 8361 \\
\hline 10 & $1.5 \mathrm{PPA}$ & 95.6 & 95.8 & 8343 & 8373 & 8308 & 8343 \\
\hline 11 & $2.00 \mathrm{PPA}$ & 95.6 & 95.7 & 8300 & 8356 & 8218 & 8299 \\
\hline 12 & $.5 \mathrm{ppa}$ & 95.6 & 95.7 & 8308 & 8367 & 8239 & 8309 \\
\hline 13 & $.75 \mathrm{ppa}$ & 95.6 & 95.8 & 8324 & 8360 & 8296 & 8323 \\
\hline 14 & $1.0 \mathrm{ppa}$ & 95.6 & 95.7 & 8330 & 8355 & 8305 & 8331 \\
\hline 15 & $1.5 \mathrm{ppa}$ & 95.6 & 95.8 & 8257 & 8329 & 8186 & 8256 \\
\hline 16 & $2.0 \mathrm{ppa}$ & 95.6 & 95.7 & 8276 & 8310 & 8204 & 8277 \\
\hline 17 & $2.5 \mathrm{ppa}$ & 95.6 & 95.8 & 8330 & 8372 & 8302 & 8330 \\
\hline 18 & $3.0 \mathrm{ppa}$ & 95.6 & 95.8 & 8318 & 8350 & 8281 & 8317 \\
\hline 19 & $3.0 \mathrm{ppa}$ & 95.6 & 95.7 & 8319 & 8379 & 8294 & 8321 \\
\hline 20 & $4.0 \mathrm{ppa}$ & 95.6 & 95.7 & 8426 & 8484 & 8379 & 8431 \\
\hline 21 & Flush & 95.4 & 95.7 & 8726 & 8961 & 7582 & 8669 \\
\hline
\end{tabular}


Table 134: Water-Oil Table

\begin{tabular}{|c|c|c|}
\hline \multicolumn{3}{|c|}{ Water-Oil Table } \\
\hline Sw & krw & krow \\
\hline 0.0000 & 0.0000 & 1.0000 \\
\hline 0.0527 & 0.0027 & 0.8777 \\
\hline 0.1101 & 0.0083 & 0.7642 \\
\hline 0.1643 & 0.0199 & 0.6627 \\
\hline 0.2186 & 0.0255 & 0.5612 \\
\hline 0.2744 & 0.0371 & 0.4805 \\
\hline 0.3287 & 0.0576 & 0.3939 \\
\hline 0.3814 & 0.0751 & 0.3192 \\
\hline 0.4372 & 0.0956 & 0.2504 \\
\hline 0.4915 & 0.1191 & 0.1905 \\
\hline 0.5504 & 0.1485 & 0.1396 \\
\hline 0.6016 & 0.1780 & 0.1006 \\
\hline 0.6558 & 0.2134 & 0.0646 \\
\hline 0.7132 & 0.2517 & 0.0374 \\
\hline 0.7628 & 0.2901 & 0.0133 \\
\hline 0.8202 & 0.3285 & 0.0011 \\
\hline 0.8744 & 0.3817 & 0.0007 \\
\hline 0.9380 & 0.4319 & 0.0006 \\
\hline 1.0000 & 0.4940 & 0.0000 \\
\hline
\end{tabular}

Table 135: Liquid-Gas Table

\begin{tabular}{|c|c|c|}
\hline \multicolumn{3}{|c|}{ Liquid-Gas Table: Gas Saturation } \\
\hline Sg & krg & krog \\
\hline 0.0000 & 0.0000 & 1.0000 \\
\hline 0.0431 & 0.0032 & 0.8786 \\
\hline 0.0873 & 0.0122 & 0.7658 \\
\hline 0.1303 & 0.0270 & 0.6676 \\
\hline 0.1745 & 0.0505 & 0.5636 \\
\hline 0.2163 & 0.0711 & 0.4769 \\
\hline 0.2628 & 0.1092 & 0.3932 \\
\hline 0.3046 & 0.1500 & 0.3181 \\
\hline 0.3475 & 0.1909 & 0.2518 \\
\hline 0.3916 & 0.2492 & 0.1941 \\
\hline 0.4346 & 0.3046 & 0.1423 \\
\hline 0.4787 & 0.3658 & 0.1049 \\
\hline 0.5227 & 0.4386 & 0.0705 \\
\hline 0.5644 & 0.5142 & 0.0418 \\
\hline 0.6097 & 0.5928 & 0.0218 \\
\hline 0.6525 & 0.6743 & 0.0077 \\
\hline 0.6953 & 0.7703 & 0.0060 \\
\hline 0.7477 & 0.8895 & 0.0053 \\
\hline 0.7940 & 1.0000 & 0.0000 \\
\hline
\end{tabular}


Table 136: Well Data and Calculated BHP

\begin{tabular}{|c|c|c|c|c|c|c|c|c|c|}
\hline \multicolumn{9}{|c|}{ SCADA DATA } & \multirow{2}{*}{$\begin{array}{c}\text { Calculated BHP } \\
\text { BHP } \\
\text { (PSIG) }\end{array}$} \\
\hline Date & $\begin{array}{l}\text { Flow Time } \\
\text { (hh:mm:ss) }\end{array}$ & $\begin{array}{c}\text { Stat } \\
\text { (PSIA) }\end{array}$ & $\begin{array}{c}\text { Diff } \\
(\text { inH2O) }\end{array}$ & $\begin{array}{l}\text { Temp } \\
\left({ }^{\circ} \mathrm{F}\right)\end{array}$ & $\begin{array}{c}\text { Gas } \\
\text { (MCFD) }\end{array}$ & $\begin{array}{l}\text { Water } \\
\text { (BBL/D) }\end{array}$ & $\begin{array}{l}P_{\text {casing }} \\
\text { (PSIG) }\end{array}$ & $\begin{array}{l}\text { Ptubing } \\
\text { (PSIG) }\end{array}$ & \\
\hline $12 / 12 / 2015$ & 16:03:08 & 213.44 & 2.19 & 66.8 & 665 & 1,395 & 3,654 & 2,393 & 4,737 \\
\hline $12 / 13 / 2015$ & $24: 00: 00$ & 210.85 & 19.52 & 80.5 & 2,944 & 1,097 & 3,496 & 2,638 & 4,564 \\
\hline $12 / 14 / 2015$ & $23: 42: 21$ & 224.59 & 37.64 & 65.4 & 4,305 & 676 & 3,404 & 2,752 & 4,463 \\
\hline $12 / 15 / 2015$ & $24: 00: 00$ & 228.23 & 41.78 & 56.5 & 4,740 & 424 & 3,270 & 2,617 & 4,316 \\
\hline $12 / 16 / 2015$ & 23:14:34 & 217.59 & 61.69 & 49.2 & 5,462 & 181 & 3,612 & 3,327 & 4,692 \\
\hline $12 / 17 / 2015$ & $16: 46: 23$ & 213.58 & 8.13 & 60.3 & 1,354 & 167 & 3,582 & 3,209 & 4,659 \\
\hline $12 / 18 / 2015$ & 21:40:50 & 213.86 & 63.69 & 58.1 & 1,945 & 196 & 3,486 & 3,071 & 4,553 \\
\hline $12 / 19 / 2015$ & $24: 00: 00$ & 225.85 & 130.53 & 57.0 & 3,197 & 145 & 3,483 & 3,094 & 4,550 \\
\hline $12 / 20 / 2015$ & $24: 00: 00$ & 218.86 & 115.1 & 79.9 & 2,896 & 88 & 3,484 & 3,112 & 4,551 \\
\hline $12 / 21 / 2015$ & $24: 00: 00$ & 213.01 & 123.43 & 79.0 & 2,917 & 71 & 3,534 & 3,187 & 4,606 \\
\hline $12 / 22 / 2015$ & $24: 00: 00$ & 215.78 & 69.81 & 86.1 & 2,225 & 72 & 3,509 & 3,160 & 4,579 \\
\hline $12 / 23 / 2015$ & 23:12:47 & 212.17 & 93.88 & 73.7 & 2,475 & 86 & 3,542 & 3,213 & 4,615 \\
\hline $12 / 24 / 2015$ & $20: 47: 14$ & 207.49 & 83.02 & 90.0 & 2,036 & 72 & 3,531 & 3,212 & 4,603 \\
\hline $12 / 25 / 2015$ & $24: 00: 00$ & 227.27 & 80.98 & 83.3 & 2,366 & 88 & 3,576 & 3,285 & 4,652 \\
\hline $12 / 26 / 2015$ & $20: 58: 17$ & 223.74 & 61.54 & 78.6 & 1,800 & 46 & 3,508 & 3,174 & 4,577 \\
\hline $12 / 27 / 2015$ & $24: 00: 00$ & 227.52 & 91.28 & 72.5 & 2,591 & 80 & 3,461 & 3,145 & 4,526 \\
\hline $12 / 28 / 2015$ & $24: 00: 00$ & 226.69 & 130.29 & 59.6 & 3,196 & 98 & 3,391 & 3,051 & 4,449 \\
\hline $12 / 29 / 2015$ & 22:52:42 & 239.93 & 193.36 & 58.7 & 3,800 & 150 & 3,514 & 983 & 4,584 \\
\hline $12 / 30 / 2015$ & 14:31:19 & 225.88 & 256.92 & 55.9 & 2,659 & 91 & 3,391 & 3,077 & 4,449 \\
\hline $12 / 31 / 2015$ & $22: 35: 15$ & 224.59 & 231.82 & 45.6 & 3,924 & 100 & 3,203 & 2,763 & 4,242 \\
\hline $1 / 1 / 2016$ & $24: 00: 00$ & 236.1 & 425.01 & 41.8 & 5,917 & 190 & 3,124 & 2,688 & 4,154 \\
\hline $1 / 2 / 2016$ & $24: 00: 00$ & 238.45 & 451.88 & 40.1 & 6,145 & 168 & 3,210 & 2,941 & 4,249 \\
\hline $1 / 3 / 2016$ & $24: 00: 00$ & 232.25 & 223.93 & 59.6 & 4,143 & 64 & 3,205 & 2,923 & 4,244 \\
\hline $1 / 4 / 2016$ & $24: 00: 00$ & 236.61 & 205.95 & 63.2 & 4,074 & 115 & 3,190 & 2,890 & 4,228 \\
\hline $1 / 5 / 2016$ & $24: 00: 00$ & 276.44 & 188.8 & 66.0 & 4,058 & 89 & 3,197 & 2,934 & 4,236 \\
\hline $1 / 6 / 2016$ & $24: 00: 00$ & 252.25 & 177.26 & 66.7 & 3,850 & 68 & 3,195 & 2,936 & 4,233 \\
\hline $1 / 7 / 2016$ & $24: 00: 00$ & 237.59 & 174.25 & 67.0 & 3,751 & 63 & 3,208 & 2,962 & 4,248 \\
\hline $1 / 8 / 2016$ & $24: 00: 00$ & 237.53 & 151.76 & 69.7 & 3,495 & 55 & 3,210 & 2,970 & 4,250 \\
\hline $1 / 9 / 2016$ & $24: 00: 00$ & 249.55 & 146.35 & 69.1 & 3,462 & 51 & 3,234 & 3,009 & 4,276 \\
\hline $1 / 10 / 2016$ & $24: 00: 00$ & 227.64 & 128.92 & 70.1 & 3,157 & 39 & 3,234 & 2,997 & 4,276 \\
\hline $1 / 11 / 2016$ & $24: 00: 00$ & 232.72 & 130.92 & 68.3 & 3,219 & 50 & 3,270 & 3,202 & 4,316 \\
\hline $1 / 12 / 2016$ & 16:07:31 & 271.33 & 204.39 & 60.4 & 2,899 & 45 & 3,323 & 3,092 & 4,375 \\
\hline $1 / 13 / 2016$ & 19:30:38 & 240.55 & 104.84 & 64.8 & 2,378 & 12 & 3,236 & 3,015 & 4,210 \\
\hline $1 / 14 / 2016$ & $24: 00: 00$ & 242.56 & 163.13 & 58.1 & 3,703 & 44 & 3,232 & 3,021 & 4,274 \\
\hline $1 / 15 / 2016$ & $24: 00: 00$ & 235.25 & 138.42 & 64.2 & 3,342 & 35 & 3,242 & 3,041 & 4,285 \\
\hline $1 / 16 / 2016$ & $24: 00: 00$ & 232.17 & 121.64 & 66.8 & 3,108 & 33 & 3,246 & 3,047 & 4,289 \\
\hline $1 / 17 / 2016$ & $24: 00: 00$ & 231.38 & 115.92 & 67.3 & 3,029 & 31 & 3,244 & 3,033 & 4,287 \\
\hline $1 / 18 / 2016$ & $24: 00: 00$ & 232.15 & 119 & 67.0 & 3,069 & 36 & 3,168 & 2,768 & 4,203 \\
\hline $1 / 19 / 2016$ & $24: 00: 00$ & 243.74 & 201.54 & 58.1 & 4,119 & 49 & 3,123 & 2,837 & 4,153 \\
\hline $1 / 20 / 2016$ & $24: 00: 00$ & 267.95 & 217.18 & 57.7 & 4,445 & 49 & 3,113 & 2,779 & 4,142 \\
\hline $1 / 21 / 2016$ & $24: 00: 00$ & 259.63 & 208.77 & 59.1 & 4,275 & 47 & 3,079 & 2,805 & 4,104 \\
\hline $1 / 22 / 2016$ & $24: 00: 00$ & 246.89 & 237.4 & 57.2 & 4,496 & 53 & 3,058 & 2,782 & 4,080 \\
\hline $1 / 23 / 2016$ & $24: 00: 00$ & 247.2 & 237.32 & 58.1 & 4,494 & 50 & 3,035 & 2,758 & 4,056 \\
\hline $1 / 24 / 2016$ & $24: 00: 00$ & 247.38 & 244.24 & 57.2 & 4,564 & 51 & 3,020 & 2,745 & 4,038 \\
\hline $1 / 25 / 2016$ & $24: 00: 00$ & 246.63 & 242.58 & 57.3 & 4,541 & 48 & 3,000 & 2,716 & 4,016 \\
\hline $1 / 26 / 2016$ & $24: 00: 00$ & 249.16 & 252.71 & 57.1 & 4,657 & 52 & 2,984 & 2,705 & 3,998 \\
\hline $1 / 27 / 2016$ & 24:00:00 & 248.99 & 250.46 & 57.6 & 4,633 & 49 & 2,969 & 2,693 & 3,982 \\
\hline $1 / 28 / 2016$ & 24:00:00 & 249.04 & 247.43 & 57.4 & 4,607 & 48 & 2,950 & 2,665 & 3,960 \\
\hline $1 / 29 / 2016$ & 24:00:00 & 253.62 & 255.63 & 57.1 & 4,725 & 51 & 2,931 & 2,644 & 3,939 \\
\hline $1 / 30 / 2016$ & $24: 00: 00$ & 254.76 & 257.94 & 57.7 & 4,754 & 50 & 2,983 & 2,767 & 3,998 \\
\hline $1 / 31 / 2016$ & $24: 00: 00$ & 250.2 & 181.04 & 61.9 & 3,920 & 32 & 3,072 & 2,922 & 4,097 \\
\hline $2 / 1 / 2016$ & $24: 00: 00$ & 239.35 & 107.12 & 70.0 & 2,906 & 15 & 3,086 & 2,922 & 4,043 \\
\hline $2 / 2 / 2016$ & $24: 00: 00$ & 228.94 & 121.96 & 64.8 & 3,098 & 21 & 3,095 & 2,928 & 4,122 \\
\hline $2 / 3 / 2016$ & 24:00:00 & 229.63 & 124.81 & 63.9 & 3,141 & 21 & 3,100 & 2,935 & 4,128 \\
\hline $2 / 4 / 2016$ & 24:00:00 & 230.17 & 123.93 & 63.1 & 3,136 & 22 & 2,955 & 2,567 & 3,967 \\
\hline $2 / 5 / 2016$ & $24: 00: 00$ & 250.41 & 331.69 & 52.1 & 5,227 & 67 & 2,937 & 2,677 & 3,946 \\
\hline $2 / 6 / 2016$ & $24: 00: 00$ & 255.55 & 249.22 & 58.5 & 4,602 & 38 & 2,928 & 2,667 & 3,936 \\
\hline $2 / 7 / 2016$ & $24: 00: 00$ & 246.9 & 232.77 & 58.7 & 4,446 & 43 & 2,912 & 2,646 & 3,918 \\
\hline $2 / 8 / 2016$ & $24: 00: 00$ & 247.25 & 238.14 & 58.8 & 4,499 & 44 & 2,898 & 2,632 & 3,903 \\
\hline $2 / 9 / 2016$ & $24: 00: 00$ & 248.63 & 240.44 & 57.9 & 4,537 & 43 & 2,836 & 2,508 & 3,833 \\
\hline $2 / 10 / 2016$ & $24: 00: 00$ & 259.45 & 318.78 & 55.1 & 5,328 & 57 & 2,775 & 2,406 & 3,765 \\
\hline $2 / 11 / 2016$ & $24: 00: 00$ & 267.71 & 373.14 & 55.4 & 5,839 & 59 & 2,762 & 2,431 & 3,750 \\
\hline $2 / 12 / 2016$ & $24: 00: 00$ & 267.07 & 331.56 & 57.5 & 5,497 & 48 & 2,806 & 2,526 & 3,800 \\
\hline $2 / 13 / 2016$ & $20: 33: 28$ & 259.18 & 293.66 & 59.5 & 4,310 & 32 & 2,826 & 2,584 & 3,821 \\
\hline $2 / 14 / 2016$ & $24: 00: 00$ & 251.6 & 215.69 & 65.9 & 4,291 & 31 & 2,828 & 2,583 & 3,824 \\
\hline $2 / 15 / 2016$ & $24: 00: 00$ & 250.54 & 216.18 & 66.6 & 4,283 & 35 & 2,821 & 2,571 & 3,816 \\
\hline $2 / 16 / 2016$ & 27:19:37 & 249.93 & 194.67 & 66.2 & 5,292 & 37 & 2,814 & 2,562 & 3,808 \\
\hline $2 / 17 / 2016$ & $20: 34: 17$ & 250.96 & 222.99 & 66.5 & 3,731 & 39 & 2,807 & 2,554 & 3,800 \\
\hline $2 / 18 / 2016$ & $24: 00: 00$ & 251.31 & 222.84 & 67.3 & 4,351 & 38 & 2,801 & 2,551 & 3,794 \\
\hline $2 / 19 / 2016$ & $24: 00: 00$ & 250.94 & 221.33 & 68.1 & 4,329 & 39 & 2,917 & 2,830 & 3,923 \\
\hline $2 / 20 / 2016$ & $23: 00: 53$ & 251.92 & 127.31 & 80.8 & 2,982 & 22 & 2,989 & 2,886 & 4,005 \\
\hline $2 / 21 / 2016$ & $20: 58: 36$ & 242.13 & 83.96 & 81.1 & 2,226 & 7 & 3,011 & 2,902 & 3,890 \\
\hline $2 / 22 / 2016$ & $24: 00: 00$ & 224.51 & 88.88 & 79.9 & 2,584 & 10 & 2,981 & 2,836 & 3,856 \\
\hline $2 / 23 / 2016$ & $26: 26: 58$ & 248.5 & 148.1 & 67.2 & 3,882 & 17 & 2,962 & 2,806 & 3,904 \\
\hline $2 / 24 / 2016$ & 20:01:10 & 242.02 & 77.19 & 67.9 & 2,927 & 21 & 2,997 & 2,873 & 4,012 \\
\hline $2 / 25 / 2016$ & 22:06:41 & 247.23 & 58.56 & 65.5 & 2,769 & 14 & 2,932 & 2,739 & 3,871 \\
\hline $2 / 26 / 2016$ & $24: 00: 00$ & 247.04 & 96.65 & 63.2 & 3,981 & 28 & 2,906 & 2,711 & 3,912 \\
\hline
\end{tabular}




\begin{tabular}{|c|c|c|c|c|c|c|c|c|c|}
\hline $2 / 27 / 2016$ & $24: 00: 00$ & 243.58 & 95.38 & 65.1 & 3,920 & 26 & 2,897 & 2,704 & 3,901 \\
\hline $2 / 28 / 2016$ & $24: 00: 00$ & 242.32 & 90.84 & 66.5 & 3,812 & 27 & 3,112 & 3,110 & 4,141 \\
\hline $2 / 29 / 2016$ & $7: 50: 33$ & 288.72 & 68.32 & 70.4 & 1,104 & 6 & 3,033 & 2,939 & 3,915 \\
\hline $3 / 1 / 2016$ & $23: 59: 38$ & 236.33 & 51.24 & 57.2 & 2,847 & 11 & 3,139 & 3,138 & 4,102 \\
\hline $3 / 2 / 2016$ & 10:48:01 & 237.08 & 43.44 & 45.4 & 1,179 & 6 & 3,034 & 2,908 & 3,916 \\
\hline $3 / 3 / 2016$ & $23: 30: 47$ & 246.55 & 75.28 & 46.2 & 3,502 & 15 & 2,996 & 2,851 & 3,942 \\
\hline $3 / 4 / 2016$ & $24: 00: 00$ & 246.29 & 73.87 & 47.8 & 3,540 & 13 & 2,968 & 2,805 & 3,911 \\
\hline $3 / 5 / 2016$ & $24: 00: 00$ & 244.1 & 79.1 & 56.0 & 3,612 & 23 & 2,966 & 2,844 & 3,978 \\
\hline $3 / 6 / 2016$ & 21:01:06 & 242 & 75.19 & 47.8 & 3,087 & 19 & 3,094 & 3,083 & 4,052 \\
\hline $3 / 7 / 2016$ & 11:57:29 & 242.73 & 76.59 & 37.9 & 1,790 & 11 & 3,030 & 2,924 & 3,981 \\
\hline $3 / 8 / 2016$ & $24: 00: 00$ & 235.58 & 62.86 & 61.3 & 3,147 & 11 & 3,029 & 2,912 & 3,979 \\
\hline $3 / 9 / 2016$ & 23:01:59 & 235.2 & 43.91 & 66.1 & 2,496 & 9 & 3,052 & 2,953 & 3,936 \\
\hline $3 / 10 / 2016$ & $24: 00: 00$ & 220.12 & 42.95 & 73.1 & 2,492 & 8 & 3,103 & 3,074 & 3,994 \\
\hline $3 / 11 / 2016$ & 16:31:50 & 232.83 & 42.38 & 72.9 & 1,727 & 6 & 3,093 & 3,002 & 3,982 \\
\hline $3 / 12 / 2016$ & $23: 10: 34$ & 220.73 & 41.31 & 68.8 & 2,372 & 6 & 3,089 & 2,990 & 3,978 \\
\hline $3 / 13 / 2016$ & $24: 00: 00$ & 221.27 & 42.97 & 68.1 & 2,511 & 8 & 3,087 & 2,984 & 3,975 \\
\hline $3 / 14 / 2016$ & $24: 00: 00$ & 222.62 & 43.92 & 68.3 & 2,545 & 9 & 3,182 & 3,177 & 4,082 \\
\hline $3 / 15 / 2016$ & 9:48:27 & 238.69 & 36.96 & 72.4 & 963 & 3 & 3,147 & 3,076 & 3,973 \\
\hline $3 / 16 / 2016$ & $24: 00: 00$ & 219.13 & 28.39 & 80.0 & 2,009 & 3 & 3,148 & 3,065 & 3,974 \\
\hline $3 / 17 / 2016$ & $24: 00: 00$ & 222.16 & 29.09 & 76.0 & 2,056 & 4 & 3,131 & 3,031 & 3,955 \\
\hline $3 / 18 / 2016$ & $24: 00: 00$ & 225.25 & 41.87 & 66.7 & 2,503 & 9 & 3,116 & 3,012 & 4,008 \\
\hline $3 / 19 / 2016$ & $24: 00: 00$ & 228.46 & 50.3 & 63.3 & 2,772 & 11 & 3,099 & 2,996 & 4,058 \\
\hline $3 / 20 / 2016$ & $24: 00: 00$ & 229.82 & 50.85 & 63.2 & 2,796 & 10 & 3,086 & 2,974 & 4,043 \\
\hline $3 / 21 / 2016$ & $24: 00: 00$ & 231.13 & 54.73 & 61.0 & 2,914 & 54 & 3,068 & 2,956 & 4,092 \\
\hline $3 / 22 / 2016$ & $24: 00: 00$ & 231.97 & 55.68 & 60.7 & 2,945 & 11 & 3,062 & 2,944 & 4,016 \\
\hline $3 / 23 / 2016$ & $24: 00: 00$ & 236.32 & 54.51 & 61.4 & 2,939 & 11 & 3,054 & 2,938 & 4,007 \\
\hline $3 / 24 / 2016$ & $24: 00: 00$ & 244.72 & 52.44 & 63.0 & 2,928 & 0 & 3,136 & 3,127 & 3,884 \\
\hline $3 / 25 / 2016$ & $14: 38: 36$ & 252.64 & 45.17 & 58.6 & 1,656 & 2 & 3,107 & 3,033 & 3,851 \\
\hline $3 / 26 / 2016$ & $24: 00: 00$ & 241.77 & 36.49 & 70.2 & 2,413 & 3 & 3,097 & 3,002 & 3,916 \\
\hline $3 / 27 / 2016$ & $24: 00: 00$ & 242.65 & 37.65 & 74.2 & 2,445 & 5 & 3,173 & 3,172 & 4,002 \\
\hline $3 / 28 / 2016$ & $23: 10: 52$ & 242.53 & 37.7 & 74.6 & 2,362 & 11 & 3,137 & 2,170 & 4,100 \\
\hline $3 / 29 / 2016$ & $20: 49: 43$ & 198.23 & 39.68 & 61.4 & 1,991 & 7 & 3,147 & 3,062 & 4,043 \\
\hline $3 / 30 / 2016$ & $24: 00: 00$ & 212.42 & 40.11 & 78.3 & 2,250 & 5 & 3,091 & 2,994 & 3,980 \\
\hline $3 / 31 / 2016$ & $25: 01: 29$ & 227.53 & 39.49 & 63.5 & 3,307 & 4 & 3,092 & 2,877 & 3,911 \\
\hline $4 / 1 / 2016$ & $21: 47: 15$ & 248.03 & 40.63 & 66.3 & 2,340 & 9 & 3,069 & 2,929 & 3,955 \\
\hline $4 / 2 / 2016$ & $23: 53: 25$ & 243.22 & 61.84 & 74.5 & 3,085 & 10 & 3,026 & 2,856 & 3,907 \\
\hline $4 / 3 / 2016$ & $24: 00: 00$ & 237.87 & 82.93 & 72.3 & 3,583 & 15 & 3,011 & 2,857 & 3,959 \\
\hline $4 / 4 / 2016$ & $24: 00: 00$ & 245.01 & 74.98 & 76.4 & 3,447 & 12 & 2,987 & 2,820 & 3,932 \\
\hline $4 / 5 / 2016$ & $23: 58: 12$ & 243.04 & 75.88 & 71.3 & 3,468 & 17 & 2,954 & 2,754 & 3,895 \\
\hline $4 / 6 / 2016$ & $24: 00: 00$ & 242.19 & 94.06 & 71.5 & 3,854 & 56 & 2,911 & 2,682 & 3,917 \\
\hline $4 / 7 / 2016$ & $24: 00: 00$ & 245.17 & 115.93 & 65.5 & 4,329 & 26 & 2,844 & 2,551 & 3,842 \\
\hline $4 / 8 / 2016$ & $24: 00: 00$ & 252.33 & 150.84 & 57.9 & 5,036 & 34 & 2,798 & 2,504 & 3,791 \\
\hline $4 / 9 / 2016$ & $24: 00: 00$ & 253.3 & 164.02 & 57.3 & 5,265 & 32 & 2,764 & 2,470 & 3,752 \\
\hline $4 / 10 / 2016$ & $24: 00: 00$ & 253.56 & 164.21 & 58.6 & 5,263 & 31 & 2,819 & 2,654 & 3,814 \\
\hline $4 / 11 / 2016$ & $24: 00: 00$ & 253.75 & 110.1 & 72.6 & 4,162 & 19 & 2,859 & 2,711 & 3,788 \\
\hline $4 / 12 / 2016$ & $24: 00: 00$ & 246.84 & 67.25 & 87.3 & 3,150 & 6 & 2,833 & 2,663 & 3,689 \\
\hline $4 / 13 / 2016$ & $24: 00: 00$ & 246.25 & 91.37 & 75.1 & 3,815 & 64 & 2,800 & 2,574 & 3,792 \\
\hline $4 / 14 / 2016$ & $24: 00: 00$ & 248.39 & 105.02 & 72.1 & 4,104 & 19 & 2,885 & 2,800 & 3,818 \\
\hline $4 / 15 / 2016$ & $23: 37: 21$ & 243.24 & 55 & 94.9 & 2,748 & 5 & 2,924 & 2,831 & 3,792 \\
\hline $4 / 16 / 2016$ & $23: 39: 05$ & 239.57 & 42.91 & 105.7 & 2,411 & 5 & 2,943 & 2,848 & 3,740 \\
\hline $4 / 17 / 2016$ & $24: 00: 00$ & 239.06 & 44.12 & 100.7 & 2,507 & 5 & 2,971 & 2,891 & 3,845 \\
\hline $4 / 18 / 2016$ & $24: 00: 00$ & 244.92 & 33.27 & 110.4 & 2,159 & 3 & 2,991 & 2,924 & 3,795 \\
\hline $4 / 19 / 2016$ & $24: 00: 00$ & 242.32 & 32.83 & 109.4 & 2,149 & 46 & 2,979 & 2,875 & 3,993 \\
\hline $4 / 20 / 2016$ & $24: 00: 00$ & 225.91 & 47.02 & 96.5 & 2,570 & 7 & 2,957 & 2,859 & 3,830 \\
\hline $4 / 21 / 2016$ & $24: 00: 00$ & 248.44 & 63.68 & 86.3 & 3,109 & 10 & 2,959 & 2,848 & 3,900 \\
\hline $4 / 22 / 2016$ & $24: 00: 00$ & 249.94 & 48.81 & 95.0 & 2,710 & 8 & 2,976 & 2,845 & 3,851 \\
\hline $4 / 23 / 2016$ & 21:31:51 & 242.47 & 52.85 & 97.6 & 2,441 & 4 & 2,956 & 2,829 & 3,754 \\
\hline $4 / 24 / 2016$ & $24: 00: 00$ & 249.13 & 54.18 & 96.3 & 2,837 & 9 & 2,962 & 2,859 & 3,835 \\
\hline $4 / 25 / 2016$ & 23:58:09 & 250.48 & 47.14 & 101.6 & 2,593 & 6 & 3,026 & 2,974 & 3,908 \\
\hline $4 / 26 / 2016$ & $20: 52: 26$ & 245.61 & 24.73 & 125.6 & 1,533 & 44 & 3,028 & 2,963 & 4,048 \\
\hline $4 / 27 / 2016$ & $22: 49: 57$ & 236.32 & 30.77 & 115.2 & 1,939 & 1 & 3,029 & 2,945 & 3,762 \\
\hline $4 / 28 / 2016$ & $23: 57: 23$ & 228.61 & 30.14 & 90.9 & 2,066 & 2 & 3,028 & 2,943 & 3,837 \\
\hline $4 / 29 / 2016$ & $23: 58: 08$ & 229.63 & 33.17 & 90.3 & 2,176 & 3 & 3,027 & 2,939 & 3,836 \\
\hline $4 / 30 / 2016$ & $24: 00: 00$ & 228.74 & 35.07 & 89.1 & 2,241 & 4 & 3,034 & 2,937 & 3,844 \\
\hline $5 / 1 / 2016$ & $23: 00: 25$ & 229.85 & 35.05 & 88.2 & 2,146 & 3 & 3,042 & 2,955 & 3,854 \\
\hline $5 / 2 / 2016$ & $22: 39: 45$ & 233.59 & 32.84 & 90.0 & 2,033 & 3 & 3,046 & 2,959 & 3,858 \\
\hline $5 / 3 / 2016$ & $23: 29: 18$ & 232.98 & 31.23 & 92.7 & 2,051 & 3 & 3,036 & 2,945 & 3,846 \\
\hline $5 / 4 / 2016$ & $23: 59: 22$ & 227.52 & 36.08 & 85.1 & 2,287 & 4 & 3,020 & 2,921 & 3,828 \\
\hline $5 / 5 / 2016$ & $23: 59: 08$ & 238.24 & 45.44 & 80.1 & 2,625 & 9 & 3,003 & 2,901 & 3,881 \\
\hline $5 / 6 / 2016$ & $24: 00: 00$ & 237.47 & 50.86 & 76.9 & 2,787 & 7 & 3,008 & 2,920 & 3,887 \\
\hline $5 / 7 / 2016$ & $20: 30: 30$ & 240.02 & 51.11 & 74.8 & 2,373 & 6 & 3,073 & 3,025 & 3,960 \\
\hline $5 / 8 / 2016$ & $12: 25: 51$ & 246.89 & 41.28 & 93.2 & 1,211 & 2 & 3,043 & 2,975 & 3,855 \\
\hline $5 / 9 / 2016$ & 18:18:07 & 242.91 & 53.09 & 78.7 & 2,123 & 5 & 3,010 & 2,907 & 3,817 \\
\hline $5 / 10 / 2016$ & $24: 00: 00$ & 241.66 & 56.72 & 76.0 & 2,962 & 8 & 3,022 & 2,922 & 3,902 \\
\hline $5 / 11 / 2016$ & $18: 39: 45$ & 237.4 & 61.51 & 74.5 & 2,326 & 7 & 3,041 & 2,939 & 3,924 \\
\hline $5 / 12 / 2016$ & $16: 44: 14$ & 249.37 & 53.11 & 80.3 & 1,928 & 2 & 3,083 & 3,017 & 3,900 \\
\hline $5 / 13 / 2016$ & $11: 17: 09$ & 252.77 & 45.07 & 93.1 & 1,148 & 1 & 3,080 & 2,998 & 3,821 \\
\hline $5 / 14 / 2016$ & $15: 28: 49$ & 250.44 & 44.55 & 86.7 & 1,620 & 3 & 3,013 & 2,890 & 3,820 \\
\hline $5 / 15 / 2016$ & $23: 52: 48$ & 240.25 & 62.93 & 69.0 & 3,122 & 10 & 2,978 & 2,843 & 3,853 \\
\hline $5 / 16 / 2016$ & $24: 00: 00$ & 236.93 & 69.93 & 67.3 & 3,308 & 11 & 2,984 & 2,870 & 3,929 \\
\hline $5 / 17 / 2016$ & $23: 55: 49$ & 241.56 & 53.95 & 78.5 & 2,836 & 9 & 2,981 & 2,862 & 3,857 \\
\hline $5 / 18 / 2016$ & $23: 59: 49$ & 242.45 & 50.85 & 80.8 & 2,754 & 8 & 2,945 & 2,796 & 3,816 \\
\hline
\end{tabular}




\begin{tabular}{|c|c|c|c|c|c|c|c|c|c|}
\hline $5 / 19 / 2016$ & $24: 00: 00$ & 237.85 & 68.75 & 69.2 & 3,278 & 13 & 2,975 & 2,871 & 3,919 \\
\hline $5 / 20 / 2016$ & $20: 01: 36$ & 244.47 & 52.79 & 80.8 & 2,315 & 5 & 3,030 & 2,921 & 3,840 \\
\hline $5 / 21 / 2016$ & $12: 01: 52$ & 252.87 & 55.07 & 83.4 & 1,429 & 2 & 3,031 & 2,950 & 3,764 \\
\hline $5 / 22 / 2016$ & $23: 11: 19$ & 248.33 & 34.07 & 90.7 & 1,955 & 4 & 3,012 & 2,929 & 3,819 \\
\hline $5 / 23 / 2016$ & $23: 47: 43$ & 254.05 & 38.45 & 89.2 & 2,364 & 4 & 3,043 & 3,019 & 3,855 \\
\hline $5 / 24 / 2016$ & $17: 18: 28$ & 295.96 & 29.89 & 97.6 & 1,619 & 1 & 3,041 & 2,961 & 3,775 \\
\hline $5 / 25 / 2016$ & $23: 41: 07$ & 231.43 & 27.38 & 88.8 & 1,936 & 1 & 3,046 & 2,943 & 3,781 \\
\hline $5 / 26 / 2016$ & $23: 40: 46$ & 227.43 & 26.79 & 83.7 & 1,924 & 2 & 3,049 & 2,955 & 3,784 \\
\hline $5 / 27 / 2016$ & 23:37:41 & 226.89 & 27.29 & 81.6 & 1,937 & 1 & 3,039 & 2,951 & 3,773 \\
\hline $5 / 28 / 2016$ & $35: 08: 34$ & 240.08 & 29.19 & 77.3 & 3,115 & 2 & 3,037 & 2,949 & 3,771 \\
\hline $5 / 29 / 2016$ & $12: 51: 22$ & 247.13 & 27.97 & 76.7 & 1,134 & 2 & 3,116 & 3,046 & 3,938 \\
\hline $5 / 30 / 2016$ & 5:42:04 & 294.45 & 25.15 & 79.3 & 503 & 0 & 3,107 & 3,095 & 3,852 \\
\hline $5 / 31 / 2016$ & 23:16:00 & 220.91 & 13.43 & 112.0 & 1,306 & 1 & 3,114 & 3,086 & 3,860 \\
\hline $6 / 1 / 2016$ & $24: 00: 00$ & 221.06 & 12.3 & 113.8 & 1,287 & 1 & 3,123 & 3,080 & 3,869 \\
\hline $6 / 2 / 2016$ & $24: 00: 00$ & 221.32 & 11.8 & 114.4 & 1,261 & 1 & 3,132 & 3,131 & 3,880 \\
\hline $6 / 3 / 2016$ & $16: 44: 50$ & 266.78 & 21.85 & 104.2 & 1,177 & 0 & 3,124 & 3,118 & 3,871 \\
\hline $6 / 4 / 2016$ & 23:05:08 & 236.39 & 21.47 & 98.9 & 1,587 & 1 & 3,116 & 3,069 & 3,862 \\
\hline $6 / 5 / 2016$ & 21:57:06 & 232.47 & 22.67 & 92.1 & 1,606 & 1 & 3,111 & 3,040 & 3,857 \\
\hline $6 / 6 / 2016$ & $22: 16: 13$ & 227.37 & 25.26 & 80.0 & 1,741 & 1 & 3,114 & 3,070 & 3,859 \\
\hline $6 / 7 / 2016$ & 21:38:54 & 231 & 23.51 & 80.9 & 1,640 & 1 & 3,104 & 3,035 & 3,848 \\
\hline $6 / 8 / 2016$ & 21:32:49 & 231.01 & 27.62 & 66.3 & 1,827 & 4 & 3,091 & 3,016 & 3,909 \\
\hline $6 / 9 / 2016$ & $24: 00: 00$ & 222.01 & 28.57 & 60.1 & 2,071 & 1 & 3,090 & 3,017 & 3,832 \\
\hline $6 / 10 / 2016$ & $23: 24: 00$ & 223.6 & 27.17 & 59.4 & 1,962 & 2 & 3,098 & 3,017 & 3,917 \\
\hline $6 / 11 / 2016$ & $23: 45: 45$ & 234.68 & 23.6 & 107.2 & 1,779 & 1 & 3,105 & 3,018 & 3,849 \\
\hline $6 / 12 / 2016$ & $23: 45: 25$ & 237.26 & 20.26 & 109.7 & 1,626 & 1 & 3,096 & 3,022 & 3,839 \\
\hline $6 / 13 / 2016$ & $23: 45: 34$ & 234.7 & 25.49 & 103.9 & 1,855 & 2 & 3,100 & 3,040 & 3,843 \\
\hline $6 / 14 / 2016$ & $21: 51: 13$ & 235.12 & 29.78 & 102.3 & 1,811 & 2 & 3,100 & 3,030 & 3,843 \\
\hline $6 / 15 / 2016$ & 21:43:06 & 235.76 & 29.28 & 102.7 & 1,794 & 1 & 3,106 & 3,023 & 3,850 \\
\hline $6 / 16 / 2016$ & $\begin{array}{l}21: 54: 40 \\
\end{array}$ & 235.81 & 26.6 & 103.8 & 1,668 & 1 & 3,100 & 3,001 & 3,843 \\
\hline $6 / 17 / 2016$ & $22: 16: 02$ & 235.48 & 28.86 & 100.8 & 1,823 & 2 & 3,102 & 3,018 & 3,846 \\
\hline $6 / 18 / 2016$ & $22: 12: 16$ & 236.5 & 27.37 & 103.1 & 1,752 & 2 & 3,110 & 3,001 & 3,854 \\
\hline $6 / 19 / 2016$ & $21: 20: 57$ & 237.45 & 25.06 & 106.6 & 1,575 & 1 & 3,116 & 3,035 & 3,862 \\
\hline $6 / 20 / 2016$ & $21: 14: 20$ & 238.25 & 22.47 & 110.2 & 1,478 & 1 & 3,116 & 3,028 & 3,861 \\
\hline $6 / 21 / 2016$ & $21: 17: 23$ & 237.42 & 25.48 & 104.6 & 1,603 & 1 & 3,115 & 3,016 & 3,861 \\
\hline $6 / 22 / 2016$ & $20: 54: 05$ & 236.95 & 26.57 & 103.9 & 1,614 & 1 & 3,115 & 3,031 & 3,861 \\
\hline $6 / 23 / 2016$ & $21: 21: 36$ & 236.69 & 26.42 & 103.4 & 1,650 & 1 & 3,113 & 3,020 & 3,858 \\
\hline $6 / 24 / 2016$ & $21: 53: 00$ & 236.47 & 27.51 & 100.7 & 1,750 & 2 & 3,116 & 3,023 & 3,862 \\
\hline $6 / 25 / 2016$ & $20: 37: 38$ & 236.61 & 27.8 & 101.9 & 1,617 & 1 & 3,120 & 2,991 & 3,867 \\
\hline $6 / 26 / 2016$ & $19: 47: 34$ & 236.68 & 27.1 & 105.1 & 1,531 & 1 & 3,083 & 2,985 & 3,824 \\
\hline $6 / 27 / 2016$ & $24: 00: 00$ & 226.23 & 42.82 & 84.1 & 2,492 & 5 & 3,066 & 2,958 & 3,953 \\
\hline $6 / 28 / 2016$ & $24: 00: 00$ & 225.72 & 43.83 & 84.1 & 2,518 & 5 & 3,047 & 2,931 & 3,859 \\
\hline $6 / 29 / 2016$ & $24: 00: 00$ & 226.76 & 48.88 & 81.1 & 2,673 & 8 & 3,039 & 2,931 & 3,922 \\
\hline $6 / 30 / 2016$ & $22: 08: 22$ & 230.27 & 53.23 & 79.2 & 2,569 & 7 & 3,074 & 2,949 & 3,961 \\
\hline $7 / 1 / 2016$ & 18:07:11 & 238.67 & 36.21 & 95.2 & 1,613 & 2 & 3,086 & 2,974 & 3,828 \\
\hline $7 / 2 / 2016$ & $16: 25: 28$ & 239.83 & 39.69 & 90.6 & 1,546 & 1 & 3,093 & 2,966 & 3,835 \\
\hline $7 / 3 / 2016$ & 16:30:09 & 239.84 & 39.15 & 91.0 & 1,532 & 1 & 3,098 & 2,992 & 3,841 \\
\hline $7 / 4 / 2016$ & 18:08:51 & 240.24 & 32.31 & 96.5 & 1,511 & 2 & 3,080 & 2,994 & 3,820 \\
\hline $7 / 5 / 2016$ & $19: 45: 04$ & 231.74 & 52.13 & 77.5 & 2,248 & 6 & 3,094 & 3,007 & 3,984 \\
\hline $7 / 6 / 2016$ & $16: 08: 21$ & 240.67 & 39.1 & 91.8 & 1,533 & 1 & 3,081 & 2,995 & 3,822 \\
\hline $7 / 7 / 2016$ & $19: 39: 54$ & 250.41 & 42.08 & 86.0 & 2,028 & 3 & 3,092 & 2,970 & 3,910 \\
\hline $7 / 8 / 2016$ & $16: 16: 54$ & 243.63 & 41.18 & 89.8 & 1,623 & 1 & 3,085 & 2,992 & 3,827 \\
\hline $7 / 9 / 2016$ & $18: 26: 54$ & 242.22 & 41.28 & 87.2 & 1,812 & 1 & 3,090 & 3,009 & 3,832 \\
\hline $7 / 10 / 2016$ & 18:39:11 & 240.1 & 37.78 & 92.1 & 1,731 & 1 & 3,074 & 2,984 & 3,813 \\
\hline $7 / 11 / 2016$ & 21:31:09 & 245.08 & 33.24 & 93.6 & 1,968 & 2 & 3,074 & 3,016 & 3,814 \\
\hline $7 / 12 / 2016$ & $23: 05: 19$ & 244.52 & 29.12 & 99.0 & 1,962 & 2 & 3,084 & 2,985 & 3,901 \\
\hline $7 / 13 / 2016$ & $18: 49: 32$ & 244.8 & 35.36 & 94.2 & 1,735 & 2 & 3,089 & 3,005 & 3,831 \\
\hline $7 / 14 / 2016$ & 19:09:49 & 244.3 & 32.25 & 96.7 & 1,662 & 0 & 3,088 & 3,011 & 3,829 \\
\hline $7 / 15 / 2016$ & $21: 32: 58$ & 243.21 & 26.53 & 103.6 & 1,685 & 2 & 3,084 & 3,012 & 3,824 \\
\hline $7 / 16 / 2016$ & $22: 49: 06$ & 279.08 & 22.55 & 104.9 & 1,722 & 1 & 3,087 & 3,038 & 3,829 \\
\hline $7 / 17 / 2016$ & $18: 49: 21$ & 271.65 & 24.92 & 103.2 & 1,523 & 1 & 3,158 & 3,121 & 3,910 \\
\hline $7 / 18 / 2016$ & $0: 00: 02$ & -0.78 & 1.04 & 91.0 & 0 & 0 & 3,108 & 3,081 & 3,853 \\
\hline $7 / 19 / 2016$ & $18: 57: 41$ & 244.02 & 35.05 & 107.0 & 1,805 & 1 & 3,086 & 3,061 & 3,827 \\
\hline $7 / 20 / 2016$ & $24: 00: 00$ & 243.14 & 29.06 & 101.4 & 2,092 & 0 & 3,069 & 2,980 & 3,808 \\
\hline $7 / 21 / 2016$ & $23: 53: 08$ & 242.74 & 31.64 & 98.9 & 2,148 & 3 & 3,092 & 3,065 & 3,911 \\
\hline $7 / 22 / 2016$ & $23: 53: 32$ & 241.46 & 19.1 & 126.9 & 1,539 & 1 & 3,089 & 3,036 & 3,831 \\
\hline $7 / 23 / 2016$ & $23: 59: 56$ & 242.53 & 17.56 & 105.1 & 1,605 & 0 & 3,091 & 2,997 & 3,833 \\
\hline $7 / 24 / 2016$ & $24: 00: 00$ & 243.3 & 17.98 & 100.7 & 1,648 & 1 & 3,087 & 3,015 & 3,828 \\
\hline $7 / 25 / 2016$ & $24: 00: 00$ & 243.42 & 21.24 & 95.3 & 1,791 & 2 & 3,140 & 3,132 & 3,889 \\
\hline $7 / 26 / 2016$ & $8: 18: 08$ & 242.7 & 25.11 & 86.4 & 680 & 0 & 3,098 & 3,073 & 3,841 \\
\hline $7 / 27 / 2016$ & $20: 15: 04$ & 243.36 & 18.68 & -11.5 & 1,602 & 0 & 3,094 & 3,037 & 3,836 \\
\hline $7 / 28 / 2016$ & $17: 49: 58$ & 244.32 & 28.95 & 88.5 & 1,566 & 0 & 3,122 & 3,073 & 3,868 \\
\hline $7 / 29 / 2016$ & $15: 02: 15$ & 240.25 & 27.43 & 100.3 & 1,234 & 0 & 3,108 & 3,069 & 3,852 \\
\hline $7 / 30 / 2016$ & $15: 59: 08$ & 264.52 & 46.5 & 95.9 & 1,805 & 0 & 3,088 & 3,017 & 3,830 \\
\hline $7 / 31 / 2016$ & $12: 11: 16$ & 231.31 & 27.42 & 104.2 & 1,121 & 0 & & & $\# \mathrm{~N} / \mathrm{A}$ \\
\hline $8 / 1 / 2016$ & $20: 25: 51$ & 230.74 & 21.21 & 90.8 & 1,473 & 0 & 3,103 & 3,055 & 3,847 \\
\hline $8 / 2 / 2016$ & $23: 57: 17$ & 238.33 & 21.52 & 96.6 & 1,766 & 0 & 3,109 & 3,030 & 3,854 \\
\hline $8 / 3 / 2016$ & $26: 50: 08$ & 220.55 & 13.31 & 123.8 & 1,668 & 0 & & & $\# \mathrm{~N} / \mathrm{A}$ \\
\hline $8 / 4 / 2016$ & $18: 36: 08$ & 218.52 & 13.63 & 128.1 & 993 & 0 & 3,102 & 3,060 & 3,846 \\
\hline $8 / 5 / 2016$ & $21: 45: 38$ & 239.18 & 26.45 & 48.1 & 1,797 & 0 & 3,149 & 177 & 3,900 \\
\hline $8 / 6 / 2016$ & $9: 25: 00$ & 232 & 23.3 & 33.8 & 668 & 0 & 3,104 & 3,078 & 3,848 \\
\hline $8 / 7 / 2016$ & $20: 54: 36$ & 241.99 & 31.99 & 102.2 & 1,897 & 0 & 3,098 & 2,948 & 3,841 \\
\hline $8 / 8 / 2016$ & $22: 09: 54$ & 242.13 & 25.29 & 80.3 & 1,773 & 0 & 3,093 & 3,016 & 3,835 \\
\hline
\end{tabular}




\begin{tabular}{|c|c|c|c|c|c|c|c|c|c|}
\hline $8 / 9 / 2016$ & $21: 39: 19$ & 242.3 & 31.14 & 108.4 & 1,876 & 0 & 3,115 & 3,097 & 3,861 \\
\hline $8 / 10 / 2016$ & $16: 03: 13$ & 256.45 & 19.53 & 25.8 & 1,168 & 0 & 3,103 & 3,048 & 3,847 \\
\hline $8 / 11 / 2016$ & $23: 41: 09$ & 240.4 & 21.66 & 120.5 & 1,682 & 0 & 3,106 & 3,040 & 3,851 \\
\hline $8 / 12 / 2016$ & $23: 18: 45$ & 240.6 & 19.28 & 113.6 & 1,554 & 0 & 3,110 & 3,012 & 3,855 \\
\hline $8 / 13 / 2016$ & $20: 36: 47$ & 240.83 & 21.53 & 91.6 & 1,458 & 0 & 3,138 & 3,109 & 3,887 \\
\hline $8 / 14 / 2016$ & $\begin{array}{l}11: 16: 09 \\
\end{array}$ & 250.79 & 20.75 & 92.6 & 764 & 0 & 3,116 & 3,082 & 3,861 \\
\hline $8 / 15 / 2016$ & 16:19:06 & 241.54 & 30.47 & 94.3 & 1,420 & 0 & 3,120 & 3,028 & 3,866 \\
\hline $8 / 16 / 2016$ & $20: 49: 41$ & 240.1 & 23.83 & 105.7 & 1,528 & 0 & 3,123 & 3,108 & 3,869 \\
\hline $8 / 17 / 2016$ & $20: 41: 49$ & 238.05 & 17.37 & 52.4 & 1,307 & 0 & 3,132 & 2,772 & 3,880 \\
\hline $8 / 18 / 2016$ & 19:18:24 & 237.79 & 18.41 & 63.4 & 1,245 & 0 & 3,123 & 3,037 & 3,870 \\
\hline $8 / 19 / 2016$ & $20: 56: 08$ & 238.05 & 24.24 & 68.0 & 1,601 & 0 & 3,120 & 3,012 & 3,867 \\
\hline $8 / 20 / 2016$ & $20: 46: 15$ & 238.78 & 23.17 & 67.6 & 1,534 & 0 & 3,116 & 3,039 & 3,862 \\
\hline $8 / 21 / 2016$ & 21:08:49 & 240.09 & 23.7 & 56.6 & 1,620 & 0 & 3,111 & 3,034 & 3,856 \\
\hline $8 / 22 / 2016$ & $22: 58: 28$ & 237.9 & 17.85 & 31.4 & 1,559 & 0 & 3,162 & 3,122 & 3,914 \\
\hline $8 / 23 / 2016$ & $5: 07: 49$ & 234.25 & 24.19 & 31.1 & 394 & 0 & 3,119 & 3,097 & 3,866 \\
\hline $8 / 24 / 2016$ & $23: 56: 36$ & 239.26 & 24.91 & 84.3 & 1,910 & 0 & 3,117 & 3,061 & 3,863 \\
\hline $8 / 25 / 2016$ & 22:31:11 & 239.4 & 20.57 & 53.2 & 1,652 & 0 & 3,118 & 3,016 & 3,864 \\
\hline $8 / 26 / 2016$ & $22: 14: 49$ & 239.55 & 17.97 & 37.9 & 1,520 & 0 & 3,165 & 3,074 & 3,918 \\
\hline $8 / 27 / 2016$ & 5:09:00 & 237.79 & 16.31 & 36.7 & 324 & 0 & 3,132 & 3,120 & 3,880 \\
\hline $8 / 28 / 2016$ & $23: 30: 52$ & 241.13 & 18.08 & 62.2 & 1,599 & 0 & 3,128 & 3,097 & 3,876 \\
\hline $8 / 29 / 2016$ & $20: 34: 11$ & 240.63 & 21.27 & 52.6 & 1,490 & 0 & 3,136 & 3,062 & 3,884 \\
\hline $8 / 30 / 2016$ & $17: 00: 30$ & 240.96 & 21.83 & 45.0 & 1,271 & 0 & 3,115 & 3,053 & 3,861 \\
\hline $8 / 31 / 2016$ & $23: 55: 06$ & 239.92 & 18.68 & 34.2 & 1,711 & 2 & 3,134 & 3,114 & 3,882 \\
\hline $9 / 1 / 2016$ & $21: 29: 49$ & 234.88 & 7.77 & -25.2 & 1,009 & 1 & 3,116 & 3,094 & 3,862 \\
\hline $9 / 2 / 2016$ & $\begin{array}{l}17: 07: 21 \\
\end{array}$ & 236.78 & 35.49 & 88.4 & 1,575 & 3 & 3,112 & 3,062 & 3,934 \\
\hline $9 / 3 / 2016$ & $22: 17: 00$ & 237.97 & 23.17 & 82.0 & 1,683 & 1 & 3,114 & 3,075 & 3,859 \\
\hline $9 / 4 / 2016$ & $19: 28: 09$ & 239.93 & 24.26 & 109.6 & 1,480 & 0 & 3,115 & 2,987 & 3,860 \\
\hline 9/5/2016 & $21: 49: 28$ & 239.53 & 23.49 & 109.0 & 1,578 & 1 & 3,107 & 3,006 & 3,852 \\
\hline $9 / 6 / 2016$ & $23: 27: 11$ & 238.66 & 23.79 & 108.4 & 1,730 & 2 & 3,103 & 3,002 & 3,846 \\
\hline $9 / 7 / 2016$ & $21: 16: 09$ & 240.56 & 30.37 & 96.7 & 1,806 & 2 & 3,107 & 2,985 & 3,928 \\
\hline $9 / 8 / 2016$ & $20: 15: 00$ & 237.35 & 26.87 & 105.0 & 1,545 & 1 & 3,109 & 2,995 & 3,854 \\
\hline 9/9/2016 & $19: 20: 49$ & 236.82 & 28.54 & 99.1 & 1,546 & 1 & 3,104 & 2,998 & 3,848 \\
\hline $9 / 10 / 2016$ & $20: 51: 06$ & 237.19 & 27.32 & 88.6 & 1,642 & 1 & 3,098 & 2,980 & 3,841 \\
\hline $9 / 11 / 2016$ & $22: 41: 49$ & 238.86 & 25.19 & 89.9 & 1,747 & 1 & 3,079 & 2,972 & 3,819 \\
\hline $9 / 12 / 2016$ & $23: 56: 32$ & 243.1 & 29.86 & 69.8 & 2,132 & 1 & 3,113 & 3,106 & 3,858 \\
\hline $9 / 13 / 2016$ & $13: 36: 00$ & 236.7 & 28.21 & 74.8 & 1,151 & 3 & 3,085 & 3,001 & 3,903 \\
\hline $9 / 14 / 2016$ & $23: 49: 22$ & 237.53 & 27.13 & 73.8 & 1,990 & 1 & 3,075 & 2,963 & 3,815 \\
\hline $9 / 15 / 2016$ & $21: 18: 15$ & 236.44 & 33.62 & 80.5 & 1,972 & 0 & 3,093 & 2,907 & 3,835 \\
\hline $9 / 16 / 2016$ & $14: 45: 06$ & 235.86 & 30.51 & 67.6 & 1,267 & 2 & 3,107 & 3,090 & 3,928 \\
\hline $9 / 17 / 2016$ & 1:11:45 & 213.9 & 14.89 & 127.2 & 471 & 0 & 3,134 & 3,129 & 3,882 \\
\hline $9 / 18 / 2016$ & $12: 31: 48$ & 216.54 & 12.74 & 102.1 & 719 & 0 & 3,130 & 3,124 & 3,877 \\
\hline $9 / 19 / 2016$ & $24: 00: 00$ & 218.64 & 10.6 & 77.2 & 1,233 & 0 & 3,106 & 3,083 & 3,851 \\
\hline $9 / 20 / 2016$ & $23: 02: 52$ & 242.5 & 23.53 & 44.3 & 1,866 & 0 & 3,103 & 3,009 & 3,846 \\
\hline $9 / 21 / 2016$ & $20: 57: 52$ & 262.55 & 23.77 & 71.0 & 1,650 & 0 & 3,100 & 2,997 & 3,843 \\
\hline $9 / 22 / 2016$ & $22: 02: 58$ & 259.85 & 22.56 & 82.1 & 1,648 & 1 & 3,092 & 2,974 & 3,834 \\
\hline $9 / 23 / 2016$ & $22: 39: 58$ & 245.9 & 25.97 & 72.7 & 1,821 & 2 & 3,097 & 2,977 & 3,840 \\
\hline $9 / 24 / 2016$ & $21: 58: 43$ & 265.35 & 19.75 & 67.5 & 1,563 & 1 & 3,089 & 2,972 & 3,831 \\
\hline $9 / 25 / 2016$ & $22: 13: 41$ & 247.75 & 22.13 & 50.8 & 1,697 & 2 & 3,087 & 2,970 & 3,905 \\
\hline $9 / 26 / 2016$ & $22: 24: 43$ & 248.36 & 21.46 & 49.7 & 1,660 & 3 & 3,085 & 2,956 & 3,902 \\
\hline $9 / 27 / 2016$ & $20: 58: 39$ & 252.15 & 24.69 & 43.5 & 1,721 & 3 & 3,071 & 2,953 & 3,887 \\
\hline $9 / 28 / 2016$ & $22: 12: 13$ & 239.29 & 25.72 & 27.5 & 1,877 & 3 & 3,106 & 2,287 & 3,927 \\
\hline $9 / 29 / 2016$ & $12: 22: 38$ & 252.52 & 28.19 & 22.5 & 1,105 & 2 & 3,148 & 2,038 & 3,973 \\
\hline $9 / 30 / 2016$ & $0: 00: 00$ & -0.73 & 0 & 77.7 & 0 & 0 & 3,160 & 3,161 & 3,912 \\
\hline $10 / 1 / 2016$ & 11:19:04 & 207.87 & 1.99 & -8.9 & 243 & 0 & 3,177 & 3,178 & 3,932 \\
\hline $10 / 2 / 2016$ & $0: 57: 26$ & 200.41 & 1.1 & -14.0 & 17 & 0 & 3,189 & 3,189 & 3,945 \\
\hline $10 / 3 / 2016$ & 0:02:04 & 238.01 & 1.17 & 59.7 & 1 & 0 & 3,160 & 3,156 & 3,913 \\
\hline $10 / 4 / 2016$ & $20: 08: 32$ & 269.89 & 10.26 & 84.8 & 1,034 & 1 & 3,104 & 3,069 & 3,848 \\
\hline $10 / 5 / 2016$ & $23: 24: 35$ & 659.82 & 20.97 & 84.4 & 2,514 & 0 & 3,108 & 3,076 & 3,853 \\
\hline $10 / 6 / 2016$ & 1:31:11 & 514.11 & 14.68 & 95.7 & 180 & 0 & & & \#N/A \\
\hline $10 / 7 / 2016$ & $21: 13: 22$ & 228.86 & 25.11 & 102.8 & 1,637 & 0 & 3,107 & 3,081 & 3,852 \\
\hline $10 / 8 / 2016$ & $23: 50: 09$ & 227.04 & 27.95 & 101.0 & 1,941 & 0 & 3,073 & 3,041 & 3,812 \\
\hline $10 / 9 / 2016$ & $24: 00: 00$ & 226.09 & 38.01 & 83.8 & 2,349 & 0 & 3,051 & 2,939 & 3,787 \\
\hline $10 / 10 / 2016$ & $23: 59: 13$ & 227.14 & 42.89 & 78.5 & 2,509 & 0 & 3,001 & 2,819 & 3,728 \\
\hline $10 / 11 / 2016$ & $24: 00: 00$ & 239.23 & 68.63 & 54.7 & 3,336 & 1 & 2,972 & 2,781 & 3,695 \\
\hline $10 / 12 / 2016$ & $24: 00: 00$ & 248.31 & 73.45 & 47.3 & 3,541 & 5 & 2,960 & 2,782 & 3,833 \\
\hline $10 / 13 / 2016$ & $24: 00: 00$ & 253.31 & 63.26 & 53.2 & 3,291 & 2 & 2,933 & 2,748 & 3,650 \\
\hline $10 / 14 / 2016$ & $24: 00: 00$ & 252.2 & 75.38 & 42.1 & 3,638 & 4 & 2,913 & 2,731 & 3,705 \\
\hline $10 / 15 / 2016$ & $24: 00: 00$ & 249.5 & 78.85 & 43.3 & 3,696 & 3 & 2,913 & 2,749 & 3,705 \\
\hline $10 / 16 / 2016$ & $24: 00: 00$ & 255.96 & 62.64 & 56.6 & 3,273 & 1 & 2,984 & 2,984 & 3,708 \\
\hline $10 / 17 / 2016$ & $17: 16: 36$ & 249.34 & 46.38 & 22.3 & 1,898 & 4 & 2,943 & 2,871 & 3,740 \\
\hline $10 / 18 / 2016$ & $22: 58: 28$ & 262.13 & 48.58 & 29.7 & 2,802 & 2 & 2,930 & 2,814 & 3,725 \\
\hline $10 / 19 / 2016$ & $24: 00: 00$ & 264.33 & 48.18 & 39.1 & 2,914 & 1 & 3,002 & 2,946 & 3,730 \\
\hline $10 / 20 / 2016$ & $10: 37: 08$ & 296.94 & 34.38 & 79.1 & 1,055 & 0 & 2,986 & 2,928 & 3,712 \\
\hline $10 / 21 / 2016$ & $20: 47: 21$ & 275.38 & 28.45 & 37.9 & 1,854 & 1 & 3,010 & 2,976 & 3,739 \\
\hline $10 / 22 / 2016$ & $20: 02: 24$ & 274.02 & 17.96 & 35.0 & 1,280 & 1 & 3,018 & 3,016 & 3,749 \\
\hline $10 / 23 / 2016$ & $21: 36: 32$ & 254.5 & 13.09 & -105.5 & 1,449 & 0 & 3,031 & 3,027 & 3,764 \\
\hline $10 / 24 / 2016$ & $22: 19: 15$ & 342.18 & 6.23 & -54.9 & 1,256 & 0 & 3,014 & 2,968 & 3,744 \\
\hline $10 / 25 / 2016$ & $15: 18: 51$ & 299.36 & 70.07 & 45.4 & 2,108 & 1 & 3,006 & 2,968 & 3,735 \\
\hline $10 / 26 / 2016$ & $18: 03: 02$ & 251.23 & 23.99 & 22.2 & 1,529 & 1 & 2,974 & 2,940 & 3,697 \\
\hline $10 / 27 / 2016$ & $23: 58: 43$ & 265.42 & 24.44 & 35.6 & 2,099 & 1 & 2,982 & 2,891 & 3,706 \\
\hline $10 / 28 / 2016$ & $23: 51: 28$ & 265.22 & 11.97 & 53.9 & 1,397 & 2 & 2,976 & 2,897 & 3,699 \\
\hline $10 / 29 / 2016$ & $21: 32: 45$ & 264.74 & 29.55 & 74.9 & 1,983 & 1 & 2,923 & 2,772 & 3,637 \\
\hline
\end{tabular}




\begin{tabular}{|c|c|c|c|c|c|c|c|c|c|}
\hline $10 / 30 / 2016$ & $24: 00: 00$ & 689.24 & 25.74 & 58.6 & 3,391 & 0 & 2,902 & 2,752 & 3,613 \\
\hline $10 / 31 / 2016$ & $23: 44: 47$ & 719.88 & 22.44 & 53.5 & 3,319 & 2 & 2,937 & 2,846 & 3,733 \\
\hline $11 / 1 / 2016$ & $23: 45: 28$ & 266.71 & 26.4 & 65.6 & 2,044 & 1 & 3,006 & 464 & 3,734 \\
\hline $11 / 2 / 2016$ & $10: 50: 38$ & 479.48 & 11.87 & 70.1 & 878 & 0 & 3,056 & 3,057 & 3,793 \\
\hline $11 / 3 / 2016$ & $0: 00: 04$ & 235 & 1.16 & 102.1 & 0 & 0 & 3,065 & 3,011 & 3,803 \\
\hline $11 / 4 / 2016$ & $7: 44: 52$ & 246.95 & 16.32 & 80.7 & 459 & 0 & 3,032 & 2,909 & 3,764 \\
\hline $11 / 5 / 2016$ & $\begin{array}{l}19: 13: 58 \\
\end{array}$ & 243.72 & 27.69 & 79.9 & 1,608 & 0 & 3,072 & 2,530 & 3,811 \\
\hline $11 / 6 / 2016$ & $7: 23: 13$ & 242.44 & 17.21 & 42.2 & 511 & 0 & 3,097 & 3,003 & 3,840 \\
\hline $11 / 7 / 2016$ & 0:00:08 & 47.72 & 1.16 & 57.1 & 0 & 0 & 3,110 & 2,593 & 3,854 \\
\hline $11 / 8 / 2016$ & 1:03:39 & 252.98 & 18.98 & 52.7 & 74 & 0 & 3,122 & 1,422 & 3,869 \\
\hline $11 / 9 / 2016$ & 0:00:09 & 254.98 & 1.1 & 57.9 & 0 & 0 & 3,132 & 3,134 & 3,880 \\
\hline $11 / 10 / 2016$ & $0: 16: 58$ & 247.74 & 31.44 & 45.7 & 26 & 0 & 3,081 & 3,054 & 3,821 \\
\hline $11 / 11 / 2016$ & $15: 57: 30$ & 241.98 & 31.22 & 45.5 & 1,514 & 1 & 3,030 & 3,006 & 3,762 \\
\hline $11 / 12 / 2016$ & $23: 49: 43$ & 243.01 & 31.52 & 23.1 & 2,336 & 1 & 3,051 & 3,048 & 3,787 \\
\hline $11 / 13 / 2016$ & $23: 47: 56$ & 235.06 & 15.79 & -91.3 & 1,890 & 1 & 3,071 & 3,071 & 3,810 \\
\hline $11 / 14 / 2016$ & $21: 57: 39$ & 233.49 & 4.56 & -200.0 & 1,405 & 1 & 3,111 & 3,113 & 3,856 \\
\hline $11 / 15 / 2016$ & 0:00:04 & 246.04 & 1.36 & 36.4 & 0 & 0 & 3,128 & 3,044 & 3,875 \\
\hline $11 / 16 / 2016$ & 0:00:02 & 244.39 & 1.14 & 49.4 & 0 & 0 & 3,080 & 3,024 & 3,820 \\
\hline $11 / 17 / 2016$ & $22: 18: 58$ & 243.43 & 22.06 & 77.3 & 1,697 & 0 & 3,063 & 3,039 & 3,801 \\
\hline $11 / 18 / 2016$ & $22: 10: 21$ & 250.78 & 23.13 & 80.8 & 1,754 & 1 & 3,039 & 3,014 & 3,773 \\
\hline $11 / 19 / 2016$ & $24: 00: 00$ & 260.19 & 28.95 & 62.2 & 2,243 & 0 & 3,081 & 2,927 & 3,822 \\
\hline $11 / 20 / 2016$ & $13: 25: 09$ & 243.18 & 21.1 & 81.3 & 1,002 & 0 & 3,113 & 2,969 & 3,859 \\
\hline $11 / 21 / 2016$ & $0: 00: 26$ & 229.14 & 2.22 & 72.6 & 0 & 0 & 3,046 & 2,963 & 3,782 \\
\hline $11 / 22 / 2016$ & $16: 08: 00$ & 245.19 & 41.24 & 21.5 & 1,823 & 1 & 3,006 & 2,839 & 3,735 \\
\hline $11 / 23 / 2016$ & $23: 44: 00$ & 248.42 & 40.29 & 27.7 & 2,648 & 1 & 2,973 & 2,535 & 3,697 \\
\hline $11 / 24 / 2016$ & 21:16:41 & 255.45 & 34.19 & 24.7 & 2,126 & 2 & 3,074 & 871 & 3,813 \\
\hline $11 / 25 / 2016$ & $23: 09: 54$ & 230.17 & 3.59 & 48.1 & 580 & 1 & 3,027 & 2,998 & 3,759 \\
\hline $11 / 26 / 2016$ & $24: 00: 00$ & 249.62 & 26 & 51.7 & 2,094 & 1 & 3,049 & 2,987 & 3,785 \\
\hline $11 / 27 / 2016$ & $24: 00: 00$ & 237.87 & 11.48 & 99.5 & 1,278 & 1 & 2,991 & 2,894 & 3,717 \\
\hline $11 / 28 / 2016$ & $23: 41: 43$ & 249.74 & 24.35 & 33.6 & 2,019 & 1 & 3,077 & 492 & 3,817 \\
\hline $11 / 29 / 2016$ & 1:12:06 & 228.49 & 8.62 & 14.6 & 53 & 0 & 3,104 & 3,106 & 3,848 \\
\hline $11 / 30 / 2016$ & $0: 00: 17$ & 239.56 & 1.13 & 69.9 & 0 & 0 & 3,117 & 93 & 3,863 \\
\hline $12 / 1 / 2016$ & 1:04:39 & 7.48 & 1.54 & 61.0 & 6 & 0 & 3,127 & 90 & 3,874 \\
\hline $12 / 2 / 2016$ & $0: 00: 11$ & -1.35 & 1.01 & 40.3 & 0 & 0 & 3,136 & 87 & 3,885 \\
\hline $12 / 3 / 2016$ & $0: 00: 04$ & -1.25 & 1 & 38.1 & 0 & 0 & 3,144 & 83 & 3,894 \\
\hline $12 / 4 / 2016$ & $0: 00: 00$ & -1.1 & 1 & 37.2 & 0 & 0 & 3,152 & 81 & 3,903 \\
\hline $12 / 5 / 2016$ & $0: 00: 11$ & -1.09 & 1.01 & 42.5 & 0 & 0 & 3,157 & 78 & 3,909 \\
\hline $12 / 6 / 2016$ & $0: 04: 26$ & -1.13 & 1.07 & 53.4 & 0 & 0 & 3,056 & 2,992 & 3,793 \\
\hline $12 / 7 / 2016$ & $13: 54: 28$ & 239.01 & 93.77 & 36.2 & 2,297 & 1 & 2,952 & 2,831 & 3,672 \\
\hline $12 / 8 / 2016$ & $23: 57: 47$ & 240.69 & 113.3 & 40.5 & 4,328 & 2 & 2,890 & 2,651 & 3,678 \\
\hline $12 / 9 / 2016$ & $24: 00: 00$ & 241.4 & 132.79 & 43.1 & 4,705 & 15 & 2,809 & 2,497 & 3,732 \\
\hline $12 / 10 / 2016$ & $24: 00: 00$ & 246.5 & 190.78 & 45.2 & 5,669 & 26 & 2,760 & 2,450 & 3,747 \\
\hline $12 / 11 / 2016$ & $24: 00: 00$ & 245.45 & 194.01 & 46.2 & 5,697 & 24 & 2,718 & 2,404 & 3,700 \\
\hline $12 / 12 / 2016$ & $24: 00: 00$ & 244.72 & 197.15 & 48.1 & 5,721 & 22 & 2,677 & 2,358 & 3,653 \\
\hline $12 / 13 / 2016$ & $24: 00: 00$ & 250.27 & 197.92 & 49.1 & 5,787 & 22 & 2,630 & 2,293 & 3,601 \\
\hline $12 / 14 / 2016$ & $24: 00: 00$ & 245.92 & 215.11 & 49.0 & 5,979 & 25 & 2,585 & 2,228 & 3,549 \\
\hline $12 / 15 / 2016$ & $24: 00: 00$ & 246.1 & 229.84 & 54.2 & 6,141 & 22 & 2,536 & 2,159 & 3,494 \\
\hline $12 / 16 / 2016$ & $24: 00: 00$ & 245.95 & 243.45 & 54.2 & 6,315 & 21 & 2,527 & 2,252 & 3,483 \\
\hline $12 / 17 / 2016$ & $24: 00: 00$ & 250.63 & 208.73 & 54.3 & 5,889 & 22 & 2,659 & 2,573 & 3,634 \\
\hline $12 / 18 / 2016$ & $23: 55: 22$ & 255.14 & 68.97 & 90.6 & 3,206 & 3 & 2,565 & 2,293 & 3,295 \\
\hline $12 / 19 / 2016$ & $24: 00: 00$ & 254.63 & 137.59 & 59.6 & 4,801 & 13 & 2,767 & 2,724 & 3,684 \\
\hline $12 / 20 / 2016$ & $4: 11: 22$ & 251.94 & 196.71 & 45.5 & 1,010 & 86 & 2,547 & 2,220 & 3,507 \\
\hline $12 / 21 / 2016$ & $24: 00: 00$ & 281.26 & 169.05 & 46.3 & 5,666 & 9 & 2,490 & 2,174 & 3,290 \\
\hline $12 / 22 / 2016$ & $24: 00: 00$ & 290.58 & 173.71 & 58.7 & 5,787 & 18 & 2,446 & 2,101 & 3,317 \\
\hline $12 / 23 / 2016$ & $24: 00: 00$ & 287.25 & 177.13 & 60.3 & 5,800 & 19 & 2,418 & 2,100 & 3,285 \\
\hline $12 / 24 / 2016$ & $24: 00: 00$ & 293.06 & 169.96 & 63.2 & 5,724 & 18 & 2,395 & 2,072 & 3,258 \\
\hline $12 / 25 / 2016$ & $24: 00: 00$ & 298.45 & 155.96 & 64.7 & 5,530 & 18 & 2,374 & 2,054 & 3,234 \\
\hline $12 / 26 / 2016$ & $24: 00: 00$ & 298.98 & 146.17 & 54.8 & 5,419 & 18 & 2,437 & 2,262 & 3,307 \\
\hline $12 / 27 / 2016$ & $24: 00: 00$ & 300.49 & 90.32 & 53.5 & 4,245 & 9 & 2,396 & 2,117 & 3,177 \\
\hline $12 / 28 / 2016$ & $24: 00: 00$ & 299.72 & 100.95 & 36.9 & 4,588 & 15 & 2,358 & 2,068 & 3,216 \\
\hline $12 / 29 / 2016$ & $24: 00: 00$ & 299.72 & 103.05 & 29.7 & 4,680 & 18 & 2,324 & 1,985 & 3,176 \\
\hline $12 / 30 / 2016$ & $24: 00: 08$ & 345.79 & 126.25 & 27.2 & 4,934 & 10 & 2,538 & 2,541 & 3,423 \\
\hline $12 / 31 / 2016$ & $12: 31: 24$ & 357.07 & 128.96 & 26.9 & 3,015 & 3 & 2,392 & 2,114 & 3,083 \\
\hline $1 / 1 / 2017$ & $20: 31: 22$ & 270.83 & 148.08 & 50.1 & 4,451 & 0 & 2,299 & 1,933 & 2,887 \\
\hline $1 / 2 / 2017$ & $24: 00: 00$ & 274.44 & 169.68 & 60.2 & 5,549 & 0 & 2,263 & 1,900 & 2,843 \\
\hline $1 / 3 / 2017$ & $24: 00: 00$ & 274.99 & 173.99 & 63.7 & 5,604 & 0 & 2,236 & 1,858 & 2,810 \\
\hline $1 / 4 / 2017$ & $24: 00: 00$ & 274.39 & 172.22 & 64.7 & 5,562 & 0 & 2,183 & 1,760 & 2,743 \\
\hline $1 / 5 / 2017$ & $24: 00: 00$ & 268.95 & 205.49 & 62.6 & 6,020 & 0 & 2,086 & 1,397 & 2,622 \\
\hline $1 / 6 / 2017$ & $24: 00: 00$ & 288.1 & 264.47 & 68.6 & 7,002 & 0 & 2,069 & 1,392 & 2,600 \\
\hline $1 / 7 / 2017$ & $19: 38: 30$ & 303.54 & 261.27 & 53.6 & 5,941 & 25 & 2,013 & 1,339 & 2,888 \\
\hline $1 / 8 / 2017$ & $24: 00: 00$ & 304.79 & 255.83 & 59.5 & 7,168 & 33 & 1,977 & 1,316 & 2,845 \\
\hline $1 / 9 / 2017$ & $24: 00: 00$ & 301.24 & 247.11 & 59.2 & 7,008 & 33 & 1,951 & 1,319 & 2,813 \\
\hline $1 / 10 / 2017$ & $22: 41: 47$ & 306 & 265.03 & 64.7 & 6,839 & 34 & 1,958 & 1,383 & 2,822 \\
\hline $1 / 11 / 2017$ & $21: 35: 58$ & 300.06 & 216.8 & 35.7 & 5,994 & 33 & 1,988 & 1,650 & 2,858 \\
\hline $1 / 12 / 2017$ & $24: 00: 00$ & 313.54 & 179.41 & 51.2 & 6,023 & 31 & 2,104 & 1,789 & 2,995 \\
\hline $1 / 13 / 2017$ & $19: 47: 54$ & 325.62 & 92.17 & 42.1 & 3,655 & 10 & 1,981 & 1,451 & 2,755 \\
\hline $1 / 14 / 2017$ & $23: 45: 39$ & 320.33 & 172.45 & 62.2 & 5,893 & 30 & 1,958 & 1,508 & 2,822 \\
\hline $1 / 15 / 2017$ & $23: 39: 21$ & 319.66 & 172.04 & 66.9 & 5,732 & 37 & 1,937 & 1,503 & 2,796 \\
\hline $1 / 16 / 2017$ & $24: 00: 00$ & 319.79 & 173.95 & 66.8 & 5,908 & 39 & 1,863 & 1,169 & 2,706 \\
\hline $1 / 17 / 2017$ & $24: 00: 00$ & 298.56 & 232.09 & 69.7 & 6,642 & 52 & 1,867 & 1,289 & 2,710 \\
\hline $1 / 18 / 2017$ & $24: 00: 00$ & 323.41 & 190.67 & 71.1 & 6,231 & 42 & 1,863 & 1,375 & 2,705 \\
\hline $1 / 19 / 2017$ & $0: 00: 00$ & 367.97 & 155.13 & 70.2 & 5,718 & 4 & 2,074 & 1,848 & 2,681 \\
\hline
\end{tabular}




\begin{tabular}{|c|c|c|c|c|c|c|c|c|c|}
\hline $1 / 20 / 2017$ & $0: 00: 00$ & 181.96 & 65.37 & 68.9 & 174 & 5 & 1,922 & 1,533 & 2,484 \\
\hline $1 / 21 / 2017$ & $23: 58: 28$ & 322.86 & 137.87 & 58.2 & 5,398 & 20 & 1,912 & 1,443 & 2,766 \\
\hline $1 / 22 / 2017$ & $24: 00: 00$ & 330.53 & 124.51 & 66.1 & 5,113 & 24 & 1,888 & 1,181 & 2,736 \\
\hline $1 / 23 / 2017$ & 23:03:11 & 330.91 & 141.02 & 68.4 & 5,273 & 32 & 0 & 0 & \#N/A \\
\hline $1 / 24 / 2017$ & $0: 00: 00$ & 245.76 & 0 & 52.4 & 0 & 0 & 0 & 0 & \#N/A \\
\hline $1 / 25 / 2017$ & $0: 00: 00$ & 252.46 & 0 & 41.5 & 0 & 0 & 0 & 0 & \#N/A \\
\hline $1 / 26 / 2017$ & $0: 00: 00$ & 270.04 & 0 & 53.4 & 0 & 0 & 0 & 0 & \#N/A \\
\hline $1 / 27 / 2017$ & $0: 00: 00$ & 256.69 & 0 & 36.0 & 0 & 0 & 0 & 0 & \#N/A \\
\hline $1 / 28 / 2017$ & 0:00:00 & 256.05 & 0 & 29.9 & 0 & 0 & 0 & 0 & \#N/A \\
\hline $1 / 29 / 2017$ & 0:00:00 & 255.89 & 0 & 32.6 & 0 & 0 & 0 & 0 & \#N/A \\
\hline $1 / 30 / 2017$ & $0: 00: 00$ & 255.99 & 0 & 30.3 & 0 & 0 & 0 & 0 & \#N/A \\
\hline $1 / 31 / 2017$ & 0:00:00 & 256.67 & 0 & 29.0 & 0 & 0 & 0 & 0 & \#N/A \\
\hline $2 / 1 / 2017$ & $0: 00: 00$ & 256.67 & 0 & 39.1 & 0 & 0 & 0 & 0 & \#N/A \\
\hline $2 / 2 / 2017$ & $0: 00: 00$ & 256.35 & 0 & 34.6 & 0 & 0 & 0 & 0 & \#N/A \\
\hline $2 / 3 / 2017$ & $0: 00: 00$ & 256.77 & 0 & 30.9 & 0 & 0 & 2,324 & 2,326 & 2,918 \\
\hline $2 / 4 / 2017$ & $20: 21: 39$ & 262.08 & 49.12 & 38.9 & 2,541 & 14 & 2,246 & 2,247 & 3,083 \\
\hline $2 / 5 / 2017$ & $24: 00: 00$ & 263.66 & 68.18 & 38.8 & 3,544 & 25 & 2,119 & 2,120 & 3,013 \\
\hline $2 / 6 / 2017$ & $24: 00: 00$ & 276.19 & 99.35 & 40.6 & 4,352 & 44 & 2,031 & 2,031 & 2,910 \\
\hline $2 / 7 / 2017$ & $24: 00: 00$ & 297.69 & 103.23 & 48.1 & 4,578 & 58 & 1,999 & 1,999 & 2,871 \\
\hline $2 / 8 / 2017$ & $24: 00: 00$ & 308.98 & 93.57 & 51.7 & 4,402 & 59 & 1,807 & 1,806 & 2,635 \\
\hline $2 / 9 / 2017$ & $24: 00: 00$ & 289.77 & 199.01 & 49.4 & 5,938 & 112 & 2,187 & 1,198 & 3,093 \\
\hline $2 / 10 / 2017$ & $12: 51: 52$ & 268.65 & 214.55 & 33.6 & 3,338 & 20 & 2,042 & 2,043 & 2,922 \\
\hline $2 / 11 / 2017$ & 18:43:13 & 287.88 & 81.21 & 52.9 & 3,103 & 5 & 1,970 & 1,971 & 2,547 \\
\hline $2 / 12 / 2017$ & $24: 00: 00$ & 301.17 & 83.74 & 61.4 & 4,097 & 16 & 1,937 & 1,938 & 2,696 \\
\hline $2 / 13 / 2017$ & $23: 51: 17$ & 302.86 & 82.27 & 63.5 & 4,031 & 20 & 1,862 & 1,863 & 2,704 \\
\hline $2 / 14 / 2017$ & $23: 58: 34$ & 307.47 & 123.64 & 59.2 & 4,954 & 45 & 1,791 & 1,791 & 2,614 \\
\hline $2 / 15 / 2017$ & $24: 00: 00$ & 302.91 & 156.37 & 59.5 & 5,606 & 50 & 1,763 & 1,763 & 2,577 \\
\hline $2 / 16 / 2017$ & $23: 55: 00$ & 292.19 & 154.04 & 60.8 & 5,359 & 41 & 1,747 & 1,748 & 2,556 \\
\hline $2 / 17 / 2017$ & $23: 34: 24$ & 279.24 & 168.26 & 60.6 & 5,432 & 34 & 1,706 & 1,706 & 2,501 \\
\hline $2 / 18 / 2017$ & $23: 42: 41$ & 302.95 & 158.84 & 64.0 & 5,554 & 37 & 1,706 & 1,706 & 2,501 \\
\hline $2 / 19 / 2017$ & $24: 00: 00$ & 320.63 & 123.53 & 68.8 & 5,033 & 33 & 1,665 & 1,665 & 2,444 \\
\hline $2 / 20 / 2017$ & $24: 00: 00$ & 306.5 & 154.65 & 65.2 & 5,565 & 39 & 1,651 & 1,651 & 2,424 \\
\hline $2 / 21 / 2017$ & $24: 00: 00$ & 312.46 & 138.58 & 69.4 & 5,255 & 34 & 1,689 & 1,689 & 2,478 \\
\hline $2 / 22 / 2017$ & $24: 00: 00$ & 324.49 & 96.02 & 77.2 & 4,384 & 22 & 1,724 & 1,724 & 2,525 \\
\hline $2 / 23 / 2017$ & $24: 00: 00$ & 328.16 & 74.17 & 83.1 & 3,836 & 12 & 1,745 & 1,745 & 2,433 \\
\hline $2 / 24 / 2017$ & $24: 00: 00$ & 328.05 & 66.69 & 84.9 & 3,625 & 2 & 1,812 & 1,812 & 2,273 \\
\hline $2 / 25 / 2017$ & $21: 55: 38$ & 333.58 & 51.54 & 102.6 & 2,746 & 2 & 1,695 & 1,695 & 2,123 \\
\hline $2 / 26 / 2017$ & $23: 33: 22$ & 294.27 & 149.57 & 58.8 & 5,230 & 2 & 1,629 & 747 & 2,038 \\
\hline $2 / 27 / 2017$ & $15: 11: 30$ & 268 & 178.25 & 61.6 & 3,541 & 2 & 0 & 0 & $\# N / A$ \\
\hline $2 / 28 / 2017$ & 14:03:30 & 253.65 & 94.53 & 64.6 & 5,947 & 3 & 0 & 1,699 & $\# \mathrm{~N} / \mathrm{A}$ \\
\hline $3 / 1 / 2017$ & $23: 59: 30$ & 274.58 & 174.73 & 78.6 & 5,387 & 30 & 0 & 1,670 & $\# \mathrm{~N} / \mathrm{A}$ \\
\hline $3 / 2 / 2017$ & $24: 00: 00$ & 246 & 152.72 & 77.3 & 4,899 & 26 & 0 & 1,666 & $\# \mathrm{~N} / \mathrm{A}$ \\
\hline $3 / 3 / 2017$ & $24: 00: 00$ & 246.44 & 143.13 & 77.4 & 4,742 & 30 & 0 & 0 & $\# \mathrm{~N} / \mathrm{A}$ \\
\hline $3 / 4 / 2017$ & $3: 23: 34$ & 254.61 & 138.67 & 76.9 & 670 & 1 & 0 & 0 & $\# N / A$ \\
\hline $3 / 5 / 2017$ & $0: 00: 00$ & 249.28 & 0 & 58.8 & 0 & 0 & 0 & 0 & $\# N / A$ \\
\hline $3 / 6 / 2017$ & $0: 00: 00$ & 252.08 & 0 & 71.0 & 0 & 0 & 0 & 0 & $\# N / A$ \\
\hline $3 / 7 / 2017$ & $0: 00: 00$ & 253.21 & 0 & 72.4 & 0 & 0 & 2,226 & 1,984 & 2,797 \\
\hline $3 / 8 / 2017$ & $11: 39: 19$ & 292.56 & 87.05 & 95.3 & 1,895 & 56 & 2,225 & 1,953 & 3,136 \\
\hline $3 / 9 / 2017$ & $23: 57: 22$ & 254.31 & 58.63 & 96.2 & 3,039 & 32 & 2,161 & 1,836 & 3,062 \\
\hline $3 / 10 / 2017$ & $24: 00: 00$ & 272.97 & 70.7 & 90.4 & 3,460 & 31 & 1,922 & 1,186 & 2,778 \\
\hline $3 / 11 / 2017$ & $24: 00: 00$ & 282.84 & 167.28 & 65.0 & 5,568 & 58 & 1,809 & 1,063 & 2,637 \\
\hline $3 / 12 / 2017$ & $23: 35: 41$ & 283.2 & 179.2 & 44.0 & 5,798 & 1 & 1,874 & 1,430 & 2,353 \\
\hline $3 / 13 / 2017$ & $22: 35: 49$ & 245.49 & 88.41 & 16.7 & 3,753 & 32 & 1,884 & 1,497 & 2,732 \\
\hline $3 / 14 / 2017$ & $24: 00: 00$ & 250.44 & 106.94 & 85.2 & 4,119 & 23 & 1,876 & 1,477 & 2,721 \\
\hline $3 / 15 / 2017$ & $24: 00: 00$ & 248.27 & 106.62 & 82.2 & 4,108 & 24 & 1,876 & 1,486 & 2,722 \\
\hline $3 / 16 / 2017$ & $24: 00: 00$ & 265.77 & 98.75 & 84.0 & 4,081 & 2 & 1,839 & 1,405 & 2,308 \\
\hline $3 / 17 / 2017$ & $24: 00: 00$ & 271.4 & 114.9 & 81.5 & 4,457 & 1 & 1,820 & 1,379 & 2,284 \\
\hline $3 / 18 / 2017$ & $24: 00: 00$ & 278.54 & 113.71 & 66.5 & 4,559 & 18 & 1,830 & 1,463 & 2,552 \\
\hline $3 / 19 / 2017$ & $23: 47: 04$ & 307.03 & 83.84 & 23.6 & 4,210 & 21 & 1,807 & 1,414 & 2,635 \\
\hline $3 / 20 / 2017$ & $23: 50: 11$ & 304.23 & 92.87 & 25.7 & 4,469 & 20 & 1,785 & 1,354 & 2,607 \\
\hline $3 / 21 / 2017$ & $24: 00: 00$ & 302.96 & 111.55 & 86.2 & 4,616 & 21 & 1,833 & 1,508 & 2,668 \\
\hline $3 / 22 / 2017$ & $22: 55: 49$ & 318.74 & 82.6 & 98.0 & 3,779 & 13 & 1,789 & 1,381 & 2,495 \\
\hline $3 / 23 / 2017$ & $25: 33: 56$ & 253.86 & 129.59 & 102.8 & 4,804 & 18 & 1,848 & 1,545 & 2,577 \\
\hline $3 / 24 / 2017$ & 19:35:04 & 254.57 & 99.81 & 105.8 & 3,206 & 8 & 1,991 & 1,860 & 2,662 \\
\hline $3 / 25 / 2017$ & $20: 33: 52$ & 326.33 & 42.33 & 126.5 & 2,292 & 3 & 2,058 & 1,959 & 2,661 \\
\hline $3 / 26 / 2017$ & 21:08:34 & 331.3 & 28.99 & 132.6 & 1,970 & 2 & 2,053 & 1,896 & 2,580 \\
\hline $3 / 27 / 2017$ & $22: 43: 11$ & 330.21 & 42.49 & 120.1 & 2,567 & 3 & 2,084 & 1,965 & 2,694 \\
\hline $3 / 28 / 2017$ & $22: 01: 15$ & 328.38 & 38.88 & 125.1 & 2,326 & 4 & 2,039 & 1,768 & 2,636 \\
\hline $3 / 29 / 2017$ & $23: 05: 26$ & 312.52 & 59.87 & 38.2 & 3,107 & 3 & 2,198 & 2,198 & 2,840 \\
\hline $3 / 30 / 2017$ & $3: 58: 34$ & 280.11 & 71.75 & 11.3 & 628 & 3 & 2,179 & 2,147 & 2,816 \\
\hline $3 / 31 / 2017$ & $24: 00: 00$ & 298.28 & 26.67 & 129.2 & 2,035 & 1 & 2,141 & 2,021 & 2,691 \\
\hline $4 / 1 / 2017$ & $23: 45: 04$ & 300.88 & 44.68 & 112.9 & 2,609 & 2 & 2,102 & 1,988 & 2,641 \\
\hline $4 / 2 / 2017$ & $19: 59: 52$ & 292.89 & 70.64 & 103.6 & 2,924 & 6 & 2,126 & 2,027 & 2,839 \\
\hline $4 / 3 / 2017$ & $17: 41: 49$ & 272.6 & 75.57 & 104.0 & 2,524 & 68 & 2,226 & 2,214 & 3,138 \\
\hline $4 / 4 / 2017$ & $13: 04: 00$ & 281.92 & 42.28 & 87.3 & 1,356 & 1 & 2,080 & 1,843 & 2,614 \\
\hline $4 / 5 / 2017$ & $22: 19: 30$ & 253.22 & 90.43 & 77.0 & 3,560 & 8 & 2,101 & 1,973 & 2,807 \\
\hline $4 / 6 / 2017$ & $23: 58: 00$ & 293.87 & 51.29 & 101.4 & 2,921 & 8 & 1,993 & 1,726 & 2,665 \\
\hline $4 / 7 / 2017$ & $24: 00: 00$ & 258.19 & 95.49 & 76.7 & 3,959 & 11 & 1,884 & 1,462 & 2,626 \\
\hline $4 / 8 / 2017$ & $23: 55: 08$ & 299.3 & 124.8 & 79.6 & 4,829 & 55 & 1,896 & 1,535 & 2,746 \\
\hline $4 / 9 / 2017$ & $24: 00: 00$ & 300.13 & 82.18 & 92.4 & 3,836 & 9 & 1,985 & 1,848 & 2,654 \\
\hline $4 / 10 / 2017$ & $24: 00: 00$ & 300.39 & 42.63 & 110.5 & 2,691 & 5 & 2,060 & 1,965 & 2,753 \\
\hline $4 / 11 / 2017$ & $23: 18: 30$ & 300.39 & 29.57 & 119.8 & 2,122 & 2 & 2,032 & 1,859 & 2,554 \\
\hline
\end{tabular}




\begin{tabular}{|c|c|c|c|c|c|c|c|c|c|}
\hline $4 / 12 / 2017$ & $23: 59: 41$ & 300.1 & 53.11 & 101.6 & 3,041 & 5 & 1,895 & 1,511 & 2,448 \\
\hline $4 / 13 / 2017$ & $24: 00: 00$ & 295.53 & 109.09 & 85.9 & 4,395 & 57 & 1,817 & 1,466 & 2,647 \\
\hline $4 / 14 / 2017$ & $24: 00: 00$ & 296.67 & 137.03 & 89.8 & 4,949 & 26 & 1,935 & 1,749 & 2,794 \\
\hline $4 / 15 / 2017$ & $23: 46: 30$ & 300.76 & 51.51 & 105.5 & 2,914 & 7 & 2,012 & 1,918 & 2,690 \\
\hline $4 / 16 / 2017$ & $23: 43: 30$ & 300.03 & 29.63 & 118.4 & 2,217 & 2 & 2,052 & 1,945 & 2,578 \\
\hline $4 / 17 / 2017$ & $23: 01: 34$ & 300.16 & 26.36 & 120.4 & 1,995 & 3 & 2,148 & 2,094 & 2,776 \\
\hline $4 / 18 / 2017$ & $12: 19: 26$ & 289.62 & 26.43 & 120.0 & 983 & 1 & 2,241 & 2,243 & 2,816 \\
\hline $4 / 19 / 2017$ & $1: 38: 34$ & 301.95 & 9.79 & 140.3 & 82 & 2 & 2,281 & 2,265 & 2,865 \\
\hline $4 / 20 / 2017$ & $5: 44: 49$ & 272.79 & 18.6 & 119.1 & 291 & 0 & 2,242 & 2,216 & 2,817 \\
\hline $4 / 21 / 2017$ & $23: 18: 26$ & 304.3 & 22.8 & 119.3 & 1,918 & 1 & 2,196 & 2,093 & 2,760 \\
\hline $4 / 22 / 2017$ & $24: 00: 00$ & 294.21 & 35.62 & 105.3 & 2,473 & 3 & 2,139 & 2,015 & 2,765 \\
\hline $4 / 23 / 2017$ & $24: 00: 00$ & 239.45 & 59.07 & 83.1 & 3,009 & 4 & 2,111 & 1,987 & 2,729 \\
\hline $4 / 24 / 2017$ & $24: 00: 00$ & 263.96 & 53.77 & 89.4 & 2,978 & 31 & 2,087 & 1,947 & 2,975 \\
\hline $4 / 25 / 2017$ & $24: 00: 00$ & 241.09 & 59.61 & 86.9 & 3,022 & 4 & 2,166 & 2,090 & 2,799 \\
\hline $4 / 26 / 2017$ & $23: 35: 26$ & 288.65 & 22.11 & 126.5 & 1,712 & 2 & 2,275 & 2,275 & 2,857 \\
\hline $4 / 27 / 2017$ & $3: 07: 22$ & 291.06 & 13.43 & 107.9 & 190 & 0 & 2,322 & 2,321 & 2,916 \\
\hline $4 / 28 / 2017$ & 4:30:56 & 291.48 & 9.71 & 127.1 & 241 & 0 & 2,352 & 2,352 & 2,953 \\
\hline $4 / 29 / 2017$ & $5: 27: 19$ & 300.14 & 7.32 & 135.0 & 251 & 0 & 2,374 & 2,375 & 2,980 \\
\hline $4 / 30 / 2017$ & $7: 28: 56$ & 300.98 & 5.53 & 140.2 & 300 & 0 & 2,396 & 2,389 & 3,007 \\
\hline $5 / 1 / 2017$ & $4: 21: 49$ & 272.8 & 17.84 & 87.7 & 309 & 0 & 2,353 & 2,323 & 2,954 \\
\hline $5 / 2 / 2017$ & $23: 33: 49$ & 285.18 & 17.39 & 113.6 & 1,657 & 1 & 2,282 & 2,167 & 2,867 \\
\hline $5 / 3 / 2017$ & $24: 00: 00$ & 282.31 & 45.17 & 89.1 & 2,781 & 1 & 2,202 & 2,078 & 2,767 \\
\hline $5 / 4 / 2017$ & $24: 00: 00$ & 254.24 & 73.44 & 73.2 & 3,481 & 3 & 2,190 & 2,080 & 2,830 \\
\hline $5 / 5 / 2017$ & $24: 00: 00$ & 285.79 & 50.82 & 87.8 & 2,969 & 4 & 2,302 & 2,282 & 2,970 \\
\hline $5 / 6 / 2017$ & $10: 47: 04$ & 277.93 & 43.36 & 89.9 & 1,161 & 1 & 2,011 & 1,531 & 2,527 \\
\hline $5 / 7 / 2017$ & $24: 00: 00$ & 251.6 & 201.81 & 70.0 & 5,690 & 22 & 2,017 & 1,810 & 2,892 \\
\hline $5 / 8 / 2017$ & $24: 00: 00$ & 254.27 & 104.39 & 89.7 & 3,986 & 7 & 1,981 & 1,722 & 2,650 \\
\hline $5 / 9 / 2017$ & $24: 00: 00$ & 294.47 & 83.16 & 91.3 & 3,878 & 41 & 2,184 & 678 & 3,089 \\
\hline $5 / 10 / 2017$ & $10: 20: 49$ & 284.87 & 42.64 & 98.1 & 1,130 & 4 & 2,176 & 2,115 & 2,811 \\
\hline $5 / 11 / 2017$ & $24: 00: 00$ & 269.28 & 27.06 & 116.1 & 2,043 & 2 & 2,106 & 1,993 & 2,722 \\
\hline $5 / 12 / 2017$ & $24: 00: 00$ & 254.76 & 59.98 & 94.8 & 3,080 & 4 & 2,039 & 1,859 & 2,636 \\
\hline $5 / 13 / 2017$ & $24: 00: 00$ & 253.55 & 77.69 & 87.1 & 3,529 & 5 & 2,052 & 1,896 & 2,744 \\
\hline $5 / 14 / 2017$ & $23: 42: 34$ & 290.76 & 58.97 & 100.1 & 3,066 & 4 & 2,153 & 2,152 & 2,783 \\
\hline $5 / 15 / 2017$ & $\begin{array}{ll}11: 12: 30 \\
\end{array}$ & 296.38 & 61.32 & 51.1 & 1,590 & 47 & 2,012 & 1,744 & 2,887 \\
\hline $5 / 16 / 2017$ & $24: 00: 00$ & 294.75 & 91.53 & 83.5 & 4,033 & 8 & 2,009 & 1,840 & 2,686 \\
\hline $5 / 17 / 2017$ & $24: 00: 00$ & 309.42 & 62.72 & 100.5 & 3,386 & 8 & 1,977 & 1,746 & 2,644 \\
\hline $5 / 18 / 2017$ & $23: 04: 15$ & 307.39 & 76.92 & 96.5 & 3,564 & 12 & 1,969 & 1,725 & 2,739 \\
\hline $5 / 19 / 2017$ & $21: 08: 49$ & 307.94 & 74.91 & 74.6 & 3,341 & 9 & 1,897 & 1,527 & 2,536 \\
\hline $5 / 20 / 2017$ & $20: 34: 34$ & 278.56 & 145.92 & 79.3 & 4,314 & 14 & 1,853 & 1,520 & 2,584 \\
\hline $5 / 21 / 2017$ & $24: 00: 00$ & 290.57 & 119.45 & 82.3 & 4,631 & 27 & 1,809 & 1,439 & 2,637 \\
\hline $5 / 22 / 2017$ & $24: 00: 00$ & 283.78 & 132.22 & 80.6 & 4,843 & 29 & 1,805 & 1,465 & 2,633 \\
\hline $5 / 23 / 2017$ & $24: 00: 00$ & 286.9 & 114.25 & 81.7 & 4,465 & 25 & 1,802 & 1,504 & 2,628 \\
\hline $5 / 24 / 2017$ & $24: 00: 00$ & 300.95 & 101.66 & 83.1 & 4,347 & 20 & 1,791 & 1,468 & 2,614 \\
\hline $5 / 25 / 2017$ & $24: 00: 00$ & 302.02 & 99.77 & 81.9 & 4,322 & 24 & 1,772 & 1,443 & 2,589 \\
\hline $5 / 26 / 2017$ & $24: 00: 00$ & 299.4 & 104.71 & 82.0 & 4,409 & 25 & 1,788 & 1,526 & 2,610 \\
\hline $5 / 27 / 2017$ & $24: 00: 00$ & 308.96 & 85.07 & 84.7 & 4,053 & 20 & 1,806 & 1,601 & 2,633 \\
\hline $5 / 28 / 2017$ & $24: 00: 00$ & 309.9 & 73.68 & 86.4 & 3,765 & 16 & 1,820 & 1,611 & 2,539 \\
\hline $5 / 29 / 2017$ & $24: 00: 00$ & 310.19 & 66.68 & 86.3 & 3,586 & 15 & 1,805 & 1,549 & 2,517 \\
\hline $5 / 30 / 2017$ & $24: 00: 00$ & 309.68 & 74.73 & 84.7 & 3,798 & 16 & 1,799 & 1,574 & 2,509 \\
\hline $5 / 31 / 2017$ & $24: 00: 00$ & 310.64 & 72.46 & 87.0 & 3,745 & 18 & 1,765 & 1,463 & 2,461 \\
\hline $6 / 1 / 2017$ & $24: 00: 00$ & 308.27 & 88.71 & 83.0 & 4,143 & 131 & 1,930 & 213 & 2,788 \\
\hline $6 / 2 / 2017$ & $11: 08: 45$ & 308.28 & 96.6 & 84.0 & 2,016 & 12 & 2,085 & 380 & 2,887 \\
\hline $6 / 3 / 2017$ & $3: 05: 34$ & 216.36 & 55.76 & 55.0 & 292 & 2 & 1,954 & 1,845 & 2,454 \\
\hline $6 / 4 / 2017$ & $24: 00: 00$ & 307.32 & 66.31 & 74.1 & 3,576 & 2 & 1,913 & 1,774 & 2,472 \\
\hline $6 / 5 / 2017$ & $24: 00: 00$ & 309.9 & 60.5 & 80.5 & 3,448 & 4 & 1,869 & 1,676 & 2,414 \\
\hline $6 / 6 / 2017$ & $24: 00: 00$ & 308.55 & 72.67 & 79.8 & 3,745 & 12 & 1,814 & 1,520 & 2,530 \\
\hline $6 / 7 / 2017$ & $24: 00: 00$ & 309.33 & 82.01 & 81.1 & 3,989 & 16 & 1,761 & 1,472 & 2,456 \\
\hline $6 / 8 / 2017$ & $24: 00: 00$ & 308.31 & 92.09 & 82.0 & 4,231 & 24 & 1,771 & 1,525 & 2,588 \\
\hline $6 / 9 / 2017$ & $24: 00: 00$ & 309.74 & 78.15 & 84.9 & 3,893 & 21 & 1,796 & 1,611 & 2,621 \\
\hline $6 / 10 / 2017$ & $24: 00: 00$ & 309.52 & 65.63 & 88.4 & 3,532 & 16 & 1,826 & 1,671 & 2,546 \\
\hline $6 / 11 / 2017$ & $24: 00: 00$ & 309.95 & 53.38 & 92.2 & 3,172 & 8 & 1,819 & 1,637 & 2,431 \\
\hline $6 / 12 / 2017$ & $24: 00: 00$ & 309.79 & 61.91 & 89.0 & 3,424 & 9 & 1,825 & 1,660 & 2,439 \\
\hline $6 / 13 / 2017$ & $24: 00: 00$ & 309.91 & 57.44 & 91.9 & 3,282 & 9 & 1,823 & 1,652 & 2,436 \\
\hline $6 / 14 / 2017$ & $24: 00: 00$ & 309.88 & 58.41 & 89.7 & 3,317 & 11 & 1,820 & 1,638 & 2,538 \\
\hline $6 / 15 / 2017$ & $24: 00: 00$ & 309.79 & 60.07 & 90.1 & 3,354 & 12 & 1,815 & 1,645 & 2,531 \\
\hline $6 / 16 / 2017$ & $24: 00: 00$ & 309.84 & 61.93 & 89.3 & 3,408 & 13 & 1,817 & 1,653 & 2,535 \\
\hline $6 / 17 / 2017$ & $24: 00: 00$ & 309.87 & 56.78 & 90.3 & 3,262 & 12 & 1,833 & 1,684 & 2,557 \\
\hline $6 / 18 / 2017$ & $24: 00: 00$ & 309.97 & 50.6 & 94.1 & 3,056 & 10 & 1,829 & 1,664 & 2,552 \\
\hline $6 / 19 / 2017$ & $24: 00: 00$ & 309.92 & 54.54 & 90.2 & 3,193 & 12 & 1,809 & 1,610 & 2,524 \\
\hline $6 / 20 / 2017$ & $24: 00: 00$ & 309.84 & 61.57 & 86.0 & 3,412 & 12 & 1,804 & 1,620 & 2,517 \\
\hline $6 / 21 / 2017$ & $24: 00: 00$ & 309.78 & 59.41 & 87.9 & 3,345 & 11 & 1,813 & 1,648 & 2,529 \\
\hline $6 / 22 / 2017$ & $24: 00: 00$ & 309.8 & 55.98 & 90.6 & 3,224 & 11 & 1,813 & 1,646 & 2,529 \\
\hline $6 / 23 / 2017$ & $24: 00: 00$ & 309.77 & 56.13 & 90.2 & 3,228 & 11 & 1,810 & 1,645 & 2,524 \\
\hline $6 / 24 / 2017$ & $24: 00: 00$ & 309.53 & 57.27 & 88.1 & 3,265 & 11 & 1,796 & 1,625 & 2,506 \\
\hline $6 / 25 / 2017$ & $24: 00: 00$ & 301.79 & 67.14 & 87.5 & 3,407 & 13 & 1,778 & 1,568 & 2,479 \\
\hline $6 / 26 / 2017$ & $24: 00: 00$ & 309.76 & 63.23 & 84.8 & 3,484 & 12 & 1,758 & 1,511 & 2,452 \\
\hline $6 / 27 / 2017$ & $24: 00: 00$ & 310.04 & 71.08 & 82.8 & 3,717 & 19 & 1,741 & 1,496 & 2,428 \\
\hline $6 / 28 / 2017$ & $24: 00: 00$ & 310.75 & 72.94 & 82.3 & 3,782 & 17 & 1,752 & 1,557 & 2,443 \\
\hline $6 / 29 / 2017$ & $24: 00: 00$ & 310.07 & 63.91 & 85.4 & 3,503 & 13 & 1,771 & 1,607 & 2,469 \\
\hline $6 / 30 / 2017$ & $24: 00: 00$ & 309.66 & 57.93 & 89.4 & 3,291 & 10 & 1,786 & 1,646 & 2,385 \\
\hline $7 / 1 / 2017$ & $24: 00: 00$ & 309.9 & 53.17 & 91.3 & 3,126 & 11 & 1,788 & 1,644 & 2,494 \\
\hline $7 / 2 / 2017$ & $24: 00: 00$ & 310.03 & 52.07 & 88.7 & 3,125 & 11 & 1,784 & 1,591 & 2,489 \\
\hline
\end{tabular}




\begin{tabular}{|c|c|c|c|c|c|c|c|c|c|}
\hline $7 / 3 / 2017$ & $24: 00: 00$ & 308.54 & 58.93 & 87.1 & 3,283 & 13 & 1,785 & 1,635 & 2,490 \\
\hline $7 / 4 / 2017$ & $24: 00: 00$ & 309.9 & 53.33 & 90.2 & 3,137 & 12 & 1,793 & 1,639 & 2,500 \\
\hline $7 / 5 / 2017$ & $24: 00: 00$ & 309.75 & 51.59 & 90.8 & 3,077 & 11 & 1,786 & 1,628 & 2,492 \\
\hline $7 / 6 / 2017$ & $24: 00: 00$ & 309.65 & 55.53 & 87.6 & 3,214 & 10 & 1,771 & 1,597 & 2,470 \\
\hline $7 / 7 / 2017$ & $24: 00: 00$ & 309.78 & 60.12 & 85.0 & 3,365 & 15 & 1,762 & 1,578 & 2,457 \\
\hline $7 / 8 / 2017$ & $24: 00: 00$ & 309.71 & 60.29 & 84.3 & 3,376 & 16 & 1,753 & 1,572 & 2,444 \\
\hline $7 / 9 / 2017$ & $24: 00: 00$ & 309.98 & 60.73 & 83.6 & 3,403 & 16 & 1,751 & 1,580 & 2,442 \\
\hline $7 / 10 / 2017$ & $24: 00: 00$ & 309.64 & 58.73 & 84.3 & 3,347 & 16 & 1,744 & 1,539 & 2,431 \\
\hline $7 / 11 / 2017$ & $24: 00: 00$ & 309.51 & 63.98 & 82.6 & 3,495 & 16 & 1,872 & 1,865 & 2,610 \\
\hline $7 / 12 / 2017$ & $24: 00: 00$ & 302.4 & 34.67 & 122.4 & 1,850 & 9 & 1,992 & 1,992 & 2,664 \\
\hline $7 / 13 / 2017$ & $24: 00: 00$ & 307.27 & 2.39 & 127.9 & 607 & 1 & 1,909 & 1,843 & 2,398 \\
\hline $7 / 14 / 2017$ & $24: 00: 00$ & 310.17 & 47.75 & 80.3 & 2,926 & 1 & 1,870 & 1,779 & 2,348 \\
\hline $7 / 15 / 2017$ & $24: 00: 00$ & 309.8 & 50.45 & 77.2 & 3,150 & 6 & 1,810 & 1,559 & 2,418 \\
\hline $7 / 16 / 2017$ & $24: 00: 00$ & 295.36 & 78.29 & 74.5 & 3,688 & 9 & 1,788 & 1,639 & 2,389 \\
\hline $7 / 17 / 2017$ & $24: 00: 00$ & 297.62 & 74.33 & 82.0 & 3,501 & 9 & 1,778 & 1,598 & 2,374 \\
\hline $7 / 18 / 2017$ & $24: 00: 00$ & 309.53 & 61.45 & 83.5 & 3,355 & 9 & 1,784 & 1,661 & 2,382 \\
\hline $7 / 19 / 2017$ & $24: 00: 00$ & 309.9 & 53.97 & 86.3 & 3,154 & 9 & 1,786 & 1,665 & 2,386 \\
\hline $7 / 20 / 2017$ & $24: 00: 00$ & 310.3 & 48.71 & 85.8 & 3,078 & 9 & 1,782 & 1,648 & 2,380 \\
\hline $7 / 21 / 2017$ & $24: 00: 00$ & 309.37 & 50.35 & 84.1 & 3,131 & 9 & 1,777 & 1,651 & 2,373 \\
\hline $7 / 22 / 2017$ & $24: 00: 00$ & 309.71 & 51.14 & 82.9 & 3,161 & 10 & 1,822 & 1,631 & 2,541 \\
\hline $7 / 23 / 2017$ & $24: 00: 00$ & 310.06 & 51.69 & 80.9 & 3,187 & 12 & 2,136 & 1,512 & 2,951 \\
\hline $7 / 24 / 2017$ & $24: 00: 00$ & 309.17 & 64.72 & 78.9 & 3,538 & 17 & 1,744 & 1,288 & 2,431 \\
\hline $7 / 25 / 2017$ & $24: 00: 00$ & 306.78 & 95.33 & 77.1 & 4,301 & 27 & 1,716 & 1,533 & 2,515 \\
\hline $7 / 26 / 2017$ & $24: 00: 00$ & 309.6 & 62.44 & 80.8 & 3,450 & 14 & 1,688 & 1,424 & 2,351 \\
\hline $7 / 27 / 2017$ & $24: 00: 00$ & 309.48 & 68.27 & 80.5 & 3,605 & 14 & 1,704 & 1,508 & 2,374 \\
\hline $7 / 28 / 2017$ & $24: 00: 00$ & 309.6 & 64.21 & 80.7 & 3,498 & 15 & 1,723 & 1,569 & 2,401 \\
\hline $7 / 29 / 2017$ & $24: 00: 00$ & 309.9 & 57.42 & 81.0 & 3,287 & 13 & 1,722 & 1,570 & 2,400 \\
\hline $7 / 30 / 2017$ & $24: 00: 00$ & 309.75 & 54.8 & 79.5 & 3,231 & 10 & 1,738 & 1,584 & 2,423 \\
\hline $7 / 31 / 2017$ & $24: 00: 00$ & 309.94 & 49.96 & 83.2 & 3,069 & 9 & 1,737 & 1,572 & 2,317 \\
\hline $8 / 1 / 2017$ & $24: 00: 00$ & 308.38 & 56.25 & 81.7 & 3,213 & 11 & 1,730 & 1,583 & 2,412 \\
\hline $8 / 2 / 2017$ & $24: 00: 00$ & 308.21 & 59.13 & 83.0 & 3,264 & 11 & 1,741 & 1,606 & 2,427 \\
\hline $8 / 3 / 2017$ & $24: 00: 00$ & 310.16 & 48.59 & 82.9 & 3,012 & 8 & 1,729 & 1,583 & 2,307 \\
\hline $8 / 4 / 2017$ & $24: 00: 00$ & 309.87 & 53.34 & 80.3 & 3,185 & 10 & 1,731 & 1,590 & 2,414 \\
\hline $8 / 5 / 2017$ & $24: 00: 00$ & 310.06 & 52.01 & 79.8 & 3,132 & 10 & 1,726 & 1,582 & 2,302 \\
\hline $8 / 6 / 2017$ & $24: 00: 00$ & 309.86 & 52.93 & 78.6 & 3,175 & 10 & 1,727 & 1,567 & 2,407 \\
\hline $8 / 7 / 2017$ & $24: 00: 00$ & 310.1 & 51.36 & 79.4 & 3,126 & 11 & 1,724 & 1,574 & 2,403 \\
\hline $8 / 8 / 2017$ & $24: 00: 00$ & 309.9 & 53.1 & 78.7 & 3,189 & 11 & 1,713 & 1,536 & 2,387 \\
\hline $8 / 9 / 2017$ & $24: 00: 00$ & 309.94 & 53.9 & 77.4 & 3,241 & 11 & 1,697 & 1,521 & 2,364 \\
\hline $8 / 10 / 2017$ & $24: 00: 00$ & 310.13 & 57.28 & 77.6 & 3,363 & 14 & 1,604 & 1,232 & 2,229 \\
\hline $8 / 11 / 2017$ & $24: 00: 00$ & 272.21 & 120.96 & 74.4 & 4,441 & 29 & 1,677 & 1,516 & 2,461 \\
\hline $8 / 12 / 2017$ & $24: 00: 00$ & 309.93 & 55.71 & 80.1 & 3,280 & 10 & 1,677 & 1,469 & 2,234 \\
\hline $8 / 13 / 2017$ & $24: 00: 00$ & 301.83 & 67.24 & 78.0 & 3,456 & 14 & 1,564 & 1,141 & 2,168 \\
\hline $8 / 14 / 2017$ & $24: 00: 00$ & 254.01 & 150.95 & 73.9 & 4,938 & 28 & 1,531 & 1,119 & 2,247 \\
\hline $8 / 15 / 2017$ & $24: 00: 00$ & 255.02 & 150.36 & 75.9 & 4,914 & 23 & 1,558 & 1,379 & 2,288 \\
\hline $8 / 16 / 2017$ & $24: 00: 00$ & 271.29 & 120.9 & 79.4 & 4,349 & 12 & 1,612 & 1,446 & 2,240 \\
\hline $8 / 17 / 2017$ & $24: 00: 00$ & 310.21 & 59.88 & 79.4 & 3,421 & 8 & 1,646 & 1,525 & 2,191 \\
\hline $8 / 18 / 2017$ & $24: 00: 00$ & 309.88 & 51.73 & 80.0 & 3,144 & 7 & 1,656 & 1,540 & 2,206 \\
\hline $8 / 19 / 2017$ & $24: 00: 00$ & 309.84 & 51.43 & 77.7 & 3,144 & 7 & 1,664 & 1,543 & 2,216 \\
\hline $8 / 20 / 2017$ & $24: 00: 00$ & 310.05 & 50.09 & 77.2 & 3,109 & 7 & 1,666 & 1,528 & 2,219 \\
\hline $8 / 21 / 2017$ & $24: 00: 00$ & 309.93 & 51.64 & 76.8 & 3,162 & 7 & 1,661 & 1,515 & 2,213 \\
\hline $8 / 22 / 2017$ & $24: 00: 00$ & 309.91 & 54.35 & 76.6 & 3,240 & 8 & 1,659 & 1,512 & 2,209 \\
\hline $8 / 23 / 2017$ & $24: 00: 00$ & 310.3 & 53.11 & 75.9 & 3,214 & 8 & 1,639 & 1,458 & 2,181 \\
\hline $8 / 24 / 2017$ & $24: 00: 00$ & 310.11 & 59.34 & 73.0 & 3,431 & 11 & 1,627 & 1,432 & 2,261 \\
\hline $8 / 25 / 2017$ & $24: 00: 00$ & 310.44 & 61.13 & 73.3 & 3,487 & 11 & 1,621 & 1,452 & 2,254 \\
\hline $8 / 26 / 2017$ & $24: 00: 00$ & 310.33 & 59.51 & 73.8 & 3,449 & 12 & 1,625 & 1,455 & 2,259 \\
\hline $8 / 27 / 2017$ & $24: 00: 00$ & 309.96 & 55.67 & 75.1 & 3,327 & 10 & 1,624 & 1,451 & 2,257 \\
\hline $8 / 28 / 2017$ & $24: 00: 00$ & 310.27 & 57.12 & 74.6 & 3,372 & 12 & 1,608 & 1,403 & 2,233 \\
\hline $8 / 29 / 2017$ & $24: 00: 00$ & 309.46 & 64.77 & 73.8 & 3,576 & 15 & 1,595 & 1,395 & 2,214 \\
\hline $8 / 30 / 2017$ & $24: 00: 00$ & 309.68 & 65.02 & 73.4 & 3,598 & 14 & 1,603 & 1,434 & 2,226 \\
\hline $8 / 31 / 2017$ & $24: 00: 00$ & 309.97 & 58.49 & 75.5 & 3,405 & 13 & 1,592 & 1,376 & 2,211 \\
\hline $9 / 1 / 2017$ & $24: 00: 00$ & 309.57 & 63.45 & 74.0 & 3,547 & 15 & 1,555 & 1,296 & 2,155 \\
\hline $9 / 2 / 2017$ & $24: 00: 00$ & 308.2 & 83.35 & 72.3 & 4,058 & 19 & 1,582 & 1,442 & 2,196 \\
\hline $9 / 3 / 2017$ & $24: 00: 00$ & 309.76 & 62.17 & 74.1 & 3,492 & 13 & 1,591 & 1,406 & 2,209 \\
\hline $9 / 4 / 2017$ & $24: 00: 00$ & 310.08 & 55.14 & 73.2 & 3,316 & 10 & 1,601 & 1,427 & 2,223 \\
\hline $9 / 5 / 2017$ & $24: 00: 00$ & 310.4 & 54.85 & 75.3 & 3,287 & 10 & 1,586 & 1,399 & 2,107 \\
\hline $9 / 6 / 2017$ & $24: 00: 00$ & 310.09 & 60.82 & 72.7 & 3,475 & 12 & 1,556 & 1,296 & 2,156 \\
\hline $9 / 7 / 2017$ & $24: 00: 00$ & 308.61 & 72.62 & 71.8 & 3,791 & 20 & 1,533 & 1,282 & 2,122 \\
\hline $9 / 8 / 2017$ & $24: 00: 00$ & 309.03 & 80.78 & 72.8 & 3,980 & 17 & 1,486 & 1,077 & 2,051 \\
\hline $9 / 9 / 2017$ & $24: 00: 00$ & 306.26 & 122.02 & 78.3 & 4,771 & 26 & 1,411 & 773 & 2,053 \\
\hline $9 / 10 / 2017$ & $24: 00: 00$ & 295.16 & 153.99 & 82.7 & 5,279 & 29 & 1,468 & 1,129 & 2,146 \\
\hline $9 / 11 / 2017$ & $24: 00: 00$ & 310 & 81.42 & 79.3 & 3,982 & 12 & 1,480 & 1,199 & 2,042 \\
\hline $9 / 12 / 2017$ & $24: 00: 00$ & 310.18 & 80.25 & 80.1 & 3,941 & 14 & 1,491 & 1,222 & 2,060 \\
\hline $9 / 13 / 2017$ & $24: 00: 00$ & 309.93 & 75.71 & 81.2 & 3,802 & 15 & 1,497 & 1,309 & 2,068 \\
\hline $9 / 14 / 2017$ & $24: 00: 00$ & 309.91 & 69.27 & 80.9 & 3,660 & 11 & 1,487 & 1,271 & 2,054 \\
\hline $9 / 15 / 2017$ & $24: 00: 00$ & 310.26 & 74.27 & 82.9 & 3,804 & 14 & 1,483 & 1,272 & 2,048 \\
\hline $9 / 16 / 2017$ & $24: 00: 00$ & 310.02 & 75.54 & 84.5 & 3,807 & 14 & 1,496 & 1,318 & 2,067 \\
\hline $9 / 17 / 2017$ & $24: 00: 00$ & 310.2 & 63.49 & 85.2 & 3,517 & 11 & 1,471 & 1,225 & 2,029 \\
\hline $9 / 18 / 2017$ & $24: 00: 00$ & 310.67 & 71.54 & 83.4 & 3,756 & 14 & 1,446 & 1,072 & 1,992 \\
\hline $9 / 19 / 2017$ & $24: 00: 00$ & 307.39 & 83.41 & 85.0 & 4,006 & 21 & 1,460 & 1,262 & 2,133 \\
\hline $9 / 20 / 2017$ & $24: 00: 00$ & 310.77 & 71.7 & 86.8 & 3,720 & 12 & 1,476 & 1,237 & 2,037 \\
\hline $9 / 21 / 2017$ & $24: 00: 00$ & 310.06 & 70.53 & 85.5 & 3,655 & 13 & 1,382 & 988 & 1,895 \\
\hline $9 / 22 / 2017$ & $24: 00: 00$ & 308.85 & 126.25 & 87.7 & 4,912 & 27 & 1,398 & 1,112 & 2,031 \\
\hline
\end{tabular}




\begin{tabular}{|c|c|c|c|c|c|c|c|c|c|}
\hline 9/23/2017 & $24: 00: 00$ & 309.59 & 97.3 & 86.1 & 4,334 & 18 & 1,459 & 1,313 & 2,011 \\
\hline $9 / 24 / 2017$ & $24: 00: 00$ & 310.96 & 57.03 & 86.9 & 3,326 & 8 & 1,478 & 1,301 & 1,955 \\
\hline $9 / 25 / 2017$ & $24: 00: 00$ & 310.27 & 56.6 & 85.8 & 3,316 & 8 & 1,477 & 1,292 & 1,955 \\
\hline $9 / 26 / 2017$ & $24: 00: 00$ & 310.67 & 61.46 & 84.5 & 3,450 & 9 & 1,459 & 1,264 & 1,929 \\
\hline 9/27/2017 & $24: 00: 00$ & 310.39 & 69.74 & 84.2 & 3,679 & 13 & 1,432 & 1,143 & 1,971 \\
\hline 9/28/2017 & $24: 00: 00$ & 309.99 & 78.94 & 84.7 & 3,907 & 12 & 1,324 & 675 & 1,807 \\
\hline $9 / 29 / 2017$ & $24: 00: 00$ & 286.03 & 164.82 & 90.6 & 5,353 & 34 & 1,304 & 735 & 1,875 \\
\hline $9 / 30 / 2017$ & $24: 00: 00$ & 282.61 & 154.19 & 89.9 & 5,108 & 25 & 1,267 & 472 & 1,814 \\
\hline $10 / 1 / 2017$ & $24: 00: 00$ & 272.12 & 163.96 & 90.3 & 5,163 & 25 & 1,279 & 707 & 1,834 \\
\hline $10 / 2 / 2017$ & 24:00:00 & 275.93 & 151.02 & 89.9 & 4,967 & 22 & 1,294 & 819 & 1,858 \\
\hline $10 / 3 / 2017$ & 24:00:00 & 289.98 & 120.17 & 89.4 & 4,500 & 17 & 1,301 & 907 & 1,773 \\
\hline $10 / 4 / 2017$ & 24:00:00 & 282.65 & 128.44 & 90.7 & 4,542 & 17 & 1,347 & 1,132 & 1,842 \\
\hline $10 / 5 / 2017$ & 24:00:00 & 309.57 & 71.05 & 88.5 & 3,697 & 9 & 1,332 & 1,047 & 1,751 \\
\hline $10 / 6 / 2017$ & $24: 00: 00$ & 310.38 & 82.02 & 86.5 & 3,997 & 12 & 1,368 & 1,197 & 1,873 \\
\hline $10 / 7 / 2017$ & $24: 00: 00$ & 309.7 & 65.32 & 87.9 & 3,550 & 9 & 1,398 & 1,274 & 1,844 \\
\hline $10 / 8 / 2017$ & $24: 00: 00$ & 310.17 & 56.05 & 89.1 & 3,285 & 6 & 1,403 & 1,238 & 1,850 \\
\hline $10 / 9 / 2017$ & $24: 00: 00$ & 310.48 & 60.34 & 86.3 & 3,420 & 8 & 1,406 & 1,219 & 1,854 \\
\hline $10 / 10 / 2017$ & 24:00:00 & 310.91 & 62.27 & 86.5 & 3,465 & 9 & 1,401 & 1,223 & 1,847 \\
\hline $10 / 11 / 2017$ & $24: 00: 00$ & 310.65 & 62.84 & 86.2 & 3,492 & 7 & 1,394 & 1,211 & 1,838 \\
\hline $10 / 12 / 2017$ & $24: 00: 00$ & 310.1 & 68.17 & 85.4 & 3,621 & 10 & 1,263 & 466 & 1,654 \\
\hline $10 / 13 / 2017$ & $24: 00: 00$ & 260.77 & 159.67 & 88.9 & 4,932 & 27 & 1,223 & 456 & 1,740 \\
\hline $10 / 14 / 2017$ & $24: 00: 00$ & 237.12 & 185.73 & 93.7 & 5,217 & 30 & 1,327 & 1,145 & 1,913 \\
\hline $10 / 15 / 2017$ & $24: 00: 00$ & 296.12 & 85.3 & 91.0 & 3,759 & 11 & 1,336 & 1,026 & 1,825 \\
\hline $10 / 16 / 2017$ & $24: 00: 00$ & 309.14 & 73.94 & 87.0 & 3,734 & 9 & 1,216 & 454 & 1,588 \\
\hline $10 / 17 / 2017$ & $24: 00: 00$ & 261.71 & 165.31 & 90.8 & 5,124 & 24 & 1,192 & 448 & 1,688 \\
\hline $10 / 18 / 2017$ & 24:00:00 & 240.51 & 177.44 & 92.8 & 5,123 & 25 & 1,177 & 444 & 1,663 \\
\hline $10 / 19 / 2017$ & 23:59:56 & 242.18 & 171.45 & 94.7 & 5,036 & 23 & 1,200 & 631 & 1,702 \\
\hline $10 / 20 / 2017$ & 24:00:00 & 302.4 & 111.48 & 93.7 & 4,533 & 14 & 1,182 & 508 & 1,595 \\
\hline $10 / 21 / 2017$ & $24: 00: 00$ & 294.2 & 121.66 & 94.5 & 4,656 & 18 & 1,227 & 862 & 1,662 \\
\hline $10 / 22 / 2017$ & $24: 00: 00$ & 308.64 & 88.01 & 92.7 & 4,085 & 13 & 1,278 & 1,010 & 1,739 \\
\hline $10 / 23 / 2017$ & $24: 00: 00$ & 309.84 & 64.93 & 90.8 & 3,513 & 9 & 1,226 & 792 & 1,602 \\
\hline $10 / 24 / 2017$ & $24: 00: 00$ & 298.83 & 105.95 & 88.2 & 4,382 & 14 & 1,162 & 451 & 1,565 \\
\hline $10 / 25 / 2017$ & $24: 00: 00$ & 281.08 & 141.94 & 94.1 & 4,941 & 19 & 1,144 & 435 & 1,538 \\
\hline $10 / 26 / 2017$ & $24: 00: 00$ & 242.02 & 163.28 & 93.0 & 4,950 & 14 & 1,132 & 433 & 1,520 \\
\hline $10 / 27 / 2017$ & $24: 00: 00$ & 257.26 & 151.93 & 94.7 & 4,897 & 14 & 1,127 & 459 & 1,513 \\
\hline $10 / 28 / 2017$ & $24: 00: 00$ & 298.23 & 125.75 & 98.5 & 4,794 & 15 & 1,117 & 449 & 1,498 \\
\hline $10 / 29 / 2017$ & $24: 00: 00$ & 285.87 & 131.55 & 95.8 & 4,817 & 11 & 1,107 & 430 & 1,484 \\
\hline $10 / 30 / 2017$ & $24: 00: 00$ & 251.56 & 148.76 & 93.0 & 4,813 & 11 & 1,103 & 453 & 1,478 \\
\hline $10 / 31 / 2017$ & $24: 00: 00$ & 294.44 & 123.86 & 96.2 & 4,744 & 10 & 1,100 & 478 & 1,473 \\
\hline $11 / 1 / 2017$ & $24: 00: 00$ & 293.44 & 122.1 & 97.7 & 4,682 & 11 & 1,094 & 457 & 1,465 \\
\hline $11 / 2 / 2017$ & $24: 00: 00$ & 300.84 & 120.61 & 100.3 & 4,708 & 14 & 1,088 & 451 & 1,456 \\
\hline $11 / 3 / 2017$ & $24: 00: 00$ & 297.97 & 120.24 & 100.8 & 4,679 & 13 & 1,081 & 434 & 1,445 \\
\hline $11 / 4 / 2017$ & $24: 00: 00$ & 282.84 & 126.32 & 99.2 & 4,667 & 12 & 1,078 & 452 & 1,441 \\
\hline $11 / 5 / 2017$ & $24: 00: 00$ & 299.74 & 118.2 & 105.6 & 4,630 & 14 & 1,113 & 717 & 1,492 \\
\hline $11 / 6 / 2017$ & 24:00:00 & 306.94 & 96.62 & 102.4 & 4,232 & 13 & 1,074 & 443 & 1,435 \\
\hline $11 / 7 / 2017$ & 24:00:00 & 291.91 & 121.65 & 102.2 & 4,653 & 11 & 1,064 & 417 & 1,421 \\
\hline $11 / 8 / 2017$ & 24:00:00 & 254.7 & 137.82 & 100.2 & 4,629 & 10 & 1,061 & 445 & 1,373 \\
\hline $11 / 9 / 2017$ & 24:00:00 & 289.94 & 119.87 & 110.6 & 4,565 & 8 & 1,057 & 441 & 1,367 \\
\hline $11 / 10 / 2017$ & 24:00:00 & 294.86 & 116.6 & 110.8 & 4,541 & 9 & 1,050 & 422 & 1,358 \\
\hline $11 / 11 / 2017$ & $24: 00: 00$ & 276.82 & 124.19 & 108.9 & 4,542 & 8 & 1,048 & 440 & 1,354 \\
\hline $11 / 12 / 2017$ & $24: 00: 00$ & 291.55 & 116.51 & 110.5 & 4,512 & 8 & 1,045 & 441 & 1,351 \\
\hline $11 / 13 / 2017$ & $24: 00: 00$ & 297.39 & 112.89 & 111.5 & 4,484 & 8 & 1,041 & 439 & 1,345 \\
\hline $11 / 14 / 2017$ & 24:00:00 & 295.6 & 112.44 & 111.4 & 4,463 & 9 & 1,036 & 436 & 1,339 \\
\hline $11 / 15 / 2017$ & 24:00:00 & 293.69 & 112.51 & 111.4 & 4,451 & 8 & 1,034 & 437 & 1,336 \\
\hline $11 / 16 / 2017$ & $24: 00: 00$ & 295.67 & 111.06 & 111.9 & 4,435 & 8 & 1,030 & 436 & 1,330 \\
\hline $11 / 17 / 2017$ & $24: 00: 00$ & 295.16 & 110.15 & 111.6 & 4,414 & 8 & 1,027 & 436 & 1,325 \\
\hline $11 / 18 / 2017$ & $24: 00: 00$ & 295.61 & 109.45 & 111.9 & 4,402 & 8 & 1,025 & 435 & 1,323 \\
\hline $11 / 19 / 2017$ & $24: 00: 00$ & 298.98 & 107.56 & 112.1 & 4,385 & 9 & 1,018 & 412 & 1,314 \\
\hline $11 / 20 / 2017$ & $24: 00: 00$ & 274.26 & 117.81 & 110.7 & 4,397 & 7 & 1,017 & 435 & 1,312 \\
\hline $11 / 21 / 2017$ & $24: 00: 00$ & 297.98 & 106.42 & 112.1 & 4,356 & 7 & 1,015 & 439 & 1,310 \\
\hline $11 / 22 / 2017$ & $24: 00: 00$ & 304.99 & 103.41 & 112.8 & 4,334 & 9 & 1,011 & 439 & 1,304 \\
\hline $11 / 23 / 2017$ & $24: 00: 00$ & 303.66 & 103.19 & 112.0 & 4,325 & 8 & 1,008 & 434 & 1,300 \\
\hline $11 / 24 / 2017$ & $24: 00: 00$ & 303.75 & 102.5 & 112.4 & 4,310 & 8 & 1,007 & 440 & 1,298 \\
\hline $11 / 25 / 2017$ & 28:01:45 & 308.76 & 99.84 & 113.5 & 5,010 & 9 & 1,004 & 432 & 1,294 \\
\hline $11 / 26 / 2017$ & 19:58:15 & 307.54 & 100.11 & 113.1 & 3,561 & 9 & 1,001 & 436 & 1,290 \\
\hline $11 / 27 / 2017$ & $24: 00: 00$ & 305.48 & 93.5 & 78.0 & 4,276 & 9 & 999 & 451 & 1,287 \\
\hline $11 / 28 / 2017$ & $24: 00: 00$ & 305.81 & 89.02 & 66.8 & 4,194 & 9 & 1,006 & 437 & 1,297 \\
\hline $11 / 29 / 2017$ & $24: 00: 00$ & 306.64 & 88.95 & 76.9 & 4,078 & 7 & 1,000 & 433 & 1,289 \\
\hline $11 / 30 / 2017$ & $24: 00: 00$ & 305.66 & 90.97 & 73.9 & 4,219 & 8 & 995 & 431 & 1,282 \\
\hline $12 / 1 / 2017$ & $24: 00: 00$ & 305.32 & 91.67 & 70.1 & 4,266 & 9 & 991 & 427 & 1,276 \\
\hline $12 / 2 / 2017$ & $24: 00: 00$ & 301.24 & 91.63 & 65.6 & 4,262 & 8 & 987 & 428 & 1,272 \\
\hline $12 / 3 / 2017$ & $24: 00: 00$ & 301.55 & 90.78 & 65.0 & 4,247 & 8 & 984 & 425 & 1,267 \\
\hline $12 / 4 / 2017$ & $24: 00: 00$ & 301.73 & 90.07 & 65.2 & 4,231 & 8 & 983 & 428 & 1,266 \\
\hline $12 / 5 / 2017$ & $24: 00: 00$ & 303.84 & 89.16 & 66.1 & 4,218 & 8 & 979 & 423 & 1,260 \\
\hline $12 / 6 / 2017$ & $24: 00: 00$ & 300.63 & 89.19 & 63.6 & 4,210 & 8 & 976 & 422 & 1,256 \\
\hline $12 / 7 / 2017$ & $24: 00: 00$ & 296.92 & 89.67 & 63.0 & 4,202 & 7 & 973 & 421 & 1,252 \\
\hline $12 / 8 / 2017$ & $24: 00: 00$ & 296.13 & 89.25 & 63.0 & 4,188 & 7 & 1,166 & 1,145 & 1,519 \\
\hline $12 / 9 / 2017$ & $24: 00: 00$ & 309.53 & 18.4 & 32.0 & 1,713 & 1 & 1,136 & 971 & 1,402 \\
\hline $12 / 10 / 2017$ & $24: 00: 00$ & 309.77 & 38.04 & 30.1 & 2,828 & 1 & 1,120 & 841 & 1,382 \\
\hline $12 / 11 / 2017$ & $24: 00: 00$ & 308.65 & 52.06 & 38.6 & 3,343 & 2 & 1,017 & 448 & 1,279 \\
\hline $12 / 12 / 2017$ & $24: 00: 00$ & 312.49 & 109.33 & 83.6 & 4,654 & 5 & 1,135 & 686 & 1,435 \\
\hline $12 / 13 / 2017$ & 15:01:04 & 273.99 & 113.04 & 73.1 & 2,773 & 3 & 1,012 & 452 & 1,273 \\
\hline
\end{tabular}




\begin{tabular}{|c|c|c|c|c|c|c|c|c|c|}
\hline $12 / 14 / 2017$ & $24: 00: 00$ & 324.3 & 102.08 & 59.9 & 4,694 & 5 & 994 & 445 & 1,248 \\
\hline $12 / 15 / 2017$ & $28: 35: 26$ & 266.99 & 35.9 & 58.9 & 5,150 & 6 & 987 & 447 & 1,271 \\
\hline $12 / 16 / 2017$ & $19: 21: 15$ & 328.13 & 81.9 & 61.6 & 3,407 & 7 & 982 & 445 & 1,264 \\
\hline $12 / 17 / 2017$ & $24: 00: 00$ & 330.65 & 81.09 & 67.6 & 4,199 & 7 & 978 & 449 & 1,259 \\
\hline $12 / 18 / 2017$ & 24:00:00 & 335.98 & 82.1 & 86.6 & 4,169 & 7 & 975 & 443 & 1,254 \\
\hline $12 / 19 / 2017$ & $24: 00: 00$ & 337.83 & 81.05 & 86.4 & 4,154 & 7 & 971 & 441 & 1,249 \\
\hline $12 / 20 / 2017$ & $24: 00: 00$ & 337.66 & 81.75 & 93.3 & 4,141 & 7 & 966 & 443 & 1,243 \\
\hline $12 / 21 / 2017$ & $24: 00: 00$ & 332.92 & 82.37 & 94.0 & 4,129 & 11 & 965 & 447 & 1,277 \\
\hline $12 / 22 / 2017$ & $24: 00: 00$ & 336.23 & 79.29 & 83.7 & 4,112 & 10 & 963 & 454 & 1,238 \\
\hline $12 / 23 / 2017$ & $24: 00: 00$ & 340.55 & 78.01 & 84.4 & 4,098 & 8 & 958 & 442 & 1,231 \\
\hline $12 / 24 / 2017$ & $24: 00: 00$ & 335.37 & 75.84 & 66.7 & 4,092 & 12 & 955 & 439 & 1,262 \\
\hline $12 / 25 / 2017$ & $24: 00: 00$ & 333.38 & 75.19 & 62.0 & 4,085 & 12 & 951 & 438 & 1,258 \\
\hline $12 / 26 / 2017$ & $24: 00: 00$ & 331.04 & 75.07 & 61.0 & 4,075 & 13 & 949 & 438 & 1,254 \\
\hline $12 / 27 / 2017$ & $24: 00: 00$ & 330.6 & 74.81 & 61.2 & 4,064 & 12 & 946 & 437 & 1,250 \\
\hline $12 / 28 / 2017$ & $24: 00: 00$ & 331.65 & 73.87 & 60.5 & 4,048 & 12 & 945 & 442 & 1,248 \\
\hline $12 / 29 / 2017$ & $24: 00: 00$ & 338.5 & 71.76 & 60.8 & 4,030 & 13 & 943 & 444 & 1,246 \\
\hline $12 / 30 / 2017$ & $24: 00: 00$ & 341.37 & 70.72 & 61.4 & 4,016 & 14 & 941 & 441 & 1,242 \\
\hline $12 / 31 / 2017$ & $24: 00: 00$ & 339.93 & 70.56 & 60.6 & 4,007 & 15 & 938 & 440 & 1,238 \\
\hline $1 / 1 / 2018$ & $24: 00: 00$ & 339.44 & 70.34 & 60.4 & 3,999 & 17 & 937 & 447 & 1,237 \\
\hline $1 / 2 / 2018$ & $24: 00: 00$ & 347.41 & 67.97 & 59.3 & 3,979 & 16 & 942 & 436 & 1,244 \\
\hline $1 / 3 / 2018$ & $24: 00: 00$ & 329.57 & 69.92 & 57.0 & 3,826 & 13 & 940 & 450 & 1,241 \\
\hline $1 / 4 / 2018$ & $24: 00: 00$ & 343.28 & 70.26 & 60.7 & 4,000 & 17 & 931 & 397 & 1,228 \\
\hline $1 / 5 / 2018$ & $24: 00: 00$ & 292.46 & 85.28 & 57.8 & 4,083 & 19 & 926 & 392 & 1,221 \\
\hline $1 / 6 / 2018$ & $24: 00: 00$ & 267.78 & 93.15 & 57.4 & 4,094 & 21 & 922 & 390 & 1,258 \\
\hline $1 / 7 / 2018$ & $24: 00: 00$ & 266.78 & 92.67 & 57.8 & 4,075 & 21 & 928 & 457 & 1,267 \\
\hline $1 / 8 / 2018$ & $24: 00: 00$ & 346.99 & 69.5 & 62.4 & 3,969 & 9 & 927 & 449 & 1,189 \\
\hline $1 / 9 / 2018$ & $24: 00: 00$ & 353.76 & 71.26 & 96.2 & 3,953 & 8 & 926 & 454 & 1,187 \\
\hline $1 / 10 / 2018$ & $24: 00: 00$ & 355.38 & 71.64 & 101.5 & 3,948 & 11 & 924 & 451 & 1,219 \\
\hline $1 / 11 / 2018$ & $24: 00: 00$ & 356.48 & 72.48 & 110.0 & 3,943 & 10 & 928 & 496 & 1,225 \\
\hline $1 / 12 / 2018$ & $24: 00: 00$ & 356.69 & 70.65 & 110.6 & 3,892 & 8 & 921 & 447 & 1,181 \\
\hline $1 / 13 / 2018$ & $24: 00: 00$ & 355.89 & 66.98 & 75.2 & 3,929 & 14 & 917 & 444 & 1,208 \\
\hline $1 / 14 / 2018$ & $24: 00: 00$ & 350.85 & 65.78 & 60.7 & 3,931 & 15 & 913 & 413 & 1,202 \\
\hline $1 / 15 / 2018$ & $24: 00: 00$ & 322.45 & 72.53 & 60.7 & 3,942 & 19 & 940 & 545 & 1,242 \\
\hline $1 / 16 / 2018$ & $24: 00: 00$ & 348.33 & 64.82 & 59.6 & 3,653 & 14 & 914 & 395 & 1,204 \\
\hline $1 / 17 / 2018$ & $24: 00: 00$ & 302.62 & 79.52 & 59.1 & 3,930 & 18 & 913 & 431 & 1,202 \\
\hline $1 / 18 / 2018$ & $24: 00: 00$ & 328.04 & 70 & 58.2 & 3,913 & 15 & 915 & 451 & 1,205 \\
\hline $1 / 19 / 2018$ & $24: 00: 00$ & 360.77 & 61.48 & 59.9 & 3,831 & 8 & 914 & 462 & 1,172 \\
\hline $1 / 20 / 2018$ & $24: 00: 00$ & 361.3 & 66.56 & 96.7 & 3,855 & 6 & 920 & 492 & 1,179 \\
\hline $1 / 21 / 2018$ & $24: 00: 00$ & 356.89 & 64.74 & 112.9 & 3,716 & 6 & 952 & 627 & 1,223 \\
\hline $1 / 22 / 2018$ & $24: 00: 00$ & 360.09 & 51.47 & 111.3 & 3,329 & 4 & 923 & 474 & 1,156 \\
\hline $1 / 23 / 2018$ & $24: 00: 00$ & 356.45 & 69.18 & 111.8 & 3,843 & 7 & 914 & 454 & 1,171 \\
\hline $1 / 24 / 2018$ & $24: 00: 00$ & 361.02 & 70.24 & 115.7 & 3,883 & 6 & 910 & 452 & 1,166 \\
\hline $1 / 25 / 2018$ & $24: 00: 00$ & 359.89 & 69.51 & 115.3 & 3,859 & 7 & 907 & 448 & 1,162 \\
\hline $1 / 26 / 2018$ & $24: 00: 00$ & 359.43 & 68.19 & 106.5 & 3,853 & 6 & 906 & 451 & 1,160 \\
\hline $1 / 27 / 2018$ & $24: 00: 00$ & 358.92 & 66.64 & 96.5 & 3,845 & 6 & 904 & 443 & 1,158 \\
\hline $1 / 28 / 2018$ & $24: 00: 00$ & 352.64 & 67.57 & 96.7 & 3,841 & 5 & 901 & 449 & 1,154 \\
\hline $1 / 29 / 2018$ & $24: 00: 00$ & 356.44 & 66.78 & 97.5 & 3,834 & 8 & 899 & 446 & 1,151 \\
\hline $1 / 30 / 2018$ & $24: 00: 00$ & 357.69 & 67.91 & 110.8 & 3,820 & 8 & 897 & 441 & 1,148 \\
\hline $1 / 31 / 2018$ & $24: 00: 00$ & 351.02 & 65.75 & 86.3 & 3,823 & 9 & 898 & 451 & 1,150 \\
\hline $2 / 1 / 2018$ & 23:59:59 & 360.46 & 63.76 & 87.9 & 3,803 & 5 & 894 & 442 & 1,118 \\
\hline $2 / 2 / 2018$ & $24: 00: 00$ & 357.44 & 64.03 & 86.4 & 3,803 & 9 & 891 & 440 & 1,141 \\
\hline $2 / 3 / 2018$ & $24: 00: 00$ & 351.32 & 65.48 & 89.7 & 3,804 & 8 & 891 & 441 & 1,141 \\
\hline $2 / 4 / 2018$ & $24: 00: 00$ & 351.62 & 64.99 & 89.3 & 3,792 & 6 & 889 & 440 & 1,137 \\
\hline $2 / 5 / 2018$ & $24: 00: 00$ & 351.98 & 63.86 & 81.5 & 3,793 & 12 & 887 & 440 & 1,165 \\
\hline $2 / 6 / 2018$ & $24: 00: 00$ & 351.72 & 61.23 & 62.7 & 3,790 & 10 & 886 & 439 & 1,165 \\
\hline $2 / 7 / 2018$ & $24: 00: 00$ & 351.21 & 61.08 & 63.3 & 3,780 & 11 & 884 & 438 & 1,161 \\
\hline $2 / 8 / 2018$ & $24: 00: 00$ & 351.07 & 60.93 & 62.2 & 3,779 & 13 & 883 & 438 & 1,160 \\
\hline $2 / 9 / 2018$ & $24: 00: 00$ & 352.67 & 60.35 & 62.7 & 3,767 & 7 & 883 & 449 & 1,130 \\
\hline $2 / 10 / 2018$ & $24: 00: 00$ & 359.3 & 59.27 & 64.5 & 3,756 & 9 & 912 & 631 & 1,168 \\
\hline $2 / 11 / 2018$ & $24: 00: 00$ & 361.88 & 52.52 & 61.1 & 3,505 & 6 & 904 & 515 & 1,158 \\
\hline $2 / 12 / 2018$ & $24: 00: 00$ & 357.14 & 56.33 & 61.3 & 3,604 & 11 & 885 & 439 & 1,163 \\
\hline $2 / 13 / 2018$ & $24: 00: 00$ & 358.61 & 59.85 & 62.5 & 3,712 & 10 & 883 & 439 & 1,160 \\
\hline $2 / 14 / 2018$ & $24: 00: 00$ & 351.85 & 60.37 & 65.1 & 3,754 & 6 & 882 & 439 & 1,127 \\
\hline $2 / 15 / 2018$ & $24: 00: 00$ & 351.89 & 60.16 & 65.7 & 3,745 & 7 & 918 & 648 & 1,176 \\
\hline $2 / 16 / 2018$ & $24: 00: 00$ & 356.24 & 48.9 & 59.7 & 3,374 & 6 & 880 & 436 & 1,125 \\
\hline $2 / 17 / 2018$ & $24: 00: 00$ & 351.39 & 61.5 & 63.3 & 3,794 & 15 & 877 & 437 & 1,151 \\
\hline $2 / 18 / 2018$ & $24: 00: 00$ & 351.29 & 59.94 & 63.8 & 3,743 & 13 & 875 & 437 & 1,149 \\
\hline $2 / 19 / 2018$ & $24: 00: 00$ & 351.78 & 59.6 & 64.8 & 3,731 & 12 & 883 & 528 & 1,161 \\
\hline $2 / 20 / 2018$ & $24: 00: 00$ & 354.8 & 56.35 & 65.6 & 3,636 & 6 & 965 & 875 & 1,241 \\
\hline $2 / 21 / 2018$ & $24: 00: 00$ & 361.19 & 29.59 & 51.5 & 2,621 & 2 & 908 & 447 & 1,135 \\
\hline $2 / 22 / 2018$ & $24: 00: 00$ & 354.89 & 51.12 & 56.5 & 3,378 & 7 & 884 & 440 & 1,131 \\
\hline $2 / 23 / 2018$ & $24: 00: 00$ & 351.2 & 64.53 & 64.9 & 3,877 & 7 & 893 & 567 & 1,144 \\
\hline $2 / 24 / 2018$ & $24: 00: 00$ & 354.44 & 57.09 & 64.4 & 3,657 & 4 & 878 & 442 & 1,097 \\
\hline $2 / 25 / 2018$ & $24: 00: 00$ & 354.35 & 60.14 & 65.5 & 3,757 & 3 & 873 & 438 & 1,090 \\
\hline $2 / 26 / 2018$ & $24: 00: 00$ & 357 & 59.27 & 66.0 & 3,739 & 9 & 870 & 435 & 1,111 \\
\hline $2 / 27 / 2018$ & $24: 00: 00$ & 350.23 & 59.49 & 65.4 & 3,717 & 9 & 868 & 435 & 1,109 \\
\hline $2 / 28 / 2018$ & $24: 00: 00$ & 352.25 & 59.3 & 66.5 & 3,717 & 7 & 867 & 440 & 1,108 \\
\hline $3 / 1 / 2018$ & $24: 00: 00$ & 354.41 & 58.8 & 66.9 & 3,709 & 9 & 864 & 434 & 1,103 \\
\hline $3 / 2 / 2018$ & $0: 00: 00$ & 315.08 & 69.42 & 62.3 & 6,230 & 0 & 864 & 433 & 1,057 \\
\hline $3 / 3 / 2018$ & $0: 00: 00$ & 301.76 & 74.51 & 62.4 & 316 & 0 & 919 & 282 & 1,126 \\
\hline $3 / 4 / 2018$ & $0: 00: 00$ & 289.06 & 28.26 & 46.1 & 3,819 & 0 & 1,060 & 805 & 1,306 \\
\hline $3 / 5 / 2018$ & $3: 18: 53$ & 249.73 & 5.37 & 32.0 & 346 & 9 & 921 & 635 & 1,181 \\
\hline
\end{tabular}




\begin{tabular}{|c|c|c|c|c|c|c|c|c|c|}
\hline $3 / 6 / 2018$ & $24: 40: 33$ & 340.46 & 46.48 & 59.1 & 4,068 & 4 & 941 & 641 & 1,179 \\
\hline $3 / 7 / 2018$ & 23:14:53 & 354.63 & 50.93 & 54.1 & 3,281 & 4 & 899 & 446 & 1,123 \\
\hline $3 / 8 / 2018$ & $24: 00: 00$ & 337.13 & 63.25 & 55.6 & 3,390 & 17 & 892 & 445 & 1,173 \\
\hline $3 / 9 / 2018$ & $24: 00: 00$ & 360.64 & 60 & 57.6 & 3,584 & 3 & 877 & 437 & 1,096 \\
\hline $3 / 10 / 2018$ & $24: 00: 00$ & 353.66 & 63.44 & 63.2 & 3,864 & 5 & 871 & 440 & 1,113 \\
\hline $3 / 11 / 2018$ & $24: 00: 00$ & 353.07 & 60.96 & 64.5 & 3,780 & 6 & 868 & 439 & 1,109 \\
\hline $3 / 12 / 2018$ & $24: 00: 00$ & 353.78 & 59.32 & 64.0 & 3,728 & 6 & 1,014 & 892 & 1,309 \\
\hline $3 / 13 / 2018$ & $24: 00: 00$ & 322.51 & 28.21 & 48.2 & 2,139 & 3 & 910 & 452 & 1,139 \\
\hline $3 / 14 / 2018$ & $24: 00: 00$ & 333.68 & 56.21 & 49.4 & 3,543 & 3 & 878 & 440 & 1,097 \\
\hline $3 / 15 / 2018$ & 24:00:00 & 353.22 & 64.26 & 61.7 & 3,892 & 5 & 870 & 437 & 1,086 \\
\hline $3 / 16 / 2018$ & 24:00:00 & 352.57 & 60.81 & 63.2 & 3,778 & 9 & 865 & 437 & 1,105 \\
\hline $3 / 17 / 2018$ & 24:00:00 & 352.19 & 59.39 & 63.3 & 3,731 & 9 & 862 & 437 & 1,101 \\
\hline $3 / 18 / 2018$ & 24:00:00 & 352.17 & 58.71 & 63.6 & 3,709 & 9 & 859 & 435 & 1,097 \\
\hline $3 / 19 / 2018$ & $24: 00: 00$ & 352.45 & 58.54 & 65.2 & 3,698 & 8 & 858 & 436 & 1,096 \\
\hline $3 / 20 / 2018$ & $24: 00: 00$ & 351.96 & 58.18 & 65.1 & 3,685 & 4 & 855 & 432 & 1,066 \\
\hline $3 / 21 / 2018$ & $24: 00: 00$ & 351.8 & 57.73 & 62.4 & 3,681 & 8 & 852 & 434 & 1,087 \\
\hline $3 / 22 / 2018$ & $24: 00: 00$ & 351.33 & 57.41 & 62.4 & 3,669 & 7 & 850 & 433 & 1,085 \\
\hline $3 / 23 / 2018$ & 24:00:00 & 352.01 & 57.36 & 63.7 & 3,660 & 7 & 848 & 433 & 1,082 \\
\hline $3 / 24 / 2018$ & $24: 00: 00$ & 352.2 & 57.07 & 63.8 & 3,656 & 6 & 847 & 432 & 1,080 \\
\hline $3 / 25 / 2018$ & $24: 00: 00$ & 353.32 & 56.54 & 63.3 & 3,647 & 3 & 864 & 472 & 1,078 \\
\hline $3 / 26 / 2018$ & $24: 00: 00$ & 353.81 & 45.62 & 58.7 & 3,068 & 51 & 852 & 437 & 1,152 \\
\hline $3 / 27 / 2018$ & $24: 00: 00$ & 352.29 & 63.04 & 64.3 & 3,838 & 6 & 849 & 434 & 1,083 \\
\hline $3 / 28 / 2018$ & $24: 00: 00$ & 352.26 & 57.88 & 65.5 & 3,676 & 4 & 860 & 559 & 1,073 \\
\hline $3 / 29 / 2018$ & $24: 00: 00$ & 356.23 & 52.5 & 63.8 & 3,519 & 2 & 868 & 543 & 1,061 \\
\hline $3 / 30 / 2018$ & $24: 00: 00$ & 358.48 & 49.06 & 61.7 & 3,403 & 2 & 845 & 433 & 1,054 \\
\hline $3 / 31 / 2018$ & 24:00:00 & 353.39 & 57.18 & 64.0 & 3,665 & 5 & 844 & 432 & 1,052 \\
\hline $4 / 1 / 2018$ & $24: 00: 00$ & 352.93 & 56.46 & 65.7 & 3,633 & 2 & 840 & 433 & 1,026 \\
\hline $4 / 2 / 2018$ & 24:00:00 & 353.77 & 56.13 & 64.1 & 3,632 & 7 & 838 & 431 & 1,069 \\
\hline $4 / 3 / 2018$ & $24: 00: 00$ & 353.37 & 56.16 & 65.0 & 3,627 & 8 & 838 & 430 & 1,069 \\
\hline $4 / 4 / 2018$ & $24: 00: 00$ & 353.63 & 55.62 & 65.8 & 3,609 & 7 & 834 & 428 & 1,063 \\
\hline $4 / 5 / 2018$ & $24: 00: 00$ & 351.56 & 55.81 & 63.8 & 3,613 & 12 & 833 & 430 & 1,089 \\
\hline $4 / 6 / 2018$ & $24: 00: 00$ & 352.7 & 55.41 & 65.5 & 3,598 & 9 & 832 & 430 & 1,061 \\
\hline $4 / 7 / 2018$ & $24: 00: 00$ & 354.44 & 53.78 & 64.2 & 3,559 & 7 & 829 & 431 & 1,056 \\
\hline $4 / 8 / 2018$ & $24: 00: 00$ & 352.78 & 55.14 & 64.1 & 3,595 & 8 & 828 & 430 & 1,056 \\
\hline $4 / 9 / 2018$ & $24: 00: 00$ & 352.08 & 54.63 & 64.3 & 3,575 & 9 & 1,001 & 155 & 1,290 \\
\hline $4 / 10 / 2018$ & $24: 00: 00$ & 209.92 & 13.35 & 46.0 & 1,163 & 2 & 1,064 & 367 & 1,341 \\
\hline $4 / 11 / 2018$ & $24: 00: 00$ & 198.34 & 13.07 & 39.8 & 1,096 & 1 & 924 & 607 & 1,132 \\
\hline $4 / 12 / 2018$ & $24: 00: 00$ & 355.19 & 56.59 & 53.5 & 3,690 & 3 & 987 & 942 & 1,239 \\
\hline $4 / 13 / 2018$ & $24: 00: 00$ & 365.49 & 27.32 & 49.2 & 2,477 & 2 & 1,061 & 1,039 & 1,306 \\
\hline $4 / 14 / 2018$ & $24: 00: 00$ & 365.32 & 7.16 & 40.6 & 1,310 & 1 & 1,083 & 1,073 & 1,335 \\
\hline $4 / 15 / 2018$ & $24: 00: 00$ & 364.61 & 12.12 & 38.8 & 1,683 & 1 & 1,049 & 856 & 1,291 \\
\hline $4 / 16 / 2018$ & $24: 00: 00$ & 362.52 & 24.79 & 38.2 & 2,367 & 3 & 948 & 631 & 1,188 \\
\hline $4 / 17 / 2018$ & $24: 00: 00$ & 352.35 & 59.9 & 52.5 & 3,796 & 3 & 910 & 549 & 1,138 \\
\hline $4 / 18 / 2018$ & $24: 00: 00$ & 351.48 & 61.68 & 57.5 & 3,824 & 3 & 1,004 & 927 & 1,262 \\
\hline $4 / 19 / 2018$ & 24:00:00 & 364.74 & 20.85 & 40.1 & 2,228 & 2 & 915 & 573 & 1,145 \\
\hline $4 / 20 / 2018$ & 24:00:00 & 353.02 & 58.94 & 56.1 & 3,745 & 3 & 886 & 497 & 1,107 \\
\hline $4 / 21 / 2018$ & 24:00:00 & 353.26 & 61.52 & 62.4 & 3,807 & 2 & 872 & 445 & 1,089 \\
\hline $4 / 22 / 2018$ & 24:00:00 & 357.38 & 59.99 & 65.0 & 3,769 & 6 & 895 & 639 & 1,145 \\
\hline $4 / 23 / 2018$ & 24:00:00 & 363.99 & 46.41 & 59.1 & 3,350 & 6 & 905 & 646 & 1,159 \\
\hline $4 / 24 / 2018$ & $24: 00: 00$ & 362.19 & 42.1 & 56.0 & 3,185 & 4 & 873 & 517 & 1,091 \\
\hline $4 / 25 / 2018$ & $24: 00: 00$ & 360.17 & 54.29 & 62.0 & 3,604 & 6 & 864 & 437 & 1,104 \\
\hline $4 / 26 / 2018$ & $24: 00: 00$ & 356.29 & 50.61 & 60.0 & 3,436 & 5 & 864 & 526 & 1,103 \\
\hline $4 / 27 / 2018$ & 24:00:00 & 359.59 & 52.33 & 64.1 & 3,518 & 8 & 960 & 824 & 1,233 \\
\hline $4 / 28 / 2018$ & 24:00:00 & 364.43 & 19.47 & 45.1 & 2,107 & 2 & 911 & 500 & 1,139 \\
\hline $4 / 29 / 2018$ & $24: 00: 00$ & 358.85 & 41.43 & 51.3 & 3,027 & 4 & 874 & 442 & 1,092 \\
\hline $4 / 30 / 2018$ & $24: 00: 00$ & 354.66 & 56.06 & 60.7 & 3,619 & 5 & 891 & 603 & 1,141 \\
\hline $5 / 1 / 2018$ & $24: 00: 00$ & 360.13 & 47.49 & 60.7 & 3,311 & 5 & 990 & 881 & 1,275 \\
\hline $5 / 2 / 2018$ & $24: 00: 00$ & 365.28 & 19.4 & 47.0 & 1,986 & 2 & 1,055 & 1,052 & 1,298 \\
\hline $5 / 3 / 2018$ & $24: 00: 00$ & 372.68 & 11.21 & 50.3 & 1,540 & 1 & 1,134 & 1,134 & 1,400 \\
\hline $5 / 4 / 2018$ & $24: 00: 00$ & 474.28 & 2.43 & 71.8 & 816 & 0 & 1,128 & 1,115 & 1,392 \\
\hline $5 / 5 / 2018$ & $24: 00: 00$ & 359.8 & 8.33 & 41.5 & 1,382 & 0 & 1,141 & 1,112 & 1,409 \\
\hline $5 / 6 / 2018$ & $24: 00: 00$ & 360.17 & 9.48 & 42.6 & 1,366 & 0 & 1,205 & 1,180 & 1,492 \\
\hline $5 / 7 / 2018$ & $24: 00: 00$ & 327.46 & 6.58 & 49.3 & 964 & 1 & 1,190 & 1,159 & 1,471 \\
\hline $5 / 8 / 2018$ & 24:00:00 & 351.34 & 6.65 & 48.3 & 1,463 & 1 & 1,126 & 998 & 1,389 \\
\hline $5 / 9 / 2018$ & $24: 00: 00$ & 352.96 & 13.05 & 40.8 & 2,790 & 3 & 1,099 & 1,031 & 1,387 \\
\hline $5 / 10 / 2018$ & $24: 00: 00$ & 359.31 & 14.92 & 44.0 & 3,030 & 3 & 1,073 & 931 & 1,353 \\
\hline $5 / 11 / 2018$ & $24: 00: 00$ & 359.39 & 14.23 & 42.6 & 3,004 & 2 & 1,063 & 982 & 1,340 \\
\hline $5 / 12 / 2018$ & $24: 00: 00$ & 360.45 & 13.02 & 44.0 & 2,879 & 3 & 1,127 & 1,098 & 1,424 \\
\hline $5 / 13 / 2018$ & $24: 00: 00$ & 360.49 & 6.62 & 40.3 & 2,036 & 1 & 1,147 & 1,088 & 1,416 \\
\hline $5 / 14 / 2018$ & $24: 00: 00$ & 360 & 7.09 & 40.0 & 2,056 & 6 & 1,120 & 1,033 & 1,455 \\
\hline $5 / 15 / 2018$ & $24: 00: 00$ & 360.68 & 9.74 & 39.5 & 2,497 & 3 & 1,134 & 1,105 & 1,434 \\
\hline $5 / 16 / 2018$ & $24: 00: 00$ & 361.11 & 8.26 & 41.3 & 2,271 & 2 & 1,213 & 1,196 & 1,539 \\
\hline $5 / 17 / 2018$ & $24: 00: 00$ & 355.37 & 4.45 & 50.0 & 1,589 & 1 & 1,144 & 1,046 & 1,412 \\
\hline $5 / 18 / 2018$ & $24: 00: 00$ & 359.51 & 11.12 & 38.8 & 2,664 & 3 & 1,078 & 901 & 1,359 \\
\hline $5 / 19 / 2018$ & $24: 00: 00$ & 357.28 & 18.2 & 45.6 & 3,380 & 4 & 1,054 & 910 & 1,328 \\
\hline $5 / 20 / 2018$ & $24: 00: 00$ & 364.31 & 18.03 & 50.5 & 3,373 & 3 & 1,017 & 804 & 1,279 \\
\hline $5 / 21 / 2018$ & $24: 00: 00$ & 358.38 & 18.44 & 52.6 & 3,357 & 4 & 1,136 & 1,112 & 1,437 \\
\hline $5 / 22 / 2018$ & $24: 00: 00$ & 360.28 & 4.88 & 43.3 & 1,750 & 3 & 1,214 & 1,201 & 1,540 \\
\hline $5 / 23 / 2018$ & $24: 00: 00$ & 362.44 & 3.56 & 56.1 & 1,433 & 1 & 1,188 & 1,143 & 1,469 \\
\hline $5 / 24 / 2018$ & $24: 00: 00$ & 359.92 & 6.68 & 32.1 & 2,053 & 1 & 1,207 & 1,185 & 1,494 \\
\hline $5 / 25 / 2018$ & $24: 00: 00$ & 360.5 & 4.88 & 34.1 & 1,770 & 1 & 1,233 & 1,190 & 1,527 \\
\hline $5 / 26 / 2018$ & $24: 00: 00$ & 359.84 & 4.75 & 42.5 & 1,701 & 1 & 1,225 & 1,175 & 1,516 \\
\hline
\end{tabular}




\begin{tabular}{|c|c|c|c|c|c|c|c|c|c|}
\hline $5 / 27 / 2018$ & $24: 00: 00$ & 360.03 & 5.77 & 38.0 & 1,889 & 1 & 1,219 & 1,138 & 1,510 \\
\hline $5 / 28 / 2018$ & $24: 00: 00$ & 359.87 & 6.79 & 37.9 & 2,025 & 3 & 1,233 & 933 & 1,566 \\
\hline $5 / 29 / 2018$ & $24: 00: 00$ & 370.32 & 5.68 & 42.2 & 1,873 & 2 & 1,303 & 610 & 1,618 \\
\hline $5 / 30 / 2018$ & $24: 00: 00$ & 354.73 & 2.63 & 73.9 & 1,196 & 0 & 1,314 & 1,313 & 1,631 \\
\hline $5 / 31 / 2018$ & 24:00:00 & 350.53 & 2.88 & 51.7 & 1,322 & 1 & 1,338 & 1,338 & 1,662 \\
\hline $6 / 1 / 2018$ & $24: 00: 00$ & 352.58 & 2.53 & 56.1 & 1,238 & 0 & 1,352 & 1,352 & 1,681 \\
\hline $6 / 2 / 2018$ & $24: 00: 00$ & 353.14 & 2.53 & 51.4 & 1,250 & 0 & 1,368 & 1,369 & 1,701 \\
\hline $6 / 3 / 2018$ & $24: 00: 00$ & 353.54 & 2.44 & 51.7 & 1,230 & 0 & 1,376 & 1,365 & 1,711 \\
\hline $6 / 4 / 2018$ & $24: 00: 00$ & 353.21 & 3.18 & 48.8 & 1,366 & 0 & 1,341 & 1,310 & 1,666 \\
\hline $6 / 5 / 2018$ & $24: 00: 00$ & 326.3 & 5.63 & 29.1 & 1,781 & 3 & 1,363 & 1,344 & 1,739 \\
\hline $6 / 6 / 2018$ & $24: 00: 00$ & 342.5 & 4.1 & 38.5 & 1,529 & 2 & 1,347 & 1,317 & 1,674 \\
\hline $6 / 7 / 2018$ & $24: 00: 00$ & 352.07 & 4.31 & 21.1 & 1,677 & 2 & 1,388 & 1,385 & 1,773 \\
\hline $6 / 8 / 2018$ & $24: 00: 00$ & 349.59 & 2.94 & 43.4 & 1,331 & 1 & 1,377 & 1,369 & 1,713 \\
\hline $6 / 9 / 2018$ & $24: 00: 00$ & 352.55 & 4.16 & 29.1 & 1,630 & 1 & 1,400 & 1,397 & 1,742 \\
\hline $6 / 10 / 2018$ & $24: 00: 00$ & 350.87 & 3.35 & 42.4 & 1,419 & 1 & 1,396 & 1,386 & 1,738 \\
\hline $6 / 11 / 2018$ & $24: 00: 00$ & 351.93 & 4.47 & 33.1 & 1,638 & 1 & 1,400 & 1,379 & 1,743 \\
\hline $6 / 12 / 2018$ & $24: 00: 00$ & 269.45 & 5.15 & 36.9 & 1,477 & 1 & 1,389 & 1,360 & 1,728 \\
\hline $6 / 13 / 2018$ & $24: 00: 00$ & 350.86 & 5.53 & 31.0 & 1,801 & 1 & 1,381 & 1,367 & 1,718 \\
\hline $6 / 14 / 2018$ & $24: 00: 00$ & 322.73 & 7.88 & 33.2 & 1,922 & 3 & 1,413 & 1,408 & 1,807 \\
\hline $6 / 15 / 2018$ & $24: 00: 00$ & 351.6 & 3.45 & 37.4 & 1,439 & 1 & 1,424 & 1,416 & 1,773 \\
\hline $6 / 16 / 2018$ & $24: 00: 00$ & 350.73 & 3.59 & 36.7 & 1,461 & 1 & 1,417 & 1,395 & 1,764 \\
\hline $6 / 17 / 2018$ & $24: 00: 00$ & 351.13 & 5.13 & 32.1 & 1,729 & 1 & 1,409 & 1,390 & 1,754 \\
\hline $6 / 18 / 2018$ & $24: 00: 00$ & 342.16 & 6.13 & 33.1 & 1,846 & 2 & 1,433 & 1,426 & 1,785 \\
\hline $6 / 19 / 2018$ & $24: 00: 00$ & 333.37 & 3.48 & 69.4 & 1,338 & 2 & 1,441 & 1,431 & 1,795 \\
\hline $6 / 20 / 2018$ & $24: 00: 00$ & 350.67 & 3.69 & 43.9 & 1,431 & 1 & 1,464 & 1,465 & 1,825 \\
\hline $6 / 21 / 2018$ & $24: 00: 00$ & 280.7 & 2.47 & 62.9 & 1,050 & 2 & 1,484 & 1,485 & 1,851 \\
\hline $6 / 22 / 2018$ & $24: 00: 00$ & 225.21 & 1.63 & 72.2 & 793 & 1 & 1,498 & 1,499 & 1,869 \\
\hline $6 / 23 / 2018$ & $24: 00: 00$ & 223.76 & 1.67 & 70.3 & 802 & 1 & 1,510 & 1,511 & 1,884 \\
\hline $6 / 24 / 2018$ & $24: 00: 00$ & 222.62 & 1.67 & 70.5 & 800 & 1 & 1,508 & 1,509 & 1,882 \\
\hline $6 / 25 / 2018$ & $24: 00: 00$ & 289.04 & 2.09 & 50.1 & 1,017 & 1 & 1,489 & 1,471 & 1,858 \\
\hline $6 / 26 / 2018$ & $12: 34: 32$ & 353.45 & 6.99 & 103.7 & 1,002 & 0 & 1,476 & 1,453 & 1,841 \\
\hline $6 / 27 / 2018$ & $15: 37: 34$ & 353.95 & 6.99 & 99.1 & 1,225 & 0 & 1,471 & 1,465 & 1,834 \\
\hline $6 / 28 / 2018$ & $15: 38: 10$ & 332.01 & 9.92 & 114.7 & 1,326 & 1 & 1,486 & 1,473 & 1,854 \\
\hline $6 / 29 / 2018$ & $9: 25: 41$ & 356.78 & 4.99 & 67.2 & 659 & 0 & 1,488 & 1,484 & 1,856 \\
\hline $6 / 30 / 2018$ & $10: 29: 16$ & 360.72 & 5.21 & 59.2 & 766 & 0 & 1,491 & 1,483 & 1,860 \\
\hline $7 / 1 / 2018$ & \begin{tabular}{|c|}
$10: 11: 09$ \\
\end{tabular} & 360.67 & 5.18 & 60.9 & 743 & 0 & 1,495 & 1,481 & 1,865 \\
\hline $7 / 2 / 2018$ & $10: 17: 47$ & 359.97 & 5.49 & 62.5 & 769 & 0 & 1,523 & 145 & 1,901 \\
\hline $7 / 3 / 2018$ & 1:43:59 & 359.94 & 6.98 & 68.5 & 145 & 0 & 1,508 & 1,487 & 1,881 \\
\hline $7 / 4 / 2018$ & 9:46:52 & 360.19 & 6.47 & 62.3 & 796 & 0 & 1,497 & 1,462 & 1,868 \\
\hline $7 / 5 / 2018$ & $12: 51: 31$ & 359.7 & 6.5 & 67.6 & 1,042 & 1 & 1,480 & 1,423 & 1,846 \\
\hline $7 / 6 / 2018$ & $14: 32: 27$ & 359.33 & 7.48 & 62.6 & 1,266 & 1 & 1,468 & 1,407 & 1,830 \\
\hline $7 / 7 / 2018$ & $16: 14: 50$ & 357.79 & 7.43 & 54.2 & 1,415 & 1 & 1,465 & 1,441 & 1,826 \\
\hline $7 / 8 / 2018$ & $16: 00: 06$ & 354.87 & 5.79 & 58.5 & 1,152 & 1 & 1,507 & 1,488 & 1,881 \\
\hline $7 / 9 / 2018$ & 4:29:09 & 353.55 & 3.25 & 67.9 & 243 & 1 & 1,487 & 1,458 & 1,855 \\
\hline $7 / 10 / 2018$ & $15: 10: 15$ & 357.84 & 7.57 & 95.2 & 1,276 & 1 & 1,484 & 1,449 & 1,850 \\
\hline $7 / 11 / 2018$ & $15: 21: 34$ & 357.58 & 6.69 & 99.0 & 1,197 & 0 & 1,474 & 1,429 & 1,838 \\
\hline $7 / 12 / 2018$ & $16: 40: 24$ & 357.24 & 7.53 & 96.9 & 1,387 & 0 & 1,446 & 1,420 & 1,802 \\
\hline $7 / 13 / 2018$ & $20: 11: 29$ & 291.58 & 16.27 & 99.5 & 2,094 & 2 & 1,447 & 1,417 & 1,853 \\
\hline $7 / 14 / 2018$ & $0: 00: 00$ & 355.45 & 6.17 & 100.9 & 2,196 & 0 & 1,438 & 1,397 & 1,792 \\
\hline $7 / 15 / 2018$ & $22: 48: 57$ & 356.11 & 7.22 & 101.3 & 1,849 & 0 & 1,425 & 1,382 & 1,775 \\
\hline $7 / 16 / 2018$ & $23: 53: 16$ & 354.89 & 7.95 & 97.7 & 2,078 & 0 & 1,411 & 1,360 & 1,757 \\
\hline $7 / 17 / 2018$ & $24: 00: 00$ & 353.41 & 9.31 & 95.0 & 2,254 & 1 & 1,428 & 1,399 & 1,778 \\
\hline $7 / 18 / 2018$ & $18: 08: 48$ & 355.43 & 8.32 & 95.2 & 1,578 & 2 & 1,429 & 1,398 & 1,828 \\
\hline $7 / 19 / 2018$ & $22: 15: 04$ & 355.33 & 5.8 & 93.3 & 1,638 & 1 & 1,426 & 1,392 & 1,776 \\
\hline $7 / 20 / 2018$ & 21:58:08 & 355.1 & 6.57 & 93.7 & 1,713 & 1 & 1,423 & 1,392 & 1,771 \\
\hline $7 / 21 / 2018$ & 23:54:05 & 354.74 & 6.31 & 89.8 & 1,843 & 1 & 1,413 & 1,380 & 1,760 \\
\hline $7 / 22 / 2018$ & $23: 58: 08$ & 354.89 & 6.9 & 89.8 & 1,966 & 1 & 1,400 & 1,355 & 1,742 \\
\hline $7 / 23 / 2018$ & $24: 00: 00$ & 354.96 & 8.36 & 92.3 & 2,163 & 1 & 1,384 & 1,335 & 1,722 \\
\hline $7 / 24 / 2018$ & $23: 38: 14$ & 353.95 & 10.5 & 95.1 & 2,362 & 1 & 1,367 & 1,312 & 1,700 \\
\hline $7 / 25 / 2018$ & $24: 00: 00$ & 353.96 & 10.74 & 93.8 & 2,445 & 1 & 1,354 & 1,304 & 1,684 \\
\hline $7 / 26 / 2018$ & $23: 39: 27$ & 352.22 & 11.51 & 91.3 & 2,471 & 2 & 1,388 & 1,361 & 1,773 \\
\hline $7 / 27 / 2018$ & $23: 58: 21$ & 356.02 & 5.55 & 93.0 & 1,705 & 1 & 1,388 & 1,357 & 1,727 \\
\hline $7 / 28 / 2018$ & $22: 33: 58$ & 355.04 & 6.44 & 89.1 & 1,743 & 0 & 1,377 & 1,340 & 1,712 \\
\hline $7 / 29 / 2018$ & $24: 00: 00$ & 354.72 & 7.34 & 89.9 & 2,040 & 0 & 1,370 & 1,324 & 1,704 \\
\hline $7 / 30 / 2018$ & $23: 47: 49$ & 354.24 & 8.28 & 93.2 & 2,082 & 1 & 1,361 & 1,310 & 1,691 \\
\hline $7 / 31 / 2018$ & $23: 59: 49$ & 353.84 & 9.09 & 90.5 & 2,244 & 1 & 1,360 & 1,319 & 1,691 \\
\hline $8 / 1 / 2018$ & $23: 49: 38$ & 353.14 & 8.16 & 91.6 & 2,081 & 1 & 1,365 & 1,314 & 1,697 \\
\hline $8 / 2 / 2018$ & $20: 42: 30$ & 349.11 & 8.76 & 90.8 & 1,858 & 1 & 1,316 & 1,237 & 1,634 \\
\hline $8 / 3 / 2018$ & $24: 00: 00$ & 330.86 & 16.02 & 92.9 & 2,887 & 1 & 1,292 & 1,217 & 1,603 \\
\hline $8 / 4 / 2018$ & $24: 00: 00$ & 324.06 & 16.58 & 90.8 & 2,915 & 1 & 1,282 & 1,200 & 1,591 \\
\hline $8 / 5 / 2018$ & $21: 57: 34$ & 324.81 & 13.78 & 73.1 & 2,432 & 1 & 1,340 & 1,310 & 1,665 \\
\hline $8 / 6 / 2018$ & $20: 10: 38$ & 348.91 & 5.63 & 65.1 & 1,461 & 2 & 1,345 & 1,308 & 1,671 \\
\hline $8 / 7 / 2018$ & $21: 37: 14$ & 348.6 & 6.53 & 66.7 & 1,720 & 0 & 1,364 & 1,332 & 1,696 \\
\hline $8 / 8 / 2018$ & $17: 23: 56$ & 347.87 & 8.31 & 67.3 & 1,539 & 2 & 1,319 & 1,260 & 1,637 \\
\hline $8 / 9 / 2018$ & $23: 49: 01$ & 322.87 & 10.95 & 67.3 & 2,271 & 0 & 1,370 & 1,364 & 1,704 \\
\hline $8 / 10 / 2018$ & $13: 21: 23$ & 348.16 & 7.82 & 62.8 & 1,143 & 1 & 1,361 & 676 & 1,691 \\
\hline $8 / 11 / 2018$ & $14: 36: 56$ & 417.92 & 11.68 & 62.8 & 1,670 & 0 & 1,344 & 1,296 & 1,670 \\
\hline $8 / 12 / 2018$ & $21: 02: 23$ & 345.62 & 10.06 & 80.5 & 2,019 & 0 & 1,332 & 1,275 & 1,655 \\
\hline $8 / 13 / 2018$ & $22: 37: 45$ & 342.19 & 9.91 & 81.8 & 2,162 & 1 & 1,317 & 1,252 & 1,635 \\
\hline $8 / 14 / 2018$ & $22: 03: 22$ & 335.99 & 11.46 & 78.4 & 2,242 & 1 & 1,334 & 1,298 & 1,657 \\
\hline $8 / 15 / 2018$ & $17: 39: 00$ & 343.92 & 9.18 & 79.3 & 1,602 & 1 & 1,338 & 1,300 & 1,663 \\
\hline $8 / 16 / 2018$ & $18: 53: 36$ & 343.05 & 9.66 & 84.9 & 1,761 & 1 & 1,341 & 1,306 & 1,667 \\
\hline
\end{tabular}




\begin{tabular}{|c|c|c|c|c|c|c|c|c|c|}
\hline $8 / 17 / 2018$ & $19: 28: 32$ & 343.08 & 9.61 & 84.4 & 1,806 & 1 & 1,333 & 1,286 & 1,655 \\
\hline $8 / 18 / 2018$ & 19:43:56 & 338.26 & 10.5 & 82.6 & 1,901 & 1 & 1,331 & 1,293 & 1,653 \\
\hline $8 / 19 / 2018$ & $20: 33: 32$ & 335.76 & 10.67 & 81.2 & 1,984 & 1 & 1,314 & 1,263 & 1,632 \\
\hline $8 / 20 / 2018$ & $22: 42: 34$ & 342.82 & 9.74 & 81.6 & 2,143 & 1 & 1,314 & 1,256 & 1,631 \\
\hline $8 / 21 / 2018$ & $21: 25: 02$ & 342.47 & 9.78 & 83.9 & 2,018 & 1 & 1,299 & 1,230 & 1,612 \\
\hline $8 / 22 / 2018$ & $22: 16: 30$ & 339.73 & 10.87 & 80.7 & 2,222 & 1 & 1,288 & 1,220 & 1,597 \\
\hline $8 / 23 / 2018$ & $22: 37: 38$ & 337.17 & 11.22 & 80.0 & 2,296 & 1 & 1,301 & 1,254 & 1,614 \\
\hline $8 / 24 / 2018$ & $20: 50: 15$ & 343.22 & 9.4 & 77.7 & 1,939 & 1 & 1,305 & 1,233 & 1,620 \\
\hline $8 / 25 / 2018$ & 18:38:09 & 342.22 & 8.74 & 59.5 & 1,677 & 1 & 1,274 & 1,219 & 1,579 \\
\hline $8 / 26 / 2018$ & $23: 59: 38$ & 339.25 & 11.29 & 60.1 & 2,528 & 1 & 1,289 & 1,240 & 1,600 \\
\hline $8 / 27 / 2018$ & $23: 44: 26$ & 345.18 & 8.77 & 79.7 & 2,154 & 0 & 1,243 & 1,109 & 1,541 \\
\hline $8 / 28 / 2018$ & $24: 00: 00$ & 345.14 & 14.96 & 68.4 & 2,907 & 0 & 1,177 & 984 & 1,455 \\
\hline $8 / 29 / 2018$ & $24: 00: 00$ & 334.65 & 23.69 & 87.0 & 3,553 & 1 & 1,142 & 912 & 1,410 \\
\hline $8 / 30 / 2018$ & $24: 00: 00$ & 321.96 & 25.93 & 86.0 & 3,634 & 1 & 1,114 & 869 & 1,374 \\
\hline $8 / 31 / 2018$ & $24: 00: 00$ & 305.6 & 30.2 & 87.2 & 3,797 & 1 & 1,093 & 867 & 1,347 \\
\hline $9 / 1 / 2018$ & $24: 00: 00$ & 305.78 & 29.13 & 71.3 & 3,802 & 1 & 1,075 & 841 & 1,324 \\
\hline $9 / 2 / 2018$ & $24: 00: 00$ & 312.72 & 27.69 & 68.4 & 3,791 & 1 & 1,109 & 1,025 & 1,367 \\
\hline $9 / 3 / 2018$ & $24: 00: 00$ & 308.33 & 21.72 & 69.8 & 3,062 & 2 & 1,162 & 1,120 & 1,435 \\
\hline $9 / 4 / 2018$ & $24: 00: 00$ & 345.11 & 9 & 67.4 & 2,244 & 0 & 1,164 & 1,109 & 1,438 \\
\hline $9 / 5 / 2018$ & $23: 59: 19$ & 345.38 & 9.17 & 70.8 & 2,251 & 0 & 1,199 & 1,161 & 1,483 \\
\hline 9/6/2018 & $23: 59: 45$ & 345.26 & 6.06 & 89.1 & 1,777 & 1 & 1,196 & 1,153 & 1,479 \\
\hline $9 / 7 / 2018$ & $24: 00: 00$ & 345.49 & 7.63 & 69.0 & 2,049 & 0 & 1,178 & 1,116 & 1,457 \\
\hline $9 / 8 / 2018$ & $24: 00: 00$ & 345 & 10.43 & 83.9 & 2,387 & 0 & 1,138 & 1,039 & 1,405 \\
\hline $9 / 9 / 2018$ & $24: 00: 00$ & 345.37 & 14.97 & 52.9 & 2,975 & 1 & 1,115 & 1,035 & 1,376 \\
\hline $9 / 10 / 2018$ & $24: 00: 00$ & 344.1 & 16.75 & 55.8 & 3,130 & 2 & 1,271 & 1,270 & 1,617 \\
\hline $9 / 11 / 2018$ & $0: 45: 21$ & 346.03 & 10.16 & 57.1 & 76 & 0 & 1,327 & 1,325 & 1,648 \\
\hline $9 / 12 / 2018$ & $0: 00: 00$ & -1.51 & 0 & 66.3 & 0 & 0 & 1,362 & 1,361 & 1,694 \\
\hline $9 / 13 / 2018$ & $0: 00: 00$ & -1.49 & 0 & 71.6 & 0 & 0 & 1,389 & 1,388 & 1,728 \\
\hline $9 / 14 / 2018$ & $0: 00: 00$ & -1.39 & 0 & 78.9 & 0 & 0 & 1,412 & 1,411 & 1,758 \\
\hline $9 / 15 / 2018$ & $0: 00: 00$ & -1.44 & 0 & 79.4 & 0 & 0 & 1,430 & 1,429 & 1,781 \\
\hline $9 / 16 / 2018$ & $0: 00: 00$ & -1.55 & 0 & 75.9 & 0 & 0 & 1,447 & 1,446 & 1,803 \\
\hline $9 / 17 / 2018$ & $0: 00: 00$ & -1.54 & 0 & 76.2 & 0 & 0 & 1,462 & 1,461 & 1,823 \\
\hline $9 / 18 / 2018$ & $0: 00: 00$ & -1.57 & 0 & 71.5 & 0 & 0 & 1,475 & 1,475 & 1,840 \\
\hline $9 / 19 / 2018$ & $0: 00: 00$ & -1.55 & 0 & 68.0 & 0 & 0 & 1,487 & 2 & 1,855 \\
\hline $9 / 20 / 2018$ & $0: 00: 00$ & -1.55 & 0 & 68.0 & 0 & 0 & 1,499 & 3 & 1,871 \\
\hline $9 / 21 / 2018$ & $0: 00: 00$ & -1.55 & 0 & 68.0 & 0 & 0 & 1,509 & 1 & 1,883 \\
\hline $9 / 22 / 2018$ & $0: 00: 00$ & -1.55 & 0 & 68.0 & 0 & 0 & 1,520 & 1 & 1,897 \\
\hline $9 / 23 / 2018$ & $0: 00: 00$ & -1.55 & 0 & 68.0 & 0 & 0 & 1,531 & 1 & 1,911 \\
\hline $9 / 24 / 2018$ & $0: 00: 00$ & -1.55 & 0 & 68.0 & 0 & 0 & 1,541 & 746 & 1,924 \\
\hline $9 / 25 / 2018$ & $0: 00: 00$ & -1.55 & 0 & 68.0 & 0 & 0 & 1,551 & 1,552 & 1,937 \\
\hline $9 / 26 / 2018$ & $0: 00: 00$ & -1.04 & 0 & -21.3 & 0 & 0 & 1,558 & 1,560 & 1,947 \\
\hline $9 / 27 / 2018$ & $0: 00: 00$ & 0 & 0 & -200.0 & 0 & 0 & 1,567 & 1,569 & 1,957 \\
\hline $9 / 28 / 2018$ & $0: 00: 00$ & 0 & 0 & -200.0 & 0 & 0 & 1,574 & 1,576 & 1,967 \\
\hline $9 / 29 / 2018$ & $0: 00: 00$ & 0 & 0 & -200.0 & 0 & 0 & 1,582 & 1,584 & 1,977 \\
\hline $9 / 30 / 2018$ & $0: 00: 00$ & 0 & 0 & -200.0 & 0 & 0 & 1,590 & 1,591 & 1,987 \\
\hline $10 / 1 / 2018$ & $0: 00: 00$ & 0 & 0 & -200.0 & 0 & 0 & 1,597 & 1,599 & 1,997 \\
\hline $10 / 2 / 2018$ & $0: 00: 00$ & 0 & 0 & -200.0 & 0 & 0 & 1,604 & 1,606 & 2,006 \\
\hline $10 / 3 / 2018$ & 0:00:00 & 0 & 0 & -200.0 & 0 & 0 & 1,611 & 1,612 & 2,015 \\
\hline $10 / 4 / 2018$ & $0: 00: 00$ & 0 & 0 & -200.0 & 0 & 0 & 1,617 & 1,619 & 2,022 \\
\hline $10 / 5 / 2018$ & $0: 00: 00$ & 0 & 0 & -200.0 & 0 & 0 & 1,623 & 1,625 & 2,031 \\
\hline $10 / 6 / 2018$ & 0:00:00 & 0 & 0 & -200.0 & 0 & 0 & 1,629 & 1,631 & 2,038 \\
\hline $10 / 7 / 2018$ & $0: 00: 00$ & 0 & 0 & -200.0 & 0 & 0 & 1,635 & 1,637 & 2,046 \\
\hline $10 / 8 / 2018$ & $0: 00: 00$ & 0 & 0 & -200.0 & 0 & 0 & 1,641 & 1,643 & 2,054 \\
\hline $10 / 9 / 2018$ & 0:00:00 & 0 & 0 & -200.0 & 0 & 0 & 1,647 & 1,649 & 2,061 \\
\hline $10 / 10 / 2018$ & $0: 00: 00$ & 0 & 0 & -200.0 & 0 & 0 & 1,653 & 1,654 & 2,069 \\
\hline $10 / 11 / 2018$ & 0:00:00 & -0.23 & 0 & -200.0 & 0 & 0 & 1,656 & 1,659 & 2,073 \\
\hline $10 / 12 / 2018$ & $0: 00: 00$ & -0.22 & 0 & -200.0 & 0 & 0 & 1,661 & 1,664 & 2,080 \\
\hline $10 / 13 / 2018$ & $0: 00: 00$ & -0.41 & 0 & -200.0 & 0 & 0 & 1,666 & 1,669 & 2,086 \\
\hline $10 / 14 / 2018$ & $0: 00: 00$ & -0.51 & 0 & -200.0 & 0 & 0 & 1,673 & 1,675 & 2,094 \\
\hline $10 / 15 / 2018$ & $0: 00: 00$ & -0.15 & 0 & -200.0 & 0 & 0 & 1,676 & 1,679 & 2,099 \\
\hline $10 / 16 / 2018$ & $0: 00: 00$ & -0.17 & 0 & -200.0 & 0 & 0 & 1,681 & 1,684 & 2,105 \\
\hline $10 / 17 / 2018$ & $0: 00: 00$ & 0.36 & 0 & -200.0 & 0 & 0 & 1,685 & 1,689 & 2,111 \\
\hline $10 / 18 / 2018$ & $0: 00: 00$ & 1.82 & 0 & -200.0 & 0 & 0 & 1,651 & 1,623 & 2,067 \\
\hline $10 / 19 / 2018$ & $22: 14: 11$ & 332.43 & 7.09 & -200.0 & 4,303 & 1 & 1,639 & 1,583 & 2,051 \\
\hline $10 / 20 / 2018$ & $24: 00: 00$ & 343.86 & 6.94 & -200.0 & 4,913 & 1 & 1,633 & 1,545 & 2,043 \\
\hline $10 / 21 / 2018$ & $24: 00: 00$ & 336.83 & 6.28 & -200.0 & 4,477 & 1 & 1,627 & 1,498 & 2,036 \\
\hline $10 / 22 / 2018$ & $24: 00: 00$ & 286.96 & 7.36 & -200.0 & 4,013 & 1 & 1,625 & 1,456 & 2,033 \\
\hline $10 / 23 / 2018$ & $24: 00: 00$ & 339.83 & 6.08 & -200.0 & 3,276 & 1 & 1,622 & 1,425 & 2,029 \\
\hline $10 / 24 / 2018$ & $24: 00: 00$ & 328.02 & 6.14 & -200.0 & 3,116 & 1 & 1,621 & 1,387 & 2,027 \\
\hline $10 / 25 / 2018$ & $23: 59: 58$ & 306.24 & 6.23 & -200.0 & 2,775 & 1 & 1,621 & 1,367 & 2,027 \\
\hline $10 / 26 / 2018$ & $24: 00: 00$ & 329.13 & 5.59 & 57.3 & 1,318 & 0 & 1,621 & 1,364 & 2,028 \\
\hline $10 / 27 / 2018$ & $24: 00: 00$ & 297.28 & 5.86 & 60.0 & 1,264 & 1 & 1,623 & 1,328 & 2,030 \\
\hline $10 / 28 / 2018$ & $24: 00: 00$ & 269.26 & 5.86 & 60.0 & 1,198 & 1 & 1,625 & 1,300 & 2,033 \\
\hline $10 / 29 / 2018$ & $24: 00: 00$ & 256.9 & 5.58 & 60.0 & 1,145 & 1 & 1,626 & 1,283 & 2,035 \\
\hline $10 / 30 / 2018$ & $24: 00: 00$ & 224.02 & 6.1 & 60.0 & 1,125 & 1 & 1,633 & 1,617 & 2,044 \\
\hline $10 / 31 / 2018$ & $19: 13: 45$ & 250.4 & 6.03 & 60.0 & 856 & 0 & 1,570 & 1,511 & 1,962 \\
\hline $11 / 1 / 2018$ & $10: 16: 24$ & 346.06 & 17.83 & 60.0 & 932 & 1 & 1,627 & 489 & 2,035 \\
\hline $11 / 2 / 2018$ & $8: 00: 45$ & 342.69 & 9.17 & 60.0 & 519 & 0 & 1,626 & 1,622 & 2,034 \\
\hline $11 / 3 / 2018$ & $23: 59: 41$ & 320.78 & 3.05 & 60.0 & 945 & 1 & 1,628 & 1,624 & 2,036 \\
\hline $11 / 4 / 2018$ & $24: 00: 00$ & 272.09 & 4 & 60.0 & 993 & 1 & 1,655 & 1,656 & 2,072 \\
\hline $11 / 5 / 2018$ & $6: 13: 11$ & 304.84 & 4.34 & 60.0 & 277 & 0 & 1,625 & 1,594 & 2,032 \\
\hline $11 / 6 / 2018$ & $23: 59: 52$ & 344.31 & 7.88 & 60.0 & 1,564 & 0 & 1,615 & 1,585 & 2,020 \\
\hline
\end{tabular}




\begin{tabular}{|c|c|c|c|c|c|c|c|c|c|}
\hline $11 / 7 / 2018$ & $24: 00: 00$ & 341.41 & 8.9 & 60.0 & 1,678 & 1 & 1,608 & 1,573 & 2,011 \\
\hline $11 / 8 / 2018$ & $23: 59: 39$ & 314.13 & 10.82 & 60.0 & 1,717 & 1 & 1,573 & 1,531 & 1,966 \\
\hline $11 / 9 / 2018$ & $23: 58: 32$ & 341.44 & 21.46 & 60.0 & 2,599 & 1 & 1,556 & 1,512 & 1,943 \\
\hline $11 / 10 / 2018$ & $23: 59: 56$ & 344.08 & 21.19 & 60.0 & 2,593 & 1 & 1,542 & 1,500 & 1,926 \\
\hline $11 / 11 / 2018$ & 24:00:00 & 310.82 & 23.9 & 60.0 & 2,610 & 1 & 1,603 & 1,600 & 2,004 \\
\hline $11 / 12 / 2018$ & $8: 07: 00$ & 335.31 & 16.83 & 60.0 & 762 & 1 & 1,575 & 1,540 & 1,969 \\
\hline $11 / 13 / 2018$ & $17: 44: 43$ & 312.41 & 18.92 & 60.0 & 1,724 & 1 & 1,556 & 1,520 & 1,944 \\
\hline $11 / 14 / 2018$ & $24: 00: 00$ & 297.12 & 20.69 & 60.0 & 2,368 & 1 & 1,543 & 1,505 & 1,927 \\
\hline $11 / 15 / 2018$ & $24: 00: 00$ & 278.92 & 23.17 & 60.0 & 2,431 & 1 & 1,536 & 1,498 & 1,918 \\
\hline $11 / 16 / 2018$ & $23: 59: 06$ & 236.09 & 26.22 & 60.0 & 2,387 & 1 & 1,524 & 1,484 & 1,902 \\
\hline $11 / 17 / 2018$ & $24: 00: 00$ & 280.25 & 25.22 & 60.0 & 2,525 & 1 & 1,516 & 1,475 & 1,892 \\
\hline $11 / 18 / 2018$ & $24: 00: 00$ & 322.58 & 21.16 & 60.0 & 2,510 & 1 & 1,510 & 1,469 & 1,884 \\
\hline $11 / 19 / 2018$ & $23: 59: 26$ & 341.21 & 19.89 & 60.0 & 2,503 & 1 & 1,504 & 1,463 & 1,876 \\
\hline $11 / 20 / 2018$ & $23: 59: 49$ & 337.97 & 19.88 & 60.0 & 2,490 & 1 & 1,498 & 1,458 & 1,869 \\
\hline $11 / 21 / 2018$ & $24: 00: 00$ & 272.92 & 24.46 & 60.0 & 2,473 & 2 & 1,493 & 1,454 & 1,914 \\
\hline $11 / 22 / 2018$ & $24: 00: 00$ & 228.99 & 28.47 & 60.0 & 2,457 & 1 & 1,488 & 1,449 & 1,856 \\
\hline $11 / 23 / 2018$ & $24: 00: 00$ & 224.46 & 28.88 & 60.0 & 2,450 & 2 & 1,483 & 1,442 & 1,850 \\
\hline $11 / 24 / 2018$ & $24: 00: 00$ & 318.25 & 21.17 & 60.0 & 2,485 & 1 & 1,479 & 1,439 & 1,845 \\
\hline $11 / 25 / 2018$ & $24: 00: 00$ & 341.92 & 19.29 & 60.0 & 2,469 & 1 & 1,475 & 1,434 & 1,839 \\
\hline $11 / 26 / 2018$ & $24: 00: 00$ & 343.62 & 19.2 & 60.0 & 2,469 & 1 & 1,471 & 1,433 & 1,834 \\
\hline $11 / 27 / 2018$ & $24: 00: 00$ & 254.54 & 25.38 & 60.0 & 2,423 & 2 & 1,468 & 1,430 & 1,880 \\
\hline $11 / 28 / 2018$ & $24: 00: 00$ & 222.53 & 27.92 & 60.0 & 2,399 & 2 & 1,465 & 1,427 & 1,826 \\
\hline $11 / 29 / 2018$ & $24: 00: 00$ & 222.67 & 27.71 & 60.0 & 2,391 & 2 & 1,461 & 1,422 & 1,821 \\
\hline $11 / 30 / 2018$ & $24: 00: 00$ & 304.96 & 21.12 & 60.0 & 2,418 & 1 & 1,458 & 1,419 & 1,817 \\
\hline $12 / 1 / 2018$ & $24: 00: 00$ & 343.64 & 18.52 & 60.0 & 2,426 & 1 & 1,455 & 1,416 & 1,814 \\
\hline $12 / 2 / 2018$ & $24: 00: 00$ & 345.11 & 18.36 & 60.0 & 2,421 & 1 & 1,452 & 1,413 & 1,809 \\
\hline $12 / 3 / 2018$ & $24: 00: 00$ & 337.25 & 18.9 & 60.0 & 2,416 & 3 & 1,448 & 1,410 & 1,854 \\
\hline $12 / 4 / 2018$ & $24: 00: 00$ & 322.37 & 19.29 & 60.0 & 2,395 & 1 & 1,442 & 1,395 & 1,797 \\
\hline $12 / 5 / 2018$ & $24: 00: 00$ & 271.29 & 25.11 & 60.0 & 2,486 & 3 & 1,421 & 1,368 & 1,817 \\
\hline $12 / 6 / 2018$ & $24: 00: 00$ & 242.95 & 37.05 & 60.0 & 2,872 & 4 & 1,376 & 1,281 & 1,756 \\
\hline $12 / 7 / 2018$ & $24: 00: 00$ & 295.51 & 49.43 & 60.0 & 3,625 & 6 & 1,344 & 1,240 & 1,767 \\
\hline $12 / 8 / 2018$ & $24: 00: 00$ & 274.42 & 57.74 & 60.0 & 3,788 & 4 & 1,327 & 1,226 & 1,692 \\
\hline $12 / 9 / 2018$ & $24: 00: 00$ & 233.91 & 63.39 & 60.0 & 3,691 & 5 & 1,312 & 1,209 & 1,671 \\
\hline $12 / 10 / 2018$ & $24: 00: 00$ & 300.83 & 49.62 & 60.0 & 3,676 & 5 & 1,299 & 1,197 & 1,654 \\
\hline $12 / 11 / 2018$ & $24: 00: 00$ & 227.08 & 62.98 & 60.0 & 3,629 & 5 & 1,288 & 1,181 & 1,639 \\
\hline $12 / 12 / 2018$ & $24: 00: 00$ & 301.58 & 47.9 & 60.0 & 3,628 & 5 & 1,279 & 1,174 & 1,677 \\
\hline $12 / 13 / 2018$ & $24: 00: 00$ & 338.34 & 41.48 & 60.0 & 3,595 & 3 & 1,272 & 1,170 & 1,617 \\
\hline $12 / 14 / 2018$ & $24: 00: 00$ & 339.2 & 40.09 & 60.0 & 3,540 & 4 & 1,265 & 1,164 & 1,609 \\
\hline $12 / 15 / 2018$ & $24: 00: 00$ & 342.94 & 39.01 & 60.0 & 3,512 & 2 & 1,258 & 1,157 & 1,559 \\
\hline $12 / 16 / 2018$ & $24: 00: 00$ & 344.54 & 38.48 & 60.0 & 3,496 & 3 & 1,250 & 1,153 & 1,589 \\
\hline $12 / 17 / 2018$ & $24: 00: 00$ & 337.36 & 38.55 & 60.0 & 3,462 & 8 & 1,255 & 1,174 & 1,642 \\
\hline $12 / 18 / 2018$ & $24: 00: 00$ & 318 & 36.26 & 60.0 & 3,241 & 4 & 1,239 & 1,143 & 1,574 \\
\hline $12 / 19 / 2018$ & $24: 00: 00$ & 271.82 & 48.03 & 60.0 & 3,434 & 7 & 1,255 & 1,189 & 1,642 \\
\hline $12 / 20 / 2018$ & $24: 00: 00$ & 243.55 & 41.76 & 60.0 & 3,048 & 6 & 1,261 & 1,198 & 1,651 \\
\hline $12 / 21 / 2018$ & $24: 00: 00$ & 324.15 & 27.9 & 60.0 & 2,875 & 4 & 1,263 & 1,202 & 1,605 \\
\hline $12 / 22 / 2018$ & $24: 00: 00$ & 270.47 & 32.3 & 60.0 & 2,819 & 6 & 1,258 & 1,193 & 1,647 \\
\hline $12 / 23 / 2018$ & $24: 00: 00$ & 233.25 & 38.45 & 60.0 & 2,873 & 6 & 1,254 & 1,186 & 1,641 \\
\hline $12 / 24 / 2018$ & $24: 00: 00$ & 268.61 & 34.58 & 60.0 & 2,910 & 5 & 1,251 & 1,181 & 1,637 \\
\hline $12 / 25 / 2018$ & $24: 00: 00$ & 225.46 & 40.29 & 60.0 & 2,897 & 5 & 1,249 & 1,179 & 1,635 \\
\hline $12 / 26 / 2018$ & $24: 00: 00$ & 224.6 & 39.78 & 60.0 & 2,874 & 6 & 1,247 & 1,171 & 1,631 \\
\hline $12 / 27 / 2018$ & $24: 00: 00$ & 232.73 & 38.77 & 60.0 & 2,877 & 6 & 1,245 & 1,175 & 1,629 \\
\hline $12 / 28 / 2018$ & $24: 00: 00$ & 317.68 & 29.42 & 60.0 & 2,911 & 4 & 1,244 & 1,172 & 1,580 \\
\hline $12 / 29 / 2018$ & $23: 59: 56$ & 278.78 & 32.92 & 60.0 & 2,857 & 6 & 1,241 & 1,158 & 1,623 \\
\hline $12 / 30 / 2018$ & $24: 00: 00$ & 226.27 & 39.37 & 60.0 & 2,868 & 8 & 1,222 & 1,130 & 1,597 \\
\hline $12 / 31 / 2018$ & $24: 00: 00$ & 295.27 & 38.22 & 60.0 & 3,196 & 7 & 1,216 & 1,118 & 1,588 \\
\hline $1 / 1 / 2019$ & $24: 00: 00$ & 326.56 & 34.86 & 60.0 & 3,211 & 4 & 1,207 & 1,108 & 1,531 \\
\hline $1 / 2 / 2019$ & $24: 00: 00$ & 332.61 & 33.83 & 60.0 & 3,220 & 8 & 1,201 & 1,096 & 1,567 \\
\hline $1 / 3 / 2019$ & $24: 00: 00$ & 324.63 & 34.89 & 60.0 & 3,229 & 7 & 1,192 & 1,040 & 1,555 \\
\hline $1 / 4 / 2019$ & $24: 00: 00$ & 256.25 & 47.06 & 60.0 & 3,300 & 9 & 1,156 & 1,007 & 1,504 \\
\hline $1 / 5 / 2019$ & $24: 00: 00$ & 331.89 & 52 & 60.0 & 3,972 & 6 & 1,143 & 973 & 1,486 \\
\hline $1 / 6 / 2019$ & $24: 00: 00$ & 343.35 & 47.6 & 60.0 & 3,879 & 5 & 1,158 & 1,063 & 1,507 \\
\hline $1 / 7 / 2019$ & $24: 00: 00$ & 296.02 & 39.96 & 60.0 & 3,262 & 9 & 1,157 & 1,054 & 1,506 \\
\hline $1 / 8 / 2019$ & $24: 00: 00$ & 315.79 & 37.73 & 60.0 & 3,294 & 7 & 1,152 & 996 & 1,499 \\
\hline $1 / 9 / 2019$ & $24: 00: 00$ & 330.82 & 36.83 & 60.0 & 3,330 & 9 & 1,101 & 817 & 1,428 \\
\hline $1 / 10 / 2019$ & $24: 00: 00$ & 286.8 & 73.91 & 60.0 & 4,339 & 11 & 1,056 & 699 & 1,409 \\
\hline $1 / 11 / 2019$ & $24: 00: 00$ & 242.08 & 110.1 & 60.0 & 4,913 & 13 & 1,037 & 696 & 1,381 \\
\hline $1 / 12 / 2019$ & $24: 00: 00$ & 318.2 & 82.25 & 60.0 & 4,820 & 7 & 1,022 & 682 & 1,320 \\
\hline $1 / 13 / 2019$ & $24: 00: 00$ & 346.91 & 70.68 & 60.0 & 4,746 & 4 & 1,009 & 666 & 1,269 \\
\hline $1 / 14 / 2019$ & $24: 00: 00$ & 345.94 & 69.87 & 60.0 & 4,712 & 9 & 998 & 650 & 1,286 \\
\hline $1 / 15 / 2019$ & $24: 00: 00$ & 343.17 & 69.4 & 60.0 & 4,676 & 10 & 987 & 640 & 1,310 \\
\hline $1 / 16 / 2019$ & $24: 00: 00$ & 343.4 & 68.52 & 60.0 & 4,646 & 7 & 978 & 632 & 1,259 \\
\hline $1 / 17 / 2019$ & $24: 00: 00$ & 347.03 & 66.94 & 60.0 & 4,620 & 4 & 1,005 & 858 & 1,263 \\
\hline $1 / 18 / 2019$ & $24: 00: 00$ & 345.36 & 49.15 & 60.0 & 3,936 & 5 & 1,013 & 842 & 1,306 \\
\hline $1 / 19 / 2019$ & $24: 00: 00$ & 345.88 & 42.84 & 60.0 & 3,695 & 5 & 1,016 & 832 & 1,310 \\
\hline $1 / 20 / 2019$ & $24: 00: 00$ & 345.81 & 42.06 & 60.0 & 3,661 & 5 & 1,006 & 798 & 1,297 \\
\hline $1 / 21 / 2019$ & $24: 00: 00$ & 298.69 & 53.55 & 60.0 & 3,788 & 8 & 1,002 & 796 & 1,291 \\
\hline $1 / 22 / 2019$ & $24: 00: 00$ & 334.35 & 47.08 & 60.0 & 3,799 & 8 & 1,002 & 838 & 1,292 \\
\hline $1 / 23 / 2019$ & $24: 00: 00$ & 352.79 & 44.57 & 60.0 & 3,798 & 5 & 990 & 751 & 1,276 \\
\hline $1 / 24 / 2019$ & $24: 00: 00$ & 347.98 & 48.89 & 60.0 & 3,955 & 1 & 981 & 736 & 1,205 \\
\hline $1 / 25 / 2019$ & $24: 00: 00$ & 345.84 & 49.92 & 60.0 & 3,987 & 4 & 975 & 728 & 1,224 \\
\hline $1 / 26 / 2019$ & $24: 00: 00$ & 339.68 & 50.62 & 60.0 & 3,978 & 8 & 970 & 720 & 1,248 \\
\hline $1 / 27 / 2019$ & $24: 00: 00$ & 343.23 & 50.55 & 60.0 & 3,996 & 3 & 964 & 712 & 1,210 \\
\hline
\end{tabular}




\begin{tabular}{|c|c|c|c|c|c|c|c|c|c|}
\hline $1 / 28 / 2019$ & $24: 00: 00$ & 341.43 & 50.48 & 60.0 & 3,982 & 9 & 961 & 716 & 1,236 \\
\hline $1 / 29 / 2019$ & $24: 00: 00$ & 346.02 & 49.66 & 60.0 & 3,978 & 4 & 958 & 722 & 1,202 \\
\hline $1 / 30 / 2019$ & $24: 00: 00$ & 346.18 & 48.62 & 60.0 & 3,936 & 8 & 955 & 724 & 1,228 \\
\hline $1 / 31 / 2019$ & $24: 00: 00$ & 344.52 & 46.7 & 60.0 & 3,850 & 4 & 953 & 719 & 1,195 \\
\hline $2 / 1 / 2019$ & 24:00:00 & 346.37 & 46.43 & 60.0 & 3,848 & 6 & 1,091 & 82 & 1,415 \\
\hline $2 / 2 / 2019$ & $9: 13: 32$ & 405.3 & 39.68 & 60.0 & 1,467 & 1 & 1,173 & 57 & 1,450 \\
\hline $2 / 3 / 2019$ & $0: 00: 00$ & 32.04 & 0 & 60.0 & 0 & 0 & 1,206 & 375 & 1,492 \\
\hline $2 / 4 / 2019$ & $3: 33: 54$ & 321.57 & 29.48 & 60.0 & 414 & 1 & 1,132 & 954 & 1,397 \\
\hline $2 / 5 / 2019$ & $33: 19: 19$ & 291.72 & 49.6 & 60.0 & 4,439 & 4 & 1,066 & 890 & 1,343 \\
\hline $2 / 6 / 2019$ & 14:40:39 & 232.25 & 62.76 & 60.0 & 2,223 & 4 & 989 & 694 & 1,242 \\
\hline $2 / 7 / 2019$ & $26: 45: 30$ & 307.9 & 73.98 & 60.0 & 5,173 & 8 & 997 & 739 & 1,285 \\
\hline $2 / 8 / 2019$ & 20:52:41 & 299.74 & 54.66 & 75.6 & 2,959 & 10 & 930 & 387 & 1,193 \\
\hline $2 / 9 / 2019$ & $24: 00: 00$ & 244.35 & 115 & 82.6 & 4,536 & 9 & & & \#N/A \\
\hline $2 / 10 / 2019$ & $24: 00: 00$ & 240.64 & 113.1 & 83.4 & 4,474 & 0 & & & \#N/A \\
\hline $2 / 11 / 2019$ & $24: 00: 00$ & 252.5 & 83.69 & 78.2 & 3,956 & 0 & & & \#N/A \\
\hline $2 / 12 / 2019$ & $24: 00: 00$ & 325.91 & 73.61 & 84.7 & 4,187 & 2 & & & \#N/A \\
\hline $2 / 13 / 2019$ & $24: 00: 00$ & 331.98 & 72.07 & 84.8 & 4,179 & 2 & 877 & 446 & 1,095 \\
\hline $2 / 14 / 2019$ & $24: 00: 00$ & 285.01 & 85.25 & 83.2 & 4,191 & 3 & 884 & 541 & 1,104 \\
\hline $2 / 15 / 2019$ & $24: 00: 00$ & 345.69 & 60.12 & 83.0 & 3,917 & 4 & 879 & 520 & 1,098 \\
\hline $2 / 16 / 2019$ & $24: 00: 00$ & 323.21 & 62.59 & 81.9 & 3,846 & 10 & 874 & 518 & 1,147 \\
\hline $2 / 17 / 2019$ & $24: 00: 00$ & 288.89 & 70.1 & 81.0 & 3,858 & 12 & 873 & 521 & 1,145 \\
\hline $2 / 18 / 2019$ & $24: 00: 00$ & 313.2 & 62.94 & 82.3 & 3,802 & 11 & 864 & 473 & 1,133 \\
\hline $2 / 19 / 2019$ & $24: 00: 00$ & 236.22 & 85.07 & 79.7 & 3,865 & 14 & 852 & 434 & 1,117 \\
\hline $2 / 20 / 2019$ & $24: 00: 00$ & 328.55 & 66.28 & 84.6 & 3,990 & 14 & 853 & 476 & 1,117 \\
\hline $2 / 21 / 2019$ & $24: 00: 00$ & 344.42 & 59.16 & 85.3 & 3,870 & 9 & 848 & 474 & 1,083 \\
\hline $2 / 22 / 2019$ & $24: 00: 00$ & 345.1 & 57.89 & 85.0 & 3,833 & 11 & 845 & 473 & 1,106 \\
\hline $2 / 23 / 2019$ & $24: 00: 00$ & 345.16 & 57.04 & 84.5 & 3,808 & 11 & 842 & 473 & 1,102 \\
\hline $2 / 24 / 2019$ & $24: 00: 00$ & 345.66 & 56.19 & 84.5 & 3,783 & 9 & 836 & 448 & 1,066 \\
\hline $2 / 25 / 2019$ & $24: 00: 00$ & 329.75 & 59.33 & 83.6 & 3,784 & 16 & 832 & 439 & 1,088 \\
\hline $2 / 26 / 2019$ & $24: 00: 00$ & 314.92 & 62.87 & 83.9 & 3,786 & 15 & 825 & 414 & 1,079 \\
\hline $2 / 27 / 2019$ & $24: 00: 00$ & 288.5 & 69.73 & 82.9 & 3,811 & 15 & 826 & 463 & 1,080 \\
\hline $2 / 28 / 2019$ & $24: 00: 00$ & 341.17 & 55.73 & 85.7 & 3,729 & 10 & 823 & 462 & 1,076 \\
\hline $3 / 1 / 2019$ & $24: 00: 00$ & 344.23 & 54.05 & 85.0 & 3,698 & 13 & 820 & 449 & 1,071 \\
\hline $3 / 2 / 2019$ & $24: 00: 00$ & 334.24 & 55.99 & 85.2 & 3,705 & 15 & 817 & 448 & 1,066 \\
\hline $3 / 3 / 2019$ & $24: 00: 00$ & 335.66 & 55.43 & 85.3 & 3,695 & 14 & 811 & 415 & 1,059 \\
\hline $3 / 4 / 2019$ & $24: 00: 00$ & 305.57 & 62.34 & 83.2 & 3,719 & 15 & 807 & 411 & 1,053 \\
\hline $3 / 5 / 2019$ & $24: 00: 00$ & 297.66 & 63.8 & 83.8 & 3,711 & 14 & 800 & 389 & 1,043 \\
\hline $3 / 6 / 2019$ & $24: 00: 00$ & 234.45 & 82.24 & 81.1 & 3,784 & 14 & 796 & 386 & 1,037 \\
\hline $3 / 7 / 2019$ & $24: 00: 00$ & 231.33 & 82.67 & 82.5 & 3,765 & 14 & 795 & 424 & 1,036 \\
\hline $3 / 8 / 2019$ & $24: 00: 00$ & 258.31 & 73.37 & 84.3 & 3,709 & 13 & 792 & 404 & 1,032 \\
\hline $3 / 9 / 2019$ & $24: 00: 00$ & 239.5 & 77.31 & 83.5 & 3,698 & 14 & 797 & 458 & 1,039 \\
\hline $3 / 10 / 2019$ & $24: 00: 00$ & 339.04 & 51.33 & 86.2 & 3,564 & 11 & 796 & 449 & 1,037 \\
\hline $3 / 11 / 2019$ & $24: 00: 00$ & 340.46 & 50.11 & 86.9 & 3,533 & 10 & 792 & 421 & 1,007 \\
\hline $3 / 12 / 2019$ & $24: 00: 00$ & 313.09 & 56.74 & 85.8 & 3,564 & 13 & 785 & 392 & 1,022 \\
\hline $3 / 13 / 2019$ & $24: 00: 00$ & 243.3 & 74.32 & 83.7 & 3,643 & 15 & 789 & 442 & 1,027 \\
\hline $3 / 14 / 2019$ & $23: 33: 39$ & 324.2 & 54.43 & 87.6 & 3,501 & 13 & 790 & 453 & 1,029 \\
\hline $3 / 15 / 2019$ & $24: 00: 00$ & 346.88 & 48.29 & 88.7 & 3,499 & 8 & 786 & 434 & 998 \\
\hline $3 / 16 / 2019$ & $24: 00: 00$ & 328.84 & 51.8 & 87.7 & 3,498 & 12 & 777 & 391 & 1,011 \\
\hline $3 / 17 / 2019$ & $24: 00: 00$ & 239.01 & 74.41 & 85.7 & 3,617 & 15 & 775 & 391 & 1,008 \\
\hline $3 / 18 / 2019$ & $24: 00: 00$ & 245.21 & 71.88 & 86.2 & 3,594 & 13 & 771 & 389 & 1,003 \\
\hline $3 / 19 / 2019$ & $24: 00: 00$ & 233.72 & 74.96 & 85.9 & 3,592 & 13 & 768 & 389 & 999 \\
\hline $3 / 20 / 2019$ & 23:33:51 & 237.86 & 73.22 & 86.2 & 3,510 & 13 & 763 & 327 & 992 \\
\hline $3 / 21 / 2019$ & $24: 00: 00$ & 286.88 & 65.39 & 89.5 & 3,668 & 12 & 757 & 306 & 983 \\
\hline $3 / 22 / 2019$ & $24: 00: 00$ & 270.54 & 70.34 & 89.3 & 3,709 & 13 & 754 & 325 & 979 \\
\hline $3 / 23 / 2019$ & $24: 00: 00$ & 290.31 & 64.48 & 89.3 & 3,664 & 14 & 752 & 327 & 976 \\
\hline $3 / 24 / 2019$ & $24: 00: 00$ & 306.02 & 59.4 & 90.0 & 3,616 & 12 & 753 & 365 & 978 \\
\hline $3 / 25 / 2019$ & $23: 58: 43$ & 335.88 & 52.01 & 90.5 & 3,556 & 11 & 748 & 324 & 970 \\
\hline $3 / 26 / 2019$ & $24: 00: 00$ & 296.62 & 60.47 & 89.1 & 3,595 & 12 & 745 & 315 & 966 \\
\hline $3 / 27 / 2019$ & $24: 00: 00$ & 299.27 & 60.16 & 90.1 & 3,579 & 12 & 762 & 475 & 990 \\
\hline $3 / 28 / 2019$ & $23: 58: 52$ & 299.88 & 53.12 & 87.1 & 3,213 & 3 & 751 & 377 & 933 \\
\hline $3 / 29 / 2019$ & $24: 00: 02$ & 346.87 & 48.77 & 90.9 & 3,508 & 2 & 748 & 376 & 928 \\
\hline $3 / 30 / 2019$ & $24: 00: 00$ & 346.62 & 48.34 & 91.0 & 3,491 & 5 & 746 & 376 & 946 \\
\hline $3 / 31 / 2019$ & $24: 00: 00$ & 347.3 & 47.79 & 91.1 & 3,474 & 6 & 742 & 358 & 940 \\
\hline $4 / 1 / 2019$ & $24: 00: 00$ & 334.54 & 49.55 & 90.1 & 3,471 & 11 & 738 & 348 & 957 \\
\hline $4 / 2 / 2019$ & $24: 00: 00$ & 327.25 & 51.25 & 90.7 & 3,489 & 11 & 737 & 357 & 956 \\
\hline $4 / 3 / 2019$ & $24: 00: 00$ & 337.08 & 48.9 & 91.1 & 3,459 & 11 & 737 & 369 & 956 \\
\hline $4 / 4 / 2019$ & $24: 00: 00$ & 344.05 & 47.03 & 91.3 & 3,428 & 11 & 737 & 376 & 956 \\
\hline $4 / 5 / 2019$ & $24: 00: 00$ & 346.33 & 46.2 & 91.0 & 3,411 & 5 & 735 & 375 & 931 \\
\hline $4 / 6 / 2019$ & $24: 00: 00$ & 346.26 & 45.92 & 91.2 & 3,400 & 6 & 733 & 375 & 929 \\
\hline $4 / 7 / 2019$ & $24: 00: 00$ & 346.29 & 45.78 & 91.8 & 3,393 & 7 & 733 & 375 & 928 \\
\hline $4 / 8 / 2019$ & $24: 00: 00$ & 346.48 & 45.67 & 91.7 & 3,390 & 6 & 732 & 376 & 926 \\
\hline $4 / 9 / 2019$ & $24: 00: 00$ & 346.65 & 45.17 & 91.5 & 3,373 & 5 & 729 & 373 & 923 \\
\hline $4 / 10 / 2019$ & $24: 00: 00$ & 345.94 & 45 & 91.7 & 3,363 & 10 & 728 & 373 & 922 \\
\hline $4 / 11 / 2019$ & $24: 00: 00$ & 345.85 & 44.8 & 91.4 & 3,356 & 8 & 728 & 376 & 922 \\
\hline $4 / 12 / 2019$ & $24: 00: 00$ & 346.66 & 44.5 & 92.0 & 3,347 & 6 & 726 & 376 & 919 \\
\hline $4 / 13 / 2019$ & $24: 00: 00$ & 351.73 & 43.47 & 91.9 & 3,330 & 3 & 725 & 375 & 898 \\
\hline $4 / 14 / 2019$ & $23: 59: 52$ & 347 & 43.98 & 91.9 & 3,330 & 5 & 723 & 374 & 896 \\
\hline $4 / 15 / 2019$ & $24: 00: 00$ & 345.99 & 43.88 & 91.0 & 3,323 & 8 & 720 & 372 & 911 \\
\hline $4 / 16 / 2019$ & $24: 00: 00$ & 345.11 & 43.81 & 90.8 & 3,317 & 10 & 729 & 378 & 923 \\
\hline $4 / 17 / 2019$ & $22: 56: 30$ & 283.81 & 18.75 & 87.9 & 3,152 & 3 & 734 & 546 & 910 \\
\hline $4 / 18 / 2019$ & $24: 00: 00$ & 398.56 & 34.81 & 91.5 & 3,138 & 2 & 757 & 613 & 922 \\
\hline $4 / 19 / 2019$ & $24: 00: 00$ & 397.38 & 25.14 & 88.4 & 2,686 & 1 & 763 & 588 & 929 \\
\hline
\end{tabular}




\begin{tabular}{|c|c|c|c|c|c|c|c|c|c|}
\hline $4 / 20 / 2019$ & $24: 00: 00$ & 366.04 & 27.14 & 87.1 & 2,698 & 2 & 764 & 585 & 931 \\
\hline $4 / 21 / 2019$ & $24: 00: 00$ & 349.84 & 29.12 & 86.3 & 2,737 & 2 & 766 & 585 & 933 \\
\hline $4 / 22 / 2019$ & $24: 00: 00$ & 343.39 & 29.96 & 85.9 & 2,752 & 3 & 768 & 586 & 954 \\
\hline $4 / 23 / 2019$ & $24: 00: 00$ & 344.95 & 29.81 & 86.7 & 2,749 & 3 & 771 & 595 & 958 \\
\hline $4 / 24 / 2019$ & 24:00:00 & 355.31 & 28.96 & 87.2 & 2,745 & 2 & 772 & 589 & 940 \\
\hline $4 / 25 / 2019$ & $24: 00: 00$ & 345.99 & 29.83 & 86.7 & 2,755 & 1 & 774 & 591 & 943 \\
\hline $4 / 26 / 2019$ & $24: 00: 00$ & 349.92 & 29.34 & 86.4 & 2,747 & 2 & 773 & 587 & 941 \\
\hline $4 / 27 / 2019$ & $24: 00: 00$ & 341.07 & 30.05 & 85.5 & 2,748 & 5 & 773 & 592 & 961 \\
\hline $4 / 28 / 2019$ & $24: 00: 00$ & 343.75 & 29.94 & 86.1 & 2,752 & 4 & 773 & 603 & 961 \\
\hline $4 / 29 / 2019$ & $24: 00: 00$ & 320.55 & 31.92 & 85.2 & 2,736 & 6 & 783 & 624 & 995 \\
\hline $4 / 30 / 2019$ & $24: 00: 00$ & 345.15 & 26.77 & 86.6 & 2,607 & 2 & 795 & 668 & 989 \\
\hline $5 / 1 / 2019$ & $21: 26: 30$ & 345.69 & 25.66 & 85.8 & 2,282 & 2 & 807 & 705 & 984 \\
\hline $5 / 2 / 2019$ & $24: 00: 00$ & 345.85 & 21.2 & 84.0 & 2,330 & 1 & 813 & 703 & 992 \\
\hline $5 / 3 / 2019$ & $24: 00: 00$ & 352.79 & 20.33 & 84.5 & 2,301 & 2 & 818 & 709 & 1,018 \\
\hline $5 / 4 / 2019$ & $24: 00: 00$ & 362.88 & 19.91 & 84.4 & 2,304 & 2 & 821 & 707 & 1,002 \\
\hline $5 / 5 / 2019$ & $24: 00: 00$ & 346.07 & 20.93 & 83.3 & 2,317 & 2 & 823 & 711 & 1,004 \\
\hline $5 / 6 / 2019$ & $24: 00: 00$ & 345.23 & 21.13 & 82.7 & 2,327 & 1 & 929 & 200 & 1,138 \\
\hline $5 / 7 / 2019$ & 4:22:08 & 344.54 & 11.32 & 87.2 & 292 & 2 & 975 & 237 & 1,224 \\
\hline $5 / 8 / 2019$ & 1:53:49 & 344.18 & 25.95 & 67.3 & 195 & 0 & 988 & 989 & 1,214 \\
\hline $5 / 9 / 2019$ & 8:58:04 & 349.88 & 3.66 & 89.9 & 353 & 1 & 969 & 936 & 1,189 \\
\hline $5 / 10 / 2019$ & $22: 18: 08$ & 349.3 & 7.89 & 84.5 & 1,309 & 0 & 880 & 661 & 1,077 \\
\hline $5 / 11 / 2019$ & $24: 00: 00$ & 347.94 & 38.47 & 81.9 & 3,082 & 2 & 825 & 508 & 1,027 \\
\hline $5 / 12 / 2019$ & $24: 00: 00$ & 345.57 & 50.2 & 84.7 & 3,571 & 3 & 797 & 472 & 992 \\
\hline $5 / 13 / 2019$ & $24: 00: 00$ & 346.24 & 49.92 & 87.0 & 3,558 & 3 & 771 & 378 & 958 \\
\hline $5 / 14 / 2019$ & $24: 00: 00$ & 345.68 & 52.13 & 88.8 & 3,627 & 3 & 758 & 377 & 942 \\
\hline $5 / 15 / 2019$ & $24: 00: 00$ & 345.97 & 50.69 & 89.5 & 3,576 & 3 & 751 & 375 & 932 \\
\hline $5 / 16 / 2019$ & $24: 00: 00$ & 346.92 & 48.34 & 89.9 & 3,496 & 3 & 765 & 529 & 950 \\
\hline $5 / 17 / 2019$ & $24: 00: 00$ & 348.76 & 40.37 & 88.0 & 3,196 & 3 & 783 & 632 & 974 \\
\hline $5 / 18 / 2019$ & $24: 00: 00$ & 348.89 & 34.42 & 86.8 & 2,931 & 2 & 791 & 682 & 964 \\
\hline $5 / 19 / 2019$ & $24: 00: 00$ & 349.62 & 30.08 & 85.9 & 2,756 & 2 & 792 & 621 & 966 \\
\hline $5 / 20 / 2019$ & $22: 27: 34$ & 349.79 & 29.61 & 86.0 & 2,565 & 3 & 825 & 734 & 1,028 \\
\hline $5 / 21 / 2019$ & $20: 49: 34$ & 313.38 & 22.74 & 82.3 & 1,950 & 1 & 853 & 754 & 1,043 \\
\hline $5 / 22 / 2019$ & $24: 00: 00$ & 375.24 & 12.98 & 84.8 & 1,841 & 1 & 888 & 855 & 1,086 \\
\hline $5 / 23 / 2019$ & $24: 00: 00$ & 375.18 & 7.69 & 86.8 & 1,444 & 1 & 902 & 868 & 1,104 \\
\hline $5 / 24 / 2019$ & $24: 00: 00$ & 375.49 & 8.27 & 85.4 & 1,502 & 1 & 915 & 883 & 1,120 \\
\hline $5 / 25 / 2019$ & $24: 00: 00$ & 375.36 & 7.2 & 86.0 & 1,406 & 0 & 922 & 890 & 1,130 \\
\hline $5 / 26 / 2019$ & $24: 00: 00$ & 374.53 & 8.13 & 85.0 & 1,496 & 1 & 928 & 892 & 1,137 \\
\hline $5 / 27 / 2019$ & $24: 00: 00$ & 374.59 & 8.28 & 84.9 & 1,505 & 1 & 924 & 858 & 1,132 \\
\hline $5 / 28 / 2019$ & $24: 00: 00$ & 375.54 & 10.71 & 83.9 & 1,709 & 1 & 933 & 887 & 1,144 \\
\hline $5 / 29 / 2019$ & $24: 00: 00$ & 374.61 & 9.58 & 84.5 & 1,618 & 1 & 941 & 902 & 1,153 \\
\hline $5 / 30 / 2019$ & $24: 00: 00$ & 375.12 & 9.22 & 84.0 & 1,590 & 0 & 933 & 876 & 1,144 \\
\hline $5 / 31 / 2019$ & $24: 00: 00$ & 375.85 & 12.34 & 82.5 & 1,848 & 1 & 940 & 899 & 1,152 \\
\hline $6 / 1 / 2019$ & $24: 00: 00$ & 375.83 & 10.68 & 83.2 & 1,716 & 0 & 951 & 910 & 1,166 \\
\hline $6 / 2 / 2019$ & $24: 00: 00$ & 376.18 & 8.81 & 84.0 & 1,559 & 0 & 940 & 863 & 1,152 \\
\hline $6 / 3 / 2019$ & $24: 00: 00$ & 376.18 & 12.29 & 82.1 & 1,846 & 2 & 932 & 836 & 1,143 \\
\hline $6 / 4 / 2019$ & $24: 00: 00$ & 374.94 & 14.17 & 82.1 & 1,978 & 2 & 940 & 879 & 1,152 \\
\hline $6 / 5 / 2019$ & $24: 00: 00$ & 375.72 & 12.58 & 82.6 & 1,863 & 1 & 935 & 821 & 1,146 \\
\hline $6 / 6 / 2019$ & $24: 00: 00$ & 375.95 & 15.32 & 81.7 & 2,047 & 1 & 944 & 894 & 1,157 \\
\hline $6 / 7 / 2019$ & $24: 00: 00$ & 375.5 & 12.63 & 82.9 & 1,852 & 1 & 950 & 905 & 1,164 \\
\hline $6 / 8 / 2019$ & $24: 00: 00$ & 375.38 & 11.22 & 82.6 & 1,763 & 1 & 956 & 905 & 1,172 \\
\hline $6 / 9 / 2019$ & $24: 00: 00$ & 375.27 & 10.73 & 82.9 & 1,720 & 1 & 963 & 912 & 1,182 \\
\hline $6 / 10 / 2019$ & $24: 00: 00$ & 375.89 & 9.87 & 83.0 & 1,645 & 1 & 947 & 842 & 1,161 \\
\hline $6 / 11 / 2019$ & $24: 00: 00$ & 375.97 & 15.4 & 80.9 & 2,057 & 1 & 945 & 872 & 1,159 \\
\hline $6 / 12 / 2019$ & $24: 00: 00$ & 375.82 & 14.72 & 80.9 & 2,017 & 1 & 943 & 875 & 1,156 \\
\hline $6 / 13 / 2019$ & $24: 00: 00$ & 375.43 & 15.73 & 85.2 & 2,075 & 2 & 926 & 811 & 1,135 \\
\hline $6 / 14 / 2019$ & $24: 00: 00$ & 374.46 & 19.35 & 85.5 & 2,306 & 2 & 935 & 863 & 1,171 \\
\hline $6 / 15 / 2019$ & $24: 00: 00$ & 375.09 & 16.47 & 86.5 & 2,119 & 1 & 949 & 903 & 1,164 \\
\hline $6 / 16 / 2019$ & $24: 00: 00$ & 375.78 & 12.73 & 87.4 & 1,862 & 1 & 945 & 889 & 1,159 \\
\hline $6 / 17 / 2019$ & $37: 47: 56$ & 376.02 & 13.67 & 86.6 & 3,085 & 1 & 959 & 923 & 1,176 \\
\hline $6 / 18 / 2019$ & $10: 06: 56$ & 374.35 & 10.12 & 87.3 & 692 & 1 & 957 & 905 & 1,174 \\
\hline $6 / 19 / 2019$ & $24: 00: 00$ & 375.17 & 12.2 & 86.3 & 1,832 & 1 & 959 & 901 & 1,176 \\
\hline $6 / 20 / 2019$ & $24: 00: 00$ & 375.29 & 12.08 & 86.9 & 1,824 & 1 & 955 & 888 & 1,171 \\
\hline $6 / 21 / 2019$ & $24: 00: 00$ & 375.16 & 13.92 & 85.6 & 1,959 & 2 & 962 & 922 & 1,180 \\
\hline $6 / 22 / 2019$ & $24: 00: 00$ & 374.41 & 11.5 & 86.4 & 1,780 & 1 & 963 & 911 & 1,182 \\
\hline $6 / 23 / 2019$ & $24: 00: 00$ & 375.04 & 11.53 & 86.7 & 1,783 & 1 & 969 & 915 & 1,189 \\
\hline $6 / 24 / 2019$ & $24: 00: 00$ & 375.53 & 11.02 & 87.2 & 1,742 & 1 & 971 & 908 & 1,191 \\
\hline $6 / 25 / 2019$ & $24: 00: 00$ & 374.13 & 11.74 & 86.7 & 1,793 & 1 & 976 & 933 & 1,198 \\
\hline $6 / 26 / 2019$ & $24: 00: 00$ & 374.95 & 10.61 & 87.1 & 1,702 & 2 & 1,027 & 1,007 & 1,263 \\
\hline $6 / 27 / 2019$ & $14: 59: 30$ & 375.12 & 6.37 & 91.1 & 733 & 3 & 896 & 466 & 1,121 \\
\hline $6 / 28 / 2019$ & $24: 00: 00$ & 376.32 & 44.34 & 89.4 & 3,394 & 7 & 1,009 & 1,010 & 1,301 \\
\hline $6 / 29 / 2019$ & $12: 19: 34$ & 379.56 & 22.4 & 92.3 & 1,097 & 1 & 1,064 & 1,066 & 1,311 \\
\hline $6 / 30 / 2019$ & $0: 00: 00$ & 378.88 & 0 & 96.1 & 0 & 0 & 1,092 & 1,095 & 1,346 \\
\hline $7 / 1 / 2019$ & $0: 00: 00$ & 379.01 & 0 & 91.4 & 0 & 0 & 1,085 & 1,075 & 1,337 \\
\hline $7 / 2 / 2019$ & $14: 33: 11$ & 380.35 & 8.11 & 91.5 & 866 & 1 & 1,075 & 1,062 & 1,324 \\
\hline $7 / 3 / 2019$ & $20: 59: 15$ & 380.28 & 6.83 & 90.5 & 1,161 & 0 & 1,075 & 1,062 & 1,324 \\
\hline $7 / 4 / 2019$ & $23: 41: 00$ & 379.48 & 6.28 & 91.2 & 1,240 & 1 & 1,072 & 1,050 & 1,321 \\
\hline $7 / 5 / 2019$ & $24: 00: 00$ & 379.61 & 6.63 & 90.4 & 1,320 & 0 & 1,070 & 1,055 & 1,319 \\
\hline $7 / 6 / 2019$ & $23: 59: 08$ & 379.66 & 7.68 & 89.3 & 1,401 & 0 & 1,073 & 1,059 & 1,321 \\
\hline $7 / 7 / 2019$ & $23: 57: 26$ & 379.74 & 7.33 & 89.1 & 1,367 & 0 & 1,067 & 1,035 & 1,314 \\
\hline $7 / 8 / 2019$ & $24: 00: 00$ & 379.58 & 8.46 & 88.5 & 1,502 & 1 & 1,048 & 1,004 & 1,290 \\
\hline $7 / 9 / 2019$ & $24: 00: 00$ & 380.35 & 13.89 & 84.9 & 1,931 & 1 & 1,050 & 1,017 & 1,292 \\
\hline $7 / 10 / 2019$ & $24: 00: 00$ & 379.54 & 11.4 & 86.7 & 1,737 & 1 & 1,045 & 1,009 & 1,285 \\
\hline
\end{tabular}




\begin{tabular}{|c|c|c|c|c|c|c|c|c|c|}
\hline $7 / 11 / 2019$ & $24: 00: 00$ & 379.89 & 12.22 & 84.6 & 1,814 & 1 & 1,074 & 1,074 & 1,323 \\
\hline $7 / 12 / 2019$ & $15: 57: 45$ & 374.36 & 18.91 & 85.1 & 1,333 & 7 & 1,121 & 1,124 & 1,456 \\
\hline $7 / 13 / 2019$ & $1: 02: 56$ & 381.97 & 1.81 & 96.5 & 30 & 0 & 1,147 & 1,149 & 1,417 \\
\hline $7 / 14 / 2019$ & $0: 00: 00$ & 384.45 & 0 & 93.2 & 0 & 0 & 1,163 & 1,165 & 1,437 \\
\hline $7 / 15 / 2019$ & 1:01:52 & 381.94 & 9.09 & 108.7 & 64 & 0 & 1,178 & 1,180 & 1,457 \\
\hline $7 / 16 / 2019$ & $0: 17: 19$ & 379.22 & 1.27 & 101.1 & 7 & 0 & 1,192 & 1,194 & 1,474 \\
\hline $7 / 17 / 2019$ & $0: 00: 00$ & 383.67 & 0 & 88.1 & 0 & 0 & 1,203 & 1,205 & 1,489 \\
\hline $7 / 18 / 2019$ & $0: 00: 00$ & 383.66 & 0 & 85.3 & 0 & 0 & 1,214 & 1,216 & 1,503 \\
\hline $7 / 19 / 2019$ & 0:00:00 & 384.7 & 0 & 87.6 & 0 & 0 & 1,225 & 1,227 & 1,517 \\
\hline $7 / 20 / 2019$ & 0:00:00 & 384.32 & 0 & 90.6 & 0 & 0 & 1,233 & 1,235 & 1,527 \\
\hline $7 / 21 / 2019$ & $0: 00: 00$ & 383.6 & 0 & 91.6 & 0 & 0 & 1,241 & 1,243 & 1,538 \\
\hline $7 / 22 / 2019$ & $0: 00: 56$ & 379.41 & 1.13 & 100.7 & 0 & 0 & 1,249 & 1,251 & 1,547 \\
\hline $7 / 23 / 2019$ & $0: 00: 19$ & 379.16 & 1.1 & 79.2 & 0 & 0 & 1,256 & 1,259 & 1,557 \\
\hline $7 / 24 / 2019$ & $0: 00: 22$ & 379.85 & 1.09 & 80.3 & 0 & 0 & 1,263 & 1,266 & 1,566 \\
\hline $7 / 25 / 2019$ & $0: 06: 34$ & 378.9 & 1.13 & 85.2 & 3 & 0 & 1,265 & 1,258 & 1,568 \\
\hline $7 / 26 / 2019$ & $3: 42: 30$ & 381.03 & 11.63 & 85.5 & 249 & 1 & 1,259 & 1,259 & 1,560 \\
\hline $7 / 27 / 2019$ & $16: 06: 22$ & 379.49 & 2.19 & 94.9 & 513 & 1 & 1,258 & 1,259 & 1,560 \\
\hline $7 / 28 / 2019$ & $15: 33: 08$ & 380.09 & 2.44 & 94.7 & 530 & 0 & 1,253 & 1,250 & 1,553 \\
\hline $7 / 29 / 2019$ & $18: 28: 08$ & 380 & 2.59 & 93.7 & 642 & 0 & 1,248 & 1,238 & 1,547 \\
\hline $7 / 30 / 2019$ & $19: 28: 38$ & 379.94 & 4.69 & 91.2 & 876 & 1 & 1,237 & 1,219 & 1,533 \\
\hline $7 / 31 / 2019$ & 19:56:56 & 380.48 & 7.14 & 88.3 & 1,101 & 1 & 1,251 & 1,252 & 1,551 \\
\hline $8 / 1 / 2019$ & 15:11:11 & 380.19 & 4.07 & 91.6 & 637 & 1 & 1,277 & 1,279 & 1,584 \\
\hline $8 / 2 / 2019$ & 0:00:00 & 384.18 & 0 & 91.5 & 0 & 0 & 1,289 & 1,292 & 1,599 \\
\hline $8 / 3 / 2019$ & $0: 00: 00$ & 383.36 & 0 & 82.8 & 0 & 0 & 1,299 & 1,301 & 1,612 \\
\hline $8 / 4 / 2019$ & $0: 00: 00$ & 383.51 & 0 & 82.3 & 0 & 0 & 1,307 & 1,309 & 1,622 \\
\hline $8 / 5 / 2019$ & $0: 00: 00$ & 334.74 & 0 & 77.3 & 0 & 0 & 1,313 & 1,316 & 1,631 \\
\hline $8 / 6 / 2019$ & $0: 52: 26$ & 338.35 & 8.29 & 80.7 & 73 & 0 & 1,317 & 1,319 & 1,636 \\
\hline $8 / 7 / 2019$ & $3: 32: 15$ & 351.28 & 3.19 & 81.4 & 117 & 0 & 1,326 & 1,328 & 1,647 \\
\hline $8 / 8 / 2019$ & $0: 00: 00$ & 374.01 & 0 & 74.7 & 0 & 0 & 1,333 & 1,335 & 1,655 \\
\hline $8 / 9 / 2019$ & $0: 00: 00$ & 366.26 & 0 & 75.1 & 0 & 0 & 1,338 & 1,341 & 1,662 \\
\hline $8 / 10 / 2019$ & $0: 00: 00$ & 375.33 & 0 & 71.5 & 0 & 0 & 1,344 & 1,347 & 1,670 \\
\hline $8 / 11 / 2019$ & $0: 00: 00$ & 375.94 & 0 & 69.6 & 0 & 0 & 1,349 & 1,352 & 1,677 \\
\hline $8 / 12 / 2019$ & $0: 00: 00$ & 378.16 & 0 & 71.7 & 0 & 0 & 1,356 & 1,358 & 1,685 \\
\hline $8 / 13 / 2019$ & $0: 00: 00$ & 381.77 & 0 & 80.0 & 0 & 0 & 1,361 & 1,363 & 1,692 \\
\hline $8 / 14 / 2019$ & $0: 00: 00$ & 368.59 & 0 & 76.3 & 0 & 0 & 1,366 & 1,368 & 1,698 \\
\hline $8 / 15 / 2019$ & $0: 00: 00$ & 377.76 & 0 & 76.1 & 0 & 0 & 1,369 & 1,370 & 1,702 \\
\hline $8 / 16 / 2019$ & $1: 58: 19$ & 379.21 & 2.78 & 100.7 & 70 & 0 & 1,337 & 1,310 & 1,661 \\
\hline $8 / 17 / 2019$ & $23: 05: 26$ & 380.31 & 4.78 & 81.4 & 1,063 & 0 & 1,315 & 1,281 & 1,633 \\
\hline $8 / 18 / 2019$ & $24: 00: 00$ & 379.83 & 8.06 & 75.8 & 1,504 & 0 & 1,297 & 1,262 & 1,610 \\
\hline $8 / 19 / 2019$ & $24: 00: 00$ & 379.14 & 10.01 & 73.6 & 1,679 & 0 & 1,290 & 1,260 & 1,600 \\
\hline $8 / 20 / 2019$ & $24: 00: 00$ & 379.91 & 8.88 & 74.6 & 1,583 & 0 & 1,274 & 1,242 & 1,580 \\
\hline $8 / 21 / 2019$ & $24: 00: 00$ & 380.4 & 11.36 & 71.8 & 1,805 & 0 & 1,266 & 1,234 & 1,569 \\
\hline $8 / 22 / 2019$ & $24: 00: 00$ & 379.39 & 11.23 & 72.3 & 1,791 & 0 & 1,254 & 1,216 & 1,554 \\
\hline $8 / 23 / 2019$ & $24: 00: 00$ & 380.17 & 12.36 & 72.0 & 1,882 & 0 & 1,239 & 1,201 & 1,535 \\
\hline $8 / 24 / 2019$ & $24: 00: 00$ & 380.24 & 14.75 & 70.3 & 2,063 & 0 & 1,232 & 1,196 & 1,526 \\
\hline $8 / 25 / 2019$ & $24: 00: 00$ & 379.69 & 13.35 & 71.6 & 1,962 & 0 & 1,225 & 1,187 & 1,516 \\
\hline $8 / 26 / 2019$ & $24: 00: 00$ & 379.95 & 13.87 & 71.5 & 1,998 & 0 & 1,215 & 1,170 & 1,504 \\
\hline $8 / 27 / 2019$ & $24: 00: 00$ & 379.61 & 14.84 & 71.9 & 2,062 & 0 & 1,211 & 1,172 & 1,499 \\
\hline $8 / 28 / 2019$ & $24: 00: 00$ & 379.84 & 14.41 & 72.1 & 2,031 & 0 & 1,202 & 1,155 & 1,487 \\
\hline $8 / 29 / 2019$ & $24: 00: 00$ & 379.72 & 14.79 & 72.1 & 2,058 & 0 & 1,195 & 1,149 & 1,478 \\
\hline $8 / 30 / 2019$ & $24: 00: 00$ & 380.13 & 15.31 & 72.1 & 2,096 & 0 & 1,192 & 1,148 & 1,475 \\
\hline $8 / 31 / 2019$ & $24: 00: 00$ & 379.15 & 14.66 & 73.0 & 2,047 & 0 & 1,186 & 1,140 & 1,466 \\
\hline $9 / 1 / 2019$ & $24: 00: 00$ & 379.88 & 14.87 & 72.9 & 2,063 & 0 & 1,185 & 1,141 & 1,466 \\
\hline $9 / 2 / 2019$ & $24: 00: 00$ & 379.83 & 13.23 & 73.7 & 1,944 & 0 & 1,197 & 1,159 & 1,480 \\
\hline $9 / 3 / 2019$ & $24: 00: 00$ & 380.05 & 10.19 & 75.3 & 1,656 & 0 & 1,199 & 1,165 & 1,483 \\
\hline $9 / 4 / 2019$ & $24: 00: 00$ & 379.45 & 10.65 & 75.1 & 1,730 & 0 & 1,189 & 1,152 & 1,471 \\
\hline $9 / 5 / 2019$ & $24: 00: 00$ & 379.78 & 12.92 & 73.5 & 1,915 & 0 & 1,190 & 1,152 & 1,472 \\
\hline $9 / 6 / 2019$ & $24: 00: 00$ & 380.54 & 11.54 & 72.0 & 1,810 & 0 & 1,166 & 1,109 & 1,441 \\
\hline $9 / 7 / 2019$ & $24: 00: 00$ & 380.47 & 18.12 & 68.7 & 2,290 & 0 & 1,172 & 1,134 & 1,448 \\
\hline $9 / 8 / 2019$ & $24: 00: 00$ & 380.07 & 13.85 & 69.9 & 1,992 & 0 & 1,173 & 1,135 & 1,449 \\
\hline $9 / 9 / 2019$ & $24: 00: 00$ & 380 & 12.82 & 68.8 & 1,918 & 0 & 1,195 & 1,166 & 1,478 \\
\hline $9 / 10 / 2019$ & $23: 58: 34$ & 379.72 & 7.35 & 71.1 & 1,415 & 0 & 1,223 & 1,219 & 1,514 \\
\hline $9 / 11 / 2019$ & $15: 39: 52$ & 334.74 & 8.06 & 72.7 & 864 & 0 & 1,222 & 1,183 & 1,513 \\
\hline $9 / 12 / 2019$ & $24: 00: 00$ & 380.31 & 4.88 & 74.9 & 1,160 & 0 & 1,227 & 1,214 & 1,520 \\
\hline $9 / 13 / 2019$ & $24: 00: 00$ & 379.65 & 5.28 & 74.8 & 1,198 & 0 & 1,230 & 1,211 & 1,523 \\
\hline $9 / 14 / 2019$ & $24: 00: 00$ & 379.77 & 4.89 & 76.5 & 1,162 & 0 & 1,231 & 1,206 & 1,525 \\
\hline $9 / 15 / 2019$ & $23: 56: 19$ & 379.55 & 4.72 & 77.0 & 1,108 & 0 & 1,218 & 1,187 & 1,508 \\
\hline $9 / 16 / 2019$ & $24: 00: 00$ & 380.13 & 7.88 & 74.2 & 1,497 & 0 & 1,246 & 1,239 & 1,543 \\
\hline $9 / 17 / 2019$ & $15: 25: 38$ & 354.38 & 6.82 & 57.3 & 799 & 0 & 1,226 & 1,193 & 1,518 \\
\hline $9 / 18 / 2019$ & $24: 00: 00$ & 380.31 & 7.04 & 73.7 & 1,399 & 0 & 1,211 & 1,168 & 1,499 \\
\hline $9 / 19 / 2019$ & $24: 00: 00$ & 379.62 & 10.46 & 71.8 & 1,724 & 0 & 1,203 & 1,165 & 1,489 \\
\hline $9 / 20 / 2019$ & $24: 00: 00$ & 380.08 & 11.22 & 71.4 & 1,788 & 0 & 1,197 & 1,155 & 1,480 \\
\hline $9 / 21 / 2019$ & $24: 00: 00$ & 380.09 & 11.73 & 71.8 & 1,833 & 0 & 1,193 & 1,157 & 1,476 \\
\hline $9 / 22 / 2019$ & $24: 00: 00$ & 379.55 & 11.89 & 72.1 & 1,840 & 0 & 1,214 & 1,198 & 1,503 \\
\hline $9 / 23 / 2019$ & $23: 16: 41$ & 377.66 & 7.36 & 75.7 & 1,363 & 0 & 1,228 & 1,212 & 1,520 \\
\hline $9 / 24 / 2019$ & $22: 53: 08$ & 379.51 & 4.72 & 76.0 & 1,061 & 0 & 1,214 & 1,181 & 1,503 \\
\hline $9 / 25 / 2019$ & $24: 00: 00$ & 379.76 & 8.1 & 72.9 & 1,515 & 0 & 1,207 & 1,175 & 1,494 \\
\hline $9 / 26 / 2019$ & $24: 00: 00$ & 379.61 & 9.47 & 72.9 & 1,642 & 0 & 1,199 & 1,164 & 1,483 \\
\hline $9 / 27 / 2019$ & $28: 25: 37$ & 376.93 & 6.99 & 76.7 & 2,000 & 0 & 1,219 & 1,189 & 1,509 \\
\hline $9 / 28 / 2019$ & $18: 53: 56$ & 379.7 & 5.18 & 77.4 & 919 & 0 & 1,225 & 1,203 & 1,517 \\
\hline $9 / 29 / 2019$ & $23: 27: 15$ & 379.89 & 6.44 & 76.5 & 1,296 & 0 & 1,228 & 1,207 & 1,521 \\
\hline $9 / 30 / 2019$ & 23:13:11 & 379.55 & 7.42 & 75.9 & 1,348 & 0 & 1,217 & 1,189 & 1,506 \\
\hline
\end{tabular}




\begin{tabular}{|c|c|c|c|c|c|c|c|c|c|}
\hline $10 / 1 / 2019$ & $24: 00: 00$ & 379.87 & 8.6 & 73.1 & 1,560 & 0 & 1,218 & 1,189 & 1,507 \\
\hline $10 / 2 / 2019$ & $24: 00: 00$ & 379.91 & 7.3 & 75.1 & 1,437 & 0 & 1,226 & 1,184 & 1,518 \\
\hline $10 / 3 / 2019$ & $23: 59: 56$ & 380.63 & 5.99 & 76.3 & 1,296 & 0 & 1,200 & 1,139 & 1,484 \\
\hline $10 / 4 / 2019$ & $24: 00: 00$ & 379.44 & 11.97 & 72.5 & 1,814 & 0 & 1,190 & 1,144 & 1,471 \\
\hline $10 / 5 / 2019$ & $24: 00: 00$ & 379.61 & 13.11 & 69.5 & 1,937 & 0 & 1,210 & 1,183 & 1,497 \\
\hline $10 / 6 / 2019$ & $24: 00: 00$ & 379.97 & 7.81 & 73.2 & 1,468 & 0 & 1,185 & 1,112 & 1,465 \\
\hline $10 / 7 / 2019$ & $23: 46: 52$ & 378.79 & 9.03 & 69.3 & 2,009 & 1 & 1,210 & 1,174 & 1,497 \\
\hline $10 / 8 / 2019$ & $23: 49: 19$ & 379.58 & 6.3 & 63.5 & 1,327 & 0 & 1,204 & 1,169 & 1,489 \\
\hline $10 / 9 / 2019$ & $24: 00: 00$ & 380.23 & 9.12 & 61.2 & 1,624 & 0 & 1,201 & 1,164 & 1,485 \\
\hline $10 / 10 / 2019$ & $24: 00: 00$ & 379.79 & 9.16 & 61.3 & 1,614 & 0 & 1,202 & 1,165 & 1,487 \\
\hline $10 / 11 / 2019$ & $24: 00: 00$ & 380.02 & 9.3 & 61.8 & 1,623 & 0 & 1,188 & 1,149 & 1,470 \\
\hline $10 / 12 / 2019$ & $24: 00: 00$ & 380.14 & 12.7 & 61.1 & 1,930 & 0 & 1,159 & 1,094 & 1,432 \\
\hline $10 / 13 / 2019$ & $24: 00: 00$ & 379.5 & 18.5 & 59.7 & 2,328 & 0 & 1,163 & 1,045 & 1,437 \\
\hline $10 / 14 / 2019$ & $23: 41: 52$ & 379.57 & 17.88 & 66.9 & 2,093 & 2 & 1,157 & 1,121 & 1,429 \\
\hline $10 / 15 / 2019$ & $23: 58: 34$ & 379.96 & 13.54 & 63.9 & 2,063 & 1 & 1,171 & 1,136 & 1,447 \\
\hline $10 / 16 / 2019$ & $24: 00: 00$ & 380.21 & 9.6 & 74.5 & 1,644 & 0 & 1,145 & 965 & 1,413 \\
\hline $10 / 17 / 2019$ & $23: 55: 22$ & 379.84 & 22.83 & 72.0 & 2,365 & 1 & 1,196 & 1,177 & 1,479 \\
\hline $10 / 18 / 2019$ & $19: 49: 52$ & 379.73 & 4.25 & 78.1 & 857 & 1 & 1,198 & 1,166 & 1,482 \\
\hline $10 / 19 / 2019$ & $23: 56: 45$ & 379.98 & 5.73 & 76.3 & 1,227 & 0 & 1,213 & 1,188 & 1,502 \\
\hline $10 / 20 / 2019$ & $22: 30: 19$ & 380.42 & 4.18 & 79.1 & 998 & 0 & 1,216 & 1,186 & 1,506 \\
\hline $10 / 21 / 2019$ & $23: 49: 41$ & 380.04 & 4.83 & 78.5 & 1,104 & 1 & 1,235 & 1,229 & 1,529 \\
\hline $10 / 22 / 2019$ & $19: 37: 49$ & 380.26 & 3.76 & 80.3 & 826 & 1 & 1,218 & 1,182 & 1,507 \\
\hline $10 / 23 / 2019$ & $23: 58: 08$ & 380.14 & 6.71 & 75.0 & 1,330 & 0 & 1,205 & 1,168 & 1,491 \\
\hline $10 / 24 / 2019$ & $24: 00: 00$ & 379.61 & 8.68 & 71.4 & 1,547 & 0 & 1,215 & 1,158 & 1,504 \\
\hline $10 / 25 / 2019$ & $23: 53: 19$ & 380.07 & 7.67 & 74.1 & 1,328 & 1 & 1,177 & 1,052 & 1,455 \\
\hline $10 / 26 / 2019$ & $23: 07: 15$ & 379.62 & 17.65 & 71.7 & 1,897 & 0 & 1,192 & 1,165 & 1,475 \\
\hline $10 / 27 / 2019$ & 24:00:00 & 380.27 & 13.04 & 71.3 & 1,856 & 3 & 1,198 & 1,164 & 1,519 \\
\hline $10 / 28 / 2019$ & $24: 00: 00$ & 379.77 & 8.18 & 72.2 & 1,508 & 2 & 1,212 & 1,180 & 1,500 \\
\hline $10 / 29 / 2019$ & $23: 17: 11$ & 380.66 & 6.01 & 74.8 & 1,201 & 1 & 1,211 & 1,178 & 1,498 \\
\hline $10 / 30 / 2019$ & $24: 00: 00$ & 379.84 & 7.15 & 72.8 & 1,417 & 1 & 1,215 & 1,191 & 1,504 \\
\hline $10 / 31 / 2019$ & $24: 00: 00$ & 380.8 & 7.08 & 72.1 & 1,413 & 1 & 1,206 & 1,155 & 1,492 \\
\hline $11 / 1 / 2019$ & $24: 00: 00$ & 380.16 & 9.27 & 71.1 & 1,543 & 1 & 1,187 & 1,148 & 1,468 \\
\hline $11 / 2 / 2019$ & $24: 00: 00$ & 379.85 & 14.12 & 67.6 & 1,993 & 1 & 1,215 & 1,186 & 1,504 \\
\hline $11 / 3 / 2019$ & $24: 00: 00$ & 380.06 & 5.32 & 73.4 & 1,184 & 1 & 1,215 & 1,178 & 1,504 \\
\hline $11 / 4 / 2019$ & $23: 59: 49$ & 379.85 & 5.51 & 72.8 & 1,220 & 1 & 1,230 & 1,212 & 1,523 \\
\hline $11 / 5 / 2019$ & $23: 53: 26$ & 380.27 & 4.01 & 73.5 & 1,044 & 1 & 1,221 & 1,196 & 1,512 \\
\hline $11 / 6 / 2019$ & 21:48:30 & 379.7 & 7.31 & 70.4 & 1,309 & 0 & 1,256 & 1,253 & 1,556 \\
\hline $11 / 7 / 2019$ & $12: 06: 52$ & 379.45 & 2.05 & 75.3 & 375 & 0 & 1,197 & 1,121 & 1,481 \\
\hline $11 / 8 / 2019$ & $23: 14: 08$ & 379.12 & 16.02 & 66.1 & 2,015 & 2 & 1,152 & 997 & 1,457 \\
\hline $11 / 9 / 2019$ & $18: 51: 26$ & 379.14 & 31.88 & 63.6 & 2,343 & 3 & 1,128 & 1,035 & 1,425 \\
\hline $11 / 10 / 2019$ & $24: 00: 00$ & 380.32 & 28.87 & 66.0 & 2,900 & 3 & 1,109 & 1,008 & 1,400 \\
\hline $11 / 11 / 2019$ & $36: 33: 11$ & 380.06 & 23.16 & 68.7 & 4,020 & 3 & 1,096 & 928 & 1,383 \\
\hline $11 / 12 / 2019$ & $11: 26: 34$ & 378.4 & 33.8 & 67.1 & 1,474 & 5 & 1,020 & 680 & 1,283 \\
\hline $11 / 13 / 2019$ & $0: 00: 00$ & 379.92 & 55.06 & 71.4 & 3,474 & 9 & 999 & 737 & 1,288 \\
\hline $11 / 14 / 2019$ & $24: 00: 00$ & 379.88 & 50.3 & 71.0 & 3,802 & 7 & 1,004 & 881 & 1,295 \\
\hline
\end{tabular}

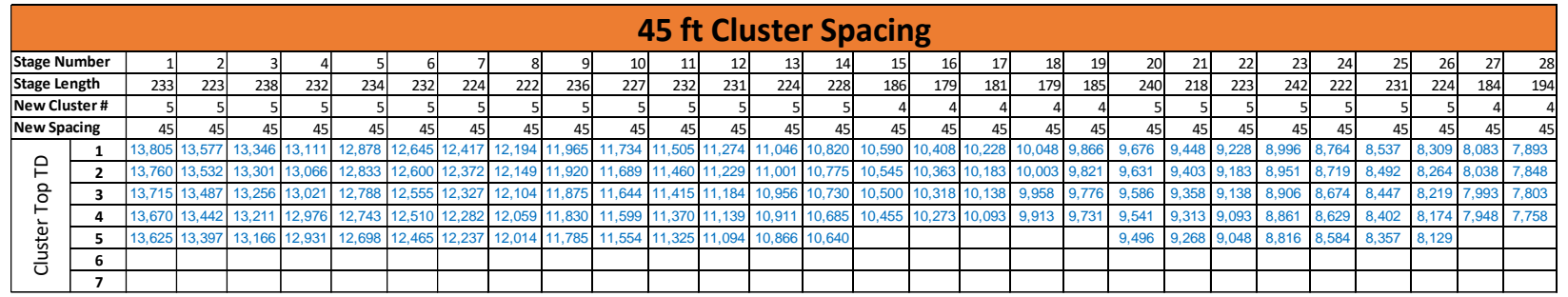

Figure 39: Case Study 1 - 45 ft Cluster Spacing Design

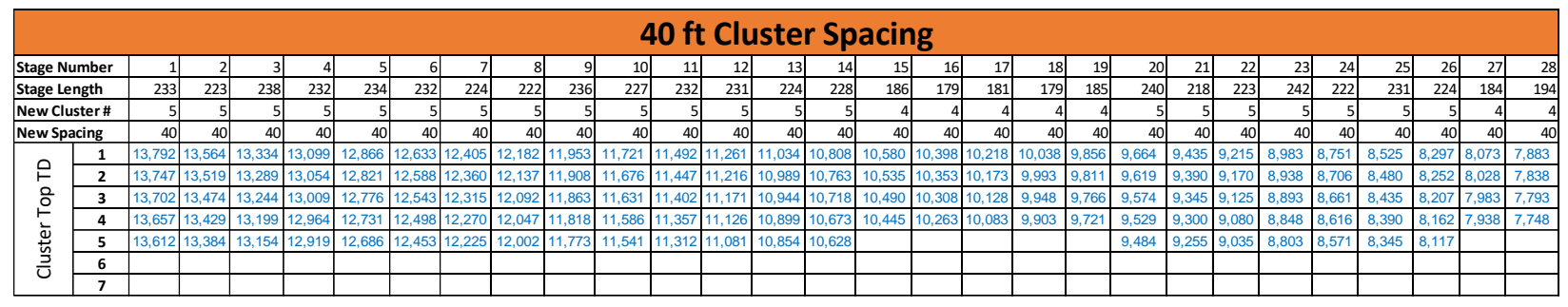

Figure 40: Case Study 1 - 40 ft Cluster Spacing Design 


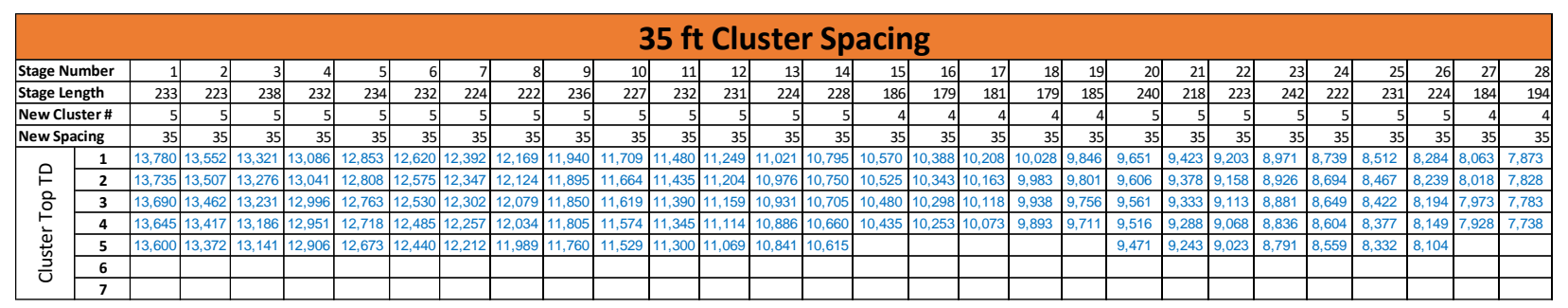

Figure 41: Case Study 1 - 35 ft Cluster Spacing Design

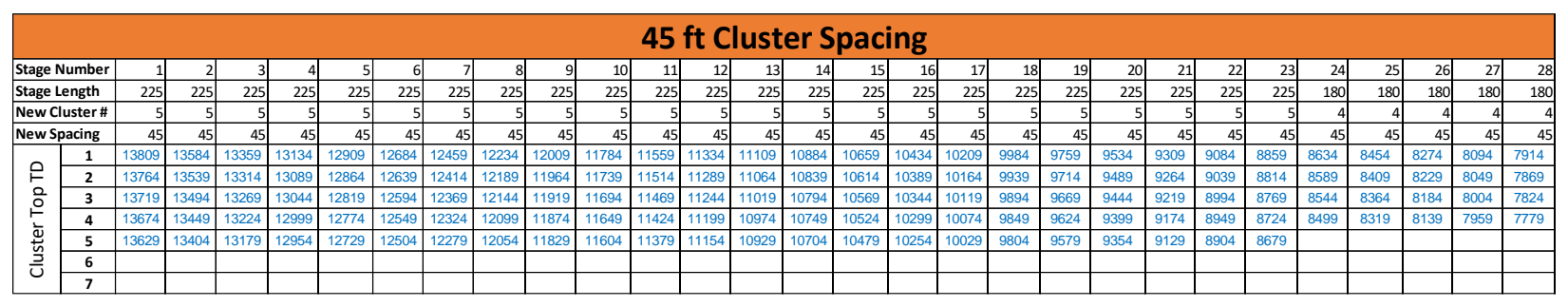

Figure 42: Case Study 2 - 45 ft Cluster Spacing Design

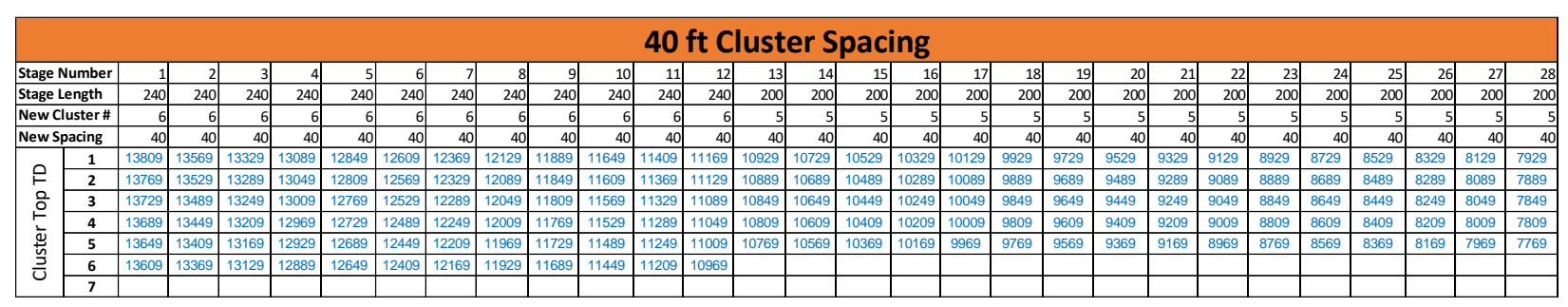

Figure 43: Case Study 2 - 40 ft Cluster Spacing Design

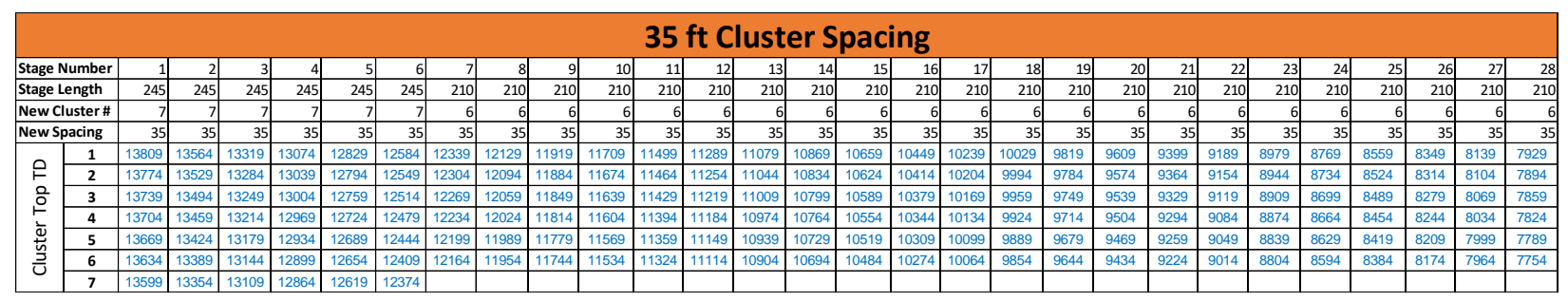

Figure 44: Case Study 2 - 35 ft Cluster Spacing Design

Table 137: Case Study 1 - 45 ft Cluster Spacing Fracture Results

\begin{tabular}{|c|c|c|c|c|c|c|c|c|}
\hline Stage Number & Fracture & $\begin{array}{l}\text { Proppant } \\
\text { Cutoff } \\
\text { Length } \\
\text { (ft) }\end{array}$ & $\begin{array}{c}\text { Est Flowing } \\
\text { Frac Length } \\
\text { (ft) }\end{array}$ & $\begin{array}{c}\text { Est Inf } \\
\text { Conductivity } \\
\text { Length } \\
\text { (ft) }\end{array}$ & $\begin{array}{c}\begin{array}{c}\text { Fracture } \\
\text { Height } \\
\text { (ft) }\end{array} \\
\end{array}$ & $\begin{array}{c}\text { Average } \\
\text { Proppant } \\
\text { Conc } \\
\left(\mathrm{lb} / \mathrm{ft}^{2}\right)\end{array}$ & $\begin{array}{l}\text { Average } \\
\text { Fracture } \\
\text { Width } \\
\text { (in) }\end{array}$ & $\begin{array}{c}\text { Max } \\
\text { Fracture } \\
\text { Width } \\
\text { (in) }\end{array}$ \\
\hline \multirow{5}{*}{1} & Transverse 5 & 120 & 10.32 & 10.27 & 155 & 0.360 & 0.322 & 0.439 \\
\hline & Transverse 4 & 40 & 7.20 & 7.13 & 110 & 0.072 & 0.030 & 0.079 \\
\hline & Transverse 3 & 80 & 9.50 & 9.43 & 120 & 0.183 & 0.174 & 0.402 \\
\hline & Transverse 2 & 40 & 7.48 & 7.40 & 110 & 0.072 & 0.027 & 0.079 \\
\hline & Transverse 1 & 100 & 10.28 & 10.23 & 155 & 0.359 & 0.314 & 0.432 \\
\hline \multirow{5}{*}{2} & Transverse 5 & 100 & 10.53 & 10.47 & 140 & 0.357 & 0.311 & 0.433 \\
\hline & Transverse 4 & 60 & 8.75 & 8.64 & 110 & 0.145 & 0.054 & 0.305 \\
\hline & Transverse 3 & 80 & 9.91 & 9.84 & 85 & 0.216 & 0.165 & 0.370 \\
\hline & Transverse 2 & 120 & 9.16 & 9.07 & 75 & 0.125 & 0.071 & 0.345 \\
\hline & Transverse 1 & 100 & 10.61 & 10.56 & 45 & 0.220 & 0.237 & 0.384 \\
\hline \multirow{5}{*}{3} & Transverse 5 & 100 & 10.97 & 10.92 & 155 & 0.455 & 0.329 & 0.446 \\
\hline & Transverse 4 & 40 & 5.47 & 5.44 & 60 & 0.168 & 0.017 & 0.239 \\
\hline & Transverse 3 & 80 & 10.01 & 9.94 & 125 & 0.179 & 0.180 & 0.391 \\
\hline & Transverse 2 & 100 & 9.23 & 9.15 & 90 & 0.255 & 0.082 & 0.294 \\
\hline & Transverse 1 & 140 & 10.63 & 10.58 & 40 & 0.344 & 0.219 & 0.367 \\
\hline 4 & Transverse 5 & 100 & 11.16 & 11.12 & 175 & 0.439 & 0.340 & 0.469 \\
\hline
\end{tabular}




\begin{tabular}{|c|c|c|c|c|c|c|c|c|}
\hline & Transverse 4 & 80 & 8.15 & 8.05 & 115 & 0.120 & 0.044 & 0.224 \\
\hline & Transverse 3 & 80 & 9.83 & 9.77 & 100 & 0.154 & 0.139 & 0.369 \\
\hline & Transverse 2 & 80 & 10.17 & 10.12 & 60 & 0.110 & 0.147 & 0.317 \\
\hline & Transverse 1 & 60 & 10.91 & 10.86 & 20 & 0.139 & 0.210 & 0.393 \\
\hline \multirow{5}{*}{5} & Transverse 5 & 120 & 10.65 & 10.59 & 145 & 0.389 & 0.307 & 0.435 \\
\hline & Transverse 4 & 60 & 8.91 & 8.81 & 110 & 0.107 & 0.073 & 0.376 \\
\hline & Transverse 3 & 60 & 10.04 & 9.97 & 90 & 0.246 & 0.176 & 0.406 \\
\hline & Transverse 2 & 80 & 9.89 & 9.82 & 50 & 0.148 & 0.136 & 0.291 \\
\hline & Transverse 1 & 100 & 10.23 & 10.17 & 30 & 0.400 & 0.218 & 0.420 \\
\hline \multirow{5}{*}{6} & Transverse 5 & 120 & 11.16 & 11.12 & 155 & 0.501 & 0.322 & 0.439 \\
\hline & Transverse 4 & 60 & 9.26 & 9.17 & 110 & 0.120 & 0.070 & 0.358 \\
\hline & Transverse 3 & 100 & 10.01 & 9.95 & 105 & 0.209 & 0.174 & 0.366 \\
\hline & Transverse 2 & 80 & 7.69 & 7.63 & 90 & 0.162 & 0.079 & 0.362 \\
\hline & Transverse 1 & 140 & 10.88 & 10.83 & 60 & 0.281 & 0.226 & 0.365 \\
\hline \multirow{5}{*}{7} & Transverse 5 & 100 & 10.73 & 10.68 & 150 & 0.318 & 0.317 & 0.446 \\
\hline & Transverse 4 & 100 & 9.12 & 9.04 & 110 & 0.114 & 0.078 & 0.331 \\
\hline & Transverse 3 & 80 & 10.07 & 10.02 & 85 & 0.186 & 0.191 & 0.419 \\
\hline & Transverse 2 & 100 & 9.45 & 9.38 & 35 & 0.131 & 0.101 & 0.299 \\
\hline & Transverse 1 & 100 & 10.24 & 10.18 & 30 & 0.334 & 0.185 & 0.393 \\
\hline \multirow{5}{*}{8} & Transverse 5 & 100 & 10.69 & 10.65 & 185 & 0.324 & 0.306 & 0.435 \\
\hline & Transverse 4 & 100 & 8.76 & 8.68 & 105 & 0.126 & 0.064 & 0.337 \\
\hline & Transverse 3 & 60 & 9.83 & 9.77 & 90 & 0.148 & 0.170 & 0.396 \\
\hline & Transverse 2 & 60 & 9.16 & 9.09 & 110 & 0.101 & 0.091 & 0.336 \\
\hline & Transverse 1 & 80 & 10.63 & 10.59 & 100 & 0.304 & 0.219 & 0.385 \\
\hline & Transverse 5 & 120 & 10.97 & 10.93 & 190 & 0.446 & 0.315 & 0.448 \\
\hline & Transverse 4 & 80 & 8.62 & 8.51 & 110 & 0.208 & 0.053 & 0.257 \\
\hline 9 & Transverse 3 & 80 & 10.27 & 10.22 & 100 & 0.222 & 0.184 & 0.424 \\
\hline & Transverse 2 & 80 & 8.98 & 8.91 & 65 & 0.169 & 0.123 & 0.306 \\
\hline & Transverse 1 & 100 & 10.43 & 10.38 & 50 & 0.304 & 0.220 & 0.387 \\
\hline & Transverse 5 & 100 & 10.80 & 10.76 & 195 & 0.442 & 0.313 & 0.448 \\
\hline & Transverse 4 & 60 & 7.96 & 7.86 & 145 & 0.149 & 0.038 & 0.231 \\
\hline 10 & Transverse 3 & 80 & 10.08 & 10.03 & 110 & 0.211 & 0.157 & 0.375 \\
\hline & Transverse 2 & 80 & 8.96 & 8.89 & 70 & 0.178 & 0.099 & 0.299 \\
\hline & Transverse 1 & 100 & 10.54 & 10.50 & 30 & 0.425 & 0.217 & 0.415 \\
\hline & Transverse 5 & 100 & 11.04 & 10.99 & 185 & 0.399 & 0.314 & 0.449 \\
\hline & Transverse 4 & 20 & 9.34 & 9.26 & 90 & 0.090 & 0.055 & 0.307 \\
\hline 11 & Transverse 3 & 80 & 10.56 & 10.50 & 85 & 0.194 & 0.137 & 0.335 \\
\hline & Transverse 2 & 60 & 8.80 & 8.73 & 65 & 0.183 & 0.129 & 0.334 \\
\hline & Transverse 1 & 80 & 10.78 & 10.72 & 25 & 0.281 & 0.202 & 0.381 \\
\hline & Transverse 5 & 100 & 10.96 & 10.92 & 190 & 0.424 & 0.319 & 0.455 \\
\hline & Transverse 4 & 40 & 5.92 & 5.90 & 70 & 0.218 & 0.038 & 0.142 \\
\hline 12 & Transverse 3 & 80 & 10.42 & 10.37 & 120 & 0.254 & 0.200 & 0.418 \\
\hline & Transverse 2 & 40 & 9.48 & 9.42 & 70 & 0.169 & 0.076 & 0.272 \\
\hline & Transverse 1 & 100 & 10.80 & 10.76 & 45 & 0.279 & 0.219 & 0.404 \\
\hline & Transverse 5 & 100 & 10.98 & 10.93 & 115 & 0.472 & 0.301 & 0.426 \\
\hline & Transverse 4 & 60 & 9.38 & 9.29 & 110 & 0.153 & 0.086 & 0.339 \\
\hline 13 & Transverse 3 & 100 & 9.92 & 9.85 & 110 & 0.268 & 0.143 & 0.324 \\
\hline & Transverse 2 & 120 & 9.99 & 9.93 & 95 & 0.152 & 0.111 & 0.323 \\
\hline & Transverse 1 & 60 & 10.19 & 10.13 & 55 & 0.168 & 0.201 & 0.411 \\
\hline & Transverse 5 & 120 & 10.80 & 10.75 & 145 & 0.376 & 0.299 & 0.428 \\
\hline & Transverse 4 & 20 & 9.01 & 8.92 & 145 & 0.117 & 0.070 & 0.376 \\
\hline 14 & Transverse 3 & 80 & 10.06 & 10.00 & 110 & 0.223 & 0.164 & 0.353 \\
\hline & Transverse 2 & 100 & 9.70 & 9.64 & 75 & 0.195 & 0.096 & 0.312 \\
\hline & Transverse 1 & 80 & 9.99 & 9.94 & 35 & 0.189 & 0.207 & 0.427 \\
\hline & Transverse 4 & 100 & 10.32 & 10.26 & 185 & 0.388 & 0.271 & 0.406 \\
\hline 15 & Transverse 3 & 80 & 5.86 & 5.84 & 105 & 0.159 & 0.052 & 0.265 \\
\hline 15 & Transverse 2 & 100 & 9.42 & 9.35 & 110 & 0.179 & 0.122 & 0.344 \\
\hline & Transverse 1 & 100 & 10.65 & 10.60 & 80 & 0.210 & 0.238 & 0.426 \\
\hline & Transverse 4 & 0 & 10.42 & 10.36 & 90 & 0.027 & 0.273 & 0.410 \\
\hline & Transverse 3 & 60 & 9.80 & 9.72 & 125 & 0.044 & 0.155 & 0.398 \\
\hline 16 & Transverse 2 & 40 & 8.05 & 7.98 & 50 & 0.170 & 0.118 & 0.302 \\
\hline & Transverse 1 & 40 & 9.97 & 9.89 & 40 & 0.006 & 0.235 & 0.401 \\
\hline & Transverse 4 & 100 & 10.40 & 10.34 & 115 & 0.422 & 0.285 & 0.411 \\
\hline & Transverse 3 & 80 & 9.24 & 9.16 & 125 & 0.157 & 0.091 & 0.332 \\
\hline 17 & Transverse 2 & 120 & 9.30 & 9.22 & 125 & 0.184 & 0.097 & 0.327 \\
\hline & Transverse 1 & 120 & 10.25 & 10.19 & 140 & 0.391 & 0.236 & 0.355 \\
\hline & Transverse 4 & 100 & 10.53 & 10.48 & 145 & 0.343 & 0.296 & 0.424 \\
\hline & Transverse 3 & 80 & 8.50 & 8.39 & 135 & 0.108 & 0.074 & 0.306 \\
\hline 18 & Transverse 2 & 80 & 9.48 & 9.41 & 90 & 0.169 & 0.125 & 0.312 \\
\hline & Transverse 1 & 80 & 10.01 & 9.95 & 25 & 0.215 & 0.227 & 0.419 \\
\hline & Transverse 4 & 120 & 10.77 & 10.73 & 160 & 0.399 & 0.295 & 0.430 \\
\hline & Transverse 3 & 80 & 9.03 & 8.95 & 95 & 0.190 & 0.071 & 0.315 \\
\hline 19 & Transverse 2 & 80 & 9.62 & 9.55 & 90 & 0.211 & 0.117 & 0.337 \\
\hline & Transverse 1 & 120 & 10.58 & 10.53 & 60 & 0.199 & 0.236 & 0.387 \\
\hline & Transverse 5 & 120 & 11.37 & 11.33 & 175 & 0.524 & 0.285 & 0.426 \\
\hline & Transverse 4 & 40 & 8.86 & 8.79 & 90 & 0.124 & 0.064 & 0.190 \\
\hline 20 & Transverse 3 & 120 & 9.68 & 9.61 & 90 & 0.449 & 0.103 & 0.331 \\
\hline & Transverse 2 & 100 & 9.21 & 9.12 & 75 & 0.161 & 0.080 & 0.283 \\
\hline & Transverse 1 & 140 & 11.26 & 11.21 & 45 & 0.376 & 0.232 & 0.418 \\
\hline & Transverse 5 & 120 & 11.62 & 11.57 & 145 & 0.570 & 0.306 & 0.425 \\
\hline 21 & Transverse 4 & 60 & 9.56 & 9.48 & 110 & 0.184 & 0.066 & 0.338 \\
\hline & Transverse 3 & 80 & 10.56 & 10.51 & 90 & 0.220 & 0.152 & 0.331 \\
\hline
\end{tabular}




\begin{tabular}{|c|c|c|c|c|c|c|c|c|}
\hline & Transverse 2 & 120 & 9.90 & 9.83 & 90 & 0.165 & 0.108 & 0.347 \\
\hline & Transverse 1 & 80 & 10.38 & 10.32 & 20 & 0.293 & 0.209 & 0.360 \\
\hline \multirow{5}{*}{22} & Transverse 5 & 120 & 10.47 & 10.42 & 215 & 0.495 & 0.320 & 0.461 \\
\hline & Transverse 4 & 80 & 8.51 & 8.41 & 130 & 0.159 & 0.049 & 0.269 \\
\hline & Transverse 3 & 80 & 9.80 & 9.74 & 110 & 0.236 & 0.144 & 0.383 \\
\hline & Transverse 2 & 80 & 9.48 & 9.41 & 70 & 0.190 & 0.121 & 0.330 \\
\hline & Transverse 1 & 80 & 9.86 & 9.80 & 30 & 0.442 & 0.178 & 0.391 \\
\hline \multirow{5}{*}{23} & Transverse 5 & 140 & 10.78 & 10.74 & 175 & 0.505 & 0.282 & 0.405 \\
\hline & Transverse 4 & 60 & 9.02 & 8.95 & 140 & 0.209 & 0.089 & 0.358 \\
\hline & Transverse 3 & 120 & 9.31 & 9.25 & 110 & 0.228 & 0.128 & 0.324 \\
\hline & Transverse 2 & 80 & 9.57 & 9.51 & 50 & 0.168 & 0.103 & 0.309 \\
\hline & Transverse 1 & 80 & 10.61 & 10.57 & 25 & 0.343 & 0.226 & 0.372 \\
\hline \multirow{5}{*}{24} & Transverse 5 & 100 & 10.61 & 10.56 & 195 & 0.366 & 0.280 & 0.415 \\
\hline & Transverse 4 & 20 & 8.58 & 8.48 & 130 & 0.096 & 0.041 & 0.268 \\
\hline & Transverse 3 & 80 & 10.03 & 9.98 & 110 & 0.207 & 0.174 & 0.344 \\
\hline & Transverse 2 & 80 & 9.35 & 9.28 & 65 & 0.140 & 0.088 & 0.307 \\
\hline & Transverse 1 & 100 & 10.22 & 10.17 & 30 & 0.370 & 0.214 & 0.394 \\
\hline \multirow{5}{*}{25} & Transverse 5 & 100 & 10.78 & 10.73 & 200 & 0.372 & 0.276 & 0.414 \\
\hline & Transverse 4 & 80 & 9.01 & 8.92 & 110 & 0.195 & 0.054 & 0.356 \\
\hline & Transverse 3 & 80 & 10.26 & 10.20 & 90 & 0.190 & 0.156 & 0.331 \\
\hline & Transverse 2 & 80 & 9.37 & 9.29 & 80 & 0.157 & 0.102 & 0.365 \\
\hline & Transverse 1 & 80 & 10.58 & 10.53 & 30 & 0.432 & 0.212 & 0.368 \\
\hline \multirow{5}{*}{26} & Transverse 5 & 120 & 11.08 & 11.03 & 155 & 0.432 & 0.261 & 0.401 \\
\hline & Transverse 4 & 40 & 9.04 & 8.95 & 110 & 0.125 & 0.055 & 0.249 \\
\hline & Transverse 3 & 80 & 10.19 & 10.13 & 90 & 0.222 & 0.153 & 0.353 \\
\hline & Transverse 2 & 100 & 9.33 & 9.26 & 90 & 0.258 & 0.109 & 0.311 \\
\hline & Transverse 1 & 100 & 10.88 & 10.83 & 30 & 0.457 & 0.207 & 0.370 \\
\hline \multirow{4}{*}{27} & Transverse 4 & 120 & 11.79 & 11.75 & 150 & 0.672 & 0.299 & 0.437 \\
\hline & Transverse 3 & 100 & 10.09 & 10.03 & 90 & 0.380 & 0.130 & 0.300 \\
\hline & Transverse 2 & 100 & 11.08 & 11.04 & 70 & 0.491 & 0.201 & 0.429 \\
\hline & Transverse 1 & 100 & 11.06 & 11.02 & 30 & 0.622 & 0.208 & 0.381 \\
\hline \multirow{4}{*}{28} & Transverse 4 & 100 & 10.88 & 10.84 & 150 & 0.557 & 0.313 & 0.452 \\
\hline & Transverse 3 & 100 & 9.31 & 9.24 & 120 & 0.127 & 0.076 & 0.404 \\
\hline & Transverse 2 & 100 & 10.20 & 10.15 & 100 & 0.285 & 0.198 & 0.417 \\
\hline & Transverse 1 & 60 & 10.26 & 10.21 & 30 & 0.362 & 0.191 & 0.380 \\
\hline
\end{tabular}

Table 138: Case Study 1 - 40 ft Cluster Spacing Fracture Results

\begin{tabular}{|c|c|c|c|c|c|c|c|c|}
\hline Stage Number & Fracture & $\begin{array}{l}\text { Proppant } \\
\text { Cutoff } \\
\text { Length } \\
\text { (ft) }\end{array}$ & $\begin{array}{c}\text { Est Flowing } \\
\text { Frac Length } \\
\text { (ft) }\end{array}$ & $\begin{array}{c}\text { Est Inf } \\
\text { Conductivity } \\
\text { Length } \\
\text { (ft) }\end{array}$ & $\begin{array}{c}\text { Fracture } \\
\text { Height } \\
\text { (ft) }\end{array}$ & $\begin{array}{c}\text { Average } \\
\text { Proppant } \\
\text { Conc } \\
\left(\mathrm{lb} / \mathrm{ft}^{2}\right)\end{array}$ & $\begin{array}{l}\text { Average } \\
\text { Fracture } \\
\text { Width } \\
\text { (in) }\end{array}$ & $\begin{array}{c}\text { Max } \\
\text { Fracture } \\
\text { Width } \\
\text { (in) }\end{array}$ \\
\hline \multirow{5}{*}{1} & Transverse 5 & 120 & 10.35 & 10.29 & 155 & 0.360 & 0.327 & 0.446 \\
\hline & Transverse 4 & 40 & 7.67 & 7.58 & 110 & 0.081 & 0.026 & 0.088 \\
\hline & Transverse 3 & 80 & 9.47 & 9.40 & 115 & 0.163 & 0.161 & 0.391 \\
\hline & Transverse 2 & 40 & 7.49 & 7.41 & 110 & 0.076 & 0.027 & 0.082 \\
\hline & Transverse 1 & 100 & 10.40 & 10.35 & 155 & 0.371 & 0.322 & 0.440 \\
\hline \multirow{5}{*}{2} & Transverse 5 & 100 & 10.61 & 10.55 & 145 & 0.346 & 0.305 & 0.424 \\
\hline & Transverse 4 & 80 & 8.49 & 8.37 & 105 & 0.140 & 0.052 & 0.346 \\
\hline & Transverse 3 & 80 & 9.81 & 9.74 & 80 & 0.169 & 0.153 & 0.364 \\
\hline & Transverse 2 & 120 & 9.35 & 9.26 & 95 & 0.147 & 0.084 & 0.340 \\
\hline & Transverse 1 & 120 & 10.62 & 10.57 & 40 & 0.264 & 0.240 & 0.376 \\
\hline \multirow{5}{*}{3} & Transverse 5 & 100 & 11.00 & 10.95 & 155 & 0.441 & 0.324 & 0.440 \\
\hline & Transverse 4 & 60 & 8.50 & 8.39 & 125 & 0.120 & 0.047 & 0.349 \\
\hline & Transverse 3 & 80 & 10.14 & 10.08 & 105 & 0.224 & 0.182 & 0.399 \\
\hline & Transverse 2 & 120 & 8.99 & 8.91 & 75 & 0.125 & 0.067 & 0.288 \\
\hline & Transverse 1 & 140 & 10.54 & 10.48 & 45 & 0.292 & 0.211 & 0.398 \\
\hline \multirow{5}{*}{4} & Transverse 5 & 100 & 11.02 & 10.98 & 175 & 0.408 & 0.324 & 0.450 \\
\hline & Transverse 4 & 140 & 9.31 & 9.22 & 110 & 0.131 & 0.076 & 0.384 \\
\hline & Transverse 3 & 80 & 9.99 & 9.93 & 85 & 0.229 & 0.157 & 0.346 \\
\hline & Transverse 2 & 120 & 9.70 & 9.63 & 45 & 0.188 & 0.068 & 0.381 \\
\hline & Transverse 1 & 60 & 10.37 & 10.31 & 30 & 0.142 & 0.215 & 0.367 \\
\hline \multirow{5}{*}{5} & Transverse 5 & 100 & 10.60 & 10.55 & 145 & 0.375 & 0.298 & 0.428 \\
\hline & Transverse 4 & 60 & 8.17 & 8.06 & 95 & 0.126 & 0.041 & 0.224 \\
\hline & Transverse 3 & 80 & 9.97 & 9.90 & 100 & 0.160 & 0.179 & 0.398 \\
\hline & Transverse 2 & 100 & 9.32 & 9.24 & 95 & 0.197 & 0.086 & 0.304 \\
\hline & Transverse 1 & 100 & 10.33 & 10.27 & 30 & 0.357 & 0.224 & 0.374 \\
\hline \multirow{5}{*}{6} & Transverse 5 & 100 & 11.16 & 11.12 & 155 & 0.499 & 0.305 & 0.431 \\
\hline & Transverse 4 & 40 & 9.13 & 9.04 & 90 & 0.098 & 0.058 & 0.354 \\
\hline & Transverse 3 & 100 & 9.91 & 9.85 & 105 & 0.255 & 0.151 & 0.344 \\
\hline & Transverse 2 & 100 & 7.71 & 7.65 & 60 & 0.173 & 0.075 & 0.340 \\
\hline & Transverse 1 & 140 & 11.13 & 11.09 & 55 & 0.308 & 0.242 & 0.391 \\
\hline \multirow{5}{*}{7} & Transverse 5 & 100 & 10.79 & 10.74 & 145 & 0.336 & 0.320 & 0.449 \\
\hline & Transverse 4 & 60 & 7.77 & 7.67 & 105 & 0.137 & 0.019 & 0.203 \\
\hline & Transverse 3 & 80 & 10.30 & 10.25 & 110 & 0.150 & 0.202 & 0.414 \\
\hline & Transverse 2 & 80 & 9.72 & 9.66 & 55 & 0.119 & 0.111 & 0.296 \\
\hline & Transverse 1 & 100 & 10.48 & 10.43 & 30 & 0.303 & 0.202 & 0.387 \\
\hline \multirow{3}{*}{8} & Transverse 5 & 100 & 10.90 & 10.85 & 195 & 0.351 & 0.327 & 0.468 \\
\hline & Transverse 4 & 0 & 8.31 & 8.18 & 110 & 0.085 & 0.036 & 0.238 \\
\hline & Transverse 3 & 60 & 9.92 & 9.86 & 85 & 0.135 & 0.185 & 0.395 \\
\hline
\end{tabular}




\begin{tabular}{|c|c|c|c|c|c|c|c|c|}
\hline & Transverse 2 & 60 & 8.98 & 8.90 & 90 & 0.087 & 0.086 & 0.338 \\
\hline & Transverse 1 & 80 & 10.80 & 10.75 & 90 & 0.316 & 0.215 & 0.401 \\
\hline \multirow{5}{*}{9} & Transverse 5 & 120 & 10.93 & 10.89 & 190 & 0.430 & 0.315 & 0.449 \\
\hline & Transverse 4 & 80 & 8.59 & 8.49 & 105 & 0.221 & 0.048 & 0.291 \\
\hline & Transverse 3 & 100 & 10.22 & 10.16 & 110 & 0.227 & 0.193 & 0.423 \\
\hline & Transverse 2 & 100 & 8.75 & 8.69 & 75 & 0.214 & 0.110 & 0.314 \\
\hline & Transverse 1 & 100 & 10.58 & 10.53 & 30 & 0.440 & 0.213 & 0.400 \\
\hline \multirow{5}{*}{10} & Transverse 5 & 100 & 10.76 & 10.72 & 195 & 0.416 & 0.300 & 0.437 \\
\hline & Transverse 4 & 100 & 9.52 & 9.45 & 105 & 0.148 & 0.073 & 0.377 \\
\hline & Transverse 3 & 80 & 10.38 & 10.33 & 110 & 0.236 & 0.166 & 0.327 \\
\hline & Transverse 2 & 40 & 9.16 & 9.08 & 65 & 0.197 & 0.085 & 0.290 \\
\hline & Transverse 1 & 100 & 10.38 & 10.33 & 30 & 0.406 & 0.209 & 0.376 \\
\hline \multirow{5}{*}{11} & Transverse 5 & 100 & 10.88 & 10.84 & 185 & 0.385 & 0.310 & 0.449 \\
\hline & Transverse 4 & 80 & 9.22 & 9.14 & 105 & 0.178 & 0.046 & 0.332 \\
\hline & Transverse 3 & 80 & 10.50 & 10.45 & 85 & 0.245 & 0.194 & 0.358 \\
\hline & Transverse 2 & 80 & 9.48 & 9.40 & 55 & 0.153 & 0.077 & 0.295 \\
\hline & Transverse 1 & 80 & 10.97 & 10.92 & 30 & 0.304 & 0.218 & 0.385 \\
\hline \multirow{5}{*}{12} & Transverse 5 & 100 & 11.15 & 11.10 & 195 & 0.437 & 0.332 & 0.472 \\
\hline & Transverse 4 & 40 & 6.15 & 6.08 & 75 & 0.201 & 0.019 & 0.125 \\
\hline & Transverse 3 & 100 & 10.34 & 10.29 & 100 & 0.311 & 0.178 & 0.398 \\
\hline & Transverse 2 & 60 & 9.67 & 9.60 & 60 & 0.164 & 0.058 & 0.288 \\
\hline & Transverse 1 & 100 & 10.72 & 10.67 & 40 & 0.319 & 0.223 & 0.397 \\
\hline & Transverse 5 & 100 & 11.12 & 11.08 & 115 & 0.473 & 0.292 & 0.402 \\
\hline & Transverse 4 & 100 & 9.33 & 9.25 & 110 & 0.152 & 0.078 & 0.353 \\
\hline 13 & Transverse 3 & 120 & 9.88 & 9.81 & 90 & 0.287 & 0.162 & 0.399 \\
\hline & Transverse 2 & 120 & 10.21 & 10.14 & 80 & 0.161 & 0.150 & 0.341 \\
\hline & Transverse 1 & 80 & 10.64 & 10.59 & 55 & 0.241 & 0.213 & 0.419 \\
\hline & Transverse 5 & 100 & 10.54 & 10.49 & 145 & 0.369 & 0.293 & 0.420 \\
\hline & Transverse 4 & 140 & 9.04 & 8.96 & 160 & 0.130 & 0.090 & 0.338 \\
\hline 14 & Transverse 3 & 60 & 9.49 & 9.43 & 140 & 0.171 & 0.128 & 0.308 \\
\hline & Transverse 2 & 60 & 9.46 & 9.40 & 90 & 0.236 & 0.098 & 0.374 \\
\hline & Transverse 1 & 100 & 9.94 & 9.88 & 35 & 0.310 & 0.193 & 0.363 \\
\hline & Transverse 4 & 100 & 10.72 & 10.67 & 145 & 0.406 & 0.293 & 0.426 \\
\hline & Transverse 3 & 140 & 9.92 & 9.85 & 125 & 0.205 & 0.165 & 0.428 \\
\hline 15 & Transverse 2 & 60 & 9.09 & 9.00 & 80 & 0.210 & 0.061 & 0.314 \\
\hline & Transverse 1 & 120 & 10.52 & 10.46 & 55 & 0.250 & 0.235 & 0.402 \\
\hline & Transverse 4 & 40 & 10.55 & 10.49 & 90 & 0.113 & 0.282 & 0.415 \\
\hline & Transverse 3 & 60 & 9.69 & 9.62 & 130 & 0.049 & 0.141 & 0.398 \\
\hline 16 & Transverse 2 & 20 & 9.99 & 9.92 & 60 & 0.043 & 0.128 & 0.326 \\
\hline & Transverse 1 & 60 & 10.05 & 9.98 & 50 & 0.021 & 0.238 & 0.408 \\
\hline & Transverse 4 & 100 & 10.31 & 10.25 & 145 & 0.384 & 0.270 & 0.398 \\
\hline & Transverse 3 & 60 & 9.30 & 9.22 & 90 & 0.153 & 0.081 & 0.307 \\
\hline 17 & Transverse 2 & 80 & 9.91 & 9.85 & 90 & 0.115 & 0.116 & 0.352 \\
\hline & Transverse 1 & 120 & 10.19 & 10.13 & 125 & 0.348 & 0.223 & 0.398 \\
\hline & Transverse 4 & 100 & 10.32 & 10.26 & 140 & 0.304 & 0.273 & 0.399 \\
\hline 18 & Transverse 3 & 100 & 9.62 & 9.55 & 130 & 0.176 & 0.143 & 0.418 \\
\hline 18 & Transverse 2 & 80 & 8.82 & 8.72 & 85 & 0.130 & 0.081 & 0.336 \\
\hline & Transverse 1 & 80 & 10.20 & 10.14 & 35 & 0.209 & 0.223 & 0.367 \\
\hline & Transverse 4 & 140 & 10.71 & 10.67 & 150 & 0.402 & 0.297 & 0.437 \\
\hline 10 & Transverse 3 & 120 & 9.53 & 9.46 & 120 & 0.188 & 0.122 & 0.402 \\
\hline 19 & Transverse 2 & 100 & 9.06 & 8.99 & 90 & 0.327 & 0.101 & 0.315 \\
\hline & Transverse 1 & 120 & 10.41 & 10.35 & 50 & 0.237 & 0.234 & 0.362 \\
\hline & Transverse 5 & 120 & 11.32 & 11.28 & 170 & 0.461 & 0.282 & 0.411 \\
\hline & Transverse 4 & 80 & 9.05 & 8.96 & 115 & 0.221 & 0.072 & 0.291 \\
\hline 20 & Transverse 3 & 100 & 9.98 & 9.91 & 90 & 0.222 & 0.129 & 0.332 \\
\hline & Transverse 2 & 100 & 9.26 & 9.18 & 75 & 0.190 & 0.084 & 0.294 \\
\hline & Transverse 1 & 80 & 10.96 & 10.91 & 30 & 0.461 & 0.223 & 0.391 \\
\hline & Transverse 5 & 120 & 11.25 & 11.20 & 195 & 0.487 & 0.270 & 0.412 \\
\hline & Transverse 4 & 80 & 9.25 & 9.17 & 110 & 0.216 & 0.066 & 0.262 \\
\hline 21 & Transverse 3 & 100 & 10.42 & 10.37 & 100 & 0.318 & 0.163 & 0.363 \\
\hline & Transverse 2 & 100 & 9.73 & 9.66 & 55 & 0.186 & 0.112 & 0.351 \\
\hline & Transverse 1 & 140 & 11.10 & 11.05 & 20 & 0.585 & 0.206 & 0.374 \\
\hline & Transverse 5 & 120 & 10.42 & 10.37 & 215 & 0.491 & 0.321 & 0.462 \\
\hline & Transverse 4 & 20 & 8.73 & 8.65 & 125 & 0.138 & 0.045 & 0.312 \\
\hline 22 & Transverse 3 & 80 & 10.01 & 9.96 & 110 & 0.239 & 0.173 & 0.363 \\
\hline & Transverse 2 & 100 & 7.47 & 7.41 & 80 & 0.198 & 0.105 & 0.377 \\
\hline & Transverse 1 & 80 & 9.80 & 9.75 & 50 & 0.302 & 0.179 & 0.402 \\
\hline & Transverse 5 & 120 & 10.70 & 10.66 & 185 & 0.477 & 0.276 & 0.407 \\
\hline & Transverse 4 & 40 & 8.48 & 8.38 & 130 & 0.130 & 0.051 & 0.265 \\
\hline 23 & Transverse 3 & 100 & 10.19 & 10.14 & 110 & 0.296 & 0.184 & 0.394 \\
\hline & Transverse 2 & 100 & 9.45 & 9.38 & 65 & 0.132 & 0.094 & 0.294 \\
\hline & Transverse 1 & 80 & 9.99 & 9.94 & 30 & 0.362 & 0.217 & 0.363 \\
\hline & Transverse 5 & 100 & 10.53 & 10.48 & 195 & 0.357 & 0.280 & 0.412 \\
\hline & Transverse 4 & 40 & 8.55 & 8.46 & 130 & 0.122 & 0.048 & 0.261 \\
\hline 24 & Transverse 3 & 80 & 10.04 & 9.98 & 110 & 0.212 & 0.174 & 0.388 \\
\hline & Transverse 2 & 80 & 9.06 & 9.00 & 65 & 0.121 & 0.092 & 0.304 \\
\hline & Transverse 1 & 80 & 10.37 & 10.32 & 30 & 0.361 & 0.212 & 0.384 \\
\hline & Transverse 5 & 100 & 10.86 & 10.81 & 180 & 0.344 & 0.281 & 0.410 \\
\hline & Transverse 4 & 100 & 9.62 & 9.55 & 105 & 0.138 & 0.094 & 0.393 \\
\hline 25 & Transverse 3 & 80 & 9.25 & 9.18 & 110 & 0.207 & 0.120 & 0.326 \\
\hline & Transverse 2 & 80 & 9.71 & 9.65 & 75 & 0.192 & 0.122 & 0.330 \\
\hline & Transverse 1 & 80 & 10.78 & 10.73 & 35 & 0.274 & 0.220 & 0.396 \\
\hline
\end{tabular}




\begin{tabular}{|c|c|r|r|r|r|r|r|r|}
\multirow{5}{*}{5} & Transverse 5 & 120 & 11.12 & 11.08 & 180 & 0.457 & 0.279 & 0.412 \\
\cline { 2 - 9 } 26 & Transverse 4 & 80 & 8.90 & 8.81 & 100 & 0.237 & 0.044 & 0.298 \\
\cline { 2 - 9 } & Transverse 3 & 80 & 10.69 & 10.64 & 90 & 0.310 & 0.195 & 0.376 \\
\cline { 2 - 9 } & Transverse 2 & 100 & 9.97 & 9.91 & 55 & 0.286 & 0.109 & 0.285 \\
\cline { 2 - 9 } & Transverse 1 & 80 & 10.45 & 10.39 & 40 & 0.311 & 0.209 & 0.390 \\
\hline \multirow{3}{*}{27} & Transverse 4 & 120 & 11.73 & 11.69 & 150 & 0.644 & 0.301 & 0.432 \\
\cline { 2 - 9 } & Transverse 3 & 100 & 9.64 & 9.57 & 110 & 0.240 & 0.100 & 0.309 \\
\cline { 2 - 9 } & Transverse 2 & 100 & 10.37 & 10.31 & 70 & 0.280 & 0.136 & 0.313 \\
\cline { 2 - 9 } & Transverse 1 & 100 & 10.82 & 10.77 & 30 & 0.574 & 0.199 & 0.377 \\
\hline \multirow{3}{*}{28} & Transverse 4 & 100 & 11.07 & 11.03 & 150 & 0.570 & 0.316 & 0.448 \\
\cline { 2 - 9 } & Transverse 3 & 100 & 10.05 & 9.99 & 125 & 0.345 & 0.174 & 0.447 \\
\cline { 2 - 9 } & Transverse 2 & 100 & 8.59 & 8.52 & 55 & 0.224 & 0.141 & 0.341 \\
\cline { 2 - 9 } & Transverse 1 & 60 & 10.89 & 10.85 & 25 & 0.369 & 0.219 & 0.395 \\
\hline
\end{tabular}

Table 139: Case Study 1 - 35 ft Cluster Spacing Fracture Results

\begin{tabular}{|c|c|c|c|c|c|c|c|c|}
\hline Stage Number & Fracture & $\begin{array}{c}\text { Proppant } \\
\text { Cutoff } \\
\text { Length } \\
\text { (ft) }\end{array}$ & $\begin{array}{c}\text { Est Flowing } \\
\text { Frac Length } \\
\text { (ft) } \\
\end{array}$ & $\begin{array}{c}\text { Est Inf } \\
\text { Conductivity } \\
\text { Length } \\
\text { (ft) } \\
\end{array}$ & $\begin{array}{c}\text { Fracture } \\
\text { Height } \\
\text { (ft) }\end{array}$ & $\begin{array}{c}\text { Average } \\
\text { Proppant } \\
\text { Conc } \\
\left(\mathrm{lb} / \mathrm{ft}^{2}\right)\end{array}$ & $\begin{array}{c}\text { Average } \\
\text { Fracture } \\
\text { Width } \\
\text { (in) }\end{array}$ & $\begin{array}{c}\text { Max } \\
\text { Fracture } \\
\text { Width } \\
\text { (in) } \\
\end{array}$ \\
\hline \multirow{5}{*}{1} & Transverse 5 & 120 & 10.33 & 10.28 & 155 & 0.358 & 0.327 & 0.445 \\
\hline & Transverse 4 & 40 & 7.75 & 7.66 & 110 & 0.084 & 0.029 & 0.110 \\
\hline & Transverse 3 & 80 & 9.45 & 9.38 & 115 & 0.169 & 0.160 & 0.383 \\
\hline & Transverse 2 & 40 & 7.50 & 7.43 & 110 & 0.077 & 0.027 & 0.082 \\
\hline & Transverse 1 & 100 & 10.37 & 10.32 & 155 & 0.369 & 0.321 & 0.439 \\
\hline \multirow{5}{*}{2} & Transverse 5 & 120 & 10.75 & 10.70 & 145 & 0.362 & 0.313 & 0.435 \\
\hline & Transverse 4 & 40 & 8.74 & 8.64 & 90 & 0.091 & 0.048 & 0.152 \\
\hline & Transverse 3 & 80 & 10.10 & 10.03 & 85 & 0.152 & 0.165 & 0.389 \\
\hline & Transverse 2 & 100 & 9.29 & 9.20 & 85 & 0.193 & 0.062 & 0.300 \\
\hline & Transverse 1 & 120 & 10.73 & 10.67 & 45 & 0.233 & 0.247 & 0.385 \\
\hline \multirow{5}{*}{3} & Transverse 5 & 120 & 10.74 & 10.69 & 145 & 0.387 & 0.311 & 0.437 \\
\hline & Transverse 4 & 80 & 8.90 & 8.81 & 145 & 0.140 & 0.067 & 0.385 \\
\hline & Transverse 3 & 100 & 10.14 & 10.09 & 130 & 0.302 & 0.200 & 0.405 \\
\hline & Transverse 2 & 120 & 9.97 & 9.91 & 50 & 0.188 & 0.094 & 0.359 \\
\hline & Transverse 1 & 120 & 10.38 & 10.33 & 30 & 0.426 & 0.198 & 0.390 \\
\hline \multirow{5}{*}{4} & Transverse 5 & 100 & 11.06 & 11.01 & 170 & 0.388 & 0.316 & 0.437 \\
\hline & Transverse 4 & 140 & 8.86 & 8.77 & 105 & 0.144 & 0.061 & 0.319 \\
\hline & Transverse 3 & 100 & 10.34 & 10.28 & 90 & 0.257 & 0.178 & 0.373 \\
\hline & Transverse 2 & 60 & 5.67 & 5.64 & 50 & 0.155 & 0.032 & 0.277 \\
\hline & Transverse 1 & 140 & 10.86 & 10.81 & 40 & 0.274 & 0.234 & 0.413 \\
\hline \multirow{5}{*}{5} & Transverse 5 & 100 & 10.67 & 10.62 & 145 & 0.378 & 0.299 & 0.430 \\
\hline & Transverse 4 & 60 & 8.14 & 8.01 & 110 & 0.167 & 0.043 & 0.234 \\
\hline & Transverse 3 & 60 & 10.03 & 9.96 & 105 & 0.195 & 0.195 & 0.400 \\
\hline & Transverse 2 & 100 & 8.36 & 8.25 & 60 & 0.168 & 0.064 & 0.293 \\
\hline & Transverse 1 & 140 & 10.43 & 10.37 & 30 & 0.388 & 0.223 & 0.374 \\
\hline \multirow{5}{*}{6} & Transverse 5 & 140 & 10.91 & 10.86 & 170 & 0.425 & 0.293 & 0.419 \\
\hline & Transverse 4 & 80 & 9.45 & 9.38 & 110 & 0.156 & 0.117 & 0.373 \\
\hline & Transverse 3 & 80 & 9.17 & 9.09 & 85 & 0.133 & 0.089 & 0.303 \\
\hline & Transverse 2 & 120 & 8.91 & 8.83 & 90 & 0.244 & 0.082 & 0.305 \\
\hline & Transverse 1 & 120 & 10.55 & 10.50 & 35 & 0.334 & 0.237 & 0.363 \\
\hline \multirow{5}{*}{7} & Transverse 5 & 120 & 11.09 & 11.05 & 155 & 0.393 & 0.344 & 0.484 \\
\hline & Transverse 4 & 80 & 9.72 & 9.65 & 80 & 0.104 & 0.116 & 0.402 \\
\hline & Transverse 3 & 80 & 9.73 & 9.66 & 85 & 0.086 & 0.100 & 0.363 \\
\hline & Transverse 2 & 60 & 8.08 & 8.01 & 65 & 0.118 & 0.099 & 0.350 \\
\hline & Transverse 1 & 100 & 10.64 & 10.59 & 45 & 0.228 & 0.207 & 0.410 \\
\hline \multirow{5}{*}{8} & Transverse 5 & 100 & 10.87 & 10.82 & 190 & 0.357 & 0.325 & 0.463 \\
\hline & Transverse 4 & 40 & 8.38 & 8.28 & 125 & 0.099 & 0.050 & 0.294 \\
\hline & Transverse 3 & 60 & 10.13 & 10.07 & 95 & 0.148 & 0.159 & 0.397 \\
\hline & Transverse 2 & 80 & 9.07 & 8.99 & 110 & 0.080 & 0.101 & 0.340 \\
\hline & Transverse 1 & 60 & 10.39 & 10.34 & 90 & 0.313 & 0.203 & 0.401 \\
\hline \multirow{5}{*}{9} & Transverse 5 & 100 & 10.66 & 10.61 & 180 & 0.403 & 0.291 & 0.428 \\
\hline & Transverse 4 & 20 & 8.44 & 8.34 & 140 & 0.128 & 0.051 & 0.233 \\
\hline & Transverse 3 & 80 & 10.14 & 10.09 & 110 & 0.213 & 0.185 & 0.392 \\
\hline & Transverse 2 & 80 & 8.50 & 8.44 & 80 & 0.202 & 0.116 & 0.297 \\
\hline & Transverse 1 & 100 & 10.54 & 10.49 & 40 & 0.337 & 0.216 & 0.404 \\
\hline \multirow{5}{*}{10} & Transverse 5 & 100 & 11.03 & 10.98 & 195 & 0.448 & 0.313 & 0.447 \\
\hline & Transverse 4 & 40 & 8.57 & 8.48 & 90 & 0.095 & 0.029 & 0.169 \\
\hline & Transverse 3 & 100 & 10.26 & 10.20 & 105 & 0.255 & 0.189 & 0.418 \\
\hline & Transverse 2 & 80 & 9.24 & 9.17 & 55 & 0.166 & 0.066 & 0.292 \\
\hline & Transverse 1 & 100 & 10.82 & 10.77 & 30 & 0.410 & 0.220 & 0.424 \\
\hline \multirow{5}{*}{11} & Transverse 5 & 100 & 10.80 & 10.75 & 180 & 0.374 & 0.303 & 0.445 \\
\hline & Transverse 4 & 20 & 8.02 & 7.92 & 110 & 0.089 & 0.021 & 0.244 \\
\hline & Transverse 3 & 80 & 10.39 & 10.34 & 105 & 0.235 & 0.199 & 0.360 \\
\hline & Transverse 2 & 80 & 8.58 & 8.50 & 55 & 0.144 & 0.058 & 0.299 \\
\hline & Transverse 1 & 100 & 10.61 & 10.56 & 30 & 0.307 & 0.213 & 0.391 \\
\hline \multirow{5}{*}{12} & Transverse 5 & 100 & 10.99 & 10.95 & 195 & 0.429 & 0.329 & 0.469 \\
\hline & Transverse 4 & 40 & 5.70 & 5.68 & 75 & 0.206 & 0.017 & 0.130 \\
\hline & Transverse 3 & 60 & 10.14 & 10.08 & 110 & 0.226 & 0.171 & 0.363 \\
\hline & Transverse 2 & 80 & 10.30 & 10.25 & 60 & 0.192 & 0.099 & 0.294 \\
\hline & Transverse 1 & 100 & 10.60 & 10.55 & 40 & 0.353 & 0.222 & 0.421 \\
\hline
\end{tabular}




\begin{tabular}{|c|c|c|c|c|c|c|c|c|}
\hline \multirow{5}{*}{13} & Transverse 5 & 100 & 10.48 & 10.43 & 135 & 0.415 & 0.266 & 0.389 \\
\hline & Transverse 4 & 40 & 8.99 & 8.90 & 135 & 0.125 & 0.066 & 0.390 \\
\hline & Transverse 3 & 80 & 9.95 & 9.89 & 110 & 0.232 & 0.143 & 0.398 \\
\hline & Transverse 2 & 100 & 9.42 & 9.34 & 80 & 0.280 & 0.112 & 0.300 \\
\hline & Transverse 1 & 60 & 10.85 & 10.80 & 25 & 0.235 & 0.212 & 0.426 \\
\hline \multirow{5}{*}{14} & Transverse 5 & 100 & 10.57 & 10.52 & 145 & 0.365 & 0.291 & 0.417 \\
\hline & Transverse 4 & 100 & 9.32 & 9.24 & 150 & 0.172 & 0.090 & 0.398 \\
\hline & Transverse 3 & 60 & 9.98 & 9.92 & 145 & 0.169 & 0.150 & 0.388 \\
\hline & Transverse 2 & 100 & 8.79 & 8.70 & 85 & 0.115 & 0.075 & 0.280 \\
\hline & Transverse 1 & 80 & 10.40 & 10.35 & 40 & 0.265 & 0.219 & 0.421 \\
\hline \multirow{4}{*}{15} & Transverse 4 & 100 & 10.61 & 10.56 & 145 & 0.403 & 0.291 & 0.423 \\
\hline & Transverse 3 & 120 & 9.67 & 9.60 & 145 & 0.192 & 0.147 & 0.369 \\
\hline & Transverse 2 & 80 & 9.36 & 9.28 & 70 & 0.191 & 0.067 & 0.295 \\
\hline & Transverse 1 & 120 & 10.46 & 10.41 & 65 & 0.237 & 0.229 & 0.402 \\
\hline \multirow{4}{*}{16} & Transverse 4 & 0 & 10.26 & 10.20 & 80 & 0.010 & 0.282 & 0.416 \\
\hline & Transverse 3 & 20 & 9.81 & 9.73 & 105 & 0.063 & 0.135 & 0.348 \\
\hline & Transverse 2 & 20 & 9.96 & 9.89 & 65 & 0.063 & 0.104 & 0.299 \\
\hline & Transverse 1 & 60 & 10.14 & 10.07 & 30 & 0.065 & 0.222 & 0.360 \\
\hline \multirow{4}{*}{17} & Transverse 4 & 100 & 10.26 & 10.20 & 145 & 0.367 & 0.263 & 0.387 \\
\hline & Transverse 3 & 40 & 9.56 & 9.49 & 90 & 0.188 & 0.102 & 0.350 \\
\hline & Transverse 2 & 20 & 9.13 & 9.05 & 110 & 0.129 & 0.081 & 0.319 \\
\hline & Transverse 1 & 120 & 10.15 & 10.10 & 145 & 0.369 & 0.225 & 0.334 \\
\hline \multirow{4}{*}{18} & Transverse 4 & 80 & 10.18 & 10.12 & 140 & 0.279 & 0.268 & 0.392 \\
\hline & Transverse 3 & 80 & 9.68 & 9.61 & 130 & 0.184 & 0.139 & 0.397 \\
\hline & Transverse 2 & 80 & 9.43 & 9.35 & 80 & 0.171 & 0.098 & 0.320 \\
\hline & Transverse 1 & 80 & 10.03 & 9.96 & 40 & 0.180 & 0.220 & 0.386 \\
\hline \multirow{4}{*}{19} & Transverse 4 & 100 & 10.70 & 10.65 & 160 & 0.395 & 0.295 & 0.432 \\
\hline & Transverse 3 & 40 & 9.48 & 9.41 & 110 & 0.127 & 0.106 & 0.370 \\
\hline & Transverse 2 & 100 & 8.76 & 8.69 & 90 & 0.191 & 0.091 & 0.330 \\
\hline & Transverse 1 & 120 & 10.08 & 10.03 & 60 & 0.264 & 0.247 & 0.424 \\
\hline \multirow{5}{*}{20} & Transverse 5 & 120 & 11.31 & 11.26 & 170 & 0.447 & 0.276 & 0.409 \\
\hline & Transverse 4 & 60 & 9.06 & 8.99 & 90 & 0.165 & 0.057 & 0.137 \\
\hline & Transverse 3 & 80 & 10.27 & 10.21 & 90 & 0.180 & 0.155 & 0.336 \\
\hline & Transverse 2 & 100 & 8.63 & 8.57 & 90 & 0.197 & 0.081 & 0.281 \\
\hline & Transverse 1 & 140 & 11.11 & 11.06 & 65 & 0.392 & 0.213 & 0.364 \\
\hline & Transverse 5 & 120 & 11.34 & 11.30 & 140 & 0.505 & 0.277 & 0.418 \\
\hline & Transverse 4 & 80 & 8.95 & 8.86 & 110 & 0.230 & 0.050 & 0.267 \\
\hline 21 & Transverse 3 & 100 & 10.32 & 10.26 & 90 & 0.300 & 0.161 & 0.382 \\
\hline & Transverse 2 & 100 & 9.43 & 9.37 & 80 & 0.287 & 0.113 & 0.301 \\
\hline & Transverse 1 & 140 & 10.85 & 10.80 & 40 & 0.399 & 0.193 & 0.359 \\
\hline & Transverse 5 & 120 & 10.22 & 10.17 & 215 & 0.480 & 0.321 & 0.467 \\
\hline & Transverse 4 & 40 & 8.22 & 8.14 & 140 & 0.141 & 0.033 & 0.280 \\
\hline 22 & Transverse 3 & 80 & 9.95 & 9.90 & 140 & 0.236 & 0.198 & 0.380 \\
\hline & Transverse 2 & 120 & 7.73 & 7.67 & 75 & 0.154 & 0.133 & 0.340 \\
\hline & Transverse 1 & 100 & 10.04 & 9.99 & 35 & 0.408 & 0.191 & 0.401 \\
\hline & Transverse 5 & 140 & 10.77 & 10.73 & 170 & 0.514 & 0.291 & 0.417 \\
\hline & Transverse 4 & 40 & 8.80 & 8.72 & 130 & 0.124 & 0.055 & 0.322 \\
\hline 23 & Transverse 3 & 100 & 10.25 & 10.20 & 110 & 0.389 & 0.189 & 0.351 \\
\hline & Transverse 2 & 80 & 9.47 & 9.41 & 35 & 0.088 & 0.082 & 0.290 \\
\hline & Transverse 1 & 80 & 10.49 & 10.44 & 30 & 0.308 & 0.224 & 0.403 \\
\hline & Transverse 5 & 100 & 10.62 & 10.57 & 195 & 0.367 & 0.280 & 0.417 \\
\hline & Transverse 4 & 20 & 8.98 & 8.90 & 105 & 0.078 & 0.047 & 0.346 \\
\hline 24 & Transverse 3 & 80 & 9.92 & 9.86 & 110 & 0.213 & 0.176 & 0.349 \\
\hline & Transverse 2 & 60 & 8.72 & 8.65 & 65 & 0.158 & 0.089 & 0.363 \\
\hline & Transverse 1 & 100 & 10.14 & 10.09 & 30 & 0.329 & 0.199 & 0.352 \\
\hline & Transverse 5 & 100 & 10.79 & 10.74 & 200 & 0.369 & 0.274 & 0.411 \\
\hline & Transverse 4 & 60 & 8.98 & 8.89 & 100 & 0.119 & 0.056 & 0.257 \\
\hline 25 & Transverse 3 & 80 & 10.43 & 10.38 & 90 & 0.199 & 0.161 & 0.393 \\
\hline & Transverse 2 & 80 & 9.34 & 9.26 & 75 & 0.212 & 0.092 & 0.293 \\
\hline & Transverse 1 & 80 & 10.86 & 10.82 & 30 & 0.389 & 0.223 & 0.370 \\
\hline & Transverse 5 & 120 & 11.49 & 11.45 & 120 & 0.516 & 0.282 & 0.426 \\
\hline & Transverse 4 & 40 & 9.24 & 9.16 & 110 & 0.163 & 0.067 & 0.254 \\
\hline 26 & Transverse 3 & 100 & 10.53 & 10.47 & 90 & 0.213 & 0.156 & 0.360 \\
\hline & Transverse 2 & 100 & 9.99 & 9.93 & 105 & 0.225 & 0.090 & 0.347 \\
\hline & Transverse 1 & 100 & 9.31 & 9.25 & 60 & 0.356 & 0.209 & 0.377 \\
\hline & Transverse 4 & 120 & 11.85 & 11.81 & 150 & 0.691 & 0.303 & 0.439 \\
\hline 27 & Transverse 3 & 100 & 10.00 & 9.94 & 120 & 0.218 & 0.107 & 0.330 \\
\hline 21 & Transverse 2 & 80 & 9.65 & 9.59 & 90 & 0.391 & 0.135 & 0.339 \\
\hline & Transverse 1 & 100 & 11.18 & 11.14 & 30 & 0.507 & 0.198 & 0.387 \\
\hline & Transverse 4 & 100 & 10.36 & 10.32 & 215 & 0.470 & 0.253 & 0.387 \\
\hline 28 & Transverse 3 & 140 & 9.06 & 8.99 & 85 & 0.188 & 0.071 & 0.315 \\
\hline 28 & Transverse 2 & 100 & 10.21 & 10.16 & 85 & 0.339 & 0.185 & 0.414 \\
\hline & Transverse 1 & 80 & 9.84 & 9.78 & 30 & 0.342 & 0.176 & 0.344 \\
\hline
\end{tabular}

Table 140: Case Study 2 - 45 ft Cluster Spacing Fracture Results

\begin{tabular}{|c|c|c|c|c|c|c|c|c|}
\hline Stage Number & Fracture & $\begin{array}{l}\text { Proppant } \\
\text { Cutoff } \\
\text { Length } \\
\text { (ft) }\end{array}$ & $\begin{array}{c}\text { Est Flowing } \\
\text { Frac Length } \\
\text { (ft) }\end{array}$ & $\begin{array}{c}\text { Est Inf } \\
\text { Conductivity } \\
\text { Length } \\
\text { (ft) }\end{array}$ & $\begin{array}{c}\text { Fracture } \\
\text { Height } \\
\text { (ft) }\end{array}$ & $\begin{array}{c}\text { Average } \\
\text { Proppant } \\
\text { Conc } \\
\left(\mathrm{lb} / \mathrm{ft}^{2}\right)\end{array}$ & $\begin{array}{c}\text { Average } \\
\text { Fracture } \\
\text { Width } \\
\text { (in) }\end{array}$ & $\begin{array}{c}\text { Max } \\
\text { Fracture } \\
\text { Width } \\
\text { (in) }\end{array}$ \\
\hline
\end{tabular}




\begin{tabular}{|c|c|c|c|c|c|c|c|c|}
\hline \multirow{5}{*}{1} & Transverse 5 & 120 & 11.15 & 11.11 & 120 & 0.574 & 0.314 & 0.415 \\
\hline & Transverse 4 & 20 & 8.21 & 8.10 & 110 & 0.122 & 0.028 & 0.122 \\
\hline & Transverse 3 & 60 & 10.08 & 10.01 & 90 & 0.277 & 0.171 & 0.387 \\
\hline & Transverse 2 & 40 & 8.23 & 8.13 & 110 & 0.132 & 0.031 & 0.122 \\
\hline & Transverse 1 & 120 & 11.14 & 11.10 & 120 & 0.585 & 0.316 & 0.409 \\
\hline \multirow{5}{*}{2} & Transverse 5 & 120 & 11.36 & 11.31 & 150 & 0.611 & 0.329 & 0.449 \\
\hline & Transverse 4 & 80 & 8.93 & 8.83 & 125 & 0.213 & 0.031 & 0.353 \\
\hline & Transverse 3 & 120 & 10.33 & 10.27 & 125 & 0.377 & 0.213 & 0.422 \\
\hline & Transverse 2 & 80 & 9.56 & 9.48 & 30 & 0.219 & 0.103 & 0.317 \\
\hline & Transverse 1 & 80 & 10.31 & 10.25 & 20 & 0.272 & 0.220 & 0.384 \\
\hline \multirow{5}{*}{3} & Transverse 5 & 120 & 11.22 & 11.18 & 145 & 0.533 & 0.312 & 0.444 \\
\hline & Transverse 4 & 100 & 9.67 & 9.60 & 125 & 0.204 & 0.100 & 0.412 \\
\hline & Transverse 3 & 100 & 9.82 & 9.75 & 105 & 0.274 & 0.127 & 0.389 \\
\hline & Transverse 2 & 100 & 10.75 & 10.70 & 60 & 0.207 & 0.199 & 0.357 \\
\hline & Transverse 1 & 60 & 10.62 & 10.56 & 20 & 0.250 & 0.150 & 0.372 \\
\hline \multirow{5}{*}{4} & Transverse 5 & 120 & 11.33 & 11.29 & 170 & 0.597 & 0.317 & 0.443 \\
\hline & Transverse 4 & 60 & 9.40 & 9.32 & 105 & 0.250 & 0.074 & 0.332 \\
\hline & Transverse 3 & 100 & 9.91 & 9.85 & 90 & 0.318 & 0.120 & 0.346 \\
\hline & Transverse 2 & 100 & 10.48 & 10.42 & 50 & 0.245 & 0.182 & 0.400 \\
\hline & Transverse 1 & 80 & 10.43 & 10.38 & 10 & 0.303 & 0.151 & 0.337 \\
\hline & Transverse 5 & 120 & 11.38 & 11.33 & 155 & 0.594 & 0.316 & 0.433 \\
\hline & Transverse 4 & 100 & 9.83 & 9.76 & 90 & 0.188 & 0.079 & 0.346 \\
\hline 5 & Transverse 3 & 100 & 9.91 & 9.85 & 95 & 0.302 & 0.155 & 0.376 \\
\hline & Transverse 2 & 100 & 10.43 & 10.38 & 40 & 0.155 & 0.176 & 0.332 \\
\hline & Transverse 1 & 80 & 7.28 & 7.25 & 20 & 0.229 & 0.221 & 0.394 \\
\hline & Transverse 5 & 120 & 11.09 & 11.05 & 175 & 0.527 & 0.309 & 0.448 \\
\hline & Transverse 4 & 60 & 9.13 & 9.05 & 135 & 0.193 & 0.080 & 0.343 \\
\hline 6 & Transverse 3 & 80 & 9.59 & 9.52 & 120 & 0.283 & 0.137 & 0.284 \\
\hline & Transverse 2 & 80 & 9.79 & 9.73 & 40 & 0.160 & 0.142 & 0.370 \\
\hline & Transverse 1 & 60 & 10.47 & 10.42 & 20 & 0.355 & 0.162 & 0.404 \\
\hline & Transverse 5 & 120 & 11.20 & 11.16 & 145 & 0.560 & 0.316 & 0.433 \\
\hline & Transverse 4 & 40 & 9.01 & 8.93 & 115 & 0.116 & 0.053 & 0.369 \\
\hline 7 & Transverse 3 & 100 & 10.45 & 10.40 & 105 & 0.282 & 0.170 & 0.365 \\
\hline & Transverse 2 & 80 & 9.58 & 9.52 & 75 & 0.244 & 0.153 & 0.305 \\
\hline & Transverse 1 & 80 & 9.92 & 9.86 & 35 & 0.338 & 0.182 & 0.415 \\
\hline & Transverse 5 & 120 & 11.19 & 11.15 & 170 & 0.441 & 0.300 & 0.435 \\
\hline & Transverse 4 & 20 & 8.68 & 8.58 & 110 & 0.143 & 0.053 & 0.290 \\
\hline 8 & Transverse 3 & 80 & 10.07 & 10.01 & 90 & 0.284 & 0.166 & 0.354 \\
\hline & Transverse 2 & 80 & 8.55 & 8.46 & 55 & 0.193 & 0.095 & 0.298 \\
\hline & Transverse 1 & 80 & 10.22 & 10.17 & 30 & 0.444 & 0.196 & 0.404 \\
\hline & Transverse 5 & 120 & 11.20 & 11.15 & 165 & 0.497 & 0.299 & 0.431 \\
\hline & Transverse 4 & 40 & 8.74 & 8.64 & 105 & 0.129 & 0.062 & 0.380 \\
\hline 9 & Transverse 3 & 80 & 9.61 & 9.54 & 90 & 0.239 & 0.144 & 0.377 \\
\hline & Transverse 2 & 80 & 9.60 & 9.53 & 65 & 0.122 & 0.125 & 0.332 \\
\hline & Transverse 1 & 100 & 10.26 & 10.21 & 45 & 0.381 & 0.155 & 0.399 \\
\hline & Transverse 5 & 120 & 11.38 & 11.34 & 150 & 0.491 & 0.303 & 0.442 \\
\hline & Transverse 4 & 20 & 8.59 & 8.50 & 90 & 0.120 & 0.051 & 0.274 \\
\hline 10 & Transverse 3 & 60 & 9.94 & 9.87 & 90 & 0.143 & 0.099 & 0.324 \\
\hline & Transverse 2 & 80 & 10.64 & 10.59 & 85 & 0.236 & 0.161 & 0.413 \\
\hline & Transverse 1 & 80 & 10.30 & 10.24 & 60 & 0.314 & 0.199 & 0.410 \\
\hline & Transverse 5 & 120 & 11.29 & 11.25 & 165 & 0.476 & 0.305 & 0.437 \\
\hline & Transverse 4 & 100 & 8.58 & 8.48 & 110 & 0.216 & 0.050 & 0.305 \\
\hline 11 & Transverse 3 & 80 & 9.60 & 9.53 & 100 & 0.229 & 0.153 & 0.347 \\
\hline & Transverse 2 & 80 & 9.02 & 8.93 & 95 & 0.181 & 0.108 & 0.373 \\
\hline & Transverse 1 & 80 & 10.08 & 10.02 & 55 & 0.394 & 0.158 & 0.401 \\
\hline & Transverse 5 & 120 & 11.20 & 11.16 & 150 & 0.441 & 0.306 & 0.440 \\
\hline & Transverse 4 & 80 & 8.50 & 8.39 & 105 & 0.186 & 0.045 & 0.272 \\
\hline 12 & Transverse 3 & 80 & 10.35 & 10.30 & 80 & 0.273 & 0.166 & 0.405 \\
\hline & Transverse 2 & 100 & 9.32 & 9.24 & 70 & 0.225 & 0.089 & 0.342 \\
\hline & Transverse 1 & 80 & 10.89 & 10.84 & 50 & 0.309 & 0.222 & 0.397 \\
\hline & Transverse 5 & 120 & 11.17 & 11.13 & 155 & 0.443 & 0.305 & 0.440 \\
\hline & Transverse 4 & 60 & 7.72 & 7.65 & 110 & 0.204 & 0.058 & 0.265 \\
\hline 13 & Transverse 3 & 60 & 10.37 & 10.31 & 80 & 0.235 & 0.162 & 0.397 \\
\hline & Transverse 2 & 80 & 9.24 & 9.16 & 80 & 0.157 & 0.096 & 0.314 \\
\hline & Transverse 1 & 80 & 10.51 & 10.46 & 60 & 0.426 & 0.170 & 0.393 \\
\hline & Transverse 5 & 100 & 11.18 & 11.13 & 150 & 0.403 & 0.307 & 0.442 \\
\hline & Transverse 4 & 80 & 8.54 & 8.44 & 110 & 0.218 & 0.052 & 0.267 \\
\hline 14 & Transverse 3 & 60 & 9.91 & 9.85 & 85 & 0.196 & 0.150 & 0.398 \\
\hline & Transverse 2 & 100 & 9.56 & 9.49 & 75 & 0.182 & 0.106 & 0.326 \\
\hline & Transverse 1 & 100 & 10.85 & 10.80 & 50 & 0.405 & 0.194 & 0.428 \\
\hline & Transverse 5 & 120 & 11.18 & 11.14 & 155 & 0.414 & 0.304 & 0.438 \\
\hline & Transverse 4 & 80 & 8.53 & 8.42 & 115 & 0.163 & 0.040 & 0.343 \\
\hline 15 & Transverse 3 & 100 & 10.15 & 10.09 & 90 & 0.219 & 0.173 & 0.381 \\
\hline & Transverse 2 & 80 & 8.69 & 8.60 & 85 & 0.112 & 0.070 & 0.312 \\
\hline & Transverse 1 & 100 & 10.23 & 10.17 & 65 & 0.449 & 0.170 & 0.416 \\
\hline & Transverse 5 & 120 & 11.19 & 11.15 & 145 & 0.373 & 0.298 & 0.441 \\
\hline & Transverse 4 & 100 & 9.33 & 9.24 & 105 & 0.161 & 0.047 & 0.312 \\
\hline 16 & Transverse 3 & 80 & 9.85 & 9.78 & 80 & 0.192 & 0.156 & 0.394 \\
\hline & Transverse 2 & 60 & 8.83 & 8.75 & 90 & 0.077 & 0.080 & 0.318 \\
\hline & Transverse 1 & 80 & 10.43 & 10.37 & 100 & 0.344 & 0.187 & 0.406 \\
\hline 17 & Transverse 5 & 120 & 11.18 & 11.13 & 155 & 0.420 & 0.300 & 0.442 \\
\hline 17 & Transverse 4 & 100 & 9.10 & 9.01 & 115 & 0.136 & 0.064 & 0.382 \\
\hline
\end{tabular}




\begin{tabular}{|c|c|c|c|c|c|c|c|c|}
\hline & Transverse 3 & 80 & 10.04 & 9.98 & 85 & 0.179 & 0.176 & 0.407 \\
\hline & Transverse 2 & 80 & 9.42 & 9.35 & 85 & 0.131 & 0.089 & 0.295 \\
\hline & Transverse 1 & 100 & 10.09 & 10.03 & 80 & 0.448 & 0.158 & 0.403 \\
\hline \multirow{5}{*}{18} & Transverse 5 & 120 & 11.04 & 10.99 & 180 & 0.358 & 0.291 & 0.416 \\
\hline & Transverse 4 & 60 & 9.21 & 9.14 & 100 & 0.139 & 0.081 & 0.333 \\
\hline & Transverse 3 & 80 & 9.67 & 9.60 & 80 & 0.164 & 0.141 & 0.377 \\
\hline & Transverse 2 & 60 & 8.92 & 8.83 & 85 & 0.170 & 0.082 & 0.311 \\
\hline & Transverse 1 & 80 & 10.38 & 10.33 & 75 & 0.467 & 0.168 & 0.399 \\
\hline \multirow{5}{*}{19} & Transverse 5 & 120 & 11.16 & 11.12 & 140 & 0.331 & 0.292 & 0.432 \\
\hline & Transverse 4 & 100 & 9.83 & 9.76 & 90 & 0.154 & 0.088 & 0.378 \\
\hline & Transverse 3 & 80 & 10.10 & 10.03 & 100 & 0.180 & 0.147 & 0.343 \\
\hline & Transverse 2 & 60 & 9.31 & 9.23 & 90 & 0.197 & 0.097 & 0.363 \\
\hline & Transverse 1 & 100 & 10.86 & 10.81 & 90 & 0.386 & 0.198 & 0.405 \\
\hline \multirow{5}{*}{20} & Transverse 5 & 120 & 10.95 & 10.90 & 175 & 0.370 & 0.288 & 0.425 \\
\hline & Transverse 4 & 80 & 9.41 & 9.33 & 85 & 0.126 & 0.074 & 0.337 \\
\hline & Transverse 3 & 80 & 9.67 & 9.60 & 75 & 0.192 & 0.163 & 0.353 \\
\hline & Transverse 2 & 80 & 9.07 & 8.99 & 85 & 0.103 & 0.105 & 0.330 \\
\hline & Transverse 1 & 100 & 10.29 & 10.24 & 110 & 0.404 & 0.206 & 0.405 \\
\hline \multirow{5}{*}{21} & Transverse 5 & 120 & 10.96 & 10.91 & 155 & 0.331 & 0.283 & 0.415 \\
\hline & Transverse 4 & 20 & 8.43 & 8.35 & 85 & 0.085 & 0.046 & 0.272 \\
\hline & Transverse 3 & 80 & 10.02 & 9.96 & 80 & 0.197 & 0.174 & 0.389 \\
\hline & Transverse 2 & 60 & 8.97 & 8.88 & 90 & 0.066 & 0.092 & 0.334 \\
\hline & Transverse 1 & 60 & 10.48 & 10.43 & 105 & 0.349 & 0.200 & 0.380 \\
\hline \multirow{5}{*}{22} & Transverse 5 & 100 & 11.04 & 10.99 & 160 & 0.278 & 0.277 & 0.409 \\
\hline & Transverse 4 & 40 & 9.03 & 8.94 & 90 & 0.124 & 0.050 & 0.276 \\
\hline & Transverse 3 & 80 & 10.04 & 9.98 & 80 & 0.185 & 0.172 & 0.402 \\
\hline & Transverse 2 & 100 & 9.64 & 9.57 & 105 & 0.116 & 0.107 & 0.358 \\
\hline & Transverse 1 & 80 & 10.11 & 10.05 & 90 & 0.441 & 0.184 & 0.376 \\
\hline \multirow{5}{*}{23} & Transverse 5 & 120 & 10.87 & 10.82 & 115 & 0.285 & 0.283 & 0.409 \\
\hline & Transverse 4 & 100 & 8.78 & 8.70 & 80 & 0.139 & 0.067 & 0.396 \\
\hline & Transverse 3 & 80 & 10.62 & 10.57 & 75 & 0.185 & 0.192 & 0.370 \\
\hline & Transverse 2 & 60 & 8.47 & 8.38 & 105 & 0.087 & 0.069 & 0.310 \\
\hline & Transverse 1 & 100 & 10.28 & 10.23 & 90 & 0.474 & 0.192 & 0.395 \\
\hline \multirow{4}{*}{24} & Transverse 4 & 120 & 10.76 & 10.72 & 190 & 0.312 & 0.285 & 0.421 \\
\hline & Transverse 3 & 100 & 9.69 & 9.63 & 120 & 0.189 & 0.158 & 0.404 \\
\hline & Transverse 2 & 80 & 8.38 & 8.29 & 110 & 0.083 & 0.086 & 0.298 \\
\hline & Transverse 1 & 80 & 9.32 & 9.25 & 130 & 0.327 & 0.171 & 0.379 \\
\hline \multirow{4}{*}{25} & Transverse 4 & 120 & 11.01 & 10.96 & 135 & 0.351 & 0.293 & 0.446 \\
\hline & Transverse 3 & 100 & 10.04 & 9.98 & 85 & 0.137 & 0.109 & 0.416 \\
\hline & Transverse 2 & 80 & 9.52 & 9.45 & 60 & 0.095 & 0.116 & 0.342 \\
\hline & Transverse 1 & 100 & 10.10 & 10.05 & 90 & 0.468 & 0.184 & 0.389 \\
\hline \multirow{4}{*}{26} & Transverse 4 & 120 & 10.71 & 10.66 & 210 & 0.392 & 0.281 & 0.421 \\
\hline & Transverse 3 & 80 & 8.85 & 8.77 & 75 & 0.104 & 0.074 & 0.307 \\
\hline & Transverse 2 & 80 & 9.38 & 9.31 & 65 & 0.094 & 0.159 & 0.385 \\
\hline & Transverse 1 & 80 & 9.29 & 9.22 & 130 & 0.406 & 0.185 & 0.383 \\
\hline \multirow{4}{*}{27} & Transverse 4 & 120 & 11.00 & 10.96 & 215 & 0.280 & 0.293 & 0.441 \\
\hline & Transverse 3 & 60 & 9.60 & 9.53 & 75 & 0.126 & 0.116 & 0.346 \\
\hline & Transverse 2 & 60 & 8.24 & 8.16 & 85 & 0.062 & 0.089 & 0.318 \\
\hline & Transverse 1 & 80 & 10.07 & 10.01 & 90 & 0.352 & 0.193 & 0.425 \\
\hline \multirow{4}{*}{28} & Transverse 4 & 120 & 10.69 & 10.65 & 215 & 0.323 & 0.296 & 0.445 \\
\hline & Transverse 3 & 80 & 9.28 & 9.21 & 80 & 0.119 & 0.100 & 0.326 \\
\hline & Transverse 2 & 40 & 8.70 & 8.61 & 100 & 0.126 & 0.081 & 0.366 \\
\hline & Transverse 1 & 100 & 10.29 & 10.24 & 90 & 0.483 & 0.205 & 0.383 \\
\hline
\end{tabular}

Table 141: Case Study 2 - 40 ft Cluster Spacing Fracture Results

\begin{tabular}{|c|c|c|c|c|c|c|c|c|}
\hline Stage Number & Fracture & $\begin{array}{c}\text { Proppant } \\
\text { Cutoff } \\
\text { Length } \\
\text { (ft) }\end{array}$ & $\begin{array}{c}\text { Est Flowing } \\
\text { Frac Length } \\
(\mathrm{ft}) \\
\end{array}$ & $\begin{array}{c}\text { Est Inf } \\
\text { Conductivity } \\
\text { Length } \\
\text { (ft) } \\
\end{array}$ & $\begin{array}{c}\begin{array}{c}\text { Fracture } \\
\text { Height }\end{array} \\
\text { (ft) } \\
\end{array}$ & $\begin{array}{c}\text { Average } \\
\text { Proppant } \\
\text { Conc } \\
\left(\mathrm{lb} / \mathrm{ft}^{2}\right) \\
\end{array}$ & $\begin{array}{c}\text { Average } \\
\text { Fracture } \\
\text { Width } \\
\text { (in) }\end{array}$ & $\begin{array}{c}\text { Max } \\
\text { Fracture } \\
\text { Width } \\
\text { (in) } \\
\end{array}$ \\
\hline \multirow{6}{*}{1} & Transverse 6 & 120 & 11.20 & 11.16 & 120 & 0.565 & 0.308 & 0.399 \\
\hline & Transverse 5 & 40 & 6.81 & 6.76 & 110 & 0.144 & 0.035 & 0.119 \\
\hline & Transverse 4 & 60 & 9.69 & 9.61 & 90 & 0.221 & 0.121 & 0.310 \\
\hline & Transverse 3 & 60 & 9.71 & 9.64 & 90 & 0.220 & 0.121 & 0.162 \\
\hline & Transverse 2 & 40 & 6.94 & 6.88 & 110 & 0.129 & 0.031 & 0.119 \\
\hline & Transverse 1 & 120 & 11.15 & 11.11 & 120 & 0.554 & 0.302 & 0.393 \\
\hline \multirow{6}{*}{2} & Transverse 6 & 120 & 11.11 & 11.06 & 145 & 0.511 & 0.295 & 0.424 \\
\hline & Transverse 5 & 80 & 8.03 & 7.94 & 150 & 0.190 & 0.056 & 0.270 \\
\hline & Transverse 4 & 100 & 9.74 & 9.67 & 125 & 0.314 & 0.142 & 0.320 \\
\hline & Transverse 3 & 80 & 8.99 & 8.90 & 60 & 0.190 & 0.105 & 0.335 \\
\hline & Transverse 2 & 80 & 8.94 & 8.85 & 40 & 0.193 & 0.101 & 0.359 \\
\hline & Transverse 1 & 100 & 9.95 & 9.89 & 45 & 0.325 & 0.246 & 0.397 \\
\hline \multirow{6}{*}{3} & Transverse 6 & 120 & 11.29 & 11.25 & 150 & 0.589 & 0.313 & 0.425 \\
\hline & Transverse 5 & 100 & 9.17 & 9.08 & 90 & 0.150 & 0.048 & 0.300 \\
\hline & Transverse 4 & 100 & 9.91 & 9.85 & 90 & 0.258 & 0.136 & 0.353 \\
\hline & Transverse 3 & 100 & 10.16 & 10.10 & 85 & 0.160 & 0.132 & 0.324 \\
\hline & Transverse 2 & 100 & 9.86 & 9.79 & 35 & 0.184 & 0.172 & 0.381 \\
\hline & Transverse 1 & 60 & 10.38 & 10.32 & 20 & 0.307 & 0.146 & 0.326 \\
\hline \multirow{2}{*}{4} & Transverse 6 & 120 & 11.32 & 11.27 & 150 & 0.598 & 0.317 & 0.437 \\
\hline & Transverse 5 & 40 & 5.47 & 5.41 & 65 & 0.139 & 0.034 & 0.268 \\
\hline
\end{tabular}




\begin{tabular}{|c|c|c|c|c|c|c|c|c|}
\hline & Transverse 4 & 100 & 9.88 & 9.81 & 110 & 0.178 & 0.118 & 0.351 \\
\hline & Transverse 3 & 100 & 10.23 & 10.17 & 110 & 0.323 & 0.136 & 0.302 \\
\hline & Transverse 2 & 100 & 10.05 & 9.98 & 35 & 0.190 & 0.149 & 0.366 \\
\hline & Transverse 1 & 80 & 10.49 & 10.43 & 20 & 0.303 & 0.182 & 0.330 \\
\hline \multirow{6}{*}{5} & Transverse 6 & 120 & 11.44 & 11.39 & 155 & 0.584 & 0.321 & 0.443 \\
\hline & Transverse 5 & 60 & 8.29 & 8.17 & 105 & 0.169 & 0.039 & 0.303 \\
\hline & Transverse 4 & 80 & 10.22 & 10.16 & 90 & 0.239 & 0.147 & 0.348 \\
\hline & Transverse 3 & 80 & 9.63 & 9.56 & 75 & 0.200 & 0.122 & 0.306 \\
\hline & Transverse 2 & 80 & 10.07 & 10.00 & 40 & 0.191 & 0.134 & 0.340 \\
\hline & Transverse 1 & 40 & 10.54 & 10.49 & 15 & 0.153 & 0.145 & 0.393 \\
\hline \multirow{6}{*}{6} & Transverse 6 & 120 & 11.26 & 11.21 & 145 & 0.514 & 0.309 & 0.443 \\
\hline & Transverse 5 & 80 & 9.64 & 9.57 & 140 & 0.160 & 0.073 & 0.375 \\
\hline & Transverse 4 & 100 & 10.61 & 10.56 & 85 & 0.351 & 0.180 & 0.388 \\
\hline & Transverse 3 & 60 & 8.37 & 8.29 & 55 & 0.160 & 0.072 & 0.298 \\
\hline & Transverse 2 & 80 & 10.54 & 10.48 & 25 & 0.166 & 0.133 & 0.371 \\
\hline & Transverse 1 & 80 & 10.22 & 10.16 & 20 & 0.330 & 0.140 & 0.406 \\
\hline \multirow{6}{*}{7} & Transverse 6 & 120 & 11.51 & 11.47 & 150 & 0.570 & 0.316 & 0.435 \\
\hline & Transverse 5 & 40 & 7.75 & 7.64 & 130 & 0.124 & 0.036 & 0.228 \\
\hline & Transverse 4 & 60 & 9.50 & 9.42 & 110 & 0.154 & 0.105 & 0.316 \\
\hline & Transverse 3 & 100 & 10.23 & 10.17 & 100 & 0.292 & 0.142 & 0.344 \\
\hline & Transverse 2 & 80 & 9.36 & 9.28 & 40 & 0.150 & 0.129 & 0.376 \\
\hline & Transverse 1 & 60 & 10.76 & 10.71 & 30 & 0.424 & 0.159 & 0.416 \\
\hline \multirow{6}{*}{8} & Transverse 6 & 120 & 11.10 & 11.05 & 165 & 0.444 & 0.281 & 0.413 \\
\hline & Transverse 5 & 80 & 9.10 & 9.01 & 110 & 0.110 & 0.057 & 0.343 \\
\hline & Transverse 4 & 40 & 9.17 & 9.08 & 90 & 0.150 & 0.066 & 0.258 \\
\hline & Transverse 3 & 60 & 10.88 & 10.83 & 90 & 0.211 & 0.163 & 0.392 \\
\hline & Transverse 2 & 60 & 7.16 & 7.12 & 75 & 0.213 & 0.093 & 0.294 \\
\hline & Transverse 1 & 80 & 9.69 & 9.64 & 55 & 0.340 & 0.191 & 0.400 \\
\hline & Transverse 6 & 120 & 11.09 & 11.05 & 150 & 0.432 & 0.289 & 0.423 \\
\hline & Transverse 5 & 60 & 9.41 & 9.32 & 110 & 0.192 & 0.051 & 0.311 \\
\hline 9 & Transverse 4 & 80 & 9.84 & 9.77 & 95 & 0.178 & 0.129 & 0.390 \\
\hline J & Transverse 3 & 60 & 10.73 & 10.68 & 85 & 0.203 & 0.131 & 0.313 \\
\hline & Transverse 2 & 80 & 9.15 & 9.08 & 80 & 0.159 & 0.140 & 0.379 \\
\hline & Transverse 1 & 80 & 10.37 & 10.31 & 55 & 0.359 & 0.146 & 0.379 \\
\hline & Transverse 6 & 120 & 11.29 & 11.25 & 165 & 0.457 & 0.287 & 0.416 \\
\hline & Transverse 5 & 60 & 8.09 & 7.97 & 100 & 0.220 & 0.039 & 0.243 \\
\hline 10 & Transverse 4 & 60 & 10.06 & 9.99 & 90 & 0.177 & 0.128 & 0.327 \\
\hline 10 & Transverse 3 & 60 & 9.02 & 8.94 & 90 & 0.168 & 0.119 & 0.328 \\
\hline & Transverse 2 & 80 & 7.72 & 7.66 & 70 & 0.334 & 0.071 & 0.300 \\
\hline & Transverse 1 & 100 & 10.77 & 10.72 & 55 & 0.429 & 0.172 & 0.398 \\
\hline & Transverse 6 & 120 & 10.95 & 10.90 & 145 & 0.386 & 0.274 & 0.411 \\
\hline & Transverse 5 & 100 & 9.41 & 9.32 & 115 & 0.168 & 0.080 & 0.389 \\
\hline 11 & Transverse 4 & 80 & 9.98 & 9.92 & 80 & 0.204 & 0.134 & 0.372 \\
\hline 11 & Transverse 3 & 80 & 9.41 & 9.33 & 75 & 0.182 & 0.087 & 0.318 \\
\hline & Transverse 2 & 80 & 9.66 & 9.58 & 55 & 0.177 & 0.103 & 0.328 \\
\hline & Transverse 1 & 80 & 10.10 & 10.03 & 30 & 0.398 & 0.153 & 0.375 \\
\hline & Transverse 6 & 120 & 11.11 & 11.06 & 180 & 0.425 & 0.274 & 0.404 \\
\hline & Transverse 5 & 60 & 7.92 & 7.83 & 135 & 0.237 & 0.042 & 0.269 \\
\hline 12 & Transverse 4 & 80 & 9.65 & 9.59 & 105 & 0.176 & 0.131 & 0.351 \\
\hline 12 & Transverse 3 & 80 & 10.59 & 10.54 & 75 & 0.215 & 0.164 & 0.403 \\
\hline & Transverse 2 & 100 & 10.40 & 10.35 & 110 & 0.204 & 0.147 & 0.412 \\
\hline & Transverse 1 & 60 & 7.60 & 7.56 & 80 & 0.420 & 0.179 & 0.358 \\
\hline & Transverse 5 & 120 & 11.24 & 11.20 & 155 & 0.471 & 0.317 & 0.453 \\
\hline & Transverse 4 & 60 & 8.52 & 8.44 & 95 & 0.090 & 0.044 & 0.302 \\
\hline 13 & Transverse 3 & 40 & 9.82 & 9.76 & 105 & 0.187 & 0.146 & 0.377 \\
\hline & Transverse 2 & 40 & 5.37 & 5.31 & 110 & 0.060 & 0.121 & 0.389 \\
\hline & Transverse 1 & 80 & 8.36 & 8.30 & 80 & 0.261 & 0.198 & 0.413 \\
\hline & Transverse 5 & 100 & 11.12 & 11.08 & 165 & 0.412 & 0.308 & 0.449 \\
\hline & Transverse 4 & 40 & 7.22 & 7.13 & 110 & 0.170 & 0.032 & 0.259 \\
\hline 14 & Transverse 3 & 60 & 9.55 & 9.48 & 85 & 0.145 & 0.147 & 0.357 \\
\hline & Transverse 2 & 40 & 8.08 & 8.01 & 85 & 0.300 & 0.092 & 0.281 \\
\hline & Transverse 1 & 100 & 10.07 & 10.01 & 65 & 0.407 & 0.163 & 0.400 \\
\hline & Transverse 5 & 120 & 11.21 & 11.17 & 155 & 0.410 & 0.310 & 0.452 \\
\hline & Transverse 4 & 60 & 8.96 & 8.87 & 140 & 0.170 & 0.076 & 0.297 \\
\hline 15 & Transverse 3 & 100 & 9.63 & 9.56 & 100 & 0.191 & 0.114 & 0.327 \\
\hline & Transverse 2 & 80 & 9.21 & 9.13 & 85 & 0.201 & 0.129 & 0.347 \\
\hline & Transverse 1 & 100 & 11.07 & 11.03 & 55 & 0.453 & 0.189 & 0.425 \\
\hline & Transverse 5 & 100 & 11.33 & 11.28 & 155 & 0.373 & 0.305 & 0.447 \\
\hline & Transverse 4 & 60 & 8.80 & 8.72 & 90 & 0.098 & 0.036 & 0.297 \\
\hline 16 & Transverse 3 & 60 & 10.66 & 10.60 & 80 & 0.189 & 0.170 & 0.372 \\
\hline & Transverse 2 & 40 & 8.55 & 8.46 & 85 & 0.195 & 0.064 & 0.306 \\
\hline & Transverse 1 & 80 & 10.02 & 9.95 & 90 & 0.426 & 0.184 & 0.404 \\
\hline & Transverse 5 & 120 & 11.19 & 11.15 & 165 & 0.434 & 0.302 & 0.441 \\
\hline & Transverse 4 & 60 & 8.94 & 8.85 & 85 & 0.138 & 0.074 & 0.325 \\
\hline 17 & Transverse 3 & 80 & 9.96 & 9.89 & 100 & 0.146 & 0.152 & 0.415 \\
\hline & Transverse 2 & 60 & 8.80 & 8.72 & 85 & 0.151 & 0.071 & 0.285 \\
\hline & Transverse 1 & 80 & 8.54 & 8.49 & 90 & 0.469 & 0.180 & 0.417 \\
\hline & Transverse 5 & 120 & 11.14 & 11.10 & 140 & 0.403 & 0.298 & 0.439 \\
\hline & Transverse 4 & 100 & 9.29 & 9.21 & 90 & 0.127 & 0.054 & 0.328 \\
\hline 18 & Transverse 3 & 80 & 10.20 & 10.14 & 100 & 0.147 & 0.176 & 0.408 \\
\hline & Transverse 2 & 80 & 9.31 & 9.23 & 90 & 0.192 & 0.095 & 0.289 \\
\hline & Transverse 1 & 60 & 7.54 & 7.48 & 90 & 0.319 & 0.196 & 0.403 \\
\hline
\end{tabular}




\begin{tabular}{|c|c|c|c|c|c|c|c|c|}
\hline \multirow{5}{*}{19} & Transverse 5 & 100 & 10.86 & 10.81 & 175 & 0.307 & 0.286 & 0.429 \\
\hline & Transverse 4 & 100 & 8.54 & 8.44 & 100 & 0.131 & 0.047 & 0.322 \\
\hline & Transverse 3 & 80 & 10.08 & 10.02 & 85 & 0.199 & 0.180 & 0.419 \\
\hline & Transverse 2 & 40 & 9.62 & 9.55 & 90 & 0.158 & 0.086 & 0.294 \\
\hline & Transverse 1 & 80 & 10.32 & 10.27 & 90 & 0.376 & 0.178 & 0.388 \\
\hline \multirow{5}{*}{20} & Transverse 5 & 120 & 11.10 & 11.05 & 145 & 0.343 & 0.295 & 0.441 \\
\hline & Transverse 4 & 80 & 10.05 & 9.98 & 75 & 0.097 & 0.056 & 0.326 \\
\hline & Transverse 3 & 60 & 9.69 & 9.62 & 85 & 0.137 & 0.156 & 0.411 \\
\hline & Transverse 2 & 60 & 9.56 & 9.48 & 95 & 0.262 & 0.079 & 0.273 \\
\hline & Transverse 1 & 80 & 7.88 & 7.85 & 90 & 0.427 & 0.198 & 0.411 \\
\hline \multirow{5}{*}{21} & Transverse 5 & 120 & 10.95 & 10.91 & 165 & 0.284 & 0.290 & 0.434 \\
\hline & Transverse 4 & 80 & 9.31 & 9.24 & 70 & 0.102 & 0.066 & 0.337 \\
\hline & Transverse 3 & 60 & 10.03 & 9.97 & 120 & 0.165 & 0.144 & 0.366 \\
\hline & Transverse 2 & 40 & 9.37 & 9.30 & 125 & 0.088 & 0.135 & 0.317 \\
\hline & Transverse 1 & 80 & 7.62 & 7.56 & 110 & 0.377 & 0.190 & 0.387 \\
\hline \multirow{5}{*}{22} & Transverse 5 & 120 & 11.09 & 11.04 & 165 & 0.362 & 0.291 & 0.435 \\
\hline & Transverse 4 & 80 & 9.41 & 9.34 & 70 & 0.089 & 0.061 & 0.339 \\
\hline & Transverse 3 & 100 & 9.97 & 9.91 & 95 & 0.183 & 0.139 & 0.375 \\
\hline & Transverse 2 & 40 & 9.14 & 9.05 & 100 & 0.126 & 0.092 & 0.282 \\
\hline & Transverse 1 & 80 & 9.50 & 9.45 & 90 & 0.419 & 0.182 & 0.390 \\
\hline \multirow{5}{*}{23} & Transverse 5 & 100 & 10.75 & 10.71 & 170 & 0.229 & 0.273 & 0.418 \\
\hline & Transverse 4 & 80 & 9.39 & 9.32 & 100 & 0.107 & 0.066 & 0.333 \\
\hline & Transverse 3 & 40 & 10.49 & 10.44 & 75 & 0.142 & 0.190 & 0.354 \\
\hline & Transverse 2 & 60 & 9.54 & 9.47 & 150 & 0.165 & 0.132 & 0.396 \\
\hline & Transverse 1 & 80 & 9.38 & 9.31 & 110 & 0.407 & 0.169 & 0.383 \\
\hline \multirow{5}{*}{24} & Transverse 5 & 100 & 10.92 & 10.88 & 130 & 0.276 & 0.279 & 0.424 \\
\hline & Transverse 4 & 100 & 8.72 & 8.62 & 95 & 0.113 & 0.041 & 0.332 \\
\hline & Transverse 3 & 60 & 10.28 & 10.23 & 80 & 0.168 & 0.164 & 0.406 \\
\hline & Transverse 2 & 80 & 9.02 & 8.93 & 85 & 0.093 & 0.076 & 0.303 \\
\hline & Transverse 1 & 100 & 10.48 & 10.43 & 90 & 0.490 & 0.183 & 0.398 \\
\hline \multirow{5}{*}{25} & Transverse 5 & 100 & 10.86 & 10.82 & 145 & 0.268 & 0.276 & 0.413 \\
\hline & Transverse 4 & 100 & 9.01 & 8.92 & 90 & 0.124 & 0.061 & 0.375 \\
\hline & Transverse 3 & 60 & 9.62 & 9.55 & 85 & 0.146 & 0.129 & 0.335 \\
\hline & Transverse 2 & 80 & 9.45 & 9.38 & 75 & 0.096 & 0.111 & 0.345 \\
\hline & Transverse 1 & 100 & 10.45 & 10.40 & 110 & 0.477 & 0.184 & 0.397 \\
\hline \multirow{5}{*}{26} & Transverse 5 & 100 & 10.71 & 10.66 & 170 & 0.267 & 0.288 & 0.443 \\
\hline & Transverse 4 & 40 & 8.73 & 8.63 & 85 & 0.085 & 0.052 & 0.304 \\
\hline & Transverse 3 & 100 & 10.12 & 10.06 & 95 & 0.206 & 0.171 & 0.390 \\
\hline & Transverse 2 & 40 & 7.59 & 7.51 & 105 & 0.075 & 0.115 & 0.314 \\
\hline & Transverse 1 & 80 & 8.02 & 8.00 & 90 & 0.476 & 0.200 & 0.394 \\
\hline \multirow{5}{*}{27} & Transverse 5 & 120 & 11.19 & 11.15 & 145 & 0.278 & 0.296 & 0.448 \\
\hline & Transverse 4 & 60 & 8.76 & 8.67 & 85 & 0.078 & 0.036 & 0.303 \\
\hline & Transverse 3 & 60 & 9.92 & 9.86 & 70 & 0.127 & 0.134 & 0.384 \\
\hline & Transverse 2 & 60 & 9.32 & 9.23 & 100 & 0.101 & 0.088 & 0.322 \\
\hline & Transverse 1 & 100 & 10.71 & 10.66 & 110 & 0.444 & 0.225 & 0.405 \\
\hline \multirow{5}{*}{28} & Transverse 5 & 100 & 10.95 & 10.90 & 190 & 0.333 & 0.297 & 0.434 \\
\hline & Transverse 4 & 60 & 8.69 & 8.60 & 95 & 0.090 & 0.041 & 0.323 \\
\hline & Transverse 3 & 80 & 9.59 & 9.53 & 110 & 0.117 & 0.135 & 0.380 \\
\hline & Transverse 2 & 60 & 8.42 & 8.32 & 95 & 0.083 & 0.056 & 0.320 \\
\hline & Transverse 1 & 100 & 9.87 & 9.81 & 110 & 0.491 & 0.197 & 0.421 \\
\hline
\end{tabular}

Table 142: Case Study 2 - 35 ft Cluster Spacing Fracture Results

\begin{tabular}{|c|c|c|c|c|c|c|c|c|}
\hline Stage Number & Fracture & $\begin{array}{c}\text { Proppant } \\
\text { Cutoff } \\
\text { Length } \\
\text { (ft) }\end{array}$ & $\begin{array}{c}\text { Est Flowing } \\
\text { Frac Length } \\
(\mathrm{ft}) \\
\end{array}$ & $\begin{array}{c}\text { Est Inf } \\
\text { Conductivity } \\
\text { Length } \\
\text { (ft) } \\
\end{array}$ & 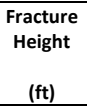 & $\begin{array}{c}\text { Average } \\
\text { Proppant } \\
\text { Conc } \\
\left(\mathrm{lb} / \mathrm{ft}^{2}\right) \\
\end{array}$ & $\begin{array}{c}\text { Average } \\
\text { Fracture } \\
\text { Width } \\
\text { (in) } \\
\end{array}$ & $\begin{array}{c}\text { Max } \\
\text { Fracture } \\
\text { Width } \\
\text { (in) } \\
\end{array}$ \\
\hline \multirow{7}{*}{1} & Transverse 7 & 120 & 11.12 & 11.07 & 110 & 0.556 & 0.288 & 0.376 \\
\hline & Transverse 6 & 60 & 6.77 & 6.72 & 115 & 0.168 & 0.044 & 0.222 \\
\hline & Transverse 5 & 40 & 9.43 & 9.34 & 110 & 0.175 & 0.086 & 0.300 \\
\hline & Transverse 4 & 40 & 9.34 & 9.26 & 110 & 0.189 & 0.097 & 0.248 \\
\hline & Transverse 3 & 40 & 9.27 & 9.19 & 90 & 0.181 & 0.097 & 0.187 \\
\hline & Transverse 2 & 60 & 7.67 & 7.58 & 95 & 0.200 & 0.038 & 0.254 \\
\hline & Transverse 1 & 120 & 11.31 & 11.27 & 110 & 0.534 & 0.322 & 0.408 \\
\hline \multirow{7}{*}{2} & Transverse 7 & 120 & 11.22 & 11.18 & 145 & 0.496 & 0.286 & 0.413 \\
\hline & Transverse 6 & 60 & 8.71 & 8.62 & 110 & 0.229 & 0.049 & 0.243 \\
\hline & Transverse 5 & 40 & 9.94 & 9.87 & 90 & 0.204 & 0.122 & 0.373 \\
\hline & Transverse 4 & 60 & 9.03 & 9.01 & 90 & 0.493 & 0.058 & 0.335 \\
\hline & Transverse 3 & 100 & 10.40 & 10.34 & 85 & 0.344 & 0.166 & 0.349 \\
\hline & Transverse 2 & 140 & 7.12 & 7.09 & 30 & 0.191 & 0.078 & 0.262 \\
\hline & Transverse 1 & 80 & 10.84 & 10.79 & 20 & 0.424 & 0.253 & 0.403 \\
\hline \multirow{7}{*}{3} & Transverse 7 & 120 & 11.15 & 11.11 & 145 & 0.500 & 0.291 & 0.409 \\
\hline & Transverse 6 & 40 & 5.03 & 4.98 & 100 & 0.112 & 0.021 & 0.127 \\
\hline & Transverse 5 & 80 & 8.97 & 8.90 & 110 & 0.145 & 0.067 & 0.299 \\
\hline & Transverse 4 & 80 & 9.88 & 9.81 & 110 & 0.227 & 0.109 & 0.355 \\
\hline & Transverse 3 & 100 & 10.76 & 10.71 & 80 & 0.334 & 0.198 & 0.333 \\
\hline & Transverse 2 & 100 & 10.78 & 10.73 & 40 & 0.261 & 0.132 & 0.348 \\
\hline & Transverse 1 & 40 & 7.65 & 7.60 & 20 & 0.168 & 0.133 & 0.334 \\
\hline \multirow{2}{*}{4} & Transverse 7 & 120 & 10.87 & 10.82 & 140 & 0.430 & 0.276 & 0.396 \\
\hline & Transverse 6 & 40 & 5.38 & 5.34 & 120 & 0.142 & 0.038 & 0.224 \\
\hline
\end{tabular}




\begin{tabular}{|c|c|c|c|c|c|c|c|c|}
\hline & Transverse 5 & 60 & 9.52 & 9.45 & 130 & 0.236 & 0.112 & 0.347 \\
\hline & Transverse 4 & 100 & 8.95 & 8.86 & 90 & 0.421 & 0.082 & 0.334 \\
\hline & Transverse 3 & 100 & 11.00 & 10.95 & 90 & 0.379 & 0.165 & 0.344 \\
\hline & Transverse 2 & 80 & 10.18 & 10.12 & 45 & 0.218 & 0.188 & 0.341 \\
\hline & Transverse 1 & 80 & 10.25 & 10.19 & 20 & 0.294 & 0.140 & 0.349 \\
\hline \multirow{7}{*}{5} & Transverse 7 & 120 & 10.98 & 10.93 & 145 & 0.464 & 0.276 & 0.403 \\
\hline & Transverse 6 & 80 & 8.83 & 8.74 & 135 & 0.194 & 0.049 & 0.252 \\
\hline & Transverse 5 & 100 & 9.57 & 9.50 & 90 & 0.224 & 0.104 & 0.335 \\
\hline & Transverse 4 & 80 & 10.35 & 10.29 & 90 & 0.327 & 0.141 & 0.331 \\
\hline & Transverse 3 & 60 & 10.32 & 10.27 & 35 & 0.171 & 0.168 & 0.341 \\
\hline & Transverse 2 & 80 & 9.01 & 8.95 & 60 & 0.317 & 0.241 & 0.388 \\
\hline & Transverse 1 & 40 & 10.13 & 10.08 & 10 & 0.204 & 0.127 & 0.341 \\
\hline \multirow{7}{*}{6} & Transverse 7 & 120 & 11.15 & 11.10 & 140 & 0.462 & 0.281 & 0.404 \\
\hline & Transverse 6 & 40 & 9.57 & 9.49 & 90 & 0.149 & 0.062 & 0.346 \\
\hline & Transverse 5 & 40 & 10.03 & 9.96 & 110 & 0.162 & 0.090 & 0.308 \\
\hline & Transverse 4 & 100 & 10.37 & 10.31 & 90 & 0.215 & 0.110 & 0.364 \\
\hline & Transverse 3 & 100 & 10.13 & 10.06 & 75 & 0.256 & 0.172 & 0.328 \\
\hline & Transverse 2 & 80 & 8.77 & 8.69 & 35 & 0.167 & 0.096 & 0.339 \\
\hline & Transverse 1 & 80 & 10.75 & 10.69 & 20 & 0.389 & 0.165 & 0.389 \\
\hline \multirow{6}{*}{7} & Transverse 6 & 120 & 10.99 & 10.95 & 150 & 0.514 & 0.300 & 0.424 \\
\hline & Transverse 5 & 40 & 8.39 & 8.31 & 145 & 0.141 & 0.058 & 0.350 \\
\hline & Transverse 4 & 80 & 8.96 & 8.88 & 105 & 0.107 & 0.080 & 0.294 \\
\hline & Transverse 3 & 100 & 10.37 & 10.32 & 120 & 0.237 & 0.181 & 0.361 \\
\hline & Transverse 2 & 20 & 10.19 & 10.14 & 80 & 0.108 & 0.117 & 0.341 \\
\hline & Transverse 1 & 80 & 10.18 & 10.13 & 30 & 0.413 & 0.176 & 0.384 \\
\hline \multirow{6}{*}{8} & Transverse 6 & 120 & 11.04 & 10.99 & 165 & 0.457 & 0.281 & 0.414 \\
\hline & Transverse 5 & 80 & 9.49 & 9.41 & 105 & 0.142 & 0.094 & 0.387 \\
\hline & Transverse 4 & 60 & 8.45 & 8.37 & 105 & 0.133 & 0.073 & 0.300 \\
\hline & Transverse 3 & 100 & 10.20 & 10.14 & 95 & 0.198 & 0.146 & 0.328 \\
\hline & Transverse 2 & 80 & 10.22 & 10.16 & 70 & 0.249 & 0.133 & 0.389 \\
\hline & Transverse 1 & 80 & 10.44 & 10.38 & 30 & 0.376 & 0.174 & 0.403 \\
\hline & Transverse 6 & 120 & 11.07 & 11.03 & 115 & 0.207 & 0.280 & 0.405 \\
\hline & Transverse 5 & 100 & 9.51 & 9.44 & 130 & 0.173 & 0.095 & 0.365 \\
\hline & Transverse 4 & 80 & 9.20 & 9.12 & 100 & 0.126 & 0.072 & 0.374 \\
\hline 9 & Transverse 3 & 80 & 10.82 & 10.77 & 75 & 0.280 & 0.215 & 0.410 \\
\hline & Transverse 2 & 20 & 4.28 & 4.22 & 90 & 0.079 & 0.115 & 0.355 \\
\hline & Transverse 1 & 80 & 9.70 & 9.64 & 55 & 0.398 & 0.139 & 0.390 \\
\hline & Transverse 6 & 120 & 10.85 & 10.80 & 140 & 0.395 & 0.283 & 0.423 \\
\hline & Transverse 5 & 40 & 5.52 & 5.49 & 85 & 0.145 & 0.043 & 0.121 \\
\hline & Transverse 4 & 100 & 10.12 & 10.05 & 120 & 0.245 & 0.127 & 0.404 \\
\hline 10 & Transverse 3 & 60 & 10.02 & 9.96 & 85 & 0.178 & 0.133 & 0.375 \\
\hline & Transverse 2 & 60 & 8.38 & 8.31 & 110 & 0.121 & 0.073 & 0.326 \\
\hline & Transverse 1 & 80 & 10.92 & 10.87 & 110 & 0.475 & 0.232 & 0.381 \\
\hline & Transverse 6 & 100 & 10.90 & 10.85 & 150 & 0.317 & 0.264 & 0.404 \\
\hline & Transverse 5 & 60 & 8.71 & 8.61 & 110 & 0.255 & 0.052 & 0.318 \\
\hline & Transverse 4 & 80 & 9.68 & 9.61 & 85 & 0.167 & 0.102 & 0.320 \\
\hline 11 & Transverse 3 & 60 & 10.33 & 10.27 & 70 & 0.221 & 0.140 & 0.352 \\
\hline & Transverse 2 & 20 & 9.41 & 9.34 & 120 & 0.096 & 0.138 & 0.352 \\
\hline & Transverse 1 & 60 & 10.56 & 10.50 & 80 & 0.351 & 0.176 & 0.345 \\
\hline & Transverse 6 & 100 & 10.76 & 10.71 & 150 & 0.339 & 0.269 & 0.414 \\
\hline & Transverse 5 & 60 & 8.10 & 8.02 & 155 & 0.139 & 0.047 & 0.294 \\
\hline 12 & Transverse 4 & 100 & 9.57 & 9.50 & 95 & 0.383 & 0.104 & 0.355 \\
\hline 12 & Transverse 3 & 60 & 10.72 & 10.67 & 75 & 0.199 & 0.171 & 0.389 \\
\hline & Transverse 2 & 20 & 4.85 & 4.80 & 100 & 0.126 & 0.065 & 0.337 \\
\hline & Transverse 1 & 80 & 7.68 & 7.66 & 60 & 0.376 & 0.197 & 0.386 \\
\hline & Transverse 6 & 100 & 10.92 & 10.88 & 145 & 0.332 & 0.271 & 0.412 \\
\hline & Transverse 5 & 20 & 9.20 & 9.11 & 125 & 0.088 & 0.044 & 0.342 \\
\hline 13 & Transverse 4 & 60 & 9.59 & 9.52 & 80 & 0.219 & 0.139 & 0.338 \\
\hline 13 & Transverse 3 & 100 & 9.93 & 9.87 & 115 & 0.236 & 0.147 & 0.405 \\
\hline & Transverse 2 & 40 & 9.80 & 9.74 & 90 & 0.139 & 0.144 & 0.289 \\
\hline & Transverse 1 & 80 & 7.71 & 7.69 & 75 & 0.395 & 0.177 & 0.380 \\
\hline & Transverse 6 & 100 & 11.06 & 11.01 & 155 & 0.341 & 0.276 & 0.419 \\
\hline & Transverse 5 & 100 & 9.68 & 9.61 & 95 & 0.134 & 0.075 & 0.334 \\
\hline & Transverse 4 & 80 & 9.73 & 9.65 & 85 & 0.168 & 0.098 & 0.403 \\
\hline 14 & Transverse 3 & 40 & 10.12 & 10.05 & 75 & 0.162 & 0.128 & 0.378 \\
\hline & Transverse 2 & 100 & 7.25 & 7.22 & 80 & 0.245 & 0.058 & 0.294 \\
\hline & Transverse 1 & 80 & 10.27 & 10.21 & 75 & 0.421 & 0.162 & 0.373 \\
\hline & Transverse 6 & 120 & 11.18 & 11.13 & 140 & 0.360 & 0.279 & 0.419 \\
\hline & Transverse 5 & 40 & 5.45 & 5.42 & 65 & 0.151 & 0.049 & 0.265 \\
\hline & Transverse 4 & 40 & 10.06 & 9.99 & 95 & 0.153 & 0.121 & 0.312 \\
\hline 15 & Transverse 3 & 60 & 9.95 & 9.88 & 75 & 0.169 & 0.137 & 0.404 \\
\hline & Transverse 2 & 80 & 7.84 & 7.77 & 85 & 0.223 & 0.072 & 0.292 \\
\hline & Transverse 1 & 80 & 9.97 & 9.90 & 80 & 0.424 & 0.150 & 0.392 \\
\hline & Transverse 6 & 100 & 11.01 & 10.96 & 140 & 0.314 & 0.270 & 0.407 \\
\hline & Transverse 5 & 20 & 9.16 & 9.07 & 85 & 0.104 & 0.047 & 0.304 \\
\hline & Transverse 4 & 100 & 10.26 & 10.20 & 110 & 0.218 & 0.129 & 0.343 \\
\hline 16 & Transverse 3 & 80 & 9.87 & 9.80 & 75 & 0.133 & 0.117 & 0.408 \\
\hline & Transverse 2 & 100 & 9.81 & 9.74 & 90 & 0.227 & 0.105 & 0.314 \\
\hline & Transverse 1 & 100 & 7.94 & 7.89 & 70 & 0.329 & 0.167 & 0.372 \\
\hline & Transverse 6 & 100 & 11.06 & 11.01 & 145 & 0.303 & 0.277 & 0.419 \\
\hline 17 & Transverse 5 & 100 & 9.18 & 9.09 & 100 & 0.141 & 0.074 & 0.328 \\
\hline & Transverse 4 & 60 & 9.49 & 9.41 & 85 & 0.145 & 0.101 & 0.325 \\
\hline
\end{tabular}




\begin{tabular}{|c|c|c|c|c|c|c|c|c|}
\hline & Transverse 3 & 60 & 9.91 & 9.84 & 80 & 0.146 & 0.140 & 0.397 \\
\hline & Transverse 2 & 100 & 8.60 & 8.50 & 90 & 0.335 & 0.070 & 0.295 \\
\hline & Transverse 1 & 80 & 9.73 & 9.66 & 75 & 0.397 & 0.131 & 0.369 \\
\hline \multirow{6}{*}{18} & Transverse 6 & 100 & 11.10 & 11.06 & 135 & 0.384 & 0.288 & 0.434 \\
\hline & Transverse 5 & 60 & 8.77 & 8.67 & 125 & 0.159 & 0.061 & 0.299 \\
\hline & Transverse 4 & 100 & 9.85 & 9.78 & 120 & 0.232 & 0.115 & 0.397 \\
\hline & Transverse 3 & 60 & 9.78 & 9.72 & 75 & 0.136 & 0.132 & 0.401 \\
\hline & Transverse 2 & 100 & 8.29 & 8.21 & 95 & 0.251 & 0.071 & 0.282 \\
\hline & Transverse 1 & 80 & 10.47 & 10.42 & 90 & 0.413 & 0.180 & 0.377 \\
\hline \multirow{6}{*}{19} & Transverse 6 & 120 & 11.00 & 10.96 & 135 & 0.366 & 0.294 & 0.437 \\
\hline & Transverse 5 & 20 & 8.23 & 8.15 & 115 & 0.090 & 0.035 & 0.285 \\
\hline & Transverse 4 & 60 & 9.39 & 9.32 & 85 & 0.128 & 0.095 & 0.311 \\
\hline & Transverse 3 & 80 & 10.21 & 10.15 & 70 & 0.215 & 0.169 & 0.420 \\
\hline & Transverse 2 & 80 & 10.24 & 10.19 & 115 & 0.210 & 0.120 & 0.369 \\
\hline & Transverse 1 & 80 & 10.03 & 9.97 & 80 & 0.439 & 0.160 & 0.392 \\
\hline \multirow{6}{*}{20} & Transverse 6 & 100 & 11.11 & 11.06 & 135 & 0.242 & 0.290 & 0.436 \\
\hline & Transverse 5 & 20 & 8.61 & 8.49 & 105 & 0.068 & 0.038 & 0.284 \\
\hline & Transverse 4 & 100 & 10.28 & 10.22 & 95 & 0.187 & 0.122 & 0.333 \\
\hline & Transverse 3 & 80 & 9.91 & 9.84 & 75 & 0.132 & 0.132 & 0.359 \\
\hline & Transverse 2 & 60 & 7.82 & 7.76 & 85 & 0.276 & 0.069 & 0.300 \\
\hline & Transverse 1 & 80 & 10.17 & 10.11 & 90 & 0.432 & 0.152 & 0.380 \\
\hline \multirow{6}{*}{21} & Transverse 6 & 100 & 10.94 & 10.89 & 150 & 0.195 & 0.283 & 0.423 \\
\hline & Transverse 5 & 40 & 8.01 & 7.92 & 90 & 0.089 & 0.027 & 0.297 \\
\hline & Transverse 4 & 80 & 9.47 & 9.39 & 75 & 0.142 & 0.111 & 0.353 \\
\hline & Transverse 3 & 80 & 9.33 & 9.25 & 80 & 0.137 & 0.125 & 0.322 \\
\hline & Transverse 2 & 40 & 8.88 & 8.79 & 100 & 0.175 & 0.097 & 0.308 \\
\hline & Transverse 1 & 80 & 10.89 & 10.84 & 110 & 0.396 & 0.207 & 0.387 \\
\hline \multirow{6}{*}{22} & Transverse 6 & 80 & 10.87 & 10.82 & 170 & 0.269 & 0.272 & 0.410 \\
\hline & Transverse 5 & 100 & 9.69 & 9.62 & 100 & 0.117 & 0.094 & 0.368 \\
\hline & Transverse 4 & 80 & 9.46 & 9.39 & 90 & 0.117 & 0.091 & 0.339 \\
\hline & Transverse 3 & 40 & 10.05 & 9.99 & 85 & 0.103 & 0.138 & 0.365 \\
\hline & Transverse 2 & 80 & 9.11 & 9.02 & 110 & 0.172 & 0.088 & 0.285 \\
\hline & Transverse 1 & 100 & 10.57 & 10.52 & 90 & 0.391 & 0.161 & 0.364 \\
\hline \multirow{6}{*}{23} & Transverse 6 & 100 & 11.15 & 11.11 & 135 & 0.270 & 0.287 & 0.417 \\
\hline & Transverse 5 & 20 & 8.15 & 8.08 & 80 & 0.088 & 0.056 & 0.303 \\
\hline & Transverse 4 & 100 & 9.56 & 9.49 & 105 & 0.155 & 0.091 & 0.356 \\
\hline & Transverse 3 & 60 & 9.87 & 9.80 & 65 & 0.119 & 0.189 & 0.376 \\
\hline & Transverse 2 & 80 & 6.65 & 6.61 & 125 & 0.128 & 0.096 & 0.359 \\
\hline & Transverse 1 & 80 & 7.51 & 7.49 & 100 & 0.389 & 0.181 & 0.384 \\
\hline \multirow{6}{*}{24} & Transverse 6 & 100 & 11.04 & 10.99 & 170 & 0.223 & 0.281 & 0.412 \\
\hline & Transverse 5 & 20 & 8.35 & 8.28 & 90 & 0.081 & 0.033 & 0.290 \\
\hline & Transverse 4 & 80 & 9.55 & 9.48 & 75 & 0.144 & 0.114 & 0.336 \\
\hline & Transverse 3 & 80 & 9.64 & 9.57 & 80 & 0.112 & 0.124 & 0.387 \\
\hline & Transverse 2 & 60 & 8.60 & 8.52 & 110 & 0.081 & 0.070 & 0.289 \\
\hline & Transverse 1 & 80 & 9.66 & 9.59 & 110 & 0.433 & 0.171 & 0.379 \\
\hline \multirow{6}{*}{25} & Transverse 6 & 100 & 10.94 & 10.89 & 140 & 0.249 & 0.276 & 0.407 \\
\hline & Transverse 5 & 40 & 8.45 & 8.35 & 95 & 0.134 & 0.036 & 0.258 \\
\hline & Transverse 4 & 80 & 9.66 & 9.59 & 75 & 0.141 & 0.111 & 0.359 \\
\hline & Transverse 3 & 80 & 10.10 & 10.03 & 65 & 0.118 & 0.146 & 0.359 \\
\hline & Transverse 2 & 120 & 10.28 & 10.22 & 90 & 0.111 & 0.178 & 0.379 \\
\hline & Transverse 1 & 80 & 7.82 & 7.80 & 90 & 0.426 & 0.153 & 0.401 \\
\hline \multirow{6}{*}{26} & Transverse 6 & 120 & 11.29 & 11.25 & 110 & 0.417 & 0.288 & 0.435 \\
\hline & Transverse 5 & 80 & 9.30 & 9.22 & 65 & 0.107 & 0.061 & 0.337 \\
\hline & Transverse 4 & 100 & 9.58 & 9.50 & 80 & 0.119 & 0.091 & 0.395 \\
\hline & Transverse 3 & 60 & 9.60 & 9.54 & 60 & 0.123 & 0.174 & 0.329 \\
\hline & Transverse 2 & 100 & 9.88 & 9.82 & 130 & 0.156 & 0.127 & 0.369 \\
\hline & Transverse 1 & 100 & 11.60 & 11.56 & 105 & 0.401 & 0.202 & 0.422 \\
\hline \multirow{6}{*}{27} & Transverse 6 & 100 & 11.13 & 11.08 & 120 & 0.246 & 0.275 & 0.419 \\
\hline & Transverse 5 & 40 & 5.74 & 5.71 & 65 & 0.188 & 0.025 & 0.137 \\
\hline & Transverse 4 & 40 & 10.09 & 10.03 & 75 & 0.113 & 0.126 & 0.402 \\
\hline & Transverse 3 & 20 & 10.04 & 9.98 & 70 & 0.087 & 0.120 & 0.394 \\
\hline & Transverse 2 & 100 & 8.82 & 8.73 & 90 & 0.117 & 0.072 & 0.298 \\
\hline & Transverse 1 & 80 & 10.07 & 10.00 & 90 & 0.444 & 0.175 & 0.378 \\
\hline & Transverse 6 & 120 & 11.48 & 11.44 & 100 & 0.359 & 0.326 & 0.447 \\
\hline & Transverse 5 & 20 & 9.89 & 9.83 & 95 & 0.079 & 0.122 & 0.399 \\
\hline 28 & Transverse 4 & 60 & 8.95 & 8.87 & 90 & 0.094 & 0.073 & 0.317 \\
\hline 28 & Transverse 3 & 0 & 9.56 & 9.49 & 90 & 0.068 & 0.083 & 0.298 \\
\hline & Transverse 2 & 100 & 8.84 & 8.75 & 70 & 0.094 & 0.075 & 0.307 \\
\hline & Transverse 1 & 100 & 10.12 & 10.06 & 105 & 0.431 & 0.194 & 0.391 \\
\hline
\end{tabular}


Table 143: Case Study 1 - 45 ft Cluster Spacing Average Fracture Results

\begin{tabular}{|c|c|c|c|c|c|c|}
\hline \multicolumn{7}{|c|}{$45 \mathrm{ft}$ Cluster Spacing } \\
\hline $\begin{array}{l}\text { Stage } \\
\text { Number }\end{array}$ & $\begin{array}{l}\text { Proppant } \\
\text { Cutoff } \\
\text { Length } \\
\text { (ft) }\end{array}$ & $\begin{array}{c}\text { Est } \\
\text { Flowing } \\
\text { Frac } \\
\text { Length } \\
\text { (ft) }\end{array}$ & $\begin{array}{c}\text { Est Inf } \\
\text { Conductivity } \\
\text { Length } \\
\text { (ft) }\end{array}$ & $\begin{array}{c}\text { Fracture } \\
\text { Height } \\
\text { (ft) }\end{array}$ & $\begin{array}{c}\text { Average } \\
\text { Proppant } \\
\text { Conc } \\
\left(\mathrm{lb} / \mathrm{ft}^{2}\right)\end{array}$ & $\begin{array}{c}\text { Average } \\
\text { Fracture } \\
\text { Width } \\
\text { (in) }\end{array}$ \\
\hline 1 & 76 & 8.96 & 8.89 & 130 & 0.209 & 0.173 \\
\hline 2 & 92 & 9.79 & 9.71 & 91 & 0.213 & 0.168 \\
\hline 3 & 92 & 9.26 & 9.21 & 94 & 0.280 & 0.165 \\
\hline 4 & 80 & 10.04 & 9.98 & 94 & 0.192 & 0.176 \\
\hline 5 & 84 & 9.94 & 9.87 & 85 & 0.258 & 0.182 \\
\hline 6 & 100 & 9.80 & 9.74 & 104 & 0.255 & 0.174 \\
\hline 7 & 96 & 9.92 & 9.86 & 82 & 0.216 & 0.174 \\
\hline 8 & 80 & 9.81 & 9.75 & 118 & 0.200 & 0.170 \\
\hline 9 & 92 & 9.86 & 9.79 & 103 & 0.270 & 0.179 \\
\hline 10 & 84 & 9.67 & 9.61 & 110 & 0.281 & 0.165 \\
\hline 11 & 68 & 10.10 & 10.04 & 90 & 0.230 & 0.167 \\
\hline 12 & 72 & 9.52 & 9.47 & 99 & 0.269 & 0.170 \\
\hline 13 & 88 & 10.09 & 10.02 & 97 & 0.243 & 0.168 \\
\hline 14 & 80 & 9.91 & 9.85 & 102 & 0.220 & 0.167 \\
\hline 15 & 95 & 9.06 & 9.01 & 120 & 0.234 & 0.171 \\
\hline 16 & 35 & 9.56 & 9.49 & 76 & 0.062 & 0.195 \\
\hline 17 & 105 & 9.80 & 9.73 & 126 & 0.289 & 0.177 \\
\hline 18 & 85 & 9.63 & 9.56 & 99 & 0.209 & 0.180 \\
\hline 19 & 100 & 10.00 & 9.94 & 101 & 0.250 & 0.180 \\
\hline 20 & 104 & 10.08 & 10.01 & 95 & 0.327 & 0.153 \\
\hline 21 & 92 & 10.40 & 10.34 & 91 & 0.286 & 0.168 \\
\hline 22 & 88 & 9.62 & 9.56 & 111 & 0.304 & 0.163 \\
\hline 23 & 96 & 9.86 & 9.80 & 100 & 0.290 & 0.166 \\
\hline 24 & 76 & 9.76 & 9.70 & 106 & 0.236 & 0.160 \\
\hline 25 & 84 & 10.00 & 9.93 & 102 & 0.269 & 0.160 \\
\hline 26 & 88 & 10.10 & 10.04 & 95 & 0.299 & 0.157 \\
\hline 27 & 105 & 11.00 & 10.96 & 85 & 0.541 & 0.209 \\
\hline 28 & 90 & 10.16 & 10.11 & 100 & 0.333 & 0.194 \\
\hline
\end{tabular}

Table 144: Case Study 1 - 40 ft Cluster Spacing Average Fracture Results

\begin{tabular}{|c|c|c|c|c|c|c|}
\hline \multicolumn{7}{|c|}{ 40ft Cluster Spacing } \\
\hline $\begin{array}{l}\text { Stage } \\
\text { Number }\end{array}$ & $\begin{array}{l}\text { Proppant } \\
\text { Cutoff } \\
\text { Length } \\
\text { (ft) }\end{array}$ & $\begin{array}{c}\text { Est } \\
\text { Flowing } \\
\text { Frac } \\
\text { Length } \\
\text { (ft) } \\
\end{array}$ & $\begin{array}{c}\text { Est Inf } \\
\text { Conductivity } \\
\text { Length } \\
\text { (ft) }\end{array}$ & $\begin{array}{c}\text { Fracture } \\
\text { Height } \\
(\mathrm{ft})\end{array}$ & $\begin{array}{c}\text { Average } \\
\text { Proppant } \\
\text { Conc } \\
\left(\mathrm{lb} / \mathrm{ft}^{2}\right)\end{array}$ & $\begin{array}{c}\text { Average } \\
\text { Fracture } \\
\text { Width } \\
\text { (in) }\end{array}$ \\
\hline 1 & 76 & 9.07 & 9.01 & 129 & 0.210 & 0.173 \\
\hline 2 & 100 & 9.78 & 9.70 & 93 & 0.213 & 0.167 \\
\hline 3 & 100 & 9.83 & 9.76 & 101 & 0.240 & 0.166 \\
\hline 4 & 100 & 10.08 & 10.01 & 89 & 0.220 & 0.168 \\
\hline 5 & 88 & 9.68 & 9.60 & 93 & 0.243 & 0.166 \\
\hline 6 & 96 & 9.81 & 9.75 & 93 & 0.267 & 0.166 \\
\hline 7 & 84 & 9.81 & 9.75 & 89 & 0.209 & 0.171 \\
\hline 8 & 60 & 9.78 & 9.71 & 114 & 0.195 & 0.170 \\
\hline 9 & 100 & 9.81 & 9.75 & 102 & 0.306 & 0.176 \\
\hline
\end{tabular}




\begin{tabular}{|r|r|r|r|r|r|r|}
\hline 10 & 84 & 10.04 & 9.98 & 101 & 0.281 & 0.166 \\
\hline 11 & 84 & 10.21 & 10.15 & 92 & 0.253 & 0.169 \\
\hline 12 & 80 & 9.61 & 9.55 & 94 & 0.286 & 0.162 \\
\hline 13 & 104 & 10.24 & 10.17 & 90 & 0.263 & 0.179 \\
\hline 14 & 92 & 9.69 & 9.63 & 114 & 0.243 & 0.161 \\
\hline 15 & 105 & 10.06 & 10.00 & 101 & 0.268 & 0.188 \\
\hline 16 & 45 & 10.07 & 10.00 & 83 & 0.056 & 0.197 \\
\hline 17 & 90 & 9.93 & 9.86 & 113 & 0.250 & 0.173 \\
\hline 18 & 90 & 9.74 & 9.67 & 98 & 0.204 & 0.180 \\
\hline 19 & 120 & 9.93 & 9.87 & 103 & 0.289 & 0.189 \\
\hline 20 & 96 & 10.11 & 10.05 & 96 & 0.311 & 0.158 \\
\hline 21 & 108 & 10.35 & 10.29 & 96 & 0.358 & 0.163 \\
\hline 22 & 80 & 9.29 & 9.23 & 116 & 0.274 & 0.165 \\
\hline 23 & 88 & 9.76 & 9.70 & 104 & 0.279 & 0.164 \\
\hline 24 & 76 & 9.71 & 9.65 & 106 & 0.235 & 0.161 \\
\hline 25 & 88 & 10.04 & 9.98 & 101 & 0.231 & 0.167 \\
\hline 26 & 92 & 10.23 & 10.17 & 93 & 0.320 & 0.167 \\
\hline 27 & 105 & 10.64 & 10.59 & 90 & 0.434 & 0.184 \\
\hline 28 & 90 & 10.15 & 10.10 & 89 & 0.377 & 0.213 \\
\hline
\end{tabular}

Table 145: Case Study 1 - 35 ft Cluster Spacing Average Fracture Results

\begin{tabular}{|c|c|c|c|c|c|c|}
\hline \multicolumn{7}{|c|}{$35 \mathrm{ft}$ Cluster Spacing } \\
\hline $\begin{array}{c}\text { Stage } \\
\text { Number }\end{array}$ & $\begin{array}{l}\text { Proppant } \\
\text { Cutoff } \\
\text { Length } \\
(\mathrm{ft})\end{array}$ & $\begin{array}{c}\text { Est } \\
\text { Flowing } \\
\text { Frac } \\
\text { Length } \\
\text { (ft) }\end{array}$ & $\begin{array}{l}\text { Est Inf } \\
\text { Conductivity } \\
\text { Length } \\
\text { (ft) }\end{array}$ & $\begin{array}{c}\text { Fracture } \\
\text { Height } \\
\text { (ft) }\end{array}$ & $\begin{array}{c}\text { Average } \\
\text { Proppant } \\
\text { Conc } \\
\left(\mathrm{lb} / \mathrm{ft}^{2}\right)\end{array}$ & $\begin{array}{c}\text { Average } \\
\text { Fracture } \\
\text { Width } \\
\text { (in) }\end{array}$ \\
\hline 1 & 76 & 9.08 & 9.01 & 129 & 0.211 & 0.173 \\
\hline 2 & 92 & 9.92 & 9.85 & 90 & 0.206 & 0.167 \\
\hline 3 & 108 & 10.02 & 9.96 & 100 & 0.289 & 0.174 \\
\hline 4 & 108 & 9.36 & 9.30 & 91 & 0.243 & 0.164 \\
\hline 5 & 92 & 9.53 & 9.44 & 90 & 0.259 & 0.165 \\
\hline 6 & 108 & 9.80 & 9.73 & 98 & 0.258 & 0.164 \\
\hline 7 & 88 & 9.85 & 9.79 & 86 & 0.186 & 0.173 \\
\hline 8 & 68 & 9.77 & 9.70 & 122 & 0.200 & 0.168 \\
\hline 9 & 76 & 9.66 & 9.59 & 110 & 0.256 & 0.172 \\
\hline 10 & 84 & 9.98 & 9.92 & 95 & 0.275 & 0.163 \\
\hline 11 & 76 & 9.68 & 9.61 & 96 & 0.230 & 0.159 \\
\hline 12 & 76 & 9.55 & 9.50 & 96 & 0.281 & 0.168 \\
\hline 13 & 76 & 9.94 & 9.87 & 97 & 0.257 & 0.160 \\
\hline 14 & 88 & 9.81 & 9.75 & 113 & 0.217 & 0.165 \\
\hline 15 & 105 & 10.03 & 9.96 & 106 & 0.256 & 0.183 \\
\hline 16 & 25 & 10.04 & 9.97 & 70 & 0.050 & 0.186 \\
\hline 17 & 70 & 9.77 & 9.71 & 123 & 0.263 & 0.168 \\
\hline 18 & 80 & 9.83 & 9.76 & 98 & 0.203 & 0.181 \\
\hline 19 & 90 & 9.76 & 9.70 & 105 & 0.244 & 0.185 \\
\hline 20 & 100 & 10.08 & 10.02 & 101 & 0.276 & 0.156 \\
\hline 21 & 108 & 10.18 & 10.12 & 92 & 0.344 & 0.159 \\
\hline 22 & 92 & 9.23 & 9.17 & 121 & 0.284 & 0.175 \\
\hline 23 & 88 & 9.96 & 9.90 & 95 & 0.285 & 0.168 \\
\hline 24 & 72 & 9.68 & 9.62 & 101 & 0.229 & 0.158 \\
\hline 25 & 80 & 10.08 & 10.02 & 99 & 0.258 & 0.161 \\
\hline 26 & 92 & 10.11 & 10.05 & 97 & 0.295 & 0.161 \\
\hline
\end{tabular}




\begin{tabular}{|r|r|r|r|r|r|r|}
27 & 100 & 10.67 & 10.62 & 98 & 0.452 & 0.186 \\
\hline 28 & 105 & 9.87 & 9.81 & 104 & 0.335 & 0.171 \\
\hline
\end{tabular}

Table 146: Case Study 2 - 45 ft Cluster Spacing Average Fracture Results

\begin{tabular}{|c|c|c|c|c|c|c|}
\hline \multicolumn{7}{|c|}{$45 \mathrm{ft}$ Cluster Spacing } \\
\hline $\begin{array}{l}\text { Stage } \\
\text { Number }\end{array}$ & $\begin{array}{l}\text { Proppant } \\
\text { Cutoff } \\
\text { Length } \\
\text { (ft) }\end{array}$ & $\begin{array}{c}\text { Est } \\
\text { Flowing } \\
\text { Frac } \\
\text { Length } \\
\text { (ft) } \\
\end{array}$ & $\begin{array}{c}\text { Est Inf } \\
\text { Conductivity } \\
\text { Length } \\
\text { (ft) }\end{array}$ & $\begin{array}{c}\text { Fracture } \\
\text { Height } \\
\text { (ft) }\end{array}$ & $\begin{array}{c}\text { Average } \\
\text { Proppant } \\
\text { Conc } \\
\left(\mathrm{lb} / \mathrm{ft}^{2}\right)\end{array}$ & $\begin{array}{c}\text { Average } \\
\text { Fracture } \\
\text { Width } \\
\text { (in) }\end{array}$ \\
\hline 1 & 72 & 9.76 & 9.69 & 110 & 0.338 & 0.172 \\
\hline 2 & 96 & 10.10 & 10.03 & 90 & 0.339 & 0.179 \\
\hline 3 & 96 & 10.41 & 10.36 & 91 & 0.294 & 0.177 \\
\hline 4 & 92 & 10.31 & 10.25 & 85 & 0.342 & 0.169 \\
\hline 5 & 100 & 9.77 & 9.71 & 80 & 0.294 & 0.189 \\
\hline 6 & 80 & 10.02 & 9.96 & 98 & 0.304 & 0.166 \\
\hline 7 & 84 & 10.03 & 9.97 & 95 & 0.308 & 0.175 \\
\hline 8 & 76 & 9.74 & 9.67 & 91 & 0.301 & 0.162 \\
\hline 9 & 84 & 9.88 & 9.81 & 94 & 0.274 & 0.157 \\
\hline 10 & 72 & 10.17 & 10.11 & 95 & 0.261 & 0.163 \\
\hline 11 & 92 & 9.72 & 9.64 & 105 & 0.299 & 0.155 \\
\hline 12 & 92 & 10.05 & 9.99 & 91 & 0.287 & 0.166 \\
\hline 13 & 80 & 9.80 & 9.74 & 97 & 0.293 & 0.158 \\
\hline 14 & 88 & 10.01 & 9.94 & 94 & 0.281 & 0.162 \\
\hline 15 & 96 & 9.76 & 9.68 & 102 & 0.271 & 0.151 \\
\hline 16 & 88 & 9.93 & 9.86 & 104 & 0.229 & 0.153 \\
\hline 17 & 96 & 9.97 & 9.90 & 104 & 0.263 & 0.157 \\
\hline 18 & 80 & 9.84 & 9.78 & 104 & 0.260 & 0.153 \\
\hline 19 & 92 & 10.25 & 10.19 & 102 & 0.250 & 0.165 \\
\hline 20 & 92 & 9.88 & 9.81 & 106 & 0.239 & 0.167 \\
\hline 21 & 68 & 9.77 & 9.71 & 103 & 0.206 & 0.159 \\
\hline 22 & 80 & 9.97 & 9.90 & 105 & 0.229 & 0.158 \\
\hline 23 & 92 & 9.80 & 9.74 & 93 & 0.234 & 0.161 \\
\hline 24 & 95 & 9.54 & 9.47 & 138 & 0.228 & 0.175 \\
\hline 25 & 100 & 10.17 & 10.11 & 93 & 0.263 & 0.175 \\
\hline 26 & 90 & 9.56 & 9.49 & 120 & 0.249 & 0.175 \\
\hline 27 & 80 & 9.73 & 9.67 & 116 & 0.205 & 0.173 \\
\hline 28 & 85 & 9.74 & 9.68 & 121 & 0.263 & 0.171 \\
\hline
\end{tabular}

Table 147: Case Study 2 - 40 ft Cluster Spacing Average Fracture Results

\begin{tabular}{|c|r|r|r|r|r|r|}
\hline \multicolumn{2}{|c|}{ 40ft Cluster Spacing } \\
\hline $\begin{array}{c}\text { Stage } \\
\text { Number }\end{array}$ & $\begin{array}{c}\text { Proppant } \\
\text { Cutoff } \\
\text { Length } \\
(\mathrm{ft})\end{array}$ & $\begin{array}{c}\text { Est } \\
\text { Flowing } \\
\text { Frac } \\
\text { Length } \\
(\mathrm{ft})\end{array}$ & $\begin{array}{c}\text { Est Inf } \\
\text { Conductivity } \\
\text { Length } \\
(\mathrm{ft})\end{array}$ & $\begin{array}{c}\text { Fracture } \\
\text { Height } \\
(\mathrm{ft})\end{array}$ & $\begin{array}{c}\text { Average } \\
\text { Proppant } \\
\text { Conc } \\
\left(\mathrm{lb} / \mathrm{ft}^{2}\right)\end{array}$ & $\begin{array}{c}\text { Average } \\
\text { Fracture } \\
\text { Width } \\
\text { (in) }\end{array}$ \\
\hline 1 & 73 & 9.25 & 9.19 & 107 & 0.306 & 0.153 \\
\hline 2 & 93 & 9.46 & 9.39 & 94 & 0.287 & 0.157 \\
\hline 3 & 97 & 10.13 & 10.06 & 78 & 0.275 & 0.158 \\
\hline 4 & 90 & 9.57 & 9.51 & 82 & 0.288 & 0.156 \\
\hline 5 & 77 & 10.03 & 9.96 & 80 & 0.256 & 0.151 \\
\hline
\end{tabular}




\begin{tabular}{|c|c|c|c|c|c|c|}
\hline 6 & 87 & 10.11 & 10.04 & 78 & 0.280 & 0.151 \\
\hline 7 & 77 & 9.85 & 9.78 & 93 & 0.286 & 0.148 \\
\hline 8 & 73 & 9.52 & 9.45 & 98 & 0.245 & 0.142 \\
\hline 9 & 80 & 10.10 & 10.04 & 96 & 0.254 & 0.148 \\
\hline 10 & 80 & 9.49 & 9.42 & 95 & 0.297 & 0.136 \\
\hline 11 & 90 & 9.92 & 9.85 & 83 & 0.252 & 0.139 \\
\hline 12 & 83 & 9.55 & 9.49 & 114 & 0.279 & 0.156 \\
\hline 13 & 68 & 8.66 & 8.60 & 109 & 0.214 & 0.165 \\
\hline 14 & 68 & 9.21 & 9.14 & 102 & 0.287 & 0.148 \\
\hline 15 & 92 & 10.02 & 9.95 & 107 & 0.285 & 0.164 \\
\hline 16 & 68 & 9.87 & 9.80 & 100 & 0.256 & 0.152 \\
\hline 17 & 80 & 9.49 & 9.42 & 105 & 0.268 & 0.156 \\
\hline 18 & 88 & 9.49 & 9.43 & 102 & 0.238 & 0.164 \\
\hline 19 & 80 & 9.88 & 9.82 & 108 & 0.234 & 0.155 \\
\hline 20 & 80 & 9.66 & 9.60 & 98 & 0.253 & 0.157 \\
\hline 21 & 76 & 9.46 & 9.40 & 118 & 0.203 & 0.165 \\
\hline 22 & 84 & 9.82 & 9.76 & 104 & 0.236 & 0.153 \\
\hline 23 & 72 & 9.91 & 9.85 & 121 & 0.210 & 0.166 \\
\hline 24 & 88 & 9.88 & 9.82 & 96 & 0.228 & 0.148 \\
\hline 25 & 88 & 9.88 & 9.81 & 101 & 0.222 & 0.152 \\
\hline 26 & 72 & 9.03 & 8.97 & 109 & 0.222 & 0.165 \\
\hline 27 & 80 & 9.98 & 9.91 & 102 & 0.205 & 0.156 \\
\hline 28 & 80 & 9.50 & 9.43 & 120 & 0.223 & 0.145 \\
\hline
\end{tabular}

Table 148: Case Study 2 - 35 ft Cluster Spacing Average Fracture Results

\begin{tabular}{|c|c|c|c|c|c|c|}
\hline \multicolumn{7}{|c|}{$35 \mathrm{ft}$ Cluster Spacing } \\
\hline $\begin{array}{l}\text { Stage } \\
\text { Number }\end{array}$ & $\begin{array}{l}\text { Proppant } \\
\text { Cutoff } \\
\text { Length } \\
\text { (ft) }\end{array}$ & $\begin{array}{c}\text { Est } \\
\text { Flowing } \\
\text { Frac } \\
\text { Length } \\
\text { (ft) }\end{array}$ & $\begin{array}{c}\text { Est Inf } \\
\text { Conductivity } \\
\text { Length } \\
\text { (ft) }\end{array}$ & $\begin{array}{c}\text { Fracture } \\
\text { Height } \\
(\mathrm{ft})\end{array}$ & $\begin{array}{c}\text { Average } \\
\text { Proppant } \\
\text { Conc } \\
\left(\mathrm{lb} / \mathrm{ft}^{2}\right)\end{array}$ & $\begin{array}{c}\text { Average } \\
\text { Fracture } \\
\text { Width } \\
\text { (in) }\end{array}$ \\
\hline 1 & 69 & 9.27 & 9.20 & 106 & 0.286 & 0.139 \\
\hline 2 & 86 & 9.61 & 9.56 & 81 & 0.340 & 0.144 \\
\hline 3 & 80 & 9.17 & 9.12 & 86 & 0.250 & 0.136 \\
\hline 4 & 83 & 9.45 & 9.39 & 91 & 0.303 & 0.143 \\
\hline 5 & 80 & 9.88 & 9.82 & 81 & 0.272 & 0.158 \\
\hline 6 & 80 & 10.11 & 10.04 & 80 & 0.257 & 0.140 \\
\hline 7 & 73 & 9.85 & 9.79 & 105 & 0.253 & 0.152 \\
\hline 8 & 87 & 9.97 & 9.91 & 95 & 0.259 & 0.150 \\
\hline 9 & 80 & 9.10 & 9.04 & 94 & 0.211 & 0.153 \\
\hline 10 & 77 & 9.30 & 9.25 & 108 & 0.260 & 0.148 \\
\hline 11 & 63 & 9.93 & 9.86 & 103 & 0.234 & 0.145 \\
\hline 12 & 70 & 8.61 & 8.56 & 106 & 0.260 & 0.142 \\
\hline 13 & 67 & 9.53 & 9.47 & 105 & 0.235 & 0.153 \\
\hline 14 & 83 & 9.69 & 9.63 & 94 & 0.245 & 0.133 \\
\hline 15 & 70 & 9.07 & 9.01 & 90 & 0.247 & 0.135 \\
\hline 16 & 83 & 9.67 & 9.61 & 95 & 0.221 & 0.139 \\
\hline 17 & 83 & 9.66 & 9.59 & 96 & 0.244 & 0.132 \\
\hline 18 & 83 & 9.71 & 9.64 & 107 & 0.262 & 0.141 \\
\hline 19 & 73 & 9.85 & 9.79 & 100 & 0.241 & 0.146 \\
\hline 20 & 73 & 9.65 & 9.58 & 98 & 0.223 & 0.134 \\
\hline 21 & 70 & 9.59 & 9.51 & 101 & 0.189 & 0.141 \\
\hline 22 & 80 & 9.96 & 9.89 & 108 & 0.195 & 0.141 \\
\hline
\end{tabular}




\begin{tabular}{|r|r|r|r|r|r|r|}
23 & 73 & 8.82 & 8.76 & 102 & 0.192 & 0.150 \\
\hline 24 & 70 & 9.47 & 9.41 & 106 & 0.179 & 0.132 \\
\hline 25 & 83 & 9.54 & 9.48 & 93 & 0.197 & 0.150 \\
\hline 26 & 93 & 10.21 & 10.15 & 92 & 0.221 & 0.157 \\
\hline 27 & 63 & 9.32 & 9.26 & 85 & 0.199 & 0.132 \\
\hline 28 & 67 & 9.81 & 9.74 & 92 & 0.188 & 0.146 \\
\hline
\end{tabular}

Table 149: No Stress Shadowing Average Fracture Results

\begin{tabular}{|c|c|c|c|c|c|c|}
\hline \multicolumn{7}{|c|}{ No Stress Shadowing } \\
\hline $\begin{array}{c}\text { Stage } \\
\#\end{array}$ & $\begin{array}{l}\text { Fracture } \\
\text { Length } \\
(\mathrm{ft})\end{array}$ & $\begin{array}{l}\text { Est } \\
\text { Flowing } \\
\text { Frac } \\
\text { Length } \\
(\mathrm{ft})\end{array}$ & $\begin{array}{l}\text { Est Inf } \\
\text { Conductivity } \\
\text { Length }(\mathrm{ft})\end{array}$ & $\begin{array}{l}\text { Fracture } \\
\text { Height } \\
(\mathrm{ft})\end{array}$ & $\begin{array}{l}\text { Average } \\
\text { Proppant } \\
\text { Conc } \\
\left(\mathrm{lb} / \mathrm{ft}^{2}\right)\end{array}$ & $\begin{array}{l}\text { Average } \\
\text { Fracture } \\
\text { Width } \\
\text { (in) }\end{array}$ \\
\hline 1 & 76 & 9.07 & 9.00 & 130 & 0.211 & 0.174 \\
\hline 2 & 68 & 9.24 & 9.17 & 103 & 0.220 & 0.174 \\
\hline 3 & 96 & 9.62 & 9.55 & 108 & 0.244 & 0.177 \\
\hline 4 & 100 & 9.59 & 9.51 & 112 & 0.230 & 0.172 \\
\hline 5 & 72 & 9.27 & 9.19 & 108 & 0.219 & 0.168 \\
\hline 6 & 104 & 9.36 & 9.30 & 116 & 0.288 & 0.175 \\
\hline 7 & 52 & 9.32 & 9.25 & 96 & 0.171 & 0.171 \\
\hline 8 & 48 & 9.42 & 9.36 & 115 & 0.184 & 0.172 \\
\hline 9 & 72 & 9.64 & 9.58 & 112 & 0.239 & 0.172 \\
\hline 10 & 68 & 9.40 & 9.33 & 117 & 0.252 & 0.168 \\
\hline 11 & 56 & 9.83 & 9.76 & 107 & 0.225 & 0.166 \\
\hline 12 & 64 & 9.21 & 9.16 & 127 & 0.227 & 0.172 \\
\hline 13 & 84 & 9.66 & 9.59 & 108 & 0.328 & 0.170 \\
\hline 14 & 60 & 9.35 & 9.28 & 119 & 0.223 & 0.167 \\
\hline 15 & 80 & 9.68 & 9.62 & 128 & 0.279 & 0.195 \\
\hline 16 & 65 & 9.57 & 9.49 & 71 & 0.059 & 0.188 \\
\hline 17 & 120 & 9.88 & 9.83 & 165 & 0.428 & 0.298 \\
\hline 18 & 60 & 9.71 & 9.64 & 108 & 0.263 & 0.201 \\
\hline 19 & 65 & 9.78 & 9.72 & 125 & 0.270 & 0.198 \\
\hline 20 & 84 & 10.04 & 9.97 & 104 & 0.333 & 0.171 \\
\hline 21 & 84 & 10.02 & 9.95 & 104 & 0.340 & 0.169 \\
\hline 22 & 96 & 9.21 & 9.14 & 142 & 0.277 & 0.162 \\
\hline 23 & 96 & 9.47 & 9.41 & 119 & 0.320 & 0.167 \\
\hline 24 & 64 & 9.61 & 9.55 & 114 & 0.225 & 0.166 \\
\hline 25 & 76 & 9.95 & 9.89 & 110 & 0.234 & 0.169 \\
\hline 26 & 76 & 10.06 & 10.00 & 98 & 0.321 & 0.170 \\
\hline 27 & 100 & 10.43 & 10.38 & 104 & 0.452 & 0.192 \\
\hline 28 & 90 & 9.74 & 9.68 & 114 & 0.389 & 0.205 \\
\hline
\end{tabular}

Table 150: Stress Shadowing Average Fracture Results

Stress Shadowing

\begin{tabular}{|c|l|l|l|r|l|l|}
\hline $\begin{array}{c}\text { Stage } \\
\#\end{array}$ & $\begin{array}{l}\text { Fracture } \\
\text { Length } \\
(\mathrm{ft})\end{array}$ & $\begin{array}{l}\text { Est } \\
\begin{array}{l}\text { Flowing } \\
\text { Frac } \\
\text { Length } \\
(\mathrm{ft})\end{array}\end{array}$ & $\begin{array}{l}\text { Est Inf } \\
\text { Conductivity } \\
\text { Length }(\mathrm{ft})\end{array}$ & $\begin{array}{l}\text { Fracture } \\
\text { Height } \\
(\mathrm{ft})\end{array}$ & $\begin{array}{l}\text { Average } \\
\text { Proppant } \\
\text { Conc } \\
\left(\mathrm{lb} / \mathrm{ft}^{2}\right)\end{array}$ & $\begin{array}{l}\text { Average } \\
\text { Fracture } \\
\text { Width } \\
(\mathrm{in})\end{array}$ \\
\hline 1 & 76 & 9.07 & 9.00 & 130 & 0.211 & 0.174 \\
\hline 2 & 88 & 9.71 & 9.63 & 91 & 0.196 & 0.161 \\
\hline
\end{tabular}




\begin{tabular}{|r|r|r|r|r|r|r|}
3 & 92 & 9.97 & 9.90 & 95 & 0.280 & 0.173 \\
\hline 4 & 108 & 10.28 & 10.22 & 95 & 0.264 & 0.179 \\
\hline 5 & 92 & 9.98 & 9.91 & 81 & 0.259 & 0.183 \\
\hline 6 & 108 & 10.03 & 9.96 & 89 & 0.298 & 0.180 \\
\hline 7 & 76 & 9.22 & 9.16 & 91 & 0.204 & 0.177 \\
\hline 8 & 60 & 9.75 & 9.69 & 115 & 0.185 & 0.161 \\
\hline 9 & 88 & 10.11 & 10.05 & 100 & 0.292 & 0.175 \\
\hline 10 & 68 & 9.90 & 9.84 & 105 & 0.277 & 0.167 \\
\hline 11 & 80 & 10.28 & 10.22 & 99 & 0.234 & 0.169 \\
\hline 12 & 88 & 10.18 & 10.12 & 96 & 0.302 & 0.171 \\
\hline 13 & 104 & 9.89 & 9.82 & 99 & 0.331 & 0.156 \\
\hline 14 & 84 & 9.66 & 9.59 & 115 & 0.205 & 0.160 \\
\hline 15 & 100 & 9.75 & 9.69 & 104 & 0.268 & 0.172 \\
\hline 16 & 30 & 10.05 & 9.97 & 60 & 0.065 & 0.204 \\
\hline 17 & 110 & 9.88 & 9.83 & 165 & 0.412 & 0.278 \\
\hline 18 & 70 & 9.66 & 9.59 & 91 & 0.183 & 0.178 \\
\hline 19 & 90 & 10.12 & 10.06 & 100 & 0.258 & 0.189 \\
\hline 20 & 88 & 10.13 & 10.06 & 100 & 0.306 & 0.157 \\
\hline 21 & 100 & 10.34 & 10.28 & 84 & 0.345 & 0.165 \\
\hline 22 & 104 & 9.57 & 9.51 & 107 & 0.291 & 0.176 \\
\hline 23 & 92 & 9.74 & 9.68 & 94 & 0.314 & 0.173 \\
\hline 24 & 72 & 9.74 & 9.67 & 108 & 0.243 & 0.161 \\
\hline 25 & 68 & 10.09 & 10.03 & 104 & 0.267 & 0.167 \\
\hline 26 & 92 & 10.42 & 10.36 & 96 & 0.319 & 0.165 \\
\hline 27 & 100 & 10.62 & 10.57 & 95 & 0.448 & 0.193 \\
\hline 28 & 90 & 10.08 & 10.02 & 86 & 0.342 & 0.190 \\
\hline
\end{tabular}

Table 151: Case Study 1-CMG Results

\begin{tabular}{|c|c|c|c|c|c|c|c|c|c|c|}
\hline \multirow[b]{2}{*}{ Time (day) } & \multirow[b]{2}{*}{ Date } & \multicolumn{3}{|c|}{ 45ft Cluster Spacing } & \multicolumn{3}{|c|}{ 40ft Cluster Spacing } & \multicolumn{3}{|c|}{$35 \mathrm{ft}$ Cluster Spacing } \\
\hline & & $\begin{array}{l}\text { Well Bottom- } \\
\text { hole } \\
\text { Pressure (psi) }\end{array}$ & $\begin{array}{l}\text { Cumulative } \\
\text { Gas SC }\left(\mathrm{ft}^{3}\right)\end{array}$ & $\begin{array}{l}\text { Gas Rate } \\
\left(\mathrm{ft}^{3} / \text { day }\right)\end{array}$ & $\begin{array}{l}\text { Well Bottom- } \\
\text { hole } \\
\text { Pressure (psi) }\end{array}$ & $\begin{array}{l}\text { Cumulative } \\
\text { Gas } \mathrm{SC}\left(\mathrm{ft}^{3}\right)\end{array}$ & $\begin{array}{l}\text { Gas Rate } \\
\left(\mathrm{ft}^{3} / \mathrm{day}^{2}\right)\end{array}$ & $\begin{array}{l}\text { Well Bottom- } \\
\text { hole } \\
\text { Pressure (psi) }\end{array}$ & $\begin{array}{l}\text { Cumulative } \\
\text { Gas } \mathrm{SC}\left(\mathrm{ft}^{3}\right)\end{array}$ & $\begin{array}{l}\text { Gas Rate } \\
\left(\mathrm{ft}^{3} / \text { day }\right)\end{array}$ \\
\hline 0 & $12 / 12 / 2015$ & & 0 & 0 & & 0 & 0 & & 0 & 0 \\
\hline 1 & $12 / 13 / 2015$ & 3850 & 27445836 & 27445836 & 3850 & 28324074 & 28324074 & 3850 & 27370108 & 27370108 \\
\hline 2 & $12 / 14 / 2015$ & 3850 & 53403064 & 25957226 & 3850 & 55330708 & 27006630 & 3850 & 52147436 & 24777334 \\
\hline 3 & $12 / 15 / 2015$ & 3850 & 72381336 & 18978272 & 3850 & 74886616 & 19555910 & 3850 & 70451432 & 18303990 \\
\hline 4 & $12 / 16 / 2015$ & 3850 & 88656720 & 16275382 & 3850 & 91234952 & 16348335 & 3850 & 85811720 & 15360288 \\
\hline 5 & $12 / 17 / 2015$ & 3850 & 103088448 & 14431731 & 3850 & 105744280 & 14509332 & 3850 & 99540704 & 13728979 \\
\hline 6 & $12 / 18 / 2015$ & 3850 & 116144896 & 13056449 & 3850 & 118862440 & 13118156 & 3850 & 112022536 & 12481834 \\
\hline 7 & $12 / 19 / 2015$ & 3850 & 128121984 & 11977088 & 3850 & 130882872 & 12020429 & 3850 & 123513688 & 11491153 \\
\hline 8 & $12 / 20 / 2015$ & 3850 & 139221216 & 11099226 & 3850 & 142007712 & 11124840 & 3850 & 134192256 & 10678568 \\
\hline 9 & $12 / 21 / 2015$ & 3850 & 149585904 & 10364696 & 3850 & 152381632 & 10373940 & 3850 & 144186320 & 9994058 \\
\hline 10 & $12 / 22 / 2015$ & 3850 & 159321584 & 9735684 & 3850 & 162111712 & 9730063 & 3850 & 153590960 & 9404647 \\
\hline 11 & $12 / 23 / 2015$ & 3850 & 168508336 & 9186745 & 3850 & 171279344 & 9167628 & 3850 & 162478816 & 8887851 \\
\hline 12 & $12 / 24 / 2015$ & 3850 & 177208576 & 8700234 & 3850 & 179948752 & 8669406 & 3850 & 170907360 & 8428552 \\
\hline 13 & $12 / 25 / 2015$ & 3850 & 185472720 & 8264147 & 3850 & 188171568 & 8222829 & 3850 & 178922992 & 8015629 \\
\hline 14 & $12 / 26 / 2015$ & 3850 & 193342144 & 7869420 & 3850 & 195990416 & 7818839 & 3850 & 186564048 & 7641060 \\
\hline 15 & $12 / 27 / 2015$ & 3850 & 200851568 & 7509429 & 3850 & 203441136 & 7450725 & 3850 & 193862960 & 7298905 \\
\hline 16 & $12 / 28 / 2015$ & 3850 & 208030720 & 7179157 & 3850 & 210554512 & 7113378 & 3850 & 200847584 & 6984612 \\
\hline 17 & $12 / 29 / 2015$ & 3850 & 214905456 & 6874728 & 3850 & 217357328 & 6802808 & 3850 & 207542192 & 6694621 \\
\hline 18 & $12 / 30 / 2015$ & 3850 & 221498496 & 6593041 & 3850 & 223873168 & 6515837 & 3850 & 213968256 & 6426085 \\
\hline 19 & $12 / 31 / 2015$ & 3850 & 227830080 & 6331581 & 3850 & 230123024 & 6249863 & 3850 & 220144944 & 6176670 \\
\hline 20 & $1 / 1 / 2016$ & 3850 & 233918336 & 6088261 & 3850 & 236125744 & 6002719 & 3850 & 226089376 & 5944432 \\
\hline 21 & $1 / 2 / 2016$ & 3850 & 239779648 & 5861320 & 3850 & 241898320 & 5772577 & 3850 & 231817104 & 5727723 \\
\hline 22 & $1 / 3 / 2016$ & 3850 & 245428896 & 5649249 & 3850 & 247456176 & 5557859 & 3850 & 237342224 & 5525125 \\
\hline 23 & $1 / 4 / 2016$ & 3850 & 250879632 & 5450735 & 3850 & 252813376 & 5357200 & 3850 & 242677632 & 5335402 \\
\hline 24 & $1 / 5 / 2016$ & 3850 & 256144272 & 5264634 & 3850 & 257982768 & 5169398 & 3850 & 247835088 & 5157468 \\
\hline 25 & $1 / 6 / 2016$ & 3850 & 261234192 & 5089927 & 3850 & 262976064 & 4993283 & 3850 & 252825344 & 4990253 \\
\hline 26 & $1 / 7 / 2016$ & 3850 & 266159792 & 4925601 & 3850 & 267804160 & 4828098 & 3850 & 257658544 & 4833203 \\
\hline 27 & $1 / 8 / 2016$ & 3850 & 270930944 & 4771140 & 3850 & 272477088 & 4672925 & 3850 & 262343728 & 4685181 \\
\hline 28 & $1 / 9 / 2016$ & 3850 & 275556384 & 4625456 & 3850 & 277004064 & 4526982 & 3850 & 266889456 & 4545725 \\
\hline 29 & $1 / 10 / 2016$ & 3850 & 280044512 & 4488113 & 3850 & 281393632 & 4389568 & 3850 & 271303616 & 4414153 \\
\hline 30 & $1 / 11 / 2016$ & 3850 & 284402976 & 4358459 & 3850 & 285653664 & 4260050 & 3850 & 275593504 & 4289883 \\
\hline 31 & $1 / 12 / 2016$ & 3850 & 288638912 & 4235934 & 3850 & 289791520 & 4137853 & 3850 & 279765888 & 4172390 \\
\hline 32 & $1 / 13 / 2016$ & 3850 & 292758944 & 4120033 & 3850 & 293813984 & 4022453 & 3850 & 283827072 & 4061195 \\
\hline
\end{tabular}




\begin{tabular}{|c|c|c|c|c|c|c|c|c|c|c|}
\hline 33 & $1 / 14 / 2016$ & 3850 & 296769216 & 4010297 & 3850 & 297727360 & 3913371 & 3850 & 287782944 & 3955862 \\
\hline 34 & $1 / 15 / 2016$ & 3850 & 300675520 & 3906305 & 3850 & 301537536 & 3810166 & 3850 & 291638912 & 3855992 \\
\hline 35 & $1 / 16 / 2016$ & 3850 & 304483200 & 3807669 & 3850 & 305249952 & 3712437 & 3850 & 295400160 & 3761217 \\
\hline 36 & $1 / 17 / 2016$ & 3850 & 308197248 & 3714036 & 3850 & 308869760 & 3619813 & 3850 & 299071360 & 3671202 \\
\hline 37 & $1 / 18 / 2016$ & 3850 & 311822304 & 3625077 & 3850 & 312401728 & 3531954 & 3850 & 302656992 & 3585634 \\
\hline 38 & $1 / 19 / 2016$ & 3850 & 315362784 & 3540491 & 3850 & 315850272 & 3448545 & 3850 & 306161216 & 3504228 \\
\hline 39 & $1 / 20 / 2016$ & 3850 & 318822816 & 3459999 & 3850 & 319219584 & 3369297 & 3850 & 309587936 & 3426719 \\
\hline 40 & $1 / 21 / 2016$ & 3850 & 322206144 & 3383345 & 3850 & 322513504 & 3293942 & 3850 & 312940800 & 3352863 \\
\hline 41 & $1 / 22 / 2016$ & 3850 & 325516448 & 3310289 & 3850 & 325735744 & 3222232 & 3850 & 316223232 & 3282433 \\
\hline 42 & $1 / 23 / 2016$ & 3850 & 328757056 & 3240611 & 3850 & 3288896966 & 3153939 & 3850 & 319438432 & 3215219 \\
\hline 43 & $1 / 24 / 2016$ & 3850 & 331931136 & 3174105 & 3850 & 331978528 & 3088851 & 3850 & 322589472 & 3151028 \\
\hline 44 & $1 / 25 / 2016$ & 3850 & 335041728 & 3110583 & 3850 & 335005312 & 3026770 & 3850 & 325679136 & 3089679 \\
\hline 45 & $1 / 26 / 2016$ & 3850 & 338091616 & 3049868 & 3850 & 337972800 & 2967514 & 3850 & 328710144 & 3031004 \\
\hline 46 & $1 / 27 / 2016$ & 3850 & 341083392 & 2991794 & 3850 & 340883744 & 2910915 & 3850 & 331684992 & 2974848 \\
\hline 47 & $1 / 28 / 2016$ & 3850 & 344019616 & 2936211 & 3850 & 343740544 & 2856813 & 3850 & 334606080 & 2921065 \\
\hline 48 & $1 / 29 / 2016$ & 3850 & 346902560 & 2882974 & 3850 & 346545600 & 2805063 & 3850 & 337475584 & 2869521 \\
\hline 49 & $1 / 30 / 2016$ & 3850 & 349734528 & 2831952 & 3850 & 349301120 & 2755528 & 3850 & 340295680 & 2820090 \\
\hline 50 & $1 / 31 / 2016$ & 3850 & 352517568 & 2783022 & 3850 & 352009216 & 2708082 & 3850 & 343068320 & 2772654 \\
\hline 51 & $2 / 1 / 2016$ & 3850 & 355253632 & 2736066 & 3850 & 354671808 & 2662604 & 3850 & 345795424 & 2727104 \\
\hline 52 & $2 / 2 / 2016$ & 3850 & 357944608 & 2690979 & 3850 & 357290816 & 2618986 & 3850 & 348478784 & 2683338 \\
\hline 53 & $2 / 3 / 2016$ & 3850 & 360592256 & 2647658 & 3850 & 359867936 & 2577124 & 3850 & 351120032 & 2641259 \\
\hline 54 & $2 / 4 / 2016$ & 3850 & 363198272 & 2606010 & 3850 & 362404864 & 2536922 & 3850 & 353720800 & 2600779 \\
\hline 55 & $2 / 5 / 2016$ & 3850 & 365764224 & 2565946 & 3850 & 364903136 & 2498289 & 3850 & 356282624 & 2561814 \\
\hline 56 & $2 / 6 / 2016$ & 3850 & 368291584 & 2527384 & 3850 & 367364288 & 2461141 & 3850 & 358806912 & 2524285 \\
\hline 57 & $2 / 7 / 2016$ & 3850 & 370781856 & 2490247 & 3850 & 369789696 & 2425399 & 3850 & 361295040 & 2488118 \\
\hline 58 & $2 / 8 / 2016$ & 3850 & 373236320 & 2454461 & 3850 & 372180672 & 2390990 & 3850 & 363748256 & 2453245 \\
\hline 59 & $2 / 9 / 2016$ & 3850 & 375656256 & 2419958 & 3850 & 374538528 & 2357844 & 3850 & 366167840 & 2419601 \\
\hline 60 & $2 / 10 / 2016$ & 3850 & 378042944 & 2386675 & 3850 & 376864416 & 2325897 & 3850 & 368555008 & 2387126 \\
\hline 61 & $2 / 11 / 2016$ & 3850 & 380397504 & 2354551 & 3850 & 379159488 & 2295087 & 3850 & 370910752 & 2355761 \\
\hline 62 & $2 / 12 / 2016$ & 3850 & 382721056 & 2323530 & 3850 & 381424864 & 2265357 & 3850 & 373236224 & 2325454 \\
\hline 63 & $2 / 13 / 2016$ & 3850 & 385014560 & 2293558 & 3850 & 383661504 & 2236654 & 3850 & 375532352 & 2296153 \\
\hline 64 & $2 / 14 / 2016$ & 3850 & 387279168 & 2264586 & 3850 & 385870432 & 2208927 & 3850 & 377800192 & 2267812 \\
\hline 65 & $2 / 15 / 2016$ & 3850 & 389515712 & 2236566 & 3850 & 388052576 & 2182129 & 3850 & 380040576 & 2240384 \\
\hline 66 & $2 / 16 / 2016$ & 3850 & 391725184 & 2209453 & 3850 & 390208768 & 2156215 & 3850 & 382254400 & 2213829 \\
\hline 67 & $2 / 17 / 2016$ & 3850 & 393908384 & 2183207 & 3850 & 392339936 & 2131143 & 3850 & 384442496 & 2188107 \\
\hline 68 & $2 / 18 / 2016$ & 3850 & 396066176 & 2157787 & 3850 & 394446784 & 2106873 & 3850 & 386605664 & 2163179 \\
\hline 69 & $2 / 19 / 2016$ & 3850 & 398199328 & 2133157 & 3850 & 396530176 & 2083369 & 3850 & 388744672 & 2139010 \\
\hline 70 & $2 / 20 / 2016$ & 3850 & 400308608 & 2109280 & 3850 & 398590752 & 2060594 & 3850 & 390860256 & 2115566 \\
\hline 71 & $2 / 21 / 2016$ & 3850 & 402394720 & 2086124 & 3850 & 400629280 & 2038516 & 3850 & 392953056 & 2092817 \\
\hline 72 & $2 / 22 / 2016$ & 3850 & 404458368 & 2063656 & 3850 & 402646368 & 2017104 & 3850 & 395023808 & 2070732 \\
\hline 73 & $2 / 23 / 2016$ & 3850 & 406500192 & 2041849 & 3850 & 404642720 & 1996328 & 3850 & 397073088 & 2049282 \\
\hline 74 & $2 / 24 / 2016$ & 3850 & 408520928 & 2020672 & 3850 & 406618880 & 1976159 & 3850 & 399101536 & 2028441 \\
\hline 75 & $2 / 25 / 2016$ & 3850 & 410521024 & 2000100 & 3850 & 408575424 & 1956572 & 3850 & 401109696 & 2008183 \\
\hline 76 & $2 / 26 / 2016$ & 3850 & 412501120 & 1980107 & 3850 & 410512992 & 1937542 & 3850 & 403098176 & 1988485 \\
\hline 77 & $2 / 27 / 2016$ & 3850 & 414461792 & 1960669 & 3850 & 412432032 & 1919044 & 3850 & 405067520 & 1969323 \\
\hline 78 & $2 / 28 / 2016$ & 3850 & 416403584 & 1941763 & 3850 & 414333056 & 1901056 & 3850 & 407018176 & 1950675 \\
\hline 79 & $2 / 29 / 2016$ & 3850 & 418326912 & 1923369 & 3850 & 416216640 & 1883558 & 3850 & 408950720 & 1932522 \\
\hline 80 & $3 / 1 / 2016$ & 3850 & 420232384 & 1905465 & 3850 & 418083168 & 1866529 & 3850 & 410865568 & 1914843 \\
\hline 81 & $3 / 2 / 2016$ & 3850 & 422120416 & 1888032 & 3850 & 419933120 & 1849949 & 3850 & 412763168 & 1897621 \\
\hline 82 & $3 / 3 / 2016$ & 3850 & 423991456 & 1871052 & 3850 & 421766912 & 1833802 & 3850 & 414644032 & 1880837 \\
\hline 83 & $3 / 4 / 2016$ & 3850 & 425845984 & 1854506 & 3850 & 423584992 & 1818069 & 3850 & 416508480 & 1864474 \\
\hline 84 & $3 / 5 / 2016$ & 3850 & 427684352 & 1838379 & 3850 & 425387712 & 1802734 & 3850 & 418356992 & 1848517 \\
\hline 85 & $3 / 6 / 2016$ & 3850 & 429507008 & 1822655 & 3850 & 427175488 & 1787783 & 3850 & 420189952 & 1832951 \\
\hline 86 & $3 / 7 / 2016$ & 3850 & 431314336 & 1807317 & 3850 & 428948704 & 1773199 & 3850 & 422007712 & 1817760 \\
\hline 87 & $3 / 8 / 2016$ & 3850 & 433106688 & 1792353 & 3850 & 430707680 & 1758970 & 3850 & 423810656 & 1802932 \\
\hline 88 & $3 / 9 / 2016$ & 3850 & 434884416 & 1777748 & 3850 & 432452768 & 1745081 & 3850 & 425599136 & 1788453 \\
\hline 89 & $3 / 10 / 2016$ & 3850 & 436647904 & 1763488 & 3850 & 434184288 & 1731521 & 3850 & 427373408 & 1774310 \\
\hline 90 & $3 / 11 / 2016$ & 3850 & 438397472 & 1749563 & 3850 & 435902560 & 1718277 & 3850 & 429133856 & 1760492 \\
\hline 91 & $3 / 12 / 2016$ & 3850 & 440133440 & 1735959 & 3850 & 437607872 & 1705337 & 3850 & 430880896 & 1746987 \\
\hline 92 & $3 / 13 / 2016$ & 3850 & 441856096 & 1722665 & 3850 & 439300576 & 1692690 & 3850 & 432614656 & 1733784 \\
\hline 93 & $3 / 14 / 2016$ & 3850 & 443565760 & 1709672 & 3850 & 440980896 & 1680327 & 3850 & 434335552 & 1720873 \\
\hline 94 & $3 / 15 / 2016$ & 3850 & 445262752 & 1696967 & 3850 & 442649152 & 1668237 & 3850 & 436043776 & 1708244 \\
\hline 95 & $3 / 16 / 2016$ & 3850 & 446947264 & 1684541 & 3850 & 444305568 & 1656411 & 3850 & 437739680 & 1695888 \\
\hline 96 & $3 / 17 / 2016$ & 3850 & 448619648 & 1672385 & 3850 & 445950400 & 1644838 & 3850 & 439423456 & 1683795 \\
\hline 97 & $3 / 18 / 2016$ & 3850 & 450280160 & 1660491 & 3850 & 447583904 & 1633512 & 3850 & 441095424 & 1671956 \\
\hline 98 & $3 / 19 / 2016$ & 3850 & 451928992 & 1648848 & 3850 & 449206336 & 1622424 & 3850 & 442755776 & 1660364 \\
\hline 99 & $3 / 20 / 2016$ & 3850 & 453566464 & 1637449 & 3850 & 450817888 & 1611565 & 3850 & 444404832 & 1649010 \\
\hline 100 & $3 / 21 / 2016$ & 3850 & 455192736 & 1626286 & 3850 & 452418816 & 1600928 & 3850 & 446042688 & 1637887 \\
\hline 101 & $3 / 22 / 2016$ & 3850 & 456808096 & 1615351 & 3850 & 454009312 & 1590506 & 3850 & 447669664 & 1626987 \\
\hline 102 & $3 / 23 / 2016$ & 3850 & 458412736 & 1604637 & 3850 & 455589600 & 1580291 & 3850 & 449285984 & 1616304 \\
\hline 103 & $3 / 24 / 2016$ & 3850 & 460006848 & 1594137 & 3850 & 457159904 & 1570278 & 3850 & 450891808 & 1605830 \\
\hline 104 & $3 / 25 / 2016$ & 3850 & 461590720 & 1583844 & 3850 & 458720352 & 1560460 & 3850 & 452487360 & 1595560 \\
\hline 105 & $3 / 26 / 2016$ & 3850 & 463164448 & 1573753 & 3850 & 460271200 & 1550830 & 3850 & 454072864 & 1585486 \\
\hline 106 & $3 / 27 / 2016$ & 3850 & 464728352 & 1563856 & 3850 & 461812576 & 1541384 & 3850 & 455648448 & 1575603 \\
\hline 107 & $3 / 28 / 2016$ & 3850 & 466282464 & 1554148 & 3850 & 463344672 & 1532114 & 3850 & 457214368 & 1565906 \\
\hline 108 & $3 / 29 / 2016$ & 3850 & 467827072 & 1544623 & 3850 & 464867712 & 1523017 & 3850 & 458770752 & 1556388 \\
\hline 109 & $3 / 30 / 2016$ & 3850 & 469362368 & 1535276 & 3850 & 466381792 & 1514086 & 3850 & 460317792 & 1547046 \\
\hline 110 & $3 / 31 / 2016$ & 3850 & 470888448 & 1526101 & 3850 & 467887104 & 1505317 & 3850 & 461855680 & 1537872 \\
\hline 111 & $4 / 1 / 2016$ & 3850 & 472405568 & 1517093 & 3850 & 469383808 & 1496705 & 3850 & 463384544 & 1528863 \\
\hline 112 & $4 / 2 / 2016$ & 3850 & 473913792 & 1508249 & 3850 & 470872064 & 1488246 & 3850 & 464904544 & 1520014 \\
\hline 113 & $4 / 3 / 2016$ & 3850 & 475413376 & 1499562 & 3850 & 472352000 & 1479934 & 3850 & 466415872 & 1511321 \\
\hline 114 & $4 / 4 / 2016$ & 3850 & 476904384 & 1491028 & 3850 & 473823744 & 1471767 & 3850 & 467918656 & 1502779 \\
\hline
\end{tabular}




\begin{tabular}{|c|c|c|c|c|c|c|c|c|c|c|}
\hline 115 & $4 / 5 / 2016$ & 3850 & 478387040 & 1482644 & 3850 & 475287488 & 1463739 & 3850 & 469413024 & 1494383 \\
\hline 116 & $4 / 6 / 2016$ & 3850 & 479861440 & 1474404 & 3850 & 476743328 & 1455847 & 3850 & 470899136 & 1486130 \\
\hline 117 & $4 / 7 / 2016$ & 3850 & 481327744 & 1466305 & 3850 & 478191424 & 1448087 & 3850 & 472377184 & 1478017 \\
\hline 118 & $4 / 8 / 2016$ & 3850 & 482786080 & 1458344 & 3850 & 479631872 & 1440456 & 3850 & 473847168 & 1470038 \\
\hline 119 & $4 / 9 / 2016$ & 3850 & 484236608 & 1450516 & 3850 & 481064832 & 1432950 & 3850 & 475309408 & 1462191 \\
\hline 120 & $4 / 10 / 2016$ & 3850 & 485679424 & 1442817 & 3850 & 482490400 & 1425565 & 3850 & 476763872 & 1454472 \\
\hline 121 & $4 / 11 / 2016$ & 3850 & 487114656 & 1435245 & 3850 & 483908736 & 1418299 & 3850 & 478210784 & 1446878 \\
\hline 122 & $4 / 12 / 2016$ & 3850 & 488542464 & 1427796 & 3850 & 485319808 & 1411148 & 3850 & 479650176 & 1439406 \\
\hline 123 & $4 / 13 / 2016$ & 3850 & 489962976 & 1420467 & 3850 & 486723968 & 1404109 & 3850 & 481082208 & 1432052 \\
\hline 124 & $4 / 14 / 2016$ & 3850 & 491376192 & 1413254 & 3850 & 488121120 & 1397180 & 3850 & 482507040 & 1424813 \\
\hline 125 & $4 / 15 / 2016$ & 3850 & 492782336 & 1406155 & 3850 & 489511488 & 1390357 & 3850 & 483924704 & 1417687 \\
\hline 126 & $4 / 16 / 2016$ & 3850 & 494181504 & 1399167 & 3850 & 490895136 & 1383638 & 3850 & 485335392 & 1410670 \\
\hline 127 & $4 / 17 / 2016$ & 3850 & 495573792 & 1392286 & 3850 & 492272160 & 1377021 & 3850 & 486739136 & 1403760 \\
\hline 128 & $4 / 18 / 2016$ & 3850 & 496959296 & 1385511 & 3850 & 493642656 & 1370502 & 3850 & 488136096 & 1396955 \\
\hline 129 & $4 / 19 / 2016$ & 3850 & 498338144 & 1378839 & 3850 & 495006720 & 1364080 & 3850 & 489526336 & 1390251 \\
\hline 130 & $4 / 20 / 2016$ & 3850 & 499710400 & 1372267 & 3850 & 496364480 & 1357751 & 3850 & 490909984 & 1383647 \\
\hline 131 & $4 / 21 / 2016$ & 3850 & 501076192 & 1365792 & 3850 & 497716000 & 1351514 & 3850 & 492287136 & 1377139 \\
\hline 132 & $4 / 22 / 2016$ & 3850 & 502435616 & 1359413 & 3850 & 499061376 & 1345367 & 3850 & 493657856 & 1370726 \\
\hline 133 & $4 / 23 / 2016$ & 3850 & 503788736 & 1353127 & 3850 & 500400672 & 1339307 & 3850 & 495022272 & 1364405 \\
\hline 134 & $4 / 24 / 2016$ & 3850 & 505135680 & 1346931 & 3850 & 501734016 & 1333332 & 3850 & 496380448 & 1358175 \\
\hline 135 & $4 / 25 / 2016$ & 3850 & 506476480 & 1340824 & 3850 & 503061440 & 1327441 & 3850 & 497732480 & 1352032 \\
\hline 136 & $4 / 26 / 2016$ & 3850 & 507811296 & 1334804 & 3850 & 504383072 & 1321631 & 3850 & 499078464 & 1345976 \\
\hline 137 & $4 / 27 / 2016$ & 3850 & 509140160 & 1328869 & 3850 & 505698976 & 1315900 & 3850 & 500418464 & 1340004 \\
\hline 138 & $4 / 28 / 2016$ & 3850 & 510463168 & 1323016 & 3850 & 507009216 & 1310247 & 3850 & 501752576 & 1334114 \\
\hline 139 & $4 / 29 / 2016$ & 3850 & 511780448 & 1317244 & 3850 & 508313888 & 1304671 & 3850 & 503080864 & 1328304 \\
\hline 140 & $4 / 30 / 2016$ & 3850 & 513092000 & 1311551 & 3850 & 509613056 & 1299168 & 3850 & 504403456 & 1322573 \\
\hline 141 & $5 / 1 / 2016$ & 3850 & 514397920 & 1305935 & 3850 & 510906784 & 1293738 & 3850 & 505720352 & 1316918 \\
\hline 142 & $5 / 2 / 2016$ & 3850 & 515698304 & 1300394 & 3850 & 512195168 & 1288380 & 3850 & 507031712 & 1311339 \\
\hline 143 & $5 / 3 / 2016$ & 3850 & 516993216 & 1294927 & 3850 & 513478240 & 1283090 & 3850 & 508337536 & 1305833 \\
\hline 144 & $5 / 4 / 2016$ & 3850 & 518282752 & 1289533 & 3850 & 514756128 & 1277869 & 3850 & 509637920 & 1300399 \\
\hline 145 & $5 / 5 / 2016$ & 3850 & 519566976 & 1284209 & 3850 & 516028864 & 1272714 & 3850 & 510932960 & 1295036 \\
\hline 146 & $5 / 6 / 2016$ & 3850 & 520845920 & 1278954 & 3850 & 517296480 & 1267624 & 3850 & 512222720 & 1289741 \\
\hline 147 & $5 / 7 / 2016$ & 3850 & 522119680 & 1273767 & 3850 & 518559072 & 1262598 & 3850 & 513507264 & 1284514 \\
\hline 148 & $5 / 8 / 2016$ & 3850 & 523388352 & 1268646 & 3850 & 519816704 & 1257635 & 3850 & 514786560 & 1279352 \\
\hline 149 & $5 / 9 / 2016$ & 3850 & 524651936 & 1263589 & 3850 & 521069440 & 1252733 & 3850 & 516060832 & 1274256 \\
\hline 150 & $5 / 10 / 2016$ & 3850 & 525910528 & 1258597 & 3850 & 522317344 & 1247890 & 3850 & 517330048 & 1269223 \\
\hline 151 & $5 / 11 / 2016$ & 3850 & 527164192 & 1253666 & 3850 & 523560448 & 1243106 & 3850 & 518594304 & 1264251 \\
\hline 152 & $5 / 12 / 2016$ & 3850 & 528412992 & 1248796 & 3850 & 524798816 & 1238380 & 3850 & 519853664 & 1259341 \\
\hline 153 & $5 / 13 / 2016$ & 3850 & 529656960 & 1243986 & 3850 & 526032512 & 1233710 & 3850 & 521108128 & 1254490 \\
\hline 154 & $5 / 14 / 2016$ & 3850 & 530896224 & 1239234 & 3850 & 527261632 & 1229095 & 3850 & 522357824 & 1249697 \\
\hline 155 & $5 / 15 / 2016$ & 3850 & 532130752 & 1234539 & 3850 & 528486144 & 1224534 & 3850 & 523602784 & 1244962 \\
\hline 156 & $5 / 16 / 2016$ & 3850 & 533360640 & 1229900 & 3850 & 529706208 & 1220027 & 3850 & 524843072 & 1240283 \\
\hline 157 & $5 / 17 / 2016$ & 3850 & 534585952 & 1225317 & 3850 & 530921760 & 1215571 & 3850 & 526078720 & 1235658 \\
\hline 158 & $5 / 18 / 2016$ & 3850 & 535806752 & 1220787 & 3850 & 532132896 & 1211166 & 3850 & 527309824 & 1231088 \\
\hline 159 & $5 / 19 / 2016$ & 3850 & 537023040 & 1216310 & 3850 & 533339744 & 1206812 & 3850 & 528536384 & 1226570 \\
\hline 160 & $5 / 20 / 2016$ & 3850 & 538234944 & 1211885 & 3850 & 534542240 & 1202507 & 3850 & 529758496 & 1222104 \\
\hline 161 & $5 / 21 / 2016$ & 3850 & 539442432 & 1207511 & 3850 & 535740480 & 1198249 & 3850 & 530976192 & 1217690 \\
\hline 162 & $5 / 22 / 2016$ & 3850 & 540645632 & 1203186 & 3850 & 536934528 & 1194039 & 3850 & 532189536 & 1213325 \\
\hline 163 & $5 / 23 / 2016$ & 3850 & 541844544 & 1198911 & 3850 & 538124416 & 1189876 & 3850 & 533398528 & 1209009 \\
\hline 164 & $5 / 24 / 2016$ & 3850 & 543039232 & 1194683 & 3850 & 539310144 & 1185758 & 3850 & 534603264 & 1204741 \\
\hline 165 & $5 / 25 / 2016$ & 3850 & 544229760 & 1190503 & 3850 & 540491840 & 1181684 & 3850 & 535803776 & 1200520 \\
\hline 166 & $5 / 26 / 2016$ & 3850 & 545416128 & 1186368 & 3850 & 541669504 & 1177655 & 3850 & 537000128 & 1196346 \\
\hline 167 & $5 / 27 / 2016$ & 3850 & 546598400 & 1182279 & 3850 & 542843200 & 1173669 & 3850 & 538192320 & 1192217 \\
\hline 168 & $5 / 28 / 2016$ & 3850 & 547776640 & 1178235 & 3850 & 544012864 & 1169725 & 3850 & 539380480 & 1188132 \\
\hline 169 & $5 / 29 / 2016$ & 3850 & 548950848 & 1174234 & 3850 & 545178688 & 1165822 & 3850 & 540564544 & 1184092 \\
\hline 170 & $5 / 30 / 2016$ & 3850 & 550121152 & 1170276 & 3850 & 546340672 & 1161961 & 3850 & 541744640 & 1180095 \\
\hline 171 & $5 / 31 / 2016$ & 3850 & 551287488 & 1166361 & 3850 & 547498816 & 1158139 & 3850 & 542920768 & 1176140 \\
\hline 172 & $6 / 1 / 2016$ & 3850 & 552449984 & 1162487 & 3850 & 548653184 & 1154357 & 3850 & 544093056 & 1172226 \\
\hline 173 & $6 / 2 / 2016$ & 3850 & 553608640 & 1158653 & 3850 & 549803776 & 1150614 & 3850 & 545261376 & 1168354 \\
\hline 174 & $6 / 3 / 2016$ & 3850 & 554763520 & 1154860 & 3850 & 550950720 & 1146909 & 3850 & 546425920 & 1164521 \\
\hline 175 & $6 / 4 / 2016$ & 3850 & 555914624 & 1151105 & 3850 & 552093952 & 1143242 & 3850 & 547586624 & 1160728 \\
\hline 176 & $6 / 5 / 2016$ & 3850 & 557062016 & 1147390 & 3850 & 553233536 & 1139611 & 3850 & 548743616 & 1156974 \\
\hline 177 & $6 / 6 / 2016$ & 3850 & 558205696 & 1143712 & 3850 & 554369536 & 1136016 & 3850 & 549896832 & 1153258 \\
\hline 178 & $6 / 7 / 2016$ & 3850 & 559345792 & 1140072 & 3850 & 555502016 & 1132457 & 3850 & 551046464 & 1149579 \\
\hline 179 & $6 / 8 / 2016$ & 3850 & 560482240 & 1136468 & 3850 & 556630976 & 1128933 & 3850 & 552192384 & 1145937 \\
\hline 180 & $6 / 9 / 2016$ & 3850 & 561615168 & 1132900 & 3850 & 557756416 & 1125443 & 3850 & 553334720 & 1142331 \\
\hline 181 & $6 / 10 / 2016$ & 3850 & 562744512 & 1129368 & 3850 & 558878400 & 1121988 & 3850 & 554473472 & 1138761 \\
\hline 182 & $6 / 11 / 2016$ & 3850 & 563870400 & 1125870 & 3850 & 559996928 & 1118565 & 3850 & 555608704 & 1135226 \\
\hline 183 & $6 / 12 / 2016$ & 3850 & 564992768 & 1122407 & 3850 & 561112128 & 1115175 & 3850 & 556740416 & 1131726 \\
\hline 184 & $6 / 13 / 2016$ & 3850 & 566111744 & 1118977 & 3850 & 562223936 & 1111818 & 3850 & 557868672 & 1128259 \\
\hline 185 & $6 / 14 / 2016$ & 3850 & 567227328 & 1115581 & 3850 & 563332416 & 1108492 & 3850 & 558993472 & 1124826 \\
\hline 186 & $6 / 15 / 2016$ & 3850 & 568339584 & 1112217 & 3850 & 564437632 & 1105198 & 3850 & 560114944 & 1121425 \\
\hline 187 & $6 / 16 / 2016$ & 3850 & 569448448 & 1108885 & 3850 & 565539584 & 1101934 & 3850 & 561232960 & 1118057 \\
\hline 188 & $6 / 17 / 2016$ & 3850 & 570554048 & 1105585 & 3850 & 566638272 & 1098700 & 3850 & 562347712 & 1114721 \\
\hline 189 & $6 / 18 / 2016$ & 3850 & 571656320 & 1102316 & 3850 & 567733760 & 1095497 & 3850 & 563459136 & 1111416 \\
\hline 190 & $6 / 19 / 2016$ & 3850 & 572755392 & 1099078 & 3850 & 568826112 & 1092322 & 3850 & 564567296 & 1108142 \\
\hline 191 & $6 / 20 / 2016$ & 3850 & 573851264 & 1095869 & 3850 & 569915264 & 1089177 & 3850 & 565672192 & 1104898 \\
\hline 192 & $6 / 21 / 2016$ & 3850 & 574944000 & 1092690 & 3850 & 571001344 & 1086060 & 3850 & 566773824 & 1101684 \\
\hline 193 & $6 / 22 / 2016$ & 3850 & 576033536 & 1089541 & 3850 & 572084288 & 1082971 & 3850 & 567872320 & 1098499 \\
\hline 194 & $6 / 23 / 2016$ & 3850 & 577119936 & 1086420 & 3850 & 573164224 & 1079909 & 3850 & 568967680 & 1095343 \\
\hline 195 & $6 / 24 / 2016$ & 3850 & 578203264 & 1083327 & 3850 & 574241088 & 1076875 & 3850 & 570059904 & 1092216 \\
\hline 196 & $6 / 25 / 2016$ & 3850 & 579283520 & 1080262 & 3850 & 575314944 & 1073868 & 3850 & 571148992 & 1089116 \\
\hline
\end{tabular}




\begin{tabular}{|c|c|c|c|c|c|c|c|c|c|c|}
\hline 197 & $6 / 26 / 2016$ & 3850 & 580360768 & 1077225 & 3850 & 576385856 & 1070886 & 3850 & 572235072 & 1086045 \\
\hline 198 & $6 / 27 / 2016$ & 3850 & 581434944 & 1074215 & 3850 & 577453760 & 1067931 & 3850 & 573318080 & 1083000 \\
\hline 199 & $6 / 28 / 2016$ & 3850 & 582506176 & 1071231 & 3850 & 578518784 & 1065002 & 3850 & 574398016 & 1079983 \\
\hline 200 & $6 / 29 / 2016$ & 3850 & 583574464 & 1068273 & 3850 & 579580864 & 1062097 & 3850 & 575475008 & 1076992 \\
\hline 201 & $6 / 30 / 2016$ & 3850 & 584639808 & 1065341 & 3850 & 580640064 & 1059218 & 3850 & 576549056 & 1074027 \\
\hline 202 & $7 / 1 / 2016$ & 3850 & 585702272 & 1062435 & 3850 & 581696448 & 1056363 & 3850 & 577620160 & 1071087 \\
\hline 203 & $7 / 2 / 2016$ & 3850 & 586761792 & 1059554 & 3850 & 582749952 & 1053532 & 3850 & 578688320 & 1068173 \\
\hline 204 & $7 / 3 / 2016$ & 3850 & 587818496 & 1056697 & 3850 & 583800704 & 1050725 & 3850 & 579753600 & 1065284 \\
\hline 205 & $7 / 4 / 2016$ & 3850 & 588872384 & 1053865 & 3850 & 584848640 & 1047941 & 3850 & 580816000 & 1062419 \\
\hline 206 & $7 / 5 / 2016$ & 3850 & 589923392 & 1051057 & 3850 & 585893824 & 1045181 & 3850 & 581875584 & 1059579 \\
\hline 207 & $7 / 6 / 2016$ & 3850 & 590971712 & 1048272 & 3850 & 586936256 & 1042443 & 3850 & 582932352 & 1056763 \\
\hline 208 & $7 / 7 / 2016$ & 3850 & 592017216 & 1045511 & 3850 & 587976000 & 1039728 & 3850 & 583986368 & 1053970 \\
\hline 209 & $7 / 8 / 2016$ & 3850 & 593059968 & 1042773 & 3850 & 589013056 & 1037035 & 3850 & 585037568 & 1051200 \\
\hline 210 & $7 / 9 / 2016$ & 3850 & 594100032 & 1040057 & 3850 & 590047424 & 1034365 & 3850 & 586086016 & 1048453 \\
\hline 211 & $7 / 10 / 2016$ & 3850 & 595137408 & 1037364 & 3850 & 591079104 & 1031715 & 3850 & 587131712 & 1045729 \\
\hline 212 & $7 / 11 / 2016$ & 3850 & 596172096 & 1034693 & 3850 & 592108224 & 1029087 & 3850 & 588174720 & 1043027 \\
\hline 213 & $7 / 12 / 2016$ & 3850 & 597204160 & 1032044 & 3850 & 593134720 & 1026480 & 3850 & 589215104 & 1040347 \\
\hline 214 & $7 / 13 / 2016$ & 3850 & 598233536 & 1029416 & 3850 & 594158592 & 1023894 & 3850 & 590252800 & 1037688 \\
\hline 215 & $7 / 14 / 2016$ & 3850 & 599260352 & 1026809 & 3850 & 595179904 & 1021328 & 3850 & 591287808 & 1035051 \\
\hline 216 & $7 / 15 / 2016$ & 3850 & 600284608 & 1024223 & 3850 & 596198656 & 1018782 & 3850 & 592320256 & 1032435 \\
\hline 217 & $7 / 16 / 2016$ & 3850 & 601306240 & 1021657 & 3850 & 597214976 & 1016256 & 3850 & 593350080 & 1029840 \\
\hline 218 & $7 / 17 / 2016$ & 3850 & 602325376 & 1019112 & 3850 & 598228672 & 1013750 & 3850 & 594377344 & 1027265 \\
\hline 219 & $7 / 18 / 2016$ & 3850 & 603341952 & 1016587 & 3850 & 599239936 & 1011263 & 3850 & 595402112 & 1024711 \\
\hline 220 & $7 / 19 / 2016$ & 3850 & 604356096 & 1014081 & 3850 & 600248768 & 1008796 & 3850 & 596424256 & 1022176 \\
\hline 221 & $7 / 20 / 2016$ & 3850 & 605367616 & 1011596 & 3850 & 601255104 & 1006347 & 3850 & 597443904 & 1019661 \\
\hline 222 & $7 / 21 / 2016$ & 3850 & 606376768 & 1009129 & 3850 & 602259008 & 1003917 & 3850 & 598461120 & 1017166 \\
\hline 223 & $7 / 22 / 2016$ & 3850 & 607383424 & 1006681 & 3850 & 603260544 & 1001505 & 3850 & 599475776 & 1014690 \\
\hline 224 & $7 / 23 / 2016$ & 3850 & 608387648 & 1004252 & 3850 & 604259648 & 999112 & 3850 & 600488000 & 1012233 \\
\hline 225 & $7 / 24 / 2016$ & 3850 & 609389504 & 1001842 & 3850 & 605256384 & 996736 & 3850 & 601497792 & 1009794 \\
\hline 226 & $7 / 25 / 2016$ & 3850 & 610388992 & 999450 & 3850 & 606250752 & 994378 & 3850 & 602505152 & 1007374 \\
\hline 227 & $7 / 26 / 2016$ & 3850 & 611385984 & 997076 & 3850 & 607242752 & 992038 & 3850 & 603510144 & 1004972 \\
\hline 228 & $7 / 27 / 2016$ & 3850 & 612380736 & 994719 & 3850 & 608232512 & 989715 & 3850 & 604512768 & 1002589 \\
\hline 229 & $7 / 28 / 2016$ & 3850 & 613373120 & 992380 & 3850 & 609219904 & 987409 & 3850 & 605512960 & 1000223 \\
\hline 230 & $7 / 29 / 2016$ & 3850 & 614363200 & 990059 & 3850 & 610204992 & 985120 & 3850 & 606510848 & 997874 \\
\hline 231 & $7 / 30 / 2016$ & 3850 & 615350976 & 987755 & 3850 & 611187840 & 982847 & 3850 & 607506368 & 995543 \\
\hline 232 & $7 / 31 / 2016$ & 3850 & 616336448 & 985467 & 3850 & 612168448 & 980591 & 3850 & 608499584 & 993229 \\
\hline 233 & $8 / 1 / 2016$ & 3850 & 617319616 & 983196 & 3850 & 613146816 & 978351 & 3850 & 609490560 & 990932 \\
\hline 234 & $8 / 2 / 2016$ & 3850 & 618300544 & 980942 & 3850 & 614122944 & 976127 & 3850 & 610479104 & 988651 \\
\hline 235 & $8 / 3 / 2016$ & 3850 & 619279296 & 978704 & 3850 & 615096832 & 973919 & 3850 & 611465600 & 986387 \\
\hline 236 & $8 / 4 / 2016$ & 3850 & 620255744 & 976483 & 3850 & 616068608 & 971727 & 3850 & 612449664 & 984140 \\
\hline 237 & $8 / 5 / 2016$ & 3850 & 621230080 & 974276 & 3850 & 617038144 & 969550 & 3850 & 613431552 & 981908 \\
\hline 238 & $8 / 6 / 2016$ & 3850 & 622202112 & 972086 & 3850 & 618005568 & 967389 & 3850 & 614411328 & 979693 \\
\hline 239 & $8 / 7 / 2016$ & 3850 & 623172032 & 969912 & 3850 & 618970752 & 965242 & 3850 & 615388800 & 977493 \\
\hline 240 & $8 / 8 / 2016$ & 3850 & 624139776 & 967752 & 3850 & 619933888 & 963111 & 3850 & 616364096 & 975308 \\
\hline 241 & $8 / 9 / 2016$ & 3850 & 625105408 & 965608 & 3850 & 620894848 & 960994 & 3850 & 617337344 & 973139 \\
\hline 242 & $8 / 10 / 2016$ & 3850 & 626068864 & 963479 & 3850 & 621853760 & 958892 & 3850 & 618308224 & 970985 \\
\hline 243 & $8 / 11 / 2016$ & 3850 & 627030208 & 961364 & 3850 & 622810624 & 956804 & 3850 & 619277120 & 968846 \\
\hline 244 & $8 / 12 / 2016$ & 3850 & 627989504 & 959264 & 3850 & 623765312 & 954730 & 3850 & 620243840 & 966722 \\
\hline 245 & $8 / 13 / 2016$ & 3850 & 628946688 & 957179 & 3850 & 624717952 & 952671 & 3850 & 621208448 & 964612 \\
\hline 246 & $8 / 14 / 2016$ & 3850 & 629901760 & 955108 & 3850 & 625668608 & 950625 & 3850 & 622170944 & 962517 \\
\hline 247 & $8 / 15 / 2016$ & 3850 & 630854848 & 953051 & 3850 & 626617216 & 948594 & 3850 & 623131392 & 960436 \\
\hline 248 & $8 / 16 / 2016$ & 3850 & 631805824 & 951007 & 3850 & 627563776 & 946575 & 3850 & 624089792 & 958370 \\
\hline 249 & $8 / 17 / 2016$ & 3850 & 632754880 & 948978 & 3850 & 628508352 & 944571 & 3850 & 625046080 & 956317 \\
\hline 250 & $8 / 18 / 2016$ & 3850 & 633701760 & 946962 & 3850 & 629450944 & 942579 & 3850 & 626000384 & 954278 \\
\hline 251 & $8 / 19 / 2016$ & 3850 & 634646720 & 944960 & 3850 & 630391552 & 940601 & 3850 & 626952576 & 952253 \\
\hline 252 & $8 / 20 / 2016$ & 3850 & 635589696 & 942971 & 3850 & 631330176 & 938636 & 3850 & 627902848 & 950241 \\
\hline 253 & $8 / 21 / 2016$ & 3850 & 636530688 & 940995 & 3850 & 632266816 & 936683 & 3850 & 628851072 & 948242 \\
\hline 254 & $8 / 22 / 2016$ & 3850 & 637469760 & 939033 & 3850 & 633201600 & 934744 & 3850 & 629797312 & 946257 \\
\hline 255 & $8 / 23 / 2016$ & 3850 & 638406848 & 937083 & 3850 & 634134400 & 932816 & 3850 & 630741632 & 944285 \\
\hline 256 & $8 / 24 / 2016$ & 3850 & 639341952 & 935146 & 3850 & 635065280 & 930902 & 3850 & 631683968 & 942325 \\
\hline 257 & $8 / 25 / 2016$ & 3850 & 640275200 & 933221 & 3850 & 635994304 & 928999 & 3850 & 632624320 & 940379 \\
\hline 258 & $8 / 26 / 2016$ & 3850 & 641206528 & 931309 & 3850 & 636921408 & 927109 & 3850 & 633562752 & 938445 \\
\hline 259 & $8 / 27 / 2016$ & 3850 & 642135936 & 929409 & 3850 & 637846656 & 925231 & 3850 & 634499264 & 936523 \\
\hline 260 & $8 / 28 / 2016$ & 3850 & 643063424 & 927522 & 3850 & 638769984 & 923365 & 3850 & 635433920 & 934614 \\
\hline 261 & $8 / 29 / 2016$ & 3850 & 643989056 & 925646 & 3850 & 639691520 & 921510 & 3850 & 636366592 & 932717 \\
\hline 262 & $8 / 30 / 2016$ & 3850 & 644912832 & 923782 & 3850 & 640611264 & 919667 & 3850 & 637297472 & 930832 \\
\hline 263 & $8 / 31 / 2016$ & 3850 & 645834816 & 921931 & 3850 & 641528960 & 917836 & 3850 & 638226432 & 928959 \\
\hline 264 & $9 / 1 / 2016$ & 3850 & 646754880 & 920090 & 3850 & 642445056 & 916016 & 3850 & 639153536 & 927098 \\
\hline 265 & $9 / 2 / 2016$ & 3850 & 647673152 & 918262 & 3850 & 6433599232 & 914207 & 3850 & 640078784 & 925248 \\
\hline 266 & $9 / 3 / 2016$ & 3850 & 648589568 & 916444 & 3850 & 644271616 & 912410 & 3850 & 641002176 & 923411 \\
\hline 267 & $9 / 4 / 2016$ & 3850 & 649504192 & 914639 & 3850 & 645182272 & 910623 & 3850 & 641923840 & 921584 \\
\hline 268 & $9 / 5 / 2016$ & 3850 & 650417088 & 912844 & 3850 & 646091136 & 908848 & 3850 & 642843520 & 919769 \\
\hline 269 & $9 / 6 / 2016$ & 3850 & 651328128 & 911060 & 3850 & 646998144 & 907083 & 3850 & 643761472 & 917965 \\
\hline 270 & $9 / 7 / 2016$ & 3850 & 652237440 & 909287 & 3850 & 647903552 & 905329 & 3850 & 644677632 & 916172 \\
\hline 271 & $9 / 8 / 2016$ & 3850 & 653144960 & 907525 & 3850 & 648807104 & 903586 & 3850 & 645592064 & 914390 \\
\hline 272 & $9 / 9 / 2016$ & 3850 & 654050624 & 905774 & 3850 & 649708992 & 901853 & 3850 & 646504640 & 912619 \\
\hline 273 & $9 / 10 / 2016$ & 3850 & 654954752 & 904033 & 3850 & 650609088 & 900131 & 3850 & 647415552 & 910859 \\
\hline 274 & $9 / 11 / 2016$ & 3850 & 655857024 & 902303 & 3850 & 651507520 & 898418 & 3850 & 648324608 & 909109 \\
\hline 275 & $9 / 12 / 2016$ & 3850 & 656757632 & 900583 & 3850 & 652404224 & 896716 & 3850 & 649231936 & 907370 \\
\hline 276 & $9 / 13 / 2016$ & 3850 & 657656512 & 898874 & 3850 & 653299328 & 895024 & 3850 & 650137664 & 905642 \\
\hline 277 & $9 / 14 / 2016$ & 3850 & 658553728 & 897174 & 3850 & 654192576 & 893342 & 3850 & 651041600 & 903923 \\
\hline 278 & $9 / 15 / 2016$ & 3850 & 659449152 & 895485 & 3850 & 655084288 & 891670 & 3850 & 651943808 & 902215 \\
\hline
\end{tabular}




\begin{tabular}{|c|c|c|c|c|c|c|c|c|c|c|}
\hline 279 & $9 / 16 / 2016$ & 3850 & 660342976 & 893806 & 3850 & 655974272 & 890008 & 3850 & 652844288 & 900517 \\
\hline 280 & $9 / 17 / 2016$ & 3850 & 661235136 & 892137 & 3850 & 656862720 & 888355 & 3850 & 653743104 & 898829 \\
\hline 281 & $9 / 18 / 2016$ & 3850 & 662125568 & 890477 & 3850 & 657749312 & 886712 & 3850 & 654640256 & 897151 \\
\hline 282 & $9 / 19 / 2016$ & 3850 & 663014464 & 888827 & 3850 & 658634432 & 885079 & 3850 & 655535744 & 895482 \\
\hline 283 & $9 / 20 / 2016$ & 3850 & 663901568 & 887186 & 3850 & 659517888 & 883455 & 3850 & 656429568 & 893824 \\
\hline 284 & $9 / 21 / 2016$ & 3850 & 664787136 & 885556 & 3850 & 660399744 & 881840 & 3850 & 657321792 & 892175 \\
\hline 285 & $9 / 22 / 2016$ & 3850 & 665671104 & 883934 & 3850 & 661279936 & 880234 & 3850 & 658212288 & 890535 \\
\hline 286 & $9 / 23 / 2016$ & 3850 & 666553408 & 882322 & 3850 & 662158592 & 878638 & 3850 & 659101184 & 888905 \\
\hline 287 & $9 / 24 / 2016$ & 3850 & 667434112 & 880719 & 3850 & 663035648 & 877050 & 3850 & 659988480 & 887284 \\
\hline 288 & $9 / 25 / 2016$ & 3850 & 668313280 & 879125 & 3850 & 663911104 & 875472 & 3850 & 660874176 & 885673 \\
\hline 289 & $9 / 26 / 2016$ & 3850 & 669190784 & 877540 & 3850 & 664785024 & 873902 & 3850 & 661758208 & 884071 \\
\hline 290 & $9 / 27 / 2016$ & 3850 & 670066752 & 875964 & 3850 & 665657344 & 872341 & 3850 & 662640704 & 882477 \\
\hline 291 & $9 / 28 / 2016$ & 3850 & 670941184 & 874397 & 3850 & 666528128 & 870789 & 3850 & 663521600 & 880893 \\
\hline 292 & $9 / 29 / 2016$ & 3850 & 671814016 & 872839 & 3850 & 667397376 & 869245 & 3850 & 664400896 & 879318 \\
\hline 293 & $9 / 30 / 2016$ & 3850 & 672685184 & 871289 & 3850 & 668265088 & 867710 & 3850 & 665278656 & 877751 \\
\hline 294 & $10 / 1 / 2016$ & 3850 & 673555008 & 869748 & 3850 & 669131264 & 866184 & 3850 & 666154880 & 876193 \\
\hline 295 & $10 / 2 / 2016$ & 3850 & 674423232 & 868216 & 3850 & 669996032 & 864666 & 3850 & 667029504 & 874644 \\
\hline 296 & $10 / 3 / 2016$ & 3850 & 675289984 & 866692 & 3850 & 670859072 & 863156 & 3850 & 667902592 & 873103 \\
\hline 297 & $10 / 4 / 2016$ & 3850 & 676155136 & 865176 & 3850 & 671720768 & 861654 & 3850 & 668774208 & 871571 \\
\hline 298 & $10 / 5 / 2016$ & 3850 & 677018752 & 863669 & 3850 & 672580864 & 860161 & 3850 & 669644224 & 870047 \\
\hline 299 & $10 / 6 / 2016$ & 3850 & 677880960 & 862169 & 3850 & 673439616 & 858675 & 3850 & 670512768 & 868532 \\
\hline 300 & $10 / 7 / 2016$ & 3850 & 678741632 & 860678 & 3850 & 674296768 & 857198 & 3850 & 671379776 & 867025 \\
\hline 301 & $10 / 8 / 2016$ & 3850 & 679600832 & 859195 & 3850 & 675152512 & 855728 & 3850 & 672245312 & 865525 \\
\hline 302 & $10 / 9 / 2016$ & 3850 & 680458560 & 857720 & 3850 & 676006784 & 854267 & 3850 & 673109312 & 864035 \\
\hline 303 & $10 / 10 / 2016$ & 3850 & 681314816 & 856253 & 3850 & 676859648 & 852813 & 3850 & 673971968 & 862552 \\
\hline 304 & $10 / 11 / 2016$ & 3850 & 682169600 & 854794 & 3850 & 677710976 & 851367 & 3850 & 674832960 & 861077 \\
\hline 305 & $10 / 12 / 2016$ & 3850 & 683022976 & 853343 & 3850 & 678560896 & 849928 & 3850 & 675692608 & 859610 \\
\hline 306 & $10 / 13 / 2016$ & 3850 & 683874816 & 851899 & 3850 & 679409408 & 848497 & 3850 & 676550656 & 858150 \\
\hline 307 & $10 / 14 / 2016$ & 3850 & 684725312 & 850463 & 3850 & 680256448 & 847074 & 3850 & 677407424 & 856699 \\
\hline 308 & $10 / 15 / 2016$ & 3850 & 685574336 & 849034 & 3850 & 681102080 & 845658 & 3850 & 678262656 & 855255 \\
\hline 309 & $10 / 16 / 2016$ & 3850 & 686421952 & 847613 & 3850 & 681946368 & 844249 & 3850 & 679116480 & 853819 \\
\hline 310 & $10 / 17 / 2016$ & 3850 & 687268224 & 846199 & 3850 & 682789248 & 842848 & 3850 & 679968832 & 852390 \\
\hline 311 & $10 / 18 / 2016$ & 3850 & 688112960 & 844793 & 3850 & 683630656 & 841454 & 3850 & 680819776 & 850968 \\
\hline 312 & $10 / 19 / 2016$ & 3850 & 688956352 & 843394 & 3850 & 684470720 & 840067 & 3850 & 681669376 & 849555 \\
\hline 313 & $10 / 20 / 2016$ & 3850 & 689798336 & 842002 & 3850 & 685309440 & 838687 & 3850 & 682517568 & 848148 \\
\hline 314 & $10 / 21 / 2016$ & 3850 & 690638976 & 840618 & 3850 & 686146752 & 837314 & 3850 & 683364288 & 846749 \\
\hline 315 & $10 / 22 / 2016$ & 3850 & 691478208 & 839240 & 3850 & 686982720 & 835949 & 3850 & 684209664 & 845357 \\
\hline 316 & $10 / 23 / 2016$ & 3850 & 692316032 & 837870 & 3850 & 687817280 & 834590 & 3850 & 685053632 & 843972 \\
\hline 317 & $10 / 24 / 2016$ & 3850 & 693152576 & 836507 & 3850 & 688650496 & 833238 & 3850 & 685896320 & 842594 \\
\hline 318 & $10 / 25 / 2016$ & 3850 & 693987712 & 835150 & 3850 & 689482432 & 831893 & 3850 & 686737472 & 841223 \\
\hline 319 & $10 / 26 / 2016$ & 3850 & 694821504 & 833800 & 3850 & 690312960 & 830555 & 3850 & 687577344 & 839859 \\
\hline 320 & $10 / 27 / 2016$ & 3850 & 695653952 & 832457 & 3850 & 691142208 & 829223 & 3850 & 688415808 & 838502 \\
\hline 321 & $10 / 28 / 2016$ & 3850 & 696485120 & 831121 & 3850 & 691970112 & 827898 & 3850 & 689252992 & 837152 \\
\hline 322 & $10 / 29 / 2016$ & 3850 & 697314880 & 829792 & 3850 & 692796736 & 826580 & 3850 & 690088768 & 835808 \\
\hline 323 & $10 / 30 / 2016$ & 3850 & 698143360 & 828469 & 3850 & 693621952 & 825268 & 3850 & 690923264 & 834472 \\
\hline 324 & $10 / 31 / 2016$ & 3850 & 698970496 & 827153 & 3850 & 694445888 & 823963 & 3850 & 691756416 & 833142 \\
\hline 325 & $11 / 1 / 2016$ & 3850 & 699796352 & 825843 & 3850 & 695268544 & 822664 & 3850 & 692588224 & 831818 \\
\hline 326 & $11 / 2 / 2016$ & 3850 & 700620864 & 824540 & 3850 & 696089920 & 821371 & 3850 & 693418688 & 830502 \\
\hline 327 & $11 / 3 / 2016$ & 3850 & 701444160 & 823243 & 3850 & 696910016 & 820085 & 3850 & 694247936 & 829191 \\
\hline 328 & $11 / 4 / 2016$ & 3850 & 702266112 & 821953 & 3850 & 697728832 & 818805 & 3850 & 695075776 & 827887 \\
\hline 329 & $11 / 5 / 2016$ & 3850 & 703086784 & 820669 & 3850 & 698546368 & 817531 & 3850 & 695902400 & 826590 \\
\hline 330 & $11 / 6 / 2016$ & 3850 & 703906176 & 819391 & 3850 & 699362624 & 816264 & 3850 & 696727680 & 825299 \\
\hline 331 & $11 / 7 / 2016$ & 3850 & 704724288 & 818119 & 3850 & 700177600 & 815002 & 3850 & 697551680 & 824014 \\
\hline 332 & $11 / 8 / 2016$ & 3850 & 705541120 & 816853 & 3850 & 700991360 & 813747 & 3850 & 698374400 & 822735 \\
\hline 333 & $11 / 9 / 2016$ & 3850 & 706356736 & 815594 & 3850 & 701803840 & 812497 & 3850 & 699195904 & 821463 \\
\hline 334 & $11 / 10 / 2016$ & 3850 & 707171072 & 814341 & 3850 & 702615104 & 811254 & 3850 & 700016064 & 820196 \\
\hline 335 & $11 / 11 / 2016$ & 3850 & 707984128 & 813093 & 3850 & 703425152 & 810016 & 3850 & 700835008 & 818936 \\
\hline 336 & $11 / 12 / 2016$ & 3850 & 708795968 & 811852 & 3850 & 704233920 & 808785 & 3850 & 701652736 & 817682 \\
\hline 337 & $11 / 13 / 2016$ & 3850 & 709606656 & 810616 & 3850 & 705041472 & 807559 & 3850 & 702469120 & 816434 \\
\hline 338 & $11 / 14 / 2016$ & 3850 & 710416000 & 809386 & 3850 & 705847808 & 806339 & 3850 & 703284352 & 815191 \\
\hline 339 & $11 / 15 / 2016$ & 3850 & 711224192 & 808163 & 3850 & 706652928 & 805124 & 3850 & 704098304 & 813955 \\
\hline 340 & $11 / 16 / 2016$ & 3850 & 712031104 & 806944 & 3850 & 707456832 & 803916 & 3850 & 704911040 & 812724 \\
\hline 341 & $11 / 17 / 2016$ & 3850 & 712836864 & 805732 & 3850 & 708259584 & 802713 & 3850 & 705722496 & 811500 \\
\hline 342 & $11 / 18 / 2016$ & 3850 & 713641344 & 804525 & 3850 & 709061056 & 801516 & 3850 & 706532736 & 810281 \\
\hline 343 & $11 / 19 / 2016$ & 3850 & 714444672 & 803324 & 3850 & 709861376 & 800324 & 3850 & 707341888 & 809067 \\
\hline 344 & $11 / 20 / 2016$ & 3850 & 715246848 & 802129 & 3850 & 710660544 & 799137 & 3850 & 708149696 & 807860 \\
\hline 345 & $11 / 21 / 2016$ & 3850 & 716047744 & 800939 & 3850 & 711458560 & 797956 & 3850 & 708956352 & 806657 \\
\hline 346 & $11 / 22 / 2016$ & 3850 & 716847488 & 799754 & 3850 & 712255296 & 796781 & 3850 & 709761856 & 805461 \\
\hline 347 & $11 / 23 / 2016$ & 3850 & 717646080 & 798575 & 3850 & 713050880 & 795611 & 3850 & 710566080 & 804270 \\
\hline 348 & $11 / 24 / 2016$ & 3850 & 718443456 & 797402 & 3850 & 713845312 & 794446 & 3850 & 711369152 & 803085 \\
\hline 349 & $11 / 25 / 2016$ & 3850 & 719239744 & 796233 & 3850 & 714638656 & 793287 & 3850 & 712171008 & 801905 \\
\hline 350 & $11 / 26 / 2016$ & 3850 & 720034816 & 795070 & 3850 & 715430784 & 792133 & 3850 & 712971840 & 800730 \\
\hline 351 & $11 / 27 / 2016$ & 3850 & 720828608 & 793913 & 3850 & 716221760 & 790984 & 3850 & 713771456 & 799561 \\
\hline 352 & $11 / 28 / 2016$ & 3850 & 721621440 & 792760 & 3850 & 717011648 & 789840 & 3850 & 714569792 & 798397 \\
\hline 353 & $11 / 29 / 2016$ & 3850 & 722413056 & 791613 & 3850 & 717800256 & 788701 & 3850 & 715367040 & 797238 \\
\hline 354 & $11 / 30 / 2016$ & 3850 & 723203520 & 790471 & 3850 & 718587840 & 787568 & 3850 & 716163072 & 796084 \\
\hline 355 & $12 / 1 / 2016$ & 3850 & 723992896 & 789334 & 3850 & 719374272 & 786439 & 3850 & 716958016 & 794936 \\
\hline 356 & $12 / 2 / 2016$ & 3850 & 724781056 & 788202 & 3850 & 720159616 & 785316 & 3850 & 717751808 & 793793 \\
\hline 357 & $12 / 3 / 2016$ & 3850 & 725568128 & 787076 & 3850 & 720943808 & 784197 & 3850 & 718544512 & 792655 \\
\hline 358 & $12 / 4 / 2016$ & 3850 & 726354112 & 785954 & 3850 & 721726912 & 783084 & 3850 & 719336000 & 791522 \\
\hline 359 & $12 / 5 / 2016$ & 3850 & 727138944 & 784837 & 3850 & 722508864 & 781975 & 3850 & 720126400 & 790394 \\
\hline 360 & $12 / 6 / 2016$ & 3850 & 727922752 & 783725 & 3850 & 723289728 & 780872 & 3850 & 720915648 & 789271 \\
\hline
\end{tabular}




\begin{tabular}{|c|c|c|c|c|c|c|c|c|c|c|}
\hline 361 & $12 / 7 / 2016$ & 3850 & 728705280 & 782618 & 3850 & 724069568 & 779773 & 3850 & 721703808 & 788153 \\
\hline 362 & $12 / 8 / 2016$ & 3850 & 729486784 & 781516 & 3850 & 724848256 & 778679 & 3850 & 722490880 & 787040 \\
\hline 363 & $12 / 9 / 2016$ & 3850 & 730267200 & 780419 & 3850 & 725625792 & 777589 & 3850 & 723276800 & 785932 \\
\hline 364 & $12 / 10 / 2016$ & 3850 & 731046528 & 779326 & 3850 & 726402304 & 776505 & 3850 & 724061632 & 784829 \\
\hline 365 & $12 / 11 / 2016$ & 3850 & 731824768 & 778239 & 3850 & 727177728 & 775425 & 3850 & 724845376 & 783730 \\
\hline 366 & $12 / 12 / 2016$ & 3850 & 732601920 & 777156 & 3850 & 727952000 & 774350 & 3850 & 725627968 & 782637 \\
\hline 367 & $12 / 13 / 2016$ & 3850 & 733378048 & 776077 & 3850 & 728725312 & 773279 & 3850 & 726409536 & 781548 \\
\hline 368 & $12 / 14 / 2016$ & 3850 & 734153024 & 775004 & 3850 & 729497536 & 772213 & 3850 & 727190016 & 780464 \\
\hline 369 & $12 / 15 / 2016$ & 3850 & 734926976 & 773935 & 3850 & 730268672 & 771152 & 3850 & 727969408 & 779384 \\
\hline 370 & $12 / 16 / 2016$ & 3850 & 735699840 & 772870 & 3850 & 731038784 & 770095 & 3850 & 728747712 & 778309 \\
\hline 371 & $12 / 17 / 2016$ & 3850 & 736471616 & 771810 & 3850 & 731807808 & 769042 & 3850 & 729524928 & 777239 \\
\hline 372 & $12 / 18 / 2016$ & 3850 & 737242432 & 770755 & 3850 & 732575808 & 767994 & 3850 & 730301120 & 776173 \\
\hline 373 & $12 / 19 / 2016$ & 3850 & 738012096 & 769704 & 3850 & 733342784 & 766951 & 3850 & 731076224 & 775112 \\
\hline 374 & $12 / 20 / 2016$ & 3850 & 738780736 & 768658 & 3850 & 734108672 & 765912 & 3850 & 731850304 & 774056 \\
\hline 375 & $12 / 21 / 2016$ & 2660 & 774362944 & 35582176 & 2660 & 770871680 & 36763004 & 2660 & 780184384 & 48334104 \\
\hline 376 & $12 / 22 / 2016$ & 2660 & 806998144 & 32635222 & 2660 & 803250560 & 32378840 & 2660 & 806933632 & 26749232 \\
\hline 377 & $12 / 23 / 2016$ & 2660 & 831353152 & 24355000 & 2660 & 828692800 & 25442264 & 2660 & 828863872 & 21930340 \\
\hline 378 & $12 / 24 / 2016$ & 2660 & 852405056 & 21051922 & 2660 & 850271936 & 21579168 & 2660 & 847996288 & 19132324 \\
\hline 379 & $12 / 25 / 2016$ & 2660 & 871245440 & 18840366 & 2660 & 869472960 & 19201012 & 2660 & 865377024 & 17380740 \\
\hline 380 & $12 / 26 / 2016$ & 2660 & 888441792 & 17196354 & 2660 & 886935104 & 17462120 & 2660 & 881387520 & 16010493 \\
\hline 381 & $12 / 27 / 2016$ & 2660 & 904343232 & 15901431 & 2660 & 903037952 & 16102846 & 2660 & 896291584 & 14904082 \\
\hline 382 & $12 / 28 / 2016$ & 2660 & 919188608 & 14845389 & 2660 & 918036352 & 14998380 & 2660 & 910278208 & 13986602 \\
\hline 383 & $12 / 29 / 2016$ & 2660 & 933150272 & 13961694 & 2660 & 932112704 & 14076408 & 2660 & 923486912 & 13208693 \\
\hline 384 & $12 / 30 / 2016$ & 2660 & 946356800 & 13206471 & 2660 & 945402816 & 13290092 & 2660 & 936023488 & 12536576 \\
\hline 385 & $12 / 31 / 2016$ & 2660 & 958906368 & 12549580 & 2660 & 958010176 & 12607399 & 2660 & 947970048 & 11946548 \\
\hline 386 & $1 / 1 / 2017$ & 2660 & 970876032 & 11969656 & 2660 & 970015936 & 12005727 & 2660 & 959391616 & 11421572 \\
\hline 387 & $1 / 2 / 2017$ & 2660 & 982327296 & 11451266 & 2660 & 981484736 & 11468783 & 2660 & 970340544 & 10948974 \\
\hline 388 & $1 / 3 / 2017$ & 2660 & 993310080 & 10982782 & 2660 & 992469056 & 10984293 & 2660 & 980860608 & 10520039 \\
\hline 389 & $1 / 4 / 2017$ & 2660 & 1003866240 & 10556199 & 2660 & 1003012800 & 10543799 & 2660 & 990988160 & 10127554 \\
\hline 390 & $1 / 5 / 2017$ & 2660 & 1014031104 & 10164811 & 2660 & 1013153088 & 10140247 & 2660 & 1000754240 & 9766086 \\
\hline 391 & $1 / 6 / 2017$ & 2660 & 1023834624 & 9803550 & 2660 & 1022921344 & 9768301 & 2660 & 1010185664 & 9431428 \\
\hline 392 & $1 / 7 / 2017$ & 2660 & 1033303104 & 9468448 & 2660 & 1032345152 & 9423783 & 2660 & 1019305920 & 9120219 \\
\hline 393 & $1 / 8 / 2017$ & 2660 & 1042459392 & 9156330 & 2660 & 1041448512 & 9103349 & 2660 & 1028135616 & 8829748 \\
\hline 394 & $1 / 9 / 2017$ & 2660 & 1051324032 & 8864613 & 2660 & 1050252800 & 8804285 & 2660 & 1036693440 & 8557791 \\
\hline 395 & $1 / 10 / 2017$ & 2660 & 1059915200 & 8591180 & 2660 & 1058777152 & 8524343 & 2660 & 1044995904 & 8302492 \\
\hline 396 & $1 / 11 / 2017$ & 2660 & 1068249472 & 8334241 & 2660 & 1067038784 & 8261654 & 2660 & 1053058240 & 8062283 \\
\hline 397 & $1 / 12 / 2017$ & 2660 & 1076341760 & 8092283 & 2660 & 1075053440 & 8014618 & 2660 & 1060894016 & 7835828 \\
\hline 398 & $1 / 13 / 2017$ & 2660 & 1084205696 & 7864007 & 2660 & 1082835328 & 7781865 & 2660 & 1068516032 & 7621966 \\
\hline 399 & $1 / 14 / 2017$ & 2660 & 1091853952 & 7648283 & 2660 & 1090397440 & 7562206 & 2660 & 1075935744 & 7419683 \\
\hline 400 & $1 / 15 / 2017$ & 2660 & 1099298176 & 7444122 & 2660 & 1097752064 & 7354599 & 2660 & 1083163776 & 7228086 \\
\hline 401 & $1 / 16 / 2017$ & 2660 & 1106548736 & 7250648 & 2660 & 1104910208 & 7158119 & 2660 & 1090210176 & 7046382 \\
\hline 402 & $1 / 17 / 2017$ & 2660 & 1113615872 & 7067081 & 2660 & 1111882112 & 6971947 & 2660 & 1097084032 & 6873861 \\
\hline 403 & $1 / 18 / 2017$ & 2660 & 1120508544 & 6892721 & 2660 & 1118677504 & 6795344 & 2660 & 1103793920 & 6709882 \\
\hline 404 & $1 / 19 / 2017$ & 2660 & 1127235584 & 6726938 & 2660 & 1125305088 & 6627646 & 2660 & 1110347776 & 6553871 \\
\hline 405 & $1 / 20 / 2017$ & 2660 & 1133804672 & 6569160 & 2660 & 1131773440 & 6468252 & 2660 & 1116753024 & 6405299 \\
\hline 406 & $1 / 21 / 2017$ & 2660 & 1140223488 & 6418864 & 2660 & 1138089856 & 6316461 & 2660 & 1123016576 & 6263534 \\
\hline 407 & $1 / 22 / 2017$ & 2660 & 1146498944 & 6275419 & 2660 & 1144262016 & 6172211 & 2660 & 1129145216 & 6128579 \\
\hline 408 & $1 / 23 / 2017$ & 2660 & 1152637824 & 6138836 & 2660 & 1150296576 & 6034509 & 2660 & 1135144704 & 5999495 \\
\hline 409 & $1 / 24 / 2017$ & 2660 & 1158646016 & 6008171 & 2660 & 1156199808 & 5903283 & 2660 & 1141020928 & 5876259 \\
\hline 410 & $1 / 25 / 2017$ & 2660 & 1164529536 & 5883557 & 2660 & 1161977856 & 5778067 & 2660 & 1146779392 & 5758455 \\
\hline 411 & $1 / 26 / 2017$ & 2660 & 1170293632 & 5764165 & 2660 & 1167636352 & 5658489 & 2660 & 1152425088 & 5645751 \\
\hline 412 & $1 / 27 / 2017$ & 2660 & 1175943680 & 5650004 & 2660 & 1173180672 & 5544211 & 2660 & 1157963008 & 5537848 \\
\hline 413 & $1 / 28 / 2017$ & 2660 & 1181484416 & 5540715 & 2660 & 1178615552 & 5434924 & 2660 & 1163397504 & 5434472 \\
\hline 414 & $1 / 29 / 2017$ & 2660 & 1186920448 & 5436010 & 2660 & 1183945856 & 5330341 & 2660 & 1168732800 & 5335370 \\
\hline 415 & $1 / 30 / 2017$ & 2660 & 1192256000 & 5335633 & 2660 & 1189176064 & 5230199 & 2660 & 1173973120 & 5240306 \\
\hline 416 & $1 / 31 / 2017$ & 2660 & 1197495424 & 5239344 & 2660 & 1194310272 & 5134249 & 2660 & 1179122176 & 5149061 \\
\hline 417 & $2 / 1 / 2017$ & 2660 & 1202642304 & 5146923 & 2660 & 1199352576 & 5042263 & 2660 & 1184183680 & 5061431 \\
\hline 418 & $2 / 2 / 2017$ & 2660 & 1207700480 & 5058164 & 2660 & 1204306560 & 4954026 & 2660 & 1189160832 & 4977224 \\
\hline 419 & $2 / 3 / 2017$ & 2660 & 1212673408 & 4972876 & 2660 & 1209175936 & 4869338 & 2660 & 1194057088 & 4896264 \\
\hline 420 & $2 / 4 / 2017$ & 2660 & 1217564288 & 4890879 & 2660 & 1213963904 & 4788011 & 2660 & 1198875648 & 4818384 \\
\hline 421 & $2 / 5 / 2017$ & 2660 & 1222376192 & 4812004 & 2660 & 1218673792 & 4709870 & 2660 & 1203618944 & 4743426 \\
\hline 422 & $2 / 6 / 2017$ & 2660 & 1227112320 & 4736093 & 2660 & 1223308544 & 4634752 & 2660 & 1208290176 & 4671244 \\
\hline 423 & $2 / 7 / 2017$ & 2660 & 1231775232 & 4662999 & 2660 & 1227871104 & 4562502 & 2660 & 1212891776 & 4601700 \\
\hline 424 & $2 / 8 / 2017$ & 2660 & 1236367872 & 4592584 & 2660 & 1232364032 & 4492975 & 2660 & 1217426560 & 4534665 \\
\hline 425 & $2 / 9 / 2017$ & 2660 & 1240892672 & 4524715 & 2660 & 1236790144 & 4426036 & 2660 & 1221896448 & 4470017 \\
\hline 426 & $2 / 10 / 2017$ & 2660 & 1245351936 & 4459270 & 2660 & 1241151616 & 4361558 & 2660 & 1226304000 & 4407642 \\
\hline 427 & $2 / 11 / 2017$ & 2660 & 1249748096 & 4396135 & 2660 & 1245451008 & 4299420 & 2660 & 1230651520 & 4347433 \\
\hline 428 & $2 / 12 / 2017$ & 2660 & 1254083200 & 4335198 & 2660 & 1249690624 & 4239509 & 2660 & 1234940928 & 4289286 \\
\hline 429 & $2 / 13 / 2017$ & 2660 & 1258359552 & 4276359 & 2660 & 1253872256 & 4181719 & 2660 & 1239174016 & 4233108 \\
\hline 430 & $2 / 14 / 2017$ & 2660 & 1262578944 & 4219519 & 2660 & 1257998208 & 4125950 & 2660 & 1243352832 & 4178806 \\
\hline 431 & $2 / 15 / 2017$ & 2660 & 1266743680 & 4164588 & 2660 & 1262070272 & 4072106 & 2660 & 1247479168 & 4126297 \\
\hline 432 & $2 / 16 / 2017$ & 2660 & 1270855168 & 4111478 & 2660 & 1266090496 & 4020100 & 2660 & 1251554560 & 4075500 \\
\hline 433 & $2 / 17 / 2017$ & 2660 & 1274915328 & 4060109 & 2660 & 1270060288 & 3969845 & 2660 & 1255580928 & 4026338 \\
\hline 434 & $2 / 18 / 2017$ & 2660 & 1278925696 & 4010402 & 2660 & 1273981568 & 3921263 & 2660 & 1259559680 & 3978739 \\
\hline 435 & $2 / 19 / 2017$ & 2660 & 1282887936 & 3962286 & 2660 & 1277855872 & 3874278 & 2660 & 1263492352 & 3932635 \\
\hline 436 & $2 / 20 / 2017$ & 2660 & 1286803712 & 3915689 & 2660 & 1281684608 & 3828819 & 2660 & 1267380224 & 3887961 \\
\hline 437 & $2 / 21 / 2017$ & 2660 & 1290674176 & 3870548 & 2660 & 1285469440 & 3784819 & 2660 & 1271225088 & 3844657 \\
\hline 438 & $2 / 22 / 2017$ & 2660 & 1294501120 & 3826800 & 2660 & 1289211648 & 3742213 & 2660 & 1275027584 & 3802664 \\
\hline 439 & $2 / 23 / 2017$ & 2660 & 1298285440 & 3784386 & 2660 & 1292912640 & 3700941 & 2660 & 1278789504 & 3761927 \\
\hline 440 & $2 / 24 / 2017$ & 2660 & 1302028800 & 3743249 & 2660 & 1296573568 & 3660947 & 2660 & 1282511872 & 3722395 \\
\hline 441 & $2 / 25 / 2017$ & 2660 & 1305731968 & 3703338 & 2660 & 1300195712 & 3622174 & 2660 & 1286195840 & 3684017 \\
\hline 442 & $2 / 26 / 2017$ & 2660 & 1309396608 & 3664601 & 2660 & 1303780352 & 3584573 & 2660 & 1289842688 & 3646746 \\
\hline
\end{tabular}




\begin{tabular}{|c|c|c|c|c|c|c|c|c|c|c|}
\hline 443 & $2 / 27 / 2017$ & 2660 & 1313023616 & 3626990 & 2660 & 1307328384 & 3548093 & 2660 & 1293453184 & 3610538 \\
\hline 444 & $2 / 28 / 2017$ & 2660 & 1316614016 & 3590462 & 2660 & 1310840960 & 3512688 & 2660 & 1297028608 & 3575351 \\
\hline 445 & $3 / 1 / 2017$ & 2660 & 1320168832 & 3554971 & 2660 & 1314319360 & 3478315 & 2660 & 1300569728 & 3541143 \\
\hline 446 & $3 / 2 / 2017$ & 2660 & 1323689472 & 3520476 & 2660 & 1317764352 & 3444931 & 2660 & 1304077568 & 3507876 \\
\hline 447 & $3 / 3 / 2017$ & 2660 & 1327176448 & 3486940 & 2660 & 1321176832 & 3412496 & 2660 & 1307553280 & 3475514 \\
\hline 448 & $3 / 4 / 2017$ & 2660 & 1330630784 & 3454323 & 2660 & 1324557824 & 3380973 & 2660 & 1310997120 & 3444023 \\
\hline 449 & $3 / 5 / 2017$ & 2660 & 1334053376 & 3422592 & 2660 & 1327907968 & 3350324 & 2660 & 1314410496 & 3413367 \\
\hline 450 & $3 / 6 / 2017$ & 2660 & 1337444992 & 3391711 & 2660 & 1331228800 & 3320517 & 2660 & 1317794048 & 3383517 \\
\hline 451 & $3 / 7 / 2017$ & 2660 & 1340806656 & 3361649 & 2660 & 1334520192 & 3291517 & 2660 & 1321148416 & 3354441 \\
\hline 452 & $3 / 8 / 2017$ & 2660 & 1344139136 & 3332375 & 2660 & 1337783424 & 3263294 & 2660 & 1324474624 & 3326112 \\
\hline 453 & $3 / 9 / 2017$ & 2660 & 1347442944 & 3303859 & 2660 & 1341019264 & 3235819 & 2660 & 1327772928 & 3298500 \\
\hline 454 & $3 / 10 / 2017$ & 2660 & 1350719104 & 3276074 & 2660 & 1344228352 & 3209062 & 2660 & 1331044480 & 3271581 \\
\hline 455 & $3 / 11 / 2017$ & 2660 & 1353968000 & 3248993 & 2660 & 1347411328 & 3182996 & 2660 & 1334289920 & 3245330 \\
\hline 456 & $3 / 12 / 2017$ & 2660 & 1357190528 & 3222590 & 2660 & 1350569088 & 3157597 & 2660 & 1337509760 & 3219721 \\
\hline 457 & $3 / 13 / 2017$ & 2660 & 1360387456 & 3196841 & 2660 & 1353701760 & 3132838 & 2660 & 1340704384 & 3194733 \\
\hline 458 & $3 / 14 / 2017$ & 2660 & 1363559168 & 3171722 & 2660 & 1356810496 & 3108698 & 2660 & 1343874816 & 3170343 \\
\hline 459 & $3 / 15 / 2017$ & 2660 & 1366706304 & 3147212 & 2660 & 1359895680 & 3085153 & 2660 & 1347021312 & 3146531 \\
\hline 460 & $3 / 16 / 2017$ & 2660 & 1369829632 & 3123288 & 2660 & 1362957824 & 3062181 & 2660 & 1350144640 & 3123277 \\
\hline 461 & $3 / 17 / 2017$ & 2660 & 1372929536 & 3099931 & 2660 & 1365997568 & 3039763 & 2660 & 1353245184 & 3100560 \\
\hline 462 & $3 / 18 / 2017$ & 2660 & 1376006656 & 3077121 & 2660 & 1369015424 & 3017878 & 2660 & 1356323456 & 3078364 \\
\hline 463 & $3 / 19 / 2017$ & 2660 & 1379061504 & 3054839 & 2660 & 1372011904 & 2996509 & 2660 & 1359380224 & 3056671 \\
\hline 464 & $3 / 20 / 2017$ & 2660 & 1382094592 & 3033067 & 2660 & 1374987648 & 2975636 & 2660 & 1362415488 & 3035463 \\
\hline 465 & $3 / 21 / 2017$ & 2660 & 1385106432 & 3011789 & 2660 & 1377942784 & 2955243 & 2660 & 1365430400 & 3014725 \\
\hline 466 & $3 / 22 / 2017$ & 2660 & 1388097408 & 2990987 & 2660 & 1380878208 & 2935314 & 2660 & 1368424832 & 2994441 \\
\hline 467 & $3 / 23 / 2017$ & 2660 & 1391068032 & 2970646 & 2660 & 1383794048 & 2915832 & 2660 & 1371399424 & 2974596 \\
\hline 468 & $3 / 24 / 2017$ & 2660 & 1394018816 & 2950751 & 2660 & 1386690816 & 2896783 & 2660 & 1374354560 & 2955177 \\
\hline 469 & $3 / 25 / 2017$ & 2660 & 1396950016 & 2931288 & 2660 & 1389568896 & 2878152 & 2660 & 1377290752 & 2936169 \\
\hline 470 & $3 / 26 / 2017$ & 2660 & 1399862272 & 2912242 & 2660 & 1392428800 & 2859925 & 2660 & 1380208256 & 2917560 \\
\hline 471 & $3 / 27 / 2017$ & 2660 & 1402755968 & 2893600 & 2660 & 1395270912 & 2842090 & 2660 & 1383107584 & 2899336 \\
\hline 472 & $3 / 28 / 2017$ & 2660 & 1405631232 & 2875350 & 2660 & 1398095616 & 2824632 & 2660 & 1385989120 & 2881487 \\
\hline 473 & $3 / 29 / 2017$ & 2660 & 1408488704 & 2857478 & 2660 & 1400903168 & 2807541 & 2660 & 1388853120 & 2863999 \\
\hline 474 & $3 / 30 / 2017$ & 2660 & 1411328768 & 2839974 & 2660 & 1403693952 & 2790804 & 2660 & 1391699968 & 2846863 \\
\hline 475 & $3 / 31 / 2017$ & 2660 & 1414151552 & 2822825 & 2660 & 1406468352 & 2774410 & 2660 & 1394530048 & 2830067 \\
\hline 476 & $4 / 1 / 2017$ & 2660 & 1416957696 & 2806022 & 2660 & 1409226752 & 2758349 & 2660 & 1397343616 & 2813602 \\
\hline 477 & $4 / 2 / 2017$ & 2660 & 1419747072 & 2789553 & 2660 & 1411969280 & 2742610 & 2660 & 1400141056 & 2797456 \\
\hline 478 & $4 / 3 / 2017$ & 2660 & 1422520576 & 2773408 & 2660 & 1414696448 & 2727182 & 2660 & 1402922752 & 2781622 \\
\hline 479 & $4 / 4 / 2017$ & 2660 & 1425278080 & 2757578 & 2660 & 1417408512 & 2712057 & 2660 & 14056888832 & 2766088 \\
\hline 480 & $4 / 5 / 2017$ & 2660 & 1428019968 & 2742053 & 2660 & 1420105728 & 2697225 & 2660 & 1408439680 & 2750848 \\
\hline 481 & $4 / 6 / 2017$ & 2660 & 1430747008 & 2726825 & 2660 & 1422788480 & 2682678 & 2660 & 1411175552 & 2735892 \\
\hline 482 & $4 / 7 / 2017$ & 2660 & 1433458816 & 2711884 & 2660 & 1425456896 & 2668406 & 2660 & 1413896832 & 2721212 \\
\hline 483 & $4 / 8 / 2017$ & 2660 & 1436156160 & 2697222 & 2660 & 1428111232 & 2654402 & 2660 & 1416603520 & 2706800 \\
\hline 484 & $4 / 9 / 2017$ & 2660 & 1438838912 & 2682832 & 2660 & 1430751872 & 2640658 & 2660 & 1419296256 & 2692649 \\
\hline 485 & $4 / 10 / 2017$ & 2660 & 1441507584 & 2668705 & 2660 & 1433379072 & 2627166 & 2660 & 1421975040 & 2678751 \\
\hline 486 & $4 / 11 / 2017$ & 2660 & 1444162560 & 2654835 & 2660 & 1435992960 & 2613919 & 2660 & 1424640128 & 2665100 \\
\hline 487 & $4 / 12 / 2017$ & 2660 & 1446803712 & 2641213 & 2660 & 1438593920 & 2600909 & 2660 & 1427291776 & 2651688 \\
\hline 488 & $4 / 13 / 2017$ & 2660 & 1449431552 & 2627833 & 2660 & 1441182080 & 2588131 & 2660 & 1429930240 & 2638509 \\
\hline 489 & $4 / 14 / 2017$ & 2660 & 1452046208 & 2614689 & 2660 & 1443757568 & 2575578 & 2660 & 1432555904 & 2625556 \\
\hline 490 & $4 / 15 / 2017$ & 2660 & 1454647936 & 2601773 & 2660 & 1446320896 & 2563242 & 2660 & 1435168640 & 2612824 \\
\hline 491 & $4 / 16 / 2017$ & 2660 & 1457236992 & 2589080 & 2660 & 1448871936 & 2551119 & 2660 & 1437768960 & 2600306 \\
\hline 492 & $4 / 17 / 2017$ & 2660 & 1459813632 & 2576604 & 2660 & 1451411200 & 2539202 & 2660 & 1440356992 & 2587998 \\
\hline 493 & $4 / 18 / 2017$ & 2660 & 1462377984 & 2564339 & 2660 & 1453938688 & 2527486 & 2660 & 1442932864 & 2575892 \\
\hline 494 & $4 / 19 / 2017$ & 2660 & 1464930304 & 2552278 & 2660 & 1456454656 & 2515965 & 2660 & 1445496832 & 2563985 \\
\hline 495 & $4 / 20 / 2017$ & 2660 & 1467470720 & 2540418 & 2660 & 1458959232 & 2504635 & 2660 & 1448049152 & 2552271 \\
\hline 496 & $4 / 21 / 2017$ & 2660 & 1469999360 & 2528752 & 2660 & 1461452672 & 2493489 & 2660 & 1450589824 & 2540744 \\
\hline 497 & $4 / 22 / 2017$ & 2660 & 1472516736 & 2517275 & 2660 & 1463935232 & 2482523 & 2660 & 1453119232 & 2529401 \\
\hline 498 & $4 / 23 / 2017$ & 2660 & 1475022720 & 2505984 & 2660 & 1466407040 & 2471733 & 2660 & 1455637504 & 2518236 \\
\hline 499 & $4 / 24 / 2017$ & 2660 & 1477517568 & 2494872 & 2660 & 1468868096 & 2461113 & 2660 & 1458144768 & 2507246 \\
\hline 500 & $4 / 25 / 2017$ & 2660 & 1480001536 & 2483935 & 2660 & 1471318784 & 2450660 & 2660 & 1460641152 & 2496425 \\
\hline 501 & $4 / 26 / 2017$ & 2660 & 1482474624 & 2473170 & 2660 & 1473759104 & 2440369 & 2660 & 1463126912 & 2485769 \\
\hline 502 & $4 / 27 / 2017$ & 2660 & 1484937216 & 2462571 & 2660 & 1476189312 & 2430236 & 2660 & 1465602176 & 2475276 \\
\hline 503 & $4 / 28 / 2017$ & 2660 & 1487389312 & 2452135 & 2660 & 1478609664 & 2420257 & 2660 & 1468067200 & 2464939 \\
\hline 504 & $4 / 29 / 2017$ & 2660 & 1489831168 & 2441857 & 2660 & 1481020032 & 2410428 & 2660 & 1470521856 & 2454756 \\
\hline 505 & $4 / 30 / 2017$ & 2660 & 1492262912 & 2431734 & 2660 & 1483420800 & 2400746 & 2660 & 1472966656 & 2444724 \\
\hline 506 & $5 / 1 / 2017$ & 2660 & 1494684672 & 2421762 & 2660 & 1485811968 & 2391207 & 2660 & 1475401472 & 2434838 \\
\hline 507 & $5 / 2 / 2017$ & 2660 & 1497096704 & 2411938 & 2660 & 1488193792 & 2381806 & 2660 & 1477826560 & 2425095 \\
\hline 508 & $5 / 3 / 2017$ & 2660 & 1499498880 & 2402257 & 2660 & 1490566400 & 2372542 & 2660 & 1480242048 & 2415491 \\
\hline 509 & $5 / 4 / 2017$ & 2660 & 1501891584 & 2392716 & 2660 & 1492929792 & 2363411 & 2660 & 1482648064 & 2406024 \\
\hline 510 & $5 / 5 / 2017$ & 2660 & 1504274944 & 2383312 & 2660 & 1495284096 & 2354409 & 2660 & 1485044736 & 2396690 \\
\hline 511 & $5 / 6 / 2017$ & 2660 & 1506648960 & 2374043 & 2660 & 1497629696 & 2345534 & 2660 & 1487432192 & 2387486 \\
\hline 512 & $5 / 7 / 2017$ & 2660 & 1509013888 & 2364905 & 2660 & 1499966464 & 2336782 & 2660 & 1489810688 & 2378410 \\
\hline 513 & $5 / 8 / 2017$ & 2660 & 1511369728 & 2355894 & 2660 & 1502294656 & 2328151 & 2660 & 1492180096 & 2369458 \\
\hline 514 & $5 / 9 / 2017$ & 2660 & 1513716736 & 2347008 & 2660 & 1504614272 & 2319638 & 2660 & 1494540800 & 2360628 \\
\hline 515 & $5 / 10 / 2017$ & 2660 & 1516054912 & 2338244 & 2660 & 1506925568 & 2311240 & 2660 & 1496892672 & 2351916 \\
\hline 516 & $5 / 11 / 2017$ & 2660 & 1518384640 & 2329599 & 2660 & 1509228416 & 2302955 & 2660 & 1499235968 & 2343320 \\
\hline 517 & $5 / 12 / 2017$ & 2660 & 1520705664 & 2321071 & 2660 & 1511523200 & 2294779 & 2660 & 1501570816 & 2334839 \\
\hline 518 & $5 / 13 / 2017$ & 2660 & 1523018368 & 2312657 & 2660 & 1513809920 & 2286712 & 2660 & 1503897344 & 2326468 \\
\hline 519 & $5 / 14 / 2017$ & 2660 & 1525322752 & 2304355 & 2660 & 1516088704 & 2278749 & 2660 & 1506215552 & 2318207 \\
\hline 520 & $5 / 15 / 2017$ & 2660 & 1527618944 & 2296162 & 2660 & 1518359552 & 2270889 & 2660 & 1508525568 & 2310051 \\
\hline 521 & $5 / 16 / 2017$ & 2660 & 1529906944 & 2288075 & 2660 & 1520622720 & 2263130 & 2660 & 1510827520 & 2302000 \\
\hline 522 & $5 / 17 / 2017$ & 2660 & 1532187008 & 2280093 & 2660 & 1522878208 & 2255470 & 2660 & 1513121536 & 2294051 \\
\hline 523 & $5 / 18 / 2017$ & 2660 & 1534459264 & 2272214 & 2660 & 1525126144 & 2247906 & 2660 & 1515407744 & 2286202 \\
\hline 524 & $5 / 19 / 2017$ & 2660 & 1536723712 & 2264434 & 2660 & 1527366528 & 2240436 & 2660 & 1517686272 & 2278451 \\
\hline
\end{tabular}




\begin{tabular}{|c|c|c|c|c|c|c|c|c|c|c|}
\hline 525 & $5 / 20 / 2017$ & 2660 & 1538980608 & 2256752 & 2660 & 1529599616 & 2233058 & 2660 & 1519956992 & 2270795 \\
\hline 526 & $5 / 21 / 2017$ & 2660 & 1541229568 & 2249166 & 2660 & 1531825408 & 2225771 & 2660 & 1522220288 & 2263234 \\
\hline 527 & $5 / 22 / 2017$ & 2660 & 1543471360 & 2241674 & 2660 & 1534043776 & 2218572 & 2660 & 1524475904 & 2255764 \\
\hline 528 & $5 / 23 / 2017$ & 2660 & 1545705600 & 2234274 & 2660 & 1536255360 & 2211459 & 2660 & 1526724480 & 2248384 \\
\hline 529 & $5 / 24 / 2017$ & 2660 & 1547932544 & 2226964 & 2660 & 1538459776 & 2204432 & 2660 & 1528965504 & 2241092 \\
\hline 530 & $5 / 25 / 2017$ & 2660 & 1550152320 & 2219742 & 2660 & 1540657280 & 2197487 & 2660 & 1531199360 & 2233887 \\
\hline 531 & $5 / 26 / 2017$ & 2660 & 1552364928 & 2212607 & 2660 & 1542848128 & 2190624 & 2660 & 1533426176 & 2226766 \\
\hline 532 & $5 / 27 / 2017$ & 2660 & 1554570368 & 2205556 & 2660 & 1545031808 & 2183841 & 2660 & 1535645952 & 2219729 \\
\hline 533 & $5 / 28 / 2017$ & 2660 & 1556769024 & 2198589 & 2660 & 1547208960 & 2177136 & 2660 & 1537858688 & 2212773 \\
\hline 534 & $5 / 29 / 2017$ & 2660 & 1558960768 & 2191703 & 2660 & 1549379456 & 2170508 & 2660 & 1540064512 & 2205897 \\
\hline 535 & $5 / 30 / 2017$ & 2660 & 1561145600 & 2184896 & 2660 & 1551543424 & 2163955 & 2660 & 1542263680 & 2199099 \\
\hline 536 & $5 / 31 / 2017$ & 2660 & 1563323776 & 2178168 & 2660 & 1553700864 & 2157475 & 2660 & 1544456064 & 2192378 \\
\hline 537 & $6 / 1 / 2017$ & 2660 & 1565495424 & 2171517 & 2660 & 1555851904 & 2151068 & 2660 & 1546641792 & 2185732 \\
\hline 538 & $6 / 2 / 2017$ & 2660 & 1567660288 & 2164941 & 2660 & 1557996672 & 2144731 & 2660 & 1548820992 & 2179160 \\
\hline 539 & $6 / 3 / 2017$ & 2660 & 1569818624 & 2158438 & 2660 & 1560135168 & 2138464 & 2660 & 1550993664 & 2172661 \\
\hline 540 & $6 / 4 / 2017$ & 2660 & 1571970688 & 2152008 & 2660 & 1562267520 & 2132266 & 2660 & 1553159808 & 2166232 \\
\hline 541 & $6 / 5 / 2017$ & 2660 & 1574116352 & 2145649 & 2660 & 1564393344 & 2126134 & 2660 & 1555319680 & 2159874 \\
\hline 542 & $6 / 6 / 2017$ & 2660 & 1576255744 & 2139360 & 2660 & 1566513536 & 2120068 & 2660 & 1557473280 & 2153584 \\
\hline 543 & $6 / 7 / 2017$ & 2660 & 1578388864 & 2133139 & 2660 & 1568627712 & 2114066 & 2660 & 1559620608 & 2147362 \\
\hline 544 & $6 / 8 / 2017$ & 2660 & 1580515840 & 2126986 & 2660 & 1570735744 & 2108127 & 2660 & 1561761792 & 2141205 \\
\hline 545 & $6 / 9 / 2017$ & 2660 & 1582636672 & 2120898 & 2660 & 1572838016 & 2102251 & 2660 & 1563896960 & 2135114 \\
\hline 546 & $6 / 10 / 2017$ & 2660 & 1584751616 & 2114875 & 2660 & 1574934528 & 2096435 & 2660 & 1566026112 & 2129086 \\
\hline 547 & $6 / 11 / 2017$ & 2660 & 1586860544 & 2108915 & 2660 & 1577025152 & 2090680 & 2660 & 1568149248 & 2123121 \\
\hline 548 & $6 / 12 / 2017$ & 2660 & 1588963712 & 2103018 & 2660 & 1579110144 & 2084983 & 2660 & 1570266368 & 2117217 \\
\hline 549 & $6 / 13 / 2017$ & 2660 & 1591060736 & 2097182 & 2660 & 1581189504 & 2079344 & 2660 & 1572377728 & 2111374 \\
\hline 550 & $6 / 14 / 2017$ & 2660 & 1593152128 & 2091407 & 2660 & 1583263360 & 2073761 & 2660 & 1574483328 & 2105590 \\
\hline 551 & $6 / 15 / 2017$ & 2660 & 1595237760 & 2085690 & 2660 & 1585331456 & 2068234 & 2660 & 1576583168 & 2099864 \\
\hline 552 & $6 / 16 / 2017$ & 2660 & 1597317888 & 2080032 & 2660 & 1587394176 & 2062762 & 2660 & 1578677376 & 2094196 \\
\hline 553 & $6 / 17 / 2017$ & 2660 & 1599392256 & 2074430 & 2660 & 1589451520 & 2057344 & 2660 & 1580765952 & 2088584 \\
\hline 554 & $6 / 18 / 2017$ & 2660 & 1601461120 & 2068885 & 2660 & 1591503616 & 2051979 & 2660 & 1582849024 & 2083027 \\
\hline 555 & $6 / 19 / 2017$ & 2660 & 1603524608 & 2063395 & 2660 & 1593550208 & 2046666 & 2660 & 1584926592 & 2077525 \\
\hline 556 & $6 / 20 / 2017$ & 2660 & 1605582464 & 2057959 & 2660 & 1595591680 & 2041404 & 2660 & 1586998656 & 2072076 \\
\hline 557 & $6 / 21 / 2017$ & 2660 & 1607635072 & 2052576 & 2660 & 1597627776 & 2036192 & 2660 & 1589065344 & 2066680 \\
\hline 558 & $6 / 22 / 2017$ & 2660 & 1609682304 & 2047245 & 2660 & 1599658880 & 2031029 & 2660 & 1591126656 & 2061336 \\
\hline 559 & $6 / 23 / 2017$ & 2660 & 1611724288 & 2041966 & 2660 & 1601684736 & 2025915 & 2660 & 1593182720 & 2056042 \\
\hline 560 & $6 / 24 / 2017$ & 2660 & 1613761024 & 2036738 & 2660 & 1603705600 & 2020849 & 2660 & 1595233536 & 2050799 \\
\hline 561 & $6 / 25 / 2017$ & 2660 & 1615792640 & 2031559 & 2660 & 1605721472 & 2015830 & 2660 & 1597279104 & 2045605 \\
\hline 562 & $6 / 26 / 2017$ & 2660 & 1617819008 & 2026430 & 2660 & 1607732352 & 2010857 & 2660 & 1599319552 & 2040459 \\
\hline 563 & $6 / 27 / 2017$ & 2660 & 1619840384 & 2021348 & 2660 & 1609738240 & 2005929 & 2660 & 1601354880 & 2035361 \\
\hline 564 & $6 / 28 / 2017$ & 2660 & 1621856640 & 2016314 & 2660 & 1611739264 & 2001047 & 2660 & 1603385216 & 2030310 \\
\hline 565 & $6 / 29 / 2017$ & 2660 & 1623867904 & 2011327 & 2660 & 1613735424 & 1996208 & 2660 & 1605410560 & 2025305 \\
\hline 566 & $6 / 30 / 2017$ & 2660 & 1625874432 & 2006385 & 2660 & 1615726848 & 1991412 & 2660 & 1607430912 & 2020345 \\
\hline 567 & $7 / 1 / 2017$ & 2660 & 1627875840 & 2001488 & 2660 & 1617713664 & 1986660 & 2660 & 1609446272 & 2015430 \\
\hline 568 & $7 / 2 / 2017$ & 2660 & 1629872384 & 1996636 & 2660 & 1619695488 & 1981949 & 2660 & 1611456896 & 2010559 \\
\hline 569 & $7 / 3 / 2017$ & 2660 & 1631864320 & 1991828 & 2660 & 1621672832 & 1977279 & 2660 & 1613462528 & 2005732 \\
\hline 570 & $7 / 4 / 2017$ & 2660 & 1633851392 & 1987062 & 2660 & 1623645440 & 1972651 & 2660 & 1615463552 & 2000947 \\
\hline 571 & $7 / 5 / 2017$ & 2660 & 1635833728 & 1982339 & 2660 & 1625613440 & 1968062 & 2660 & 1617459712 & 1996204 \\
\hline 572 & $7 / 6 / 2017$ & 2660 & 1637811456 & 1977658 & 2660 & 1627576960 & 1963513 & 2660 & 1619451264 & 1991502 \\
\hline 573 & $7 / 7 / 2017$ & 2660 & 1639784448 & 1973018 & 2660 & 1629536000 & 1959002 & 2660 & 1621438080 & 1986841 \\
\hline 574 & $7 / 8 / 2017$ & 2660 & 1641752832 & 1968418 & 2660 & 1631490560 & 1954530 & 2660 & 1623420288 & 1982221 \\
\hline 575 & $7 / 9 / 2017$ & 2660 & 1643716736 & 1963858 & 2660 & 1633440640 & 1950096 & 2660 & 1625397888 & 1977639 \\
\hline 576 & $7 / 10 / 2017$ & 2660 & 1645676032 & 1959337 & 2660 & 1635386368 & 1945699 & 2660 & 1627371008 & 1973097 \\
\hline 577 & $7 / 11 / 2017$ & 2660 & 1647630720 & 1954855 & 2660 & 1637327616 & 1941338 & 2660 & 1629339648 & 1968593 \\
\hline 578 & $7 / 12 / 2017$ & 2660 & 1649581312 & 1950410 & 2660 & 1639264640 & 1937013 & 2660 & 1631303808 & 1964127 \\
\hline 579 & $7 / 13 / 2017$ & 2660 & 1651527296 & 1946004 & 2660 & 1641197440 & 1932724 & 2660 & 1633263488 & 1959698 \\
\hline 580 & $7 / 14 / 2017$ & 2660 & 1653468800 & 1941634 & 2660 & 1643125888 & 1928470 & 2660 & 1635218816 & 1955306 \\
\hline 581 & $7 / 15 / 2017$ & 2660 & 1655406208 & 1937300 & 2660 & 1645050112 & 1924250 & 2660 & 1637169664 & 1950950 \\
\hline 582 & $7 / 16 / 2017$ & 2660 & 1657339264 & 1933003 & 2660 & 1646970240 & 1920065 & 2660 & 1639116288 & 1946630 \\
\hline 583 & $7 / 17 / 2017$ & 2660 & 1659267968 & 1928740 & 2660 & 1648886144 & 1915913 & 2660 & 1641058688 & 1942344 \\
\hline 584 & $7 / 18 / 2017$ & 2660 & 1661192448 & 1924513 & 2660 & 1650797952 & 1911794 & 2660 & 1642996736 & 1938094 \\
\hline 585 & $7 / 19 / 2017$ & 2660 & 1663112832 & 1920320 & 2660 & 1652705664 & 1907707 & 2660 & 1644930560 & 1933877 \\
\hline 586 & $7 / 20 / 2017$ & 2660 & 1665028992 & 1916160 & 2660 & 1654609280 & 1903653 & 2660 & 1646860416 & 1929694 \\
\hline 587 & $7 / 21 / 2017$ & 2660 & 1666941184 & 1912034 & 2660 & 1656508928 & 1899630 & 2660 & 1648785792 & 1925545 \\
\hline 588 & $7 / 22 / 2017$ & 2660 & 1668849024 & 1907941 & 2660 & 1658404480 & 1895639 & 2660 & 1650707456 & 1921428 \\
\hline 589 & $7 / 23 / 2017$ & 2660 & 1670752640 & 1903880 & 2660 & 1660296192 & 1891678 & 2660 & 1652624640 & 1917343 \\
\hline 590 & $7 / 24 / 2017$ & 2660 & 1672652672 & 1899851 & 2660 & 1662183936 & 1887748 & 2660 & 1654537984 & 1913291 \\
\hline 591 & $7 / 25 / 2017$ & 2660 & 1674548480 & 1895854 & 2660 & 1664067840 & 1883847 & 2660 & 1656447360 & 1909269 \\
\hline 592 & $7 / 26 / 2017$ & 2660 & 1676440448 & 1891888 & 2660 & 1665947776 & 1879976 & 2660 & 1658352512 & 1905279 \\
\hline 593 & $7 / 27 / 2017$ & 2660 & 1678328320 & 1887952 & 2660 & 1667823872 & 1876135 & 2660 & 1660253824 & 1901319 \\
\hline 594 & $7 / 28 / 2017$ & 2660 & 1680212480 & 1884047 & 2660 & 1669696256 & 1872322 & 2660 & 1662151168 & 1897389 \\
\hline 595 & $7 / 29 / 2017$ & 2660 & 1682092544 & 1880172 & 2660 & 1671564800 & 1868538 & 2660 & 1664044672 & 1893489 \\
\hline 596 & $7 / 30 / 2017$ & 2660 & 1683968896 & 1876326 & 2660 & 1673429504 & 1864781 & 2660 & 1665934336 & 1889618 \\
\hline 597 & $7 / 31 / 2017$ & 2660 & 1685841408 & 1872509 & 2660 & 1675290624 & 1861052 & 2660 & 1667820032 & 1885777 \\
\hline 598 & $8 / 1 / 2017$ & 2660 & 1687710208 & 1868720 & 2660 & 1677147904 & 1857351 & 2660 & 1669702016 & 1881964 \\
\hline 599 & $8 / 2 / 2017$ & 2660 & 1689575168 & 1864960 & 2660 & 1679001600 & 1853676 & 2660 & 1671580288 & 1878179 \\
\hline 600 & $8 / 3 / 2017$ & 2660 & 1691436160 & 1861228 & 2660 & 1680851712 & 1850028 & 2660 & 1673454720 & 1874421 \\
\hline 601 & $8 / 4 / 2017$ & 2660 & 1693293824 & 1857523 & 2660 & 1682698112 & 1846406 & 2660 & 1675325184 & 1870692 \\
\hline 602 & $8 / 5 / 2017$ & 2660 & 1695147648 & 1853845 & 2660 & 1684540928 & 1842811 & 2660 & 1677192320 & 1866989 \\
\hline 603 & $8 / 6 / 2017$ & 2660 & 1696997888 & 1850194 & 2660 & 1686380160 & 1839240 & 2660 & 1679055616 & 1863314 \\
\hline 604 & $8 / 7 / 2017$ & 2660 & 1698844416 & 1846570 & 2660 & 1688215808 & 1835695 & 2660 & 1680915328 & 1859664 \\
\hline 605 & $8 / 8 / 2017$ & 2660 & 1700687488 & 1842972 & 2660 & 1690047872 & 1832175 & 2660 & 1682771328 & 1856041 \\
\hline 606 & $8 / 9 / 2017$ & 2660 & 1702526848 & 1839400 & 2660 & 1691876608 & 1828680 & 2660 & 1684623744 & 1852443 \\
\hline
\end{tabular}




\begin{tabular}{|c|c|c|c|c|c|c|c|c|c|c|}
\hline 607 & $8 / 10 / 2017$ & 2660 & 1704362752 & 1835853 & 2660 & 1693701888 & 1825208 & 2660 & 1686472704 & 1848871 \\
\hline 608 & $8 / 11 / 2017$ & 2660 & 1706195072 & 1832331 & 2660 & 1695523584 & 1821761 & 2660 & 1688317952 & 1845324 \\
\hline 609 & $8 / 12 / 2017$ & 2660 & 1708023808 & 1828834 & 2660 & 1697341952 & 1818338 & 2660 & 1690159872 & 1841802 \\
\hline 610 & $8 / 13 / 2017$ & 2660 & 1709849088 & 1825361 & 2660 & 1699156864 & 1814938 & 2660 & 1691998208 & 1838305 \\
\hline 611 & $8 / 14 / 2017$ & 2660 & 1711671040 & 1821913 & 2660 & 1700968448 & 1811561 & 2660 & 1693832960 & 1834831 \\
\hline 612 & $8 / 15 / 2017$ & 2660 & 1713489664 & 1818488 & 2660 & 1702776704 & 1808207 & 2660 & 1695664256 & 1831382 \\
\hline 613 & $8 / 16 / 2017$ & 2660 & 1715304704 & 1815088 & 2660 & 1704581504 & 1804875 & 2660 & 1697492224 & 1827956 \\
\hline 614 & $8 / 17 / 2017$ & 2660 & 1717116544 & 1811710 & 2660 & 1706383104 & 1801566 & 2660 & 1699316864 & 1824553 \\
\hline 615 & $8 / 18 / 2017$ & 2660 & 1718924672 & 1808356 & 2660 & 1708181248 & 1798279 & 2660 & 1701138048 & 1821174 \\
\hline 616 & $8 / 19 / 2017$ & 2660 & 1720729856 & 1805024 & 2660 & 1709976448 & 1795014 & 2660 & 1702955776 & 1817817 \\
\hline 617 & $8 / 20 / 2017$ & 2660 & 1722531456 & 1801715 & 2660 & 1711768192 & 1791770 & 2660 & 1704770304 & 1814483 \\
\hline 618 & $8 / 21 / 2017$ & 2660 & 1724329984 & 1798428 & 2660 & 1713556736 & 1788548 & 2660 & 1706581504 & 1811170 \\
\hline 619 & $8 / 22 / 2017$ & 2660 & 1726125056 & 1795163 & 2660 & 1715342080 & 1785346 & 2660 & 1708389376 & 1807880 \\
\hline 620 & $8 / 23 / 2017$ & 2660 & 1727917056 & 1791919 & 2660 & 1717124224 & 1782165 & 2660 & 1710193920 & 1804612 \\
\hline 621 & $8 / 24 / 2017$ & 2660 & 1729705728 & 1788697 & 2660 & 1718903296 & 1779005 & 2660 & 1711995264 & 1801365 \\
\hline 622 & $8 / 25 / 2017$ & 2660 & 1731491200 & 1785496 & 2660 & 1720679168 & 1775865 & 2660 & 1713793408 & 1798139 \\
\hline 623 & $8 / 26 / 2017$ & 2660 & 1733273472 & 1782316 & 2660 & 1722451840 & 1772745 & 2660 & 1715588352 & 1794934 \\
\hline 624 & $8 / 27 / 2017$ & 2660 & 1735052672 & 1779157 & 2660 & 1724221568 & 1769645 & 2660 & 1717380096 & 1791750 \\
\hline 625 & $8 / 28 / 2017$ & 2660 & 1736828672 & 1776018 & 2660 & 1725988096 & 1766565 & 2660 & 1719168768 & 1788587 \\
\hline 626 & $8 / 29 / 2017$ & 2660 & 1738601600 & 1772900 & 2660 & 1727751552 & 1763504 & 2660 & 1720954240 & 1785443 \\
\hline 627 & $8 / 30 / 2017$ & 2660 & 1740371456 & 1769801 & 2660 & 1729512064 & 1760462 & 2660 & 1722736512 & 1782320 \\
\hline 628 & $8 / 31 / 2017$ & 2660 & 1742138112 & 1766722 & 2660 & 1731269504 & 1757439 & 2660 & 1724515712 & 1779217 \\
\hline 629 & 9/1/2017 & 2660 & 1743901824 & 1763663 & 2660 & 1733023872 & 1754435 & 2660 & 1726291840 & 1776133 \\
\hline 630 & $9 / 2 / 2017$ & 2660 & 1745662464 & 1760623 & 2660 & 1734775168 & 1751450 & 2660 & 1728064896 & 1773068 \\
\hline 631 & $9 / 3 / 2017$ & 2660 & 1747420032 & 1757602 & 2660 & 1736523776 & 1748483 & 2660 & 1729834880 & 1770022 \\
\hline 632 & 9/4/2017 & 2660 & 1749174656 & 1754600 & 2660 & 1738269312 & 1745534 & 2660 & 1731601792 & 1766996 \\
\hline 633 & 9/5/2017 & 2660 & 1750926208 & 1751616 & 2660 & 1740012032 & 1742602 & 2660 & 1733365888 & 1763988 \\
\hline 634 & 9/6/2017 & 2660 & 1752674944 & 1748651 & 2660 & 1741751680 & 1739689 & 2660 & 1735126912 & 1760998 \\
\hline 635 & 9/7/2017 & 2660 & 1754420608 & 1745704 & 2660 & 1743488512 & 1736793 & 2660 & 1736884864 & 1758027 \\
\hline 636 & 9/8/2017 & 2660 & 1756163328 & 1742775 & 2660 & 1745222400 & 1733915 & 2660 & 1738640128 & 1755074 \\
\hline 637 & 9/9/2017 & 2660 & 1757903232 & 1739864 & 2660 & 1746953472 & 1731053 & 2660 & 1740392192 & 1752139 \\
\hline 638 & $9 / 10 / 2017$ & 2660 & 1759640192 & 1736971 & 2660 & 1748681600 & 1728209 & 2660 & 1742141440 & 1749222 \\
\hline 639 & $9 / 11 / 2017$ & 2660 & 1761374336 & 1734095 & 2660 & 1750407040 & 1725382 & 2660 & 1743887744 & 1746322 \\
\hline 640 & $9 / 12 / 2017$ & 2660 & 1763105536 & 1731237 & 2660 & 1752129536 & 1722571 & 2660 & 1745631104 & 1743439 \\
\hline 641 & $9 / 13 / 2017$ & 2660 & 1764833920 & 1728395 & 2660 & 1753849344 & 1719776 & 2660 & 1747371776 & 1740574 \\
\hline 642 & $9 / 14 / 2017$ & 2660 & 1766559616 & 1725571 & 2660 & 1755566336 & 1716998 & 2660 & 1749109504 & 1737726 \\
\hline 643 & $9 / 15 / 2017$ & 2660 & 1768282240 & 1722763 & 2660 & 1757280640 & 1714236 & 2660 & 1750844288 & 1734894 \\
\hline 644 & $9 / 16 / 2017$ & 2660 & 1770002176 & 1719971 & 2660 & 1758992128 & 1711490 & 2660 & 1752576384 & 1732079 \\
\hline 645 & $9 / 17 / 2017$ & 2660 & 1771719424 & 1717197 & 2660 & 1760700800 & 1708760 & 2660 & 1754305664 & 1729281 \\
\hline 646 & $9 / 18 / 2017$ & 2660 & 1773433856 & 1714438 & 2660 & 1762407040 & 1706045 & 2660 & 1756032256 & 1726498 \\
\hline 647 & $9 / 19 / 2017$ & 2660 & 1775145600 & 1711695 & 2660 & 1764110336 & 1703346 & 2660 & 1757755904 & 1723732 \\
\hline 648 & $9 / 20 / 2017$ & 2660 & 1776854528 & 1708968 & 2660 & 1765810944 & 1700662 & 2660 & 1759476864 & 1720982 \\
\hline 649 & $9 / 21 / 2017$ & 2660 & 1778560768 & 1706257 & 2660 & 1767508864 & 1697994 & 2660 & 1761195136 & 1718247 \\
\hline 650 & $9 / 22 / 2017$ & 2660 & 1780264320 & 1703561 & 2660 & 1769204224 & 1695340 & 2660 & 1762910720 & 1715528 \\
\hline 651 & $9 / 23 / 2017$ & 2660 & 1781965184 & 1700881 & 2660 & 1770896896 & 1692701 & 2660 & 1764623488 & 1712825 \\
\hline 652 & $9 / 24 / 2017$ & 1196 & 1824334976 & 42369656 & 1196 & 1814824832 & 43927836 & 1196 & 1827917824 & 63294348 \\
\hline 653 & $9 / 25 / 2017$ & 1196 & 1862870144 & 38535284 & 1196 & 1854324352 & 39499560 & 1196 & 1858807040 & 30889202 \\
\hline 654 & $9 / 26 / 2017$ & 1196 & 1891658880 & 28788796 & 1196 & 1883940608 & 29616340 & 1196 & 1884089472 & 25282374 \\
\hline 655 & $9 / 27 / 2017$ & 1196 & 1916359296 & 24700354 & 1196 & 1909252480 & 25311806 & 1196 & 1906375680 & 22286302 \\
\hline 656 & $9 / 28 / 2017$ & 1196 & 1938794240 & 22434982 & 1196 & 1932044288 & 22791798 & 1196 & 1926884352 & 20508666 \\
\hline 657 & $9 / 29 / 2017$ & 1196 & 1959496192 & 20701956 & 1196 & 1953056512 & 21012154 & 1196 & 1945981312 & 19097090 \\
\hline 658 & $9 / 30 / 2017$ & 1196 & 1978817408 & 19321148 & 1196 & 1972641024 & 19584572 & 1196 & 1963926272 & 17944726 \\
\hline 659 & $10 / 1 / 2017$ & 1196 & 1997002368 & 18184942 & 1196 & 1991051264 & 18410258 & 1196 & 1980907136 & 16980876 \\
\hline 660 & $10 / 2 / 2017$ & 1196 & 2014230784 & 17228470 & 1196 & 2008472832 & 17421522 & 1196 & 1997066112 & 16159070 \\
\hline 661 & $10 / 3 / 2017$ & 1196 & 2030639360 & 16408594 & 1196 & 2025046656 & 16573884 & 1196 & 2012513280 & 15447053 \\
\hline 662 & $10 / 4 / 2017$ & 1196 & 2046334464 & 15695076 & 1196 & 2040882816 & 15836162 & 1196 & 2027334912 & 14821652 \\
\hline 663 & $10 / 5 / 2017$ & 1196 & 2061400448 & 15065991 & 1196 & 2056068608 & 15185764 & 1196 & 2041600640 & 14265786 \\
\hline 664 & $10 / 6 / 2017$ & 1196 & 2075905536 & 14505065 & 1196 & 2070674432 & 14605917 & 1196 & 2055367296 & 13766623 \\
\hline 665 & $10 / 7 / 2017$ & 1196 & 2089905536 & 13999995 & 1196 & 2084758400 & 14083940 & 1196 & 2068681600 & 13314376 \\
\hline 666 & $10 / 8 / 2017$ & 1196 & 2103446912 & 13541341 & 1196 & 2098368000 & 13609609 & 1196 & 2081582720 & 12901028 \\
\hline 667 & $10 / 9 / 2017$ & 1196 & 2116568192 & 13121310 & 1196 & 2111544320 & 13176303 & 1196 & 2094104192 & 12521484 \\
\hline 668 & $10 / 10 / 2017$ & 1196 & 2129303168 & 12734985 & 1196 & 2124321792 & 12777626 & 1196 & 2106274816 & 12170651 \\
\hline 669 & $10 / 11 / 2017$ & 1196 & 2141680512 & 12377372 & 1196 & 2136730752 & 12408723 & 1196 & 2118119424 & 11844658 \\
\hline 670 & $10 / 12 / 2017$ & 1196 & 2153725184 & 12044677 & 1196 & 2148796416 & 12065715 & 1196 & 2129659904 & 11540397 \\
\hline 671 & $10 / 13 / 2017$ & 1196 & 2165458944 & 11733832 & 1196 & 2160541952 & 11745446 & 1196 & 2140915200 & 11255321 \\
\hline 672 & $10 / 14 / 2017$ & 1196 & 2176901376 & 11442324 & 1196 & 2171987200 & 11445308 & 1196 & 2151902464 & 10987319 \\
\hline 673 & $10 / 15 / 2017$ & 1196 & 2188069376 & 11168062 & 1196 & 2183150336 & 11163134 & 1196 & 2162637056 & 10734624 \\
\hline 674 & $10 / 16 / 2017$ & 1196 & 2198978816 & 10909289 & 1196 & 2194047488 & 10897097 & 1196 & 2173132800 & 10495736 \\
\hline 675 & $10 / 17 / 2017$ & 1196 & 2209643264 & 10664513 & 1196 & 2204692992 & 10645648 & 1196 & 2183402240 & 10269381 \\
\hline 676 & $10 / 18 / 2017$ & 1196 & 2220075776 & 10432456 & 1196 & 2215100416 & 10407453 & 1196 & 2193456640 & 10054452 \\
\hline 677 & $10 / 19 / 2017$ & 1196 & 2230287616 & 10212017 & 1196 & 2225281792 & 10181366 & 1196 & 2203306752 & 9849996 \\
\hline 678 & $10 / 20 / 2017$ & 1196 & 2240290048 & 10002238 & 1196 & 2235248384 & 9966386 & 1196 & 2212961792 & 9655176 \\
\hline 679 & $10 / 21 / 2017$ & 1196 & 2250092288 & 9802277 & 1196 & 2245009920 & 9761636 & 1196 & 2222431232 & 9469258 \\
\hline 680 & $10 / 22 / 2017$ & 1196 & 2259703552 & 9611396 & 1196 & 2254576128 & 9566344 & 1196 & 2231722752 & 9291591 \\
\hline 681 & $10 / 23 / 2017$ & 1196 & 2269132544 & 9428935 & 1196 & 2263955968 & 9379822 & 1196 & 2240844288 & 9121594 \\
\hline 682 & $10 / 24 / 2017$ & 1196 & 2278386944 & 9254313 & 1196 & 2273157632 & 9201456 & 1196 & 2249803008 & 8958747 \\
\hline 683 & $10 / 25 / 2017$ & 1196 & 2287473920 & 9087000 & 1196 & 2282188288 & 9030699 & 1196 & 2258605568 & 8802583 \\
\hline 684 & $10 / 26 / 2017$ & 1196 & 2296400384 & 8926525 & 1196 & 2291055360 & 8867052 & 1196 & 2267258368 & 8652678 \\
\hline 685 & $10 / 27 / 2017$ & 1196 & 2305172736 & 8772457 & 1196 & 2299765248 & 8710066 & 1196 & 2275767040 & 8508648 \\
\hline 686 & $10 / 28 / 2017$ & 1196 & 2313797376 & 8624407 & 1196 & 2308324608 & 8559333 & 1196 & 2284137216 & 8370145 \\
\hline 687 & $10 / 29 / 2017$ & 1196 & 2322279168 & 8482019 & 1196 & 2316739072 & 8414480 & 1196 & 2292374016 & 8236845 \\
\hline 688 & $10 / 30 / 2017$ & 1196 & 2330624256 & 8344968 & 1196 & 2325014272 & 8275166 & 1196 & 2300482304 & 8108455 \\
\hline
\end{tabular}




\begin{tabular}{|c|c|c|c|c|c|c|c|c|c|c|}
\hline 689 & $10 / 31 / 2017$ & 1196 & 2338837248 & 8212954 & 1196 & 2333155328 & 8141077 & 1196 & 2308466944 & 7984417 \\
\hline 690 & $11 / 1 / 2017$ & 1196 & 2346923008 & 8085700 & 1196 & 2341167104 & 8011643 & 1196 & 2316332032 & 7865304 \\
\hline 691 & $11 / 2 / 2017$ & 1196 & 2354885632 & 7962670 & 1196 & 2349054464 & 7887404 & 1196 & 2324081920 & 7749878 \\
\hline 692 & $11 / 3 / 2017$ & 1196 & 2362729984 & 7844440 & 1196 & 2356821504 & 7767123 & 1196 & 2331720960 & 7638874 \\
\hline 693 & $11 / 4 / 2017$ & 1196 & 2370459904 & 7729795 & 1196 & 2364472832 & 7651223 & 1196 & 2339251968 & 7531155 \\
\hline 694 & $11 / 5 / 2017$ & 1196 & 2378079232 & 7619470 & 1196 & 2372012544 & 7539628 & 1196 & 2346679296 & 7427169 \\
\hline 695 & $11 / 6 / 2017$ & 1196 & 2385591552 & 7512351 & 1196 & 2379443712 & 7431334 & 1196 & 2354005760 & 7326612 \\
\hline 696 & $11 / 7 / 2017$ & 1196 & 2393000448 & 7408885 & 1196 & 2386770688 & 7326797 & 1196 & 2361235200 & 7229295 \\
\hline 697 & $11 / 8 / 2017$ & 1196 & 2400309248 & 7308779 & 1196 & 2393996288 & 7225721 & 1196 & 2368370176 & 7135057 \\
\hline 698 & $11 / 9 / 2017$ & 1196 & 2407521024 & 7211849 & 1196 & 2401124096 & 7127919 & 1196 & 2375414016 & 7043751 \\
\hline 699 & $11 / 10 / 2017$ & 1196 & 2414639104 & 7117942 & 1196 & 2408157440 & 7033232 & 1196 & 2382369280 & 6955244 \\
\hline 700 & $11 / 11 / 2017$ & 1196 & 2421666048 & 7026916 & 1196 & 2415098880 & 6941513 & 1196 & 2389238528 & 6869410 \\
\hline 701 & $11 / 12 / 2017$ & 1196 & 2428604672 & 6938643 & 1196 & 2421951488 & 6852628 & 1196 & 2396024832 & 6786132 \\
\hline 702 & $11 / 13 / 2017$ & 1196 & 2435457536 & 6853002 & 1196 & 2428718080 & 6766450 & 1196 & 2402729984 & 6705299 \\
\hline 703 & $11 / 14 / 2017$ & 1196 & 2442227712 & 6769879 & 1196 & 2435400960 & 6682862 & 1196 & 2409356800 & 6626808 \\
\hline 704 & $11 / 15 / 2017$ & 1196 & 2448916736 & 6689167 & 1196 & 2442002688 & 6601752 & 1196 & 2415907328 & 6550560 \\
\hline 705 & $11 / 16 / 2017$ & 1196 & 2455527424 & 6610766 & 1196 & 2448525568 & 6523014 & 1196 & 2422383872 & 6476463 \\
\hline 706 & $11 / 17 / 2017$ & 1196 & 2462062080 & 6534581 & 1196 & 2454972160 & 6446551 & 1196 & 2428788224 & 6404428 \\
\hline 707 & $11 / 18 / 2017$ & 1196 & 2468522496 & 6460520 & 1196 & 2461344512 & 6372266 & 1196 & 2435122688 & 6334372 \\
\hline 708 & $11 / 19 / 2017$ & 1196 & 2474910976 & 6388500 & 1196 & 2467644672 & 6300073 & 1196 & 2441388800 & 6266217 \\
\hline 709 & $11 / 20 / 2017$ & 1196 & 2481229568 & 6318439 & 1196 & 2473874432 & 6229886 & 1196 & 2447588864 & 6199889 \\
\hline 710 & $11 / 21 / 2017$ & 1196 & 2487479808 & 6250261 & 1196 & 2480036096 & 6161626 & 1196 & 2453724160 & 6135316 \\
\hline 711 & $11 / 22 / 2017$ & 1196 & 2493663744 & 6183892 & 1196 & 2486130944 & 6095218 & 1196 & 2459796480 & 6072432 \\
\hline 712 & $11 / 23 / 2017$ & 1196 & 2499782912 & 6119265 & 1196 & 2492161792 & 6030590 & 1196 & 2465807616 & 6011173 \\
\hline 713 & $11 / 24 / 2017$ & 1196 & 2505839104 & 6056313 & 1196 & 2498129408 & 5967673 & 1196 & 2471759104 & 5951477 \\
\hline 714 & $11 / 25 / 2017$ & 1196 & 2511834112 & 5994973 & 1196 & 2504035840 & 5906403 & 1196 & 2477652480 & 5893288 \\
\hline 715 & $11 / 26 / 2017$ & 1196 & 2517769216 & 5935187 & 1196 & 2509882624 & 5846718 & 1196 & 2483489024 & 5836550 \\
\hline 716 & $11 / 27 / 2017$ & 1196 & 2523646208 & 5876898 & 1196 & 2515671296 & 5788560 & 1196 & 2489270272 & 5781211 \\
\hline 717 & $11 / 28 / 2017$ & 1196 & 2529466368 & 5820053 & 1196 & 2521403136 & 5731872 & 1196 & 2494997504 & 5727222 \\
\hline 718 & $11 / 29 / 2017$ & 1196 & 2535230976 & 5764599 & 1196 & 2527079680 & 5676603 & 1196 & 2500672000 & 5674534 \\
\hline 719 & $11 / 30 / 2017$ & 1196 & 2540941312 & 5710487 & 1196 & 2532702464 & 5622701 & 1196 & 2506295296 & 5623102 \\
\hline 720 & $12 / 1 / 2017$ & 1196 & 2546599168 & 5657672 & 1196 & 2538272512 & 5570116 & 1196 & 2511867904 & 5572884 \\
\hline 721 & $12 / 2 / 2017$ & 1196 & 2552205312 & 5606108 & 1196 & 2543791360 & 5518805 & 1196 & 2517391872 & 5523837 \\
\hline 722 & $12 / 3 / 2017$ & 1196 & 2557761024 & 5555753 & 1196 & 2549260032 & 5468722 & 1196 & 2522867712 & 5475922 \\
\hline 723 & $12 / 4 / 2017$ & 1196 & 2563267584 & 5506566 & 1196 & 2554680064 & 5419825 & 1196 & 2528296704 & 5429101 \\
\hline 724 & $12 / 5 / 2017$ & 1196 & 2568726016 & 5458509 & 1196 & 2560051968 & 5372075 & 1196 & 2533680128 & 5383339 \\
\hline 725 & $12 / 6 / 2017$ & 1196 & 2574137600 & 5411543 & 1196 & 2565377280 & 5325432 & 1196 & 2539018752 & 5338600 \\
\hline 726 & $12 / 7 / 2017$ & 1196 & 2579503104 & 5365633 & 1196 & 2570657024 & 5279860 & 1196 & 2544313600 & 5294851 \\
\hline 727 & $12 / 8 / 2017$ & 1196 & 2584824064 & 5320745 & 1196 & 2575892480 & 5235324 & 1196 & 2549565696 & 5252061 \\
\hline 728 & $12 / 9 / 2017$ & 1196 & 2590100736 & 5276847 & 1196 & 2581084416 & 5191790 & 1196 & 2554775808 & 5210199 \\
\hline 729 & $12 / 10 / 2017$ & 1196 & 2595334656 & 5233907 & 1196 & 2586233856 & 5149225 & 1196 & 2559944960 & 5169237 \\
\hline 730 & $12 / 11 / 2017$ & 1196 & 2600526592 & 5191894 & 1196 & 2591341056 & 5107600 & 1196 & 2565073920 & 5129145 \\
\hline 731 & $12 / 12 / 2017$ & 1196 & 2605677312 & 5150780 & 1196 & 2596408064 & 5066883 & 1196 & 2570164224 & 5089898 \\
\hline 732 & $12 / 13 / 2017$ & 1196 & 2610787840 & 5110539 & 1196 & 2601435136 & 5027047 & 1196 & 2575215616 & 5051469 \\
\hline 733 & $12 / 14 / 2017$ & 1196 & 2615858944 & 5071140 & 1196 & 2606423040 & 4988064 & 1196 & 2580229376 & 5013834 \\
\hline 734 & $12 / 15 / 2017$ & 1196 & 2620891648 & 5032562 & 1196 & 2611373056 & 4949908 & 1196 & 2585206272 & 4976969 \\
\hline 735 & $12 / 16 / 2017$ & 1196 & 2625886464 & 4994778 & 1196 & 2616285696 & 4912554 & 1196 & 2590147328 & 4940851 \\
\hline 736 & $12 / 17 / 2017$ & 1196 & 2630844160 & 4957765 & 1196 & 2621161472 & 4875978 & 1196 & 2595052800 & 4905459 \\
\hline 737 & $12 / 18 / 2017$ & 1196 & 2635765760 & 4921500 & 1196 & 2626001664 & 4840156 & 1196 & 2599923456 & 4870769 \\
\hline 738 & $12 / 19 / 2017$ & 1196 & 2640651264 & 4885962 & 1196 & 2630806784 & 4805066 & 1196 & 2604760320 & 4836764 \\
\hline 739 & $12 / 20 / 2017$ & 1196 & 2645502720 & 4851130 & 1196 & 2635577600 & 4770686 & 1196 & 2609563648 & 4803423 \\
\hline 740 & $12 / 21 / 2017$ & 1196 & 2650319616 & 4816982 & 1196 & 2640314368 & 4736996 & 1196 & 2614334208 & 4770727 \\
\hline 741 & $12 / 22 / 2017$ & 1196 & 2655103232 & 4783501 & 1196 & 2645018368 & 4703976 & 1196 & 2619073024 & 4738659 \\
\hline 742 & $12 / 23 / 2017$ & 1196 & 2659853824 & 4750666 & 1196 & 2649690112 & 4671607 & 1196 & 2623780352 & 4707199 \\
\hline 743 & $12 / 24 / 2017$ & 1196 & 2664572416 & 4718461 & 1196 & 2654329856 & 4639870 & 1196 & 2628456448 & 4676332 \\
\hline 744 & $12 / 25 / 2017$ & 1196 & 2669259264 & 4686867 & 1196 & 2658938624 & 4608747 & 1196 & 2633102592 & 4646042 \\
\hline 745 & $12 / 26 / 2017$ & 1196 & 2673915136 & 4655868 & 1196 & 2663516928 & 4578222 & 1196 & 2637719040 & 4616313 \\
\hline 746 & $12 / 27 / 2017$ & 1196 & 2678540544 & 4625448 & 1196 & 2668065280 & 4548277 & 1196 & 2642306048 & 4587130 \\
\hline 747 & $12 / 28 / 2017$ & 1196 & 2683136000 & 4595591 & 1196 & 2672584192 & 4518898 & 1196 & 2646864384 & 4558477 \\
\hline 748 & $12 / 29 / 2017$ & 1196 & 2687702784 & 4566281 & 1196 & 2677074176 & 4490067 & 1196 & 2651395072 & 4530341 \\
\hline 749 & $12 / 30 / 2017$ & 1196 & 2692239872 & 4537505 & 1196 & 2681536000 & 4461771 & 1196 & 2655897600 & 4502709 \\
\hline 750 & $12 / 31 / 2017$ & 1196 & 2696749056 & 4509248 & 1196 & 2685969920 & 4433995 & 1196 & 2660373248 & 4475567 \\
\hline 751 & $1 / 1 / 2018$ & 1196 & 2701230848 & 4481497 & 1196 & 2690376704 & 4406726 & 1196 & 2664822272 & 4448903 \\
\hline 752 & $1 / 2 / 2018$ & 1196 & 2705684992 & 4454238 & 1196 & 2694756608 & 4379950 & 1196 & 2669244672 & 4422704 \\
\hline 753 & $1 / 3 / 2018$ & 1196 & 2710112256 & 4427459 & 1196 & 2699110144 & 4353654 & 1196 & 2673641728 & 4396958 \\
\hline 754 & $1 / 4 / 2018$ & 1196 & 2714513152 & 4401148 & 1196 & 2703438080 & 4327825 & 1196 & 2678013440 & 4371654 \\
\hline 755 & $1 / 5 / 2018$ & 1196 & 2718888704 & 4375293 & 1196 & 2707740416 & 4302452 & 1196 & 2682360064 & 4346781 \\
\hline 756 & $1 / 6 / 2018$ & 1196 & 2723238656 & 4349882 & 1196 & 2712017920 & 4277523 & 1196 & 2686682368 & 4322328 \\
\hline 757 & $1 / 7 / 2018$ & 1196 & 2727563520 & 4324904 & 1196 & 2716271104 & 4253027 & 1196 & 2690981120 & 4298284 \\
\hline 758 & $1 / 8 / 2018$ & 1196 & 2731863808 & 4300349 & 1196 & 2720500224 & 4228952 & 1196 & 2695255296 & 4274641 \\
\hline 759 & $1 / 9 / 2018$ & 1196 & 2736140032 & 4276206 & 1196 & 2724705280 & 4205288 & 1196 & 2699506688 & 4251387 \\
\hline 760 & $1 / 10 / 2018$ & 1196 & 2740392704 & 4252464 & 1196 & 2728887296 & 4182026 & 1196 & 2703735296 & 4228513 \\
\hline 761 & $1 / 11 / 2018$ & 1196 & 2744621824 & 4229115 & 1196 & 2733046528 & 4159154 & 1196 & 2707941376 & 4206011 \\
\hline 762 & $1 / 12 / 2018$ & 1196 & 2748827904 & 4206149 & 1196 & 2737183232 & 4136664 & 1196 & 2712125184 & 4183872 \\
\hline 763 & $1 / 13 / 2018$ & 1196 & 2753011456 & 4183556 & 1196 & 2741297664 & 4114547 & 1196 & 2716287232 & 4162085 \\
\hline 764 & $1 / 14 / 2018$ & 1196 & 2757172736 & 4161329 & 1196 & 2745390848 & 4092792 & 1196 & 2720428032 & 4140646 \\
\hline 765 & $1 / 15 / 2018$ & 1196 & 2761312256 & 4139457 & 1196 & 2749461760 & 4071392 & 1196 & 2724547328 & 4119543 \\
\hline 766 & $1 / 16 / 2018$ & 1196 & 2765430016 & 4117933 & 1196 & 2753512192 & 4050339 & 1196 & 2728646144 & 4098770 \\
\hline 767 & $1 / 17 / 2018$ & 1196 & 2769526784 & 4096749 & 1196 & 2757541888 & 4029623 & 1196 & 2732724224 & 4078319 \\
\hline 768 & $1 / 18 / 2018$ & 1196 & 2773602816 & 4075897 & 1196 & 2761551104 & 4009237 & 1196 & 2736783104 & 4058182 \\
\hline 769 & $1 / 19 / 2018$ & 1196 & 2777658112 & 4055369 & 1196 & 2765540352 & 3989173 & 1196 & 2740820992 & 4038354 \\
\hline 770 & $1 / 20 / 2018$ & 1196 & 2781693184 & 4035158 & 1196 & 2769509632 & 3969424 & 1196 & 2744839936 & 4018825 \\
\hline
\end{tabular}




\begin{tabular}{|c|c|c|c|c|c|c|c|c|c|c|}
\hline 771 & $1 / 21 / 2018$ & 1196 & 2785708544 & 4015256 & 1196 & 2773459712 & 3949983 & 1196 & 2748839424 & 3999591 \\
\hline 772 & $1 / 22 / 2018$ & 1196 & 2789704192 & 3995658 & 1196 & 2777390592 & 3930842 & 1196 & 2752819968 & 3980644 \\
\hline 773 & $1 / 23 / 2018$ & 1196 & 2793680640 & 3976356 & 1196 & 2781302528 & 3911995 & 1196 & 2756782080 & 3961978 \\
\hline 774 & $1 / 24 / 2018$ & 1196 & 2797637888 & 3957343 & 1196 & 2785196032 & 3893435 & 1196 & 2760725760 & 3943587 \\
\hline 775 & $1 / 25 / 2018$ & 1196 & 2801576448 & 3938613 & 1196 & 2789071104 & 3875156 & 1196 & 2764651264 & 3925465 \\
\hline 776 & $1 / 26 / 2018$ & 1196 & 2805496576 & 3920159 & 1196 & 2792928256 & 3857151 & 1196 & 2768558848 & 3907606 \\
\hline 777 & $1 / 27 / 2018$ & 1196 & 2809398784 & 3901977 & 1196 & 2796767744 & 3839414 & 1196 & 2772448768 & 3890004 \\
\hline 778 & $1 / 28 / 2018$ & 1196 & 2813282816 & 3884060 & 1196 & 2800589568 & 3821940 & 1196 & 2776321536 & 3872654 \\
\hline 779 & $1 / 29 / 2018$ & 1196 & 2817149184 & 3866402 & 1196 & 2804394240 & 3804722 & 1196 & 2780176896 & 3855550 \\
\hline 780 & $1 / 30 / 2018$ & 1196 & 2820997888 & 3848997 & 1196 & 2808182016 & 3787756 & 1196 & 2784015616 & 3838688 \\
\hline 781 & $1 / 31 / 2018$ & 1196 & 2824829952 & 3831841 & 1196 & 2811953152 & 3771035 & 1196 & 2787837952 & 3822061 \\
\hline 782 & $2 / 1 / 2018$ & 1196 & 2828644864 & 3814928 & 1196 & 2815707648 & 3754555 & 1196 & 2791643392 & 3805666 \\
\hline 783 & $2 / 2 / 2018$ & 1196 & 2832443136 & 3798254 & 1196 & 2819446272 & 3738310 & 1196 & 2795432960 & 3789498 \\
\hline 784 & $2 / 3 / 2018$ & 1196 & 2836225024 & 3781812 & 1196 & 2823168256 & 3722296 & 1196 & 2799206400 & 3773551 \\
\hline 785 & $2 / 4 / 2018$ & 1196 & 2839990528 & 3765598 & 1196 & 2826874880 & 3706507 & 1196 & 2802963968 & 3757822 \\
\hline 786 & $2 / 5 / 2018$ & 1196 & 2843740160 & 3749608 & 1196 & 2830565632 & 3690938 & 1196 & 2806706688 & 3742305 \\
\hline 787 & $2 / 6 / 2018$ & 1196 & 2847473920 & 3733837 & 1196 & 2834241280 & 3675586 & 1196 & 2810433536 & 3726997 \\
\hline 788 & $2 / 7 / 2018$ & 1196 & 2851192320 & 3718280 & 1196 & 2837901824 & 3660446 & 1196 & 2814145536 & 3711893 \\
\hline 789 & $2 / 8 / 2018$ & 1196 & 2854895104 & 3702934 & 1196 & 2841547264 & 3645512 & 1196 & 2817842176 & 3696989 \\
\hline 790 & $2 / 9 / 2018$ & 1196 & 2858583040 & 3687793 & 1196 & 2845178112 & 3630782 & 1196 & 2821524736 & 3682282 \\
\hline 791 & $2 / 10 / 2018$ & 1196 & 2862255872 & 3672855 & 1196 & 2848794368 & 3616251 & 1196 & 2825192448 & 3667767 \\
\hline 792 & $2 / 11 / 2018$ & 1196 & 2865913856 & 3658114 & 1196 & 2852396544 & 3601915 & 1196 & 2828845824 & 3653440 \\
\hline 793 & $2 / 12 / 2018$ & 1196 & 2869557504 & 3643567 & 1196 & 2855983872 & 3587770 & 1196 & 2832485120 & 3639299 \\
\hline 794 & $2 / 13 / 2018$ & 1196 & 2873186816 & 3629210 & 1196 & 2859557888 & 3573812 & 1196 & 2836110592 & 3625338 \\
\hline 795 & $2 / 14 / 2018$ & 1196 & 2876801792 & 3615040 & 1196 & 2863118080 & 3560038 & 1196 & 2839722240 & 3611556 \\
\hline 796 & $2 / 15 / 2018$ & 1196 & 2880402944 & 3601052 & 1196 & 2866664192 & 3546443 & 1196 & 2843320064 & 3597948 \\
\hline 797 & $2 / 16 / 2018$ & 1196 & 2883990016 & 3587243 & 1196 & 2870197248 & 3533025 & 1196 & 2846904576 & 3584511 \\
\hline 798 & $2 / 17 / 2018$ & 1196 & 2887563776 & 3573610 & 1196 & 2873716992 & 3519780 & 1196 & 2850475776 & 3571242 \\
\hline 799 & $2 / 18 / 2018$ & 1196 & 2891123968 & 3560150 & 1196 & 2877223680 & 3506704 & 1196 & 2854033920 & 3558137 \\
\hline 800 & $2 / 19 / 2018$ & 1196 & 2894670592 & 3546859 & 1196 & 2880717568 & 3493795 & 1196 & 2857579264 & 3545194 \\
\hline 801 & $2 / 20 / 2018$ & 1196 & 2898204416 & 3533734 & 1196 & 2884198656 & 3481048 & 1196 & 2861111552 & 3532409 \\
\hline 802 & $2 / 21 / 2018$ & 1196 & 2901725184 & 3520771 & 1196 & 2887666944 & 3468462 & 1196 & 2864631296 & 3519780 \\
\hline 803 & $2 / 22 / 2018$ & 1196 & 2905233152 & 3507969 & 1196 & 2891123456 & 3456033 & 1196 & 2868138752 & 3507304 \\
\hline 804 & $2 / 23 / 2018$ & 1196 & 2908728576 & 3495323 & 1196 & 2894566912 & 3443757 & 1196 & 2871633664 & 3494977 \\
\hline 805 & $2 / 24 / 2018$ & 1196 & 2912211456 & 3482831 & 1196 & 2897998592 & 3431633 & 1196 & 2875116544 & 3482798 \\
\hline 806 & $2 / 25 / 2018$ & 1196 & 2915681792 & 3470490 & 1196 & 2901418240 & 3419657 & 1196 & 2878587136 & 3470763 \\
\hline 807 & $2 / 26 / 2018$ & 1196 & 2919140096 & 3458298 & 1196 & 2904826112 & 3407826 & 1196 & 2882045952 & 3458870 \\
\hline 808 & $2 / 27 / 2018$ & 1196 & 2922586368 & 3446251 & 1196 & 2908222208 & 3396139 & 1196 & 2885493248 & 3447117 \\
\hline 809 & $2 / 28 / 2018$ & 1196 & 2926020608 & 3434347 & 1196 & 2911606784 & 3384591 & 1196 & 2888928768 & 3435500 \\
\hline 810 & $3 / 1 / 2018$ & 1196 & 2929443328 & 3422584 & 1196 & 2914979840 & 3373180 & 1196 & 2892352768 & 3424018 \\
\hline 811 & $3 / 2 / 2018$ & 1196 & 2932854272 & 3410958 & 1196 & 2918341888 & 3361905 & 1196 & 2895765504 & 3412667 \\
\hline 812 & $3 / 3 / 2018$ & 1196 & 2936253696 & 3399468 & 1196 & 2921692672 & 3350763 & 1196 & 2899166720 & 3401446 \\
\hline 813 & $3 / 4 / 2018$ & 1196 & 2939641856 & 3388111 & 1196 & 2925032192 & 3339751 & 1196 & 2902557184 & 3390353 \\
\hline 814 & $3 / 5 / 2018$ & 1196 & 2943018752 & 3376884 & 1196 & 2928361216 & 3328867 & 1196 & 2905936640 & 3379384 \\
\hline 815 & $3 / 6 / 2018$ & 1196 & 2946384384 & 3365786 & 1196 & 2931679232 & 3318108 & 1196 & 2909305088 & 3368539 \\
\hline 816 & $3 / 7 / 2018$ & 1196 & 2949739264 & 3354815 & 1196 & 2934986752 & 3307473 & 1196 & 2912662784 & 3357815 \\
\hline 817 & $3 / 8 / 2018$ & 1196 & 2953083392 & 3343967 & 1196 & 2938283776 & 3296959 & 1196 & 2916010240 & 3347209 \\
\hline 818 & $3 / 9 / 2018$ & 1196 & 2956416512 & 3333241 & 1196 & 2941570304 & 3286565 & 1196 & 2919346944 & 3336721 \\
\hline 819 & $3 / 10 / 2018$ & 1196 & 2959739136 & 3322634 & 1196 & 2944846592 & 3276287 & 1196 & 2922673152 & 3326347 \\
\hline 820 & $3 / 11 / 2018$ & 1196 & 2963051520 & 3312146 & 1196 & 2948112640 & 3266124 & 1196 & 2925989376 & 3316086 \\
\hline 821 & $3 / 12 / 2018$ & 1196 & 2966353152 & 3301773 & 1196 & 2951368704 & 3256075 & 1196 & 2929295104 & 3305935 \\
\hline 822 & $3 / 13 / 2018$ & 1196 & 2969644544 & 3291514 & 1196 & 2954614784 & 3246136 & 1196 & 2932591104 & 3295894 \\
\hline 823 & $3 / 14 / 2018$ & 1196 & 2972925952 & 3281366 & 1196 & 2957851136 & 3236306 & 1196 & 2935877120 & 3285960 \\
\hline 824 & $3 / 15 / 2018$ & 1196 & 2976197376 & 3271329 & 1196 & 2961077760 & 3226584 & 1196 & 2939153152 & 3276132 \\
\hline 825 & $3 / 16 / 2018$ & 1196 & 2979458816 & 3261399 & 1196 & 2964294656 & 3216967 & 1196 & 2942419712 & 3266408 \\
\hline 826 & $3 / 17 / 2018$ & 1196 & 2982710272 & 3251576 & 1196 & 2967502336 & 3207454 & 1196 & 2945676288 & 3256785 \\
\hline 827 & $3 / 18 / 2018$ & 1196 & 2985952000 & 3241857 & 1196 & 2970700288 & 3198043 & 1196 & 2948923648 & 3247263 \\
\hline 828 & $3 / 19 / 2018$ & 1196 & 2989184256 & 3232241 & 1196 & 2973889024 & 3188732 & 1196 & 2952161536 & 3237840 \\
\hline 829 & $3 / 20 / 2018$ & 1196 & 2992407040 & 3222727 & 1196 & 2977068544 & 3179520 & 1196 & 2955389696 & 3228514 \\
\hline 830 & $3 / 21 / 2018$ & 1196 & 2995620352 & 3213311 & 1196 & 2980238848 & 3170405 & 1196 & 2958609152 & 3219283 \\
\hline 831 & $3 / 22 / 2018$ & 1196 & 2998824192 & 3203994 & 1196 & 2983400192 & 3161385 & 1196 & 2961819392 & 3210146 \\
\hline 832 & $3 / 23 / 2018$ & 1196 & 3002019072 & 3194773 & 1196 & 2986552832 & 3152459 & 1196 & 2965020672 & 3201103 \\
\hline 833 & $3 / 24 / 2018$ & 1196 & 3005204736 & 3185646 & 1196 & 2989696256 & 3143625 & 1196 & 2968212736 & 3192150 \\
\hline 834 & $3 / 25 / 2018$ & 1196 & 3008381184 & 3176613 & 1196 & 2992831232 & 3134881 & 1196 & 2971395840 & 3183286 \\
\hline 835 & $3 / 26 / 2018$ & 1196 & 3011549184 & 3167672 & 1196 & 2995957504 & 3126228 & 1196 & 2974570496 & 3174511 \\
\hline 836 & $3 / 27 / 2018$ & 1196 & 3014707968 & 3158820 & 1196 & 2999075072 & 3117661 & 1196 & 2977736192 & 3165823 \\
\hline 837 & $3 / 28 / 2018$ & 1196 & 3017858048 & 3150058 & 1196 & 3002184448 & 3109182 & 1196 & 2980893440 & 3157221 \\
\hline 838 & $3 / 29 / 2018$ & 1196 & 3020999424 & 3141383 & 1196 & 3005285120 & 3100787 & 1196 & 2984042240 & 3148702 \\
\hline 839 & $3 / 30 / 2018$ & 1196 & 3024132096 & 3132795 & 1196 & 3008377600 & 3092477 & 1196 & 2987182592 & 3140267 \\
\hline 840 & $3 / 31 / 2018$ & 1196 & 3027256320 & 3124290 & 1196 & 3011461888 & 3084248 & 1196 & 2990314496 & 3131913 \\
\hline 841 & $4 / 1 / 2018$ & 1196 & 3030372352 & 3115870 & 1196 & 3014537984 & 3076101 & 1196 & 2993437952 & 3123639 \\
\hline 842 & $4 / 2 / 2018$ & 1196 & 3033479936 & 3107531 & 1196 & 3017605888 & 3068033 & 1196 & 2996553472 & 3115445 \\
\hline 843 & $4 / 3 / 2018$ & 1196 & 3036579072 & 3099274 & 1196 & 3020666112 & 3060044 & 1196 & 2999660800 & 3107328 \\
\hline 844 & $4 / 4 / 2018$ & 1196 & 3039670272 & 3091096 & 1196 & 3023718144 & 3052133 & 1196 & 3002759936 & 3099288 \\
\hline 845 & $4 / 5 / 2018$ & 1196 & 3042753280 & 3082997 & 1196 & 3026762496 & 3044298 & 1196 & 3005851392 & 3091324 \\
\hline 846 & $4 / 6 / 2018$ & 1196 & 3045828096 & 3074974 & 1196 & 3029798912 & 3036537 & 1196 & 3008935168 & 3083435 \\
\hline 847 & $4 / 7 / 2018$ & 1196 & 3048895232 & 3067028 & 1196 & 3032827904 & 3028851 & 1196 & 3012010496 & 3075618 \\
\hline 848 & $4 / 8 / 2018$ & 1196 & 3051954432 & 3059157 & 1196 & 3035848960 & 3021237 & 1196 & 3015078400 & 3067874 \\
\hline 849 & $4 / 9 / 2018$ & 1196 & 3055005696 & 3051360 & 1196 & 3038862848 & 3013695 & 1196 & 3018138624 & 3060202 \\
\hline 850 & $4 / 10 / 2018$ & 1196 & 3058049280 & 3043635 & 1196 & 3041869056 & 3006223 & 1196 & 3021191168 & 3052599 \\
\hline 851 & $4 / 11 / 2018$ & 1196 & 3061085440 & 3035982 & 1196 & 3044867840 & 2998821 & 1196 & 3024236288 & 3045065 \\
\hline 852 & $4 / 12 / 2018$ & 1196 & 3064113664 & 3028400 & 1196 & 3047859200 & 2991488 & 1196 & 3027273728 & 3037600 \\
\hline
\end{tabular}




\begin{tabular}{|c|c|c|c|c|c|c|c|c|c|c|}
\hline 853 & $4 / 13 / 2018$ & 1196 & 3067134720 & 3020887 & 1196 & 3050843648 & 2984222 & 1196 & 3030304000 & 3030202 \\
\hline 854 & 4/14/2018 & 1196 & 3070148096 & 3013443 & 1196 & 3053820672 & 2977022 & 1196 & 3033326848 & 3022870 \\
\hline 855 & $4 / 15 / 2018$ & 1196 & 3073154048 & 3006066 & 1196 & 3056790528 & 2969887 & 1196 & 3036342528 & 3015603 \\
\hline 856 & $4 / 16 / 2018$ & 1196 & 3076152576 & 2998756 & 1196 & 3059753216 & 2962818 & 1196 & 3039350784 & 3008401 \\
\hline 857 & $4 / 17 / 2018$ & 1196 & 3079144448 & 2991512 & 1196 & 3062708992 & 2955811 & 1196 & 3042352128 & 3001262 \\
\hline 858 & $4 / 18 / 2018$ & 1196 & 3082128384 & 2984332 & 1196 & 3065657856 & 2948867 & 1196 & 3045346560 & 2994186 \\
\hline 859 & $4 / 19 / 2018$ & 1196 & 3085105920 & 2977216 & 1196 & 3068599808 & 2941986 & 1196 & 3048333568 & 2987171 \\
\hline 860 & $4 / 20 / 2018$ & 1196 & 3088076032 & 2970163 & 1196 & 3071535360 & 2935165 & 1196 & 3051313664 & 2980217 \\
\hline 861 & $4 / 21 / 2018$ & 1196 & 3091038976 & 2963171 & 1196 & 3074463488 & 2928404 & 1196 & 3054287104 & 2973322 \\
\hline 862 & $4 / 22 / 2018$ & 1196 & 3093995520 & 2956241 & 1196 & 3077385216 & 2921701 & 1196 & 3057253632 & 2966487 \\
\hline 863 & $4 / 23 / 2018$ & 1196 & 3096944896 & 2949371 & 1196 & 3080300288 & 2915058 & 1196 & 3060213248 & 2959710 \\
\hline 864 & $4 / 24 / 2018$ & 1196 & 3099887360 & 2942560 & 1196 & 3083208448 & 2908471 & 1196 & 3063166208 & 2952991 \\
\hline 865 & $4 / 25 / 2018$ & 1196 & 3102823168 & 2935808 & 1196 & 3086110464 & 2901942 & 1196 & 3066112512 & 2946328 \\
\hline 866 & $4 / 26 / 2018$ & 1196 & 3105752320 & 2929114 & 1196 & 3089006080 & 2895468 & 1196 & 3069052160 & 2939721 \\
\hline 867 & $4 / 27 / 2018$ & 1196 & 3108674560 & 2922477 & 1196 & 3091895040 & 2889049 & 1196 & 3071985408 & 2933169 \\
\hline 868 & $4 / 28 / 2018$ & 1196 & 3111590656 & 2915895 & 1196 & 3094777856 & 2882684 & 1196 & 3074912000 & 2926672 \\
\hline 869 & $4 / 29 / 2018$ & 1196 & 3114500096 & 2909370 & 1196 & 3097654272 & 2876373 & 1196 & 3077832448 & 2920228 \\
\hline 870 & $4 / 30 / 2018$ & 1196 & 3117403136 & 2902898 & 1196 & 3100524288 & 2870115 & 1196 & 3080746240 & 2913837 \\
\hline 871 & $5 / 1 / 2018$ & 1196 & 3120299520 & 2896481 & 1196 & 3103388160 & 2863908 & 1196 & 3083653632 & 2907498 \\
\hline 872 & $5 / 2 / 2018$ & 1665 & 3120299520 & 102 & 1665 & 3103388416 & 115 & 1665 & 3083653632 & 0 \\
\hline 873 & $5 / 3 / 2018$ & 1665 & 3120299776 & 102 & 1665 & 3103388416 & 116 & 1665 & 3083653632 & 0 \\
\hline 874 & $5 / 4 / 2018$ & 1665 & 3120315648 & 16019 & 1665 & 3103410944 & 22260 & 1665 & 3083676928 & 23262 \\
\hline 875 & $5 / 5 / 2018$ & 1665 & 3120354816 & 39206 & 1665 & 3103445248 & 34612 & 1665 & 3083724544 & 47501 \\
\hline 876 & $5 / 6 / 2018$ & 1665 & 3120420096 & 65128 & 1665 & 3103505920 & 60656 & 1665 & 3083796224 & 71726 \\
\hline 877 & $5 / 7 / 2018$ & 1665 & 3120498432 & 78015 & 1665 & 3103579136 & 73004 & 1665 & 3083880448 & 84168 \\
\hline 878 & $5 / 8 / 2018$ & 1665 & 3120584960 & 86908 & 1665 & 3103660288 & 81399 & 1665 & 3083973120 & 92878 \\
\hline 879 & $5 / 9 / 2018$ & 1665 & 3120678912 & 93914 & 1665 & 3103748352 & 87974 & 1665 & 3084072960 & 99796 \\
\hline 880 & $5 / 10 / 2018$ & 1665 & 3120778752 & 99789 & 1665 & 3103841536 & 93476 & 1665 & 3084178688 & 105609 \\
\hline 881 & $5 / 11 / 2018$ & 1665 & 3120883456 & 104851 & 1665 & 3103940096 & 98214 & 1665 & 3084289280 & 110626 \\
\hline 882 & $5 / 12 / 2018$ & 1665 & 3120992768 & 109275 & 1665 & 3104042496 & 102357 & 1665 & 3084404224 & 115010 \\
\hline 883 & $5 / 13 / 2018$ & 1665 & 3121105920 & 113173 & 1665 & 3104148480 & 106009 & 1665 & 3084523008 & 118873 \\
\hline 884 & $5 / 14 / 2018$ & 1665 & 3121222656 & 116631 & 1665 & 3104257792 & 109251 & 1665 & 3084645376 & 122301 \\
\hline 885 & $5 / 15 / 2018$ & 1665 & 3121342464 & 119716 & 1665 & 3104369920 & 112146 & 1665 & 3084770816 & 125359 \\
\hline 886 & $5 / 16 / 2018$ & 1665 & 3121464832 & 122483 & 1665 & 3104484608 & 114747 & 1665 & 3084898816 & 128101 \\
\hline 887 & $5 / 17 / 2018$ & 1665 & 3121589760 & 124979 & 1665 & 3104601600 & 117095 & 1665 & 3085029376 & 130576 \\
\hline 888 & $5 / 18 / 2018$ & 1665 & 3121716992 & 127246 & 1665 & 3104720896 & 119231 & 1665 & 3085162240 & 132824 \\
\hline 889 & $5 / 19 / 2018$ & 1665 & 3121846272 & 129310 & 1665 & 3104841984 & 121179 & 1665 & 3085297152 & 134870 \\
\hline 890 & $5 / 20 / 2018$ & 1665 & 3121977600 & 131202 & 1665 & 3104965120 & 122967 & 1665 & 3085433856 & 136746 \\
\hline 891 & $5 / 21 / 2018$ & 1665 & 3122110464 & 132945 & 1665 & 3105089792 & 124618 & 1665 & 3085572352 & 138474 \\
\hline 892 & $5 / 22 / 2018$ & 1665 & 3122245120 & 134560 & 1665 & 3105215744 & 126149 & 1665 & 3085712640 & 140075 \\
\hline 893 & $5 / 23 / 2018$ & 1665 & 3122381056 & 136065 & 1665 & 3105343488 & 127577 & 1665 & 3085853952 & 141565 \\
\hline 894 & $5 / 24 / 2018$ & 1665 & 3122518528 & 137471 & 1665 & 3105472256 & 128915 & 1665 & 3085997056 & 142958 \\
\hline 895 & $5 / 25 / 2018$ & 1665 & 3122657280 & 138794 & 1665 & 3105602560 & 130175 & 1665 & 3086141184 & 144267 \\
\hline 896 & $5 / 26 / 2018$ & 1665 & 3122797312 & 140041 & 1665 & 3105733888 & 131365 & 1665 & 3086286848 & 145501 \\
\hline 897 & $5 / 27 / 2018$ & 1665 & 3122939136 & 141791 & 1665 & 3105866240 & 132495 & 1665 & 3086433536 & 146670 \\
\hline 898 & $5 / 28 / 2018$ & 1665 & 3123084032 & 144820 & 1665 & 3105999872 & 133569 & 1665 & 3086581248 & 147780 \\
\hline 899 & $5 / 29 / 2018$ & 1665 & 3123230976 & 147156 & 1665 & 3106134528 & 134595 & 1665 & 3086729984 & 148838 \\
\hline 900 & $5 / 30 / 2018$ & 1665 & 3123380480 & 149212 & 1665 & 3106270208 & 135578 & 1665 & 3086880000 & 149849 \\
\hline 901 & $5 / 31 / 2018$ & 1665 & 3123531520 & 151100 & 1665 & 3106406656 & 136521 & 1665 & 3087030784 & 150818 \\
\hline 902 & $6 / 1 / 2018$ & 1665 & 3123684352 & 152866 & 1665 & 3106544128 & 137429 & 1665 & 3087184640 & 153838 \\
\hline 903 & $6 / 2 / 2018$ & 1665 & 3123838976 & 154534 & 1665 & 3106682368 & 138304 & 1665 & 3087341312 & 156673 \\
\hline 904 & $6 / 3 / 2018$ & 1665 & 3123995136 & 156120 & 1665 & 3106821376 & 139149 & 1665 & 3087501824 & 160500 \\
\hline 905 & $6 / 4 / 2018$ & 1665 & 3124152576 & 157633 & 1665 & 3106961408 & 139966 & 1665 & 3087665152 & 163470 \\
\hline 906 & $6 / 5 / 2018$ & 1665 & 3124311808 & 159083 & 1665 & 3107102976 & 141596 & 1665 & 3087831808 & 166368 \\
\hline 907 & $6 / 6 / 2018$ & 1665 & 3124472320 & 160475 & 1665 & 3107247104 & 143946 & 1665 & 3088002816 & 171324 \\
\hline 908 & $6 / 7 / 2018$ & 1665 & 3124634112 & 161816 & 1665 & 3107393024 & 145812 & 1665 & 3088179968 & 177131 \\
\hline 909 & $6 / 8 / 2018$ & 1665 & 3124797440 & 163366 & 1665 & 3107539968 & 147482 & 1665 & 3088363776 & 183685 \\
\hline 910 & $6 / 9 / 2018$ & 1665 & 3124962816 & 165712 & 1665 & 3107689216 & 149038 & 1665 & 3088552704 & 189115 \\
\hline 911 & $6 / 10 / 2018$ & 1665 & 3125130752 & 167757 & 1665 & 3107839744 & 150512 & 1665 & 3088748544 & 195761 \\
\hline 912 & $6 / 11 / 2018$ & 1665 & 3125301760 & 170786 & 1665 & 3107992064 & 152114 & 1665 & 3088952832 & 204232 \\
\hline 913 & $6 / 12 / 2018$ & 1665 & 3125478144 & 176596 & 1665 & 3108146688 & 154786 & 1665 & 3089164032 & 211284 \\
\hline 914 & $6 / 13 / 2018$ & 1665 & 3125662720 & 184464 & 1665 & 3108304640 & 157988 & 1665 & 3089382144 & 217962 \\
\hline 915 & $6 / 14 / 2018$ & 1665 & 3125855232 & 192634 & 1665 & 3108466688 & 161884 & 1665 & 3089607680 & 225539 \\
\hline 916 & $6 / 15 / 2018$ & 1665 & 3126054656 & 199343 & 1665 & 3108633344 & 166638 & 1665 & 3089842688 & 235216 \\
\hline 917 & $6 / 16 / 2018$ & 1665 & 3126259968 & 205387 & 1665 & 3108806656 & 173334 & 1665 & 3090089216 & 246537 \\
\hline 918 & $6 / 17 / 2018$ & 1665 & 3126471168 & 211231 & 1665 & 3108986368 & 179790 & 1665 & 3090347776 & 258474 \\
\hline 919 & $6 / 18 / 2018$ & 1665 & 3126690304 & 218912 & 1665 & 3109176064 & 189639 & 1665 & 3090617344 & 269573 \\
\hline 920 & $6 / 19 / 2018$ & 1665 & 3126916352 & 226002 & 1665 & 3109374464 & 198535 & 1665 & 3090897152 & 280136 \\
\hline 921 & $6 / 20 / 2018$ & 1665 & 3127148800 & 232447 & 1665 & 3109582848 & 208337 & 1665 & 3091190528 & 292912 \\
\hline 922 & $6 / 21 / 2018$ & 1665 & 3127389952 & 241155 & 1665 & 3109804288 & 221458 & 1665 & 3091495168 & 304608 \\
\hline 923 & $6 / 22 / 2018$ & 1665 & 3127642112 & 252161 & 1665 & 3110040320 & 235928 & 1665 & 3091813120 & 318007 \\
\hline 924 & $6 / 23 / 2018$ & 1665 & 3127905536 & 263562 & 1665 & 3110288896 & 248679 & 1665 & 3092145664 & 332533 \\
\hline 925 & $6 / 24 / 2018$ & 1665 & 3128178944 & 273332 & 1665 & 3110551040 & 262109 & 1665 & 3092491776 & 346310 \\
\hline 926 & $6 / 25 / 2018$ & 1665 & 3128465664 & 286723 & 1665 & 3110827008 & 275868 & 1665 & 3092854528 & 362561 \\
\hline 927 & $6 / 26 / 2018$ & 1665 & 3128768000 & 302508 & 1665 & 3111115520 & 288661 & 1665 & 3093234944 & 380432 \\
\hline 928 & $6 / 27 / 2018$ & 1665 & 3129088512 & 320445 & 1665 & 3111416576 & 300876 & 1665 & 3093634560 & 399632 \\
\hline 929 & $6 / 28 / 2018$ & 1665 & 3129426176 & 337597 & 1665 & 3111730688 & 314178 & 1665 & 3094052608 & 418026 \\
\hline 930 & $6 / 29 / 2018$ & 1665 & 3129778944 & 352837 & 1665 & 3112060928 & 330206 & 1665 & 3094488064 & 435509 \\
\hline 931 & $6 / 30 / 2018$ & 1665 & 3130146304 & 367201 & 1665 & 3112407552 & 346700 & 1665 & 3094942720 & 454663 \\
\hline 932 & $7 / 1 / 2018$ & 1665 & 3130527232 & 380923 & 1665 & 3112769536 & 362044 & 1665 & 3095419136 & 476372 \\
\hline 933 & $7 / 2 / 2018$ & 1665 & 3130922240 & 395049 & 1665 & 3113146368 & 376765 & 1665 & 3095916288 & 497254 \\
\hline 934 & $7 / 3 / 2018$ & 1665 & 3131331328 & 409271 & 1665 & 3113536768 & 390511 & 1665 & 3096433152 & 516695 \\
\hline
\end{tabular}




\begin{tabular}{|c|c|c|c|c|c|c|c|c|c|c|}
\hline 935 & $7 / 4 / 2018$ & 1665 & 3131753984 & 422526 & 1665 & 3113940480 & 403645 & 1665 & 3096969984 & 536916 \\
\hline 936 & $7 / 5 / 2018$ & 1665 & 3132191488 & 437534 & 1665 & 3114357504 & 417107 & 1665 & 3097526528 & 556505 \\
\hline 937 & $7 / 6 / 2018$ & 1665 & 3132642560 & 451021 & 1665 & 3114788096 & 430522 & 1665 & 3098103040 & 576809 \\
\hline 938 & $7 / 7 / 2018$ & 1665 & 3133109504 & 467039 & 1665 & 3115231744 & 443382 & 1665 & 3098701312 & 598138 \\
\hline 939 & $7 / 8 / 2018$ & 1665 & 3133593600 & 483991 & 1665 & 3115688960 & 457331 & 1665 & 3099321600 & 620255 \\
\hline 940 & $7 / 9 / 2018$ & 1665 & 3134096384 & 502753 & 1665 & 3116160256 & 471335 & 1665 & 3099961856 & 640096 \\
\hline 941 & $7 / 10 / 2018$ & 1665 & 3134620672 & 524484 & 1665 & 3116647424 & 487244 & 1665 & 3100621824 & 659939 \\
\hline 942 & $7 / 11 / 2018$ & 1665 & 3135166208 & 545651 & 1665 & 3117148672 & 501380 & 1665 & 3101301760 & 679971 \\
\hline 943 & $7 / 12 / 2018$ & 1665 & 3135731712 & 565225 & 1665 & 3117665792 & 516873 & 1665 & 3102001152 & 699575 \\
\hline 944 & $7 / 13 / 2018$ & 1665 & 3136316672 & 585103 & 1665 & 3118199296 & 533640 & 1665 & 3102719744 & 718546 \\
\hline 945 & $7 / 14 / 2018$ & 1665 & 3136921856 & 605196 & 1665 & 3118750720 & 551294 & 1665 & 3103458304 & 738531 \\
\hline 946 & $7 / 15 / 2018$ & 1665 & 3137547264 & 625456 & 1665 & 3119319808 & 569370 & 1665 & 3104216832 & 758404 \\
\hline 947 & $7 / 16 / 2018$ & 1665 & 3138192640 & 645210 & 1665 & 3119907840 & 587883 & 1665 & 3104994816 & 778205 \\
\hline 948 & $7 / 17 / 2018$ & 1665 & 3138857728 & 664994 & 1665 & 3120516608 & 608676 & 1665 & 3105792000 & 796958 \\
\hline 949 & $7 / 18 / 2018$ & 1665 & 3139542528 & 684811 & 1665 & 3121146112 & 629472 & 1665 & 3106609152 & 817322 \\
\hline 950 & $7 / 19 / 2018$ & 1665 & 3140246016 & 703556 & 1665 & 3121796096 & 650050 & 1665 & 3107447808 & 838672 \\
\hline 951 & $7 / 20 / 2018$ & 1665 & 3140968192 & 722195 & 1665 & 3122466816 & 670806 & 1665 & 3108306432 & 858626 \\
\hline 952 & $7 / 21 / 2018$ & 1665 & 3141709056 & 740941 & 1665 & 3123158528 & 691641 & 1665 & 3109184256 & 877659 \\
\hline 953 & $7 / 22 / 2018$ & 1665 & 3142469120 & 760091 & 1665 & 3123871744 & 713294 & 1665 & 3110081792 & 897722 \\
\hline 954 & $7 / 23 / 2018$ & 1665 & 3143249920 & 780646 & 1665 & 3124606720 & 734921 & 1665 & 3110998784 & 916860 \\
\hline 955 & $7 / 24 / 2018$ & 1665 & 3144050688 & 800751 & 1665 & 3125364992 & 758336 & 1665 & 3111933440 & 934724 \\
\hline 956 & $7 / 25 / 2018$ & 1665 & 3144872192 & 821509 & 1665 & 3126145280 & 780239 & 1665 & 3112885760 & 952309 \\
\hline 957 & $7 / 26 / 2018$ & 1665 & 3145713152 & 841142 & 1665 & 3126946816 & 801493 & 1665 & 3113855488 & 969715 \\
\hline 958 & $7 / 27 / 2018$ & 1665 & 3146574080 & 860740 & 1665 & 3127768832 & 821996 & 1665 & 3114843392 & 987966 \\
\hline 959 & $7 / 28 / 2018$ & 1665 & 3147454464 & 880429 & 1665 & 3128611328 & 842437 & 1665 & 3115850496 & 1006968 \\
\hline 960 & $7 / 29 / 2018$ & 1665 & 3148354816 & 900384 & 1665 & 3129475584 & 864329 & 1665 & 3116874496 & 1024119 \\
\hline 961 & $7 / 30 / 2018$ & 1665 & 3149276160 & 921302 & 1665 & 3130360832 & 885106 & 1665 & 3117914880 & 1040417 \\
\hline 962 & $7 / 31 / 2018$ & 1665 & 3150217472 & 941277 & 1665 & 3131266816 & 906161 & 1665 & 3118972160 & 1057187 \\
\hline 963 & $8 / 1 / 2018$ & 1665 & 3151177984 & 960664 & 1665 & 3132194560 & 927705 & 1665 & 3120046080 & 1073875 \\
\hline 964 & $8 / 2 / 2018$ & 1665 & 3152157440 & 979395 & 1665 & 3133143808 & 949375 & 1665 & 3121135616 & 1089501 \\
\hline 965 & $8 / 3 / 2018$ & 1665 & 3153155072 & 997611 & 1665 & 3134114816 & 970777 & 1665 & 3122240256 & 1104826 \\
\hline 966 & $8 / 4 / 2018$ & 1665 & 3154171392 & 1016227 & 1665 & 3135106048 & 991297 & 1665 & 3123360000 & 1119546 \\
\hline 967 & $8 / 5 / 2018$ & 1665 & 3155205120 & 1033836 & 1665 & 3136116480 & 1010530 & 1665 & 3124494080 & 1133960 \\
\hline 968 & $8 / 6 / 2018$ & 1665 & 3156255488 & 1050451 & 1665 & 3137145600 & 1029164 & 1665 & 3125641728 & 1147965 \\
\hline 969 & $8 / 7 / 2018$ & 1665 & 3157321984 & 1066436 & 1665 & 3138192640 & 1046935 & 1665 & 3126804224 & 1162120 \\
\hline 970 & $8 / 8 / 2018$ & 1665 & 3158404864 & 1082758 & 1665 & 3139256576 & 1063862 & 1665 & 3127980544 & 1176563 \\
\hline 971 & $8 / 9 / 2018$ & 1665 & 3159503104 & 1098539 & 1665 & 3140336896 & 1080361 & 1665 & 3129170432 & 1189921 \\
\hline 972 & $8 / 10 / 2018$ & 1665 & 3160617216 & 1113904 & 1665 & 3141433344 & 1096465 & 1665 & 3130373632 & 1203312 \\
\hline 973 & $8 / 11 / 2018$ & 1665 & 3161745664 & 1128468 & 1665 & 3142545152 & 1111820 & 1665 & 3131589888 & 1216021 \\
\hline 974 & $8 / 12 / 2018$ & 1665 & 3162888704 & 1143038 & 1665 & 3143672064 & 1126945 & 1665 & 3132817920 & 1228405 \\
\hline 975 & $8 / 13 / 2018$ & 1665 & 3164045568 & 1156963 & 1665 & 3144814080 & 1142079 & 1665 & 3134058496 & 1240371 \\
\hline 976 & $8 / 14 / 2018$ & 1665 & 3165216000 & 1170343 & 1665 & 3145971200 & 1157059 & 1665 & 3135310592 & 1251959 \\
\hline 977 & $8 / 15 / 2018$ & 1665 & 3166400256 & 1184103 & 1665 & 3147142400 & 1171499 & 1665 & 3136573952 & 1263150 \\
\hline 978 & $8 / 16 / 2018$ & 1665 & 3167597568 & 1197311 & 1665 & 3148327936 & 1185206 & 1665 & 3137847296 & 1273992 \\
\hline 979 & $8 / 17 / 2018$ & 1665 & 3168807424 & 1209961 & 1665 & 3149526528 & 1198396 & 1665 & 3139131904 & 1284508 \\
\hline 980 & $8 / 18 / 2018$ & 1665 & 3170029568 & 1222130 & 1665 & 3150737152 & 1211137 & 1665 & 3140426752 & 1294717 \\
\hline 981 & $8 / 19 / 2018$ & 1665 & 3171263488 & 1233893 & 1665 & 3151960832 & 1223466 & 1665 & 3141731840 & 1304882 \\
\hline 982 & $8 / 20 / 2018$ & 1665 & 3172508672 & 1245344 & 1665 & 3153199104 & 1238130 & 1665 & 3143046656 & 1314944 \\
\hline 983 & $8 / 21 / 2018$ & 1665 & 3173765376 & 1256572 & 1665 & 3154446848 & 1247931 & 1665 & 3144371712 & 1325105 \\
\hline 984 & $8 / 22 / 2018$ & 1665 & 3175033088 & 1267705 & 1665 & 3155706368 & 1259455 & 1665 & 3145705984 & 1334211 \\
\hline 985 & $8 / 23 / 2018$ & 1665 & 3176311552 & 1278429 & 1665 & 3156977152 & 1270687 & 1665 & 3147051008 & 1344910 \\
\hline 986 & $8 / 24 / 2018$ & 1665 & 3177600256 & 1288750 & 1665 & 3158258688 & 1281607 & 1665 & 3148403456 & 1352549 \\
\hline 987 & $8 / 25 / 2018$ & 1665 & 3178898944 & 1298816 & 1665 & 3159550976 & 1292141 & 1665 & 3149764608 & 1361259 \\
\hline 988 & $8 / 26 / 2018$ & 1665 & 3180207616 & 1308599 & 1665 & 3160853248 & 1302343 & 1665 & 3151134464 & 1369702 \\
\hline 989 & $8 / 27 / 2018$ & 1665 & 3181525760 & 1318063 & 1665 & 3162165504 & 1312235 & 1665 & 3152512256 & 1377895 \\
\hline 990 & $8 / 28 / 2018$ & 1421 & 3189381888 & 7856143 & 1421 & 3170250240 & 8084807 & 1421 & 3160112128 & 7599708 \\
\hline 991 & $8 / 29 / 2018$ & 1421 & 3196961792 & 7580077 & 1421 & 3178019328 & 7769016 & 1421 & 3167486208 & 7374213 \\
\hline 992 & $8 / 30 / 2018$ & 1421 & 3202659840 & 5697930 & 1421 & 3183827200 & 5808009 & 1421 & 3173061632 & 5575129 \\
\hline 993 & $8 / 31 / 2018$ & 1421 & 3207714560 & 5054619 & 1421 & 3188965376 & 5138026 & 1421 & 3178020608 & 4959343 \\
\hline 994 & $9 / 1 / 2018$ & 1421 & 3212395264 & 4680733 & 1421 & 3193714432 & 4749014 & 1421 & 3182621696 & 4600977 \\
\hline 995 & $9 / 2 / 2018$ & 1421 & 3216819200 & 4424062 & 1421 & 3198196480 & 4482177 & 1421 & 3186976512 & 4354796 \\
\hline 996 & $9 / 3 / 2018$ & 1421 & 3221046528 & 4227249 & 1421 & 3202473984 & 4277676 & 1421 & 3191142912 & 4166382 \\
\hline 997 & $9 / 4 / 2018$ & 1421 & 3225113344 & 4066821 & 1421 & 3206585088 & 4111023 & 1421 & 3195155712 & 4012847 \\
\hline 998 & $9 / 5 / 2018$ & 1421 & 3229044736 & 3931444 & 1421 & 3210555392 & 3970420 & 1421 & 3199038976 & 3883338 \\
\hline 999 & $9 / 6 / 2018$ & 1421 & 3232859648 & 3814898 & 1421 & 3214405120 & 3849393 & 1421 & 3202810880 & 3771860 \\
\hline 1000 & $9 / 7 / 2018$ & 1421 & 3236572928 & 3713242 & 1421 & 3218148608 & 3743842 & 1421 & 3206485504 & 3674616 \\
\hline 1001 & $9 / 8 / 2018$ & 1421 & 3240196608 & 3623590 & 1421 & 3221799680 & 3650773 & 1421 & 3210074368 & 3588824 \\
\hline 1002 & $9 / 9 / 2018$ & 1421 & 3243740672 & 3544066 & 1421 & 3225367808 & 3568222 & 1421 & 3213586944 & 3512686 \\
\hline 1003 & $9 / 10 / 2018$ & 1421 & 3247213568 & 3472957 & 1421 & 3228861952 & 3494411 & 1421 & 3217031680 & 3444558 \\
\hline 1004 & $9 / 11 / 2018$ & 1873 & 3247213568 & 84 & 1873 & 3228862464 & 96 & 1873 & 3217031680 & 0 \\
\hline 1005 & $9 / 12 / 2018$ & 1873 & 3247213824 & 85 & 1873 & 3228862464 & 96 & 1873 & 3217031680 & 0 \\
\hline 1006 & $9 / 13 / 2018$ & 1873 & 3247216640 & 2846 & 1873 & 3228872704 & 10261 & 1873 & 3217039360 & 7891 \\
\hline 1007 & $9 / 14 / 2018$ & 1873 & 3247236608 & 19938 & 1873 & 3228895232 & 22513 & 1873 & 3217062912 & 23545 \\
\hline 1008 & $9 / 15 / 2018$ & 1873 & 3247280128 & 43765 & 1873 & 3228935168 & 39862 & 1873 & 3217112576 & 49209 \\
\hline 1009 & $9 / 16 / 2018$ & 1873 & 3247339008 & 58723 & 1873 & 3228990464 & 55452 & 1873 & 3217175296 & 63169 \\
\hline 1010 & $9 / 17 / 2018$ & 1873 & 3247407104 & 68020 & 1873 & 3229054976 & 64434 & 1873 & 3217247744 & 72225 \\
\hline 1011 & $9 / 18 / 2018$ & 1873 & 3247482112 & 75090 & 1873 & 3229126144 & 71130 & 1873 & 3217326848 & 79192 \\
\hline 1012 & $9 / 19 / 2018$ & 1873 & 3247563008 & 80878 & 1873 & 3229202688 & 76573 & 1873 & 3217411840 & 84922 \\
\hline 1013 & $9 / 20 / 2018$ & 1873 & 3247648512 & 85766 & 1873 & 3229283840 & 81160 & 1873 & 3217501440 & 89767 \\
\hline 1014 & $9 / 21 / 2018$ & 1873 & 3247738624 & 89959 & 1873 & 3229368832 & 85092 & 1873 & 3217595392 & 93927 \\
\hline 1015 & $9 / 22 / 2018$ & 1873 & 3247832320 & 93593 & 1873 & 3229457408 & 88501 & 1873 & 3217692928 & 97533 \\
\hline 1016 & $9 / 23 / 2018$ & 1873 & 3247929088 & 96768 & 1873 & 3229548800 & 91482 & 1873 & 3217793536 & 100683 \\
\hline
\end{tabular}




\begin{tabular}{|c|c|c|c|c|c|c|c|c|c|c|}
\hline 1017 & $9 / 24 / 2018$ & 1873 & 3248028672 & 99562 & 1873 & 3229643008 & 94108 & 1873 & 3217897216 & 103457 \\
\hline 1018 & $9 / 25 / 2018$ & 1873 & 3248130560 & 102038 & 1873 & 3229739520 & 96437 & 1873 & 3218002944 & 105916 \\
\hline 1019 & $9 / 26 / 2018$ & 1873 & 3248235008 & 104253 & 1873 & 3229838080 & 98522 & 1873 & 3218111232 & 108114 \\
\hline 1020 & $9 / 27 / 2018$ & 1873 & 3248341248 & 106242 & 1873 & 3229938432 & 100398 & 1873 & 3218221312 & 110090 \\
\hline 1021 & $9 / 28 / 2018$ & 1873 & 3248449280 & 108043 & 1873 & 3230040576 & 102100 & 1873 & 3218333184 & 111879 \\
\hline 1022 & $9 / 29 / 2018$ & 1873 & 3248558848 & 109686 & 1873 & 3230144256 & 103654 & 1873 & 3218446592 & 113510 \\
\hline 1023 & $9 / 30 / 2018$ & 1873 & 3248669952 & 111195 & 1873 & 3230249216 & 105084 & 1873 & 3218561536 & 115008 \\
\hline 1024 & $10 / 1 / 2018$ & 1873 & 3248782592 & 112590 & 1873 & 3230355712 & 106407 & 1873 & 3218678016 & 116393 \\
\hline 1025 & $10 / 2 / 2018$ & 1873 & 3248896512 & 113887 & 1873 & 3230462976 & 107639 & 1873 & 3218795776 & 117680 \\
\hline 1026 & $10 / 3 / 2018$ & 1873 & 3249011712 & 115100 & 1873 & 3230572032 & 108792 & 1873 & 3218914560 & 118884 \\
\hline 1027 & $10 / 4 / 2018$ & 1873 & 3249127936 & 116241 & 1873 & 3230681856 & 109878 & 1873 & 3219034624 & 120014 \\
\hline 1028 & $10 / 5 / 2018$ & 1873 & 3249245184 & 117319 & 1873 & 3230792704 & 110906 & 1873 & 3219155712 & 121082 \\
\hline 1029 & $10 / 6 / 2018$ & 1873 & 3249363456 & 118341 & 1873 & 3230904576 & 111881 & 1873 & 3219278080 & 122095 \\
\hline 1030 & $10 / 7 / 2018$ & 1873 & 3249482752 & 119314 & 1873 & 3231017472 & 112811 & 1873 & 3219400960 & 123058 \\
\hline 1031 & $10 / 8 / 2018$ & 1873 & 3249603072 & 120245 & 1873 & 3231131136 & 113701 & 1873 & 3219524864 & 123979 \\
\hline 1032 & $10 / 9 / 2018$ & 1873 & 3249724160 & 121137 & 1873 & 3231246080 & 114554 & 1873 & 3219649792 & 124860 \\
\hline 1033 & $10 / 10 / 2018$ & 1873 & 3249846272 & 121993 & 1873 & 3231361024 & 115374 & 1873 & 3219775488 & 125706 \\
\hline 1034 & $10 / 11 / 2018$ & 1873 & 3249969152 & 122819 & 1873 & 3231477248 & 116165 & 1873 & 3219901952 & 126521 \\
\hline 1035 & $10 / 12 / 2018$ & 1873 & 3250092288 & 123616 & 1873 & 3231594240 & 116929 & 1873 & 3220029184 & 127307 \\
\hline 1036 & $10 / 13 / 2018$ & 1873 & 3250217728 & 125169 & 1873 & 3231712000 & 117668 & 1873 & 3220157184 & 128066 \\
\hline 1037 & $10 / 14 / 2018$ & 1873 & 3250344960 & 127143 & 1873 & 3231830272 & 118384 & 1873 & 3220285952 & 128800 \\
\hline 1038 & $10 / 15 / 2018$ & 1873 & 3250473728 & 128738 & 1873 & 3231949312 & 119080 & 1873 & 3220415488 & 129512 \\
\hline 1039 & $10 / 16 / 2018$ & 1873 & 3250603776 & 130178 & 1873 & 3232069120 & 119755 & 1873 & 3220545792 & 130202 \\
\hline 1040 & $10 / 17 / 2018$ & 1873 & 3250735360 & 131524 & 1873 & 3232189440 & 120614 & 1873 & 3220676608 & 130873 \\
\hline 1041 & $10 / 18 / 2018$ & 1873 & 3250868224 & 132800 & 1873 & 3232312576 & 122892 & 1873 & 3220808448 & 131752 \\
\hline 1042 & $10 / 19 / 2018$ & 1873 & 3251002368 & 134210 & 1873 & 3232438016 & 125019 & 1873 & 3220942336 & 133818 \\
\hline 1043 & $10 / 20 / 2018$ & 1873 & 3251138560 & 136011 & 1873 & 3232564480 & 126801 & 1873 & 3221077504 & 135395 \\
\hline 1044 & $10 / 21 / 2018$ & 1873 & 3251276032 & 137562 & 1873 & 3232692992 & 128422 & 1873 & 3221216000 & 138403 \\
\hline 1045 & $10 / 22 / 2018$ & 1873 & 3251415040 & 138996 & 1873 & 3232822784 & 129940 & 1873 & 3221357312 & 141232 \\
\hline 1046 & $10 / 23 / 2018$ & 1873 & 3251555328 & 140355 & 1873 & 3232954112 & 131382 & 1873 & 3221502208 & 144898 \\
\hline 1047 & $10 / 24 / 2018$ & 1873 & 3251696896 & 141656 & 1873 & 3233087744 & 133548 & 1873 & 3221650944 & 148867 \\
\hline 1048 & $10 / 25 / 2018$ & 1873 & 3251840000 & 142908 & 1873 & 3233223680 & 135855 & 1873 & 3221805056 & 154102 \\
\hline 1049 & $10 / 26 / 2018$ & 1873 & 3251984128 & 144117 & 1873 & 3233361408 & 137745 & 1873 & 3221964800 & 159657 \\
\hline 1050 & $10 / 27 / 2018$ & 1873 & 3252129536 & 145456 & 1873 & 3233500928 & 139480 & 1873 & 3222131200 & 166512 \\
\hline 1051 & $10 / 28 / 2018$ & 1873 & 3252277504 & 148046 & 1873 & 3233643008 & 142161 & 1873 & 3222303744 & 172453 \\
\hline 1052 & $10 / 29 / 2018$ & 1873 & 3252427520 & 150049 & 1873 & 3233788416 & 145387 & 1873 & 3222481664 & 177974 \\
\hline 1053 & $10 / 30 / 2018$ & 1873 & 3252579584 & 152042 & 1873 & 3233936384 & 148014 & 1873 & 3222664704 & 183048 \\
\hline 1054 & $10 / 31 / 2018$ & 1873 & 3252734208 & 154576 & 1873 & 3234086656 & 150357 & 1873 & 3222854400 & 189528 \\
\hline 1055 & $11 / 1 / 2018$ & 1873 & 3252892416 & 158177 & 1873 & 3234240000 & 153314 & 1873 & 3223051264 & 196993 \\
\hline 1056 & $11 / 2 / 2018$ & 1873 & 3253055488 & 163060 & 1873 & 3234397696 & 157530 & 1873 & 3223256064 & 204814 \\
\hline 1057 & $11 / 3 / 2018$ & 1873 & 3253223424 & 168135 & 1873 & 3234560000 & 162123 & 1873 & 3223469824 & 213642 \\
\hline 1058 & $11 / 4 / 2018$ & 1873 & 3253398016 & 174674 & 1873 & 3234725632 & 165967 & 1873 & 3223692288 & 222434 \\
\hline 1059 & $11 / 5 / 2018$ & 1873 & 3253579008 & 180841 & 1873 & 3234895872 & 170288 & 1873 & 3223923712 & 231594 \\
\hline 1060 & $11 / 6 / 2018$ & 1873 & 3253766144 & 187034 & 1873 & 3235073024 & 177016 & 1873 & 3224164352 & 240678 \\
\hline 1061 & $11 / 7 / 2018$ & 1873 & 3253960960 & 194790 & 1873 & 3235258880 & 185953 & 1873 & 3224414208 & 249629 \\
\hline 1062 & $11 / 8 / 2018$ & 1873 & 3254162944 & 201955 & 1873 & 3235456256 & 197204 & 1873 & 3224673024 & 259033 \\
\hline 1063 & $11 / 9 / 2018$ & 1873 & 3254372352 & 209561 & 1873 & 3235663104 & 207032 & 1873 & 3224941568 & 268493 \\
\hline 1064 & $11 / 10 / 2018$ & 1873 & 3254590464 & 218149 & 1873 & 3235882240 & 218946 & 1873 & 3225222400 & 280819 \\
\hline 1065 & $11 / 11 / 2018$ & 1873 & 3254817280 & 226584 & 1873 & 3236111872 & 229485 & 1873 & 3225516544 & 294083 \\
\hline 1066 & $11 / 12 / 2018$ & 1873 & 3255055872 & 238820 & 1873 & 3236352512 & 240869 & 1873 & 3225825280 & 308701 \\
\hline 1067 & $11 / 13 / 2018$ & 1873 & 3255307264 & 251363 & 1873 & 3236604160 & 251730 & 1873 & 3226148096 & 323202 \\
\hline 1068 & $11 / 14 / 2018$ & 1873 & 3255571712 & 264543 & 1873 & 3236866304 & 262077 & 1873 & 3226486272 & 337733 \\
\hline 1069 & $11 / 15 / 2018$ & 1873 & 3255848960 & 276973 & 1873 & 3237139456 & 273089 & 1873 & 3226838528 & 352458 \\
\hline 1070 & $11 / 16 / 2018$ & 1873 & 3256137728 & 288886 & 1873 & 3237423872 & 284496 & 1873 & 3227206400 & 367967 \\
\hline 1071 & $11 / 17 / 2018$ & 1873 & 3256438016 & 300155 & 1873 & 3237721088 & 297325 & 1873 & 3227590912 & 384553 \\
\hline 1072 & $11 / 18 / 2018$ & 1873 & 3256750080 & 312053 & 1873 & 3238031104 & 309944 & 1873 & 3227992832 & 401622 \\
\hline 1073 & $11 / 19 / 2018$ & 1873 & 3257073664 & 323807 & 1873 & 3238352640 & 321387 & 1873 & 3228411904 & 419454 \\
\hline 1074 & $11 / 20 / 2018$ & 1873 & 3257410048 & 336280 & 1873 & 3238684672 & 332215 & 1873 & 3228849152 & 436961 \\
\hline 1075 & $11 / 21 / 2018$ & 1873 & 3257758208 & 348202 & 1873 & 3239027456 & 342601 & 1873 & 3229303808 & 454642 \\
\hline 1076 & $11 / 22 / 2018$ & 1873 & 3258117632 & 359284 & 1873 & 3239380736 & 353418 & 1873 & 3229775616 & 472146 \\
\hline 1077 & $11 / 23 / 2018$ & 1873 & 3258490880 & 373324 & 1873 & 3239746304 & 365649 & 1873 & 3230265856 & 489922 \\
\hline 1078 & $11 / 24 / 2018$ & 1873 & 3258880000 & 389153 & 1873 & 3240125184 & 378678 & 1873 & 3230772224 & 506454 \\
\hline 1079 & $11 / 25 / 2018$ & 1873 & 3259285760 & 405796 & 1873 & 3240516352 & 391260 & 1873 & 3231294720 & 522359 \\
\hline 1080 & $11 / 26 / 2018$ & 1873 & 3259709952 & 424181 & 1873 & 3240919296 & 403017 & 1873 & 3231833344 & 538609 \\
\hline 1081 & $11 / 27 / 2018$ & 1873 & 3260151552 & 441548 & 1873 & 3241336320 & 416856 & 1873 & 3232390400 & 557145 \\
\hline 1082 & $11 / 28 / 2018$ & 1873 & 3260609792 & 458264 & 1873 & 3241767680 & 431374 & 1873 & 3232965120 & 574556 \\
\hline 1083 & $11 / 29 / 2018$ & 1873 & 3261084928 & 475202 & 1873 & 3242214400 & 446843 & 1873 & 3233556992 & 591886 \\
\hline 1084 & $11 / 30 / 2018$ & 1873 & 3261577216 & 492283 & 1873 & 3242678528 & 464004 & 1873 & 3234164992 & 608170 \\
\hline 1085 & $12 / 1 / 2018$ & 1873 & 3262085376 & 508213 & 1873 & 3243162368 & 483839 & 1873 & 3234789376 & 624312 \\
\hline 1086 & $12 / 2 / 2018$ & 1873 & 3262608896 & 523368 & 1873 & 3243663360 & 501175 & 1873 & 3235429120 & 639882 \\
\hline 1087 & $12 / 3 / 2018$ & 1873 & 3263147264 & 538507 & 1873 & 3244181760 & 518376 & 1873 & 3236084992 & 655810 \\
\hline 1088 & $12 / 4 / 2018$ & 1873 & 3263701760 & 554494 & 1873 & 3244716544 & 535101 & 1873 & 3236756992 & 671854 \\
\hline 1089 & $12 / 5 / 2018$ & 1873 & 3264271616 & 569753 & 1873 & 3245268992 & 551980 & 1873 & 3237444864 & 687869 \\
\hline 1090 & $12 / 6 / 2018$ & 1873 & 3264857856 & 586157 & 1873 & 3245837568 & 568580 & 1873 & 3238148608 & 703948 \\
\hline 1091 & $12 / 7 / 2018$ & 1873 & 3265461504 & 604084 & 1873 & 3246424320 & 586615 & 1873 & 3238867968 & 719229 \\
\hline 1092 & $12 / 8 / 2018$ & 1873 & 3266082560 & 620815 & 1873 & 3247028736 & 604680 & 1873 & 3239601664 & 733613 \\
\hline 1093 & $12 / 9 / 2018$ & 1873 & 3266719232 & 636529 & 1873 & 3247651072 & 622174 & 1873 & 3240348928 & 747384 \\
\hline 1094 & $12 / 10 / 2018$ & 1873 & 3267372288 & 653076 & 1873 & 3248290560 & 639585 & 1873 & 3241110272 & 761379 \\
\hline 1095 & $12 / 11 / 2018$ & 1873 & 3268042496 & 670335 & 1873 & 3248947712 & 657291 & 1873 & 3241885440 & 775182 \\
\hline 1096 & $12 / 12 / 2018$ & 1873 & 3268730368 & 687885 & 1873 & 3249622528 & 674781 & 1873 & 3242674688 & 789234 \\
\hline 1097 & $12 / 13 / 2018$ & 1873 & 3269435136 & 704713 & 1873 & 3250314240 & 691580 & 1873 & 3243478016 & 803164 \\
\hline 1098 & $12 / 14 / 2018$ & 1873 & 3270157824 & 722549 & 1873 & 3251023104 & 709000 & 1873 & 3244295424 & 817432 \\
\hline
\end{tabular}




\begin{tabular}{|c|c|c|c|c|c|c|c|c|c|c|}
\hline 1099 & $12 / 15 / 2018$ & 1873 & 3270897152 & 739512 & 1873 & 3251750656 & 727540 & 1873 & 3245126912 & 831616 \\
\hline 1100 & $12 / 16 / 2018$ & 1873 & 3271652608 & 755247 & 1873 & 3252496640 & 745858 & 1873 & 3245971968 & 844961 \\
\hline 1101 & $12 / 17 / 2018$ & 1873 & 3272422656 & 770228 & 1873 & 3253260288 & 763649 & 1873 & 3246829824 & 857979 \\
\hline 1102 & $12 / 18 / 2018$ & 1873 & 3273208064 & 785419 & 1873 & 3254040320 & 780206 & 1873 & 3247700224 & 870224 \\
\hline 1103 & $12 / 19 / 2018$ & 1873 & 3274008320 & 800129 & 1873 & 3254836224 & 795725 & 1873 & 3248582400 & 882392 \\
\hline 1104 & $12 / 20 / 2018$ & 1873 & 3274822656 & 814364 & 1873 & 3255646976 & 810693 & 1873 & 3249476864 & 894224 \\
\hline 1105 & $12 / 21 / 2018$ & 1873 & 3275651584 & 828622 & 1873 & 3256471808 & 825014 & 1873 & 3250382592 & 905830 \\
\hline 1106 & $12 / 22 / 2018$ & 1873 & 3276493312 & 842058 & 1873 & 3257310976 & 839095 & 1873 & 3251301888 & 919383 \\
\hline 1107 & $12 / 23 / 2018$ & 1873 & 3277348352 & 855074 & 1873 & 3258163712 & 852653 & 1873 & 3252235264 & 933233 \\
\hline 1108 & $12 / 24 / 2018$ & 1873 & 3278216192 & 867829 & 1873 & 3259029504 & 865940 & 1873 & 3253174784 & 939509 \\
\hline 1109 & $12 / 25 / 2018$ & 1873 & 3279096320 & 880149 & 1873 & 3259908352 & 879146 & 1873 & 3254124544 & 949949 \\
\hline 1110 & $12 / 26 / 2018$ & 1873 & 3279988736 & 892376 & 1873 & 3260800256 & 891853 & 1873 & 3255084800 & 960173 \\
\hline 1111 & $12 / 27 / 2018$ & 1873 & 3280892672 & 903965 & 1873 & 3261704704 & 904052 & 1873 & 3256054528 & 969979 \\
\hline 1112 & $12 / 28 / 2018$ & 1873 & 3281808384 & 915615 & 1873 & 3262620416 & 915880 & 1873 & 3257034240 & 979456 \\
\hline 1113 & $12 / 29 / 2018$ & 1873 & 3282735104 & 926730 & 1873 & 3263547648 & 927205 & 1873 & 3258022912 & 988635 \\
\hline 1114 & $12 / 30 / 2018$ & 1873 & 3283672576 & 937386 & 1873 & 3264485888 & 938175 & 1873 & 3259020544 & 997593 \\
\hline 1115 & $12 / 31 / 2018$ & 1873 & 3284620032 & 947675 & 1873 & 3265434880 & 949083 & 1873 & 3260026880 & 1006357 \\
\hline 1116 & $1 / 1 / 2019$ & 1873 & 3285577728 & 957635 & 1873 & 3266394624 & 959689 & 1873 & 3261041664 & 1014876 \\
\hline 1117 & $1 / 2 / 2019$ & 1129 & 3307536128 & 21958320 & 1129 & 3289091328 & 22696610 & 1129 & 3282215680 & 21173996 \\
\hline 1118 & $1 / 3 / 2019$ & 1129 & 3326380288 & 18844306 & 1129 & 3308480000 & 19388864 & 1129 & 3300668928 & 18453310 \\
\hline 1119 & $1 / 4 / 2019$ & 1129 & 3340399104 & 14018748 & 1129 & 3323070464 & 14590504 & 1129 & 3314017536 & 13348538 \\
\hline 1120 & $1 / 5 / 2019$ & 1129 & 3352513792 & 12114754 & 1129 & 3335363840 & 12293335 & 1129 & 3325542144 & 11524580 \\
\hline 1121 & $1 / 6 / 2019$ & 1129 & 3363475712 & 10961778 & 1129 & 3346513408 & 11149399 & 1129 & 3336074496 & 10532442 \\
\hline 1122 & $1 / 7 / 2019$ & 1129 & 3373630464 & 10154798 & 1129 & 3356840448 & 10327043 & 1129 & 3345874944 & 9800127 \\
\hline 1123 & $1 / 8 / 2019$ & 1129 & 3383162624 & 9532059 & 1129 & 3366527232 & 9686824 & 1129 & 3355096832 & 9222072 \\
\hline 1124 & $1 / 9 / 2019$ & 1129 & 3392187392 & 9024763 & 1129 & 3375690240 & 9163130 & 1129 & 3363842816 & 8746125 \\
\hline 1125 & $1 / 10 / 2019$ & 1129 & 3400785408 & 8598193 & 1129 & 3384412160 & 8721909 & 1129 & 3372186624 & 8343851 \\
\hline 1126 & $1 / 11 / 2019$ & 1129 & 3409017600 & 8232134 & 1129 & 3392755200 & 8342908 & 1129 & 3380184320 & 7997664 \\
\hline 1127 & $1 / 12 / 2019$ & 1129 & 3416930560 & 7913061 & 1129 & 3400767488 & 8012404 & 1129 & 3387879936 & 7695397 \\
\hline 1128 & $1 / 13 / 2019$ & 1129 & 3424562944 & 7632148 & 1129 & 3408488960 & 7721331 & 1129 & 3395308800 & 7428927 \\
\hline 1129 & $1 / 14 / 2019$ & 1129 & 3431944960 & 7382187 & 1129 & 3415951104 & 7462295 & 1129 & 3402500352 & 7191576 \\
\hline 1130 & $1 / 15 / 2019$ & 1129 & 3439102720 & 7157815 & 1129 & 3423181056 & 7229763 & 1129 & 3409478912 & 6978319 \\
\hline 1131 & $1 / 16 / 2019$ & 1129 & 3446057728 & 6954850 & 1129 & 3430200320 & 7019416 & 1129 & 3416263936 & 6785237 \\
\hline 1132 & $1 / 17 / 2019$ & 1129 & 3452827648 & 6769976 & 1129 & 3437028096 & 6827829 & 1129 & 3422873344 & 6609211 \\
\hline 1133 & $1 / 18 / 2019$ & 1129 & 3459428096 & 6600530 & 1129 & 3443680512 & 6652243 & 1129 & 3429320960 & 6447737 \\
\hline 1134 & $1 / 19 / 2019$ & 1129 & 3465872640 & 6444341 & 1129 & 3450170880 & 6490421 & 1129 & 3435619584 & 6298780 \\
\hline 1135 & $1 / 20 / 2019$ & 1129 & 3472172288 & 6299638 & 1129 & 3456511488 & 6340524 & 1129 & 3441780224 & 6160673 \\
\hline 1136 & $1 / 21 / 2019$ & 1129 & 3478337024 & 6164954 & 1129 & 3462712320 & 6201036 & 1129 & 3447812352 & 6032038 \\
\hline 1137 & $1 / 22 / 2019$ & 1129 & 3484376064 & 6039071 & 1129 & 3468783104 & 6070695 & 1129 & 3453724160 & 5911728 \\
\hline 1138 & $1 / 23 / 2019$ & 1129 & 3490296832 & 5920751 & 1129 & 3474731264 & 5948234 & 1129 & 3459522560 & 5798577 \\
\hline 1139 & $1 / 24 / 2019$ & 1129 & 3496106752 & 5809753 & 1129 & 3480564480 & 5833150 & 1129 & 3465214720 & 5692153 \\
\hline 1140 & $1 / 25 / 2019$ & 1129 & 3501811200 & 5704602 & 1129 & 3486289152 & 5724555 & 1129 & 3470806528 & 5591642 \\
\hline 1141 & $1 / 26 / 2019$ & 1129 & 3507416576 & 5605153 & 1129 & 3491910912 & 5621771 & 1129 & 3476302848 & 5496428 \\
\hline 1142 & $1 / 27 / 2019$ & 1129 & 3512927232 & 5510768 & 1129 & 3497435136 & 5524232 & 1129 & 3481708800 & 5405995 \\
\hline 1143 & $1 / 28 / 2019$ & 1129 & 3518348288 & 5420964 & 1129 & 3502866432 & 5431456 & 1129 & 3487028736 & 5319905 \\
\hline 1144 & $1 / 29 / 2019$ & 1129 & 3523683584 & 5335333 & 1129 & 3508209664 & 5343028 & 1129 & 3492266496 & 5237779 \\
\hline 1145 & $1 / 30 / 2019$ & 1129 & 3528936960 & 5253523 & 1129 & 3513468160 & 5258585 & 1129 & 3497425920 & 5159290 \\
\hline 1146 & $1 / 31 / 2019$ & 1129 & 3534112256 & 5175230 & 1129 & 3518646016 & 5177809 & 1129 & 3502509824 & 5084150 \\
\hline 1147 & $2 / 1 / 2019$ & 1129 & 3539212544 & 5100183 & 1129 & 3523746304 & 5100423 & 1129 & 3507521792 & 5012103 \\
\hline 1148 & $2 / 2 / 2019$ & 1129 & 3544240640 & 5028146 & 1129 & 3528772608 & 5026180 & 1129 & 3512464896 & 4942928 \\
\hline 1149 & $2 / 3 / 2019$ & 1129 & 3549199616 & 4958907 & 1129 & 3533727488 & 4954857 & 1129 & 3517341440 & 4876423 \\
\hline 1150 & $2 / 4 / 2019$ & 1129 & 3554091776 & 4892279 & 1129 & 3538613760 & 4886262 & 1129 & 3522153728 & 4812410 \\
\hline 1151 & $2 / 5 / 2019$ & 1129 & 3558919936 & 4828093 & 1129 & 3543433984 & 4820216 & 1129 & 3526904576 & 4750731 \\
\hline 1152 & $2 / 6 / 2019$ & 1129 & 3563686144 & 4766196 & 1129 & 3548190464 & 4756563 & 1129 & 3531595776 & 4691239 \\
\hline 1153 & $2 / 7 / 2019$ & 1129 & 3568392448 & 4706451 & 1129 & 3552885504 & 4695156 & 1129 & 3536229632 & 4633807 \\
\hline 1154 & $2 / 8 / 2019$ & 1129 & 3573041152 & 4648734 & 1129 & 3557521152 & 4635868 & 1129 & 3540807936 & 4578313 \\
\hline 1155 & $2 / 9 / 2019$ & 1129 & 3577634304 & 4592932 & 1129 & 3562099968 & 4578579 & 1129 & 3545332224 & 4524652 \\
\hline 1156 & $2 / 10 / 2019$ & 1129 & 3582173184 & 4538940 & 1129 & 3566623232 & 4523181 & 1129 & 3549805312 & 4472725 \\
\hline 1157 & $2 / 11 / 2019$ & 1129 & 3586659840 & 4486665 & 1129 & 3571092736 & 4469574 & 1129 & 3554227712 & 4422440 \\
\hline 1158 & $2 / 12 / 2019$ & 1129 & 3591095808 & 4436018 & 1129 & 3575510528 & 4417667 & 1129 & 3558601216 & 4373715 \\
\hline 1159 & $2 / 13 / 2019$ & 1129 & 3595482624 & 4386918 & 1129 & 3579877888 & 4367376 & 1129 & 3562927872 & 4326474 \\
\hline 1160 & $2 / 14 / 2019$ & 1129 & 3599822080 & 4339293 & 1129 & 3584196352 & 4318622 & 1129 & 3567208448 & 4280644 \\
\hline 1161 & $2 / 15 / 2019$ & 1129 & 3604115200 & 4293072 & 1129 & 3588467712 & 4271333 & 1129 & 3571444736 & 4236161 \\
\hline 1162 & $2 / 16 / 2019$ & 1129 & 3608363264 & 4248191 & 1129 & 3592693248 & 4225441 & 1129 & 3575637760 & 4192962 \\
\hline 1163 & $2 / 17 / 2019$ & 1129 & 3612567808 & 4204590 & 1129 & 3596874240 & 4180885 & 1129 & 3579788544 & 4150991 \\
\hline 1164 & $2 / 18 / 2019$ & 1129 & 3616730112 & 4162214 & 1129 & 3601011712 & 4137604 & 1129 & 35838988880 & 4110194 \\
\hline 1165 & $2 / 19 / 2019$ & 1129 & 3620851200 & 4121011 & 1129 & 3605107200 & 4095546 & 1129 & 3587969280 & 4070522 \\
\hline 1166 & $2 / 20 / 2019$ & 1129 & 3624932096 & 4080931 & 1129 & 3609161984 & 4054657 & 1129 & 3592001280 & 4031927 \\
\hline 1167 & $2 / 21 / 2019$ & 1129 & 3628974080 & 4041928 & 1129 & 3613176832 & 4014890 & 1129 & 3595995648 & 3994367 \\
\hline 1168 & $2 / 22 / 2019$ & 1129 & 3632977920 & 4003960 & 1129 & 3617153024 & 3976199 & 1129 & 3599953408 & 3957798 \\
\hline 1169 & $2 / 23 / 2019$ & 1129 & 3636944896 & 3966985 & 1129 & 3621091584 & 3938542 & 1129 & 3603875584 & 3922182 \\
\hline 1170 & $2 / 24 / 2019$ & 1129 & 3640875776 & 3930965 & 1129 & 3624993536 & 3901878 & 1129 & 3607763200 & 3887483 \\
\hline 1171 & $2 / 25 / 2019$ & 1129 & 3644771840 & 3895864 & 1129 & 3628859648 & 3866169 & 1129 & 3611616768 & 3853665 \\
\hline 1172 & $2 / 26 / 2019$ & 1129 & 3648633344 & 3861647 & 1129 & 3632690944 & 3831379 & 1129 & 3615437568 & 3820695 \\
\hline 1173 & $2 / 27 / 2019$ & 1129 & 3652461568 & 3828282 & 1129 & 3636488448 & 3797474 & 1129 & 3619226112 & 3788543 \\
\hline 1174 & $2 / 28 / 2019$ & 1129 & 3656257280 & 3795737 & 1129 & 3640252672 & 3764420 & 1129 & 3622983168 & 3757178 \\
\hline 1175 & $3 / 1 / 2019$ & 1129 & 3660021504 & 3763984 & 1129 & 3643985152 & 3732188 & 1129 & 3626709760 & 3726573 \\
\hline 1176 & $3 / 2 / 2019$ & 1129 & 3663754496 & 3732994 & 1129 & 3647685888 & 3700749 & 1129 & 3630406400 & 3696701 \\
\hline 1177 & $3 / 3 / 2019$ & 1129 & 3667457024 & 3702741 & 1129 & 3651355904 & 3670073 & 1129 & 3634074112 & 3667535 \\
\hline 1178 & $3 / 4 / 2019$ & 1129 & 3671130368 & 3673200 & 1129 & 3654995968 & 3640135 & 1129 & 3637713152 & 3639053 \\
\hline 1179 & $3 / 5 / 2019$ & 1129 & 3674774784 & 3644347 & 1129 & 3658606848 & 3610910 & 1129 & 3641324288 & 3611231 \\
\hline 1180 & $3 / 6 / 2019$ & 1129 & 3678390784 & 3616158 & 1129 & 3662189312 & 3582373 & 1129 & 3644908288 & 3584047 \\
\hline
\end{tabular}




\begin{tabular}{|c|c|c|c|c|c|c|c|c|c|c|}
\hline 1181 & $3 / 7 / 2019$ & 1129 & 3681979392 & 3588611 & 1129 & 3665743872 & 3554501 & 1129 & 3648465920 & 3557479 \\
\hline 1182 & $3 / 8 / 2019$ & 1129 & 3685541120 & 3561686 & 1129 & 3669271040 & 3527273 & 1129 & 3651997440 & 3531508 \\
\hline 1183 & $3 / 9 / 2019$ & 1129 & 3689076480 & 3535363 & 1129 & 3672771840 & 3500667 & 1129 & 3655503360 & 3506115 \\
\hline 1184 & $3 / 10 / 2019$ & 1129 & 3692585984 & 3509623 & 1129 & 3676246528 & 3474663 & 1129 & 3658984704 & 3481281 \\
\hline 1185 & $3 / 11 / 2019$ & 1129 & 3696070656 & 3484446 & 1129 & 3679695616 & 3449242 & 1129 & 3662441728 & 3456988 \\
\hline 1186 & $3 / 12 / 2019$ & 1129 & 3699530240 & 3459816 & 1129 & 3683119872 & 3424386 & 1129 & 3665874944 & 3433219 \\
\hline 1187 & $3 / 13 / 2019$ & 1129 & 3702966016 & 3435715 & 1129 & 3686520064 & 3400076 & 1129 & 3669284864 & 3409959 \\
\hline 1188 & $3 / 14 / 2019$ & 1129 & 3706378240 & 3412128 & 1129 & 3689896448 & 3376297 & 1129 & 3672672000 & 3387192 \\
\hline 1189 & $3 / 15 / 2019$ & 1129 & 3709766912 & 3389039 & 1129 & 3693249536 & 3353031 & 1129 & 3676037120 & 3364902 \\
\hline 1190 & $3 / 16 / 2019$ & 1129 & 3713133568 & 3366432 & 1129 & 3696579584 & 3330263 & 1129 & 3679379968 & 3343076 \\
\hline 1191 & $3 / 17 / 2019$ & 1129 & 3716477952 & 3344294 & 1129 & 3699887616 & 3307979 & 1129 & 3682701824 & 3321700 \\
\hline 1192 & $3 / 18 / 2019$ & 1129 & 3719800576 & 3322612 & 1129 & 3703173888 & 3286163 & 1129 & 3686002432 & 3300761 \\
\hline 1193 & $3 / 19 / 2019$ & 1129 & 3723101952 & 3301370 & 1129 & 3706438656 & 3264802 & 1129 & 3689282816 & 3280246 \\
\hline 1194 & $3 / 20 / 2019$ & 1129 & 3726382848 & 3280558 & 1129 & 3709682688 & 3243883 & 1129 & 3692542976 & 3260142 \\
\hline 1195 & $3 / 21 / 2019$ & 1129 & 3729642752 & 3260163 & 1129 & 3712905984 & 3223393 & 1129 & 3695783680 & 3240439 \\
\hline 1196 & $3 / 22 / 2019$ & 1129 & 3732882944 & 3240172 & 1129 & 3716109312 & 3203318 & 1129 & 3699004416 & 3221124 \\
\hline 1197 & $3 / 23 / 2019$ & 1129 & 3736103424 & 3220575 & 1129 & 3719292928 & 3183649 & 1129 & 3702206464 & 3202187 \\
\hline 1198 & $3 / 24 / 2019$ & 1129 & 3739304704 & 3201361 & 1129 & 3722457344 & 3164374 & 1129 & 3705390336 & 3183617 \\
\hline 1199 & $3 / 25 / 2019$ & 1129 & 3742487296 & 3182518 & 1129 & 3725602816 & 3145480 & 1129 & 3708555776 & 3165405 \\
\hline 1200 & $3 / 26 / 2019$ & 1129 & 3745651456 & 3164038 & 1129 & 3728729600 & 3126958 & 1129 & 3711703296 & 3147540 \\
\hline 1201 & $3 / 27 / 2019$ & 1129 & 3748797184 & 3145910 & 1129 & 3731838464 & 3108797 & 1129 & 3714833152 & 3130013 \\
\hline 1202 & $3 / 28 / 2019$ & 1129 & 3751924992 & 3128124 & 1129 & 3734929408 & 3090988 & 1129 & 3717946112 & 3112815 \\
\hline 1203 & $3 / 29 / 2019$ & 1129 & 3755036160 & 3110672 & 1129 & 3738002944 & 3073521 & 1129 & 3721041920 & 3095937 \\
\hline 1204 & $3 / 30 / 2019$ & 1129 & 3758129664 & 3093544 & 1129 & 3741059328 & 3056387 & 1129 & 3724121344 & 3079370 \\
\hline 1205 & $3 / 31 / 2019$ & 1129 & 3761206272 & 3076733 & 1129 & 3744099072 & 3039577 & 1129 & 3727184384 & 3063108 \\
\hline 1206 & $4 / 1 / 2019$ & 1129 & 3764266496 & 3060229 & 1129 & 3747121920 & 3023082 & 1129 & 3730231552 & 3047140 \\
\hline 1207 & $4 / 2 / 2019$ & 1129 & 3767310848 & 3044026 & 1129 & 3750128896 & 3006895 & 1129 & 3733263104 & 3031462 \\
\hline 1208 & $4 / 3 / 2019$ & 1129 & 3770338560 & 3028115 & 1129 & 3753120000 & 2991006 & 1129 & 3736279040 & 3016063 \\
\hline 1209 & $4 / 4 / 2019$ & 1129 & 3773351168 & 3012488 & 1129 & 3756095232 & 2975410 & 1129 & 3739280128 & 3000938 \\
\hline 1210 & $4 / 5 / 2019$ & 1129 & 3776348416 & 2997140 & 1129 & 3759055360 & 2960096 & 1129 & 3742266112 & 2986080 \\
\hline 1211 & $4 / 6 / 2019$ & 1129 & 3779330304 & 2982062 & 1129 & 3762000640 & 2945060 & 1129 & 3745237760 & 2971482 \\
\hline 1212 & $4 / 7 / 2019$ & 1129 & 3782297600 & 2967248 & 1129 & 3764930816 & 2930293 & 1129 & 3748194816 & 2957137 \\
\hline 1213 & $4 / 8 / 2019$ & 1129 & 3785250304 & 2952691 & 1129 & 3767846656 & 2915789 & 1129 & 3751137792 & 2943039 \\
\hline 1214 & $4 / 9 / 2019$ & 1129 & 3788188672 & 2938386 & 1129 & 3770748160 & 2901542 & 1129 & 3754066944 & 2929183 \\
\hline 1215 & $4 / 10 / 2019$ & 1129 & 3791113216 & 2924325 & 1129 & 3773635328 & 2887545 & 1129 & 3756982528 & 2915562 \\
\hline 1216 & $4 / 11 / 2019$ & 1129 & 3794023424 & 2910504 & 1129 & 3776509440 & 2873791 & 1129 & 3759884800 & 2902170 \\
\hline 1217 & $4 / 12 / 2019$ & 1129 & 3796920320 & 2896916 & 1129 & 3779369728 & 2860276 & 1129 & 3762773760 & 2889003 \\
\hline 1218 & $4 / 13 / 2019$ & 1129 & 3799803904 & 2883556 & 1129 & 3782216704 & 2846992 & 1129 & 3765649408 & 2876054 \\
\hline 1219 & $4 / 14 / 2019$ & 1129 & 3802674432 & 2870418 & 1129 & 3785050624 & 2833936 & 1129 & 3768513024 & 2863319 \\
\hline 1220 & $4 / 15 / 2019$ & 1129 & 3805531904 & 2857497 & 1129 & 3787871744 & 2821100 & 1129 & 3771363840 & 2850793 \\
\hline 1221 & $4 / 16 / 2019$ & 1129 & 3808376576 & 2844789 & 1129 & 3790680320 & 2808480 & 1129 & 3774202368 & 2838470 \\
\hline 1222 & $4 / 17 / 2019$ & 1129 & 3811208960 & 2832287 & 1129 & 3793476352 & 2796071 & 1129 & 3777028352 & 2826346 \\
\hline 1223 & $4 / 18 / 2019$ & 1129 & 3814028800 & 2819988 & 1129 & 3796260096 & 2783868 & 1129 & 3779843072 & 2814416 \\
\hline 1224 & $4 / 19 / 2019$ & 1129 & 3816836864 & 2807887 & 1129 & 3799032064 & 2771866 & 1129 & 3782645760 & 2802677 \\
\hline 1225 & $4 / 20 / 2019$ & 1129 & 3819632896 & 2795979 & 1129 & 3801792000 & 2760060 & 1129 & 3785436928 & 2791123 \\
\hline 1226 & $4 / 21 / 2019$ & 1129 & 3822417152 & 2784259 & 1129 & 3804540672 & 2748446 & 1129 & 3788216320 & 2779751 \\
\hline 1227 & $4 / 22 / 2019$ & 1129 & 3825189888 & 2772725 & 1129 & 3807277568 & 2737020 & 1129 & 3790985216 & 2768555 \\
\hline 1228 & $4 / 23 / 2019$ & 1129 & 3827951104 & 2761371 & 1129 & 3810003456 & 2725776 & 1129 & 3793742848 & 2757534 \\
\hline 1229 & $4 / 24 / 2019$ & 1129 & 3830701312 & 2750193 & 1129 & 3812718080 & 2714712 & 1129 & 3796489472 & 2746682 \\
\hline 1230 & $4 / 25 / 2019$ & 1129 & 3833440512 & 2739188 & 1129 & 3815421952 & 2703823 & 1129 & 3799225344 & 2735996 \\
\hline 1231 & $4 / 26 / 2019$ & 1129 & 3836168960 & 2728352 & 1129 & 3818115072 & 2693104 & 1129 & 3801950976 & 2725472 \\
\hline 1232 & $4 / 27 / 2019$ & 1129 & 3838886656 & 2717681 & 1129 & 3820797440 & 2682554 & 1129 & 3804666112 & 2715106 \\
\hline 1233 & $4 / 28 / 2019$ & 1129 & 3841593856 & 2707171 & 1129 & 3823469824 & 2672166 & 1129 & 3807371008 & 2704896 \\
\hline 1234 & $4 / 29 / 2019$ & 1129 & 3844290560 & 2696819 & 1129 & 3826131712 & 2661939 & 1129 & 3810065664 & 2694838 \\
\hline 1235 & $4 / 30 / 2019$ & 1129 & 3846977280 & 2686623 & 1129 & 3828783616 & 2651869 & 1129 & 3812750592 & 2684928 \\
\hline 1236 & $5 / 1 / 2019$ & 1129 & 3849653760 & 2676577 & 1129 & 3831425536 & 2641951 & 1129 & 3815425792 & 2675164 \\
\hline 1237 & $5 / 2 / 2019$ & 1129 & 3852320512 & 2666680 & 1129 & 3834057728 & 2632183 & 1129 & 3818091520 & 2665543 \\
\hline 1238 & $5 / 3 / 2019$ & 1129 & 3854977280 & 2656927 & 1129 & 3836680192 & 2622562 & 1129 & 3820747520 & 2656060 \\
\hline 1239 & $5 / 4 / 2019$ & 1129 & 3857624832 & 2647317 & 1129 & 3839293440 & 2613085 & 1129 & 3823394048 & 2646715 \\
\hline 1240 & $5 / 5 / 2019$ & 1129 & 3860262656 & 2637846 & 1129 & 3841896960 & 2603748 & 1129 & 3826031616 & 2637502 \\
\hline 1241 & $5 / 6 / 2019$ & 1129 & 3862891264 & 2628510 & 1129 & 3844491520 & 2594548 & 1129 & 3828659968 & 2628421 \\
\hline 1242 & $5 / 7 / 2019$ & 1129 & 3865510400 & 2619309 & 1129 & 3847077120 & 2585483 & 1129 & 3831279616 & 2619468 \\
\hline 1243 & $5 / 8 / 2019$ & 1129 & 3868120576 & 2610238 & 1129 & 3849653760 & 2576550 & 1129 & 3833890304 & 2610641 \\
\hline 1244 & $5 / 9 / 2019$ & 1129 & 3870722048 & 2601295 & 1129 & 3852221440 & 2567746 & 1129 & 3836492288 & 2601937 \\
\hline 1245 & $5 / 10 / 2019$ & 1129 & 3873314304 & 2592477 & 1129 & 3854780416 & 2559068 & 1129 & 3839085568 & 2593353 \\
\hline 1246 & $5 / 11 / 2019$ & 1129 & 3875898368 & 2583782 & 1129 & 3857330944 & 2550514 & 1129 & 3841670400 & 2584888 \\
\hline 1247 & $5 / 12 / 2019$ & 1129 & 3878473472 & 2575208 & 1129 & 3859873024 & 2542081 & 1129 & 3844247040 & 2576538 \\
\hline 1248 & $5 / 13 / 2019$ & 1129 & 3881040128 & 2566751 & 1129 & 3862406912 & 2533768 & 1129 & 3846815232 & 2568302 \\
\hline 1249 & $5 / 14 / 2019$ & 1129 & 3883598592 & 2558410 & 1129 & 3864932352 & 2525570 & 1129 & 3849375488 & 2560177 \\
\hline 1250 & $5 / 15 / 2019$ & 1129 & 3886148608 & 2550183 & 1129 & 3867449856 & 2517487 & 1129 & 3851927552 & 2552161 \\
\hline 1251 & $5 / 16 / 2019$ & 1129 & 3888690688 & 2542067 & 1129 & 3869959424 & 2509515 & 1129 & 3854471936 & 2544252 \\
\hline 1252 & $5 / 17 / 2019$ & 1129 & 3891224832 & 2534059 & 1129 & 3872461056 & 2501652 & 1129 & 3857008384 & 2536448 \\
\hline 1253 & $5 / 18 / 2019$ & 1129 & 3893751040 & 2526159 & 1129 & 3874955008 & 2493898 & 1129 & 3859536896 & 2528746 \\
\hline 1254 & $5 / 19 / 2019$ & 1129 & 3896269312 & 2518364 & 1129 & 3877441280 & 2486248 & 1129 & 3862058240 & 2521146 \\
\hline 1255 & $5 / 20 / 2019$ & 1129 & 3898780160 & 2510670 & 1129 & 3879919872 & 2478702 & 1129 & 3864571904 & 2513644 \\
\hline 1256 & $5 / 21 / 2019$ & 1129 & 3901283072 & 2503078 & 1129 & 3882391040 & 2471257 & 1129 & 3867078144 & 2506239 \\
\hline 1257 & $5 / 22 / 2019$ & 1129 & 3903778816 & 2495585 & 1129 & 3884855296 & 2463911 & 1129 & 3869576960 & 2498930 \\
\hline 1258 & $5 / 23 / 2019$ & 1129 & 3906266880 & 2488189 & 1129 & 3887311616 & 2456663 & 1129 & 3872068608 & 2491714 \\
\hline 1259 & $5 / 24 / 2019$ & 1129 & 3908747776 & 2480888 & 1129 & 3889761280 & 2449509 & 1129 & 3874553344 & 2484590 \\
\hline 1260 & $5 / 25 / 2019$ & 1129 & 3911221504 & 2473681 & 1129 & 3892203776 & 2442450 & 1129 & 3877030912 & 2477555 \\
\hline 1261 & $5 / 26 / 2019$ & 1129 & 3913688064 & 2466565 & 1129 & 3894639104 & 2435482 & 1129 & 3879501312 & 2470609 \\
\hline 1262 & $5 / 27 / 2019$ & 1129 & 3916147456 & 2459539 & 1129 & 3897067776 & 2428604 & 1129 & 3881965056 & 2463749 \\
\hline
\end{tabular}




\begin{tabular}{|c|c|c|c|c|c|c|c|c|c|c|}
\hline 1263 & $5 / 28 / 2019$ & 1129 & 3918600192 & 2452601 & 1129 & 3899489536 & 2421815 & 1129 & 3884422144 & 2456975 \\
\hline 1264 & $5 / 29 / 2019$ & 1129 & 3921046016 & 2445750 & 1129 & 3901904640 & 2415112 & 1129 & 3886872320 & 2450283 \\
\hline 1265 & $5 / 30 / 2019$ & 1129 & 3923484928 & 2438984 & 1129 & 3904313088 & 2408495 & 1129 & 3889316096 & 2443674 \\
\hline 1266 & $5 / 31 / 2019$ & 1129 & 3925917184 & 2432302 & 1129 & 3906715136 & 2401961 & 1129 & 3891753216 & 2437145 \\
\hline 1267 & $6 / 1 / 2019$ & 1129 & 3928343040 & 2425702 & 1129 & 3909110528 & 2395509 & 1129 & 3894183936 & 2430696 \\
\hline 1268 & $6 / 2 / 2019$ & 1129 & 3930761984 & 2419182 & 1129 & 3911499776 & 2389137 & 1129 & 3896608256 & 2424323 \\
\hline 1269 & $6 / 3 / 2019$ & 1129 & 3933174784 & 2412741 & 1129 & 3913882624 & 2382844 & 1129 & 3899026176 & 2418027 \\
\hline 1270 & $6 / 4 / 2019$ & 1129 & 3935581184 & 2406378 & 1129 & 3916259328 & 2376629 & 1129 & 3901438208 & 2411806 \\
\hline 1271 & $6 / 5 / 2019$ & 1129 & 3937981184 & 2400091 & 1129 & 3918629632 & 2370491 & 1129 & 3903843840 & 2405658 \\
\hline 1272 & $6 / 6 / 2019$ & 1129 & 3940375296 & 2393879 & 1129 & 3920994304 & 2364426 & 1129 & 3906243328 & 2399583 \\
\hline 1273 & $6 / 7 / 2019$ & 1129 & 3942763008 & 2387741 & 1129 & 3923352576 & 2358435 & 1129 & 3908636928 & 2393578 \\
\hline 1274 & $6 / 8 / 2019$ & 1129 & 3945144576 & 2381675 & 1129 & 3925705216 & 2352517 & 1129 & 3911024640 & 2387644 \\
\hline 1275 & $6 / 9 / 2019$ & 1129 & 3947520256 & 2375680 & 1129 & 3928051712 & 2346669 & 1129 & 3913406464 & 2381777 \\
\hline 1276 & $6 / 10 / 2019$ & 1129 & 3949890048 & 2369755 & 1129 & 3930392576 & 2340890 & 1129 & 3915782400 & 2375978 \\
\hline 1277 & $6 / 11 / 2019$ & 1129 & 3952253952 & 2363899 & 1129 & 3932727808 & 2335180 & 1129 & 3918152448 & 2370245 \\
\hline 1278 & $6 / 12 / 2019$ & 1129 & 3954611968 & 2358110 & 1129 & 3935057408 & 2329537 & 1129 & 3920517120 & 2364577 \\
\hline 1279 & $6 / 13 / 2019$ & 1129 & 3956964352 & 2352387 & 1129 & 3937381376 & 2323960 & 1129 & 3922876160 & 2358973 \\
\hline 1280 & $6 / 14 / 2019$ & 1129 & 3959311104 & 2346730 & 1129 & 3939699712 & 2318447 & 1129 & 3925229568 & 2353432 \\
\hline 1281 & $6 / 15 / 2019$ & 1129 & 3961652224 & 2341136 & 1129 & 3942012928 & 2312999 & 1129 & 3927577856 & 2347952 \\
\hline 1282 & $6 / 16 / 2019$ & 1129 & 3963987968 & 2335605 & 1129 & 3944320512 & 2307612 & 1129 & 3929920000 & 2342533 \\
\hline 1283 & $6 / 17 / 2019$ & 1129 & 3966318080 & 2330137 & 1129 & 3946622720 & 2302288 & 1129 & 3932257280 & 2337174 \\
\hline 1284 & $6 / 18 / 2019$ & 1129 & 3968642816 & 2324728 & 1129 & 3948919808 & 2297023 & 1129 & 3934589184 & 2331874 \\
\hline 1285 & $6 / 19 / 2019$ & 1129 & 3970962176 & 2319381 & 1129 & 3951211520 & 2291818 & 1129 & 3936915712 & 2326631 \\
\hline 1286 & $6 / 20 / 2019$ & 1129 & 3973276160 & 2314091 & 1129 & 3953498112 & 2286672 & 1129 & 3939237120 & 2321445 \\
\hline 1287 & $6 / 21 / 2019$ & 1129 & 3975585024 & 2308860 & 1129 & 3955779840 & 2281582 & 1129 & 3941553408 & 2316314 \\
\hline 1288 & $6 / 22 / 2019$ & 1129 & 3977888768 & 2303685 & 1129 & 3958056448 & 2276549 & 1129 & 3943864832 & 2311239 \\
\hline 1289 & $6 / 23 / 2019$ & 1129 & 3980187136 & 2298566 & 1129 & 3960327936 & 2271572 & 1129 & 3946170880 & 2306218 \\
\hline 1290 & $6 / 24 / 2019$ & 1129 & 3982480896 & 2293503 & 1129 & 3962594560 & 2266649 & 1129 & 3948472064 & 2301250 \\
\hline 1291 & $6 / 25 / 2019$ & 1129 & 3984769280 & 2288493 & 1129 & 3964856576 & 2261780 & 1129 & 3950768640 & 2296334 \\
\hline 1292 & $6 / 26 / 2019$ & 1129 & 3987052800 & 2283537 & 1129 & 3967113216 & 2256963 & 1129 & 3953060352 & 2291469 \\
\hline 1293 & $6 / 27 / 2019$ & 1129 & 3989331456 & 2278633 & 1129 & 3969365504 & 2252198 & 1129 & 3955346688 & 2286655 \\
\hline 1294 & $6 / 28 / 2019$ & 1129 & 3991605248 & 2273781 & 1129 & 3971612928 & 2247484 & 1129 & 3957628416 & 2281891 \\
\hline 1295 & $6 / 29 / 2019$ & 1129 & 3993874176 & 2268979 & 1129 & 3973855744 & 2242820 & 1129 & 3959905792 & 2277175 \\
\hline 1296 & $6 / 30 / 2019$ & 1129 & 3996138496 & 2264227 & 1129 & 3976093952 & 2238206 & 1129 & 3962178304 & 2272508 \\
\hline 1297 & $7 / 1 / 2019$ & 1129 & 3998397952 & 2259524 & 1129 & 3978327552 & 2233640 & 1129 & 3964446208 & 2267889 \\
\hline 1298 & $7 / 2 / 2019$ & 1129 & 4000652800 & 2254870 & 1129 & 3980556800 & 2229122 & 1129 & 3966709504 & 2263316 \\
\hline 1299 & $7 / 3 / 2019$ & 1129 & 4002903040 & 2250263 & 1129 & 3982781440 & 2224651 & 1129 & 3968968192 & 2258789 \\
\hline 1300 & $7 / 4 / 2019$ & 1129 & 4005148672 & 2245703 & 1129 & 3985001728 & 2220226 & 1129 & 3971222528 & 2254307 \\
\hline 1301 & $7 / 5 / 2019$ & 1129 & 4007389952 & 2241189 & 1129 & 3987217408 & 2215846 & 1129 & 3973472512 & 2249869 \\
\hline 1302 & $7 / 6 / 2019$ & 1129 & 4009626880 & 2236721 & 1129 & 3989428992 & 2211512 & 1129 & 3975717888 & 2245476 \\
\hline 1303 & $7 / 7 / 2019$ & 1129 & 4011858944 & 2232297 & 1129 & 3991636224 & 2207221 & 1129 & 3977959168 & 2241126 \\
\hline 1304 & $7 / 8 / 2019$ & 1129 & 4014086912 & 2227917 & 1129 & 3993839104 & 2202973 & 1129 & 3980195840 & 2236818 \\
\hline 1305 & $7 / 9 / 2019$ & 1129 & 4016310528 & 2223580 & 1129 & 3996037888 & 2198769 & 1129 & 3982428416 & 2232552 \\
\hline 1306 & $7 / 10 / 2019$ & 1129 & 4018529536 & 2219286 & 1129 & 3998232576 & 2194606 & 1129 & 3984656640 & 2228327 \\
\hline 1307 & $7 / 11 / 2019$ & 1129 & 4020744960 & 2215034 & 1129 & 4000423168 & 2190484 & 1129 & 3986880768 & 2224143 \\
\hline 1308 & $7 / 12 / 2019$ & 1129 & 4022955776 & 2210823 & 1129 & 4002609408 & 2186404 & 1129 & 3989100800 & 2219999 \\
\hline 1309 & $7 / 13 / 2019$ & 1486 & 4022955776 & 98 & 1486 & 4002609408 & 110 & 1486 & 3989100800 & 0 \\
\hline 1310 & $7 / 14 / 2019$ & 1486 & 4022962176 & 6319 & 1486 & 4002622208 & 12529 & 1486 & 3989112320 & 11555 \\
\hline 1311 & $7 / 15 / 2019$ & 1486 & 4022998016 & 35768 & 1486 & 4002654464 & 32315 & 1486 & 3989154304 & 41805 \\
\hline 1312 & $7 / 16 / 2019$ & 1486 & 4023062528 & 64860 & 1486 & 4002715392 & 60836 & 1486 & 3989223936 & 69760 \\
\hline 1313 & $7 / 17 / 2019$ & 1486 & 4023140352 & 77695 & 1486 & 4002788352 & 73080 & 1486 & 3989306368 & 82309 \\
\hline 1314 & $7 / 18 / 2019$ & 1486 & 4023226112 & 85657 & 1486 & 4002868992 & 80603 & 1486 & 3989396480 & 90168 \\
\hline 1315 & $7 / 19 / 2019$ & 1486 & 4023317504 & 91507 & 1486 & 4002955008 & 86102 & 1486 & 3989492480 & 95973 \\
\hline 1316 & $7 / 20 / 2019$ & 1486 & 4023413760 & 96248 & 1486 & 4003045632 & 90549 & 1486 & 3989593088 & 100692 \\
\hline 1317 & $7 / 21 / 2019$ & 1486 & 4023514112 & 100284 & 1486 & 4003139840 & 94331 & 1486 & 3989698048 & 104709 \\
\hline 1318 & $7 / 22 / 2019$ & 1486 & 4023618048 & 103806 & 1486 & 4003237632 & 97631 & 1486 & 3989806080 & 108216 \\
\hline 1319 & $7 / 23 / 2019$ & 1486 & 4023724800 & 106920 & 1486 & 4003338240 & 100550 & 1486 & 3989917440 & 111316 \\
\hline 1320 & $7 / 24 / 2019$ & 1486 & 4023834368 & 109695 & 1486 & 4003441152 & 103151 & 1486 & 3990031360 & 114078 \\
\hline 1321 & $7 / 25 / 2019$ & 1486 & 4023946752 & 112181 & 1486 & 4003546624 & 105483 & 1486 & 3990148096 & 116553 \\
\hline 1322 & $7 / 26 / 2019$ & 1486 & 4024061184 & 114423 & 1486 & 4003654400 & 107588 & 1486 & 3990266624 & 118783 \\
\hline 1323 & $7 / 27 / 2019$ & 1486 & 4024177664 & 116448 & 1486 & 4003763712 & 109491 & 1486 & 3990387456 & 120798 \\
\hline 1324 & $7 / 28 / 2019$ & 1486 & 4024295936 & 118285 & 1486 & 4003875328 & 111220 & 1486 & 3990510080 & 122625 \\
\hline 1325 & $7 / 29 / 2019$ & 1486 & 4024415744 & 119961 & 1486 & 4003987968 & 112798 & 1486 & 3990634496 & 124292 \\
\hline 1326 & $7 / 30 / 2019$ & 1486 & 4024537344 & 121496 & 1486 & 4004102144 & 114246 & 1486 & 3990760192 & 125819 \\
\hline 1327 & $7 / 31 / 2019$ & 1486 & 4024660224 & 122909 & 1486 & 4004217600 & 115581 & 1486 & 3990887424 & 127223 \\
\hline 1328 & $8 / 1 / 2019$ & 1486 & 4024784384 & 124214 & 1486 & 4004334592 & 116816 & 1486 & 3991015936 & 128521 \\
\hline 1329 & $8 / 2 / 2019$ & 1486 & 4024910336 & 125842 & 1486 & 4004452352 & 117965 & 1486 & 3991145728 & 129726 \\
\hline 1330 & $8 / 3 / 2019$ & 1486 & 4025039104 & 128793 & 1486 & 4004571392 & 119037 & 1486 & 3991276544 & 130849 \\
\hline 1331 & $8 / 4 / 2019$ & 1486 & 4025170176 & 131031 & 1486 & 4004691456 & 120043 & 1486 & 3991408384 & 131900 \\
\hline 1332 & $8 / 5 / 2019$ & 1486 & 4025303040 & 132969 & 1486 & 4004812544 & 120989 & 1486 & 3991541504 & 132887 \\
\hline 1333 & $8 / 6 / 2019$ & 1486 & 4025437952 & 134721 & 1486 & 4004934400 & 121884 & 1486 & 3991675136 & 133819 \\
\hline 1334 & $8 / 7 / 2019$ & 1486 & 4025574144 & 136339 & 1486 & 4005057024 & 122732 & 1486 & 3991809792 & 134700 \\
\hline 1335 & $8 / 8 / 2019$ & 1486 & 4025712128 & 137850 & 1486 & 4005180672 & 123539 & 1486 & 3991945472 & 135536 \\
\hline 1336 & $8 / 9 / 2019$ & 1486 & 4025851392 & 139270 & 1486 & 4005305088 & 124503 & 1486 & 3992082432 & 137085 \\
\hline 1337 & $8 / 10 / 2019$ & 1486 & 4025992192 & 140613 & 1486 & 4005432064 & 126771 & 1486 & 3992221952 & 139348 \\
\hline 1338 & $8 / 11 / 2019$ & 1486 & 4026134016 & 141887 & 1486 & 4005560832 & 128543 & 1486 & 3992364288 & 142318 \\
\hline 1339 & $8 / 12 / 2019$ & 1486 & 4026276864 & 143100 & 1486 & 4005690624 & 130083 & 1486 & 3992509184 & 144976 \\
\hline 1340 & $8 / 13 / 2019$ & 1486 & 4026421248 & 144258 & 1486 & 4005821952 & 131490 & 1486 & 3992657408 & 148048 \\
\hline 1341 & $8 / 14 / 2019$ & 1486 & 4026567168 & 145998 & 1486 & 4005955328 & 133239 & 1486 & 3992811520 & 154405 \\
\hline 1342 & $8 / 15 / 2019$ & 1486 & 4026715136 & 148095 & 1486 & 4006090240 & 134902 & 1486 & 3992971008 & 159503 \\
\hline 1343 & $8 / 16 / 2019$ & 1486 & 4026867200 & 152075 & 1486 & 4006226688 & 136404 & 1486 & 3993138688 & 167459 \\
\hline 1344 & $8 / 17 / 2019$ & 1486 & 4027022592 & 155296 & 1486 & 4006364416 & 137811 & 1486 & 3993312512 & 174046 \\
\hline
\end{tabular}




\begin{tabular}{|c|c|c|c|c|c|c|c|c|c|c|}
\hline 1345 & $8 / 18 / 2019$ & 1486 & 4027181568 & 158875 & 1486 & 4006505216 & 140669 & 1486 & 3993492224 & 179687 \\
\hline 1346 & $8 / 19 / 2019$ & 1486 & 4027344896 & 163127 & 1486 & 4006649088 & 143800 & 1486 & 3993677056 & 184829 \\
\hline 1347 & $8 / 20 / 2019$ & 1486 & 4027512320 & 167601 & 1486 & 4006796032 & 147197 & 1486 & 3993867008 & 189755 \\
\hline 1348 & $8 / 21 / 2019$ & 1486 & 4027684096 & 171888 & 1486 & 4006947072 & 150922 & 1486 & 3994062080 & 195202 \\
\hline 1349 & $8 / 22 / 2019$ & 1486 & 4027861760 & 177698 & 1486 & 4007102720 & 155730 & 1486 & 3994265344 & 203207 \\
\hline 1350 & $8 / 23 / 2019$ & 1486 & 4028044288 & 182541 & 1486 & 4007262976 & 160167 & 1486 & 3994477824 & 212536 \\
\hline 1351 & $8 / 24 / 2019$ & 1486 & 4028231424 & 186938 & 1486 & 4007426816 & 164000 & 1486 & 3994699008 & 221140 \\
\hline 1352 & $8 / 25 / 2019$ & 1486 & 4028422912 & 191513 & 1486 & 4007595520 & 168543 & 1486 & 3994928384 & 229457 \\
\hline 1353 & $8 / 26 / 2019$ & 1486 & 4028620800 & 197986 & 1486 & 4007770624 & 175279 & 1486 & 3995165696 & 237257 \\
\hline 1354 & $8 / 27 / 2019$ & 1486 & 4028824576 & 203819 & 1486 & 4007953152 & 182420 & 1486 & 3995410944 & 245380 \\
\hline 1355 & $8 / 28 / 2019$ & 1486 & 4029033472 & 208973 & 1486 & 4008142336 & 189194 & 1486 & 3995665152 & 254066 \\
\hline 1356 & $8 / 29 / 2019$ & 1486 & 4029249536 & 215913 & 1486 & 4008340224 & 197790 & 1486 & 3995929344 & 264097 \\
\hline 1357 & $8 / 30 / 2019$ & 1486 & 4029473536 & 224100 & 1486 & 4008549120 & 209101 & 1486 & 3996204800 & 275584 \\
\hline 1358 & $8 / 31 / 2019$ & 1486 & 4029705472 & 232003 & 1486 & 4008769280 & 219929 & 1486 & 3996491264 & 286502 \\
\hline 1359 & $9 / 1 / 2019$ & 1486 & 4029944832 & 239286 & 1486 & 4008998912 & 229873 & 1486 & 3996788224 & 297022 \\
\hline 1360 & $9 / 2 / 2019$ & 1486 & 4030193152 & 248349 & 1486 & 4009238272 & 239117 & 1486 & 3997096448 & 308026 \\
\hline 1361 & $9 / 3 / 2019$ & 1486 & 4030454528 & 261207 & 1486 & 4009486848 & 248691 & 1486 & 3997417472 & 321221 \\
\hline 1362 & $9 / 4 / 2019$ & 1486 & 4030729216 & 274665 & 1486 & 4009745408 & 258609 & 1486 & 3997751552 & 333890 \\
\hline 1363 & $9 / 5 / 2019$ & 1486 & 4031016704 & 287489 & 1486 & 4010014976 & 269599 & 1486 & 3998099968 & 348491 \\
\hline 1364 & $9 / 6 / 2019$ & 1486 & 4031315456 & 298880 & 1486 & 4010296576 & 281639 & 1486 & 3998461952 & 362056 \\
\hline 1365 & 9/7/2019 & 1486 & 4031624960 & 309505 & 1486 & 4010589952 & 293375 & 1486 & 3998838016 & 375961 \\
\hline 1366 & $9 / 8 / 2019$ & 1486 & 4031944448 & 319589 & 1486 & 4010894336 & 304262 & 1486 & 3999229440 & 391550 \\
\hline 1367 & 9/9/2019 & 1486 & 4032274176 & 329625 & 1486 & 4011208960 & 314669 & 1486 & 3999636480 & 407058 \\
\hline 1368 & $9 / 10 / 2019$ & 1486 & 4032613888 & 339737 & 1486 & 4011535360 & 326318 & 1486 & 4000057600 & 421105 \\
\hline 1369 & $9 / 11 / 2019$ & 1486 & 4032963840 & 349912 & 1486 & 4011872512 & 337214 & 1486 & 4000492800 & 435073 \\
\hline 1370 & $9 / 12 / 2019$ & 1486 & 4033323776 & 360025 & 1486 & 4012219904 & 347397 & 1486 & 4000943360 & 450618 \\
\hline 1371 & $9 / 13 / 2019$ & 1486 & 4033694720 & 370749 & 1486 & 4012577024 & 357142 & 1486 & 4001409024 & 465756 \\
\hline 1372 & $9 / 14 / 2019$ & 1486 & 4034078464 & 383827 & 1486 & 4012943616 & 366556 & 1486 & 4001889280 & 480102 \\
\hline 1373 & $9 / 15 / 2019$ & 1486 & 4034477824 & 399468 & 1486 & 4013319424 & 375847 & 1486 & 4002383872 & 494620 \\
\hline 1374 & $9 / 16 / 2019$ & 1486 & 4034892288 & 414410 & 1486 & 4013704960 & 385500 & 1486 & 4002893056 & 509542 \\
\hline 1375 & $9 / 17 / 2019$ & 1486 & 4035319552 & 427486 & 1486 & 4014101760 & 396721 & 1486 & 4003418112 & 524741 \\
\hline 1376 & $9 / 18 / 2019$ & 1486 & 4035761408 & 441853 & 1486 & 4014509568 & 407857 & 1486 & 4003958528 & 540306 \\
\hline 1377 & $9 / 19 / 2019$ & 1486 & 4036219648 & 457904 & 1486 & 4014928640 & 419044 & 1486 & 4004513024 & 554663 \\
\hline 1378 & $9 / 20 / 2019$ & 1486 & 4036691968 & 472387 & 1486 & 4015359232 & 430596 & 1486 & 4005081600 & 568552 \\
\hline 1379 & $9 / 21 / 2019$ & 1486 & 4037178368 & 486296 & 1486 & 4015803136 & 443863 & 1486 & 4005664000 & 582332 \\
\hline 1380 & $9 / 22 / 2019$ & 1486 & 4037677824 & 499523 & 1486 & 4016260096 & 457170 & 1486 & 4006259968 & 595971 \\
\hline 1381 & $9 / 23 / 2019$ & 1486 & 4038190848 & 513066 & 1486 & 4016730880 & 470717 & 1486 & 4006871040 & 610948 \\
\hline 1382 & $9 / 24 / 2019$ & 1486 & 4038718976 & 528023 & 1486 & 4017215232 & 484161 & 1486 & 4007496704 & 625715 \\
\hline 1383 & $9 / 25 / 2019$ & 1486 & 4039262208 & 543408 & 1486 & 4017714688 & 499702 & 1486 & 4008137216 & 640619 \\
\hline 1384 & $9 / 26 / 2019$ & 1486 & 4039820288 & 558035 & 1486 & 4018228992 & 514171 & 1486 & 4008792064 & 654857 \\
\hline 1385 & $9 / 27 / 2019$ & 1486 & 4040391936 & 571554 & 1486 & 4018757632 & 528629 & 1486 & 4009461760 & 669681 \\
\hline 1386 & $9 / 28 / 2019$ & 1486 & 4040976640 & 584596 & 1486 & 4019301120 & 543396 & 1486 & 4010146816 & 685110 \\
\hline 1387 & $9 / 29 / 2019$ & 1486 & 4041574400 & 597852 & 1486 & 4019858176 & 557227 & 1486 & 4010846976 & 700199 \\
\hline 1388 & $9 / 30 / 2019$ & 1486 & 4042184960 & 610728 & 1486 & 4020429824 & 571677 & 1486 & 4011562240 & 715105 \\
\hline 1389 & $10 / 1 / 2019$ & 1486 & 4042809600 & 624586 & 1486 & 4021016832 & 586859 & 1486 & 4012291840 & 729679 \\
\hline 1390 & $10 / 2 / 2019$ & 1486 & 4043448320 & 638703 & 1486 & 4021619712 & 603172 & 1486 & 4013035264 & 743274 \\
\hline 1391 & $10 / 3 / 2019$ & 1486 & 4044102400 & 654029 & 1486 & 4022238464 & 618444 & 1486 & 4013791744 & 756484 \\
\hline 1392 & $10 / 4 / 2019$ & 1486 & 4044770560 & 668315 & 1486 & 4022873088 & 634692 & 1486 & 4014561024 & 769553 \\
\hline 1393 & $10 / 5 / 2019$ & 1486 & 4045453824 & 683268 & 1486 & 4023524352 & 651286 & 1486 & 4015343616 & 782344 \\
\hline 1394 & $10 / 6 / 2019$ & 1486 & 4046151168 & 697341 & 1486 & 4024190976 & 666612 & 1486 & 4016138752 & 795231 \\
\hline 1395 & $10 / 7 / 2019$ & 1486 & 4046863104 & 711778 & 1486 & 4024872960 & 681973 & 1486 & 4016947200 & 808439 \\
\hline 1396 & $10 / 8 / 2019$ & 1486 & 4047590400 & 727426 & 1486 & 4025570816 & 697877 & 1486 & 4017768960 & 821704 \\
\hline 1397 & $10 / 9 / 2019$ & 1486 & 4048333312 & 742748 & 1486 & 4026284544 & 713745 & 1486 & 4018603520 & 834380 \\
\hline 1398 & $10 / 10 / 2019$ & 1486 & 4049090304 & 757161 & 1486 & 4027013376 & 728739 & 1486 & 4019450368 & 847053 \\
\hline 1399 & $10 / 11 / 2019$ & 1486 & 4049862656 & 772362 & 1486 & 4027758080 & 744703 & 1486 & 4020310016 & 859680 \\
\hline 1400 & $10 / 12 / 2019$ & 1486 & 4050649088 & 786270 & 1486 & 4028518656 & 760662 & 1486 & 4021181440 & 871510 \\
\hline 1401 & $10 / 13 / 2019$ & 1486 & 4051449088 & 799975 & 1486 & 4029294336 & 775756 & 1486 & 4022064640 & 882929 \\
\hline 1402 & $10 / 14 / 2019$ & 1486 & 4052262912 & 813911 & 1486 & 4030085632 & 791250 & 1486 & 4022958592 & 894050 \\
\hline 1403 & $10 / 15 / 2019$ & 1486 & 4053089792 & 826960 & 1486 & 4030891520 & 805885 & 1486 & 4023868160 & 909630 \\
\hline 1404 & $10 / 16 / 2019$ & 1486 & 4053928960 & 839484 & 1486 & 4031711232 & 820049 & 1486 & 4024783872 & 915681 \\
\hline 1405 & $10 / 17 / 2019$ & 1486 & 4054781184 & 851760 & 1486 & 4032545024 & 833500 & 1486 & 4025709824 & 925956 \\
\hline 1406 & $10 / 18 / 2019$ & 1486 & 4055644928 & 863511 & 1486 & 4033391360 & 846619 & 1486 & 4026646272 & 936516 \\
\hline 1407 & $10 / 19 / 2019$ & 1486 & 4056529664 & 885001 & 1486 & 4034251008 & 859237 & 1486 & 4027593216 & 946819 \\
\hline 1408 & $10 / 20 / 2019$ & 1486 & 4057416960 & 887308 & 1486 & 4035122432 & 871351 & 1486 & 4028550144 & 957260 \\
\hline 1409 & $10 / 21 / 2019$ & 1486 & 4058320640 & 903719 & 1486 & 4036005376 & 883176 & 1486 & 4029517568 & 967270 \\
\hline 1410 & $10 / 22 / 2019$ & 1486 & 4059230976 & 910283 & 1486 & 4036900608 & 894998 & 1486 & 4030494720 & 977146 \\
\hline 1411 & $10 / 23 / 2019$ & 1486 & 4060152064 & 921106 & 1486 & 4037806848 & 906412 & 1486 & 4031481344 & 986561 \\
\hline 1412 & $10 / 24 / 2019$ & 1486 & 4061086720 & 934703 & 1486 & 4038724608 & 917716 & 1486 & 4032476928 & 995662 \\
\hline 1413 & $10 / 25 / 2019$ & 1486 & 4062028800 & 942058 & 1486 & 4039653376 & 928865 & 1486 & 4033481728 & 1004498 \\
\hline 1414 & $10 / 26 / 2019$ & 1486 & 4062980864 & 952006 & 1486 & 4040595456 & 941888 & 1486 & 4034494720 & 1013219 \\
\hline 1415 & $10 / 27 / 2019$ & 1486 & 4063942400 & 961661 & 1486 & 4041545472 & 950179 & 1486 & 4035516416 & 1021712 \\
\hline 1416 & $10 / 28 / 2019$ & 1486 & 4064913920 & 971433 & 1486 & 4042505984 & 960420 & 1486 & 4036546304 & 1029968 \\
\hline 1417 & $10 / 29 / 2019$ & 1486 & 4065894912 & 980926 & 1486 & 4043476224 & 970284 & 1486 & 4037584384 & 1037987 \\
\hline 1418 & $10 / 30 / 2019$ & 1486 & 4066884864 & 990039 & 1486 & 4044456192 & 979844 & 1486 & 4038630400 & 1045960 \\
\hline 1419 & $10 / 31 / 2019$ & 1486 & 4067883776 & 998878 & 1486 & 4045448192 & 992141 & 1486 & 4039684096 & 1053809 \\
\hline 1420 & $11 / 1 / 2019$ & 1486 & 4068891136 & 1007475 & 1486 & 4046447616 & 999402 & 1486 & 4040745728 & 1061515 \\
\hline 1421 & $11 / 2 / 2019$ & 1486 & 4069907200 & 1016005 & 1486 & 4047455488 & 1007740 & 1486 & 4041815040 & 1069235 \\
\hline 1422 & $11 / 3 / 2019$ & 1486 & 4070931456 & 1024299 & 1486 & 4048471808 & 1016514 & 1486 & 4042891008 & 1076360 \\
\hline 1423 & $11 / 4 / 2019$ & 1486 & 4071964160 & 1032548 & 1486 & 4049496832 & 1025029 & 1486 & 4043974912 & 1083536 \\
\hline 1424 & $11 / 5 / 2019$ & 1486 & 4073004544 & 1040496 & 1486 & 4050530304 & 1033309 & 1486 & 4045065472 & 1090574 \\
\hline 1425 & $11 / 6 / 2019$ & 1486 & 4074052864 & 1048213 & 1486 & 4051571712 & 1041483 & 1486 & 4046162944 & 1097470 \\
\hline 1426 & $11 / 7 / 2019$ & 1486 & 4075108608 & 1055725 & 1486 & 4052621056 & 1049428 & 1486 & 4047267072 & 1104143 \\
\hline
\end{tabular}




\begin{tabular}{|c|c|c|c|c|c|c|c|c|c|c|}
\hline 1427 & $11 / 8 / 2019$ & 1486 & 4076171520 & 1063047 & 1486 & 4053678336 & 1057144 & 1486 & 4048377600 & 1110639 \\
\hline 1428 & $11 / 9 / 2019$ & 1486 & 4077241856 & 1070222 & 1486 & 4054743040 & 1064657 & 1486 & 4049494528 & 1116971 \\
\hline 1429 & $11 / 10 / 2019$ & 1486 & 4078319104 & 1077263 & 1486 & 4055814912 & 1071978 & 1486 & 4050617856 & 1123149 \\
\hline 1430 & $11 / 11 / 2019$ & 1486 & 4079403008 & 1084106 & 1486 & 4056893696 & 1079116 & 1486 & 4051746816 & 1129179 \\
\hline 1431 & $11 / 12 / 2019$ & 1486 & 4080493824 & 1090776 & 1486 & 4057980160 & 1086079 & 1486 & 4052881920 & 1135067 \\
\hline 1432 & $11 / 13 / 2019$ & 1486 & 4081591296 & 1097285 & 1486 & 4059073280 & 1092871 & 1486 & 4054022912 & 1140866 \\
\hline 1433 & $11 / 14 / 2019$ & 1486 & 4082694912 & 1103640 & 1486 & 4060172544 & 1099500 & 1486 & 4055169536 & 1146562 \\
\hline 1434 & $11 / 15 / 2019$ & 1486 & 4083804672 & 1109846 & 1486 & 4061278464 & 1105969 & 1486 & 4056321536 & 1152096 \\
\hline
\end{tabular}

Table 152: Case Study 2 - CMG Results

\begin{tabular}{|c|c|c|c|c|c|c|c|c|c|c|}
\hline \multirow[b]{2}{*}{ Time (day) } & \multirow[b]{2}{*}{ Date } & \multicolumn{3}{|c|}{ 45ft Cluster Spacing } & \multicolumn{3}{|c|}{ 40ft Cluster Spacing } & \multicolumn{3}{|c|}{ 35ft Cluster Spacing } \\
\hline & & $\begin{array}{l}\text { Well Bottom- } \\
\text { hole } \\
\text { Pressure (psi) }\end{array}$ & $\begin{array}{l}\text { Cumulative } \\
\text { Gas SC }\left(\mathrm{ft}^{3}\right)\end{array}$ & $\begin{array}{l}\text { Gas Rate } \\
\left(\mathrm{ft}^{3} / \text { day) }\right.\end{array}$ & $\begin{array}{l}\text { Well Bottom- } \\
\text { hole } \\
\text { Pressure (psi) }\end{array}$ & $\begin{array}{l}\text { Cumulative } \\
\text { Gas SC }\left(\mathrm{ft}^{3}\right)\end{array}$ & $\begin{array}{l}\text { Gas Rate } \\
\left(\mathrm{ft}^{3} / \text { day) }\right.\end{array}$ & $\begin{array}{l}\text { Well Bottom- } \\
\text { hole } \\
\text { Pressure (psi) }\end{array}$ & $\begin{array}{l}\text { Cumulative } \\
\text { Gas SC }\left(\mathrm{ft}^{3}\right)\end{array}$ & $\begin{array}{l}\text { Gas Rate } \\
\left(\mathrm{ft}^{3} / \text { day) }\right.\end{array}$ \\
\hline 0 & $12 / 12 / 2015$ & & 0 & 0 & & 0 & 0 & & 0 & 0 \\
\hline 1 & $12 / 13 / 2015$ & 3850 & 29656842 & 29656842 & 3850 & 31805480 & 31805480 & 3850 & 35216988 & 35216988 \\
\hline 2 & $12 / 14 / 2015$ & 3850 & 57732324 & 28075484 & 3850 & 61466144 & 29660664 & 3850 & 65820860 & 30603874 \\
\hline 3 & $12 / 15 / 2015$ & 3850 & 77920296 & 20187970 & 3850 & 82695848 & 21229702 & 3850 & 87389848 & 21568988 \\
\hline 4 & $12 / 16 / 2015$ & 3850 & 94910456 & 16990160 & 3850 & 100259536 & 17563688 & 3850 & 105706880 & 18317032 \\
\hline 5 & $12 / 17 / 2015$ & 3850 & 109953712 & 15043259 & 3850 & 115722808 & 15463278 & 3850 & 121714616 & 16007735 \\
\hline 6 & $12 / 18 / 2015$ & 3850 & 123526672 & 13572959 & 3850 & 129596176 & 13873367 & 3850 & 135987168 & 14272561 \\
\hline 7 & $12 / 19 / 2015$ & 3850 & 135940128 & 12413447 & 3850 & 142215728 & 12619545 & 3850 & 148905200 & 12918002 \\
\hline 8 & $12 / 20 / 2015$ & 3850 & 147407872 & 11467746 & 3850 & 153813904 & 11598165 & 3850 & 160733648 & 11828472 \\
\hline 9 & $12 / 21 / 2015$ & 3850 & 158083008 & 10675151 & 3850 & 164557856 & 10743969 & 3850 & 171663536 & 10929884 \\
\hline 10 & $12 / 22 / 2015$ & 3850 & 168078944 & 9995927 & 3850 & 174571952 & 10014098 & 3850 & 181836352 & 10172821 \\
\hline 11 & $12 / 23 / 2015$ & 3850 & 177485616 & 9406664 & 3850 & 183951296 & 9379340 & 3850 & 191359776 & 9523426 \\
\hline 12 & $12 / 24 / 2015$ & 3850 & 186366976 & 8881370 & 3850 & 192771216 & 8819926 & 3850 & 200309088 & 8949311 \\
\hline 13 & $12 / 25 / 2015$ & 3850 & 194775984 & 8409001 & 3850 & 201093888 & 8322682 & 3850 & 208763248 & 8454156 \\
\hline 14 & $12 / 26 / 2015$ & 3850 & 202763440 & 7987464 & 3850 & 208966928 & 7873018 & 3850 & 216773232 & 8009983 \\
\hline 15 & $12 / 27 / 2015$ & 3850 & 210364112 & 7600680 & 3850 & 216435120 & 7468200 & 3850 & 224383728 & 7610500 \\
\hline 16 & $12 / 28 / 2015$ & 3850 & 217610544 & 7246422 & 3850 & 223532608 & 7097492 & 3850 & 231632752 & 7249027 \\
\hline 17 & $12 / 29 / 2015$ & 3850 & 224533712 & 6923168 & 3850 & 230293040 & 6760427 & 3850 & 238552720 & 6919974 \\
\hline 18 & $12 / 30 / 2015$ & 3850 & 231157184 & 6623470 & 3850 & 236742256 & 6449225 & 3850 & 245171600 & 6618872 \\
\hline 19 & $12 / 31 / 2015$ & 3850 & 237503312 & 6346123 & 3850 & 242906640 & 6164379 & 3850 & 251513760 & 6342157 \\
\hline 20 & $1 / 1 / 2016$ & 3850 & 243592176 & 6088868 & 3850 & 248806528 & 5899906 & 3850 & 257600720 & 6086961 \\
\hline 21 & $1 / 2 / 2016$ & 3850 & 249441920 & 5849748 & 3850 & 254463216 & 5656669 & 3850 & 263451616 & 5850895 \\
\hline 22 & $1 / 3 / 2016$ & 3850 & 255068864 & 5626940 & 3850 & 259893104 & 5429888 & 3850 & 269083552 & 5631950 \\
\hline 23 & $1 / 4 / 2016$ & 3850 & 260487952 & 5419095 & 3850 & 265113648 & 5220537 & 3850 & 274511968 & 5428419 \\
\hline 24 & $1 / 5 / 2016$ & 3850 & 265712688 & 5224733 & 3850 & 270138240 & 5024602 & 3850 & 279750816 & 5238819 \\
\hline 25 & $1 / 6 / 2016$ & 3850 & 270755808 & 5043121 & 3850 & 274980960 & 4842703 & 3850 & 284812672 & 5061880 \\
\hline 26 & $1 / 7 / 2016$ & 3850 & 275628544 & 4872743 & 3850 & 279654048 & 4673095 & 3850 & 289709152 & 4896467 \\
\hline 27 & $1 / 8 / 2016$ & 3850 & 280341568 & 4713020 & 3850 & 284168736 & 4514710 & 3850 & 294450752 & 4741598 \\
\hline 28 & $1 / 9 / 2016$ & 3850 & 284904576 & 4563008 & 3850 & 288535360 & 4366614 & 3850 & 299047136 & 4596383 \\
\hline 29 & $1 / 10 / 2016$ & 3850 & 289326592 & 4422002 & 3850 & 292763328 & 4227972 & 3850 & 303507168 & 4460044 \\
\hline 30 & $1 / 11 / 2016$ & 3850 & 293615840 & 4289270 & 3850 & 296861376 & 4098027 & 3850 & 307839040 & 4331865 \\
\hline 31 & $1 / 12 / 2016$ & 3850 & 297780096 & 4164241 & 3850 & 300837504 & 3976097 & 3850 & 312050272 & 4211221 \\
\hline 32 & $1 / 13 / 2016$ & 3850 & 301826400 & 4046311 & 3850 & 304699392 & 3861922 & 3850 & 316147712 & 4097460 \\
\hline 33 & $1 / 14 / 2016$ & 3850 & 305761408 & 3935007 & 3850 & 308453600 & 3754218 & 3850 & 320137984 & 3990254 \\
\hline 34 & $1 / 15 / 2016$ & 3850 & 309591232 & 3829822 & 3850 & 312106432 & 3652842 & 3850 & 324026880 & 3888905 \\
\hline 35 & $1 / 16 / 2016$ & 3850 & 313321600 & 3730360 & 3850 & 315663712 & 3557260 & 3850 & 327820000 & 3793126 \\
\hline 36 & $1 / 17 / 2016$ & 3850 & 316957792 & 3636196 & 3850 & 319130848 & 3467151 & 3850 & 331522496 & 3702486 \\
\hline 37 & $1 / 18 / 2016$ & 3850 & 320504768 & 3546995 & 3850 & 322512864 & 3382003 & 3850 & 335139104 & 3616628 \\
\hline 38 & $1 / 19 / 2016$ & 3850 & 323967168 & 3462398 & 3850 & 325814432 & 3301588 & 3850 & 338674336 & 3535210 \\
\hline 39 & $1 / 20 / 2016$ & 3850 & 327349312 & 3382122 & 3850 & 329039872 & 3225424 & 3850 & 342132256 & 3457937 \\
\hline 40 & $1 / 21 / 2016$ & 3850 & 330655168 & 3305859 & 3850 & 332193216 & 3153358 & 3850 & 345516768 & 3384519 \\
\hline 41 & $1 / 22 / 2016$ & 3850 & 333889600 & 3234432 & 3850 & 335278208 & 3084997 & 3850 & 348831488 & 3314711 \\
\hline 42 & $1 / 23 / 2016$ & 3850 & 337054080 & 3164484 & 3850 & 338298400 & 3020190 & 3850 & 352079744 & 3248265 \\
\hline 43 & $1 / 24 / 2016$ & 3850 & 340153824 & 3099745 & 3850 & 341256960 & 2958562 & 3850 & 355264736 & 3184973 \\
\hline 44 & $1 / 25 / 2016$ & 3850 & 343190016 & 3036206 & 3850 & 344157024 & 2900050 & 3850 & 358389344 & 3124626 \\
\hline 45 & $1 / 26 / 2016$ & 3850 & 346167392 & 2977373 & 3850 & 347001344 & 2844333 & 3850 & 361456384 & 3067044 \\
\hline 46 & $1 / 27 / 2016$ & 3850 & 349086944 & 2919558 & 3850 & 349792672 & 2791283 & 3850 & 364468448 & 3012049 \\
\hline 47 & $1 / 28 / 2016$ & 3850 & 351952896 & 2865937 & 3850 & 352533408 & 2740722 & 3850 & 367427904 & 2959488 \\
\hline 48 & $1 / 29 / 2016$ & 3850 & 354766048 & 2813139 & 3850 & 355225856 & 2692483 & 3850 & 370337152 & 2909207 \\
\hline 49 & $1 / 30 / 2016$ & 3850 & 357530144 & 2764114 & 3850 & 357872256 & 2646423 & 3850 & 373198208 & 2861076 \\
\hline 50 & $1 / 31 / 2016$ & 3850 & 360246752 & 2716613 & 3850 & 360474688 & 2602403 & 3850 & 376013184 & 2814959 \\
\hline 51 & $2 / 1 / 2016$ & 3850 & 362917728 & 2670969 & 3850 & 363034976 & 2560300 & 3850 & 378783936 & 2770747 \\
\hline 52 & $2 / 2 / 2016$ & 3850 & 365544960 & 2627221 & 3850 & 365554976 & 2519996 & 3850 & 381512256 & 2728322 \\
\hline 53 & $2 / 3 / 2016$ & 3850 & 368130208 & 2585251 & 3850 & 368036352 & 2481382 & 3850 & 384199840 & 2687588 \\
\hline 54 & $2 / 4 / 2016$ & 3850 & 370675168 & 2544962 & 3850 & 370480704 & 2444359 & 3850 & 386848288 & 2648445 \\
\hline 55 & $2 / 5 / 2016$ & 3850 & 373181440 & 2506262 & 3850 & 372889536 & 2408832 & 3850 & 389459072 & 2610808 \\
\hline 56 & $2 / 6 / 2016$ & 3850 & 375650464 & 2469065 & 3850 & 375264256 & 2374716 & 3850 & 392033664 & 2574590 \\
\hline 57 & $2 / 7 / 2016$ & 3850 & 378083776 & 2433289 & 3850 & 377606176 & 2341928 & 3850 & 394573408 & 2539719 \\
\hline 58 & $2 / 8 / 2016$ & 3850 & 380482624 & 2398858 & 3850 & 379916576 & 2310394 & 3850 & 397079520 & 2506117 \\
\hline 59 & $2 / 9 / 2016$ & 3850 & 382848352 & 2365702 & 3850 & 382196608 & 2280044 & 3850 & 399553248 & 2473722 \\
\hline 60 & $2 / 10 / 2016$ & 3850 & 385182080 & 2333752 & 3850 & 384447424 & 2250812 & 3850 & 401995712 & 2442466 \\
\hline 61 & $2 / 11 / 2016$ & 3850 & 387485056 & 2302949 & 3850 & 386670080 & 2222637 & 3850 & 404408000 & 2412294 \\
\hline
\end{tabular}




\begin{tabular}{|c|c|c|c|c|c|c|c|c|c|c|}
\hline 62 & $2 / 12 / 2016$ & 3850 & 389758272 & 2273231 & 3850 & 388865536 & 2195463 & 3850 & 406791136 & 2383148 \\
\hline 63 & $2 / 13 / 2016$ & 3850 & 392002816 & 2244545 & 3850 & 391034752 & 2169236 & 3850 & 409146112 & 2354979 \\
\hline 64 & $2 / 14 / 2016$ & 3850 & 394219648 & 2216840 & 3850 & 393178688 & 2143907 & 3850 & 411473856 & 2327735 \\
\hline 65 & $2 / 15 / 2016$ & 3850 & 396409696 & 2190065 & 3850 & 395298112 & 2119429 & 3850 & 413775232 & 2301373 \\
\hline 66 & $2 / 16 / 2016$ & 3850 & 398573888 & 2164178 & 3850 & 397393856 & 2095759 & 3850 & 416051072 & 2275848 \\
\hline 67 & $2 / 17 / 2016$ & 3850 & 400713024 & 2139133 & 3850 & 399466720 & 2072856 & 3850 & 418302208 & 2251123 \\
\hline 68 & $2 / 18 / 2016$ & 3850 & 402827936 & 2114892 & 3850 & 401517408 & 2050682 & 3850 & 420529344 & 2227157 \\
\hline 69 & $2 / 19 / 2016$ & 3850 & 404919296 & 2091416 & 3850 & 403546592 & 2029201 & 3850 & 422733280 & 2203917 \\
\hline 70 & $2 / 20 / 2016$ & 3850 & 406988000 & 2068670 & 3850 & 405554976 & 2008380 & 3850 & 424914624 & 2181368 \\
\hline 71 & $2 / 21 / 2016$ & 3850 & 409034624 & 2046621 & 3850 & 407543168 & 1988187 & 3850 & 427074112 & 2159479 \\
\hline 72 & $2 / 22 / 2016$ & 3850 & 411059872 & 2025235 & 3850 & 409511744 & 1968593 & 3850 & 429212352 & 2138220 \\
\hline 73 & $2 / 23 / 2016$ & 3850 & 413064352 & 2004485 & 3850 & 411461312 & 1949569 & 3850 & 431329888 & 2117564 \\
\hline 74 & $2 / 24 / 2016$ & 3850 & 415048672 & 1984340 & 3850 & 413392416 & 1931091 & 3850 & 433427392 & 2097484 \\
\hline 75 & $2 / 25 / 2016$ & 3850 & 417013504 & 1964776 & 3850 & 415305536 & 1913132 & 3850 & 435505344 & 2077955 \\
\hline 76 & $2 / 26 / 2016$ & 3850 & 418959232 & 1945766 & 3850 & 417201216 & 1895670 & 3850 & 437564288 & 2058953 \\
\hline 77 & $2 / 27 / 2016$ & 3850 & 420886528 & 1927288 & 3850 & 419079904 & 1878684 & 3850 & 439604736 & 2040457 \\
\hline 78 & $2 / 28 / 2016$ & 3850 & 422795840 & 1909317 & 3850 & 420942048 & 1862152 & 3850 & 441627200 & 2022445 \\
\hline 79 & $2 / 29 / 2016$ & 3850 & 424687680 & 1891834 & 3850 & 422788096 & 1846055 & 3850 & 443632096 & 2004898 \\
\hline 80 & $3 / 1 / 2016$ & 3850 & 426562496 & 1874817 & 3850 & 424618496 & 1830375 & 3850 & 445619872 & 1987797 \\
\hline 81 & $3 / 2 / 2016$ & 3850 & 428420736 & 1858249 & 3850 & 426433568 & 1815095 & 3850 & 447591008 & 1971123 \\
\hline 82 & $3 / 3 / 2016$ & 3850 & 430262848 & 1842110 & 3850 & 428233792 & 1800198 & 3850 & 449545888 & 1954860 \\
\hline 83 & $3 / 4 / 2016$ & 3850 & 432089216 & 1826384 & 3850 & 430019456 & 1785668 & 3850 & 451484864 & 1938992 \\
\hline 84 & $3 / 5 / 2016$ & 3850 & 433900288 & 1811054 & 3850 & 431790944 & 1771491 & 3850 & 453408384 & 1923504 \\
\hline 85 & $3 / 6 / 2016$ & 3850 & 435696384 & 1796105 & 3850 & 433548608 & 1757654 & 3850 & 455316736 & 1908381 \\
\hline 86 & $3 / 7 / 2016$ & 3850 & 437477920 & 1781522 & 3850 & 435292736 & 1744142 & 3850 & 457210368 & 1893610 \\
\hline 87 & $3 / 8 / 2016$ & 3850 & 439245216 & 1767291 & 3850 & 437023712 & 1730944 & 3850 & 459089536 & 1879177 \\
\hline 88 & $3 / 9 / 2016$ & 3850 & 440998560 & 1753399 & 3850 & 438741728 & 1718048 & 3850 & 460954560 & 1865070 \\
\hline 89 & $3 / 10 / 2016$ & 3850 & 442738432 & 1739833 & 3850 & 440447168 & 1705442 & 3850 & 462805888 & 1851277 \\
\hline 90 & $3 / 11 / 2016$ & 3850 & 444465024 & 1726581 & 3850 & 442140288 & 1693116 & 3850 & 464643680 & 1837788 \\
\hline 91 & $3 / 12 / 2016$ & 3850 & 446178656 & 1713633 & 3850 & 443821344 & 1681059 & 3850 & 466468256 & 1824591 \\
\hline 92 & $3 / 13 / 2016$ & 3850 & 447879616 & 1700976 & 3850 & 445490592 & 1669263 & 3850 & 468279936 & 1811677 \\
\hline 93 & $3 / 14 / 2016$ & 3850 & 449568224 & 1688601 & 3850 & 447148320 & 1657717 & 3850 & 470078976 & 1799034 \\
\hline 94 & $3 / 15 / 2016$ & 3850 & 451244736 & 1676497 & 3850 & 448794752 & 1646414 & 3850 & 471865632 & 1786656 \\
\hline 95 & $3 / 16 / 2016$ & 3850 & 452909376 & 1664656 & 3850 & 450430048 & 1635344 & 3850 & 473640160 & 1774531 \\
\hline 96 & $3 / 17 / 2016$ & 3850 & 454562432 & 1653068 & 3850 & 452054624 & 1624501 & 3850 & 475402816 & 1762652 \\
\hline 97 & $3 / 18 / 2016$ & 3850 & 456204160 & 1641724 & 3850 & 453668448 & 1613876 & 3850 & 477153824 & 1751011 \\
\hline 98 & $3 / 19 / 2016$ & 3850 & 457834784 & 1630617 & 3850 & 455271904 & 1603461 & 3850 & 478893440 & 1739601 \\
\hline 99 & $3 / 20 / 2016$ & 3850 & 459454528 & 1619738 & 3850 & 456865184 & 1593251 & 3850 & 480621792 & 1728413 \\
\hline 100 & $3 / 21 / 2016$ & 3850 & 461063616 & 1609080 & 3850 & 458448384 & 1583239 & 3850 & 482339264 & 1717441 \\
\hline 101 & $3 / 22 / 2016$ & 3850 & 462662240 & 1598636 & 3850 & 460021824 & 1573418 & 3850 & 484045952 & 1706678 \\
\hline 102 & $3 / 23 / 2016$ & 3850 & 464250624 & 1588399 & 3850 & 461585600 & 1563782 & 3850 & 485742080 & 1696118 \\
\hline 103 & $3 / 24 / 2016$ & 3850 & 465828992 & 1578362 & 3850 & 463140320 & 1554717 & 3850 & 487427840 & 1685754 \\
\hline 104 & $3 / 25 / 2016$ & 3850 & 467397504 & 1568519 & 3850 & 464685408 & 1545069 & 3850 & 489103424 & 1675580 \\
\hline 105 & $3 / 26 / 2016$ & 3850 & 468956384 & 1558864 & 3850 & 466221536 & 1536148 & 3850 & 490768992 & 1665591 \\
\hline 106 & $3 / 27 / 2016$ & 3850 & 470505760 & 1549392 & 3850 & 467748544 & 1526994 & 3850 & 492424768 & 1655781 \\
\hline 107 & $3 / 28 / 2016$ & 3850 & 472045856 & 1540096 & 3850 & 469267008 & 1518468 & 3850 & 494070912 & 1646145 \\
\hline 108 & $3 / 29 / 2016$ & 3850 & 473576864 & 1530971 & 3850 & 470776576 & 1509575 & 3850 & 495707648 & 1636679 \\
\hline 109 & $3 / 30 / 2016$ & 3850 & 475098848 & 1522013 & 3850 & 472277856 & 1501286 & 3850 & 497334976 & 1627376 \\
\hline 110 & $3 / 31 / 2016$ & 3850 & 476612064 & 1513216 & 3850 & 473770624 & 1492743 & 3850 & 498953216 & 1618233 \\
\hline 111 & $4 / 1 / 2016$ & 3850 & 478116640 & 1504575 & 3850 & 475255392 & 1484771 & 3850 & 500562496 & 1609244 \\
\hline 112 & $4 / 2 / 2016$ & 3850 & 479612736 & 1496087 & 3850 & 476731872 & 1476486 & 3850 & 502162848 & 1600407 \\
\hline 113 & $4 / 3 / 2016$ & 3850 & 481100448 & 1487746 & 3850 & 478200608 & 1468753 & 3850 & 503754624 & 1591716 \\
\hline 114 & $4 / 4 / 2016$ & 3850 & 482580032 & 1479549 & 3850 & 479661376 & 1460757 & 3850 & 505337760 & 1583168 \\
\hline 115 & $4 / 5 / 2016$ & 3850 & 484051520 & 1471491 & 3850 & 481114656 & 1453293 & 3850 & 506912512 & 1574758 \\
\hline 116 & $4 / 6 / 2016$ & 3850 & 485515072 & 1463569 & 3850 & 482560224 & 1445540 & 3850 & 508478976 & 1566484 \\
\hline 117 & $4 / 7 / 2016$ & 3850 & 486970880 & 1455779 & 3850 & 483998496 & 1438301 & 3850 & 510037344 & 1558341 \\
\hline 118 & $4 / 8 / 2016$ & 3850 & 488418976 & 1448117 & 3850 & 485429312 & 1430798 & 3850 & 511587648 & 1550327 \\
\hline 119 & $4 / 9 / 2016$ & 3850 & 489859552 & 1440580 & 3850 & 486853088 & 1423793 & 3850 & 513130080 & 1542437 \\
\hline 120 & $4 / 10 / 2016$ & 3850 & 491292736 & 1433164 & 3850 & 488269632 & 1416515 & 3850 & 514664768 & 1534669 \\
\hline 121 & $4 / 11 / 2016$ & 3850 & 492718592 & 1425867 & 3850 & 489679328 & 1409719 & 3850 & 516191776 & 1527019 \\
\hline 122 & $4 / 12 / 2016$ & 3850 & 494137280 & 1418685 & 3850 & 491081984 & 1402662 & 3850 & 517711264 & 1519485 \\
\hline 123 & $4 / 13 / 2016$ & 3850 & 495548896 & 1411616 & 3850 & 492478080 & 1396072 & 3850 & 519223328 & 1512064 \\
\hline 124 & $4 / 14 / 2016$ & 3850 & 496953536 & 1404656 & 3850 & 493867264 & 1389222 & 3850 & 520728096 & 1504753 \\
\hline 125 & $4 / 15 / 2016$ & 3850 & 498351360 & 1397802 & 3850 & 495250112 & 1382824 & 3850 & 522225632 & 1497549 \\
\hline 126 & $4 / 16 / 2016$ & 3850 & 499742400 & 1391052 & 3850 & 496626272 & 1376171 & 3850 & 523716096 & 1490450 \\
\hline 127 & $4 / 17 / 2016$ & 3850 & 501127264 & 1384848 & 3850 & 497996256 & 1369958 & 3850 & 525199520 & 1483453 \\
\hline 128 & $4 / 18 / 2016$ & 3850 & 502505152 & 1377895 & 3850 & 499359744 & 1363494 & 3850 & 526676096 & 1476556 \\
\hline 129 & $4 / 19 / 2016$ & 3850 & 503876832 & 1371698 & 3850 & 500717184 & 1357456 & 3850 & 528145856 & 1469756 \\
\hline 130 & $4 / 20 / 2016$ & 3850 & 505241920 & 1365066 & 3850 & 502068384 & 1351171 & 3850 & 529608896 & 1463051 \\
\hline 131 & $4 / 21 / 2016$ & 3850 & 506600992 & 1359102 & 3850 & 503413632 & 1345302 & 3850 & 531065344 & 1456440 \\
\hline 132 & $4 / 22 / 2016$ & 3850 & 507953632 & 1352631 & 3850 & 504752864 & 1339189 & 3850 & 532515264 & 1449919 \\
\hline 133 & $4 / 23 / 2016$ & 3850 & 509300448 & 1346803 & 3850 & 506086336 & 1333479 & 3850 & 533958752 & 1443487 \\
\hline 134 & $4 / 24 / 2016$ & 3850 & 510640992 & 1340531 & 3850 & 507413856 & 1327529 & 3850 & 535395872 & 1437142 \\
\hline 135 & $4 / 25 / 2016$ & 3850 & 511975872 & 1334881 & 3850 & 508735840 & 1321972 & 3850 & 536826784 & 1430882 \\
\hline 136 & $4 / 26 / 2016$ & 3850 & 513304640 & 1328772 & 3850 & 510052032 & 1316180 & 3850 & 538251456 & 1424706 \\
\hline 137 & $4 / 27 / 2016$ & 3850 & 514627904 & 1323270 & 3850 & 511362816 & 1310770 & 3850 & 539670080 & 1418610 \\
\hline 138 & $4 / 28 / 2016$ & 3850 & 515945248 & 1317329 & 3850 & 512667904 & 1305126 & 3850 & 541082688 & 1412594 \\
\hline 139 & $4 / 29 / 2016$ & 3850 & 517257216 & 1311978 & 3850 & 513967776 & 1299855 & 3850 & 542489344 & 1406655 \\
\hline 140 & $4 / 30 / 2016$ & 3850 & 518563392 & 1306192 & 3850 & 515262112 & 1294357 & 3850 & 543890112 & 1400793 \\
\hline 141 & $5 / 1 / 2016$ & 3850 & 519864384 & 1300982 & 3850 & 516551360 & 1289220 & 3850 & 545285120 & 1395005 \\
\hline 142 & $5 / 2 / 2016$ & 3850 & 521159776 & 1295346 & 3850 & 517835200 & 1283858 & 3850 & 546674432 & 1389290 \\
\hline 143 & $5 / 3 / 2016$ & 3850 & 522449984 & 1290270 & 3850 & 519114048 & 1278850 & 3850 & 548058048 & 1383647 \\
\hline
\end{tabular}




\begin{tabular}{|c|c|c|c|c|c|c|c|c|c|c|}
\hline 144 & $5 / 4 / 2016$ & 3850 & 523734816 & 1284779 & 3850 & 520388064 & 1274000 & 3850 & 549436160 & 1378074 \\
\hline 145 & $5 / 5 / 2016$ & 3850 & 525014624 & 1279834 & 3850 & 521656672 & 1268616 & 3850 & 550808704 & 1372568 \\
\hline 146 & $5 / 6 / 2016$ & 3850 & 526289088 & 1274479 & 3850 & 522920416 & 1263754 & 3850 & 552175808 & 1367131 \\
\hline 147 & $5 / 7 / 2016$ & 3850 & 527558752 & 1269656 & 3850 & 524179424 & 1258993 & 3850 & 553537600 & 1361759 \\
\hline 148 & $5 / 8 / 2016$ & 3850 & 528823200 & 1264435 & 3850 & 525433280 & 1253898 & 3850 & 554894080 & 1356452 \\
\hline 149 & $5 / 9 / 2016$ & 3850 & 530082880 & 1259734 & 3850 & 526682528 & 1249216 & 3850 & 556245248 & 1351207 \\
\hline 150 & $5 / 10 / 2016$ & 3850 & 531337568 & 1254637 & 3850 & 527927168 & 1244628 & 3850 & 557591296 & 1346026 \\
\hline 151 & $5 / 11 / 2016$ & 3850 & 532587584 & 1250047 & 3850 & 529166816 & 1239706 & 3850 & 558932160 & 1340904 \\
\hline 152 & $5 / 12 / 2016$ & 3850 & 533832672 & 1245074 & 3850 & 530402048 & 1235187 & 3850 & 560268032 & 1335842 \\
\hline 153 & $5 / 13 / 2016$ & 3850 & 535073280 & 1240597 & 3850 & 531632800 & 1230758 & 3850 & 561598848 & 1330839 \\
\hline 154 & $5 / 14 / 2016$ & 3850 & 536308992 & 1235738 & 3850 & 532858816 & 1226003 & 3850 & 562924736 & 1325893 \\
\hline 155 & $5 / 15 / 2016$ & 3850 & 537540352 & 1231362 & 3850 & 534080448 & 1221638 & 3850 & 564245760 & 1321003 \\
\hline 156 & $5 / 16 / 2016$ & 3850 & 538766976 & 1226619 & 3850 & 535297760 & 1217358 & 3850 & 565561920 & 1316169 \\
\hline 157 & $5 / 17 / 2016$ & 3850 & 539989312 & 1222348 & 3850 & 536510560 & 1212762 & 3850 & 566873344 & 1311388 \\
\hline 158 & $5 / 18 / 2016$ & 3850 & 541207040 & 1217710 & 3850 & 537719104 & 1208543 & 3850 & 568179968 & 1306661 \\
\hline 159 & $5 / 19 / 2016$ & 3850 & 542420608 & 1213532 & 3850 & 538923520 & 1204404 & 3850 & 569481984 & 1301986 \\
\hline 160 & $5 / 20 / 2016$ & 3850 & 543629568 & 1209000 & 3850 & 540123456 & 1199957 & 3850 & 570779328 & 1297363 \\
\hline 161 & $5 / 21 / 2016$ & 3850 & 544834496 & 1204921 & 3850 & 541319360 & 1195876 & 3850 & 572072128 & 1292789 \\
\hline 162 & $5 / 22 / 2016$ & 3850 & 546035008 & 1200486 & 3850 & 542511232 & 1191871 & 3850 & 573360384 & 1288265 \\
\hline 163 & $5 / 23 / 2016$ & 3850 & 547231488 & 1196493 & 3850 & 543698816 & 1187566 & 3850 & 574644160 & 1283790 \\
\hline 164 & $5 / 24 / 2016$ & 3850 & 548424064 & 1192560 & 3850 & 544882368 & 1183615 & 3850 & 575923520 & 1279362 \\
\hline 165 & $5 / 25 / 2016$ & 3850 & 549612096 & 1188086 & 3850 & 546062144 & 1179737 & 3850 & 577198528 & 1274981 \\
\hline 166 & $5 / 26 / 2016$ & 3850 & 550796288 & 1184187 & 3850 & 547237696 & 1175566 & 3850 & 578469184 & 1270646 \\
\hline 167 & $5 / 27 / 2016$ & 3850 & 551976640 & 1180333 & 3850 & 548409408 & 1171738 & 3850 & 579735488 & 1266356 \\
\hline 168 & $5 / 28 / 2016$ & 3850 & 553152704 & 1176054 & 3850 & 549577408 & 1167981 & 3850 & 580997632 & 1262111 \\
\hline 169 & $5 / 29 / 2016$ & 3850 & 554324992 & 1172282 & 3850 & 550741376 & 1163937 & 3850 & 582255552 & 1257909 \\
\hline 170 & $5 / 30 / 2016$ & 3850 & 555493504 & 1168547 & 3850 & 551901568 & 1160227 & 3850 & 583509312 & 1253751 \\
\hline 171 & $5 / 31 / 2016$ & 3850 & 556657920 & 1164403 & 3850 & 553058176 & 1156583 & 3850 & 584758912 & 1249634 \\
\hline 172 & $6 / 1 / 2016$ & 3850 & 557818688 & 1160748 & 3850 & 554210816 & 1152661 & 3850 & 586004480 & 1245560 \\
\hline 173 & $6 / 2 / 2016$ & 3850 & 558975808 & 1157131 & 3850 & 555359872 & 1149062 & 3850 & 587246016 & 1241525 \\
\hline 174 & $6 / 3 / 2016$ & 3850 & 560128896 & 1153110 & 3850 & 556505408 & 1145527 & 3850 & 588483520 & 1237532 \\
\hline 175 & $6 / 4 / 2016$ & 3850 & 561278464 & 1149565 & 3850 & 557647104 & 1141720 & 3850 & 589717120 & 1233577 \\
\hline 176 & $6 / 5 / 2016$ & 3850 & 562424512 & 1146056 & 3850 & 558785344 & 1138227 & 3850 & 590946752 & 1229661 \\
\hline 177 & $6 / 6 / 2016$ & 3850 & 563566720 & 1142155 & 3850 & 559920128 & 1134795 & 3850 & 592172544 & 1225784 \\
\hline 178 & $6 / 7 / 2016$ & 3850 & 564705408 & 1138716 & 3850 & 561051264 & 1131097 & 3850 & 593394432 & 1221944 \\
\hline 179 & $6 / 8 / 2016$ & 3850 & 565840704 & 1135310 & 3850 & 562178944 & 1127705 & 3850 & 594612672 & 1218141 \\
\hline 180 & $6 / 9 / 2016$ & 3850 & 566972224 & 1131522 & 3850 & 563303360 & 1124372 & 3850 & 595827008 & 1214374 \\
\hline 181 & $6 / 10 / 2016$ & 3850 & 568100416 & 1128184 & 3850 & 564424128 & 1120779 & 3850 & 597037696 & 1210643 \\
\hline 182 & $6 / 11 / 2016$ & 3850 & 569225280 & 1124876 & 3850 & 565541568 & 1117483 & 3850 & 598244608 & 1206948 \\
\hline 183 & $6 / 12 / 2016$ & 3850 & 570346496 & 1121196 & 3850 & 566655808 & 1114244 & 3850 & 599447872 & 1203287 \\
\hline 184 & $6 / 13 / 2016$ & 3850 & 571464448 & 1117953 & 3850 & 567766592 & 1110749 & 3850 & 600647552 & 1199661 \\
\hline 185 & $6 / 14 / 2016$ & 3850 & 572579200 & 1114739 & 3850 & 568874112 & 1107545 & 3850 & 601843648 & 1196067 \\
\hline 186 & $6 / 15 / 2016$ & 3850 & 573690368 & 1111162 & 3850 & 569978496 & 1104395 & 3850 & 603036160 & 1192508 \\
\hline 187 & $6 / 16 / 2016$ & 3850 & 574798336 & 1108010 & 3850 & 571079552 & 1100996 & 3850 & 604225088 & 1188980 \\
\hline 188 & $6 / 17 / 2016$ & 3850 & 575903232 & 1104886 & 3850 & 572177408 & 1097880 & 3850 & 605410624 & 1185485 \\
\hline 189 & $6 / 18 / 2016$ & 3850 & 577004672 & 1101407 & 3850 & 573272192 & 1094815 & 3850 & 606592640 & 1182021 \\
\hline 190 & $6 / 19 / 2016$ & 3850 & 578102976 & 1098341 & 3850 & 574363712 & 1091507 & 3850 & 607771200 & 1178589 \\
\hline 191 & $6 / 20 / 2016$ & 3850 & 579198272 & 1095302 & 3850 & 575452224 & 1088474 & 3850 & 608946432 & 1175187 \\
\hline 192 & $6 / 21 / 2016$ & 3850 & 580290240 & 1091917 & 3850 & 576537664 & 1085491 & 3850 & 610118208 & 1171816 \\
\hline 193 & $6 / 22 / 2016$ & 3850 & 581379136 & 1088934 & 3850 & 577620288 & 1082616 & 3850 & 611286720 & 1168474 \\
\hline 194 & $6 / 23 / 2016$ & 3850 & 582465152 & 1085976 & 3850 & 578699904 & 1079579 & 3850 & 612451840 & 1165162 \\
\hline 195 & $6 / 24 / 2016$ & 3850 & 583547840 & 1082681 & 3850 & 579776512 & 1076601 & 3850 & 613613760 & 1161878 \\
\hline 196 & $6 / 25 / 2016$ & 3850 & 584627584 & 1079777 & 3850 & 580850112 & 1073616 & 3850 & 614772352 & 1158623 \\
\hline 197 & $6 / 26 / 2016$ & 3850 & 585704512 & 1076896 & 3850 & 581920768 & 1070678 & 3850 & 615927744 & 1155396 \\
\hline 198 & $6 / 27 / 2016$ & 3850 & 586778176 & 1073687 & 3850 & 582988544 & 1067748 & 3850 & 617079936 & 1152197 \\
\hline 199 & $6 / 28 / 2016$ & 3850 & 587849024 & 1070858 & 3850 & 584053376 & 1064856 & 3850 & 618228992 & 1149025 \\
\hline 200 & $6 / 29 / 2016$ & 3850 & 588917056 & 1068052 & 3850 & 585115328 & 1061976 & 3850 & 619374848 & 1145880 \\
\hline 201 & $6 / 30 / 2016$ & 3850 & 589982016 & 1064925 & 3850 & 586174464 & 1059132 & 3850 & 620517632 & 1142761 \\
\hline 202 & $7 / 1 / 2016$ & 3850 & 591044160 & 1062169 & 3850 & 587230784 & 1056300 & 3850 & 621657280 & 1139669 \\
\hline 203 & $7 / 2 / 2016$ & 3850 & 592103616 & 1059434 & 3850 & 588284288 & 1053503 & 3850 & 622793856 & 1136603 \\
\hline 204 & $7 / 3 / 2016$ & 3850 & 593160000 & 1056385 & 3850 & 589335040 & 1050718 & 3850 & 623927424 & 1133562 \\
\hline 205 & $7 / 4 / 2016$ & 3850 & 594213696 & 1053698 & 3850 & 590382976 & 1047966 & 3850 & 625058048 & 1130545 \\
\hline 206 & $7 / 5 / 2016$ & 3850 & 595264704 & 1051031 & 3850 & 591428224 & 1045227 & 3850 & 626185536 & 1127554 \\
\hline 207 & $7 / 6 / 2016$ & 3850 & 596312768 & 1048057 & 3850 & 592470720 & 1042520 & 3850 & 627310144 & 1124587 \\
\hline 208 & $7 / 7 / 2016$ & 3850 & 597358208 & 1045437 & 3850 & 593510528 & 1039825 & 3850 & 628431808 & 1121644 \\
\hline 209 & $7 / 8 / 2016$ & 3850 & 598401024 & 1042835 & 3850 & 594547712 & 1037162 & 3850 & 629550528 & 1118725 \\
\hline 210 & $7 / 9 / 2016$ & 3850 & 599440960 & 1039933 & 3850 & 595582208 & 1034510 & 3850 & 630666240 & 1115830 \\
\hline 211 & $7 / 10 / 2016$ & 3850 & 600478272 & 1037376 & 3850 & 596614080 & 1031889 & 3850 & 631779264 & 1112957 \\
\hline 212 & $7 / 11 / 2016$ & 3850 & 601513216 & 1034838 & 3850 & 597643392 & 1029279 & 3850 & 632889408 & 1110107 \\
\hline 213 & $7 / 12 / 2016$ & 3850 & 602545216 & 1032005 & 3850 & 598670080 & 1026699 & 3850 & 633996672 & 1107280 \\
\hline 214 & $7 / 13 / 2016$ & 3850 & 603574720 & 1029509 & 3850 & 599694208 & 1024130 & 3850 & 635101056 & 1104475 \\
\hline 215 & $7 / 14 / 2016$ & 3850 & 604601728 & 1027031 & 3850 & 600715840 & 1021591 & 3850 & 636202816 & 1101692 \\
\hline 216 & $7 / 15 / 2016$ & 3850 & 605625984 & 1024265 & 3850 & 601734848 & 1019061 & 3850 & 637301760 & 1098930 \\
\hline 217 & $7 / 16 / 2016$ & 3850 & 606647744 & 1021827 & 3850 & 602751424 & 1016561 & 3850 & 638397952 & 1096190 \\
\hline 218 & $7 / 17 / 2016$ & 3850 & 607667264 & 1019407 & 3850 & 603765504 & 1014070 & 3850 & 639491456 & 1093471 \\
\hline 219 & $7 / 18 / 2016$ & 3850 & 608684288 & 1017059 & 3850 & 604777088 & 1011608 & 3850 & 640582208 & 1090772 \\
\hline 220 & $7 / 19 / 2016$ & 3850 & 609698880 & 1014571 & 3850 & 605786240 & 1009155 & 3850 & 641670272 & 1088095 \\
\hline 221 & $7 / 20 / 2016$ & 3850 & 610711040 & 1012128 & 3850 & 606792960 & 1006730 & 3850 & 642755712 & 1085437 \\
\hline 222 & $7 / 21 / 2016$ & 3850 & 611720704 & 1009676 & 3850 & 607797312 & 1004314 & 3850 & 643838528 & 1082800 \\
\hline 223 & $7 / 22 / 2016$ & 3850 & 612727872 & 1007256 & 3850 & 608799232 & 1001925 & 3850 & 644918720 & 1080182 \\
\hline 224 & $7 / 23 / 2016$ & 3850 & 613732800 & 1004845 & 3850 & 609798784 & 999545 & 3850 & 645996288 & 1077584 \\
\hline 225 & $7 / 24 / 2016$ & 3850 & 614735232 & 1002458 & 3850 & 610795968 & 997192 & 3850 & 647071296 & 1075005 \\
\hline
\end{tabular}




\begin{tabular}{|c|c|c|c|c|c|c|c|c|c|c|}
\hline 226 & $7 / 25 / 2016$ & 3850 & 615735296 & 1000083 & 3850 & 611790848 & 994847 & 3850 & 648143680 & 1072445 \\
\hline 227 & $7 / 26 / 2016$ & 3850 & 616733056 & 997730 & 3850 & 612783360 & 992527 & 3850 & 649213632 & 1069904 \\
\hline 228 & $7 / 27 / 2016$ & 3850 & 617728448 & 995392 & 3850 & 613773568 & 990217 & 3850 & 650281024 & 1067382 \\
\hline 229 & $7 / 28 / 2016$ & 3850 & 618721536 & 993072 & 3850 & 614761472 & 987931 & 3850 & 651345920 & 1064877 \\
\hline 230 & $7 / 29 / 2016$ & 3850 & 619712256 & 990768 & 3850 & 615747136 & 985654 & 3850 & 652408256 & 1062367 \\
\hline 231 & $7 / 30 / 2016$ & 3850 & 620700736 & 988482 & 3850 & 616730560 & 983401 & 3850 & 653468224 & 1059924 \\
\hline 232 & $7 / 31 / 2016$ & 3850 & 621686976 & 986211 & 3850 & 617711680 & 981156 & 3850 & 654525632 & 1057452 \\
\hline 233 & $8 / 1 / 2016$ & 3850 & 622670912 & 983958 & 3850 & 618690624 & 978935 & 3850 & 655580672 & 1055041 \\
\hline 234 & $8 / 2 / 2016$ & 3850 & 623652736 & 981719 & 3850 & 619667328 & 976722 & 3850 & 656633280 & 1052603 \\
\hline 235 & $8 / 3 / 2016$ & 3850 & 624632128 & 979498 & 3850 & 620641920 & 974533 & 3850 & 657683520 & 1050227 \\
\hline 236 & $8 / 4 / 2016$ & 3850 & 625609408 & 977291 & 3850 & 621614272 & 972351 & 3850 & 658731328 & 1047824 \\
\hline 237 & $8 / 5 / 2016$ & 3850 & 626584512 & 975101 & 3850 & 622584448 & 970192 & 3850 & 659776832 & 1045481 \\
\hline 238 & $8 / 6 / 2016$ & 3850 & 627557440 & 972925 & 3850 & 623552512 & 968040 & 3850 & 660819968 & 1043111 \\
\hline 239 & $8 / 7 / 2016$ & 3850 & 628528256 & 970766 & 3850 & 624518400 & 965911 & 3850 & 661860672 & 1040800 \\
\hline 240 & $8 / 8 / 2016$ & 3850 & 629496832 & 968620 & 3850 & 625482176 & 963788 & 3850 & 662899136 & 1038463 \\
\hline 241 & $8 / 9 / 2016$ & 3850 & 630463360 & 966491 & 3850 & 626443840 & 961689 & 3850 & 663935360 & 1036183 \\
\hline 242 & $8 / 10 / 2016$ & 3850 & 631427712 & 964375 & 3850 & 627403456 & 959595 & 3850 & 664969344 & 1033878 \\
\hline 243 & $8 / 11 / 2016$ & 3850 & 632390080 & 962275 & 3850 & 628360896 & 957524 & 3850 & 666000896 & 1031630 \\
\hline 244 & $8 / 12 / 2016$ & 3850 & 633350208 & 960187 & 3850 & 629316416 & 955458 & 3850 & 667030272 & 1029356 \\
\hline 245 & $8 / 13 / 2016$ & 3850 & 634308288 & 958115 & 3850 & 630269824 & 953415 & 3850 & 668057408 & 1027138 \\
\hline 246 & $8 / 14 / 2016$ & 3850 & 635264320 & 956056 & 3850 & 631221248 & 951377 & 3850 & 669082240 & 1024894 \\
\hline 247 & $8 / 15 / 2016$ & 3850 & 636218368 & 954012 & 3850 & 632170560 & 949361 & 3850 & 670104960 & 1022706 \\
\hline 248 & $8 / 16 / 2016$ & 3850 & 637170368 & 951981 & 3850 & 633117952 & 947350 & 3850 & 671125504 & 1020492 \\
\hline 249 & $8 / 17 / 2016$ & 3850 & 638120320 & 949964 & 3850 & 634063360 & 945361 & 3850 & 672143808 & 1018333 \\
\hline 250 & $8 / 18 / 2016$ & 3850 & 639068288 & 947959 & 3850 & 635006656 & 943376 & 3850 & 673159936 & 1016148 \\
\hline 251 & $8 / 19 / 2016$ & 3850 & 640014208 & 945969 & 3850 & 635948096 & 941412 & 3850 & 674173952 & 1014017 \\
\hline 252 & $8 / 20 / 2016$ & 3850 & 640958208 & 943991 & 3850 & 636887552 & 939454 & 3850 & 675185856 & 1011861 \\
\hline 253 & $8 / 21 / 2016$ & 3850 & 641900224 & 942027 & 3850 & 637825088 & 937516 & 3850 & 676195584 & 1009757 \\
\hline 254 & $8 / 22 / 2016$ & 3850 & 642840320 & 940074 & 3850 & 638760704 & 935582 & 3850 & 677203200 & 1007629 \\
\hline 255 & $8 / 23 / 2016$ & 3850 & 643778432 & 938135 & 3850 & 639694336 & 933669 & 3850 & 678208768 & 1005552 \\
\hline 256 & $8 / 24 / 2016$ & 3850 & 644714624 & 936208 & 3850 & 640626048 & 931760 & 3850 & 679212224 & 1003452 \\
\hline 257 & $8 / 25 / 2016$ & 3850 & 645648960 & 934294 & 3850 & 641555968 & 929871 & 3850 & 680213696 & 1001401 \\
\hline 258 & $8 / 26 / 2016$ & 3850 & 646581376 & 932392 & 3850 & 642483904 & 927987 & 3850 & 681212928 & 999327 \\
\hline 259 & $8 / 27 / 2016$ & 3850 & 647511872 & 930502 & 3850 & 643410048 & 926122 & 3850 & 682210240 & 997303 \\
\hline 260 & $8 / 28 / 2016$ & 3850 & 648440448 & 928623 & 3850 & 644334336 & 924261 & 3850 & 683205504 & 995255 \\
\hline 261 & $8 / 29 / 2016$ & 3850 & 649367232 & 926758 & 3850 & 645256704 & 922419 & 3850 & 684198784 & 993256 \\
\hline 262 & $8 / 30 / 2016$ & 3850 & 650292160 & 924903 & 3850 & 646177344 & 920582 & 3850 & 685190016 & 991234 \\
\hline 263 & $8 / 31 / 2016$ & 3850 & 651215232 & 923060 & 3850 & 647096064 & 918763 & 3850 & 686179200 & 989260 \\
\hline 264 & $9 / 1 / 2016$ & 3850 & 652136448 & 921228 & 3850 & 648013056 & 916948 & 3850 & 687166528 & 987263 \\
\hline 265 & $9 / 2 / 2016$ & 3850 & 653055808 & 919409 & 3850 & 648928192 & 915151 & 3850 & 688151808 & 985313 \\
\hline 266 & $9 / 3 / 2016$ & 3850 & 653973440 & 917600 & 3850 & 649841600 & 913358 & 3850 & 689135168 & 983340 \\
\hline 267 & $9 / 4 / 2016$ & 3850 & 654889216 & 915803 & 3850 & 650753088 & 911583 & 3850 & 690116608 & 981415 \\
\hline 268 & $9 / 5 / 2016$ & 3850 & 655803264 & 914015 & 3850 & 651662912 & 909812 & 3850 & 691096064 & 979466 \\
\hline 269 & $9 / 6 / 2016$ & 3850 & 656715520 & 912240 & 3850 & 652571008 & 908059 & 3850 & 692073600 & 977563 \\
\hline 270 & $9 / 7 / 2016$ & 3850 & 657625984 & 910475 & 3850 & 653477312 & 906309 & 3850 & 693049280 & 975638 \\
\hline 271 & $9 / 8 / 2016$ & 3850 & 658534720 & 908721 & 3850 & 654381888 & 904577 & 3850 & 694023040 & 973759 \\
\hline 272 & $9 / 9 / 2016$ & 3850 & 659441664 & 906977 & 3850 & 655284736 & 902848 & 3850 & 694994880 & 971857 \\
\hline 273 & $9 / 10 / 2016$ & 3850 & 660346944 & 905244 & 3850 & 656185856 & 901137 & 3850 & 695964864 & 969980 \\
\hline 274 & $9 / 11 / 2016$ & 3850 & 661250496 & 903521 & 3850 & 657085312 & 899428 & 3850 & 696932992 & 968118 \\
\hline 275 & $9 / 12 / 2016$ & 3850 & 662152256 & 901808 & 3850 & 657983040 & 897737 & 3850 & 697899264 & 966267 \\
\hline 276 & $9 / 13 / 2016$ & 3850 & 663052352 & 900105 & 3850 & 658879104 & 896048 & 3850 & 698863680 & 964426 \\
\hline 277 & $9 / 14 / 2016$ & 3850 & 663950784 & 898413 & 3850 & 659773440 & 894377 & 3850 & 699826240 & 962596 \\
\hline 278 & $9 / 15 / 2016$ & 3850 & 664847488 & 896730 & 3850 & 660666176 & 892708 & 3850 & 700787072 & 960777 \\
\hline 279 & $9 / 16 / 2016$ & 3850 & 665742592 & 895058 & 3850 & 661557184 & 891056 & 3850 & 701745984 & 958969 \\
\hline 280 & $9 / 17 / 2016$ & 3850 & 666635968 & 893395 & 3850 & 662446592 & 889406 & 3850 & 702703168 & 957171 \\
\hline 281 & $9 / 18 / 2016$ & 3850 & 667527616 & 891742 & 3850 & 663334400 & 887773 & 3850 & 703658560 & 955383 \\
\hline 282 & $9 / 19 / 2016$ & 3850 & 668417792 & 890098 & 3850 & 664220544 & 886142 & 3850 & 704612160 & 953606 \\
\hline 283 & $9 / 20 / 2016$ & 3850 & 669306240 & 888464 & 3850 & 665105088 & 884528 & 3850 & 705564032 & 951839 \\
\hline 284 & $9 / 21 / 2016$ & 3850 & 670193088 & 886838 & 3850 & 665987968 & 882916 & 3850 & 706514112 & 950082 \\
\hline 285 & $9 / 22 / 2016$ & 3850 & 671078336 & 885223 & 3850 & 666869312 & 881319 & 3850 & 707462400 & 948335 \\
\hline 286 & $9 / 23 / 2016$ & 3850 & 671961920 & 883616 & 3850 & 667748992 & 879725 & 3850 & 708409024 & 946597 \\
\hline 287 & $9 / 24 / 2016$ & 3850 & 672843968 & 882019 & 3850 & 668627200 & 878147 & 3850 & 709353920 & 944870 \\
\hline 288 & $9 / 25 / 2016$ & 3850 & 673724352 & 880430 & 3850 & 669503744 & 876571 & 3850 & 710297024 & 943152 \\
\hline 289 & $9 / 26 / 2016$ & 3850 & 674603200 & 878851 & 3850 & 670378752 & 875010 & 3850 & 711238464 & 941444 \\
\hline 290 & $9 / 27 / 2016$ & 3850 & 675480512 & 877280 & 3850 & 671252224 & 873452 & 3850 & 712178240 & 939746 \\
\hline 291 & $9 / 28 / 2016$ & 3850 & 676356224 & 875719 & 3850 & 672124032 & 871908 & 3850 & 713116288 & 938057 \\
\hline 292 & $9 / 29 / 2016$ & 3850 & 677230400 & 874165 & 3850 & 672994496 & 870367 & 3850 & 714052672 & 936377 \\
\hline 293 & $9 / 30 / 2016$ & 3850 & 678103040 & 872621 & 3850 & 673863296 & 868840 & 3850 & 714987392 & 934707 \\
\hline 294 & $10 / 1 / 2016$ & 3850 & 678974080 & 871085 & 3850 & 674730624 & 867316 & 3850 & 715920448 & 933045 \\
\hline 295 & $10 / 2 / 2016$ & 3850 & 679843648 & 869557 & 3850 & 675596416 & 865806 & 3850 & 716851840 & 931393 \\
\hline 296 & $10 / 3 / 2016$ & 3850 & 680711680 & 868038 & 3850 & 676460736 & 864298 & 3850 & 717781568 & 929750 \\
\hline 297 & $10 / 4 / 2016$ & 3850 & 681578240 & 866527 & 3850 & 677323520 & 862804 & 3850 & 718709696 & 928116 \\
\hline 298 & $10 / 5 / 2016$ & 3850 & 682443264 & 865024 & 3850 & 678184832 & 861312 & 3850 & 719636160 & 926491 \\
\hline 299 & $10 / 6 / 2016$ & 3850 & 683306752 & 863530 & 3850 & 679044672 & 859835 & 3850 & 720561024 & 924874 \\
\hline 300 & $10 / 7 / 2016$ & 3850 & 684168832 & 862043 & 3850 & 679903040 & 858359 & 3850 & 721484288 & 923266 \\
\hline 301 & $10 / 8 / 2016$ & 3850 & 685029376 & 860564 & 3850 & 680759936 & 856897 & 3850 & 722405952 & 921667 \\
\hline 302 & $10 / 9 / 2016$ & 3850 & 685888448 & 859093 & 3850 & 681615360 & 855437 & 3850 & 723326080 & 920076 \\
\hline 303 & $10 / 10 / 2016$ & 3850 & 686746112 & 857631 & 3850 & 682469376 & 853991 & 3850 & 724244544 & 918494 \\
\hline 304 & $10 / 11 / 2016$ & 3850 & 687602240 & 856175 & 3850 & 683321920 & 852546 & 3850 & 725161472 & 916920 \\
\hline 305 & $10 / 12 / 2016$ & 3850 & 688457024 & 854728 & 3850 & 684173056 & 851115 & 3850 & 726076800 & 915354 \\
\hline 306 & $10 / 13 / 2016$ & 3850 & 689310272 & 853288 & 3850 & 685022720 & 849685 & 3850 & 726990656 & 913797 \\
\hline 307 & $10 / 14 / 2016$ & 3850 & 690162176 & 851856 & 3850 & 685870976 & 848269 & 3850 & 727902848 & 912248 \\
\hline
\end{tabular}




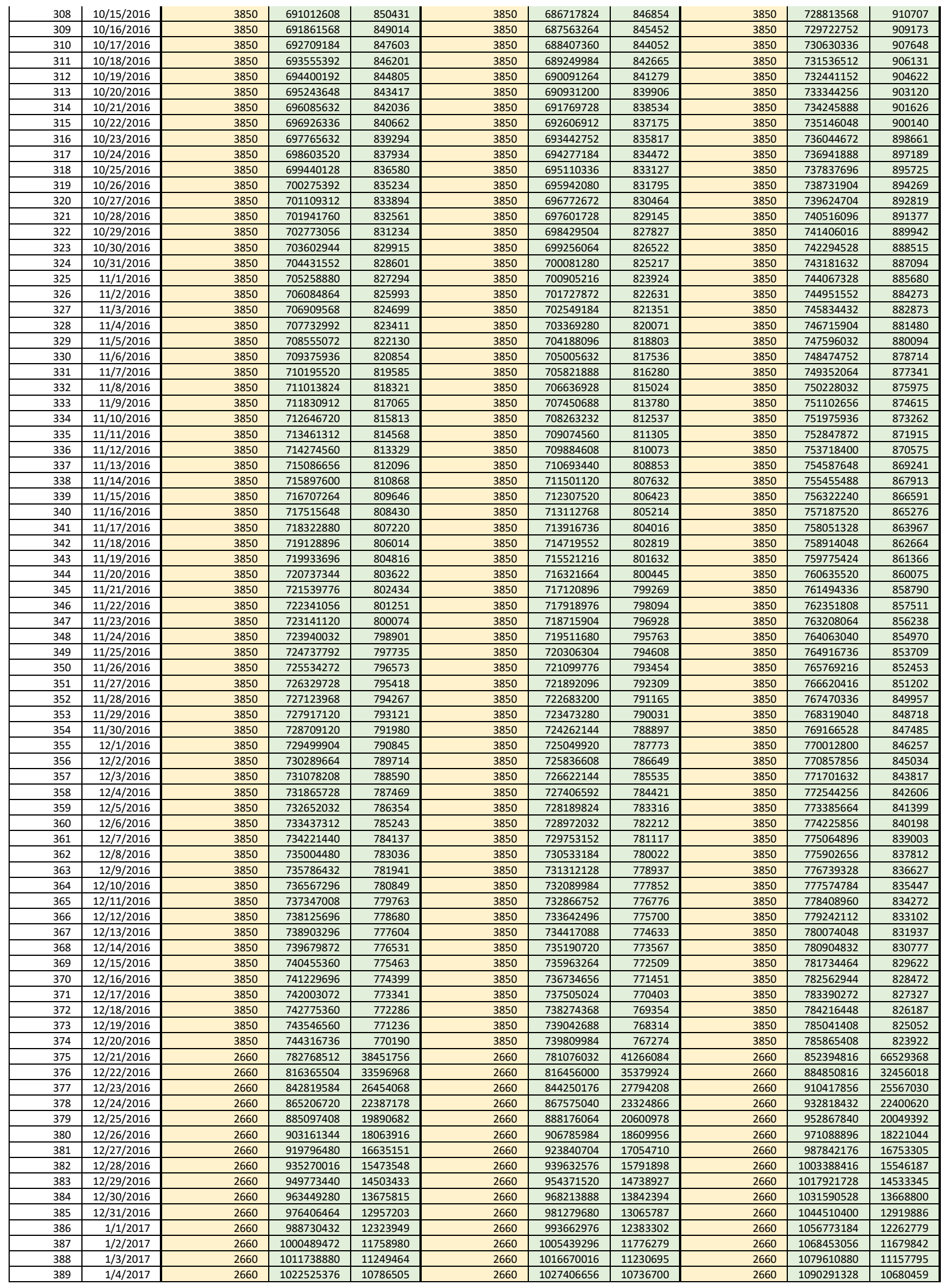




\begin{tabular}{|c|c|c|c|c|c|c|c|c|c|c|}
\hline 390 & $1 / 5 / 2017$ & 2660 & 1032891520 & 10366144 & 2660 & 1037692800 & 10286145 & 2660 & 1100548736 & 10257406 \\
\hline 391 & $1 / 6 / 2017$ & 2660 & 1042867072 & 9975518 & 2660 & 1047565632 & 9872815 & 2660 & 1110409344 & 9860586 \\
\hline 392 & $1 / 7 / 2017$ & 2660 & 1052478592 & 9611468 & 2660 & 1057057472 & 9491805 & 2660 & 1119909632 & 9500317 \\
\hline 393 & $1 / 8 / 2017$ & 2660 & 1061757184 & 9278686 & 2660 & 1066196608 & 9139173 & 2660 & 1129077504 & 9167904 \\
\hline 394 & $1 / 9 / 2017$ & 2660 & 1070722688 & 8965512 & 2660 & 1075008256 & 8811692 & 2660 & 1137937280 & 8859814 \\
\hline 395 & $1 / 10 / 2017$ & 2660 & 1079399040 & 8676374 & 2660 & 1083515008 & 8506686 & 2660 & 1146510592 & 8573264 \\
\hline 396 & $1 / 11 / 2017$ & 2660 & 1087798272 & 8399256 & 2660 & 1091736832 & 8221900 & 2660 & 1154816512 & 8305949 \\
\hline 397 & $1 / 12 / 2017$ & 2660 & 1095939712 & 8141341 & 2660 & 1099692288 & 7955417 & 2660 & 1162872448 & 8055928 \\
\hline 398 & $1 / 13 / 2017$ & 2660 & 1103838208 & 7898572 & 2660 & 1107397888 & 7705581 & 2660 & 1170695168 & 7822690 \\
\hline 399 & $1 / 14 / 2017$ & 2660 & 1111508096 & 7669909 & 2660 & 1114868864 & 7470953 & 2660 & 1178297600 & 7602427 \\
\hline 400 & $1 / 15 / 2017$ & 2660 & 1118962688 & 7454563 & 2660 & 1122119168 & 7250269 & 2660 & 1185692672 & 7395079 \\
\hline 401 & $1 / 16 / 2017$ & 2660 & 1126213376 & 7250644 & 2660 & 1129161472 & 7042411 & 2660 & 1192892288 & 7199667 \\
\hline 402 & $1 / 17 / 2017$ & 2660 & 1133270912 & 7057553 & 2660 & 1136007936 & 6846383 & 2660 & 1199907456 & 7015125 \\
\hline 403 & $1 / 18 / 2017$ & 2660 & 1140145536 & 6874656 & 2660 & 1142669184 & 6661293 & 2660 & 1206748160 & 6840658 \\
\hline 404 & $1 / 19 / 2017$ & 2660 & 1146846720 & 6701168 & 2660 & 1149155584 & 6486338 & 2660 & 1213423616 & 6675456 \\
\hline 405 & $1 / 20 / 2017$ & 2660 & 1153383040 & 6536317 & 2660 & 1155476224 & 6320642 & 2660 & 1219942400 & 6518867 \\
\hline 406 & $1 / 21 / 2017$ & 2660 & 1159762944 & 6379940 & 2660 & 1161640192 & 6163974 & 2660 & 1226312704 & 6370255 \\
\hline 407 & $1 / 22 / 2017$ & 2660 & 1165993984 & 6230961 & 2660 & 1167655424 & 6015216 & 2660 & 1232541824 & 6229074 \\
\hline 408 & $1 / 23 / 2017$ & 2660 & 1172083200 & 6089233 & 2660 & 1173529472 & 5874153 & 2660 & 1238636544 & 6094814 \\
\hline 409 & $1 / 24 / 2017$ & 2660 & 1178037504 & 5954270 & 2660 & 1179269760 & 5740210 & 2660 & 1244603648 & 5967017 \\
\hline 410 & $1 / 25 / 2017$ & 2660 & 1183863040 & 5825587 & 2660 & 1184882688 & 5612910 & 2660 & 1250448896 & 5845262 \\
\hline 411 & $1 / 26 / 2017$ & 2660 & 1189565824 & 5702840 & 2660 & 1190374528 & 5491824 & 2660 & 1256178048 & 5729163 \\
\hline 412 & $1 / 27 / 2017$ & 2660 & 1195151488 & 5585632 & 2660 & 1195751040 & 5376556 & 2660 & 1261796352 & 5618370 \\
\hline 413 & $1 / 28 / 2017$ & 2660 & 1200625152 & 5473672 & 2660 & 1201017728 & 5266747 & 2660 & 1267308928 & 5512553 \\
\hline 414 & $1 / 29 / 2017$ & 2660 & 1205991808 & 5366620 & 2660 & 1206179840 & 5162060 & 2660 & 1272720384 & 5411417 \\
\hline 415 & $1 / 30 / 2017$ & 2660 & 1211256064 & 5264227 & 2660 & 1211241984 & 5062187 & 2660 & 1278034944 & 5314582 \\
\hline 416 & $1 / 31 / 2017$ & 2660 & 1216422272 & 5166200 & 2660 & 1216208896 & 4966840 & 2660 & 1283256960 & 5222067 \\
\hline 417 & $2 / 1 / 2017$ & 2660 & 1221494528 & 5072322 & 2660 & 1221084672 & 4875754 & 2660 & 1288390272 & 5133306 \\
\hline 418 & $2 / 2 / 2017$ & 2660 & 1226478208 & 4983671 & 2660 & 1225873664 & 4789067 & 2660 & 1293438720 & 5048290 \\
\hline 419 & $2 / 3 / 2017$ & 2660 & 1231374464 & 4896189 & 2660 & 1230579456 & 4705743 & 2660 & 1298405248 & 4966767 \\
\hline 420 & $2 / 4 / 2017$ & 2660 & 1236188928 & 4814566 & 2660 & 1235205504 & 4626077 & 2660 & 1303293952 & 4888542 \\
\hline 421 & $2 / 5 / 2017$ & 2660 & 1240922880 & 4733911 & 2660 & 1239755136 & 4549653 & 2660 & 1308107392 & 4813427 \\
\hline 422 & $2 / 6 / 2017$ & 2660 & 1245581696 & 4658652 & 2660 & 1244231424 & 4476458 & 2660 & 1312848640 & 4741258 \\
\hline 423 & $2 / 7 / 2017$ & 2660 & 1250165760 & 4584206 & 2660 & 1248637824 & 4406279 & 2660 & 1317520512 & 4671874 \\
\hline 424 & $2 / 8 / 2017$ & 2660 & 1254680448 & 4514661 & 2660 & 1252976768 & 4338945 & 2660 & 1322125568 & 4605135 \\
\hline 425 & $2 / 9 / 2017$ & 2660 & 1259126016 & 4445794 & 2660 & 1257251072 & 4274302 & 2660 & 1326666496 & 4540897 \\
\hline 426 & $2 / 10 / 2017$ & 2660 & 1263507584 & 4381404 & 2660 & 1261463296 & 4212206 & 2660 & 1331145600 & 4479039 \\
\hline 427 & $2 / 11 / 2017$ & 2660 & 1267825152 & 4317557 & 2660 & 1265615872 & 4152524 & 2660 & 1335564928 & 4419436 \\
\hline 428 & $2 / 12 / 2017$ & 2660 & 1272082944 & 4257815 & 2660 & 1269710976 & 4095131 & 2660 & 1339926912 & 4361979 \\
\hline 429 & $2 / 13 / 2017$ & 2660 & 1276282496 & 4199529 & 2660 & 1273750912 & 4039908 & 2660 & 1344233472 & 4306559 \\
\hline 430 & $2 / 14 / 2017$ & 2660 & 1280425728 & 4143161 & 2660 & 1277737600 & 3986745 & 2660 & 1348486656 & 4253080 \\
\hline 431 & $2 / 15 / 2017$ & 2660 & 1284514560 & 4088774 & 2660 & 1281673216 & 3935538 & 2660 & 1352688000 & 4201445 \\
\hline 432 & $2 / 16 / 2017$ & 2660 & 1288550656 & 4036269 & 2660 & 1285559424 & 3886187 & 2660 & 1356839552 & 4151569 \\
\hline 433 & $2 / 17 / 2017$ & 2660 & 1292536320 & 3985561 & 2660 & 1289397888 & 3838600 & 2660 & 1360942976 & 4103366 \\
\hline 434 & $2 / 18 / 2017$ & 2660 & 1296472832 & 3936567 & 2660 & 1293190656 & 3792690 & 2660 & 13649996680 & 4056762 \\
\hline 435 & $2 / 19 / 2017$ & 2660 & 1300361984 & 3889209 & 2660 & 1296939008 & 3748376 & 2660 & 1369011456 & 4011678 \\
\hline 436 & $2 / 20 / 2017$ & 2660 & 1304205440 & 3843413 & 2660 & 1300644480 & 3705582 & 2660 & 1372979456 & 3968049 \\
\hline 437 & $2 / 21 / 2017$ & 2660 & 1308004480 & 3799108 & 2660 & 1304308992 & 3664236 & 2660 & 1376905216 & 3925805 \\
\hline 438 & $2 / 22 / 2017$ & 2660 & 1311760768 & 3756229 & 2660 & 1307933184 & 3624269 & 2660 & 1380790144 & 3884888 \\
\hline 439 & $2 / 23 / 2017$ & 2660 & 1315475456 & 3714711 & 2660 & 1311518720 & 3585617 & 2660 & 1384635392 & 3845235 \\
\hline 440 & $2 / 24 / 2017$ & 2660 & 1319149952 & 3674496 & 2660 & 1315067008 & 3548219 & 2660 & 1388442112 & 3806793 \\
\hline 441 & $2 / 25 / 2017$ & 2660 & 1322785536 & 3635528 & 2660 & 1318578944 & 3512017 & 2660 & 1392211712 & 3769508 \\
\hline 442 & $2 / 26 / 2017$ & 2660 & 1326383232 & 3597751 & 2660 & 1322055936 & 3476958 & 2660 & 1395944960 & 3733332 \\
\hline 443 & $2 / 27 / 2017$ & 2660 & 1329944448 & 3561116 & 2660 & 1325498880 & 3442988 & 2660 & 1399643264 & 3698214 \\
\hline 444 & $2 / 28 / 2017$ & 2660 & 1333469952 & 3525574 & 2660 & 1328908928 & 3410061 & 2660 & 1403307392 & 3664113 \\
\hline 445 & $3 / 1 / 2017$ & 2660 & 1336961024 & 3491079 & 2660 & 1332287104 & 3378130 & 2660 & 1406938240 & 3630985 \\
\hline 446 & $3 / 2 / 2017$ & 2660 & 1340418688 & 3457589 & 2660 & 1335634304 & 3347150 & 2660 & 1410537088 & 3598789 \\
\hline 447 & $3 / 3 / 2017$ & 2660 & 1343843712 & 3425061 & 2660 & 1338951296 & 3317082 & 2660 & 1414104576 & 3567487 \\
\hline 448 & $3 / 4 / 2017$ & 2660 & 1347237120 & 3393457 & 2660 & 1342239232 & 3287886 & 2660 & 1417641600 & 3537043 \\
\hline 449 & $3 / 5 / 2017$ & 2660 & 1350599936 & 3362738 & 2660 & 1345498752 & 3259525 & 2660 & 1421149056 & 3507423 \\
\hline 450 & $3 / 6 / 2017$ & 2660 & 1353932800 & 3332871 & 2660 & 1348730752 & 3231963 & 2660 & 1424627712 & 3478593 \\
\hline 451 & $3 / 7 / 2017$ & 2660 & 1357236608 & 3303821 & 2660 & 1351935872 & 3205168 & 2660 & 1428078208 & 3450522 \\
\hline 452 & $3 / 8 / 2017$ & 2660 & 1360512128 & 32755555 & 2660 & 1355115008 & 3179107 & 2660 & 1431501312 & 3423180 \\
\hline 453 & $3 / 9 / 2017$ & 2660 & 1363760128 & 3248044 & 2660 & 1358268800 & 3153750 & 2660 & 1434897920 & 3396540 \\
\hline 454 & $3 / 10 / 2017$ & 2660 & 1366981248 & 3221257 & 2660 & 1361397760 & 3129070 & 2660 & 1438268544 & 3370574 \\
\hline 455 & $3 / 11 / 2017$ & 2660 & 1370176640 & 3195169 & 2660 & 1364502784 & 3105038 & 2660 & 1441613568 & 3345256 \\
\hline 456 & $3 / 12 / 2017$ & 2660 & 1373346304 & 3169751 & 2660 & 1367584512 & 3081629 & 2660 & 1444934400 & 3320563 \\
\hline 457 & $3 / 13 / 2017$ & 2660 & 1376491392 & 3144979 & 2660 & 1370643328 & 3058818 & 2660 & 1448230784 & 3296471 \\
\hline 458 & $3 / 14 / 2017$ & 2660 & 1379612032 & 3120828 & 2660 & 1373679872 & 3036583 & 2660 & 1451503744 & 3272957 \\
\hline 459 & $3 / 15 / 2017$ & 2660 & 1382709504 & 3097277 & 2660 & 1376694784 & 3014901 & 2660 & 1454753664 & 3250001 \\
\hline 460 & $3 / 16 / 2017$ & 2660 & 1385783680 & 3074302 & 2660 & 1379688576 & 2993750 & 2660 & 1457981312 & 3227583 \\
\hline 461 & $3 / 17 / 2017$ & 2660 & 1388835584 & 3051883 & 2660 & 1382661632 & 2973111 & 2660 & 1461186944 & 3205682 \\
\hline 462 & $3 / 18 / 2017$ & 2660 & 1391865600 & 3029999 & 2660 & 1385614592 & 2952964 & 2660 & 1464371328 & 3184281 \\
\hline 463 & $3 / 19 / 2017$ & 2660 & 1394874240 & 3008633 & 2660 & 1388547840 & 2933291 & 2660 & 1467534592 & 3163361 \\
\hline 464 & $3 / 20 / 2017$ & 2660 & 1397862016 & 2987765 & 2660 & 1391462016 & 2914075 & 2660 & 1470677504 & 3142907 \\
\hline 465 & $3 / 21 / 2017$ & 2660 & 1400829440 & 2967379 & 2660 & 1394357248 & 2895299 & 2660 & 1473800448 & 3122902 \\
\hline 466 & $3 / 22 / 2017$ & 2660 & 1403776896 & 2947456 & 2660 & 1397234176 & 2876947 & 2660 & 1476903808 & 3103331 \\
\hline 467 & $3 / 23 / 2017$ & 2660 & 1406704896 & 2927982 & 2660 & 1400093184 & 2859004 & 2660 & 1479987968 & 3084179 \\
\hline 468 & $3 / 24 / 2017$ & 2660 & 1409613824 & 2908942 & 2660 & 1402934656 & 2841456 & 2660 & 1483053440 & 3065432 \\
\hline 469 & $3 / 25 / 2017$ & 2660 & 1412504064 & 2890319 & 2660 & 1405758976 & 2824289 & 2660 & 1486100480 & 3047076 \\
\hline 470 & $3 / 26 / 2017$ & 2660 & 1415376256 & 2872101 & 2660 & 1408566400 & 2807489 & 2660 & 1489129600 & 3029099 \\
\hline 471 & $3 / 27 / 2017$ & 2660 & 1418230528 & 2854274 & 2660 & 1411357440 & 2791044 & 2660 & 1492141056 & 3011489 \\
\hline
\end{tabular}




\begin{tabular}{|c|c|c|c|c|c|c|c|c|c|c|}
\hline 472 & $3 / 28 / 2017$ & 2660 & 1421067264 & 2836826 & 2660 & 1414132480 & 2774942 & 2660 & 1495135232 & 2994232 \\
\hline 473 & $3 / 29 / 2017$ & 2660 & 1423887104 & 2819743 & 2660 & 1416891648 & 2759172 & 2660 & 1498112640 & 2977319 \\
\hline 474 & $3 / 30 / 2017$ & 2660 & 1426690048 & 2803014 & 2660 & 1419635328 & 2743722 & 2660 & 1501073280 & 2960738 \\
\hline 475 & $3 / 31 / 2017$ & 2660 & 1429476736 & 2786628 & 2660 & 1422363904 & 2728581 & 2660 & 1504017792 & 2944479 \\
\hline 476 & $4 / 1 / 2017$ & 2660 & 1432247296 & 2770574 & 2660 & 1425077632 & 2713741 & 2660 & 1506946304 & 2928531 \\
\hline 477 & $4 / 2 / 2017$ & 2660 & 1435002112 & 2754841 & 2660 & 1427776896 & 2699190 & 2660 & 1509859200 & 2912886 \\
\hline 478 & $4 / 3 / 2017$ & 2660 & 1437741568 & 2739419 & 2660 & 1430461824 & 2684921 & 2660 & 1512756736 & 2897534 \\
\hline 479 & $4 / 4 / 2017$ & 2660 & 1440465792 & 2724299 & 2660 & 1433132800 & 2670923 & 2660 & 1515639168 & 2882466 \\
\hline 480 & $4 / 5 / 2017$ & 2660 & 1443175296 & 2709472 & 2660 & 1435789824 & 2657188 & 2660 & 1518506880 & 2867673 \\
\hline 481 & $4 / 6 / 2017$ & 2660 & 1445870208 & 2694928 & 2660 & 1438433536 & 2643710 & 2660 & 1521360000 & 2853148 \\
\hline 482 & $4 / 7 / 2017$ & 2660 & 1448550784 & 2680659 & 2660 & 1441064192 & 2630478 & 2660 & 1524198912 & 2838883 \\
\hline 483 & $4 / 8 / 2017$ & 2660 & 1451217536 & 2666657 & 2660 & 1443681536 & 2617486 & 2660 & 1527023744 & 2824869 \\
\hline 484 & 4/9/2017 & 2660 & 1453870464 & 2652913 & 2660 & 1446286336 & 2604727 & 2660 & 1529834880 & 2811100 \\
\hline 485 & $4 / 10 / 2017$ & 2660 & 1456509824 & 2639421 & 2660 & 1448878464 & 2592194 & 2660 & 1532632320 & 2797570 \\
\hline 486 & 4/11/2017 & 2660 & 1459136000 & 2626173 & 2660 & 1451458432 & 2579880 & 2660 & 1535416704 & 2784270 \\
\hline 487 & $4 / 12 / 2017$ & 2660 & 1461749248 & 2613162 & 2660 & 1454026112 & 2567779 & 2660 & 1538187904 & 2771195 \\
\hline 488 & $4 / 13 / 2017$ & 2660 & 1464349696 & 2600381 & 2660 & 1456582016 & 2555884 & 2660 & 1540946304 & 2758337 \\
\hline 489 & $4 / 14 / 2017$ & 2660 & 1466937344 & 2587824 & 2660 & 1459126144 & 2544190 & 2660 & 1543691904 & 2745693 \\
\hline 490 & $4 / 15 / 2017$ & 2660 & 1469512832 & 2575484 & 2660 & 1461659264 & 2533078 & 2660 & 1546425216 & 2733255 \\
\hline 491 & $4 / 16 / 2017$ & 2660 & 1472076288 & 2563355 & 2660 & 1464180736 & 2521405 & 2660 & 1549146240 & 2721018 \\
\hline 492 & $4 / 17 / 2017$ & 2660 & 1474627712 & 2551432 & 2660 & 1466691200 & 2510493 & 2660 & 1551855232 & 2708976 \\
\hline 493 & $4 / 18 / 2017$ & 2660 & 1477167360 & 2539709 & 2660 & 1469190528 & 2499323 & 2660 & 1554552320 & 2697125 \\
\hline 494 & $4 / 19 / 2017$ & 2660 & 1479695616 & 2528180 & 2660 & 1471679360 & 2488822 & 2660 & 1557237760 & 2685459 \\
\hline 495 & $4 / 20 / 2017$ & 2660 & 1482212352 & 2516841 & 2660 & 1474157312 & 2477954 & 2660 & 1559911808 & 2673974 \\
\hline 496 & $4 / 21 / 2017$ & 2660 & 1484718208 & 2505685 & 2660 & 1476625024 & 2467736 & 2660 & 1562574464 & 2662665 \\
\hline 497 & $4 / 22 / 2017$ & 2660 & 1487212800 & 2494709 & 2660 & 1479082240 & 2457239 & 2660 & 1565225984 & 2651528 \\
\hline 498 & $4 / 23 / 2017$ & 2660 & 1489696768 & 2483907 & 2660 & 1481529600 & 2447360 & 2660 & 1567866496 & 2640557 \\
\hline 499 & $4 / 24 / 2017$ & 2660 & 1492169984 & 2473276 & 2660 & 1483966592 & 2437154 & 2660 & 1570496256 & 2629749 \\
\hline 500 & $4 / 25 / 2017$ & 2660 & 1494632832 & 2462809 & 2660 & 1486394368 & 2427552 & 2660 & 1573115392 & 2619100 \\
\hline 501 & $4 / 26 / 2017$ & 2660 & 1497085312 & 2452505 & 2660 & 1488811904 & 2417662 & 2660 & 1575724032 & 2608607 \\
\hline 502 & $4 / 27 / 2017$ & 2660 & 1499527680 & 2442358 & 2660 & 1491220224 & 2408353 & 2660 & 1578322176 & 2598263 \\
\hline 503 & $4 / 28 / 2017$ & 2660 & 1501960064 & 2432363 & 2660 & 1493619200 & 2398736 & 2660 & 1580910336 & 2588069 \\
\hline 504 & $4 / 29 / 2017$ & 2660 & 1504382592 & 2422519 & 2660 & 1496008704 & 2389685 & 2660 & 1583488384 & 2578017 \\
\hline 505 & $4 / 30 / 2017$ & 2660 & 1506795264 & 2412820 & 2660 & 1498389120 & 2380347 & 2660 & 1586056448 & 2568107 \\
\hline 506 & $5 / 1 / 2017$ & 2660 & 1509198592 & 2403264 & 2660 & 1500760704 & 2371556 & 2660 & 1588614784 & 2558333 \\
\hline 507 & $5 / 2 / 2017$ & 2660 & 1511592448 & 2393846 & 2660 & 1503123072 & 2362469 & 2660 & 1591163520 & 2548694 \\
\hline 508 & $5 / 3 / 2017$ & 2660 & 1513977088 & 2384564 & 2660 & 1505476992 & 2353917 & 2660 & 1593702656 & 2539185 \\
\hline 509 & $5 / 4 / 2017$ & 2660 & 1516352256 & 2375414 & 2660 & 1507822080 & 2345080 & 2660 & 1596232448 & 2529804 \\
\hline 510 & $5 / 5 / 2017$ & 2660 & 1518718848 & 2366393 & 2660 & 1510158848 & 2336760 & 2660 & 1598753024 & 2520548 \\
\hline 511 & $5 / 6 / 2017$ & 2660 & 1521076352 & 2357498 & 2660 & 1512487040 & 2328154 & 2660 & 1601264256 & 2511415 \\
\hline 512 & $5 / 7 / 2017$ & 2660 & 1523425024 & 2348726 & 2660 & 1514807040 & 2320053 & 2660 & 1603766656 & 2502401 \\
\hline 513 & $5 / 8 / 2017$ & 2660 & 1525765120 & 2340074 & 2660 & 1517118848 & 2311674 & 2660 & 1606260224 & 2493424 \\
\hline 514 & $5 / 9 / 2017$ & 2660 & 1528096768 & 2331540 & 2660 & 1519422592 & 2303784 & 2660 & 1608744960 & 2484722 \\
\hline 515 & $5 / 10 / 2017$ & 2660 & 1530420352 & 2323627 & 2660 & 1521718144 & 2295615 & 2660 & 1611220992 & 2475980 \\
\hline 516 & $5 / 11 / 2017$ & 2660 & 1532735232 & 2314867 & 2660 & 1524006144 & 2287926 & 2660 & 1613688448 & 2467488 \\
\hline 517 & $5 / 12 / 2017$ & 2660 & 1535042176 & 2306969 & 2660 & 1526286080 & 2279964 & 2660 & 1616147328 & 2458963 \\
\hline 518 & $5 / 13 / 2017$ & 2660 & 1537340672 & 2298558 & 2660 & 1528558464 & 2272468 & 2660 & 1618598016 & 2450684 \\
\hline 519 & $5 / 14 / 2017$ & 2660 & 1539631616 & 2290923 & 2660 & 1530823168 & 2264699 & 2660 & 1621040384 & 2442368 \\
\hline 520 & $5 / 15 / 2017$ & 2660 & 1541914368 & 2282701 & 2660 & 1533080576 & 2257387 & 2660 & 1623474688 & 2434288 \\
\hline 521 & $5 / 16 / 2017$ & 2660 & 1544189568 & 2275228 & 2660 & 1535330432 & 2249808 & 2660 & 1625900928 & 2426172 \\
\hline 522 & $5 / 17 / 2017$ & 2660 & 1546456832 & 2267229 & 2660 & 1537573248 & 2242671 & 2660 & 1628319104 & 2418286 \\
\hline 523 & $5 / 18 / 2017$ & 2660 & 1548716672 & 2259957 & 2660 & 1539808384 & 2235270 & 2660 & 1630729600 & 2410362 \\
\hline 524 & $5 / 19 / 2017$ & 2660 & 1550968832 & 2252149 & 2660 & 1542036736 & 2228303 & 2660 & 1633132160 & 2402661 \\
\hline 525 & $5 / 20 / 2017$ & 2660 & 1553213952 & 2245050 & 2660 & 1544257792 & 2221076 & 2660 & 1635527168 & 2394920 \\
\hline 526 & $5 / 21 / 2017$ & 2660 & 1555451392 & 2237433 & 2660 & 1546472064 & 2214271 & 2660 & 1637914496 & 2387397 \\
\hline 527 & $5 / 22 / 2017$ & 2660 & 1557681920 & 2230508 & 2660 & 1548679168 & 2207208 & 2660 & 1640294400 & 2379834 \\
\hline 528 & $5 / 23 / 2017$ & 2660 & 1559904896 & 2223071 & 2660 & 1550879744 & 2200559 & 2660 & 1642667008 & 2372481 \\
\hline 529 & $5 / 24 / 2017$ & 2660 & 1562121216 & 2216312 & 2660 & 1553073792 & 2194072 & 2660 & 1645031936 & 2365088 \\
\hline 530 & $5 / 25 / 2017$ & 2660 & 1564330368 & 2209049 & 2660 & 1555260800 & 2187020 & 2660 & 1647389824 & 2357900 \\
\hline 531 & $5 / 26 / 2017$ & 2660 & 1566532736 & 2202445 & 2660 & 1557441408 & 2180549 & 2660 & 1649740416 & 2350670 \\
\hline 532 & $5 / 27 / 2017$ & 2660 & 1568728064 & 2195350 & 2660 & 1559615616 & 2174184 & 2660 & 1652084096 & 2343639 \\
\hline 533 & $5 / 28 / 2017$ & 2660 & 1570916992 & 2188902 & 2660 & 1561783040 & 2167463 & 2660 & 1654420864 & 2336566 \\
\hline 534 & $5 / 29 / 2017$ & 2660 & 1573099008 & 2181966 & 2660 & 1563944320 & 2161212 & 2660 & 1656750336 & 2329619 \\
\hline 535 & $5 / 30 / 2017$ & 2660 & 1575274496 & 2175660 & 2660 & 1566099328 & 2155063 & 2660 & 1659073024 & 2322759 \\
\hline 536 & $5 / 31 / 2017$ & 2660 & 1577443456 & 2168880 & 2660 & 1568247936 & 2148547 & 2660 & 1661389056 & 2315968 \\
\hline 537 & $6 / 1 / 2017$ & 2660 & 1579606272 & 2162718 & 2660 & 1570390400 & 2142494 & 2660 & 1663698304 & 2309252 \\
\hline 538 & $6 / 2 / 2017$ & 2660 & 1581762304 & 2156084 & 2660 & 1572526848 & 2136538 & 2660 & 1666000896 & 2302604 \\
\hline 539 & $6 / 3 / 2017$ & 2660 & 1583912448 & 2150053 & 2660 & 1574657152 & 2130225 & 2660 & 1668296960 & 2296027 \\
\hline 540 & $6 / 4 / 2017$ & 2660 & 1586055936 & 2143565 & 2660 & 1576781568 & 2124360 & 2660 & 1670586496 & 2289517 \\
\hline 541 & $6 / 5 / 2017$ & 2660 & 1588193536 & 2137668 & 2660 & 1578900096 & 2118587 & 2660 & 1672869504 & 2283074 \\
\hline 542 & $6 / 6 / 2017$ & 2660 & 1590324864 & 2131315 & 2660 & 1581012608 & 2112466 & 2660 & 1675146240 & 2276696 \\
\hline 543 & $6 / 7 / 2017$ & 2660 & 1592450432 & 2125539 & 2660 & 1583119360 & 2106780 & 2660 & 1677416576 & 2270382 \\
\hline 544 & $6 / 8 / 2017$ & 2660 & 1594570240 & 2119809 & 2660 & 1585220480 & 2101181 & 2660 & 1679680768 & 2264132 \\
\hline 545 & $6 / 9 / 2017$ & 2660 & 1596683520 & 2113452 & 2660 & 1587315712 & 2095241 & 2660 & 1681938688 & 2257944 \\
\hline 546 & $6 / 10 / 2017$ & 2660 & 1598791552 & 2107811 & 2660 & 1589405568 & 2089724 & 2660 & 1684190464 & 2251817 \\
\hline 547 & $6 / 11 / 2017$ & 2660 & 1600893696 & 2102207 & 2660 & 1591489792 & 2084290 & 2660 & 1686436224 & 2245750 \\
\hline 548 & $6 / 12 / 2017$ & 2660 & 1602989824 & 2096099 & 2660 & 1593568256 & 2078523 & 2660 & 1688675968 & 2239742 \\
\hline 549 & $6 / 13 / 2017$ & 2660 & 1605080448 & 2090635 & 2660 & 1595641472 & 2073166 & 2660 & 1690909952 & 2233792 \\
\hline 550 & $6 / 14 / 2017$ & 2660 & 1607165696 & 2085203 & 2660 & 1597709312 & 2067889 & 2660 & 1693137664 & 2227899 \\
\hline 551 & $6 / 15 / 2017$ & 2660 & 1609244928 & 2079273 & 2660 & 1599771648 & 2062285 & 2660 & 1695359744 & 2222062 \\
\hline 552 & $6 / 16 / 2017$ & 2660 & 1611318912 & 2073971 & 2660 & 1601828736 & 2057081 & 2660 & 1697576064 & 2216280 \\
\hline 553 & $6 / 17 / 2017$ & 2660 & 1613387648 & 2068698 & 2660 & 1603880704 & 2051952 & 2660 & 1699786624 & 2210553 \\
\hline
\end{tabular}




\begin{tabular}{|c|c|c|c|c|c|c|c|c|c|c|}
\hline 554 & $6 / 18 / 2017$ & 2660 & 1615450496 & 2062939 & 2660 & 1605927168 & 2046504 & 2660 & 1701991552 & 2204879 \\
\hline 555 & 6/19/2017 & 2660 & 1617508352 & 2057791 & 2660 & 1607968640 & 2041445 & 2660 & 1704190720 & 2199257 \\
\hline 556 & 6/20/2017 & 2660 & 1619560960 & 2052670 & 2660 & 1610005120 & 2036458 & 2660 & 1706384384 & 2193688 \\
\hline 557 & 6/21/2017 & 2660 & 1621608064 & 2047075 & 2660 & 1612036224 & 2031159 & 2660 & 1708572544 & 2188169 \\
\hline 558 & $6 / 22 / 2017$ & 2660 & 1623650176 & 2042072 & 2660 & 1614062464 & 2026237 & 2660 & 1710755200 & 2182700 \\
\hline 559 & 6/23/2017 & 2660 & 1625687296 & 2037095 & 2660 & 1616083968 & 2021385 & 2660 & 1712932480 & 2177280 \\
\hline 560 & 6/24/2017 & 2660 & 1627718912 & 2031655 & 2660 & 1618100096 & 2016227 & 2660 & 1715104512 & 2171909 \\
\hline 561 & $6 / 25 / 2017$ & 2660 & 1629745536 & 2026792 & 2660 & 1620111488 & 2011437 & 2660 & 1717271040 & 2166585 \\
\hline 562 & $6 / 26 / 2017$ & 2660 & 1631767680 & 2021952 & 2660 & 1622118272 & 2006714 & 2660 & 1719432320 & 2161308 \\
\hline 563 & $6 / 27 / 2017$ & 2660 & 1633784320 & 2016660 & 2660 & 1624119936 & 2001691 & 2660 & 1721588480 & 2156078 \\
\hline 564 & $6 / 28 / 2017$ & 2660 & 1635796224 & 2011928 & 2660 & 1626116992 & 1997027 & 2660 & 1723739264 & 2150893 \\
\hline 565 & $6 / 29 / 2017$ & 2660 & 1637803392 & 2007219 & 2660 & 1628109312 & 1992426 & 2660 & 1725885056 & 2145754 \\
\hline 566 & 6/30/2017 & 2660 & 1639805568 & 2002068 & 2660 & 1630096896 & 1987532 & 2660 & 1728025728 & 2140658 \\
\hline 567 & $7 / 1 / 2017$ & 2660 & 1641803008 & 1997463 & 2660 & 1632079872 & 1982988 & 2660 & 1730161280 & 2135606 \\
\hline 568 & $7 / 2 / 2017$ & 2660 & 1643795840 & 1992878 & 2660 & 1634058368 & 1978505 & 2660 & 1732291840 & 2130596 \\
\hline 569 & $7 / 3 / 2017$ & 2660 & 1645783680 & 1987861 & 2660 & 1636032128 & 1973734 & 2660 & 1734417536 & 2125629 \\
\hline 570 & $7 / 4 / 2017$ & 2660 & 1647767040 & 1983377 & 2660 & 1638001408 & 1969304 & 2660 & 1736538240 & 2120704 \\
\hline 571 & $7 / 5 / 2017$ & 2660 & 1649746048 & 1978910 & 2660 & 1639966336 & 1964933 & 2660 & 1738654080 & 2115820 \\
\hline 572 & $7 / 6 / 2017$ & 2660 & 1651720064 & 1974022 & 2660 & 1641927040 & 1960709 & 2660 & 1740765056 & 2110976 \\
\hline 573 & $7 / 7 / 2017$ & 2660 & 1653689728 & 1969652 & 2660 & 1643883264 & 1956295 & 2660 & 1742871168 & 2106172 \\
\hline 574 & $7 / 8 / 2017$ & 2660 & 1655655040 & 1965299 & 2660 & 1645834880 & 1951522 & 2660 & 1744972672 & 2101407 \\
\hline 575 & $7 / 9 / 2017$ & 2660 & 1657615488 & 1960534 & 2660 & 1647782272 & 1947256 & 2660 & 1747069312 & 2096680 \\
\hline 576 & $7 / 10 / 2017$ & 2660 & 1659571840 & 1956274 & 2660 & 1649725184 & 1943064 & 2660 & 1749161344 & 2091992 \\
\hline 577 & $7 / 11 / 2017$ & 2660 & 1661523840 & 1952029 & 2660 & 1651664256 & 1939004 & 2660 & 1751248640 & 2087342 \\
\hline 578 & $7 / 12 / 2017$ & 2660 & 1663471232 & 1947381 & 2660 & 1653598976 & 1934761 & 2660 & 1753331328 & 2082729 \\
\hline 579 & $7 / 13 / 2017$ & 2660 & 1665414400 & 1943226 & 2660 & 1655529600 & 1930584 & 2660 & 1755409536 & 2078152 \\
\hline 580 & $7 / 14 / 2017$ & 2660 & 1667353472 & 1939085 & 2660 & 1657456000 & 1926402 & 2660 & 1757483136 & 2073611 \\
\hline 581 & $7 / 15 / 2017$ & 2660 & 1669288192 & 1934550 & 2660 & 1659378176 & 1922276 & 2660 & 1759552256 & 2069106 \\
\hline 582 & $7 / 16 / 2017$ & 2660 & 1671218560 & 1930494 & 2660 & 1661296384 & 1918163 & 2660 & 1761616896 & 2064636 \\
\hline 583 & $7 / 17 / 2017$ & 2660 & 1673144960 & 1926453 & 2660 & 1663210496 & 1914098 & 2660 & 1763677056 & 2060201 \\
\hline 584 & $7 / 18 / 2017$ & 2660 & 1675067008 & 1922025 & 2660 & 1665120640 & 1910048 & 2660 & 1765732864 & 2055800 \\
\hline 585 & $7 / 19 / 2017$ & 2660 & 1676985088 & 1918066 & 2660 & 1667026560 & 1906044 & 2660 & 1767784320 & 2051432 \\
\hline 586 & $7 / 20 / 2017$ & 2660 & 1678899200 & 1914119 & 2660 & 1668928640 & 1902056 & 2660 & 1769831424 & 2047098 \\
\hline 587 & $7 / 21 / 2017$ & 2660 & 1680808960 & 1909795 & 2660 & 1670826752 & 1898112 & 2660 & 1771874176 & 2042796 \\
\hline 588 & $7 / 22 / 2017$ & 2660 & 1682714880 & 1905928 & 2660 & 1672720896 & 1894185 & 2660 & 1773912576 & 2038527 \\
\hline 589 & $7 / 23 / 2017$ & 2660 & 1684616960 & 1902073 & 2660 & 1674611200 & 1890299 & 2660 & 1775947008 & 2034289 \\
\hline 590 & $7 / 24 / 2017$ & 2660 & 1686514816 & 1897847 & 2660 & 1676497664 & 1886430 & 2660 & 1777977088 & 2030084 \\
\hline 591 & $7 / 25 / 2017$ & 2660 & 1688408960 & 1894069 & 2660 & 1678380288 & 1882602 & 2660 & 1780002944 & 2025909 \\
\hline 592 & $7 / 26 / 2017$ & 2660 & 1690299264 & 1890301 & 2660 & 1680259072 & 1878790 & 2660 & 1782024832 & 2021765 \\
\hline 593 & $7 / 27 / 2017$ & 2660 & 1692185344 & 1886171 & 2660 & 1682134016 & 1875018 & 2660 & 1784042496 & 2017651 \\
\hline 594 & $7 / 28 / 2017$ & 2660 & 1694067840 & 1882477 & 2660 & 1684005248 & 1871260 & 2660 & 1786055936 & 2013567 \\
\hline 595 & $7 / 29 / 2017$ & 2660 & 1695946624 & 1878793 & 2660 & 1685872896 & 1867543 & 2660 & 1788065536 & 2009513 \\
\hline 596 & $7 / 30 / 2017$ & 2660 & 1697821440 & 1874755 & 2660 & 1687736704 & 1863840 & 2660 & 1790071040 & 2005488 \\
\hline 597 & $7 / 31 / 2017$ & 2660 & 1699692544 & 1871142 & 2660 & 1689596928 & 1860176 & 2660 & 1792072448 & 2001491 \\
\hline 598 & $8 / 1 / 2017$ & 2660 & 1701560064 & 1867539 & 2660 & 1691453440 & 1856525 & 2660 & 1794070016 & 1997523 \\
\hline 599 & $8 / 2 / 2017$ & 2660 & 1703423744 & 1863588 & 2660 & 1693306368 & 1852913 & 2660 & 1796063488 & 1993583 \\
\hline 600 & $8 / 3 / 2017$ & 2660 & 1705283712 & 1860055 & 2660 & 1695155584 & 1849313 & 2660 & 1798053120 & 1989671 \\
\hline 601 & $8 / 4 / 2017$ & 2660 & 1707140224 & 1856529 & 2660 & 1697001344 & 1845751 & 2660 & 1800039040 & 1985786 \\
\hline 602 & $8 / 5 / 2017$ & 2660 & 1708993408 & 1853111 & 2660 & 1698843648 & 1842202 & 2660 & 1802020992 & 1981929 \\
\hline 603 & $8 / 6 / 2017$ & 2660 & 1710842880 & 1849523 & 2660 & 1700682368 & 1838689 & 2660 & 1803998976 & 1978097 \\
\hline 604 & $8 / 7 / 2017$ & 2660 & 1712688896 & 1845981 & 2660 & 1702517504 & 1835189 & 2660 & 1805973376 & 1974293 \\
\hline 605 & $8 / 8 / 2017$ & 2660 & 1714531328 & 1842433 & 2660 & 1704349184 & 1831725 & 2660 & 1807943936 & 1970514 \\
\hline 606 & $8 / 9 / 2017$ & 2660 & 1716370304 & 1838925 & 2660 & 1706177536 & 1828272 & 2660 & 1809910656 & 1966761 \\
\hline 607 & $8 / 10 / 2017$ & 2660 & 1718205696 & 1835429 & 2660 & 1708002304 & 1824855 & 2660 & 1811873664 & 1963034 \\
\hline 608 & $8 / 11 / 2017$ & 2660 & 1720037632 & 1831965 & 2660 & 1709823744 & 1821448 & 2660 & 1813832960 & 1959331 \\
\hline 609 & $8 / 12 / 2017$ & 2660 & 1721866112 & 1828518 & 2660 & 1711641856 & 1818077 & 2660 & 1815788672 & 1955654 \\
\hline 610 & $8 / 13 / 2017$ & 2660 & 1723691264 & 1825100 & 2660 & 1713456512 & 1814716 & 2660 & 1817740672 & 1952001 \\
\hline 611 & $8 / 14 / 2017$ & 2660 & 1725512960 & 1821701 & 2660 & 1715267968 & 1811389 & 2660 & 1819689088 & 1948372 \\
\hline 612 & $8 / 15 / 2017$ & 2660 & 1727331328 & 1818328 & 2660 & 1717076096 & 1808073 & 2660 & 1821633792 & 1944768 \\
\hline 613 & $8 / 16 / 2017$ & 2660 & 1729146240 & 1814975 & 2660 & 1718880768 & 1804790 & 2660 & 1823575040 & 1941187 \\
\hline 614 & $8 / 17 / 2017$ & 2660 & 1730957952 & 1811647 & 2660 & 1720682368 & 1801517 & 2660 & 1825512576 & 1937629 \\
\hline 615 & $8 / 18 / 2017$ & 2660 & 1732766208 & 1808338 & 2660 & 1722480640 & 1798277 & 2660 & 1827446656 & 1934095 \\
\hline 616 & $8 / 19 / 2017$ & 2660 & 1734571264 & 1805054 & 2660 & 1724275712 & 1795046 & 2660 & 1829377280 & 1930583 \\
\hline 617 & $8 / 20 / 2017$ & 2660 & 1736373120 & 1801788 & 2660 & 1726067456 & 1791847 & 2660 & 1831304448 & 1927094 \\
\hline 618 & $8 / 21 / 2017$ & 2660 & 1738171648 & 1798547 & 2660 & 1727856128 & 1788658 & 2660 & 1833228032 & 1923628 \\
\hline 619 & $8 / 22 / 2017$ & 2660 & 1739966848 & 1795325 & 2660 & 1729641728 & 1785500 & 2660 & 1835148160 & 1920183 \\
\hline 620 & $8 / 23 / 2017$ & 2660 & 1741759104 & 1792125 & 2660 & 1731424000 & 1782351 & 2660 & 1837064960 & 1916760 \\
\hline 621 & $8 / 24 / 2017$ & 2660 & 1743548160 & 1788944 & 2660 & 1733203200 & 1779234 & 2660 & 1838978304 & 1913360 \\
\hline 622 & $8 / 25 / 2017$ & 2660 & 1745333760 & 1785786 & 2660 & 1734979328 & 1776124 & 2660 & 1840888320 & 1909980 \\
\hline 623 & $8 / 26 / 2017$ & 2660 & 1747116416 & 1782646 & 2660 & 1736752384 & 1773045 & 2660 & 1842794880 & 1906622 \\
\hline 624 & $8 / 27 / 2017$ & 2660 & 1748896000 & 1779528 & 2660 & 1738522368 & 1769975 & 2660 & 1844698240 & 1903284 \\
\hline 625 & $8 / 28 / 2017$ & 2660 & 1750672384 & 1776427 & 2660 & 1740289280 & 1766934 & 2660 & 1846598144 & 1899967 \\
\hline 626 & $8 / 29 / 2017$ & 2660 & 1752445824 & 1773348 & 2660 & 1742053248 & 1763901 & 2660 & 1848494848 & 1896671 \\
\hline 627 & $8 / 30 / 2017$ & 2660 & 1754216064 & 1770286 & 2660 & 1743814144 & 1760898 & 2660 & 1850388224 & 1893395 \\
\hline 628 & $8 / 31 / 2017$ & 2660 & 1755983360 & 1767246 & 2660 & 1745571968 & 1757902 & 2660 & 1852278400 & 1890139 \\
\hline 629 & $9 / 1 / 2017$ & 2660 & 1757747584 & 1764222 & 2660 & 1747326848 & 1754936 & 2660 & 1854165248 & 1886902 \\
\hline 630 & $9 / 2 / 2017$ & 2660 & 1759508736 & 1761219 & 2660 & 1749078912 & 1751976 & 2660 & 1856049024 & 1883685 \\
\hline 631 & $9 / 3 / 2017$ & 2660 & 1761266816 & 1758232 & 2660 & 1750828032 & 1749045 & 2660 & 1857929472 & 1880488 \\
\hline 632 & $9 / 4 / 2017$ & 2660 & 1763022208 & 1755266 & 2660 & 1752574080 & 1746121 & 2660 & 1859806720 & 1877310 \\
\hline 633 & $9 / 5 / 2017$ & 2660 & 1764774528 & 1752315 & 2660 & 1754317312 & 1743225 & 2660 & 1861680896 & 1874151 \\
\hline 634 & $9 / 6 / 2017$ & 2660 & 1766523904 & 1749385 & 2660 & 1756057728 & 1740335 & 2660 & 1863551872 & 1871010 \\
\hline 635 & $9 / 7 / 2017$ & 2660 & 1768270464 & 1746470 & 2660 & 1757795200 & 1737474 & 2660 & 1865419776 & 1867888 \\
\hline
\end{tabular}




\begin{tabular}{|c|c|c|c|c|c|c|c|c|c|c|}
\hline 636 & 9/8/2017 & 2660 & 1770013952 & 1743575 & 2660 & 1759529728 & 1734618 & 2660 & 1867284608 & 1864785 \\
\hline 637 & $9 / 9 / 2017$ & 2660 & 1771754624 & 1740695 & 2660 & 1761261568 & 1731790 & 2660 & 1869146240 & 1861699 \\
\hline 638 & 9/10/2017 & 2660 & 1773492480 & 1737834 & 2660 & 1762990464 & 1728968 & 2660 & 1871004928 & 1858632 \\
\hline 639 & 9/11/2017 & 2660 & 1775227520 & 1734988 & 2660 & 1764716672 & 1726172 & 2660 & 1872860544 & 1855583 \\
\hline 640 & 9/12/2017 & 2660 & 1776959616 & 1732160 & 2660 & 1766440064 & 1723383 & 2660 & 1874713088 & 1852551 \\
\hline 641 & 9/13/2017 & 2660 & 1778689024 & 1729348 & 2660 & 1768160640 & 1720620 & 2660 & 1876562688 & 1849536 \\
\hline 642 & 9/14/2017 & 2660 & 1780415616 & 1726553 & 2660 & 1769878528 & 1717862 & 2660 & 1878408960 & 1846539 \\
\hline 643 & 9/15/2017 & 2660 & 1782139392 & 1723773 & 2660 & 1771593728 & 1715130 & 2660 & 1880252672 & 1843559 \\
\hline 644 & $9 / 16 / 2017$ & 2660 & 1783860352 & 1721011 & 2660 & 1773306112 & 1712404 & 2660 & 1882093312 & 1840596 \\
\hline 645 & 9/17/2017 & 2660 & 1785578624 & 1718263 & 2660 & 1775015808 & 1709703 & 2660 & 1883930880 & 1837650 \\
\hline 646 & 9/18/2017 & 2660 & 1787294080 & 1715533 & 2660 & 1776722816 & 1707007 & 2660 & 1885765632 & 1834720 \\
\hline 647 & 9/19/2017 & 2660 & 1789006976 & 1712816 & 2660 & 1778427136 & 1704337 & 2660 & 1887597440 & 1831806 \\
\hline 648 & 9/20/2017 & 2660 & 1790717056 & 1710116 & 2660 & 1780128768 & 1701671 & 2660 & 1889426432 & 1828909 \\
\hline 649 & $9 / 21 / 2017$ & 2660 & 1792424448 & 1707430 & 2660 & 1781827840 & 1699030 & 2660 & 1891252352 & 1826028 \\
\hline 650 & $9 / 22 / 2017$ & 2660 & 1794129280 & 1704761 & 2660 & 1783524096 & 1696394 & 2660 & 1893075584 & 1823163 \\
\hline 651 & $9 / 23 / 2017$ & 2660 & 1795831424 & 1702105 & 2660 & 1785218048 & 1693782 & 2660 & 1894895872 & 1820314 \\
\hline 652 & $9 / 24 / 2017$ & 1196 & 1841367424 & 45536012 & 1196 & 1834489216 & 49271092 & 1196 & 1975851904 & 80956008 \\
\hline 653 & $9 / 25 / 2017$ & 1196 & 1882026240 & 40658880 & 1196 & 1877508096 & 43018956 & 1196 & 2014119552 & 38267756 \\
\hline 654 & 9/26/2017 & 1196 & 1913022464 & 30996236 & 1196 & 1910233216 & 32725128 & 1196 & 2044602240 & 30482548 \\
\hline 655 & 9/27/2017 & 1196 & 1939321600 & 26299086 & 1196 & 1937755264 & 27522034 & 1196 & 2071674240 & 27071992 \\
\hline 656 & $9 / 28 / 2017$ & 1196 & 1963087616 & 23766078 & 1196 & 1962533248 & 24778086 & 1196 & 2096219392 & 24545262 \\
\hline 657 & $9 / 29 / 2017$ & 1196 & 1984935168 & 21847512 & 1196 & 1985225472 & 22692138 & 1196 & 2118799744 & 22580310 \\
\hline 658 & 9/30/2017 & 1196 & 2005260672 & 20325504 & 1196 & 2006262016 & 21036396 & 1196 & 2139789440 & 20989720 \\
\hline 659 & $10 / 1 / 2017$ & 1196 & 2024337536 & 19076844 & 1196 & 2025939968 & 19678130 & 1196 & 2159457792 & 19668394 \\
\hline 660 & $10 / 2 / 2017$ & 1196 & 2042364928 & 18027404 & 1196 & 2044477056 & 18537052 & 1196 & 2178007296 & 18549434 \\
\hline 661 & $10 / 3 / 2017$ & 1196 & 2059493760 & 17128848 & 1196 & 2062037760 & 17560712 & 1196 & 2195594240 & 17587028 \\
\hline 662 & $10 / 4 / 2017$ & 1196 & 2075841408 & 16347605 & 1196 & 2078750336 & 16712611 & 1196 & 2212342784 & 16748339 \\
\hline 663 & $10 / 5 / 2017$ & 1196 & 2091500800 & 15659445 & 1196 & 2094716800 & 15966402 & 1196 & 2228351744 & 16009127 \\
\hline 664 & $10 / 6 / 2017$ & 1196 & 2106547072 & 15046434 & 1196 & 2110019328 & 15302570 & 1196 & 2243703040 & 15351137 \\
\hline 665 & $10 / 7 / 2017$ & 1196 & 2121042304 & 14495031 & 1196 & 2124725632 & 14706377 & 1196 & 2258463232 & 14760311 \\
\hline 666 & $10 / 8 / 2017$ & 1196 & 2135036544 & 13994338 & 1196 & 2138892160 & 14166505 & 1196 & 2272688896 & 14225755 \\
\hline 667 & $10 / 9 / 2017$ & 1196 & 2148573952 & 13537308 & 1196 & 2152566016 & 13673690 & 1196 & 2286427904 & 13738866 \\
\hline 668 & $10 / 10 / 2017$ & 1196 & 2161691136 & 13117119 & 1196 & 2165787648 & 13221862 & 1196 & 2299720192 & 13292370 \\
\hline 669 & $10 / 11 / 2017$ & 1196 & 2174419712 & 12728605 & 1196 & 2178592768 & 12805061 & 1196 & 2312601856 & 12881517 \\
\hline 670 & $10 / 12 / 2017$ & 1196 & 2186787328 & 12367643 & 1196 & 2191011584 & 12418712 & 1196 & 2325103104 & 12501451 \\
\hline 671 & $10 / 13 / 2017$ & 1196 & 2198818048 & 12030868 & 1196 & 2203070720 & 12059103 & 1196 & 2337251584 & 12148379 \\
\hline 672 & $10 / 14 / 2017$ & 1196 & 2210533632 & 11715510 & 1196 & 2214793728 & 11723173 & 1196 & 2349070848 & 11819171 \\
\hline 673 & $10 / 15 / 2017$ & 1196 & 2221953024 & 11419261 & 1196 & 2226202112 & 11408366 & 1196 & 2360579584 & 11508922 \\
\hline 674 & $10 / 16 / 2017$ & 1196 & 2233093120 & 11140172 & 1196 & 2237314816 & 11112527 & 1196 & 2371801856 & 11222085 \\
\hline 675 & $10 / 17 / 2017$ & 1196 & 2243969792 & 10876590 & 1196 & 2248148480 & 10833817 & 1196 & 2382750464 & 10948405 \\
\hline 676 & $10 / 18 / 2017$ & 1196 & 2254598912 & 10629286 & 1196 & 2258719232 & 10570658 & 1196 & 2393444352 & 10694160 \\
\hline 677 & $10 / 19 / 2017$ & 1196 & 2264991744 & 10392698 & 1196 & 2269040896 & 10321680 & 1196 & 2403894528 & 10450306 \\
\hline 678 & $10 / 20 / 2017$ & 1196 & 2275159552 & 10167825 & 1196 & 2279126528 & 10085693 & 1196 & 2414115584 & 10221015 \\
\hline 679 & $10 / 21 / 2017$ & 1196 & 2285113344 & 9953800 & 1196 & 2288988160 & 9861649 & 1196 & 2424119296 & 10003711 \\
\hline 680 & $10 / 22 / 2017$ & 1196 & 2294863104 & 9749809 & 1196 & 2298636800 & 9648620 & 1196 & 2433916672 & 9797326 \\
\hline 681 & $10 / 23 / 2017$ & 1196 & 2304418304 & 9555122 & 1196 & 2308082688 & 9445785 & 1196 & 2443517440 & 9600987 \\
\hline 682 & $10 / 24 / 2017$ & 1196 & 2313787392 & 9369084 & 1196 & 2317335040 & 9252408 & 1196 & 2452931584 & 9413938 \\
\hline 683 & $10 / 25 / 2017$ & 1196 & 2322978560 & 9191107 & 1196 & 2326402816 & 9067829 & 1196 & 2462167040 & 9235504 \\
\hline 684 & $10 / 26 / 2017$ & 1196 & 2331998976 & 9020661 & 1196 & 2335294208 & 8891454 & 1196 & 2471232256 & 9065081 \\
\hline 685 & $10 / 27 / 2017$ & 1196 & 2340856320 & 8857266 & 1196 & 2344017152 & 8722743 & 1196 & 2480134400 & 8902125 \\
\hline 686 & $10 / 28 / 2017$ & 1196 & 2349556736 & 8700486 & 1196 & 2352578304 & 8561207 & 1196 & 2488880640 & 8746142 \\
\hline 687 & $10 / 29 / 2017$ & 1196 & 2358108672 & 8551861 & 1196 & 2360984576 & 8406399 & 1196 & 2497477120 & 8596684 \\
\hline 688 & $10 / 30 / 2017$ & 1196 & 2366514176 & 8405493 & 1196 & 2369242368 & 8257617 & 1196 & 2505930496 & 8453341 \\
\hline 689 & $10 / 31 / 2017$ & 1196 & 2374780160 & 8265838 & 1196 & 2377357568 & 8115332 & 1196 & 2514246144 & 8315740 \\
\hline 690 & $11 / 1 / 2017$ & 1196 & 2382912000 & 8132056 & 1196 & 2385336064 & 7978163 & 1196 & 2522429696 & 8183537 \\
\hline 691 & $11 / 2 / 2017$ & 1196 & 2390916608 & 8004444 & 1196 & 2393182464 & 7846762 & 1196 & 2530486272 & 8056417 \\
\hline 692 & $11 / 3 / 2017$ & 1196 & 2398795264 & 7878824 & 1196 & 2400902400 & 7719887 & 1196 & 2538420224 & 7934092 \\
\hline 693 & $11 / 4 / 2017$ & 1196 & 2406553856 & 7758480 & 1196 & 2408500224 & 7597907 & 1196 & 2546236672 & 7816292 \\
\hline 694 & $11 / 5 / 2017$ & 1196 & 2414198016 & 7644175 & 1196 & 2415980800 & 7480434 & 1196 & 2553939456 & 7702771 \\
\hline 695 & $11 / 6 / 2017$ & 1196 & 2421729024 & 7530975 & 1196 & 2423347968 & 7367208 & 1196 & 2561532672 & 7593300 \\
\hline 696 & $11 / 7 / 2017$ & 1196 & 2429151744 & 7422831 & 1196 & 2430606080 & 7258000 & 1196 & 2569020416 & 7487665 \\
\hline 697 & $11 / 8 / 2017$ & 1196 & 2436471552 & 7319820 & 1196 & 2437758464 & 7152602 & 1196 & 2576406016 & 7385669 \\
\hline 698 & $11 / 9 / 2017$ & 1196 & 2443689216 & 7217552 & 1196 & 2444809472 & 7050826 & 1196 & 2583693056 & 7287127 \\
\hline 699 & $11 / 10 / 2017$ & 1196 & 2450810368 & 7121115 & 1196 & 2451761920 & 6952492 & 1196 & 2590884864 & 7191867 \\
\hline 700 & $11 / 11 / 2017$ & 1196 & 2457835520 & 7025217 & 1196 & 2458619648 & 6857435 & 1196 & 2597984768 & 7099729 \\
\hline 701 & $11 / 12 / 2017$ & 1196 & 2464770304 & 6934789 & 1196 & 2465384704 & 6765499 & 1196 & 2604995072 & 7010384 \\
\hline 702 & $11 / 13 / 2017$ & 1196 & 2471614976 & 6844760 & 1196 & 2472061440 & 6676540 & 1196 & 2611919360 & 6924193 \\
\hline 703 & $11 / 14 / 2017$ & 1196 & 2478374912 & 6759816 & 1196 & 2478651648 & 6590419 & 1196 & 2618759680 & 6840419 \\
\hline 704 & $11 / 15 / 2017$ & 1196 & 2485050112 & 6675152 & 1196 & 2485158656 & 6507010 & 1196 & 2625519104 & 6759510 \\
\hline 705 & $11 / 16 / 2017$ & 1196 & 2491645184 & 6595223 & 1196 & 2491585024 & 6426190 & 1196 & 2632199936 & 6680781 \\
\hline 706 & $11 / 17 / 2017$ & 1196 & 2498160640 & 6515474 & 1196 & 2497932544 & 6347846 & 1196 & 2638804480 & 6604502 \\
\hline 707 & $11 / 18 / 2017$ & 1196 & 2504600832 & 6440144 & 1196 & 2504204544 & 6271869 & 1196 & 2645335040 & 6530498 \\
\hline 708 & $11 / 19 / 2017$ & 1196 & 2510965760 & 6364910 & 1196 & 2510402816 & 6198159 & 1196 & 2651793664 & 6458656 \\
\hline 709 & $11 / 20 / 2017$ & 1196 & 2517259264 & 6293806 & 1196 & 2516529408 & 6126618 & 1196 & 2658182400 & 6388876 \\
\hline 710 & $11 / 21 / 2017$ & 1196 & 2523482368 & 6222726 & 1196 & 2522586624 & 6057157 & 1196 & 2664503552 & 6321070 \\
\hline 711 & $11 / 22 / 2017$ & 1196 & 2529637888 & 6155514 & 1196 & 2528576256 & 5989689 & 1196 & 2670758656 & 6255155 \\
\hline 712 & $11 / 23 / 2017$ & 1196 & 2535726080 & 6088266 & 1196 & 2534500352 & 5924135 & 1196 & 2676949760 & 6191054 \\
\hline 713 & $11 / 24 / 2017$ & 1196 & 2541750784 & 6024646 & 1196 & 2540360960 & 5860415 & 1196 & 2683078400 & 6128693 \\
\hline 714 & $11 / 25 / 2017$ & 1196 & 2547711744 & 5960939 & 1196 & 2546159104 & 5798458 & 1196 & 2689146368 & 6068003 \\
\hline 715 & $11 / 26 / 2017$ & 1196 & 2553612288 & 5900641 & 1196 & 2551897600 & 5738194 & 1196 & 2695155456 & 6008919 \\
\hline 716 & $11 / 27 / 2017$ & 1196 & 2559452416 & 5840212 & 1196 & 2557576960 & 5679558 & 1196 & 2701106688 & 5951379 \\
\hline 717 & $11 / 28 / 2017$ & 1196 & 2565235456 & 5782991 & 1196 & 2563199488 & 5622486 & 1196 & 2707002112 & 5895324 \\
\hline
\end{tabular}




\begin{tabular}{|c|c|c|c|c|c|c|c|c|c|c|}
\hline 718 & $11 / 29 / 2017$ & 1196 & 2570962176 & 5726571 & 1196 & 2568766464 & 5566920 & 1196 & 2712842752 & 5840697 \\
\hline 719 & $11 / 30 / 2017$ & 1196 & 2576633600 & 5671422 & 1196 & 2574279424 & 5512803 & 1196 & 2718630144 & 5787446 \\
\hline 720 & $12 / 1 / 2017$ & 1196 & 2582251264 & 5617630 & 1196 & 2579739392 & 5460081 & 1196 & 2724365824 & 5735519 \\
\hline 721 & $12 / 2 / 2017$ & 1196 & 2587816192 & 5565153 & 1196 & 2585148416 & 5409019 & 1196 & 2730050560 & 5684870 \\
\hline 722 & $12 / 3 / 2017$ & 1196 & 2593330176 & 5513950 & 1196 & 2590507264 & 5358968 & 1196 & 2735686144 & 5635452 \\
\hline 723 & $12 / 4 / 2017$ & 1196 & 2598794240 & 5463975 & 1196 & 2595817472 & 5310132 & 1196 & 2741273344 & 5587221 \\
\hline 724 & $12 / 5 / 2017$ & 1196 & 2604209408 & 5415189 & 1196 & 2601080064 & 5262500 & 1196 & 2746813440 & 5540135 \\
\hline 725 & $12 / 6 / 2017$ & 1196 & 2609576960 & 5367550 & 1196 & 2606296064 & 5216028 & 1196 & 2752307712 & 5494156 \\
\hline 726 & $12 / 7 / 2017$ & 1196 & 2614897920 & 5321020 & 1196 & 2611466752 & 5170677 & 1196 & 2757756672 & 5449244 \\
\hline 727 & $12 / 8 / 2017$ & 1196 & 2620173568 & 5275563 & 1196 & 2616593152 & 5126409 & 1196 & 2763162112 & 5405363 \\
\hline 728 & $12 / 9 / 2017$ & 1196 & 2625404672 & 5231142 & 1196 & 2621676288 & 5083187 & 1196 & 2768525056 & 5362480 \\
\hline 729 & $12 / 10 / 2017$ & 1196 & 2630592512 & 5187724 & 1196 & 2626717184 & 5040975 & 1196 & 2773845248 & 5320560 \\
\hline 730 & $12 / 11 / 2017$ & 1196 & 2635737600 & 5145277 & 1196 & 2631716864 & 4999740 & 1196 & 2779124736 & 5279573 \\
\hline 731 & $12 / 12 / 2017$ & 1196 & 2640841472 & 5103770 & 1196 & 2636676352 & 4959449 & 1196 & 2784364288 & 5239487 \\
\hline 732 & $12 / 13 / 2017$ & 1196 & 2645904384 & 5063173 & 1196 & 2641596416 & 4920073 & 1196 & 2789564672 & 5200274 \\
\hline 733 & $12 / 14 / 2017$ & 1196 & 2650928128 & 5023457 & 1196 & 2646478080 & 4881581 & 1196 & 2794726400 & 5161905 \\
\hline 734 & $12 / 15 / 2017$ & 1196 & 2655912704 & 4984595 & 1196 & 2651322112 & 4843944 & 1196 & 2799850752 & 5124355 \\
\hline 735 & $12 / 16 / 2017$ & 1196 & 2660858880 & 4946561 & 1196 & 2656129024 & 4807136 & 1196 & 2804938496 & 5087598 \\
\hline 736 & $12 / 17 / 2017$ & 1196 & 2665768704 & 4909329 & 1196 & 2660900352 & 4771130 & 1196 & 2809989888 & 5051609 \\
\hline 737 & $12 / 18 / 2017$ & 1196 & 2670641408 & 4872875 & 1196 & 2665636096 & 4735900 & 1196 & 2815006464 & 5016364 \\
\hline 738 & $12 / 19 / 2017$ & 1196 & 2675478528 & 4837175 & 1196 & 2670337536 & 4701423 & 1196 & 2819988224 & 4981841 \\
\hline 739 & $12 / 20 / 2017$ & 1196 & 2680280832 & 4802208 & 1196 & 2675005184 & 4667675 & 1196 & 2824936192 & 4948019 \\
\hline 740 & $12 / 21 / 2017$ & 1196 & 2685048576 & 4767952 & 1196 & 2679639808 & 4634636 & 1196 & 2829851136 & 4914876 \\
\hline 741 & $12 / 22 / 2017$ & 1196 & 2689783296 & 4734386 & 1196 & 2684242176 & 4602282 & 1196 & 2834733568 & 4882392 \\
\hline 742 & $12 / 23 / 2017$ & 1196 & 2694484736 & 4701490 & 1196 & 2688812800 & 4570594 & 1196 & 2839584000 & 4850549 \\
\hline 743 & $12 / 24 / 2017$ & 1196 & 2699153920 & 4669244 & 1196 & 2693352448 & 4539552 & 1196 & 2844403456 & 4819327 \\
\hline 744 & $12 / 25 / 2017$ & 1196 & 2703791616 & 4637631 & 1196 & 2697861376 & 4509137 & 1196 & 2849192192 & 4788709 \\
\hline 745 & $12 / 26 / 2017$ & 1196 & 2708398080 & 4606632 & 1196 & 2702340608 & 4479330 & 1196 & 2853950720 & 4758678 \\
\hline 746 & $12 / 27 / 2017$ & 1196 & 2712974336 & 4576231 & 1196 & 2706790912 & 4450115 & 1196 & 2858680064 & 4729216 \\
\hline 747 & $12 / 28 / 2017$ & 1196 & 2717520896 & 4546409 & 1196 & 2711212288 & 4421474 & 1196 & 2863380224 & 4700308 \\
\hline 748 & $12 / 29 / 2017$ & 1196 & 2722038016 & 4517153 & 1196 & 2715605760 & 4393391 & 1196 & 2868052224 & 4671939 \\
\hline 749 & $12 / 30 / 2017$ & 1196 & 2726526464 & 4488445 & 1196 & 2719971584 & 4365850 & 1196 & 2872696320 & 4644095 \\
\hline 750 & $12 / 31 / 2017$ & 1196 & 2730986752 & 4460272 & 1196 & 2724310528 & 4338836 & 1196 & 2877313024 & 4616760 \\
\hline 751 & $1 / 1 / 2018$ & 1196 & 2735419392 & 4432618 & 1196 & 2728622848 & 4312335 & 1196 & 2881903104 & 4589920 \\
\hline 752 & $1 / 2 / 2018$ & 1196 & 2739824896 & 4405471 & 1196 & 2732909056 & 4286331 & 1196 & 2886466560 & 4563564 \\
\hline 753 & $1 / 3 / 2018$ & 1196 & 2744203520 & 4378816 & 1196 & 2737169920 & 4260813 & 1196 & 2891004160 & 4537677 \\
\hline 754 & $1 / 4 / 2018$ & 1196 & 2748556288 & 4352641 & 1196 & 2741405696 & 4235766 & 1196 & 2895516416 & 4512247 \\
\hline 755 & $1 / 5 / 2018$ & 1196 & 2752883200 & 4326932 & 1196 & 2745616896 & 4211178 & 1196 & 2900003584 & 4487263 \\
\hline 756 & $1 / 6 / 2018$ & 1196 & 2757184768 & 4301679 & 1196 & 2749804032 & 4187037 & 1196 & 2904466432 & 4462713 \\
\hline 757 & $1 / 7 / 2018$ & 1196 & 2761461760 & 4276870 & 1196 & 2753967360 & 4163330 & 1196 & 2908904960 & 4438586 \\
\hline 758 & $1 / 8 / 2018$ & 1196 & 2765714176 & 4252492 & 1196 & 2758107392 & 4140047 & 1196 & 2913319936 & 4414870 \\
\hline 759 & $1 / 9 / 2018$ & 1196 & 2769942784 & 4228536 & 1196 & 2762224640 & 4117177 & 1196 & 2917711616 & 4391556 \\
\hline 760 & $1 / 10 / 2018$ & 1196 & 2774147840 & 4204990 & 1196 & 2766319104 & 4094708 & 1196 & 2922080256 & 4368633 \\
\hline 761 & $1 / 11 / 2018$ & 1196 & 2778329600 & 4181844 & 1196 & 2770391808 & 4072630 & 1196 & 2926426112 & 4346092 \\
\hline 762 & $1 / 12 / 2018$ & 1196 & 2782488576 & 4159089 & 1196 & 2774442752 & 4050935 & 1196 & 2930750464 & 4323923 \\
\hline 763 & $1 / 13 / 2018$ & 1196 & 2786625280 & 4136714 & 1196 & 2778472448 & 4029610 & 1196 & 2935052288 & 4302118 \\
\hline 764 & $1 / 14 / 2018$ & 1196 & 2790739968 & 4114712 & 1196 & 2782480896 & 4008648 & 1196 & 2939332864 & 4280666 \\
\hline 765 & $1 / 15 / 2018$ & 1196 & 2794833152 & 4093071 & 1196 & 2786469120 & 3988040 & 1196 & 2943592448 & 4259560 \\
\hline 766 & $1 / 16 / 2018$ & 1196 & 2798904832 & 4071785 & 1196 & 2790436864 & 3967776 & 1196 & 2947831296 & 4238791 \\
\hline 767 & $1 / 17 / 2018$ & 1196 & 2802955776 & 4050844 & 1196 & 2794384640 & 3947848 & 1196 & 2952049664 & 4218351 \\
\hline 768 & $1 / 18 / 2018$ & 1196 & 2806985984 & 4030240 & 1196 & 2798312960 & 3928248 & 1196 & 2956247808 & 4198233 \\
\hline 769 & $1 / 19 / 2018$ & 1196 & 2810995968 & 4009965 & 1196 & 2802221824 & 3908968 & 1196 & 2960426240 & 4178429 \\
\hline 770 & $1 / 20 / 2018$ & 1196 & 2814985984 & 3990012 & 1196 & 2806112000 & 3890001 & 1196 & 2964585216 & 4158931 \\
\hline 771 & $1 / 21 / 2018$ & 1196 & 2818956288 & 3970373 & 1196 & 2809983232 & 3871338 & 1196 & 2968724992 & 4139732 \\
\hline 772 & $1 / 22 / 2018$ & 1196 & 2822907392 & 3951041 & 1196 & 2813836288 & 3852972 & 1196 & 2972845824 & 4120826 \\
\hline 773 & $1 / 23 / 2018$ & 1196 & 2826839296 & 3932008 & 1196 & 2817671168 & 3834898 & 1196 & 2976947968 & 4102206 \\
\hline 774 & $1 / 24 / 2018$ & 1196 & 2830752768 & 3913269 & 1196 & 2821488128 & 3817106 & 1196 & 2981031936 & 4083865 \\
\hline 775 & $1 / 25 / 2018$ & 1196 & 2834647552 & 3894815 & 1196 & 2825287680 & 3799592 & 1196 & 2985097728 & 4065797 \\
\hline 776 & $1 / 26 / 2018$ & 1196 & 2838524160 & 3876641 & 1196 & 2829070080 & 3782349 & 1196 & 2989145600 & 4047996 \\
\hline 777 & $1 / 27 / 2018$ & 1196 & 2842382848 & 3858741 & 1196 & 2832835584 & 3765370 & 1196 & 2993176064 & 4030456 \\
\hline 778 & $1 / 28 / 2018$ & 1196 & 2846223872 & 3841109 & 1196 & 2836584192 & 3748649 & 1196 & 2997189376 & 4013171 \\
\hline 779 & $1 / 29 / 2018$ & 1196 & 2850047744 & 3823738 & 1196 & 2840316672 & 3732181 & 1196 & 3001185536 & 3996136 \\
\hline 780 & $1 / 30 / 2018$ & 1196 & 2853854464 & 3806623 & 1196 & 2844032256 & 3715960 & 1196 & 3005164800 & 3979345 \\
\hline 781 & $1 / 31 / 2018$ & 1196 & 2857643776 & 3789758 & 1196 & 2847732224 & 3699980 & 1196 & 3009127424 & 3962792 \\
\hline 782 & $2 / 1 / 2018$ & 1196 & 2861417216 & 3773138 & 1196 & 2851416576 & 3684235 & 1196 & 3013073920 & 3946473 \\
\hline 783 & $2 / 2 / 2018$ & 1196 & 2865174016 & 3756758 & 1196 & 2855085312 & 3668722 & 1196 & 3017004288 & 3930383 \\
\hline 784 & $2 / 3 / 2018$ & 1196 & 2868914688 & 3740612 & 1196 & 2858738688 & 3653434 & 1196 & 3020919040 & 3914517 \\
\hline 785 & $2 / 4 / 2018$ & 1196 & 2872639232 & 3724695 & 1196 & 2862376960 & 3638367 & 1196 & 3024817664 & 3898869 \\
\hline 786 & $2 / 5 / 2018$ & 1196 & 2876348416 & 3709003 & 1196 & 2866000640 & 3623515 & 1196 & 3028701184 & 3883436 \\
\hline 787 & $2 / 6 / 2018$ & 1196 & 2880041472 & 3693531 & 1196 & 2869609472 & 3608875 & 1196 & 3032569344 & 3868213 \\
\hline 788 & $2 / 7 / 2018$ & 1196 & 2883720192 & 3678274 & 1196 & 2873203712 & 3594441 & 1196 & 3036422656 & 3853196 \\
\hline 789 & $2 / 8 / 2018$ & 1196 & 2887383296 & 3663228 & 1196 & 2876784128 & 3580210 & 1196 & 3040261120 & 3838379 \\
\hline 790 & $2 / 9 / 2018$ & 1196 & 2891031808 & 3648389 & 1196 & 2880350208 & 3566176 & 1196 & 3044084736 & 3823761 \\
\hline 791 & $2 / 10 / 2018$ & 1196 & 2894665472 & 3633751 & 1196 & 2883902720 & 3552336 & 1196 & 3047894016 & 3809334 \\
\hline 792 & $2 / 11 / 2018$ & 1196 & 2898284800 & 3619312 & 1196 & 2887441408 & 3538686 & 1196 & 3051689216 & 3795098 \\
\hline 793 & $2 / 12 / 2018$ & 1196 & 2901889792 & 3605067 & 1196 & 2890966528 & 3525222 & 1196 & 3055470336 & 3781047 \\
\hline 794 & $2 / 13 / 2018$ & 1196 & 2905480960 & 3591012 & 1196 & 2894478336 & 3511940 & 1196 & 3059237376 & 3767177 \\
\hline 795 & $2 / 14 / 2018$ & 1196 & 2909058048 & 3577143 & 1196 & 2897977088 & 3498835 & 1196 & 3062990848 & 3753486 \\
\hline 796 & $2 / 15 / 2018$ & 1196 & 2912621568 & 3563457 & 1196 & 2901463296 & 3485906 & 1196 & 3066731008 & 3739969 \\
\hline 797 & $2 / 16 / 2018$ & 1196 & 2916171520 & 3549950 & 1196 & 2904936448 & 3473147 & 1196 & 3070457600 & 3726623 \\
\hline 798 & $2 / 17 / 2018$ & 1196 & 2919708160 & 3536619 & 1196 & 2908396800 & 3460555 & 1196 & 3074170880 & 3713445 \\
\hline 799 & $2 / 18 / 2018$ & 1196 & 2923231488 & 3523459 & 1196 & 2911845120 & 3448128 & 1196 & 3077871360 & 3700432 \\
\hline
\end{tabular}




\begin{tabular}{|c|c|c|c|c|c|c|c|c|c|c|}
\hline 800 & $2 / 19 / 2018$ & 1196 & 2926742016 & 3510468 & 1196 & 2915280896 & 3435861 & 1196 & 3081559040 & 3687580 \\
\hline 801 & $2 / 20 / 2018$ & 1196 & 2930239744 & 3497643 & 1196 & 2918704640 & 3423752 & 1196 & 3085233920 & 3674886 \\
\hline 802 & $2 / 21 / 2018$ & 1196 & 2933724672 & 3484979 & 1196 & 2922116352 & 3411798 & 1196 & 3088896256 & 3662348 \\
\hline 803 & $2 / 22 / 2018$ & 1196 & 2937197056 & 3472475 & 1196 & 2925516544 & 3399995 & 1196 & 3092546048 & 3649961 \\
\hline 804 & $2 / 23 / 2018$ & 1196 & 2940657152 & 3460127 & 1196 & 2928904704 & 3388340 & 1196 & 3096183808 & 3637724 \\
\hline 805 & $2 / 24 / 2018$ & 1196 & 2944105216 & 3447932 & 1196 & 2932281600 & 3376831 & 1196 & 3099809536 & 3625634 \\
\hline 806 & $2 / 25 / 2018$ & 1196 & 2947540992 & 3435887 & 1196 & 2935646976 & 3365465 & 1196 & 3103423232 & 3613687 \\
\hline 807 & $2 / 26 / 2018$ & 1196 & 2950964992 & 3423989 & 1196 & 2939001344 & 3354238 & 1196 & 3107025152 & 3601882 \\
\hline 808 & $2 / 27 / 2018$ & 1196 & 2954377216 & 3412237 & 1196 & 2942344704 & 3343150 & 1196 & 3110615296 & 3590215 \\
\hline 809 & $2 / 28 / 2018$ & 1196 & 2957777920 & 3400626 & 1196 & 2945676544 & 3332195 & 1196 & 3114193920 & 3578684 \\
\hline 810 & $3 / 1 / 2018$ & 1196 & 2961167104 & 3389154 & 1196 & 2948997888 & 3321373 & 1196 & 3117761280 & 3567287 \\
\hline 811 & $3 / 2 / 2018$ & 1196 & 2964544768 & 3377819 & 1196 & 2952308736 & 3310681 & 1196 & 3121317376 & 3556020 \\
\hline 812 & $3 / 3 / 2018$ & 1196 & 2967911424 & 3366618 & 1196 & 2955608832 & 3300115 & 1196 & 3124861952 & 3544883 \\
\hline 813 & $3 / 4 / 2018$ & 1196 & 2971267072 & 3355550 & 1196 & 2958898432 & 3289675 & 1196 & 3128396032 & 3533871 \\
\hline 814 & $3 / 5 / 2018$ & 1196 & 2974611712 & 3344610 & 1196 & 2962177792 & 3279356 & 1196 & 3131919104 & 3522984 \\
\hline 815 & $3 / 6 / 2018$ & 1196 & 2977945344 & 3333798 & 1196 & 2965446912 & 3269159 & 1196 & 3135431168 & 3512218 \\
\hline 816 & $3 / 7 / 2018$ & 1196 & 2981268480 & 3323110 & 1196 & 2968706048 & 3259079 & 1196 & 3138932736 & 3501573 \\
\hline 817 & $3 / 8 / 2018$ & 1196 & 2984581120 & 3312546 & 1196 & 2971955200 & 3249115 & 1196 & 3142423808 & 3491045 \\
\hline 818 & $3 / 9 / 2018$ & 1196 & 2987883264 & 3302101 & 1196 & 2975194368 & 3239265 & 1196 & 3145904128 & 3480632 \\
\hline 819 & $3 / 10 / 2018$ & 1196 & 2991174912 & 3291775 & 1196 & 2978424064 & 3229527 & 1196 & 3149374720 & 3470333 \\
\hline 820 & $3 / 11 / 2018$ & 1196 & 2994456576 & 3281565 & 1196 & 2981643776 & 3219898 & 1196 & 3152835072 & 3460146 \\
\hline 821 & $3 / 12 / 2018$ & 1196 & 2997727744 & 3271469 & 1196 & 2984854272 & 3210377 & 1196 & 3156285184 & 3450068 \\
\hline 822 & $3 / 13 / 2018$ & 1196 & 3000989440 & 3261486 & 1196 & 2988055296 & 3200962 & 1196 & 3159725056 & 3440098 \\
\hline 823 & $3 / 14 / 2018$ & 1196 & 3004240896 & 3251612 & 1196 & 2991246848 & 3191651 & 1196 & 3163155200 & 3430234 \\
\hline 824 & $3 / 15 / 2018$ & 1196 & 3007482880 & 3241847 & 1196 & 2994429184 & 3182442 & 1196 & 3166575872 & 3420475 \\
\hline 825 & $3 / 16 / 2018$ & 1196 & 3010715136 & 3232189 & 1196 & 2997602304 & 3173333 & 1196 & 3169986560 & 3410817 \\
\hline 826 & $3 / 17 / 2018$ & 1196 & 3013937664 & 3222635 & 1196 & 3000766976 & 3164323 & 1196 & 3173388032 & 3401260 \\
\hline 827 & $3 / 18 / 2018$ & 1196 & 3017150976 & 3213184 & 1196 & 3003922432 & 3155410 & 1196 & 3176779776 & 3391802 \\
\hline 828 & $3 / 19 / 2018$ & 1196 & 3020354560 & 3203834 & 1196 & 3007068928 & 3146592 & 1196 & 3180162048 & 3382441 \\
\hline 829 & $3 / 20 / 2018$ & 1196 & 3023549440 & 3194584 & 1196 & 3010206720 & 3137867 & 1196 & 3183535360 & 3373176 \\
\hline 830 & $3 / 21 / 2018$ & 1196 & 3026734848 & 3185432 & 1196 & 3013336064 & 3129235 & 1196 & 3186899456 & 3364006 \\
\hline 831 & $3 / 22 / 2018$ & 1196 & 3029911040 & 3176376 & 1196 & 3016456704 & 3120693 & 1196 & 3190254336 & 3354927 \\
\hline 832 & $3 / 23 / 2018$ & 1196 & 3033078528 & 3167414 & 1196 & 3019568896 & 3112239 & 1196 & 3193600256 & 3345940 \\
\hline 833 & $3 / 24 / 2018$ & 1196 & 3036237056 & 3158545 & 1196 & 3022672896 & 3103874 & 1196 & 3196937216 & 3337042 \\
\hline 834 & $3 / 25 / 2018$ & 1196 & 3039387136 & 3149768 & 1196 & 3025768448 & 3095594 & 1196 & 3200265472 & 3328233 \\
\hline 835 & $3 / 26 / 2018$ & 1196 & 3042528000 & 3141081 & 1196 & 3028855808 & 3087398 & 1196 & 3203585024 & 3319510 \\
\hline 836 & $3 / 27 / 2018$ & 1196 & 3045660416 & 3132483 & 1196 & 3031935232 & 3079285 & 1196 & 3206895872 & 3310873 \\
\hline 837 & $3 / 28 / 2018$ & 1196 & 3048784384 & 3123972 & 1196 & 3035006464 & 3071255 & 1196 & 3210198016 & 3302319 \\
\hline 838 & $3 / 29 / 2018$ & 1196 & 3051899904 & 3115546 & 1196 & 3038069760 & 3063304 & 1196 & 3213491968 & 3293848 \\
\hline 839 & $3 / 30 / 2018$ & 1196 & 3055007232 & 3107205 & 1196 & 3041125120 & 3055433 & 1196 & 3216777472 & 3285459 \\
\hline 840 & $3 / 31 / 2018$ & 1196 & 3058106112 & 3098947 & 1196 & 3044172800 & 3047639 & 1196 & 3220054528 & 3277149 \\
\hline 841 & $4 / 1 / 2018$ & 1196 & 3061196800 & 3090770 & 1196 & 3047212800 & 3039922 & 1196 & 3223323648 & 3268918 \\
\hline 842 & $4 / 2 / 2018$ & 1196 & 3064279552 & 3082674 & 1196 & 3050245120 & 3032482 & 1196 & 3226584320 & 3260765 \\
\hline 843 & $4 / 3 / 2018$ & 1196 & 3067353856 & 3074657 & 1196 & 3053270016 & 3024736 & 1196 & 3229837056 & 3252689 \\
\hline 844 & $4 / 4 / 2018$ & 1196 & 3070420992 & 3066718 & 1196 & 3056287232 & 3017359 & 1196 & 3233081344 & 3244687 \\
\hline 845 & $4 / 5 / 2018$ & 1196 & 3073479680 & 3058856 & 1196 & 3059297024 & 3009809 & 1196 & 3236318464 & 3236760 \\
\hline 846 & $4 / 6 / 2018$ & 1196 & 3076530944 & 3051069 & 1196 & 3062299648 & 3002601 & 1196 & 3239547392 & 3228905 \\
\hline 847 & $4 / 7 / 2018$ & 1196 & 3079574272 & 3043356 & 1196 & 3065294592 & 2995178 & 1196 & 3242768384 & 3221122 \\
\hline 848 & $4 / 8 / 2018$ & 1196 & 3082609920 & 3035717 & 1196 & 3068282880 & 2988087 & 1196 & 3245981952 & 3213410 \\
\hline 849 & $4 / 9 / 2018$ & 1196 & 3085638144 & 3028150 & 1196 & 3071263744 & 2980812 & 1196 & 3249187584 & 3205768 \\
\hline 850 & $4 / 10 / 2018$ & 1196 & 3088658688 & 3020653 & 1196 & 3074237696 & 2973864 & 1196 & 3252385792 & 3198194 \\
\hline 851 & $4 / 11 / 2018$ & 1196 & 3091672064 & 3013227 & 1196 & 3077204480 & 2966718 & 1196 & 3255576576 & 3190688 \\
\hline 852 & $4 / 12 / 2018$ & 1196 & 3094677760 & 3005870 & 1196 & 3080164352 & 2959889 & 1196 & 3258759680 & 3183249 \\
\hline 853 & $4 / 13 / 2018$ & 1196 & 3097676288 & 2998580 & 1196 & 3083117056 & 2952876 & 1196 & 3261935616 & 3175875 \\
\hline 854 & $4 / 14 / 2018$ & 1196 & 3100667648 & 2991357 & 1196 & 3086063360 & 2946176 & 1196 & 3265104128 & 3168566 \\
\hline 855 & $4 / 15 / 2018$ & 1196 & 3103652096 & 2984200 & 1196 & 3089002496 & 2939286 & 1196 & 3268265216 & 3161320 \\
\hline 856 & $4 / 16 / 2018$ & 1196 & 3106629376 & 2977108 & 1196 & 3091935232 & 2932702 & 1196 & 3271419648 & 3154138 \\
\hline 857 & $4 / 17 / 2018$ & 1196 & 3109599232 & 2970080 & 1196 & 3094861312 & 2925936 & 1196 & 3274566656 & 3147017 \\
\hline 858 & $4 / 18 / 2018$ & 1196 & 3112562176 & 2963114 & 1196 & 3097780736 & 2919472 & 1196 & 3277706752 & 3139957 \\
\hline 859 & $4 / 19 / 2018$ & 1196 & 3115518464 & 2956211 & 1196 & 3100693504 & 2912822 & 1196 & 3280839680 & 3132957 \\
\hline 860 & $4 / 20 / 2018$ & 1196 & 3118467840 & 2949369 & 1196 & 3103599872 & 2906468 & 1196 & 3283965696 & 3126017 \\
\hline 861 & $4 / 21 / 2018$ & 1196 & 3121410560 & 2942587 & 1196 & 3106499840 & 2899934 & 1196 & 3287085056 & 3119135 \\
\hline 862 & $4 / 22 / 2018$ & 1196 & 3124346368 & 2935864 & 1196 & 3109393664 & 2893692 & 1196 & 3290196992 & 3112310 \\
\hline 863 & $4 / 23 / 2018$ & 1196 & 3127275520 & 2929200 & 1196 & 3112280832 & 2887269 & 1196 & 3293302528 & 3105543 \\
\hline 864 & $4 / 24 / 2018$ & 1196 & 3130198016 & 2922594 & 1196 & 3115162112 & 2881131 & 1196 & 3296401408 & 3098831 \\
\hline 865 & $4 / 25 / 2018$ & 1196 & 3133114112 & 2916045 & 1196 & 3118036736 & 2874817 & 1196 & 3299493632 & 3092175 \\
\hline 866 & $4 / 26 / 2018$ & 1196 & 3136023808 & 2909551 & 1196 & 3120905728 & 2868785 & 1196 & 3302579200 & 3085573 \\
\hline 867 & $4 / 27 / 2018$ & 1196 & 3138926848 & 2903113 & 1196 & 3123768064 & 2862576 & 1196 & 3305658368 & 3079024 \\
\hline 868 & $4 / 28 / 2018$ & 1196 & 3141823488 & 2896730 & 1196 & 3126624768 & 2856642 & 1196 & 3308730880 & 3072529 \\
\hline 869 & $4 / 29 / 2018$ & 1196 & 3144713984 & 2890400 & 1196 & 3129475328 & 2850535 & 1196 & 3311796736 & 3066086 \\
\hline 870 & $4 / 30 / 2018$ & 1196 & 3147597824 & 2884123 & 1196 & 3132320000 & 2844701 & 1196 & 3314856448 & 3059694 \\
\hline 871 & $5 / 1 / 2018$ & 1196 & 3150476032 & 2877898 & 1196 & 3135158784 & 2838693 & 1196 & 3317910016 & 3053354 \\
\hline 872 & $5 / 2 / 2018$ & 1665 & 3150476032 & 13 & 1665 & 3135158784 & 20 & 1665 & 3317910016 & 0 \\
\hline 873 & $5 / 3 / 2018$ & 1665 & 3150476032 & 14 & 1665 & 3135158784 & 21 & 1665 & 3317910016 & 0 \\
\hline 874 & $5 / 4 / 2018$ & 1665 & 3150490368 & 14292 & 1665 & 3135172864 & 13976 & 1665 & 3317930240 & 20286 \\
\hline 875 & $5 / 5 / 2018$ & 1665 & 3150527744 & 37425 & 1665 & 3135205888 & 33001 & 1665 & 3317973248 & 42968 \\
\hline 876 & $5 / 6 / 2018$ & 1665 & 3150591744 & 64134 & 1665 & 3135264256 & 58502 & 1665 & 3318056192 & 83108 \\
\hline 877 & $5 / 7 / 2018$ & 1665 & 3150669312 & 77240 & 1665 & 3135335168 & 70881 & 1665 & 3318164224 & 107623 \\
\hline 878 & $5 / 8 / 2018$ & 1665 & 3150755328 & 86210 & 1665 & 3135414784 & 79331 & 1665 & 3318289920 & 125938 \\
\hline 879 & $5 / 9 / 2018$ & 1665 & 3150848512 & 93246 & 1665 & 3135500544 & 85956 & 1665 & 3318429952 & 140029 \\
\hline 880 & $5 / 10 / 2018$ & 1665 & 3150947584 & 99134 & 1665 & 3135591680 & 91503 & 1665 & 3318581504 & 151683 \\
\hline 881 & $5 / 11 / 2018$ & 1665 & 3151051776 & 104201 & 1665 & 3135688192 & 96283 & 1665 & 3318743296 & 161711 \\
\hline
\end{tabular}




\begin{tabular}{|c|c|c|c|c|c|c|c|c|c|c|}
\hline 882 & $5 / 12 / 2018$ & 1665 & 3151160576 & 108627 & 1665 & 3135788800 & 100464 & 1665 & 3318913792 & 170497 \\
\hline 883 & $5 / 13 / 2018$ & 1665 & 3151272960 & 112526 & 1665 & 3135892736 & 104155 & 1665 & 3319091968 & 178273 \\
\hline 884 & $5 / 14 / 2018$ & 1665 & 3151389440 & 116214 & 1665 & 3136000256 & 107435 & 1665 & 3319277312 & 185205 \\
\hline 885 & $5 / 15 / 2018$ & 1665 & 3151508480 & 119120 & 1665 & 3136110592 & 110367 & 1665 & 3319468544 & 191418 \\
\hline 886 & $5 / 16 / 2018$ & 1665 & 3151630336 & 121870 & 1665 & 3136223744 & 113003 & 1665 & 3319665664 & 197016 \\
\hline 887 & $5 / 17 / 2018$ & 1665 & 3151754496 & 124364 & 1665 & 3136338944 & 115387 & 1665 & 3319867648 & 202084 \\
\hline 888 & $5 / 18 / 2018$ & 1665 & 3151881216 & 126626 & 1665 & 3136456704 & 117559 & 1665 & 3320074496 & 206740 \\
\hline 889 & $5 / 19 / 2018$ & 1665 & 3152009984 & 128688 & 1665 & 3136576256 & 119541 & 1665 & 3320285440 & 210966 \\
\hline 890 & $5 / 20 / 2018$ & 1665 & 3152140544 & 130573 & 1665 & 3136697600 & 121522 & 1665 & 3320500224 & 214803 \\
\hline 891 & $5 / 21 / 2018$ & 1665 & 3152273152 & 132313 & 1665 & 3136820736 & 123066 & 1665 & 3320718592 & 218407 \\
\hline 892 & $5 / 22 / 2018$ & 1665 & 3152406784 & 133922 & 1665 & 3136945408 & 124618 & 1665 & 3320940288 & 221725 \\
\hline 893 & $5 / 23 / 2018$ & 1665 & 3152542208 & 135422 & 1665 & 3137071616 & 126208 & 1665 & 3321165056 & 224774 \\
\hline 894 & $5 / 24 / 2018$ & 1665 & 3152678912 & 136822 & 1665 & 3137199104 & 127547 & 1665 & 3321392896 & 227689 \\
\hline 895 & $5 / 25 / 2018$ & 1665 & 3152817152 & 138140 & 1665 & 3137327872 & 128757 & 1665 & 3321623296 & 230404 \\
\hline 896 & $5 / 26 / 2018$ & 1665 & 3152956416 & 139381 & 1665 & 3137457920 & 129968 & 1665 & 3321856256 & 232924 \\
\hline 897 & $5 / 27 / 2018$ & 1665 & 3153096960 & 140558 & 1665 & 3137588992 & 131120 & 1665 & 3322091264 & 235370 \\
\hline 898 & $5 / 28 / 2018$ & 1665 & 3153238784 & 141675 & 1665 & 3137721088 & 132225 & 1665 & 3322329088 & 237671 \\
\hline 899 & $5 / 29 / 2018$ & 1665 & 3153381376 & 142741 & 1665 & 3137854464 & 133281 & 1665 & 3322568960 & 239824 \\
\hline 900 & $5 / 30 / 2018$ & 1665 & 3153525248 & 143760 & 1665 & 3137988864 & 134357 & 1665 & 3322810880 & 241940 \\
\hline 901 & $5 / 31 / 2018$ & 1665 & 3153669888 & 144738 & 1665 & 3138124032 & 135283 & 1665 & 3323055104 & 244165 \\
\hline 902 & $6 / 1 / 2018$ & 1665 & 3153815552 & 145676 & 1665 & 3138260224 & 136206 & 1665 & 3323302656 & 247202 \\
\hline 903 & $6 / 2 / 2018$ & 1665 & 3153962240 & 146582 & 1665 & 3138397440 & 137108 & 1665 & 3323551744 & 249376 \\
\hline 904 & $6 / 3 / 2018$ & 1665 & 3154109696 & 147454 & 1665 & 3138535424 & 138038 & 1665 & 3323803392 & 251713 \\
\hline 905 & $6 / 4 / 2018$ & 1665 & 3154257920 & 148298 & 1665 & 3138674176 & 138842 & 1665 & 3324057344 & 254035 \\
\hline 906 & $6 / 5 / 2018$ & 1665 & 3154407168 & 149114 & 1665 & 3138813952 & 139658 & 1665 & 3324313600 & 256224 \\
\hline 907 & $6 / 6 / 2018$ & 1665 & 3154556928 & 149905 & 1665 & 3138954496 & 140452 & 1665 & 3324571904 & 258328 \\
\hline 908 & $6 / 7 / 2018$ & 1665 & 3154707712 & 150673 & 1665 & 3139095552 & 141224 & 1665 & 3324832256 & 260357 \\
\hline 909 & $6 / 8 / 2018$ & 1665 & 3154859520 & 151863 & 1665 & 3139237632 & 142097 & 1665 & 3325094656 & 262272 \\
\hline 910 & $6 / 9 / 2018$ & 1665 & 3155014144 & 154284 & 1665 & 3139381504 & 143861 & 1665 & 3325359104 & 264211 \\
\hline 911 & $6 / 10 / 2018$ & 1665 & 3155170048 & 156137 & 1665 & 3139526144 & 144530 & 1665 & 3325624832 & 266060 \\
\hline 912 & $6 / 11 / 2018$ & 1665 & 3155327488 & 157789 & 1665 & 3139671552 & 145537 & 1665 & 3325892864 & 267852 \\
\hline 913 & $6 / 12 / 2018$ & 1665 & 3155486976 & 159320 & 1665 & 3139819776 & 148064 & 1665 & 3326162432 & 269594 \\
\hline 914 & $6 / 13 / 2018$ & 1665 & 3155647744 & 160770 & 1665 & 3139970048 & 150225 & 1665 & 3326433792 & 271247 \\
\hline 915 & $6 / 14 / 2018$ & 1665 & 3155810048 & 162227 & 1665 & 3140122368 & 152116 & 1665 & 3326707456 & 273760 \\
\hline 916 & $6 / 15 / 2018$ & 1665 & 3155974400 & 164258 & 1665 & 3140276224 & 154129 & 1665 & 3326984704 & 277371 \\
\hline 917 & $6 / 16 / 2018$ & 1665 & 3156142336 & 167959 & 1665 & 3140432640 & 156404 & 1665 & 3327266560 & 281754 \\
\hline 918 & $6 / 17 / 2018$ & 1665 & 3156315136 & 172751 & 1665 & 3140590848 & 158395 & 1665 & 3327554304 & 287920 \\
\hline 919 & $6 / 18 / 2018$ & 1665 & 3156491264 & 176249 & 1665 & 3140752896 & 161892 & 1665 & 3327847680 & 293374 \\
\hline 920 & $6 / 19 / 2018$ & 1665 & 3156672512 & 180921 & 1665 & 3140917504 & 164650 & 1665 & 3328146688 & 298954 \\
\hline 921 & $6 / 20 / 2018$ & 1665 & 3156859392 & 187236 & 1665 & 3141084672 & 167192 & 1665 & 3328451072 & 304199 \\
\hline 922 & $6 / 21 / 2018$ & 1665 & 3157054464 & 195149 & 1665 & 3141254656 & 169821 & 1665 & 3328760320 & 309490 \\
\hline 923 & $6 / 22 / 2018$ & 1665 & 3157256448 & 201855 & 1665 & 3141429760 & 175308 & 1665 & 3329077248 & 316801 \\
\hline 924 & $6 / 23 / 2018$ & 1665 & 3157464320 & 207829 & 1665 & 3141611520 & 181636 & 1665 & 3329401600 & 324315 \\
\hline 925 & $6 / 24 / 2018$ & 1665 & 3157677568 & 213400 & 1665 & 3141802496 & 190799 & 1665 & 3329733632 & 332254 \\
\hline 926 & $6 / 25 / 2018$ & 1665 & 3157897984 & 220465 & 1665 & 3142002176 & 200014 & 1665 & 3330075904 & 341980 \\
\hline 927 & $6 / 26 / 2018$ & 1665 & 3158127872 & 229853 & 1665 & 3142212352 & 209988 & 1665 & 3330427392 & 351632 \\
\hline 928 & $6 / 27 / 2018$ & 1665 & 3158370048 & 242047 & 1665 & 3142433024 & 220646 & 1665 & 3330788352 & 360877 \\
\hline 929 & $6 / 28 / 2018$ & 1665 & 3158624256 & 254364 & 1665 & 3142665472 & 232663 & 1665 & 3331155712 & 367433 \\
\hline 930 & $6 / 29 / 2018$ & 1665 & 3158892544 & 268167 & 1665 & 3142908928 & 243459 & 1665 & 3331529472 & 373760 \\
\hline 931 & $6 / 30 / 2018$ & 1665 & 3159175168 & 282559 & 1665 & 3143163648 & 254585 & 1665 & 3331911680 & 382306 \\
\hline 932 & $7 / 1 / 2018$ & 1665 & 3159472640 & 297549 & 1665 & 3143428864 & 265137 & 1665 & 3332302080 & 390316 \\
\hline 933 & $7 / 2 / 2018$ & 1665 & 3159783424 & 310905 & 1665 & 3143707136 & 278303 & 1665 & 3332703232 & 401011 \\
\hline 934 & $7 / 3 / 2018$ & 1665 & 3160107776 & 324190 & 1665 & 3143997440 & 290481 & 1665 & 3333113600 & 410383 \\
\hline 935 & $7 / 4 / 2018$ & 1665 & 3160445952 & 338226 & 1665 & 3144300288 & 302852 & 1665 & 3333533952 & 420500 \\
\hline 936 & $7 / 5 / 2018$ & 1665 & 3160797184 & 351163 & 1665 & 3144615424 & 315049 & 1665 & 3333963520 & 429628 \\
\hline 937 & $7 / 6 / 2018$ & 1665 & 3161160448 & 363303 & 1665 & 3144944896 & 329437 & 1665 & 3334406912 & 443290 \\
\hline 938 & $7 / 7 / 2018$ & 1665 & 3161536512 & 376090 & 1665 & 3145287680 & 342942 & 1665 & 3334861824 & 454820 \\
\hline 939 & $7 / 8 / 2018$ & 1665 & 3161925120 & 388722 & 1665 & 3145645568 & 357752 & 1665 & 3335332864 & 471068 \\
\hline 940 & $7 / 9 / 2018$ & 1665 & 3162325760 & 400633 & 1665 & 3146017792 & 372280 & 1665 & 3335820800 & 487934 \\
\hline 941 & $7 / 10 / 2018$ & 1665 & 3162739456 & 413704 & 1665 & 3146404864 & 387074 & 1665 & 3336325632 & 505010 \\
\hline 942 & $7 / 11 / 2018$ & 1665 & 3163168768 & 429513 & 1665 & 3146806016 & 401108 & 1665 & 3336846592 & 520806 \\
\hline 943 & $7 / 12 / 2018$ & 1665 & 3163614464 & 445383 & 1665 & 3147219968 & 414039 & 1665 & 3337382912 & 536375 \\
\hline 944 & $7 / 13 / 2018$ & 1665 & 3164078080 & 463607 & 1665 & 3147649280 & 429173 & 1665 & 3337937408 & 554585 \\
\hline 945 & $7 / 14 / 2018$ & 1665 & 3164561152 & 483141 & 1665 & 3148093440 & 444307 & 1665 & 3338513664 & 576092 \\
\hline 946 & $7 / 15 / 2018$ & 1665 & 3165062144 & 501038 & 1665 & 3148554240 & 460783 & 1665 & 3339110912 & 597327 \\
\hline 947 & $7 / 16 / 2018$ & 1665 & 3165580800 & 518577 & 1665 & 3149031680 & 477349 & 1665 & 3339729664 & 619093 \\
\hline 948 & $7 / 17 / 2018$ & 1665 & 3166119936 & 538995 & 1665 & 3149525504 & 493834 & 1665 & 3340370432 & 640337 \\
\hline 949 & $7 / 18 / 2018$ & 1665 & 3166679808 & 559892 & 1665 & 3150035968 & 510422 & 1665 & 3341031936 & 661692 \\
\hline 950 & $7 / 19 / 2018$ & 1665 & 3167260416 & 580648 & 1665 & 3150563840 & 527800 & 1665 & 3341715712 & 683575 \\
\hline 951 & $7 / 20 / 2018$ & 1665 & 3167862016 & 601613 & 1665 & 3151108864 & 545198 & 1665 & 3342421248 & 705633 \\
\hline 952 & $7 / 21 / 2018$ & 1665 & 3168483584 & 621722 & 1665 & 3151673088 & 563894 & 1665 & 3343145984 & 724601 \\
\hline 953 & $7 / 22 / 2018$ & 1665 & 3169126144 & 642527 & 1665 & 3152254720 & 581934 & 1665 & 3343890944 & 745126 \\
\hline 954 & $7 / 23 / 2018$ & 1665 & 3169789952 & 663723 & 1665 & 3152855040 & 600203 & 1665 & 3344660224 & 769317 \\
\hline 955 & $7 / 24 / 2018$ & 1665 & 3170474496 & 684538 & 1665 & 3153473536 & 618441 & 1665 & 3345452544 & 792345 \\
\hline 956 & $7 / 25 / 2018$ & 1665 & 3171179264 & 704787 & 1665 & 3154113280 & 639888 & 1665 & 3346267904 & 815280 \\
\hline 957 & $7 / 26 / 2018$ & 1665 & 3171905280 & 726109 & 1665 & 3154775040 & 661853 & 1665 & 3347105280 & 837307 \\
\hline 958 & $7 / 27 / 2018$ & 1665 & 3172653056 & 747785 & 1665 & 3155458304 & 683403 & 1665 & 3347961856 & 856615 \\
\hline 959 & $7 / 28 / 2018$ & 1665 & 3173421312 & 768319 & 1665 & 3156164096 & 705439 & 1665 & 3348839680 & 877846 \\
\hline 960 & $7 / 29 / 2018$ & 1665 & 3174211840 & 790273 & 1665 & 3156892160 & 728268 & 1665 & 3349738752 & 899136 \\
\hline 961 & $7 / 30 / 2018$ & 1665 & 3175025920 & 814034 & 1665 & 3157645056 & 752799 & 1665 & 3350658304 & 919548 \\
\hline 962 & $7 / 31 / 2018$ & 1665 & 3175866624 & 840800 & 1665 & 3158420992 & 775835 & 1665 & 3351599104 & 940623 \\
\hline 963 & $8 / 1 / 2018$ & 1665 & 3176731136 & 864504 & 1665 & 3159219456 & 798924 & 1665 & 3352563712 & 964743 \\
\hline
\end{tabular}




\begin{tabular}{|c|c|c|c|c|c|c|c|c|c|c|}
\hline 964 & $8 / 2 / 2018$ & 1665 & 3177619456 & 888332 & 1665 & 3160041728 & 822025 & 1665 & 3353550848 & 987168 \\
\hline 965 & $8 / 3 / 2018$ & 1665 & 3178531840 & 912285 & 1665 & 3160886016 & 844105 & 1665 & 3354559232 & 1008293 \\
\hline 966 & $8 / 4 / 2018$ & 1665 & 3179466240 & 934496 & 1665 & 3161752576 & 866541 & 1665 & 3355590656 & 1031342 \\
\hline 967 & $8 / 5 / 2018$ & 1665 & 3180421632 & 955419 & 1665 & 3162642432 & 889953 & 1665 & 3356645632 & 1054925 \\
\hline 968 & $8 / 6 / 2018$ & 1665 & 3181397504 & 975976 & 1665 & 3163554560 & 912044 & 1665 & 3357720576 & 1075144 \\
\hline 969 & $8 / 7 / 2018$ & 1665 & 3182393344 & 995792 & 1665 & 3164488960 & 934558 & 1665 & 3358816768 & 1096053 \\
\hline 970 & $8 / 8 / 2018$ & 1665 & 3183409152 & 1015617 & 1665 & 3165444864 & 955851 & 1665 & 3359930880 & 1114232 \\
\hline 971 & $8 / 9 / 2018$ & 1665 & 3184442624 & 1033655 & 1665 & 3166421760 & 976904 & 1665 & 3361062912 & 1132086 \\
\hline 972 & $8 / 10 / 2018$ & 1665 & 3185494528 & 1051793 & 1665 & 3167420416 & 998598 & 1665 & 3362214144 & 1151185 \\
\hline 973 & $8 / 11 / 2018$ & 1665 & 3186563840 & 1069406 & 1665 & 3168441600 & 1021271 & 1665 & 3363384576 & 1170501 \\
\hline 974 & $8 / 12 / 2018$ & 1665 & 3187650048 & 1086049 & 1665 & 3169485312 & 1043733 & 1665 & 3364573440 & 1188806 \\
\hline 975 & $8 / 13 / 2018$ & 1665 & 3188752128 & 1102173 & 1665 & 3170548992 & 1063635 & 1665 & 3365779456 & 1205888 \\
\hline 976 & $8 / 14 / 2018$ & 1665 & 3189870848 & 1118411 & 1665 & 3171632896 & 1083913 & 1665 & 3367001344 & 1222047 \\
\hline 977 & $8 / 15 / 2018$ & 1665 & 3191004416 & 1133826 & 1665 & 3172733952 & 1101254 & 1665 & 3368238848 & 1237509 \\
\hline 978 & $8 / 16 / 2018$ & 1665 & 3192154112 & 1149839 & 1665 & 3173853696 & 1119602 & 1665 & 3369492480 & 1253467 \\
\hline 979 & $8 / 17 / 2018$ & 1665 & 3193317632 & 1163522 & 1665 & 3174990336 & 1136623 & 1665 & 3370761216 & 1268828 \\
\hline 980 & $8 / 18 / 2018$ & 1665 & 3194496000 & 1178403 & 1665 & 3176144128 & 1153787 & 1665 & 3372045056 & 1283898 \\
\hline 981 & $8 / 19 / 2018$ & 1665 & 3195687936 & 1191880 & 1665 & 3177313024 & 1168799 & 1665 & 3373345024 & 1300005 \\
\hline 982 & $8 / 20 / 2018$ & 1665 & 3196893184 & 1205300 & 1665 & 3178497024 & 1184118 & 1665 & 33746588816 & 1313774 \\
\hline 983 & $8 / 21 / 2018$ & 1665 & 3198112000 & 1218609 & 1665 & 3179697152 & 1199952 & 1665 & 3375985408 & 1326514 \\
\hline 984 & $8 / 22 / 2018$ & 1665 & 3199343360 & 1231353 & 1665 & 3180910336 & 1213290 & 1665 & 3377325056 & 1339690 \\
\hline 985 & $8 / 23 / 2018$ & 1665 & 3200587008 & 1243465 & 1665 & 3182137600 & 1227097 & 1665 & 3378677248 & 1352119 \\
\hline 986 & $8 / 24 / 2018$ & 1665 & 3201841920 & 1255255 & 1665 & 3183377920 & 1240545 & 1665 & 3380041728 & 1364602 \\
\hline 987 & $8 / 25 / 2018$ & 1665 & 3203108608 & 1266697 & 1665 & 3184631808 & 1253741 & 1665 & 3381417984 & 1376231 \\
\hline 988 & $8 / 26 / 2018$ & 1665 & 3204386560 & 1277779 & 1665 & 3185898240 & 1266370 & 1665 & 3382806016 & 1388018 \\
\hline 989 & $8 / 27 / 2018$ & 1665 & 3205675008 & 1288522 & 1665 & 3187176704 & 1278682 & 1665 & 3384205312 & 1399123 \\
\hline 990 & $8 / 28 / 2018$ & 1421 & 3214077952 & 8403083 & 1421 & 3196148992 & 8972215 & 1421 & 3393721088 & 9515908 \\
\hline 991 & $8 / 29 / 2018$ & 1421 & 3222086144 & 8008081 & 1421 & 3204606464 & 8457424 & 1421 & 3402735872 & 9014730 \\
\hline 992 & $8 / 30 / 2018$ & 1421 & 3228056576 & 5970567 & 1421 & 3210851840 & 6245291 & 1421 & 3409403904 & 6668156 \\
\hline 993 & $8 / 31 / 2018$ & 1421 & 3233328384 & 5271635 & 1421 & 3216335616 & 5483770 & 1421 & 3415250176 & 5845884 \\
\hline 994 & $9 / 1 / 2018$ & 1421 & 3238195200 & 4867246 & 1421 & 3221376768 & 5041239 & 1421 & 3420613120 & 5363182 \\
\hline 995 & $9 / 2 / 2018$ & 1421 & 3242782208 & 4586652 & 1421 & 3226114560 & 4737838 & 1421 & 3425642496 & 5029536 \\
\hline 996 & $9 / 3 / 2018$ & 1421 & 3247154944 & 4372988 & 1421 & 3230619904 & 4505367 & 1421 & 3430415872 & 4773162 \\
\hline 997 & $9 / 4 / 2018$ & 1421 & 3251354112 & 4198822 & 1421 & 3234935808 & 4315901 & 1421 & 3434979584 & 4563766 \\
\hline 998 & $9 / 5 / 2018$ & 1421 & 3255408128 & 4054146 & 1421 & 3239091968 & 4155998 & 1421 & 3439366144 & 4386739 \\
\hline 999 & $9 / 6 / 2018$ & 1421 & 3259335680 & 3927545 & 1421 & 3243110144 & 4018296 & 1421 & 3443600384 & 4234164 \\
\hline 1000 & $9 / 7 / 2018$ & 1421 & 3263152640 & 3817000 & 1421 & 3247008256 & 3898137 & 1421 & 3447701504 & 4100992 \\
\hline 1001 & $9 / 8 / 2018$ & 1421 & 3266872320 & 3719491 & 1421 & 3250800384 & 3792138 & 1421 & 3451685120 & 3983724 \\
\hline 1002 & 9/9/2018 & 1421 & 3270505216 & 3632975 & 1421 & 3254498560 & 3698052 & 1421 & 3455564800 & 3879657 \\
\hline 1003 & $9 / 10 / 2018$ & 1421 & 3274060800 & 3555599 & 1421 & 3258112768 & 3614278 & 1421 & 3459351552 & 3786725 \\
\hline 1004 & $9 / 11 / 2018$ & 1873 & 3274060800 & 12 & 1873 & 3258112768 & 18 & 1873 & 3459351552 & 0 \\
\hline 1005 & $9 / 12 / 2018$ & 1873 & 3274060800 & 13 & 1873 & 3258112768 & 19 & 1873 & 3459351552 & 0 \\
\hline 1006 & $9 / 13 / 2018$ & 1873 & 3274062080 & 1359 & 1873 & 3258116352 & 3661 & 1873 & 3459356928 & 5332 \\
\hline 1007 & $9 / 14 / 2018$ & 1873 & 3274081280 & 19002 & 1873 & 3258134272 & 17691 & 1873 & 3459378432 & 21553 \\
\hline 1008 & $9 / 15 / 2018$ & 1873 & 3274123776 & 42490 & 1873 & 3258173440 & 39195 & 1873 & 3459424000 & 45614 \\
\hline 1009 & $9 / 16 / 2018$ & 1873 & 3274181632 & 57905 & 1873 & 3258227200 & 53911 & 1873 & 3459490304 & 66248 \\
\hline 1010 & $9 / 17 / 2018$ & 1873 & 3274248960 & 67316 & 1873 & 3258290176 & 62801 & 1873 & 3459575808 & 85540 \\
\hline 1011 & $9 / 18 / 2018$ & 1873 & 3274322944 & 74428 & 1873 & 3258359552 & 69503 & 1873 & 3459676160 & 100446 \\
\hline 1012 & $9 / 19 / 2018$ & 1873 & 3274403584 & 80235 & 1873 & 3258434560 & 74975 & 1873 & 3459788800 & 112855 \\
\hline 1013 & $9 / 20 / 2018$ & 1873 & 3274488576 & 85134 & 1873 & 3258514176 & 79595 & 1873 & 3459912192 & 122993 \\
\hline 1014 & $9 / 21 / 2018$ & 1873 & 3274578176 & 89414 & 1873 & 3258597632 & 83562 & 1873 & 3460043776 & 131602 \\
\hline 1015 & $9 / 22 / 2018$ & 1873 & 3274671104 & 93066 & 1873 & 3258684672 & 87005 & 1873 & 3460182784 & 139047 \\
\hline 1016 & $9 / 23 / 2018$ & 1873 & 3274767360 & 96068 & 1873 & 3258774784 & 90031 & 1873 & 3460328448 & 145590 \\
\hline 1017 & $9 / 24 / 2018$ & 1873 & 3274866176 & 98849 & 1873 & 3258867456 & 92678 & 1873 & 3460479744 & 151366 \\
\hline 1018 & $9 / 25 / 2018$ & 1873 & 3274967296 & 101331 & 1873 & 3258962432 & 95056 & 1873 & 3460636160 & 156472 \\
\hline 1019 & $9 / 26 / 2018$ & 1873 & 3275070976 & 103549 & 1873 & 3259059712 & 97157 & 1873 & 3460797184 & 161094 \\
\hline 1020 & $9 / 27 / 2018$ & 1873 & 3275176448 & 105543 & 1873 & 3259158784 & 99082 & 1873 & 3460962560 & 165242 \\
\hline 1021 & $9 / 28 / 2018$ & 1873 & 3275283968 & 107347 & 1873 & 3259259648 & 100829 & 1873 & 3461131520 & 169000 \\
\hline 1022 & $9 / 29 / 2018$ & 1873 & 3275392768 & 108993 & 1873 & 3259362048 & 102411 & 1873 & 3461304064 & 172428 \\
\hline 1023 & $9 / 30 / 2018$ & 1873 & 3275503360 & 110504 & 1873 & 3259465728 & 103770 & 1873 & 3461479424 & 175574 \\
\hline 1024 & $10 / 1 / 2018$ & 1873 & 3275615232 & 111901 & 1873 & 3259570944 & 105110 & 1873 & 3461658112 & 178479 \\
\hline 1025 & $10 / 2 / 2018$ & 1873 & 3275728384 & 113199 & 1873 & 3259677184 & 106372 & 1873 & 3461839104 & 181176 \\
\hline 1026 & $10 / 3 / 2018$ & 1873 & 3275842816 & 114413 & 1873 & 3259784704 & 107555 & 1873 & 3462022656 & 183693 \\
\hline 1027 & $10 / 4 / 2018$ & 1873 & 3275958528 & 115553 & 1873 & 3259893504 & 108671 & 1873 & 3462209024 & 186055 \\
\hline 1028 & $10 / 5 / 2018$ & 1873 & 3276075008 & 116631 & 1873 & 3260003072 & 109727 & 1873 & 3462397184 & 188279 \\
\hline 1029 & $10 / 6 / 2018$ & 1873 & 3276192768 & 117653 & 1873 & 3260113920 & 110732 & 1873 & 3462587648 & 190385 \\
\hline 1030 & $10 / 7 / 2018$ & 1873 & 3276311552 & 118625 & 1873 & 3260225536 & 111690 & 1873 & 3462779904 & 192384 \\
\hline 1031 & $10 / 8 / 2018$ & 1873 & 3276430848 & 119555 & 1873 & 3260338176 & 112607 & 1873 & 3462974208 & 194290 \\
\hline 1032 & $10 / 9 / 2018$ & 1873 & 3276551680 & 120445 & 1873 & 3260451584 & 113487 & 1873 & 3463170304 & 196113 \\
\hline 1033 & $10 / 10 / 2018$ & 1873 & 3276672512 & 121301 & 1873 & 3260565760 & 114333 & 1873 & 3463368448 & 198020 \\
\hline 1034 & $10 / 11 / 2018$ & 1873 & 3276794624 & 122125 & 1873 & 3260681216 & 115150 & 1873 & 3463569408 & 200829 \\
\hline 1035 & $10 / 12 / 2018$ & 1873 & 3276917760 & 122921 & 1873 & 3260797184 & 115939 & 1873 & 3463771904 & 202605 \\
\hline 1036 & $10 / 13 / 2018$ & 1873 & 3277041408 & 123690 & 1873 & 3260913664 & 116703 & 1873 & 3463976448 & 204659 \\
\hline 1037 & $10 / 14 / 2018$ & 1873 & 3277165824 & 124435 & 1873 & 3261031168 & 117443 & 1873 & 3464182784 & 206620 \\
\hline 1038 & $10 / 15 / 2018$ & 1873 & 3277291008 & 125158 & 1873 & 3261149952 & 118680 & 1873 & 3464391680 & 208504 \\
\hline 1039 & $10 / 16 / 2018$ & 1873 & 3277416704 & 125859 & 1873 & 3261269760 & 119789 & 1873 & 3464601856 & 210318 \\
\hline 1040 & $10 / 17 / 2018$ & 1873 & 3277543424 & 126541 & 1873 & 3261390336 & 120706 & 1873 & 3464814080 & 212072 \\
\hline 1041 & $10 / 18 / 2018$ & 1873 & 3277670656 & 127205 & 1873 & 3261511936 & 121612 & 1873 & 3465027840 & 213769 \\
\hline 1042 & $10 / 19 / 2018$ & 1873 & 3277798400 & 127852 & 1873 & 3261634560 & 122482 & 1873 & 3465243136 & 215414 \\
\hline 1043 & $10 / 20 / 2018$ & 1873 & 3277926912 & 128482 & 1873 & 3261757952 & 123316 & 1873 & 3465460224 & 217012 \\
\hline 1044 & $10 / 21 / 2018$ & 1873 & 3278055936 & 129097 & 1873 & 3261881856 & 124121 & 1873 & 3465678848 & 218565 \\
\hline 1045 & $10 / 22 / 2018$ & 1873 & 3278187008 & 131012 & 1873 & 3262007040 & 125068 & 1873 & 3465898752 & 220077 \\
\hline
\end{tabular}




\begin{tabular}{|c|c|c|c|c|c|c|c|c|c|c|}
\hline 1046 & $10 / 23 / 2018$ & 1873 & 3278320640 & 133668 & 1873 & 3262133760 & 126646 & 1873 & 3466120448 & 221550 \\
\hline 1047 & $10 / 24 / 2018$ & 1873 & 3278455808 & 135114 & 1873 & 3262262016 & 128453 & 1873 & 3466343424 & 222986 \\
\hline 1048 & $10 / 25 / 2018$ & 1873 & 3278592512 & 136744 & 1873 & 3262392832 & 130698 & 1873 & 3466567680 & 224387 \\
\hline 1049 & $10 / 26 / 2018$ & 1873 & 3278730496 & 138260 & 1873 & 3262525184 & 132457 & 1873 & 3466794240 & 226471 \\
\hline 1050 & $10 / 27 / 2018$ & 1873 & 3278870528 & 139694 & 1873 & 3262659328 & 134132 & 1873 & 3467023872 & 229743 \\
\hline 1051 & $10 / 28 / 2018$ & 1873 & 3279011328 & 141061 & 1873 & 3262795008 & 135710 & 1873 & 3467256576 & 232593 \\
\hline 1052 & $10 / 29 / 2018$ & 1873 & 3279153920 & 142372 & 1873 & 3262932224 & 137211 & 1873 & 3467491840 & 235191 \\
\hline 1053 & $10 / 30 / 2018$ & 1873 & 3279297536 & 143633 & 1873 & 3263070976 & 138647 & 1873 & 3467729920 & 238045 \\
\hline 1054 & $10 / 31 / 2018$ & 1873 & 3279443968 & 146541 & 1873 & 3263211008 & 140026 & 1873 & 3467972608 & 242721 \\
\hline 1055 & $11 / 1 / 2018$ & 1873 & 3279592960 & 148949 & 1873 & 3263353088 & 142047 & 1873 & 3468220160 & 247711 \\
\hline 1056 & $11 / 2 / 2018$ & 1873 & 3279743744 & 150773 & 1873 & 3263497472 & 144389 & 1873 & 3468470272 & 250112 \\
\hline 1057 & $11 / 3 / 2018$ & 1873 & 3279896320 & 152580 & 1873 & 3263643392 & 146032 & 1873 & 3468723968 & 253624 \\
\hline 1058 & $11 / 4 / 2018$ & 1873 & 3280050944 & 154366 & 1873 & 3263792640 & 148978 & 1873 & 3468981248 & 257253 \\
\hline 1059 & $11 / 5 / 2018$ & 1873 & 3280207616 & 156955 & 1873 & 3263944960 & 152544 & 1873 & 3469242112 & 261213 \\
\hline 1060 & $11 / 6 / 2018$ & 1873 & 3280369152 & 161323 & 1873 & 3264100864 & 155753 & 1873 & 3469507328 & 264941 \\
\hline 1061 & $11 / 7 / 2018$ & 1873 & 3280535296 & 166260 & 1873 & 3264259072 & 158455 & 1873 & 3469776128 & 268703 \\
\hline 1062 & $11 / 8 / 2018$ & 1873 & 3280706816 & 171463 & 1873 & 3264420096 & 161036 & 1873 & 3470050048 & 273932 \\
\hline 1063 & $11 / 9 / 2018$ & 1873 & 3280882944 & 176312 & 1873 & 3264584448 & 164278 & 1873 & 3470329600 & 279472 \\
\hline 1064 & $11 / 10 / 2018$ & 1873 & 3281064704 & 181735 & 1873 & 3264751872 & 167495 & 1873 & 3470614016 & 284638 \\
\hline 1065 & $11 / 11 / 2018$ & 1873 & 3281251328 & 186578 & 1873 & 3264925696 & 173674 & 1873 & 3470904064 & 290007 \\
\hline 1066 & $11 / 12 / 2018$ & 1873 & 3281444608 & 193041 & 1873 & 3265106944 & 181595 & 1873 & 3471199744 & 295728 \\
\hline 1067 & $11 / 13 / 2018$ & 1873 & 3281645568 & 201261 & 1873 & 3265297152 & 189778 & 1873 & 3471501568 & 301677 \\
\hline 1068 & $11 / 14 / 2018$ & 1873 & 3281856512 & 210741 & 1873 & 3265496576 & 199501 & 1873 & 3471810048 & 308442 \\
\hline 1069 & $11 / 15 / 2018$ & 1873 & 3282078464 & 221979 & 1873 & 3265705728 & 209321 & 1873 & 3472124672 & 314602 \\
\hline 1070 & $11 / 16 / 2018$ & 1873 & 3282310400 & 232062 & 1873 & 3265924096 & 218217 & 1873 & 3472445696 & 321151 \\
\hline 1071 & $11 / 17 / 2018$ & 1873 & 3282554880 & 244450 & 1873 & 3266151424 & 227406 & 1873 & 3472773888 & 328124 \\
\hline 1072 & $11 / 18 / 2018$ & 1873 & 3282812416 & 257530 & 1873 & 3266388224 & 236809 & 1873 & 3473108224 & 334357 \\
\hline 1073 & $11 / 19 / 2018$ & 1873 & 3283081728 & 269164 & 1873 & 3266634240 & 245860 & 1873 & 3473448448 & 340174 \\
\hline 1074 & $11 / 20 / 2018$ & 1873 & 3283362048 & 280362 & 1873 & 3266891008 & 256554 & 1873 & 3473794048 & 345689 \\
\hline 1075 & $11 / 21 / 2018$ & 1873 & 3283652608 & 290738 & 1873 & 3267157248 & 266446 & 1873 & 3474145536 & 351352 \\
\hline 1076 & $11 / 22 / 2018$ & 1873 & 3283954432 & 301678 & 1873 & 3267435008 & 277913 & 1873 & 3474503168 & 357715 \\
\hline 1077 & $11 / 23 / 2018$ & 1873 & 3284267520 & 312854 & 1873 & 3267725568 & 290601 & 1873 & 3474868224 & 364959 \\
\hline 1078 & $11 / 24 / 2018$ & 1873 & 3284590080 & 322963 & 1873 & 3268028928 & 303304 & 1873 & 3475243008 & 374783 \\
\hline 1079 & $11 / 25 / 2018$ & 1873 & 3284924160 & 333830 & 1873 & 3268344320 & 315719 & 1873 & 3475628032 & 385072 \\
\hline 1080 & $11 / 26 / 2018$ & 1873 & 3285270016 & 345944 & 1873 & 3268672768 & 328087 & 1873 & 3476026112 & 398043 \\
\hline 1081 & $11 / 27 / 2018$ & 1873 & 3285630720 & 360786 & 1873 & 3269012992 & 339896 & 1873 & 3476441088 & 415178 \\
\hline 1082 & $11 / 28 / 2018$ & 1873 & 3286005248 & 374383 & 1873 & 3269366272 & 353645 & 1873 & 3476871168 & 429618 \\
\hline 1083 & $11 / 29 / 2018$ & 1873 & 3286392576 & 387323 & 1873 & 3269733888 & 367468 & 1873 & 3477315840 & 445101 \\
\hline 1084 & $11 / 30 / 2018$ & 1873 & 3286795008 & 402624 & 1873 & 3270114816 & 380777 & 1873 & 3477776896 & 460952 \\
\hline 1085 & $12 / 1 / 2018$ & 1873 & 3287214848 & 419705 & 1873 & 3270508544 & 393957 & 1873 & 3478254592 & 477653 \\
\hline 1086 & $12 / 2 / 2018$ & 1873 & 3287651840 & 436969 & 1873 & 3270917120 & 408536 & 1873 & 3478747648 & 493183 \\
\hline 1087 & $12 / 3 / 2018$ & 1873 & 3288105472 & 453774 & 1873 & 3271340800 & 423808 & 1873 & 3479256320 & 508641 \\
\hline 1088 & $12 / 4 / 2018$ & 1873 & 3288575232 & 469749 & 1873 & 3271779584 & 438715 & 1873 & 3479779584 & 523229 \\
\hline 1089 & $12 / 5 / 2018$ & 1873 & 3289062400 & 487033 & 1873 & 3272233216 & 453745 & 1873 & 3480317696 & 538109 \\
\hline 1090 & $12 / 6 / 2018$ & 1873 & 3289567232 & 504934 & 1873 & 3272702464 & 469196 & 1873 & 3480871936 & 554214 \\
\hline 1091 & $12 / 7 / 2018$ & 1873 & 3290089472 & 522251 & 1873 & 3273187328 & 484748 & 1873 & 3481443840 & 571954 \\
\hline 1092 & $12 / 8 / 2018$ & 1873 & 3290629632 & 540177 & 1873 & 3273687808 & 500170 & 1873 & 3482031616 & 587731 \\
\hline 1093 & $12 / 9 / 2018$ & 1873 & 3291187712 & 558053 & 1873 & 3274204160 & 516759 & 1873 & 3482636288 & 604629 \\
\hline 1094 & $12 / 10 / 2018$ & 1873 & 3291763456 & 575750 & 1873 & 3274739712 & 535617 & 1873 & 3483260928 & 624775 \\
\hline 1095 & $12 / 11 / 2018$ & 1873 & 3292356608 & 593045 & 1873 & 3275293440 & 553547 & 1873 & 3483900416 & 639384 \\
\hline 1096 & $12 / 12 / 2018$ & 1873 & 3292966912 & 610443 & 1873 & 3275866112 & 572831 & 1873 & 3484557056 & 656679 \\
\hline 1097 & $12 / 13 / 2018$ & 1873 & 3293595648 & 628637 & 1873 & 3276456448 & 590194 & 1873 & 3485230848 & 673617 \\
\hline 1098 & $12 / 14 / 2018$ & 1873 & 3294242560 & 647008 & 1873 & 3277065728 & 609377 & 1873 & 3485921536 & 690869 \\
\hline 1099 & $12 / 15 / 2018$ & 1873 & 3294908160 & 665549 & 1873 & 3277694464 & 628666 & 1873 & 3486631936 & 710352 \\
\hline 1100 & $12 / 16 / 2018$ & 1873 & 3295592960 & 684900 & 1873 & 3278341888 & 647484 & 1873 & 3487358976 & 727090 \\
\hline 1101 & $12 / 17 / 2018$ & 1873 & 3296298752 & 705628 & 1873 & 3279008512 & 666532 & 1873 & 3488103936 & 745035 \\
\hline 1102 & $12 / 18 / 2018$ & 1873 & 3297023232 & 724631 & 1873 & 3279693312 & 685230 & 1873 & 3488865280 & 761190 \\
\hline 1103 & $12 / 19 / 2018$ & 1873 & 3297765632 & 742062 & 1873 & 3280397824 & 704097 & 1873 & 3489645056 & 779755 \\
\hline 1104 & $12 / 20 / 2018$ & 1873 & 3298524672 & 759152 & 1873 & 3281120256 & 722569 & 1873 & 3490441984 & 797006 \\
\hline 1105 & $12 / 21 / 2018$ & 1873 & 3299300608 & 775974 & 1873 & 3281860608 & 740358 & 1873 & 3491256320 & 814364 \\
\hline 1106 & $12 / 22 / 2018$ & 1873 & 3300092672 & 792209 & 1873 & 3282618880 & 758317 & 1873 & 3492087808 & 831395 \\
\hline 1107 & $12 / 23 / 2018$ & 1873 & 3300900608 & 807909 & 1873 & 3283394816 & 775891 & 1873 & 3492935424 & 847871 \\
\hline 1108 & $12 / 24 / 2018$ & 1873 & 3301724416 & 823692 & 1873 & 3284189440 & 794586 & 1873 & 3493798144 & 862475 \\
\hline 1109 & $12 / 25 / 2018$ & 1873 & 3302561792 & 837511 & 1873 & 3285002240 & 812853 & 1873 & 3494674944 & 876956 \\
\hline 1110 & $12 / 26 / 2018$ & 1873 & 3303413504 & 851787 & 1873 & 3285832448 & 830212 & 1873 & 3495566336 & 891260 \\
\hline 1111 & $12 / 27 / 2018$ & 1873 & 3304279296 & 865645 & 1873 & 3286678272 & 845940 & 1873 & 3496472320 & 905961 \\
\hline 1112 & $12 / 28 / 2018$ & 1873 & 3305157888 & 878723 & 1873 & 3287539968 & 861434 & 1873 & 3497392896 & 920600 \\
\hline 1113 & $12 / 29 / 2018$ & 1873 & 3306049792 & 891917 & 1873 & 3288416256 & 876330 & 1873 & 3498326272 & 933505 \\
\hline 1114 & $12 / 30 / 2018$ & 1873 & 3306953984 & 904432 & 1873 & 3289306624 & 890391 & 1873 & 3499272704 & 946460 \\
\hline 1115 & $12 / 31 / 2018$ & 1873 & 3307870976 & 916613 & 1873 & 3290210304 & 903959 & 1873 & 3500231936 & 959071 \\
\hline 1116 & $1 / 1 / 2019$ & 1873 & 3308798976 & 928076 & 1873 & 3291127552 & 917068 & 1873 & 3501203200 & 971309 \\
\hline 1117 & $1 / 2 / 2019$ & 1129 & 3332530688 & 23731550 & 1129 & 3316675584 & 25547968 & 1129 & 3540343296 & 39140140 \\
\hline 1118 & $1 / 3 / 2019$ & 1129 & 3352665344 & 20134640 & 1129 & 3337241600 & 20566084 & 1129 & 3559748096 & 19404740 \\
\hline 1119 & $1 / 4 / 2019$ & 1129 & 3367571712 & 14906453 & 1129 & 3353530880 & 16288919 & 1129 & 3574727680 & 14979659 \\
\hline 1120 & $1 / 5 / 2019$ & 1129 & 3380407552 & 12835911 & 1129 & 3367128832 & 13598234 & 1129 & 3588009984 & 13282192 \\
\hline 1121 & $1 / 6 / 2019$ & 1129 & 3391980800 & 11573171 & 1129 & 3379292416 & 12163566 & 1129 & 3600124928 & 12114973 \\
\hline 1122 & $1 / 7 / 2019$ & 1129 & 3402672896 & 10692215 & 1129 & 3390470144 & 11177661 & 1129 & 3611354880 & 11230043 \\
\hline 1123 & $1 / 8 / 2019$ & 1129 & 3412686336 & 10013015 & 1129 & 3400897536 & 10427442 & 1129 & 3621872384 & 10517803 \\
\hline 1124 & $1 / 9 / 2019$ & 1129 & 3422146048 & 9460024 & 1129 & 3410717952 & 9820294 & 1129 & 3631796992 & 9924221 \\
\hline 1125 & $1 / 10 / 2019$ & 1129 & 3431141120 & 8995161 & 1129 & 3420029184 & 9311447 & 1129 & 3641215744 & 9418551 \\
\hline 1126 & $1 / 11 / 2019$ & 1129 & 3439737344 & 8596255 & 1129 & 3428904704 & 8875489 & 1129 & 3650196480 & 8981105 \\
\hline 1127 & $1 / 12 / 2019$ & 1129 & 3447985920 & 8248554 & 1129 & 3437400576 & 8495885 & 1129 & 3658794496 & 8597904 \\
\hline
\end{tabular}




\begin{tabular}{|c|c|c|c|c|c|c|c|c|c|c|}
\hline 1128 & $1 / 13 / 2019$ & 1129 & 3455928320 & 7942414 & 1129 & 3445562880 & 8161854 & 1129 & 3667053824 & 8259413 \\
\hline 1129 & $1 / 14 / 2019$ & 1129 & 3463598336 & 7670007 & 1129 & 3453427200 & 7864802 & 1129 & 3675011584 & 7957708 \\
\hline 1130 & $1 / 15 / 2019$ & 1129 & 3471023872 & 7425492 & 1129 & 3461025536 & 7598323 & 1129 & 3682697728 & 7686049 \\
\hline 1131 & $1 / 16 / 2019$ & 1129 & 3478228224 & 7204321 & 1129 & 3468382976 & 7357443 & 1129 & 3690138624 & 7440856 \\
\hline 1132 & $1 / 17 / 2019$ & 1129 & 3485231104 & 7002890 & 1129 & 3475521280 & 7138223 & 1129 & 3697357056 & 7218845 \\
\hline 1133 & $1 / 18 / 2019$ & 1129 & 3492049408 & 6818302 & 1129 & 3482458880 & 6937499 & 1129 & 3704371712 & 7014372 \\
\hline 1134 & $1 / 19 / 2019$ & 1129 & 3498697728 & 6648204 & 1129 & 3489211392 & 6752704 & 1129 & 3711198720 & 6826853 \\
\hline 1135 & $1 / 20 / 2019$ & 1129 & 3505188352 & 6490669 & 1129 & 3495793152 & 6581731 & 1129 & 3717853184 & 6654619 \\
\hline 1136 & $1 / 21 / 2019$ & 1129 & 3511532288 & 6344099 & 1129 & 3502216192 & 6422841 & 1129 & 3724346880 & 6493564 \\
\hline 1137 & $1 / 22 / 2019$ & 1129 & 3517739520 & 6207177 & 1129 & 3508490752 & 6274587 & 1129 & 3730690816 & 6344096 \\
\hline 1138 & $1 / 23 / 2019$ & 1129 & 3523820544 & 6081005 & 1129 & 3514626304 & 6135537 & 1129 & 3736896256 & 6205340 \\
\hline 1139 & $1 / 24 / 2019$ & 1129 & 3529780992 & 5960378 & 1129 & 3520631296 & 6005067 & 1129 & 3742970368 & 6074059 \\
\hline 1140 & $1 / 25 / 2019$ & 1129 & 3535627264 & 5846364 & 1129 & 3526513408 & 5882166 & 1129 & 3748921344 & 5951129 \\
\hline 1141 & $1 / 26 / 2019$ & 1129 & 3541365760 & 5738465 & 1129 & 3532279552 & 5766057 & 1129 & 3754757376 & 5836086 \\
\hline 1142 & $1 / 27 / 2019$ & 1129 & 3547001856 & 5636098 & 1129 & 3537935872 & 5656088 & 1129 & 3760484096 & 5726229 \\
\hline 1143 & $1 / 28 / 2019$ & 1129 & 3552540672 & 5538764 & 1129 & 3543487232 & 5551700 & 1129 & 3766106368 & 5622664 \\
\hline 1144 & $1 / 29 / 2019$ & 1129 & 3557986560 & 5446027 & 1129 & 3548939776 & 5452410 & 1129 & 3771631616 & 5525148 \\
\hline 1145 & $1 / 30 / 2019$ & 1129 & 3563344128 & 5357507 & 1129 & 3554297344 & 5357796 & 1129 & 3777062656 & 5431202 \\
\hline 1146 & $1 / 31 / 2019$ & 1129 & 3568616960 & 5272869 & 1129 & 3559565056 & 5267488 & 1129 & 3782405120 & 5342424 \\
\hline 1147 & $2 / 1 / 2019$ & 1129 & 3573808896 & 5191820 & 1129 & 3564746240 & 5181161 & 1129 & 3787662592 & 5257640 \\
\hline 1148 & $2 / 2 / 2019$ & 1129 & 3578923008 & 5114099 & 1129 & 3569844736 & 5098522 & 1129 & 3792840192 & 5177322 \\
\hline 1149 & $2 / 3 / 2019$ & 1129 & 3583962368 & 5039472 & 1129 & 3574863872 & 5019315 & 1129 & 3797939968 & 5099609 \\
\hline 1150 & $2 / 4 / 2019$ & 1129 & 3588930048 & 4967732 & 1129 & 3579807488 & 4943308 & 1129 & 3802964992 & 5025392 \\
\hline 1151 & $2 / 5 / 2019$ & 1129 & 3593828864 & 4898694 & 1129 & 3584677632 & 4870292 & 1129 & 3807919616 & 4954409 \\
\hline 1152 & $2 / 6 / 2019$ & 1129 & 3598661120 & 4832189 & 1129 & 3589477632 & 4800080 & 1129 & 3812805888 & 4886258 \\
\hline 1153 & $2 / 7 / 2019$ & 1129 & 3603429120 & 4768065 & 1129 & 3594210048 & 4732502 & 1129 & 3817626624 & 4820782 \\
\hline 1154 & $2 / 8 / 2019$ & 1129 & 3608135168 & 4706183 & 1129 & 3598877696 & 4667402 & 1129 & 3822384384 & 4757811 \\
\hline 1155 & $2 / 9 / 2019$ & 1129 & 3612781824 & 4646419 & 1129 & 3603482112 & 4604640 & 1129 & 3827081472 & 4697191 \\
\hline 1156 & $2 / 10 / 2019$ & 1129 & 3617370368 & 4588656 & 1129 & 3608026368 & 4544085 & 1129 & 3831720448 & 4638784 \\
\hline 1157 & $2 / 11 / 2019$ & 1129 & 3621903104 & 4532790 & 1129 & 3612512256 & 4485619 & 1129 & 3836302848 & 4582462 \\
\hline 1158 & $2 / 12 / 2019$ & 1129 & 3626381824 & 4478722 & 1129 & 3616941056 & 4429133 & 1129 & 3840830976 & 4528110 \\
\hline 1159 & $2 / 13 / 2019$ & 1129 & 3630808320 & 4426364 & 1129 & 3621315584 & 4374526 & 1129 & 3845306624 & 4475621 \\
\hline 1160 & $2 / 14 / 2019$ & 1129 & 3635183872 & 4375632 & 1129 & 3625637632 & 4321995 & 1129 & 3849731328 & 4424895 \\
\hline 1161 & $2 / 15 / 2019$ & 1129 & 3639510272 & 4326450 & 1129 & 3629908480 & 4270883 & 1129 & 3854107392 & 4375841 \\
\hline 1162 & $2 / 16 / 2019$ & 1129 & 3643789056 & 4278745 & 1129 & 3634129920 & 4221378 & 1129 & 3858435584 & 4328375 \\
\hline 1163 & $2 / 17 / 2019$ & 1129 & 3648021504 & 4232451 & 1129 & 3638303232 & 4173411 & 1129 & 3862717952 & 4282418 \\
\hline 1164 & $2 / 18 / 2019$ & 1129 & 3652208896 & 4187505 & 1129 & 3642430208 & 4126916 & 1129 & 3866956032 & 4237897 \\
\hline 1165 & $2 / 19 / 2019$ & 1129 & 3656352768 & 4143849 & 1129 & 3646511872 & 4081826 & 1129 & 3871150592 & 4194744 \\
\hline 1166 & $2 / 20 / 2019$ & 1129 & 3660454400 & 4101429 & 1129 & 3650550016 & 4038081 & 1129 & 3875303424 & 4152895 \\
\hline 1167 & $2 / 21 / 2019$ & 1129 & 3664514560 & 4060192 & 1129 & 3654545664 & 3995622 & 1129 & 3879415808 & 4112291 \\
\hline 1168 & $2 / 22 / 2019$ & 1129 & 3668534528 & 4020092 & 1129 & 3658500096 & 3954397 & 1129 & 3883488512 & 4072876 \\
\hline 1169 & $2 / 23 / 2019$ & 1129 & 3672515584 & 3981081 & 1129 & 3662414336 & 3914352 & 1129 & 3887523328 & 4034598 \\
\hline 1170 & $2 / 24 / 2019$ & 1129 & 3676458752 & 3943117 & 1129 & 3666289920 & 3875440 & 1129 & 3891520768 & 3997408 \\
\hline 1171 & $2 / 25 / 2019$ & 1129 & 3680365056 & 3906160 & 1129 & 3670127360 & 3837616 & 1129 & 3895482112 & 3961260 \\
\hline 1172 & $2 / 26 / 2019$ & 1129 & 3684235008 & 3870170 & 1129 & 3673928192 & 3800836 & 1129 & 3899408128 & 3926110 \\
\hline 1173 & $2 / 27 / 2019$ & 1129 & 3688070144 & 3835113 & 1129 & 3677693440 & 3765061 & 1129 & 3903300096 & 3891918 \\
\hline 1174 & $2 / 28 / 2019$ & 1129 & 3691871232 & 3800952 & 1129 & 3681423360 & 3730250 & 1129 & 3907158784 & 3858644 \\
\hline 1175 & $3 / 1 / 2019$ & 1129 & 3695638784 & 3767655 & 1129 & 3685120000 & 3696368 & 1129 & 3910984960 & 3826254 \\
\hline 1176 & $3 / 2 / 2019$ & 1129 & 3699374080 & 3735192 & 1129 & 3688783360 & 3663379 & 1129 & 3914779648 & 3794711 \\
\hline 1177 & $3 / 3 / 2019$ & 1129 & 3703077632 & 3703532 & 1129 & 3692414720 & 3631252 & 1129 & 3918543616 & 3763984 \\
\hline 1178 & $3 / 4 / 2019$ & 1129 & 3706750208 & 3672647 & 1129 & 3696014592 & 3599952 & 1129 & 3922277632 & 3734041 \\
\hline 1179 & $3 / 5 / 2019$ & 1129 & 3710392576 & 3642511 & 1129 & 3699584000 & 3569452 & 1129 & 3925982464 & 3704853 \\
\hline 1180 & $3 / 6 / 2019$ & 1129 & 3714005760 & 3613097 & 1129 & 3703123712 & 3539722 & 1129 & 3929658624 & 3676392 \\
\hline 1181 & $3 / 7 / 2019$ & 1129 & 3717590272 & 3584382 & 1129 & 3706634496 & 3510735 & 1129 & 3933307648 & 3648632 \\
\hline 1182 & $3 / 8 / 2019$ & 1129 & 3721146624 & 3556342 & 1129 & 3710116864 & 3482466 & 1129 & 3936929024 & 3621547 \\
\hline 1183 & $3 / 9 / 2019$ & 1129 & 3724675584 & 3528954 & 1129 & 3713572096 & 3454890 & 1129 & 3940524288 & 3595114 \\
\hline 1184 & $3 / 10 / 2019$ & 1129 & 3728177664 & 3502197 & 1129 & 3716999680 & 3427983 & 1129 & 3944093440 & 3569310 \\
\hline 1185 & $3 / 11 / 2019$ & 1129 & 3731653632 & 3476052 & 1129 & 3720401152 & 3401723 & 1129 & 3947637760 & 3544113 \\
\hline 1186 & $3 / 12 / 2019$ & 1129 & 3735104256 & 3450498 & 1129 & 3723777536 & 3376088 & 1129 & 3951157248 & 3519503 \\
\hline 1187 & $3 / 13 / 2019$ & 1129 & 3738529792 & 3425516 & 1129 & 3727128576 & 3351057 & 1129 & 3954652672 & 3495459 \\
\hline 1188 & $3 / 14 / 2019$ & 1129 & 3741930752 & 3401089 & 1129 & 3730455296 & 3326612 & 1129 & 3958124544 & 3471963 \\
\hline 1189 & $3 / 15 / 2019$ & 1129 & 3745307904 & 3377200 & 1129 & 3733757952 & 3302732 & 1129 & 3961573632 & 3448996 \\
\hline 1190 & $3 / 16 / 2019$ & 1129 & 3748661760 & 3353831 & 1129 & 3737037312 & 3279400 & 1129 & 3965000192 & 3426542 \\
\hline 1191 & $3 / 17 / 2019$ & 1129 & 3751992832 & 3330967 & 1129 & 3740294144 & 3256599 & 1129 & 3968404992 & 3404584 \\
\hline 1192 & $3 / 18 / 2019$ & 1129 & 3755301376 & 3308593 & 1129 & 3743528448 & 3234311 & 1129 & 3971787776 & 3383106 \\
\hline 1193 & $3 / 19 / 2019$ & 1129 & 3758588416 & 3286694 & 1129 & 3746740736 & 3212521 & 1129 & 3975149568 & 3362093 \\
\hline 1194 & $3 / 20 / 2019$ & 1129 & 3761853440 & 3265257 & 1129 & 3749932032 & 3191213 & 1129 & 3978491392 & 3341531 \\
\hline 1195 & $3 / 21 / 2019$ & 1129 & 3765097728 & 3244267 & 1129 & 3753102336 & 3170374 & 1129 & 3981812736 & 3321405 \\
\hline 1196 & $3 / 22 / 2019$ & 1129 & 3768321280 & 3223711 & 1129 & 3756252416 & 3149987 & 1129 & 3985114624 & 3301702 \\
\hline 1197 & $3 / 23 / 2019$ & 1129 & 3771524864 & 3203577 & 1129 & 3759382528 & 3130040 & 1129 & 3988397056 & 3282411 \\
\hline 1198 & $3 / 24 / 2019$ & 1129 & 3774708736 & 3183853 & 1129 & 3762492928 & 3110520 & 1129 & 3991660544 & 3263517 \\
\hline 1199 & $3 / 25 / 2019$ & 1129 & 3777873408 & 3164528 & 1129 & 3765584384 & 3091413 & 1129 & 3994905600 & 3245009 \\
\hline 1200 & $3 / 26 / 2019$ & 1129 & 3781018880 & 3145590 & 1129 & 3768657152 & 3072710 & 1129 & 3998132480 & 3226877 \\
\hline 1201 & $3 / 27 / 2019$ & 1129 & 3784145920 & 3127028 & 1129 & 3771711488 & 3054396 & 1129 & 4001341184 & 3209109 \\
\hline 1202 & $3 / 28 / 2019$ & 1129 & 3787254784 & 3108831 & 1129 & 3774747904 & 3036461 & 1129 & 4004533248 & 3191695 \\
\hline 1203 & $3 / 29 / 2019$ & 1129 & 3790345728 & 3090991 & 1129 & 3777766912 & 3018894 & 1129 & 4007707904 & 3174625 \\
\hline 1204 & $3 / 30 / 2019$ & 1129 & 3793419264 & 3073497 & 1129 & 3780768512 & 3001686 & 1129 & 4010865408 & 3157888 \\
\hline 1205 & $3 / 31 / 2019$ & 1129 & 3796475648 & 3056339 & 1129 & 3783753472 & 2984824 & 1129 & 4014007040 & 3141475 \\
\hline 1206 & $4 / 1 / 2019$ & 1129 & 3799515136 & 3039510 & 1129 & 3786722048 & 2968301 & 1129 & 4017132544 & 3125379 \\
\hline 1207 & $4 / 2 / 2019$ & 1129 & 3802537984 & 3022999 & 1129 & 3789673728 & 2952106 & 1129 & 4020242176 & 3109589 \\
\hline 1208 & $4 / 3 / 2019$ & 1129 & 3805544960 & 3006799 & 1129 & 3792610048 & 2936231 & 1129 & 4023336192 & 3094097 \\
\hline 1209 & $4 / 4 / 2019$ & 1129 & 3808536064 & 2990901 & 1129 & 3795530752 & 2920667 & 1129 & 4026415104 & 3078896 \\
\hline
\end{tabular}




\begin{tabular}{|c|c|c|c|c|c|c|c|c|c|c|}
\hline 1210 & $4 / 5 / 2019$ & 1129 & 3811511040 & 2975298 & 1129 & 3798436352 & 2905405 & 1129 & 4029479168 & 3063978 \\
\hline 1211 & $4 / 6 / 2019$ & 1129 & 3814471168 & 2959982 & 1129 & 3801326592 & 2890437 & 1129 & 4032528384 & 3049335 \\
\hline 1212 & $4 / 7 / 2019$ & 1129 & 3817415936 & 2944946 & 1129 & 3804202240 & 2875756 & 1129 & 4035563264 & 3034959 \\
\hline 1213 & $4 / 8 / 2019$ & 1129 & 3820346112 & 2930182 & 1129 & 3807063552 & 2861353 & 1129 & 4038584320 & 3020845 \\
\hline 1214 & $4 / 9 / 2019$ & 1129 & 3823261952 & 2915683 & 1129 & 3809910784 & 2847222 & 1129 & 4041591296 & 3006984 \\
\hline 1215 & $4 / 10 / 2019$ & 1129 & 3826163456 & 2901444 & 1129 & 3812744448 & 2833355 & 1129 & 4044584704 & 2993371 \\
\hline 1216 & $4 / 11 / 2019$ & 1129 & 3829050880 & 2887457 & 1129 & 3815564032 & 2819746 & 1129 & 4047564544 & 2979999 \\
\hline 1217 & $4 / 12 / 2019$ & 1129 & 3831924480 & 2873716 & 1129 & 3818370304 & 2806387 & 1129 & 4050531328 & 2966862 \\
\hline 1218 & $4 / 13 / 2019$ & 1129 & 3834784512 & 2860216 & 1129 & 3821163520 & 2793273 & 1129 & 4053485312 & 2953954 \\
\hline 1219 & $4 / 14 / 2019$ & 1129 & 3837631744 & 2846949 & 1129 & 3823943936 & 2780397 & 1129 & 4056426752 & 2941269 \\
\hline 1220 & $4 / 15 / 2019$ & 1129 & 3840465664 & 2833911 & 1129 & 3826711808 & 2767753 & 1129 & 4059355392 & 2928802 \\
\hline 1221 & $4 / 16 / 2019$ & 1129 & 3843286784 & 2821097 & 1129 & 3829467136 & 2755335 & 1129 & 4062272000 & 2916548 \\
\hline 1222 & $4 / 17 / 2019$ & 1129 & 3846095104 & 2808500 & 1129 & 3832209920 & 2743138 & 1129 & 4065176576 & 2904501 \\
\hline 1223 & $4 / 18 / 2019$ & 1129 & 3848891392 & 2796115 & 1129 & 3834941440 & 2731156 & 1129 & 4068069120 & 2892656 \\
\hline 1224 & $4 / 19 / 2019$ & 1129 & 3851675136 & 2783938 & 1129 & 3837660672 & 2719384 & 1129 & 4070950144 & 2881009 \\
\hline 1225 & $4 / 20 / 2019$ & 1129 & 3854447104 & 2771964 & 1129 & 3840368640 & 2707817 & 1129 & 4073819648 & 2869554 \\
\hline 1226 & $4 / 21 / 2019$ & 1129 & 3857207296 & 2760188 & 1129 & 3843065088 & 2696450 & 1129 & 4076677888 & 2858287 \\
\hline 1227 & $4 / 22 / 2019$ & 1129 & 3859955968 & 2748605 & 1129 & 3845750272 & 2685277 & 1129 & 4079525120 & 2847204 \\
\hline 1228 & $4 / 23 / 2019$ & 1129 & 3862693120 & 2737210 & 1129 & 3848424448 & 2674294 & 1129 & 4082361600 & 2836300 \\
\hline 1229 & $4 / 24 / 2019$ & 1129 & 3865419520 & 2726000 & 1129 & 3851088128 & 2663498 & 1129 & 4085187072 & 2825571 \\
\hline 1230 & $4 / 25 / 2019$ & 1129 & 3868134144 & 2714971 & 1129 & 3853741056 & 2652882 & 1129 & 4088002048 & 2815012 \\
\hline 1231 & $4 / 26 / 2019$ & 1129 & 3870838272 & 2704118 & 1129 & 3856383488 & 2642443 & 1129 & 4090807040 & 2804622 \\
\hline 1232 & $4 / 27 / 2019$ & 1129 & 3873531648 & 2693437 & 1129 & 3859015680 & 2632177 & 1129 & 4093601024 & 2794394 \\
\hline 1233 & $4 / 28 / 2019$ & 1129 & 3876214784 & 2682924 & 1129 & 3861637632 & 2622080 & 1129 & 4096385536 & 2784326 \\
\hline 1234 & $4 / 29 / 2019$ & 1129 & 3878887168 & 2672576 & 1129 & 3864249856 & 2612148 & 1129 & 4099159808 & 2774414 \\
\hline 1235 & $4 / 30 / 2019$ & 1129 & 3881549312 & 2662389 & 1129 & 3866852096 & 2602377 & 1129 & 4101924608 & 2764654 \\
\hline 1236 & $5 / 1 / 2019$ & 1129 & 3884201984 & 2652359 & 1129 & 3869444864 & 2592763 & 1129 & 4104679424 & 2755043 \\
\hline 1237 & $5 / 2 / 2019$ & 1129 & 3886844416 & 2642483 & 1129 & 3872028160 & 2583303 & 1129 & 4107425280 & 2745578 \\
\hline 1238 & $5 / 3 / 2019$ & 1129 & 3889477120 & 2632758 & 1129 & 3874602240 & 2573993 & 1129 & 4110161408 & 2736255 \\
\hline 1239 & $5 / 4 / 2019$ & 1129 & 3892100352 & 2623181 & 1129 & 3877167104 & 2564830 & 1129 & 4112888576 & 2727072 \\
\hline 1240 & $5 / 5 / 2019$ & 1129 & 3894714112 & 2613747 & 1129 & 3879723008 & 2555811 & 1129 & 4115606528 & 2718025 \\
\hline 1241 & $5 / 6 / 2019$ & 1129 & 3897318656 & 2604455 & 1129 & 3882269952 & 2546932 & 1129 & 4118315520 & 2709112 \\
\hline 1242 & $5 / 7 / 2019$ & 1129 & 3899913984 & 2595301 & 1129 & 3884807936 & 2538190 & 1129 & 4121015808 & 2700329 \\
\hline 1243 & $5 / 8 / 2019$ & 1129 & 3902500096 & 2586281 & 1129 & 3887337728 & 2529582 & 1129 & 4123707648 & 2691674 \\
\hline 1244 & $5 / 9 / 2019$ & 1129 & 3905077504 & 2577395 & 1129 & 3889858816 & 2521106 & 1129 & 4126390784 & 2683144 \\
\hline 1245 & $5 / 10 / 2019$ & 1129 & 3907646208 & 2568638 & 1129 & 3892371456 & 2512758 & 1129 & 4129065472 & 2674736 \\
\hline 1246 & $5 / 11 / 2019$ & 1129 & 3910206208 & 2560007 & 1129 & 3894875904 & 2504536 & 1129 & 4131732224 & 2666448 \\
\hline 1247 & $5 / 12 / 2019$ & 1129 & 3912757760 & 2551501 & 1129 & 3897372416 & 2496436 & 1129 & 4134390272 & 2658277 \\
\hline 1248 & $5 / 13 / 2019$ & 1129 & 3915300864 & 2543117 & 1129 & 3899860992 & 2488457 & 1129 & 4137040384 & 2650221 \\
\hline 1249 & $5 / 14 / 2019$ & 1129 & 3917835776 & 2534851 & 1129 & 3902341376 & 2480596 & 1129 & 4139682816 & 2642277 \\
\hline 1250 & $5 / 15 / 2019$ & 1129 & 3920362496 & 2526703 & 1129 & 3904814336 & 2472849 & 1129 & 4142317056 & 2634444 \\
\hline 1251 & $5 / 16 / 2019$ & 1129 & 3922881280 & 2518669 & 1129 & 3907279616 & 2465216 & 1129 & 4144943616 & 2626718 \\
\hline 1252 & $5 / 17 / 2019$ & 1129 & 3925391872 & 2510746 & 1129 & 3909737216 & 2457692 & 1129 & 4147563008 & 2619099 \\
\hline 1253 & $5 / 18 / 2019$ & 1129 & 3927894784 & 2502934 & 1129 & 3912187648 & 2450277 & 1129 & 4150174464 & 2611582 \\
\hline 1254 & $5 / 19 / 2019$ & 1129 & 3930390016 & 2495229 & 1129 & 3914630400 & 2442967 & 1129 & 4152778752 & 2604167 \\
\hline 1255 & $5 / 20 / 2019$ & 1129 & 3932877568 & 2487630 & 1129 & 3917065984 & 2435761 & 1129 & 4155375616 & 2596852 \\
\hline 1256 & $5 / 21 / 2019$ & 1129 & 3935357696 & 2480134 & 1129 & 3919494912 & 2428657 & 1129 & 4157965312 & 2589634 \\
\hline 1257 & $5 / 22 / 2019$ & 1129 & 3937830400 & 2472740 & 1129 & 3921916672 & 2421652 & 1129 & 4160547328 & 2582511 \\
\hline 1258 & $5 / 23 / 2019$ & 1129 & 3940295936 & 2465444 & 1129 & 3924331264 & 2414744 & 1129 & 4163123200 & 2575482 \\
\hline 1259 & $5 / 24 / 2019$ & 1129 & 3942754048 & 2458246 & 1129 & 3926739200 & 2407932 & 1129 & 4165691648 & 2568545 \\
\hline 1260 & $5 / 25 / 2019$ & 1129 & 3945204992 & 2451144 & 1129 & 3929140736 & 2401212 & 1129 & 4168253440 & 2561698 \\
\hline 1261 & $5 / 26 / 2019$ & 1129 & 3947649536 & 2444135 & 1129 & 3931535104 & 2394585 & 1129 & 4170808320 & 2554939 \\
\hline 1262 & $5 / 27 / 2019$ & 1129 & 3950086656 & 2437219 & 1129 & 3933923072 & 2388047 & 1129 & 4173356544 & 2548266 \\
\hline 1263 & $5 / 28 / 2019$ & 1129 & 3952517120 & 2430392 & 1129 & 3936304640 & 2381597 & 1129 & 4175898368 & 2541678 \\
\hline 1264 & $5 / 29 / 2019$ & 1129 & 3954940672 & 2423653 & 1129 & 3938679808 & 2375233 & 1129 & 4178433536 & 2535174 \\
\hline 1265 & $5 / 30 / 2019$ & 1129 & 3957357568 & 2417002 & 1129 & 3941048832 & 2368953 & 1129 & 4180962560 & 2528751 \\
\hline 1266 & $5 / 31 / 2019$ & 1129 & 3959768064 & 2410435 & 1129 & 3943411712 & 2362757 & 1129 & 4183484672 & 2522408 \\
\hline 1267 & $6 / 1 / 2019$ & 1129 & 3962172160 & 2403952 & 1129 & 3945768192 & 2356641 & 1129 & 4186000896 & 2516144 \\
\hline 1268 & $6 / 2 / 2019$ & 1129 & 3964569600 & 2397551 & 1129 & 3948118784 & 2350605 & 1129 & 4188510720 & 2509956 \\
\hline 1269 & $6 / 3 / 2019$ & 1129 & 3966960896 & 2391230 & 1129 & 3950463488 & 2344647 & 1129 & 4191014656 & 2503845 \\
\hline 1270 & $6 / 4 / 2019$ & 1129 & 3969345536 & 2384988 & 1129 & 3952802304 & 2338766 & 1129 & 4193512448 & 2497807 \\
\hline 1271 & $6 / 5 / 2019$ & 1129 & 3971724544 & 2378823 & 1129 & 3955135232 & 2332959 & 1129 & 4196004352 & 2491843 \\
\hline 1272 & $6 / 6 / 2019$ & 1129 & 3974097408 & 2372735 & 1129 & 3957462528 & 2327226 & 1129 & 4198490112 & 2485950 \\
\hline 1273 & $6 / 7 / 2019$ & 1129 & 3976464128 & 2366721 & 1129 & 3959783936 & 2321565 & 1129 & 4200970240 & 2480127 \\
\hline 1274 & $6 / 8 / 2019$ & 1129 & 3978824960 & 2360780 & 1129 & 3962099968 & 2315975 & 1129 & 4203444480 & 2474373 \\
\hline 1275 & $6 / 9 / 2019$ & 1129 & 3981179904 & 2354911 & 1129 & 3964410368 & 2310455 & 1129 & 4205913344 & 2468687 \\
\hline 1276 & $6 / 10 / 2019$ & 1129 & 3983528960 & 2349113 & 1129 & 3966715392 & 2305003 & 1129 & 4208376320 & 2463067 \\
\hline 1277 & $6 / 11 / 2019$ & 1129 & 3985872384 & 2343384 & 1129 & 3969015040 & 2299617 & 1129 & 4210833920 & 2457512 \\
\hline 1278 & $6 / 12 / 2019$ & 1129 & 3988209920 & 2337724 & 1129 & 3971309312 & 2294297 & 1129 & 4213285888 & 2452022 \\
\hline 1279 & $6 / 13 / 2019$ & 1129 & 3990542080 & 2332130 & 1129 & 3973598464 & 2289042 & 1129 & 4215732480 & 2446594 \\
\hline 1280 & $6 / 14 / 2019$ & 1129 & 3992868864 & 2326602 & 1129 & 3975882240 & 2283850 & 1129 & 4218173696 & 2441228 \\
\hline 1281 & $6 / 15 / 2019$ & 1129 & 3995190016 & 2321138 & 1129 & 3978160640 & 2278719 & 1129 & 4220609792 & 2435923 \\
\hline 1282 & $6 / 16 / 2019$ & 1129 & 3997505536 & 2315738 & 1129 & 3980434688 & 2273650 & 1129 & 4223040512 & 2430678 \\
\hline 1283 & $6 / 17 / 2019$ & 1129 & 3999815936 & 2310400 & 1129 & 3982703360 & 2268641 & 1129 & 4225465856 & 2425491 \\
\hline 1284 & $6 / 18 / 2019$ & 1129 & 4002121216 & 2305123 & 1129 & 3984966912 & 2263690 & 1129 & 4227886336 & 2420361 \\
\hline 1285 & $6 / 19 / 2019$ & 1129 & 4004421120 & 2299907 & 1129 & 3987225856 & 2258797 & 1129 & 4230301440 & 2415288 \\
\hline 1286 & $6 / 20 / 2019$ & 1129 & 4006715904 & 2294750 & 1129 & 3989479936 & 2253960 & 1129 & 4232711936 & 2410271 \\
\hline 1287 & $6 / 21 / 2019$ & 1129 & 4009005312 & 2289650 & 1129 & 3991728896 & 2249179 & 1129 & 4235117056 & 2405308 \\
\hline 1288 & $6 / 22 / 2019$ & 1129 & 4011290112 & 2284608 & 1129 & 3993973248 & 2244453 & 1129 & 4237517568 & 2400399 \\
\hline 1289 & $6 / 23 / 2019$ & 1129 & 4013569792 & 2279622 & 1129 & 3996213248 & 2239780 & 1129 & 4239912960 & 2395543 \\
\hline 1290 & $6 / 24 / 2019$ & 1129 & 4015844352 & 2274691 & 1129 & 3998448384 & 2235160 & 1129 & 4242303744 & 2390739 \\
\hline 1291 & $6 / 25 / 2019$ & 1129 & 4018114304 & 2269815 & 1129 & 4000678912 & 2230592 & 1129 & 4244689664 & 2385985 \\
\hline
\end{tabular}




\begin{tabular}{|c|c|c|c|c|c|c|c|c|c|c|}
\hline 1292 & $6 / 26 / 2019$ & 1129 & 4020379136 & 2264991 & 1129 & 4002905088 & 2226074 & 1129 & 4247070976 & 2381282 \\
\hline 1293 & $6 / 27 / 2019$ & 1129 & 4022639360 & 2260220 & 1129 & 4005126656 & 2221606 & 1129 & 4249447680 & 2376628 \\
\hline 1294 & $6 / 28 / 2019$ & 1129 & 4024894976 & 2255501 & 1129 & 4007343872 & 2217188 & 1129 & 4251819776 & 2372022 \\
\hline 1295 & $6 / 29 / 2019$ & 1129 & 4027145472 & 2250832 & 1129 & 4009556224 & 2212817 & 1129 & 4254187264 & 2367464 \\
\hline 1296 & $6 / 30 / 2019$ & 1129 & 4029391872 & 2246213 & 1129 & 4011764992 & 2208494 & 1129 & 4256550144 & 2362953 \\
\hline 1297 & $7 / 1 / 2019$ & 1129 & 4031633664 & 2241643 & 1129 & 4013969152 & 2204218 & 1129 & 4258908672 & 2358488 \\
\hline 1298 & $7 / 2 / 2019$ & 1129 & 4033870848 & 2237122 & 1129 & 4016169216 & 2199987 & 1129 & 4261262592 & 2354068 \\
\hline 1299 & $7 / 3 / 2019$ & 1129 & 4036103424 & 2232648 & 1129 & 4018365184 & 2195801 & 1129 & 4263612416 & 2349693 \\
\hline 1300 & $7 / 4 / 2019$ & 1129 & 4038331648 & 2228220 & 1129 & 4020556800 & 2191758 & 1129 & 4265957376 & 2345362 \\
\hline 1301 & $7 / 5 / 2019$ & 1129 & 4040555520 & 2223838 & 1129 & 4022744320 & 2187574 & 1129 & 4268298752 & 2341074 \\
\hline 1302 & $7 / 6 / 2019$ & 1129 & 4042775040 & 2219502 & 1129 & 4024928000 & 2183578 & 1129 & 4270635520 & 2336828 \\
\hline 1303 & $7 / 7 / 2019$ & 1129 & 4044990208 & 2215210 & 1129 & 4027107328 & 2179501 & 1129 & 4272968192 & 2332624 \\
\hline 1304 & $7 / 8 / 2019$ & 1129 & 4047201024 & 2210961 & 1129 & 4029283072 & 2175600 & 1129 & 4275296768 & 2328461 \\
\hline 1305 & $7 / 9 / 2019$ & 1129 & 4049407744 & 2206756 & 1129 & 4031454720 & 2171600 & 1129 & 4277620992 & 2324339 \\
\hline 1306 & $7 / 10 / 2019$ & 1129 & 4051610368 & 2202592 & 1129 & 4033622528 & 2167770 & 1129 & 4279941376 & 2320256 \\
\hline 1307 & $7 / 11 / 2019$ & 1129 & 4053808896 & 2198471 & 1129 & 4035785984 & 2163854 & 1129 & 4282257664 & 2316213 \\
\hline 1308 & $7 / 12 / 2019$ & 1129 & 4056003328 & 2194390 & 1129 & 4037946112 & 2160106 & 1129 & 4284569856 & 2312208 \\
\hline 1309 & $7 / 13 / 2019$ & 1486 & 4056003328 & 21 & 1486 & 4037946112 & 27 & 1486 & 4284569856 & 0 \\
\hline 1310 & $7 / 14 / 2019$ & 1486 & 4056011264 & 7833 & 1486 & 4037955328 & 8764 & 1486 & 4284579072 & 9446 \\
\hline 1311 & $7 / 15 / 2019$ & 1486 & 4056044288 & 33161 & 1486 & 4037986304 & 31127 & 1486 & 4284617472 & 38347 \\
\hline 1312 & $7 / 16 / 2019$ & 1486 & 4056108032 & 63591 & 1486 & 4038044928 & 58580 & 1486 & 4284697088 & 79475 \\
\hline 1313 & $7 / 17 / 2019$ & 1486 & 4056184576 & 76859 & 1486 & 4038115840 & 71045 & 1486 & 4284801280 & 104295 \\
\hline 1314 & $7 / 18 / 2019$ & 1486 & 4056269824 & 84986 & 1486 & 4038194688 & 78684 & 1486 & 4284922368 & 121067 \\
\hline 1315 & $7 / 19 / 2019$ & 1486 & 4056360704 & 90901 & 1486 & 4038278912 & 84251 & 1486 & 4285054976 & 132868 \\
\hline 1316 & $7 / 20 / 2019$ & 1486 & 4056456448 & 95670 & 1486 & 4038367744 & 88744 & 1486 & 4285197568 & 142246 \\
\hline 1317 & $7 / 21 / 2019$ & 1486 & 4056556032 & 99676 & 1486 & 4038460160 & 92562 & 1486 & 4285347584 & 150189 \\
\hline 1318 & $7 / 22 / 2019$ & 1486 & 4056659456 & 103202 & 1486 & 4038556160 & 95894 & 1486 & 4285504768 & 157149 \\
\hline 1319 & $7 / 23 / 2019$ & 1486 & 4056765440 & 106333 & 1486 & 4038654976 & 98842 & 1486 & 4285668096 & 163312 \\
\hline 1320 & $7 / 24 / 2019$ & 1486 & 4056874496 & 109112 & 1486 & 4038756608 & 101471 & 1486 & 4285837056 & 168800 \\
\hline 1321 & $7 / 25 / 2019$ & 1486 & 4056986368 & 111605 & 1486 & 4038860288 & 103984 & 1486 & 4286010624 & 173756 \\
\hline 1322 & $7 / 26 / 2019$ & 1486 & 4057100032 & 113849 & 1486 & 4038966528 & 106066 & 1486 & 4286189056 & 178249 \\
\hline 1323 & $7 / 27 / 2019$ & 1486 & 4057216000 & 115878 & 1486 & 4039074560 & 107989 & 1486 & 4286371328 & 182326 \\
\hline 1324 & $7 / 28 / 2019$ & 1486 & 4057333760 & 117715 & 1486 & 4039184128 & 109662 & 1486 & 4286557440 & 186078 \\
\hline 1325 & $7 / 29 / 2019$ & 1486 & 4057452800 & 119392 & 1486 & 4039295488 & 111256 & 1486 & 4286746880 & 189480 \\
\hline 1326 & $7 / 30 / 2019$ & 1486 & 4057574144 & 120925 & 1486 & 4039408384 & 112835 & 1486 & 4286939392 & 192610 \\
\hline 1327 & $7 / 31 / 2019$ & 1486 & 4057696256 & 122338 & 1486 & 4039522304 & 114162 & 1486 & 4287134976 & 195499 \\
\hline 1328 & $8 / 1 / 2019$ & 1486 & 4057819904 & 123641 & 1486 & 4039637760 & 115418 & 1486 & 4287333120 & 198174 \\
\hline 1329 & $8 / 2 / 2019$ & 1486 & 4057944832 & 124853 & 1486 & 4039754240 & 116518 & 1486 & 4287533824 & 200662 \\
\hline 1330 & $8 / 3 / 2019$ & 1486 & 4058070784 & 125980 & 1486 & 4039871744 & 117605 & 1486 & 4287736832 & 202984 \\
\hline 1331 & $8 / 4 / 2019$ & 1486 & 4058197760 & 127036 & 1486 & 4039990528 & 118634 & 1486 & 4287941888 & 205160 \\
\hline 1332 & $8 / 5 / 2019$ & 1486 & 4058326016 & 128027 & 1486 & 4040110080 & 119603 & 1486 & 4288149248 & 207205 \\
\hline 1333 & $8 / 6 / 2019$ & 1486 & 4058454784 & 128964 & 1486 & 4040230656 & 120522 & 1486 & 4288357888 & 209135 \\
\hline 1334 & $8 / 7 / 2019$ & 1486 & 4058585088 & 129849 & 1486 & 4040351744 & 121391 & 1486 & 4288569344 & 211177 \\
\hline 1335 & $8 / 8 / 2019$ & 1486 & 4058715392 & 130690 & 1486 & 4040474368 & 122223 & 1486 & 4288783360 & 214045 \\
\hline 1336 & $8 / 9 / 2019$ & 1486 & 4058846976 & 131491 & 1486 & 4040597248 & 123014 & 1486 & 4288999168 & 215805 \\
\hline 1337 & $8 / 10 / 2019$ & 1486 & 4058979072 & 132257 & 1486 & 4040721152 & 123775 & 1486 & 4289217024 & 217849 \\
\hline 1338 & $8 / 11 / 2019$ & 1486 & 4059112192 & 132990 & 1486 & 4040845824 & 124502 & 1486 & 4289436928 & 219787 \\
\hline 1339 & $8 / 12 / 2019$ & 1486 & 4059245824 & 133695 & 1486 & 4040971264 & 125309 & 1486 & 4289658624 & 221635 \\
\hline 1340 & $8 / 13 / 2019$ & 1486 & 4059380992 & 135089 & 1486 & 4041097984 & 126987 & 1486 & 4289881856 & 223403 \\
\hline 1341 & $8 / 14 / 2019$ & 1486 & 4059517952 & 137062 & 1486 & 4041225472 & 127519 & 1486 & 4290107136 & 225100 \\
\hline 1342 & $8 / 15 / 2019$ & 1486 & 4059656960 & 138624 & 1486 & 4041353728 & 128422 & 1486 & 4290333696 & 226734 \\
\hline 1343 & $8 / 16 / 2019$ & 1486 & 4059796736 & 140021 & 1486 & 4041484032 & 129886 & 1486 & 4290562048 & 228310 \\
\hline 1344 & $8 / 17 / 2019$ & 1486 & 4059938560 & 141952 & 1486 & 4041615872 & 132104 & 1486 & 4290791936 & 229832 \\
\hline 1345 & $8 / 18 / 2019$ & 1486 & 4060082432 & 143601 & 1486 & 4041749760 & 133938 & 1486 & 4291023360 & 231306 \\
\hline 1346 & $8 / 19 / 2019$ & 1486 & 4060227328 & 145103 & 1486 & 4041885696 & 136084 & 1486 & 4291256064 & 232930 \\
\hline 1347 & $8 / 20 / 2019$ & 1486 & 4060376064 & 148692 & 1486 & 4042023680 & 137780 & 1486 & 4291491072 & 234903 \\
\hline 1348 & $8 / 21 / 2019$ & 1486 & 4060527104 & 151056 & 1486 & 4042162688 & 139405 & 1486 & 4291727872 & 236676 \\
\hline 1349 & $8 / 22 / 2019$ & 1486 & 4060680192 & 153027 & 1486 & 4042304000 & 140938 & 1486 & 4291966208 & 238360 \\
\hline 1350 & $8 / 23 / 2019$ & 1486 & 4060834816 & 154927 & 1486 & 4042446336 & 142398 & 1486 & 4292207872 & 241672 \\
\hline 1351 & $8 / 24 / 2019$ & 1486 & 4060992768 & 157711 & 1486 & 4042590208 & 143793 & 1486 & 4292454400 & 246887 \\
\hline 1352 & $8 / 25 / 2019$ & 1486 & 4061153024 & 160411 & 1486 & 4042735872 & 145389 & 1486 & 4292705792 & 251207 \\
\hline 1353 & $8 / 26 / 2019$ & 1486 & 4061315840 & 162819 & 1486 & 4042884096 & 148461 & 1486 & 4292960256 & 254428 \\
\hline 1354 & $8 / 27 / 2019$ & 1486 & 4061482496 & 166496 & 1486 & 4043035904 & 151770 & 1486 & 4293217792 & 257606 \\
\hline 1355 & $8 / 28 / 2019$ & 1486 & 4061653504 & 171020 & 1486 & 4043191040 & 155412 & 1486 & 4293478912 & 260960 \\
\hline 1356 & $8 / 29 / 2019$ & 1486 & 4061830656 & 177048 & 1486 & 4043349760 & 158689 & 1486 & 4293744384 & 265401 \\
\hline 1357 & $8 / 30 / 2019$ & 1486 & 4062012416 & 182017 & 1486 & 4043511808 & 161976 & 1486 & 4294014464 & 270078 \\
\hline 1358 & $8 / 31 / 2019$ & 1486 & 4062199040 & 186549 & 1486 & 4043678208 & 166472 & 1486 & 4294291968 & 277567 \\
\hline 1359 & $9 / 1 / 2019$ & 1486 & 4062390528 & 191497 & 1486 & 4043850496 & 172259 & 1486 & 4294577152 & 285358 \\
\hline 1360 & $9 / 2 / 2019$ & 1486 & 4062587136 & 196501 & 1486 & 4044029696 & 179033 & 1486 & 4294870272 & 292969 \\
\hline 1361 & $9 / 3 / 2019$ & 1486 & 4062791936 & 204955 & 1486 & 4044215552 & 185875 & 1486 & 4295170048 & 299766 \\
\hline 1362 & $9 / 4 / 2019$ & 1486 & 4063004928 & 212886 & 1486 & 4044409856 & 194421 & 1486 & 4295475712 & 305781 \\
\hline 1363 & $9 / 5 / 2019$ & 1486 & 4063226368 & 221443 & 1486 & 4044612608 & 202745 & 1486 & 4295788032 & 312039 \\
\hline 1364 & $9 / 6 / 2019$ & 1486 & 4063458304 & 232034 & 1486 & 4044823296 & 210661 & 1486 & 4296105984 & 317990 \\
\hline 1365 & $9 / 7 / 2019$ & 1486 & 4063701248 & 242816 & 1486 & 4045041920 & 218702 & 1486 & 4296429056 & 323104 \\
\hline 1366 & $9 / 8 / 2019$ & 1486 & 4063954944 & 253625 & 1486 & 4045268224 & 226254 & 1486 & 4296757248 & 328539 \\
\hline 1367 & $9 / 9 / 2019$ & 1486 & 4064218624 & 263699 & 1486 & 4045503744 & 235389 & 1486 & 4297092096 & 334642 \\
\hline 1368 & $9 / 10 / 2019$ & 1486 & 4064491776 & 273280 & 1486 & 4045749760 & 245762 & 1486 & 4297434624 & 342597 \\
\hline 1369 & $9 / 11 / 2019$ & 1486 & 4064774656 & 282875 & 1486 & 4046004480 & 255184 & 1486 & 4297784320 & 349609 \\
\hline 1370 & $9 / 12 / 2019$ & 1486 & 4065066752 & 292087 & 1486 & 4046268416 & 263831 & 1486 & 4298140672 & 356121 \\
\hline 1371 & $9 / 13 / 2019$ & 1486 & 4065368832 & 302087 & 1486 & 4046542336 & 273828 & 1486 & 4298503680 & 363124 \\
\hline 1372 & $9 / 14 / 2019$ & 1486 & 4065680896 & 312172 & 1486 & 4046826240 & 283964 & 1486 & 4298876416 & 372779 \\
\hline 1373 & $9 / 15 / 2019$ & 1486 & 4066002688 & 321780 & 1486 & 4047120640 & 294345 & 1486 & 4299260416 & 384130 \\
\hline
\end{tabular}




\begin{tabular}{|c|c|c|c|c|c|c|c|c|c|c|}
\hline 1374 & $9 / 16 / 2019$ & 1486 & 4066333696 & 330851 & 1486 & 4047425280 & 304608 & 1486 & 4299655168 & 394471 \\
\hline 1375 & 9/17/2019 & 1486 & 4066673152 & 339568 & 1486 & 4047740416 & 315346 & 1486 & 4300061184 & 406435 \\
\hline 1376 & $9 / 18 / 2019$ & 1486 & 4067023360 & 350261 & 1486 & 4048066560 & 326052 & 1486 & 4300480000 & 418457 \\
\hline 1377 & 9/19/2019 & 1486 & 4067387392 & 363948 & 1486 & 4048403712 & 337232 & 1486 & 4300910080 & 430062 \\
\hline 1378 & $9 / 20 / 2019$ & 1486 & 4067765248 & 378145 & 1486 & 4048751360 & 347567 & 1486 & 4301353472 & 443372 \\
\hline 1379 & $9 / 21 / 2019$ & 1486 & 4068156928 & 391434 & 1486 & 4049108480 & 357242 & 1486 & 4301809152 & 455884 \\
\hline 1380 & $9 / 22 / 2019$ & 1486 & 4068561664 & 404720 & 1486 & 4049475840 & 367114 & 1486 & 4302279168 & 469865 \\
\hline 1381 & $9 / 23 / 2019$ & 1486 & 4068978944 & 417601 & 1486 & 4049855488 & 379853 & 1486 & 4302763520 & 484425 \\
\hline 1382 & $9 / 24 / 2019$ & 1486 & 4069409536 & 430274 & 1486 & 4050248448 & 392962 & 1486 & 4303262720 & 499046 \\
\hline 1383 & $9 / 25 / 2019$ & 1486 & 4069853696 & 444064 & 1486 & 4050654208 & 405569 & 1486 & 4303775232 & 512650 \\
\hline 1384 & $9 / 26 / 2019$ & 1486 & 4070312448 & 458908 & 1486 & 4051071744 & 417663 & 1486 & 4304300544 & 525630 \\
\hline 1385 & $9 / 27 / 2019$ & 1486 & 4070786048 & 473543 & 1486 & 4051501056 & 429313 & 1486 & 4304840704 & 539874 \\
\hline 1386 & $9 / 28 / 2019$ & 1486 & 4071274496 & 488493 & 1486 & 4051942400 & 441269 & 1486 & 4305394176 & 553615 \\
\hline 1387 & $9 / 29 / 2019$ & 1486 & 4071777280 & 503002 & 1486 & 4052395520 & 453217 & 1486 & 4305963008 & 568743 \\
\hline 1388 & $9 / 30 / 2019$ & 1486 & 4072295168 & 517570 & 1486 & 4052862208 & 466548 & 1486 & 4306546176 & 583430 \\
\hline 1389 & $10 / 1 / 2019$ & 1486 & 4072826880 & 531785 & 1486 & 4053341952 & 479958 & 1486 & 4307143168 & 596835 \\
\hline 1390 & $10 / 2 / 2019$ & 1486 & 4073373440 & 546460 & 1486 & 4053835520 & 493410 & 1486 & 4307756544 & 613087 \\
\hline 1391 & $10 / 3 / 2019$ & 1486 & 4073934592 & 561147 & 1486 & 4054342656 & 507183 & 1486 & 4308385280 & 628787 \\
\hline 1392 & $10 / 4 / 2019$ & 1486 & 4074510592 & 576088 & 1486 & 4054863872 & 521290 & 1486 & 4309030400 & 645449 \\
\hline 1393 & $10 / 5 / 2019$ & 1486 & 4075102464 & 591857 & 1486 & 4055400448 & 536454 & 1486 & 4309692416 & 661893 \\
\hline 1394 & $10 / 6 / 2019$ & 1486 & 4075710720 & 608124 & 1486 & 4055952640 & 552448 & 1486 & 4310370304 & 678031 \\
\hline 1395 & $10 / 7 / 2019$ & 1486 & 4076333824 & 623305 & 1486 & 4056520448 & 567631 & 1486 & 4311064576 & 693821 \\
\hline 1396 & $10 / 8 / 2019$ & 1486 & 4076972800 & 638825 & 1486 & 4057103616 & 583124 & 1486 & 4311773184 & 708930 \\
\hline 1397 & $10 / 9 / 2019$ & 1486 & 4077630720 & 657720 & 1486 & 4057703680 & 600376 & 1486 & 4312497664 & 724327 \\
\hline 1398 & $10 / 10 / 2019$ & 1486 & 4078305792 & 675262 & 1486 & 4058321408 & 617355 & 1486 & 4313236480 & 739143 \\
\hline 1399 & $10 / 11 / 2019$ & 1486 & 4078999040 & 693412 & 1486 & 4058955520 & 634155 & 1486 & 4313990144 & 753579 \\
\hline 1400 & $10 / 12 / 2019$ & 1486 & 4079710976 & 711859 & 1486 & 4059607040 & 651455 & 1486 & 4314759168 & 768878 \\
\hline 1401 & $10 / 13 / 2019$ & 1486 & 4080440064 & 729058 & 1486 & 4060275712 & 668904 & 1486 & 4315543040 & 784078 \\
\hline 1402 & $10 / 14 / 2019$ & 1486 & 4081185792 & 745750 & 1486 & 4060961536 & 685611 & 1486 & 4316343296 & 800188 \\
\hline 1403 & $10 / 15 / 2019$ & 1486 & 4081947136 & 761296 & 1486 & 4061663232 & 701773 & 1486 & 4317160960 & 817367 \\
\hline 1404 & $10 / 16 / 2019$ & 1486 & 4082724096 & 776870 & 1486 & 4062381056 & 717938 & 1486 & 4317995008 & 834231 \\
\hline 1405 & $10 / 17 / 2019$ & 1486 & 4083515648 & 791633 & 1486 & 4063114752 & 733629 & 1486 & 4318844416 & 849470 \\
\hline 1406 & $10 / 18 / 2019$ & 1486 & 4084321792 & 806238 & 1486 & 4063863808 & 749085 & 1486 & 4319708160 & 863659 \\
\hline 1407 & $10 / 19 / 2019$ & 1486 & 4085142272 & 820520 & 1486 & 4064630528 & 766731 & 1486 & 4320585728 & 877732 \\
\hline 1408 & $10 / 20 / 2019$ & 1486 & 4085976320 & 834183 & 1486 & 4065414144 & 783567 & 1486 & 4321477120 & 891209 \\
\hline 1409 & $10 / 21 / 2019$ & 1486 & 4086824192 & 847668 & 1486 & 4066213632 & 799103 & 1486 & 4322381824 & 904550 \\
\hline 1410 & $10 / 22 / 2019$ & 1486 & 4087684608 & 860415 & 1486 & 4067028736 & 815524 & 1486 & 4323300352 & 918763 \\
\hline 1411 & $10 / 23 / 2019$ & 1486 & 4088558080 & 873498 & 1486 & 4067859712 & 830992 & 1486 & 4324234240 & 933608 \\
\hline 1412 & $10 / 24 / 2019$ & 1486 & 4089444352 & 886226 & 1486 & 4068706048 & 846305 & 1486 & 4325181440 & 947245 \\
\hline 1413 & $10 / 25 / 2019$ & 1486 & 4090342656 & 898248 & 1486 & 4069567488 & 861430 & 1486 & 4326141440 & 960180 \\
\hline 1414 & $10 / 26 / 2019$ & 1486 & 4091252480 & 909922 & 1486 & 4070443008 & 875344 & 1486 & 4327113728 & 972522 \\
\hline 1415 & $10 / 27 / 2019$ & 1486 & 4092173824 & 921194 & 1486 & 4071331584 & 888631 & 1486 & 4328099328 & 985133 \\
\hline 1416 & $10 / 28 / 2019$ & 1486 & 4093105920 & 932116 & 1486 & 4072233216 & 901795 & 1486 & 4329095168 & 995947 \\
\hline 1417 & $10 / 29 / 2019$ & 1486 & 4094048512 & 942716 & 1486 & 4073148160 & 914937 & 1486 & 4330102272 & 1007093 \\
\hline 1418 & $10 / 30 / 2019$ & 1486 & 4095001856 & 953268 & 1486 & 4074075648 & 927457 & 1486 & 4331120640 & 1018694 \\
\hline 1419 & $10 / 31 / 2019$ & 1486 & 4095965696 & 963786 & 1486 & 4075015168 & 939326 & 1486 & 4332151296 & 1030533 \\
\hline 1420 & $11 / 1 / 2019$ & 1486 & 4096939776 & 974064 & 1486 & 4075966464 & 951331 & 1486 & 4333192704 & 1041457 \\
\hline 1421 & $11 / 2 / 2019$ & 1486 & 4097923584 & 983837 & 1486 & 4076929792 & 963486 & 1486 & 4334244864 & 1052109 \\
\hline 1422 & $11 / 3 / 2019$ & 1486 & 4098917888 & 994244 & 1486 & 4077902848 & 972990 & 1486 & 4335307264 & 1062477 \\
\hline 1423 & $11 / 4 / 2019$ & 1486 & 4099920640 & 1002766 & 1486 & 4078886400 & 983448 & 1486 & 4336379904 & 1072360 \\
\hline 1424 & $11 / 5 / 2019$ & 1486 & 4100933120 & 1012606 & 1486 & 4079880192 & 994006 & 1486 & 4337462272 & 1082695 \\
\hline 1425 & $11 / 6 / 2019$ & 1486 & 4101954048 & 1020695 & 1486 & 4080885248 & 1004994 & 1486 & 4338554368 & 1092103 \\
\hline 1426 & $11 / 7 / 2019$ & 1486 & 4102983168 & 1029266 & 1486 & 4081899520 & 1014214 & 1486 & 4339656192 & 1101569 \\
\hline 1427 & $11 / 8 / 2019$ & 1486 & 4104020736 & 1037616 & 1486 & 4082923264 & 1023772 & 1486 & 4340766720 & 1110704 \\
\hline 1428 & $11 / 9 / 2019$ & 1486 & 4105066496 & 1045736 & 1486 & 4083956480 & 1033124 & 1486 & 4341885952 & 1119295 \\
\hline 1429 & $11 / 10 / 2019$ & 1486 & 4106120192 & 1053655 & 1486 & 4084998656 & 1042272 & 1486 & 4343013888 & 1127781 \\
\hline 1430 & $11 / 11 / 2019$ & 1486 & 4107181568 & 1061362 & 1486 & 4086049792 & 1051048 & 1486 & 4344150016 & 1136038 \\
\hline 1431 & $11 / 12 / 2019$ & 1486 & 4108250368 & 1068886 & 1486 & 4087109376 & 1059628 & 1486 & 4345293824 & 1143912 \\
\hline 1432 & $11 / 13 / 2019$ & 1486 & 4109327616 & 1077172 & 1486 & 4088177408 & 1068100 & 1486 & 4346445312 & 1151677 \\
\hline 1433 & $11 / 14 / 2019$ & 1486 & 4110411264 & 1083635 & 1486 & 4089254400 & 1077039 & 1486 & 4347604480 & 1159149 \\
\hline 1434 & $11 / 15 / 2019$ & 1486 & 4111501824 & 1090664 & 1486 & 4090338560 & 1084186 & 1486 & 4348771328 & 1166509 \\
\hline
\end{tabular}

